\title{
A Probabilistic Assessment of Health Risks Associated with Short-Term Exposure to Tropospheric Ozone
}

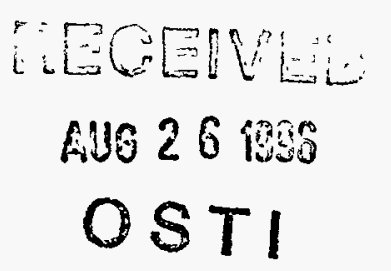

DISTRIBUTION OF THS DOCUAENT IS UNLAMTEO

Decision and Information

Sciences Division

MASTER

Argonne National Laboratory

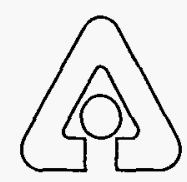

Operated by The University of Chicago, under Contract W-31-109-Eng-38, for the

United States Department of Energy 


\section{Argonne National Laboratory}

Argonne National Laboratory, with facilities in the states of Illinois and Idaho, is owned by the United States Government, and operated by the University of Chicago under the provisions of a contract with the Department of Energy.

This technical report is a product of Argonne's Decision and Information Sciences Division. For information on the division's scientific and engineering activities, contact:

Director, Decision and information

Sciences Division

Argonne National Laboratory

Argonne, Illinois 60439-4815

Telephone (708) 252-5464

http://www.dis.anl.gov

Publishing support services were provided by Argonne's Information and Publishing Division (for more information, see IPD's home page: http://www.ipd.anl.gov/).

\section{Disclaimer}

This report was prepared as an account of work sponsored by an agency of the United States Government. Neither the United States Government nor any agency thereof, nor any of their employees, makes any warranty, express or implied, or assumes any legal liability or responsibility for the accuracy, completeness, or usefulness of any information, apparatus, product, or process disclosed, or represents that its use would not infringe privately owned rights. Reference herein to any specific commercial product, process, or service by trade name, trademark, manufacturer, or otherwise, does not necessarily constitute or imply its endorsement, recommendation, or favoring by the United States Government or any agency thereof. The views and opinions of authors expressed herein do not necessarily state or reflect those of the United States Government or any agency thereof.

Reproduced directly from the best available copy.

Available to DOE and DOE contractors from the Office of Scientific and Technical Information, P.O. Box 62, Oak Ridge, TN 37831; prices available from (423) 576-8401.

Available to the public from the National Technical Information Service, U.S. Department of Commerce, 5285 Port Royal Road, Springfield, VA 22161.

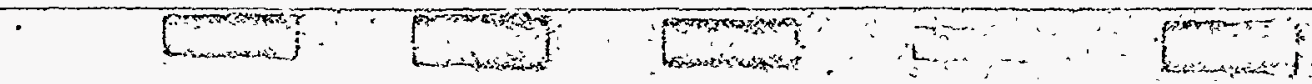




\section{A Probabilistic Assessment of Health Risks Associated with Short-Term Exposure to Tropospheric Ozone}

by R.G. Whitfield, W.F. Biller, ${ }^{*}$ M.J. Jusko, and J.M. Keisler

Decision and Information Sciences Division,

Argonne National Laboratory, 9700 South Cass Avenue, Argonne, Illinois 60439

June 1996

Work funded by the U.S. Environmental Protection Agency under Interagency Agreement DW89935085-01-3 with the U.S. Department of Energy

*Affiliated with William F. Biller Consultants, East Brunswick, New Jersey. 


\section{DISCLATMER}

This work was funded through Interagency Agreement DW89935085-01-3 between the U.S. Department of Energy and the U.S. Environmental Protection Agency. William F. Biller's contribution to this effort was supported under U.S. Environmental Protection Agency contract number 68D30092. Any opinions, findings, conclusions, or recommendations are those of the authors and do not necessarily reflect the views of reviewers, people named in the acknowledgments, the U.S. Environmental Protection Agency, the U.S. Department of Energy, or Argonne National Laboratory. Comments on the report should be addressed to Harvey Richmond, U.S. Environmental Protection Agency, Office of Air Quality Planning and Standards, MD-15, Research Triangle Park, NC 27711.

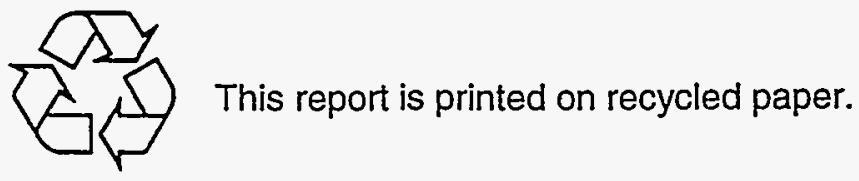




\section{DISCLAIMER}

Portions of this document may be illegible in electronic image products. Images are produced from the best available original document. 


\section{CONTENTS}

DISCLAIMER $\ldots \ldots \ldots \ldots \ldots \ldots \ldots \ldots \ldots \ldots \ldots \ldots \ldots \ldots \ldots \ldots \ldots \ldots \ldots$ ii

ACKNOWLEDGMENTS $\ldots \ldots \ldots \ldots \ldots \ldots \ldots \ldots \ldots \ldots \ldots \ldots \ldots \ldots \ldots \ldots \ldots \ldots$

NOTATION $\ldots \ldots \ldots \ldots \ldots \ldots \ldots \ldots \ldots \ldots \ldots \ldots \ldots \ldots \ldots \ldots \ldots \ldots \ldots \ldots$

ABSTRACT $\ldots \ldots \ldots \ldots \ldots \ldots \ldots \ldots \ldots \ldots \ldots \ldots \ldots \ldots \ldots \ldots \ldots \ldots \ldots \ldots \ldots$

1 INTRODUCTION $\ldots \ldots \ldots \ldots \ldots \ldots \ldots \ldots \ldots \ldots \ldots \ldots \ldots \ldots \ldots \ldots \ldots \ldots$

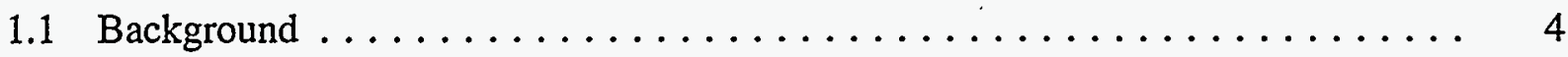

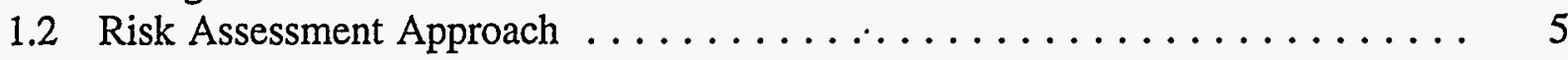

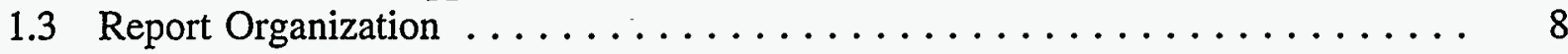

2 EXPOSURE MODEL $\ldots \ldots \ldots \ldots \ldots \ldots \ldots \ldots \ldots \ldots \ldots \ldots \ldots \ldots \ldots \ldots$

2.1 Alternative Air Quality Scenarios Included in the Analysis . . . . . . . . . 9

2.2 Exposure Modeling Methodology ..................... 9

2.2.1 Define the Study Area, Subdivisions of the Study Area, the Exposure Period, and the Population of Interest . . . . . . . 11

2.2.2 Divide the Population of Interest into an Exhaustive Set of Cohorts ........................ 12

2.2.3 Develop an Exposure Event Sequence for Each Cohort

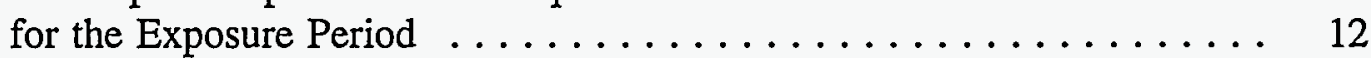

2.2.4 Estimate the Pollutant Concentration and Ventilation Rate Associated with Each Exposure Event ............... 14

2.2.5 Extrapolate the Cohort Exposures to the Population of Interest

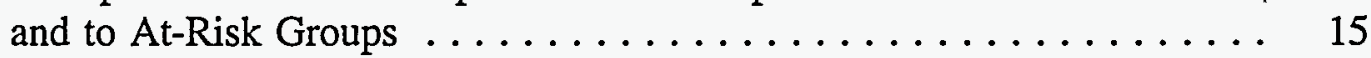

2.3 Example of the Exposure Estimates Used in the Risk Assessment . . . . . . 19

3 EXPOSURE-RESPONSE DATA AND RELATIONSHIPS $\ldots \ldots \ldots \ldots \ldots \ldots 22$

3.1 Criteria for Selecting Laboratory Data $\ldots \ldots \ldots \ldots \ldots \ldots \ldots \ldots \ldots \ldots \ldots$

3.2 Discussion of 1-Hour Data for Populations Engaged

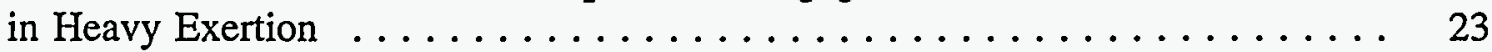

3.3 Discussion of 1-Hour Data for Populations Engaged

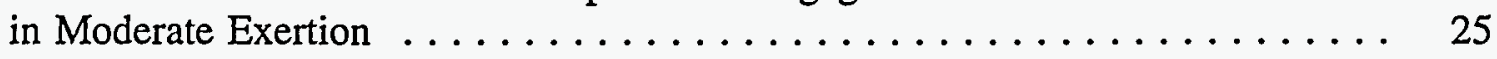

3.4 Discussion of 8-Hour Data for Populations Engaged in Moderate Exertion . ...................... 27

3.5 Exposure-Response Relationships for Acute Endpoints . . . . . . . . . . . . 29 


\section{CONTENTS (Cont.)}

4 HEADCOUNT RISK MODELS AND ILLUSTRATIVE RESULTS $\ldots \ldots \ldots$. . . . . 36

4.1 Calculation of Headcount Risk . . . . . . . . . . . . . 36

4.2 Headcount Risk Distributions for $10 \mathrm{pNEM} / \mathrm{O}_{3}$ Runs

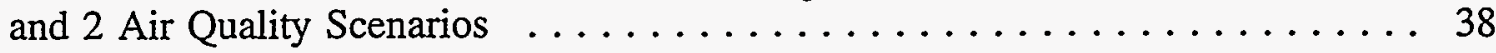

4.3 Summary of Risk Distributions for Nine Urban Areas and Nine Air Quality Scenarios . . . . . . . . . . . . . . . . 41

4.4 Significance Tests ... . . . . . . . . . . . . . . . . . 42

4.5 Ratios of Median Numbers of Both Occurrences and Responders . . . . . . . . 47

4.6 Aggregate Results for Nine Urban Areas . . . . . . . . . . . . . . . . . . . 49

4.7 Analysis Addressing Multiyear Forms of Ozone 8-Hour NAAQS . . . . . . . . . 52

5 BENCHMARK RISK MODEL AND SELECTED RESULTS . . . . . . . . . . . 56

5.1 Equation for Benchmark Risk ... . . . . . . . . . . . . 56

5.2 Graphic Format for Displaying Benchmark Risk Results . . . . . . . . . . . 57

6 MODEL FOR EXCESS HOSPITAL ADMISSIONS $\ldots \ldots \ldots \ldots \ldots \ldots$

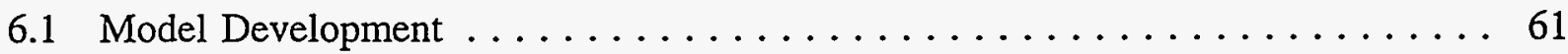

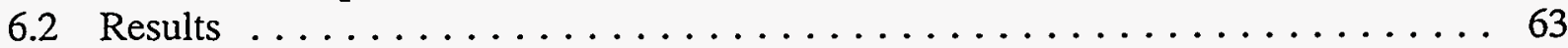

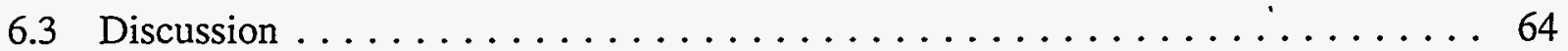

7 LIMITATIONS OF THIS RISK ASSESSMENT . . . . . . . . . . . 73

7.1 Issues Pertaining to Exposure Modeling $\ldots \ldots \ldots \ldots \ldots \ldots \ldots \ldots$

7.2 Issues Pertaining to Exposure-Response Modeling . . . . . . . . . . . 74

7.3 Issues Pertaining to Risk Modeling $\ldots \ldots \ldots \ldots \ldots \ldots \ldots$

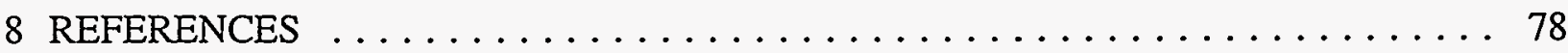

APPENDIX A: Modeling Techniques ................... 83

APPENDIX B: Probabilistic Exposure-Response Relationships

for Acute Endpoints . . . . . . . . . . . . . . . 97

APPENDIX C: Headcount Risk Results for Acute Endpoints . . . . . . . . . . . . 119

APPENDIX D: Benchmark Risk Results $\ldots \ldots \ldots \ldots \ldots \ldots \ldots \ldots$ 


\section{TABLES}

1 Characteristics of Alternative Air Quality Scenarios $\ldots \ldots \ldots \ldots \ldots$

2 Ozone Seasons for the Nine Urban Areas $\ldots \ldots \ldots \ldots \ldots \ldots \ldots \ldots$

3 Ozone Concentration Intervals for Which $\mathrm{pNEM} / \mathrm{O}_{3}$

Data Have Been Generated ....................... 20

$4 \quad$ Ozone Concentrations for Which Response Rates Need

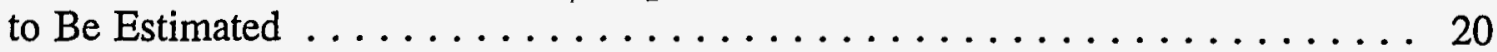

$5 \quad$ Calculating Exposure Probabilities for Outdoor Children Exposed

for Eight Hours at Moderate Exertion from $\mathrm{pNEM} / \mathrm{O}_{3}$ Estimates

for Run 2, Philadelphia, Scenario 1112, Just Attained . . . . . . . . . . 21

6 Health Endpoints Associated with Each Study $\ldots \ldots \ldots \ldots \ldots \ldots$

$7 \quad$ Summary of the Studies Used in Developing 1-h Exposure-Response

Relationships for Populations Engaged in Heavy Exertion ............ 24

8 Summary of the Study Used to Develop 1-h Exposure-Response

Relationships for Populations Engaged in Moderate Exertion . . . . . . . . 26

9 Summary of the Studies Used to Develop 8-h

Exposure-Response Relationships . . . . . . . . . . . . . . . . 27

10 Summary of Experimental Data - Forced Expiratory Volume -

Corrected for Exercise in Clean Air .................... 30

11 Summary of Experimental Data - Symptoms - Corrected

for Exercise in Clean Air $\ldots \ldots \ldots \ldots \ldots \ldots \ldots \ldots \ldots \ldots \ldots \ldots \ldots \ldots$

12 Summary of Functions Fit to Experimental Data - $\mathrm{FEV}_{1}$ Decrement -

Corrected for Exercise in Clean Air . . . . . . . . . . . . . . . . 34

13 Summary of Functions Fit to Experimental Data - Symptoms -

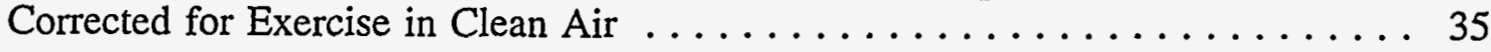

14 Calculating the Median of a Risk Distribution $\ldots \ldots \ldots \ldots \ldots \ldots \ldots$

15 Probability Distributions over the Number of Outdoor Children Having

$\mathrm{FEV}_{1}$ Decrements $\geq 20 \%$ during One Ozone Season, Philadelphia,

8-Hour Exposure, Moderate Exertion, Just Attaining Standard 1112,

Corrected for Background Ozone . . . . . . . . . . . . . . . . . . 39 


\section{TABLES (Cont.)}

16 Definitions of Letter Codes for Air Quality Scenarios ............. 45

17 Summary of KS Tests for Significance $\ldots \ldots \ldots \ldots \ldots \ldots \ldots \ldots \ldots$

18 Details about Scenarios That Are Not Significantly Different .......... 48

19 Summary of Benchmark Risk Results: Number of Urban Areas

Having Benchmark Risks $\leq 0.1,0.2$, and 0.3 for As-Is Air Quality

and Just Attaining Eight Alternative NAAQS for 5 and 10\% Benchmarks . . . . 60

20 Regression Coefficients and Standard Errors for the Hospital

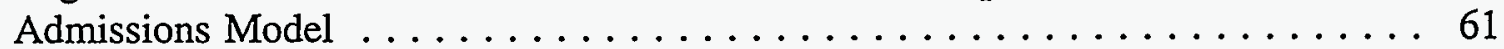

21 Monitor Site Numbers, Names, and Reasons for Inclusion

in the Hospital Admissions Model . . . . . . . . . . . . . . . 62

22 Annual Excess Hospital Admissions of Asthmatics for Monitor 9 Data . . . . . 65

23 Admissions of New York City Asthmatics - Comparison Relative

to Meeting the Current Standard $\ldots \ldots \ldots \ldots \ldots \ldots \ldots \ldots \ldots \ldots \ldots$

A.1 $\mathrm{FEV}_{1}$ Data from McDonnell et al. (1991) - Subject $12 \ldots \ldots \ldots \ldots$

A.2 Details about Probabilistic Exposure-Response Relationships

for Acute Health Endpoints for Headcount Risk Estimates . . . . . . . . . 92

D.1 Summary of Benchmark Risk Results: Number of Urban Areas Having

Benchmark Risks $\leq 0.1,0.2$, and 0.3 for As-Is Air Quality and Just

Attaining Eight Alternative NAAQS for 5 and 10\% Benchmarks . . . . . . . 196

\section{FIGURES}

$1 \quad$ Basic Risk Assessment Approach: Risk Model Incorporating Both an Exposure Model and a Health Model $\ldots \ldots \ldots \ldots \ldots \ldots \ldots \ldots$

2 Relationship between Benchmark and Headcount Risk Models $\ldots \ldots \ldots \ldots 7$

3 Major Components of the NEM Series of Exposure Models and Associated Health Risk Assessment Procedures .............. 10

$4 \quad$ Steps Used to Develop Probabilistic Exposure-Response Relationships for $\mathrm{FEV}_{1}$ Decrement $\geq 20 \%$, 6.6-h Exposure, Moderate Exertion ....... 32 


\section{FIGURES (Cont.)}

5 Comparison of 2 Sets of 10 Risk Distributions $\ldots \ldots \ldots \ldots \ldots \ldots$

6 Representative Distributions for 9 Sets of 10 Risk Distributions ......... 41

$7 \quad$ Using Rectangles to Represent Variability in 1 Set of 10 Risk

Distributions Attributable to Differences in $\mathrm{pNEM} / \mathrm{O}_{3}$ Exposure Estimates . . . . 43

8 Using Rectangles to Represent Variability in 81 Sets of 10 Risk

Distributions Attributable to Differences in $\mathrm{pNEM} / \mathrm{O}_{3}$ Exposure Estimates . . . . 44

9 Patterns of Rectangles That Appear in Summary Figures

for Headcount Risk Distributions . . . . . . . . . . . . . . . . 45

10 Ratios of Median Numbers of Occurrences and Responders Experiencing

Moderate-to-Severe Pain on Deep Inspiration, for Nine Urban Areas,

Outdoor Children, 1-h Exposures, and Moderate Exertion ........... 50

11 Ratios of Median Numbers of Occurrences and Responders

for $\mathrm{FEV}_{1}$ Decrements $\geq 15 \%$, Nine Urban Areas, Outdoor

Children, 8-h Exposures, and Moderate Exertion ............. 50

12 Ratios of Median Numbers of Occurrences and Responders

for $\mathrm{FEV}_{1}$ Decrements $\geq 20 \%$, Nine Urban Areas, Outdoor

Children, 8-h Exposures, and Moderate Exertion .............. 51

13 Aggregate Risk Results across Nine Urban Areas for Selected Health

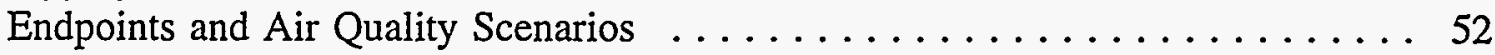

14 Risk Results for the MPD Endpoint and a Tenth-Highest, 1-h Daily

Maximum Air Quality Scenario ...................... 54

15 Risk Results for the $\mathrm{FEV}_{1}$ Decrement $\geq 15 \%$ Endpoint and a Tenth-Highest,

8-h Daily Maximum Air Quality Scenario . . . . . . . . . . . . . 54

16 Risk Results for the $\mathrm{FEV}_{1}$ Decrement $\geq 20 \%$ Endpoint and a Tenth-Highest,

8-h Daily Maximum Air Quality Scenario . . . . . . . . . . . . 55

17 Probability That the Benchmark Response for the 8-h Exposure, Moderate Exertion, FEV 1 Decrement $\geq 20 \%$ Endpoint Will Be Exceeded

Five or More Times in an Ozone Season $\ldots \ldots \ldots \ldots \ldots \ldots \ldots$

18 Uncertainty about Daily Hospital Admissions of Asthmatics

in Relation to Ozone Concentration $\ldots \ldots \ldots \ldots \ldots \ldots \ldots \ldots \ldots \ldots \ldots$ 


\section{FIGURES (Cont.)}

19 Excess Annual Hospitalizations of New York City Asthmatics for Nine Air Quality Scenarios and Monitor 9 Air Quality Data

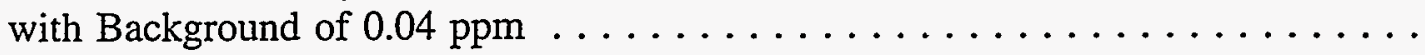

20 Excess Annual Hospitalizations of New York City Asthmatics for Nine Air Quality Scenarios and Monitor 1 Air Quality Data

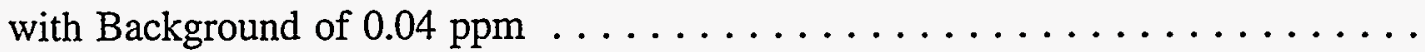

21 Excess Annual Hospitalizations of New York City Asthmatics for Nine Air Quality Scenarios and Monitor 11 Air Quality Data

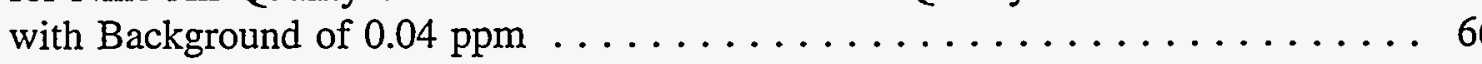

22 Excess Annual Hospitalizations of New York City Asthmatics for Nine Air Quality Scenarios and Monitor 12 Air Quality Data with Background of $0.04 \mathrm{ppm} \ldots \ldots \ldots \ldots \ldots \ldots \ldots$

23 Excess Annual Hospitalizations of New York City Respiratory Patients for Nine Air Quality Scenarios and Monitor 1 Air Quality Data with Background of $0.04 \mathrm{ppm} \ldots \ldots \ldots \ldots \ldots \ldots \ldots$

24 Excess Annual Hospitalizations of New York City Respiratory Patients for Nine Air Quality Scenarios and Monitor 9 Air Quality Data with Background of $0.04 \mathrm{ppm} \ldots \ldots \ldots \ldots \ldots \ldots$

25 Excess Annual Hospitalizations of New York City Respiratory Patients for Nine Air Quality Scenarios and Monitor 11 Air Quality Data with Background of $0.04 \mathrm{ppm} \ldots \ldots \ldots \ldots \ldots \ldots \ldots$

26 Excess Annual Hospitalizations of New York City Respiratory Patients for Nine Air Quality Scenarios and Monitor 12 Air Quality Data

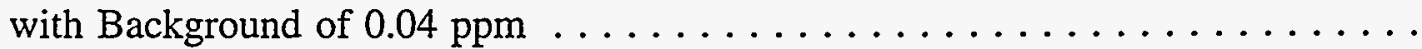

27 Median and 90\% Credible Interval Estimates of Excess Annual Hospitalizations of New York City Asthmatics for Nine Air Quality Scenarios and Air Quality Data for All Monitors ................

28 Median and 90\% Credible Interval Estimates of Excess Annual Hospitalizations of Respiratory Patients for Nine Air Quality Scenarios and Air Quality Data for All Monitors ...............

B.1 Probabilistic Exposure-Response Relationship for FEV 1 Decrement $\geq 10 \%$ for 1-h Exposures, Heavy Exertion; Includes Data, Medians, and 90\% CIs for Data and Relationship (Source: derived from Avol et al. 1984) . . . . . . . . . 


\section{FIGURES (Cont.)}

B.2 Probabilistic Exposure-Response Relationship for $\mathrm{FEV}_{1}$ Decrement $\geq 15 \%$ for 1-h Exposures, Heavy Exertion; Includes Data, Medians, and 90\% CIs for Data and Relationship (Source: derived from Avol et al. 1984) . . . . . . 100

B.3 Probabilistic Exposure-Response Relationship for $\mathrm{FEV}_{1}$ Decrement $\geq 20 \%$ for 1-h Exposures, Heavy Exertion; Includes Data, Medians, and 90\% CIs for Data and Relationship (Source: derived from Avol et al. 1984) . . . . . . 101

B.4 Probabilistic Exposure-Response Relationship for Any Lower Respiratory Symptoms for 1-h Exposures, Heavy Exertion; Includes Data, Medians, and 90\% CIs for Data and Relationship (Source: derived from Avol et al. 1984) . . 101

B.5 Probabilistic Exposure-Response Relationship for Moderate-to-Severe Lower Respiratory Symptoms for 1-h Exposures, Heavy Exertion; Includes Data, Medians, and 90\% CIs for Data and Relationship (Source: derived from Avol et al. 1984) . . . . . . . . . . . . . . . . . . . . 102

B.6 Probabilistic Exposure-Response Relationship for $\mathrm{FEV}_{1}$ Decrement $\geq 10 \%$ for 1-h Exposures, Heavy Exertion; Includes Data, Medians, and 90\% Cls for Data and Relationship (Source: derived from Kulle et al. 1984) . . . . . 102

B.7 Probabilistic Exposure-Response Relationship for $\mathrm{FEV}_{1}$ Decrement $\geq 15 \%$ for 1-h Exposures, Heavy Exertion; Includes Data, Medians, and $90 \%$ CIs for Data and Relationship (Source: derived from Kulle et al. 1984) . . . . . 103

B.8 Probabilistic Exposure-Response Relationship for FEV 1 Decrement $\geq 20 \%$ for 1-h Exposures, Heavy Exertion; Includes Data, Medians, and 90\% CIs for Data and Relationship (Source: derived from Kulle et al. 1984) . . . . . . 103

B.9 Probabilistic Exposure-Response Relationship for Any Cough for 1-h Exposures, Heavy Exertion; Includes Data, Medians, and $90 \%$ CIs for Data and Relationship (Source: derived from Kulle et al. 1984) . . . . . . . . . . . . 104

B.10 Probabilistic Exposure-Response Relationship for Moderate-to-Severe Cough for 1-h Exposures, Heavy Exertion; Includes Data, Medians, and 90\% CIs for Data and Relationship (Source: derived from Kulle et al. 1984) . . . . . . 104

B.11 Probabilistic Exposure-Response Relationship for Any Pain on Deep Inspiration for 1-h Exposures, Heavy Exertion; Includes Data, Medians, and 90\% CIs for Data and Relationship (Source: derived from Kulle et al. 1984) . . . . . . 105 


\section{FIGURES (Cont.)}

B.12 Probabilistic Exposure-Response Relationship for Moderate-to-Severe Pain on Deep Inspiration for 1-h Exposures, Heavy Exertion; Includes Data, Medians, and 90\% CIs for Data and Relationship (Source: derived from Kulle et al. 1984) . . . . . . . . . . . . . . . . . . . 105

B.13 Probabilistic Exposure-Response Relationship for $\mathrm{FEV}_{1}$ Decrement $\geq 10 \%$ for 1-h Exposures, Heavy Exertion; Includes Data, Medians, and 90\% CIs for Data and Relationship (Source: derived from McDonnell et al. 1983) . . . . 106

B.14 Probabilistic Exposure-Response Relationship for $\mathrm{FEV}_{1}$ Decrement $\geq 15 \%$ for 1-h Exposures, Heavy Exertion; Includes Data, Medians, and 90\% CIs for Data and Relationship (Source: derived from McDonnell et al. 1983) . . . . 106

B.15 Probabilistic Exposure-Response Relationship for $\mathrm{FEV}_{1}$ Decrement $\geq 20 \%$ for 1-h Exposures, Heavy Exertion; Includes Data, Medians, and 90\% CIs for Data and Relationship (Source: derived from McDonnell et al. 1983) . . . . 107

B.16 Probabilistic Exposure-Response Relationship for Any Cough for 1-h Exposures, Heavy Exertion; Includes Data, Medians, and 90\% CIs for Data and Relationship (Source: derived from McDonnell et al. 1983) . . . . . . . . . . 107

B.17 Probabilistic Exposure-Response Relationship for Moderate-to-Severe Cough for 1-h Exposures, Heavy Exertion; Includes Data, Medians, and 90\% CIs for Data and Relationship (Source: derived from McDonnell et al. 1983) . . . 108

B.18 Probabilistic Exposure-Response Relationship for Any Pain on Deep Inspiration for 1-h Exposures, Heavy Exertion; Includes Data, Medians, and 90\% CIs for Data and Relationship (Source: derived from McDonnell et al. 1983) . . 108

B.19 Probabilistic Exposure-Response Relationship for Moderate-to-Severe Pain on Deep Inspiration for 1-h Exposures, Heavy Exertion; Includes Data, Medians, and 90\% CIs for Data and Relationship (Source: derived from McDonnell et al. 1983) . . . . . . . . . . . . . . . . . . . . . 109

B.20 Probabilistic Exposure-Response Relationship for $\mathrm{FEV}_{1}$ Decrement $\geq 10 \%$ for 1-h Exposures and Moderate Exertion; Includes Data, Medians, and $90 \%$ CIs for Data and Relationship (Source: derived from Seal et al. 1993) . . . . . 109

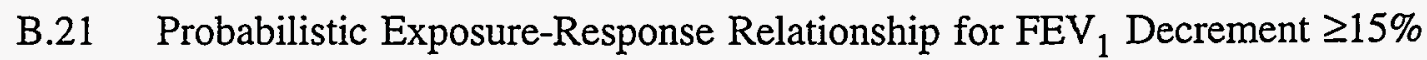
for 1-h Exposures and Moderate Exertion; Includes Data, Medians, and $90 \%$ CIs for Data and Relationship (Source: derived from Seal et al. 1993) . . . . . 110 


\section{FIGURES (Cont.)}

B.22 Probabilistic Exposure-Response Relationship for $\mathrm{FEV}_{1}$ Decrement $\geq 20 \%$. for 1-h Exposures and Moderate Exertion; Includes Data, Medians, and $90 \%$ CIs for Data and Relationship (Source: derived from Seal et al. 1993) . . . . . 110

B.23 Probabilistic Exposure-Response Relátionship for Any Cough for 1-h Exposures and Moderate Exertion; Includes Data, Medians, and 90\% CIs for Data and Relationship (Source: derived from Seal et al. 1993) . . . . . . . . . . 111

B.24 Probabilistic Exposure-Response Relationship for Moderate-to-Severe Cough for 1-h Exposures and Moderate Exertion; Includes Data, Medians, and $90 \%$ CIs for Data and Relationship (Source: derived from Seal et al. 1993) . . . . . . . . . . . . . . . . . . . . . 111

B.25 Probabilistic Exposure-Response Relationship for Any Pain on Deep Inspiration for 1-h Exposures and Moderate Exertion; Includes Data, Medians, and $90 \%$ CIs for Data and Relationship (Source: derived from Seal et al. 1993) . . . . . . . . . . . . . . . . . . . . . . . . 112

B.26 Probabilistic Exposure-Response Relationship for Moderate-to-Severe Pain on Deep Inspiration for 1-h Exposures and Moderate Exertion; Includes Data, Medians, and $90 \%$ CIs for Data and Relationship (Source: derived from Seal et al. 1993) . . . . . . . . . . . . . . . . . . . . . . . 112

B.27 Probabilistic Exposure-Response Relationship for $\mathrm{FEV}_{1}$ Decrement $\geq 10 \%$ for 8-h Exposures and Moderate Exertion; Includes Data, Medians, and 90\% CIs for Data and Relationship (Sources: derived from Folinsbee et al. 1988,

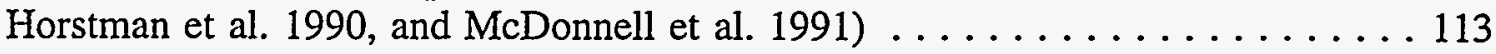

B.28 Probabilistic Exposure-Response Relationship for $\mathrm{FEV}_{1}$ Decrement $\geq 15 \%$ for 8-h Exposures and Moderate Exertion; Includes Data, Medians, and 90\% CIs for Data and Relationship (Sources: derived from Folinsbee et al. 1988,

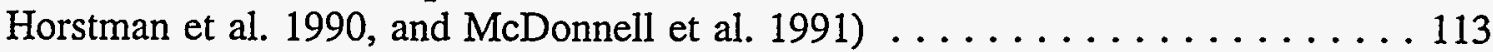

B.29 Probabilistic Exposure-Response Relationship for $\mathrm{FEV}_{1}$ Decrement $\geq 20 \%$ for 8-h Exposures and Moderate Exertion; Includes Data, Medians, and $90 \%$ CIs for Data and Relationship (Sources: derived from Folinsbee et al. 1988,

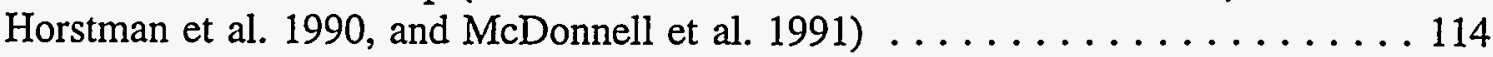

B.30 Probabilistic Exposure-Response Relationship for Any Cough for 8-h Exposures and Moderate Exertion; Includes Data, Medians, and 90\% CIs for Data and Relationship (Sources: derived from Folinsbee et al. 1988, Horstman et al. 1990, and McDonnell et al. 1991) . . . . . . . . . . 114 


\section{FIGURES (Cont.)}

B.31 Probabilistic Exposure-Response Relationship for Moderate-to-Severe Cough for 8-h Exposures and Moderate Exertion; Includes Data, Medians, and 90\% CIs for Data and Relationship (Sources: derived from Folinsbee et al. 1988, Horstman et al. 1990, and McDonnell et al. 1991) . . . . . . . . . . . . 115

B.32 Probabilistic Exposure-Response Relationship for Any Pain on Deep Inspiration for 8-h Exposures and Moderate Exertion; Includes Data, Medians, and 90\% CIs for Data and Relationship (Sources: derived from Folinsbee et al. 1988, Horstman et al. 1990, and McDonnell et al. 1991) . . . . . . . . . . . . 115

B.33 Probabilistic Exposure-Response Relationship for Moderate-to-Severe Pain on Deep Inspiration for 8-h Exposures and Moderate Exertion; Includes Data, Medians, and 90\% CIs for Data and Relationship (Sources: derived from Folinsbee et al. 1988, Horstman et al. 1990, and McDonnell et al. 1991) . . . . 116

C.1 Representative Risk Distributions for Alternative Air Quality Scenarios for FEV Decrements $\geq 15 \%$, Chicago, Outdoor Children, 8-h Exposures, and Moderate Exertion (underlying exposure-response relationship based on Folinsbee et al. 1988, Horstman et al. 1990, and McDonnell et al. 1991) . . . . . . . . . . . . 124

C.2 Representative Risk Distributions for Alternative Air Quality Scenarios for FEV Decrements $\geq 15 \%$, Denver, Outdoor Children, 8-h Exposures, and Moderate Exertion (underlying exposure-response relationship based on Folinsbee et al. 1988, Horstman et al. 1990, and McDonnell et al. 1991) . . . . . . . . . . . . 125

C.3 Representative Risk Distributions for Alternative Air Quality Scenarios for FEV Decrements $\geq 15 \%$, Houston, Outdoor Children, 8-h Exposures, and Moderate Exertion (underlying exposure-response relationship based on Folinsbee et al. 1988, Horstman et al. 1990, and McDonnell et al. 1991) . . . . . . . . . . . . 126

C.4 Representative Risk Distributions for Alternative Air Quality Scenarios for FEV Decrements $\geq 15 \%$, Los Angeles, Outdoor Children, 8-h Exposures, and Moderate Exertion (underlying exposure-response relationship based on Folinsbee et al. 1988, Horstman et al. 1990, and McDonnell et al. 1991) . . . . . . . . . . . . 127

C.5 Representative Risk Distributions for Alternative Air Quality Scenarios for FEV Decrements $\geq 15 \%$, Miami, Outdoor Children, 8-h Exposures, and Moderate Exertion (underlying exposure-response relationship based on Folinsbee et al. 1988, Horstman et al. 1990, and McDonnell et al. 1991) . . . . . . . . . . . . 128

C.6 Representative Risk Distributions for Alternative Air Quality Scenarios for FEV 1 Decrements $\geq 15 \%$, New York, Outdoor Children, 8-h Exposures, and Moderate Exertion (underlying exposure-response relationship based on Folinsbee et al. 1988, Horstman et al. 1990, and McDonnell et al. 1991) . . . . . . . . . . . . . . 129 


\section{FIGURES (Cont.)}

C.7 Representative Risk Distributions for Alternative Air Quality Scenarios for FEV Decrements $\geq 15 \%$, Philadelphia, Outdoor Children, 8-h Exposures, and Moderate Exertion (underlying exposure-response relationship based on Folinsbee et al. 1988, Horstman et al. 1990, and McDonnell et al. 1991) . . . . . . . . . . 130

C.8 Representative Risk Distributions for Alternative Air Quality Scenarios for FEV 1 Decrements $\geq 15 \%$, St. Louis, Outdoor Children, 8-h Exposures, and Moderate Exertion (underlying exposure-response relationship based on Folinsbee et al. 1988, Horstman et al. 1990, and McDonnell et al. 1991) . . . . . . . . . 131

C.9 Representative Risk Distributions for Alternative Air Quality Scenarios for FEV 1 Decrements $\geq 15 \%$, Washington DC, Outdoor Children, 8-h Exposures, and Moderate Exertion (underlying exposure-response relationship based on Folinsbee et al. 1988, Horstman et al. 1990, and McDonnell et al. 1991) . . . 132

C.10 Representative Risk Distributions for Alternative Air Quality Scenarios for FEV Decrements $\geq 20 \%$, Chicago, Outdoor Children, 8-h Exposures, and Moderate Exertion (underlying exposure-response relationship based on Folinsbee et al. 1988, Horstman et al. 1990, and McDonnell et al. 1991) . . . . . . . . . . 133

C.11 Representative Risk Distributions for Alternative Air Quality Scenarios for FEV Decrements $\geq 20 \%$, Denver, Outdoor Children, 8-h Exposures, and Moderate Exertion (underlying exposure-response relationship based on Folinsbee et al. 1988, Horstman et al. 1990, and McDonnell et al. 1991) . . . . . . . . . 134

C.12 Representative Risk Distributions for Alternative Air Quality Scenarios for FEV 1 Decrements $\geq 20 \%$, Houston, Outdoor Children, 8-h Exposures, and Moderate Exertion (underlying exposure-response relationship based on Folinsbee et al. 1988, Horstman et al. 1990, and McDonnell et al. 1991) . . . . . . . . 135

C.13 Representative Risk Distributions for Alternative Air Quality Scenarios for FEV 1 Decrements $\geq 20 \%$, Los Angeles, Outdoor Children, 8-h Exposures, and Moderate Exertion (underlying exposure-response relationship based on Folinsbee et al. 1988, Horstman et al. 1990, and McDonnell et al. 1991) . . . . . . . . 136

C.14 Representative Risk Distributions for Alternative Air Quality Scenarios for FEV Decrements $\geq 20 \%$, Miami, Outdoor Children, 8-h Exposures, and Moderate Exertion (underlying exposure-response relationship based on Folinsbee et al. 1988, Horstman et al. 1990, and McDonnell et al. 1991) . . . . . . . . . 137

C.15 Representative Risk Distributions for Alternative Air Quality Scenarios for FEV Decrements $\geq 20 \%$, New York, Outdoor Children, 8-h Exposures, and Moderate Exertion (underlying exposure-response relationship based on Folinsbee et al. 1988, Horstman et al. 1990, and McDonnell et al. 1991) . . . . . . . . 138 


\section{FIGURES (Cont.)}

C.16 Representative Risk Distributions for Alternative Air Quality Scenarios for FEV Decrements $\geq 20 \%$, Philadelphia, Outdoor Children, 8-h Exposures, and Moderate Exertion (underlying exposure-response relationship based on Folinsbee et al. 1988, Horstman et al. 1990, and McDonnell et al. 1991) . . . . . . . . . . . 139

C.17 Representative Risk Distributions for Alternative Air Quality Scenarios for FEV Decrements $\geq 20 \%$, St. Louis, Outdoor Children, 8-h Exposures, and Moderate Exertion (underlying exposure-response relationship based on Folinsbee et al. 1988, Horstman et al. 1990, and McDonnell et al. 1991) . . . . . . . . . . 140

C.18 Representative Risk Distributions for Alternative Air Quality Scenarios for FEV Decrements $\geq 20 \%$, Washington DC, Outdoor Children, 8-h Exposures, and Moderate Exertion (underlying exposure-response relationship based on Folinsbee et al. 1988, Horstman et al. 1990, and McDonnell et al. 1991) . . . . 141

C.19 Representative Risk Distributions for Alternative Air Quality Scenarios for Moderate-to-Severe Pain on Deep Inspiration, Chicago, Outdoor Children, 1-h Exposures, and Moderate Exertion (underlying exposure-response relationship based on Seal et al. 1993) . . . . . . . . . . . . . . . 142

C.20 Representative Risk Distributions for Alternative Air Quality Scenarios for Moderate-to-Severe Pain on Deep Inspiration, Denver, Outdoor Children, 1-h Exposures, and Moderate Exertion (underlying exposure-response relationship based on Seal et al. 1993) . . . . . . . . . . . . . . . 143

C.21 Representative Risk Distributions for Alternative Air Quality Scenarios for Moderate-to-Severe Pain on Deep Inspiration, Houston, Outdoor Children, 1-h Exposures, and Moderate Exertion (underlying exposure-response relationship based on Seal et al. 1993) . . . . . . . . . . . . . . . 144

C.22 Representative Risk Distributions for Alternative Air Quality Scenarios for Moderate-to-Severe Pain on Deep Inspiration, Los Angeles, Outdoor Children, 1-h Exposures, and Moderate Exertion (underlying exposure-response relationship based on Seal et al. 1993) . . . . . . . . . . . . . . 145

C.23 Representative Risk Distributions for Alternative Air Quality Scenarios for Moderate-to-Severe Pain on Deep Inspiration, Miami, Outdoor Children, 1-h Exposures, and Moderate Exertion (underlying exposure-response relationship based on Seal et al. 1993) $\ldots \ldots \ldots \ldots \ldots \ldots \ldots \ldots \ldots \ldots$

C.24 Representative Risk Distributions for Alternative Air Quality Scenarios for Moderate-to-Severe Pain on Deep Inspiration, New York, Outdoor Children, 1-h Exposures, and Moderate Exertion (underlying exposure-response relationship based on Seal et al. 1993) 


\section{FIGURES (Cont.)}

C.25 Representative Risk Distributions for Alternative Air Quality Scenarios for Moderate-to-Severe Pain on Deep Inspiration, Philadelphia, Outdoor Children, 1-h Exposures, and Moderate Exertion (underlying exposure-response relationship based on Seal et al. 1993) . . . . . . . . . . . . . . 148

C.26 Representative Risk Distributions for Alternative Air Quality Scenarios for Moderate-to-Severe Pain on Deep Inspiration, St. Louis, Outdoor Children, 1-h Exposures, and Moderate Exertion (underlying exposure-response relationship based on Seal et al. 1993) . . . . . . . . . . . . . . . 149

C.27 Representative Risk Distributions for Alternative Air Quality Scenarios for Moderate-to-Severe Pain on Deep Inspiration, Washington DC, Outdoor Children, 1-h Exposures, and Moderate Exertion (underlying exposure-response relationship based on Seal et al. 1993) . . . . . . . . 150

C.28 Ratios of Mean Number of Occurrences and Mean Number of Responders for $\mathrm{FEV}_{1}$ Decrements $\geq 15 \%$, 9 Urban Areas, Outdoor Children, 8-h Exposures, and Moderate Exertion (underlying exposure-response relationship based on Folinsbee et al. 1988, Horstman et al. 1990, and McDonnell et al. 1991) . . . 151

C.29 Ratios of Mean Number of Occurrences and Mean Number of Responders for $\mathrm{FEV}_{1}$ Decrements $\geq 20 \%$, 9 Urban Areas, Outdoor Children, 8-h Exposures, and Moderate Exertion (underlying exposure-response relationship based on Folinsbee et al. 1988, Horstman et al. 1990, and McDonnell et al. 1991) . . . . 151

C.30 Ratios of Mean Number of Occurrences and Mean Number of Responders for Moderate-to-Severe Pain on Deep Inspiration, 9 Urban Areas, Outdoor Children, 1-h Exposures, and Moderate Exertion (underlying exposure-response relationship based on Seal et al. 1993) . . . . . . . . 152

C.31 Headcount Risk Results for Percentage of Children Responding for FEV 1 Decrement $\geq 15 \%$, 1-h Exposures, and Heavy Exertion (persons basis; underlying exposure-response relationship based on Avol et al. 1984) $\ldots \ldots 153$

C.32 Headcount Risk Results for Percentage of Children Responding for FEV 1 Decrement $\geq 20 \%, 1-\mathrm{h}$ Exposures, and Heavy Exertion (persons basis; underlying exposure-response relationship based on Avol et al. 1984) $\ldots \ldots \ldots 154$

C.33 Headcount Risk Results for Percentage of Children Responding for Moderate-to-Severe Lower Respiratory Symptoms, 1-h Exposures, and Heavy Exertion (persons basis; underlying exposure-response

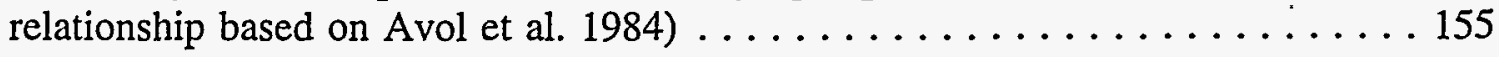




\section{FIGURES (Cont.)}

C.34 Headcount Risk Results for Percentage of Children Responding for FEV 1 Decrement $\geq 15 \%$, 1-h Exposures, and Heavy Exertion (persons basis; underlying exposure-response relationship based on Kulle et al. 1985) . . . . . 156

C.35 Headcount Risk Results for Percentage of Children Responding for FEV Decrement $\geq 20 \%$, 1-h Exposures, and Heavy Exertion (persons basis; underlying exposure-response relationship based on Kulle et al. 1985) . . . . . 157

C.36 Headcount Risk Results for Percentage of Children Responding for Moderate-to-Severe Cough, 1-h Exposures, and Heavy Exertion (persons basis; underlying exposure-response relationship based on Kulle et al. 1985) . . . . . 158

C.37 Headcount Risk Results for Percentage of Children Responding for Moderate-to-Severe.PDI, 1-h Exposures, and Heavy Exertion (persons basis; underlying exposure-response relationship based on Kulle et al. 1985) . . . . . 159

C.38 Headcount Risk Results for Percentage of Children Responding for FEV 1 Decrement $\geq 15 \%$, 1-h Exposures, and Heavy Exertion (persons basis; underlying exposure-response relationship based on McDonnell et al. 1983) . . 160

C.39 Headcount Risk Results for Percentage of Children Responding for FEV 1 Decrement $\geq 20 \%$, 1-h Exposures, and Heavy Exertion (persons basis; underlying exposure-response relationship based on McDonnell et al. 1983) . . . 161

C.40 Headcount Risk Results for Percentage of Children Responding for Moderate-to-Severe Cough, 1-h Exposures, and Heavy Exertion (persons basis; underlying exposure-response relationship based on McDonnell et al. 1983) . . . 162

C.41 Headcount Risk Results for Percentage of Children Responding for Moderate-to-Severe PDI, 1-h Exposures, and Heavy Exertion (persons basis; underlying exposure-response relationship based on McDonnell et al. 1983) . . . 163

C.42 Headcount Risk Results for Percentage of Children Responding for FEV 1 Decrement $\geq 15 \%$, 1-h Exposures, and Moderate Exertion (persons basis; underlying exposure-response relationship based on Seal et al. 1993) . . . . . . 164

C.43 Headcount Risk Results for Percentage of Children Responding for FEV 1 Decrement $\geq 20 \%$, 1-h Exposures, and Moderate Exertion (persons basis; underlying exposure-response relationship based on Seal et al. 1993) . . . . . 165

C.44 Headcount Risk Results for Percentage of Children Responding for Moderate-to-Severe Cough, 1-h Exposures, and Moderate Exertion (persons basis; underlying exposure-response relationship based on Seal et al. 1993) . . . . . 166 


\section{FIGURES (Cont.)}

C.45 Headcount Risk Results for Percentage of Children Responding for Moderate-to-Severe PDI, 1-h Exposures, and Moderate Exertion (persons basis; underlying exposure-response relationship based on Seal et al. 1993) . . . . . . . . . . . . . . . . . . . . . . . 167

C.46 Headcount Risk Results for Percentage of Children Responding for FEV 1 Decrement $\geq 15 \%$, 8-h Exposures, and Moderate Exertion (persons basis; underlying exposure-response relationship based on Folinsbee et al. 1988, Horstman et al. 1990, and McDonnell et al. 1991) . . . . . . . . . . . 168

C.47 Headcount Risk Results for Percentage of Children Responding for FEV 1 Decrement $\geq 20 \%$, 8-h Exposures, and Moderate Exertion (persons basis; underlying exposure-response relationship based on Folinsbee et al. 1988, Horstman et al. 1990, and McDonnell et al. 1991) . . . . . . . . . . . . . . 169

C.48 Headcount Risk Results for Percentage of Children Responding for Moderate-to-Severe Cough, 8-h Exposures, and Moderate Exertion (persons basis; underlying exposure-response relationship based on Folinsbee et al. 1988, Horstman et al. 1990, and McDonnell et al. 1991) . . . 170

C.49 Headcount Risk Results for Percentage of Children Responding for Moderate-to-Severe PDI, 8-h Exposures, and Moderate Exertion (persons basis; underlying exposure-response relationship based on Folinsbee et al. 1988, Horstman et al. 1990, and McDonnell et al. 1991) . . . 171

D.1 Probability That the Benchmark Response for the FEV ${ }_{1}$ Decrement $\geq 15 \%$ Endpoint Will Be Exceeded Five or More Times in an Ozone Season for 1-h Exposures, Heavy Exertion, 1-h DMax, and $\mathrm{N}$ of Nhigh $=5$ (underlying exposure-response relationship based on Avol et al. 1984) . . . . 177

D.2 Probability That the Benchmark Response for the FEV Decrement $_{1} \geq 15 \%$ Endpoint Will Be Exceeded Five or More Times in an Ozone Season for 1-h Exposures, Heavy Exertion, 1-h DMax, and $\mathrm{N}$ of Nhigh $=5$ (underlying exposure-response relationship based on Kulle et al. 1985) . . . . 178

D.3 Probability That the Benchmark Response for the $\mathrm{FEV}_{1}$ Decrement $\geq 15 \%$ Endpoint Will Be Exceeded Five or More Times in an Ozone Season for 1-h Exposures, Heavy Exertion, 1-h DMax, and $\mathrm{N}$ of Nhigh $=5$ (underlying exposure-response relationship based on McDonnell et al. 1983) . . . 179

D.4 Probability That the Benchmark Response for the $\mathrm{FEV}_{1}$ Decrement $\geq 20 \%$ Endpoint Will Be Exceeded Five or More Times in an Ozone Season for 1-h Exposures, Heavy Exertion, 1-h DMax, and $\mathrm{N}$ of Nhigh $=5$ (underlying exposure-response relationship based on Avol et al. 1984) . . . . 180 


\section{FIGURES (Cont.)}

D.5 Probability That the Benchmark Response for the $\mathrm{FEV}_{1}$ Decrement $\geq 20 \%$ Endpoint Will Be Exceeded Five or More Times in an Ozone Season for 1-h Exposures, Heavy Exertion, 1-h DMax, and $\mathrm{N}$ of Nhigh = 5 (underlying exposure-response relationship based on Kulle et al. 1985) . . . . . 181

D.6 Probability That the Benchmark Response for the FEV 1 Decrement $\geq 20 \%$ Endpoint Will Be Exceeded Five or More Times in an Ozone Season for 1-h Exposures, Heavy Exertion, 1-h DMax, and $\mathrm{N}$ of Nhigh $=5$ (underlying exposure-response relationship based on McDonnell et al. 1983) . . . 182

D.7 Probability That the Benchmark Response for the Moderate-to-Severe Lower Respiratory Endpoint Will Be Exceeded Five or More Times in an Ozone Season for 1-h Exposures, Heavy Exertion, 1-h DMax, and $\mathrm{N}$ of Nhigh $=5$ (underlying exposure-response relationship based on Avol et al. 1984) . . . . 183

D.8 Probability That the Benchmark Response for the Moderate-to-Severe Cough Endpoint Will Be Exceeded Five or More Times in an Ozone Season for 1-h Exposures, Heavy Exertion, 1-h DMax, and $\mathrm{N}$ of Nhigh $=5$ (underlying exposure-response relationship based on Kulle et al. 1985) . . . . . . . . . 184

D.9 Probability That the Benchmark Response for the Moderate-to-Severe Cough Endpoint Will Be Exceeded Five or More Times in an Ozone Season for 1-h Exposures, Heavy Exertion, 1-h DMax, and $\mathrm{N}$ of Nhigh $=5$ (underlying exposure-response relationship based on McDonnell et al. 1983) . . . . . . . 185

D.10 Probability That the Benchmark Response for the Moderate-to-Severe Pain on Deep Inspiration Endpoint Will Be Exceeded Five or More Times in an Ozone Season for 1-h Exposures, Heavy Exertion, 1-h DMax, and $\mathrm{N}$ of Nhigh $=5$ (underlying exposure-response relationship based on Kulle et al. 1985) $\ldots \ldots 186$

D.11 Probability That the Benchmark Response for the Moderate-to-Severe Pain on Deep Inspiration Endpoint Will Be Exceeded Five or More Times in an Ozone Season for 1-h Exposures, Heavy Exertion, 1-h DMax, and $\mathrm{N}$ of Nhigh $=5$ (underlying exposure-response relationship based on McDonnell et al. 1983) . . . 187

D.12 Probability That the Benchmark Response for the $\mathrm{FEV}_{1}$ Decrement $\geq 15 \%$ Endpoint Will Be Exceeded Five or More Times in an Ozone Season for 1-h Exposures, Moderate Exertion, 1-h DMax, and $\mathrm{N}$ of Nhigh $=5$ (underlying exposure-response relationship based on Seal et al. 1993) $\ldots \ldots \ldots 188$

D.13 Probability That the Benchmark Response for the FEV 1 Decrement $\geq 20 \%$ Endpoint Will Be Exceeded Five or More Times in an Ozone Season for 1-h Exposures, Moderate Exertion, 1-h DMax, and $\mathrm{N}$ of Nhigh $=5$ (underlying exposure-response relationship based on Seal et al. 1993) 


\section{FIGURES (Cont.)}

D.14 Probability That the Benchmark Response for the Moderate-to-Severe Cough Endpoint Will Be Exceeded Five or More Times in an Ozone Season for 1-h Exposures, Moderate Exertion, 1-h DMax, and $\mathrm{N}$ of Nhigh $=5$

(underlying exposure-response relationship based on Seal et al. 1993) $\ldots \ldots \ldots 190$

D.15 Probability That the Benchmark Response for the Moderate-to-Severe Pain on Deep Inspiration Endpoint Will Be Exceeded Five or More Times in an Ozone Season for 1-h Exposures, Moderate Exertion, 1-h DMax, and $\mathrm{N}$ of Nhigh $=5$ (underlying exposure-response relationship based on Seal et al. 1993) . . . . . 191

D.16 Probability That the Benchmark Response for the FEV 1 Decrement $\geq 15 \%$ Endpoint Will Be Exceeded Five or More Times in an Ozone Season for 8-h Exposures, Moderate Exercise, 8-h DMax, and $\mathrm{N}$ of Nhigh $=5$ (underlying exposure-response relationship based on Folinsbee et al. 1988, Horstman et al., 1990, and McDonnell et al. 1983) . . . . . . . . . . . . 192

D.17 Probability That the Benchmark Response for the $\mathrm{FEV}_{1}$ Decrement $\geq 20 \%$ Endpoint Will Be Exceeded Five or More Times in an Ozone Season for 8-h Exposures, Moderate Exercise, 8-h DMax, and $\mathrm{N}$ of Nhigh $=5$ (underlying exposure-response relationship based on Folinsbee et al. 1988, Horstman et al., 1990, and McDonnell et al. 1991) . . . . . . . . . . . . 193

D.18 Probability That the Benchmark Response for the Moderate-to-Severe Cough Endpoint Will Be Exceeded Five or More Times in an Ozone Season for 8-h Exposures, Moderate Exercise, 8-h DMax, and $\mathrm{N}$ of Nhigh $=5$ (underlying exposure-response relationship based on Folinsbee et al. 1988, Horstman et al., 1990, and McDonnell et al. 1991) . . . . . . . . . . . . . 194

D.19 Probability That the Benchmark Response for the Moderate-to-Severe Pain on Deep Inspiration Endpoint Will Be Exceeded Five or More Times in an Ozone Season for 8-h Exposures, Moderate Exercise, 8-h DMax, and $\mathrm{N}$ of Nhigh $=5$ (underlying exposure-response relationship based on Folinsbee et al. 1988, Horstman et al., 1990, and McDonnell et al. 1991) . . . . . . . . . . . 195 


\section{ACKNOWLEDGMENTS}

Many people at the U.S. Environmental Protection Agency assisted and guided us during this work, and the authors appreciate and acknowledge their respective contributions. We benefited from many discussions with Drs. William McDonnell, Elston Seal, Lawrence Folinsbee, and Donald Horstman about details of their controlled human exposure studies. Ted Johnson provided information for the section on the exposure models. Harvey Richmond (project officer), James Raub, Thomas McCurdy, and Drs. David McKee and Karen Martin helped to clarify issues regarding exposure, exposure response, and development of risk estimates. We also acknowledge the review of previous drafts by the Clean Air Scientific Advisory Committee Panel for the Ozone NAAQS Review.

The authors gratefully acknowledge the efforts of colleagues at Argonne National Laboratory (ANL) who were instrumental in preparing this report. Craig Burns, Michelle Romanesko, Kristy Pegues, and David Katauskas helped to generate and assemble the figures and tables. Rosemary Stanton and Sandy Schreader helped to prepare the manuscript. Argonne's Information and Publishing Division provided publishing support: Margaret Clemmons did the technical editing, and the Document Processing and Control Center (most notably, Lynette McCallum) provided document processing services.

We also acknowledge the contributions of our colleagues in earlier work on lead and ozone risk assessments: Stan Hayes, Arlene Rosenbaum, and Drs. Thomas Wallsten and Robert Winkler. Those efforts provided critical foundations for this enterprise.

The views, opinions, and conclusions of the authors expressed in this report do not necessarily state or reflect those of the U.S. government or any agency thereof, ANL, or others who assisted and guided us in this effort. 


\section{NOTATION}

The following is a list of acronyms, initialisms, and abbreviations (including units of measure) used in this document.

\section{ACRONYMS, INITIALISMS, AND ABBREVIATIONS}

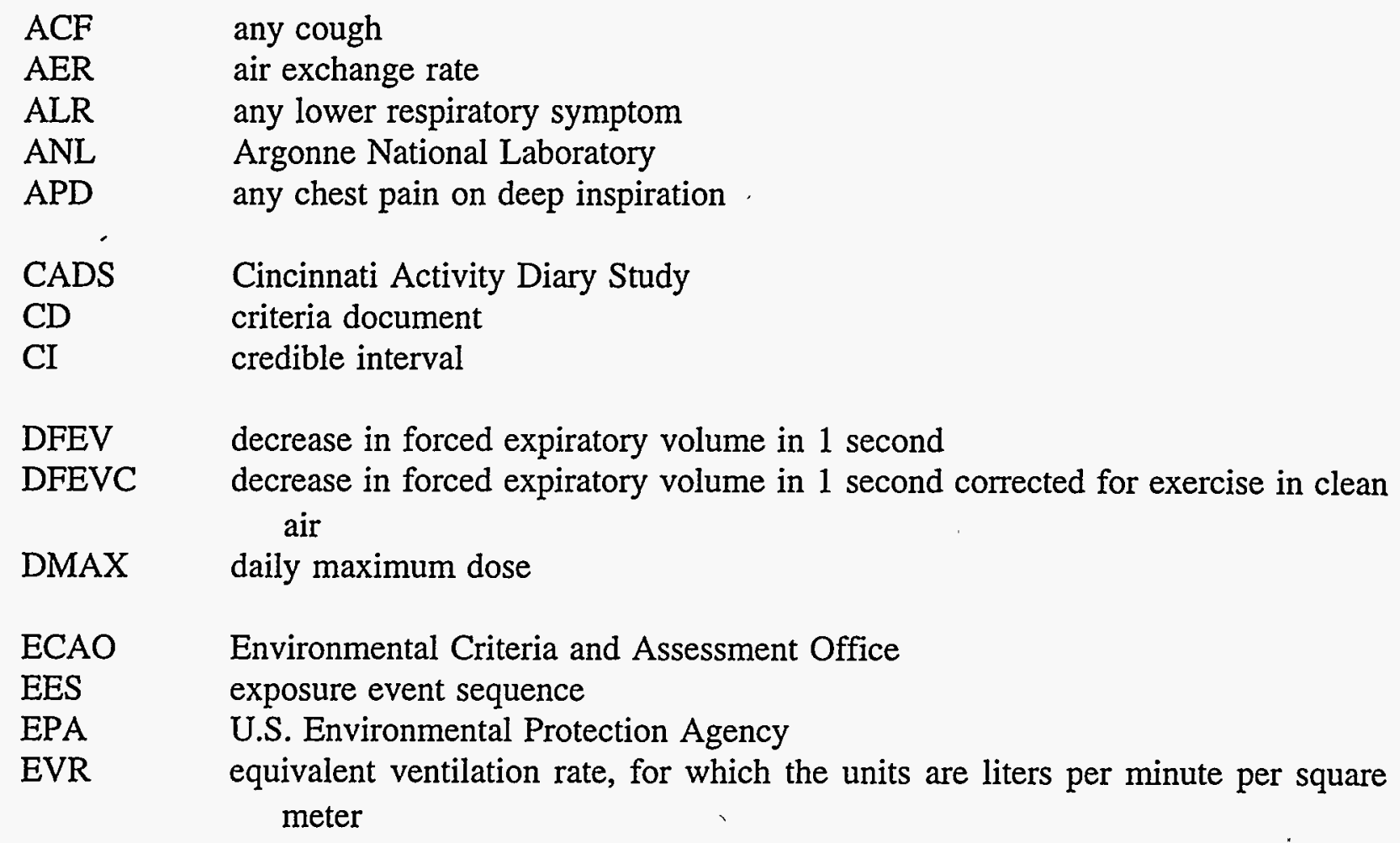

DFEV decrease in forced expiratory volume in 1 second

DFEVC decrease in forced expiratory volume in 1 second corrected for exercise in clean air

DMAX daily maximum dose

ECAO Environmental Criteria and Assessment Office

EES exposure event sequence

EPA U.S. Environmental Protection Agency

EVR equivalent ventilation rate, for which the units are liters per minute per square meter

FEV forced expiratory volume (the maximum amount of air that can be expelled from . the lungs)

$\mathrm{FEV}_{1}$ forced expiratory volume in 1 second (the maximum amount of air that can be expelled from the lungs in 1 second)

GCU geographic census unit

KS Kolmogorov-Smirnoff

MCF moderate-to-severe cough

MLR moderate-to-severe lower respiratory symptom

MPD moderate-to-severe chest discomfort

$\mathrm{M} / \mathrm{S} \quad$ moderate to severe 
NAAQS national ambient air quality standards

NEM NAAQS exposure model

$\begin{array}{ll}\mathrm{O}_{3} & \text { ozone } \\ \text { OAQPS } & \text { Office of Air Quality Planning and Standards }\end{array}$

PDI pain on deep inspiration

$\mathrm{pNEM} \mathrm{O}_{3}$ probabilistic NAAQS exposure model for ozone

s.d. $\quad$ standard deviation

s.e. standard error

As-Is As-Is air quality scenario: 1990 or 1991 air quality in a specific urban area (letter code: Z)

1112 current ozone NAAQS: 1 -h averaging time, 1 expected exceedance, $0.12 \mathrm{ppm}$ ozone (letter code: A)

1110 an alternative NAAQS: 1-h averaging time, 1 expected exceedance, $0.10 \mathrm{ppm}$ ozone (letter code: $\mathrm{H}$ )

8110 an alternative NAAQS: 8-h averaging time, 1 expected exceedance, $0.10 \mathrm{ppm}$ ozone (letter code: $\mathrm{D}$ )

8109 an alternative NAAQS: 8-h averaging time, 1 expected exceedance, $0.09 \mathrm{ppm}$ ozone (letter code: B)

8108 an alternative NAAQS: 8-h averaging time, 1 expected exceedance, $0.08 \mathrm{ppm}$ ozone (letter code: $\mathrm{C}$ )

8107 an alternative NAAQS: 8-h averaging time, 1 expected exceedance, $0.07 \mathrm{ppm}$ ozone (letter code: $\mathrm{G}$ )

8508 an alternative NAAQS: 8-h averaging time, 5 expected exceedances, $0.08 \mathrm{ppm}$ ozone (letter code: $\mathrm{F}$ )

8509 an alternative NAAQS: 8-h averaging time, 5 expected exceedances, $0.09 \mathrm{ppm}$ ozone (letter code: J)

\section{UNITS OF MEASURE}

ELPM equivalent liter(s) per minute (the units for ventilation rate adjusted for [i.e., divided by] body surface area, which is typically assumed to be $1.9 \mathrm{~m}^{2}$ for adults; the units are liters per minute per square meter $\left[\mathrm{L} / \mathrm{min} / \mathrm{m}^{2}\right]$ )

$\mathrm{h}$

$\mathrm{km} \quad$ kilometer(s)

L liter(s)

$\mathrm{m}^{2} \quad$ square meter(s)

Mbyte megabyte(s)

min minute(s)

ppm part(s) per million

$s \quad$ second(s)

yr year(s) 


\title{
A PROBABILISTIC ASSESSMENT OF HEALTH RISKS ASSOCIATED WITH SHORT-TERM EXPOSURE TO TROPOSPHERIC OZONE
}

by

\author{
R.G. Whitfield, W.F. Biller, \\ M.J. Jusko, and J.M. Keisler
}

\begin{abstract}
The work described in this report is part of a larger risk assessment sponsored by the U.S. Environmental Protection Agency. Earlier efforts developed exposure-response relationships for acute health effects among populations engaged in heavy exertion. Those efforts also developed a probabilistic national ambient air quality standards exposure model and a general methodology for integrating probabilistic exposure-response relationships and exposure estimates to calculate overall risk results. Recently published data make it possible to model additional health endpoints (for exposure at moderate exertion), including hospital admissions. New air quality and exposure estimates for alternative national ambient air quality standards for ozone are combined with exposure-response models to produce the risk results for hospital admissions and acute health effects. Sample results explain the methodology and introduce risk output formats.
\end{abstract}

\section{INTRODUCTION}

This report presents models and estimates for selected risks of acute (short-term) exposure to ozone $\left(\mathrm{O}_{3}\right)$. The risks are described in terms of two fundamental types of health endpoints: (1) hospital admissions of asthmatics or patients diagnosed with various respiratory problems and (2) the occurrence of acute effects, such as coughing, chest pain when taking a deep breath, and decreased forced expiratory volume.

This report considers two fundamental types of risk measures: headcount and benchmark. Headcount risk accounts for either (1) the number of people or the number of times an individual from a specific population experiences a particular "event" (e.g., a hospital admission, cough, chest pain, decreased lung function) or (2) the percentage of possible events estimated to occur. Headcount risk combines exposure-response relationships with exposure estimates about populations as they go about their daily activities. In contrast, benchmark risk focuses on the probability or risk of unhealthful air. Benchmark risk is a measure of the hazard posed by 
elevated ambient ozone levels. It is calculated by assuming that all members of the at-risk population are exposed outdoors under identical exposure conditions.

The risks are represented by probability distributions (called risk distributions) over a variety of risk measures (e.g., the number of outdoor children that may experience pain on deep inspiration attributable to exposure to tropospheric ozone for one year in Los Angeles). Probability distributions are generally needed to represent the risks fairly because considerable uncertainty exists regarding the amount of exposure that individuals and populations receive and the degree to which they experience various health endpoints at specific exposure levels. Factors that make up the risk measures include the following:

- Population of interest (outdoor children, outdoor workers, asthmatics, the general population);

- Type of effect (hospital admission, coughing, chest pain, decreased lung function);

- Area of residence (nine urban areas are analyzed);

- Length of exposure ( 1 or $8 \mathrm{~h})$;

- Numbers or percentages; and

- Method of counting multiple exposures or occurrences of an effect experienced by an individual.

Results are provided for alternative national ambient air quality standards (NAAQS) that have the following components:

- Averaging time (1- or 8 -h daily maximum average);

- Number of expected exceedances in one year (1 or 5); and

- Allowed ozone concentration $(0.07,0.08,0.09,0.10$, or 0.12 parts per million [ppm]).

Of the 20 possible combinations of these components, eight NAAQS are analyzed in this report ${ }^{1}$ :

- 1-h daily maximum average, 1 expected exceedance, $0.12 \mathrm{ppm}$ (the current ozone standard), which is referred to as scenario 1112 ;

1 A ninth NAAQS, 8506, was initially investigated but was subsequently dropped and is not included in this report. 
- 1-h daily maximum average, 1 expected exceedance, $0.10 \mathrm{ppm}$, which is referred to as scenario 1110 ;

- 8-h daily maximum average, 1 expected exceedance, $0.10 \mathrm{ppm}$, which is referred to as scenario 8110 ;

- 8-h daily maximum average, 1 expected exceedance, $0.09 \mathrm{ppm}$, which is referred to as scenario 8109 ;

- 8-h daily maximum average, 1 expected exceedance, $0.08 \mathrm{ppm}$, which is referred to as scenario 8108 ;

- 8-h daily maximum average, 1 expected exceedance, $0.07 \mathrm{ppm}$, which is referred to as scenario 8107 ;

- 8-h daily maximum average, 5 expected exceedances, $0.09 \mathrm{ppm}$, which is referred to as scenario 8509 ; and

- 8-h daily maximum average, 5 expected exceedances, $0.08 \mathrm{ppm}$, which is referred to as scenario 8508 .

In all cases, the average level is a "daily maximum" value (i.e., the highest daily 1- or 8-h average for each day of the ozone season), which means that the 1- or 8-h average ozone concentration can exceed $0.12 \mathrm{ppm}$ two or more times in a given day, but only one exceedance will "count" for a specific day and year. Moreover, the standard specifies such that an area is considered to be out of compliance only if the average number of exceedances during a threeyear period is $>1$ at any monitor within the urban area. Thus, to comply with the current standard, the highest 1-h daily maximum ozone concentration in an attainment area cannot exceed $0.12 \mathrm{ppm}$ more than three times in any three-year period at any monitor. ${ }^{2}$

While the current standard (or any new standard) actually addresses a three-year period in determining compliance, the results reported herein are based on only one ozone season. Air quality data were adjusted to simulate just attaining a given standard. Therefore, exposure and risk estimates reflect what is expected, on average, when air quality in an area just attains a given standard. Actual exposures and risks can be lower or higher in different years during a three-year period used to judge compliance with a standard.

2 The exposure analysis, and therefore the risk assessment, simulated air quality that just attained the specified standard level (e.g., $0.12 \mathrm{ppm}$ ). In implementing the current standard, the U.S. Environmental Protection Agency (EPA) uses a rounding convention in which only concentrations that equal or exceed 0.125 are counted as exceedances of the standard level. 
Headcount risk distributions are obtained by combining probabilistic exposure-response relationships with exposure estimates. The exposure-response relationships are derived from a variety of sources, primarily human experimental and observational data for acute effects. Exposure estimates, which are also probabilistic, were obtained from the recently developed probabilistic version of the National Ambient Air Quality Standards Exposure Model for ozone $\left(\mathrm{pNEM} / \mathrm{O}_{3}\right.$ ). The $\mathrm{pNEM} / \mathrm{O}_{3}$ output includes results for 10 separate runs of the model to gain insights about the effects of run-to-run variations on risk output.

Benchmark response is $r$, the fraction of the population that experiences the specified health effect when exposed to ozone. Benchmark risk is defined as the probability that the benchmark response is $\geq r, n$ or more times in a specified period (one ozone season) at some location within a geographic region, given a particular air quality condition (e.g., that scenario 1112 is just attained). This report uses $r$ values of 0.05 and 0.1 (sometimes referred to as 0.05 and 0.1 benchmarks, or 5 and $10 \%$ benchmarks).

The hospital admissions model has elements of both benchmark and headcount risk models. It uses air quality data, as does the benchmark risk model, and a concentration-response relationship that resembles the exposure-response relationships used in the headcount risk models. The model assumes a linear relationship between hospital admissions (the response) and the previous day's highest hourly average ozone concentration.

The motivation for this work is to (1) develop first-time risk results for some acute health endpoints (hospital admissions, acute effects for populations engaged in moderate exertion for short and prolonged periods) and (2) update previous risk results (Hayes et al. 1987, 1989) for populations exposed to ozone while engaged in heavy exertion for $1 \mathrm{~h}$. The latter involves using recent exposure estimates from $\mathrm{pNEM} \mathrm{O}_{3}$ that (3) reflect analysis of possible alternative NAAQS for ozone.

\subsection{BACKGROUND}

Ozone is a highly reactive irritant gas present in elevated amounts throughout much of the United States. The EPA estimated that in 1988 about 120 million people lived in urban areas where the current 1-h daily maximum average ozone standard of $0.12 \mathrm{ppm}$ was not attained. More recently (1993), the EPA estimated that 51 million people lived in urban areas where the current 1-h standard was not attained (EPA 1994). The current NAAQS require that the $0.12 \mathrm{ppm}$ of ozone concentration not be exceeded on average on more than one day per year. This current standard for ozone is based on concern for acute respiratory effects reported in many controlled human exposure studies, as well as on acute and longer-term effects reported in animal toxicology, human epidemiology, and human field studies.

As stated in the Clean Air Act, the EPA is required to set, review, and revise, as appropriate, the primary NAAQS for criteria pollutants. The primary standards, which are to be 
set at levels sufficient to protect public health with an adequate margin of safety, are based on scientific evidence reviewed in "criteria documents" (CDs). A CD summarizes and evaluates the human clinical and field studies and the epidemiological and animal toxicological evidence regarding physiological and adverse health effects that result from exposure to ozone and other photochemical oxidants.

To assist in its review of the NAAQS for ozone, EPA's Office of Air Quality Planning and Standards (OAQPS) entered into an interagency agreement with Argonne National Laboratory (ANL) to assess the public health risk associated with attaining alternative ozone NAAQS. The purpose of this risk assessment is to characterize, as explicitly as possible, the range and implications of uncertainties in the existing scientific database, while fully using current scientific knowledge, available animal and human experimental and observational data, and scientific expertise. This risk assessment includes the effects of acute exposures to ozone. It combines exposure-response relationships with exposure estimates to produce overall risk estimates. In addition, hourly air quality data in New York City are used to estimate excess respiratory-related hospital admissions of asthmatics and the general population during the ozone season. A summary of the results of acute risk assessment and its role in the ozone NAAQS review can be found in the OAQPS staff paper (EPA 1996a).

The acute risk assessment addresses the effects of exposure to ozone for populations engaged in either heavy or moderate exertion. The heavy exertion effects are based on 1- to 3-h controlled human exposure studies by McDonnell et al. (1983), Avol et al. (1984), and Kulle et al. (1985). The moderate exertion effects are based on results from 2-h controlled human studies by Seal et al. (1993) and from 6.6-h controlled human studies by Folinsbee et al. (1988), Horstman et al. (1990), and McDonnell et al. (1991). The hospital admissions model is based on a multiyear study of air pollution and respiratory hospital admissions in New York City (Thurston et al. 1992).

Previous risk assessments studied the acute health effects of ozone (Hayes et al. 1987). Methods developed in these assessments (Hayes et al. 1987, 1989) and earlier assessments for lead (Wallsten and Whitfield 1986; Whitfield and Wallsten 1989) provide a foundation for the current risk assessment.

\subsection{RISK ASSESSMENT APPROACH}

The basic risk assessment approach, illustrated in Figure 1, involves developing an exposure model and a health model. ${ }^{3}$ The exposure model accounts for human contact with a

${ }^{3}$ Strictly speaking, Figure 1 applies only to headcount risks for acute and chronic effects. However, the differences for benchmark risks and hospital admissions are subtle. One difference is that benchmark risks and hospital admissions are based on actual air quality data rather than on estimates of exposure. 


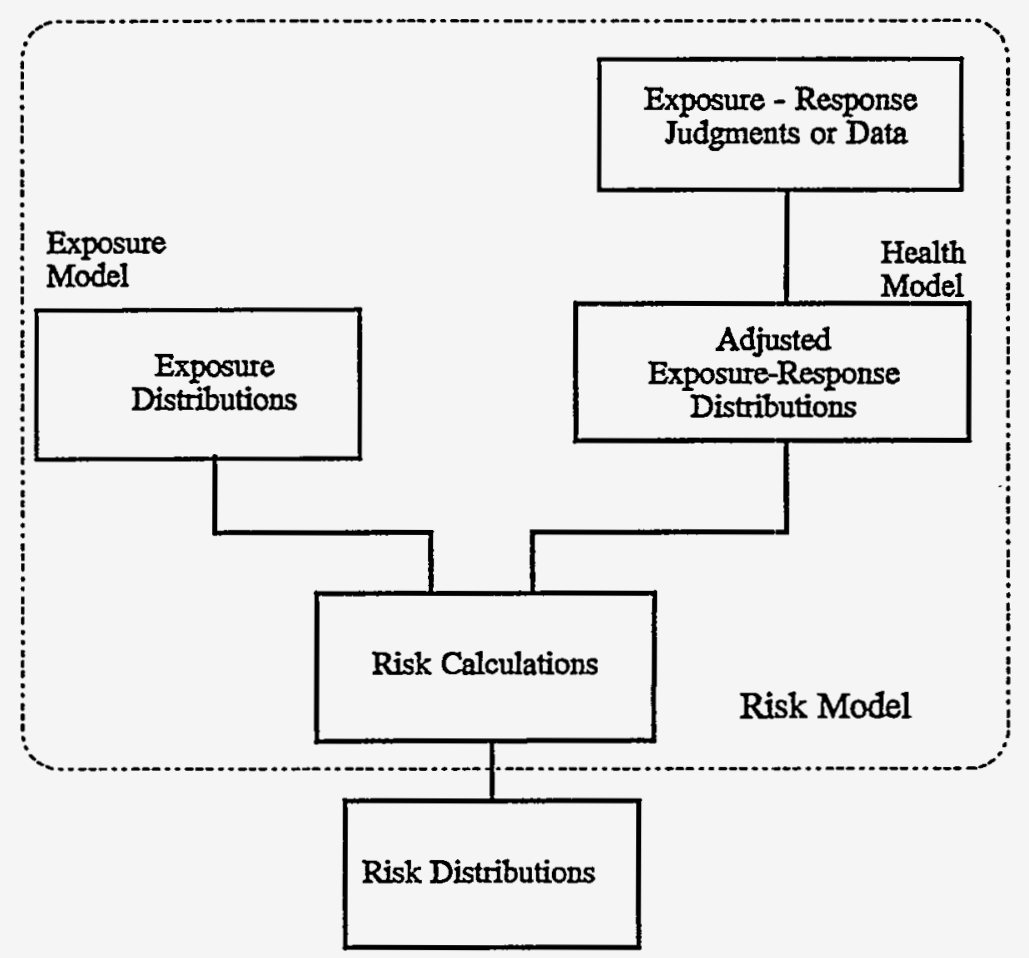

FIGURE 1 Basic Risk Assessment Approach: Risk Model Incorporating Both an Exposure Model and a Health Model

specific criteria pollutant. The contact can be described in terms of a cumulative exposure over a specified time. For this assessment, exposure estimates were generated by $\mathrm{pNEM} / \mathrm{O}_{3}$ for nine urban areas and that portion of the population thought to be potentially at greatest risk to ozone exposure. The at-risk groups are outdoor children and outdoor workers. This determination is based on the discussion of at-risk populations in the ozone CD (EPA 1996b).

The two types of risk estimates made - benchmark and headcount (Whitfield et al. 1994) - use the same exposure-response relationships, but different types of air quality data and exposure estimates. Relationships between the two risk models are indicated in Figure 2. Benchmark risk estimates measure the risk of potential harm - or hazard — posed by elevated levels of ozone. Headcount risk estimates measure the risk of actual harm incurred by individuals as they go about their daily lives, moving from one place to another and from indoors to outdoors.

In addition, two types of exposure measures can be made: people and personoccurrences. The people measure counts the number of individuals exposed one or more times per ozone season to the exposure indicator (ozone level and breathing rate) of interest. The person-occurrences measure first counts the times per ozone season that an individual is exposed to the exposure indicator of interest and then accumulates counts over all individuals. Therefore, the person-occurrences measure confounds people and occurrences: 1 occurrence for 10 people 


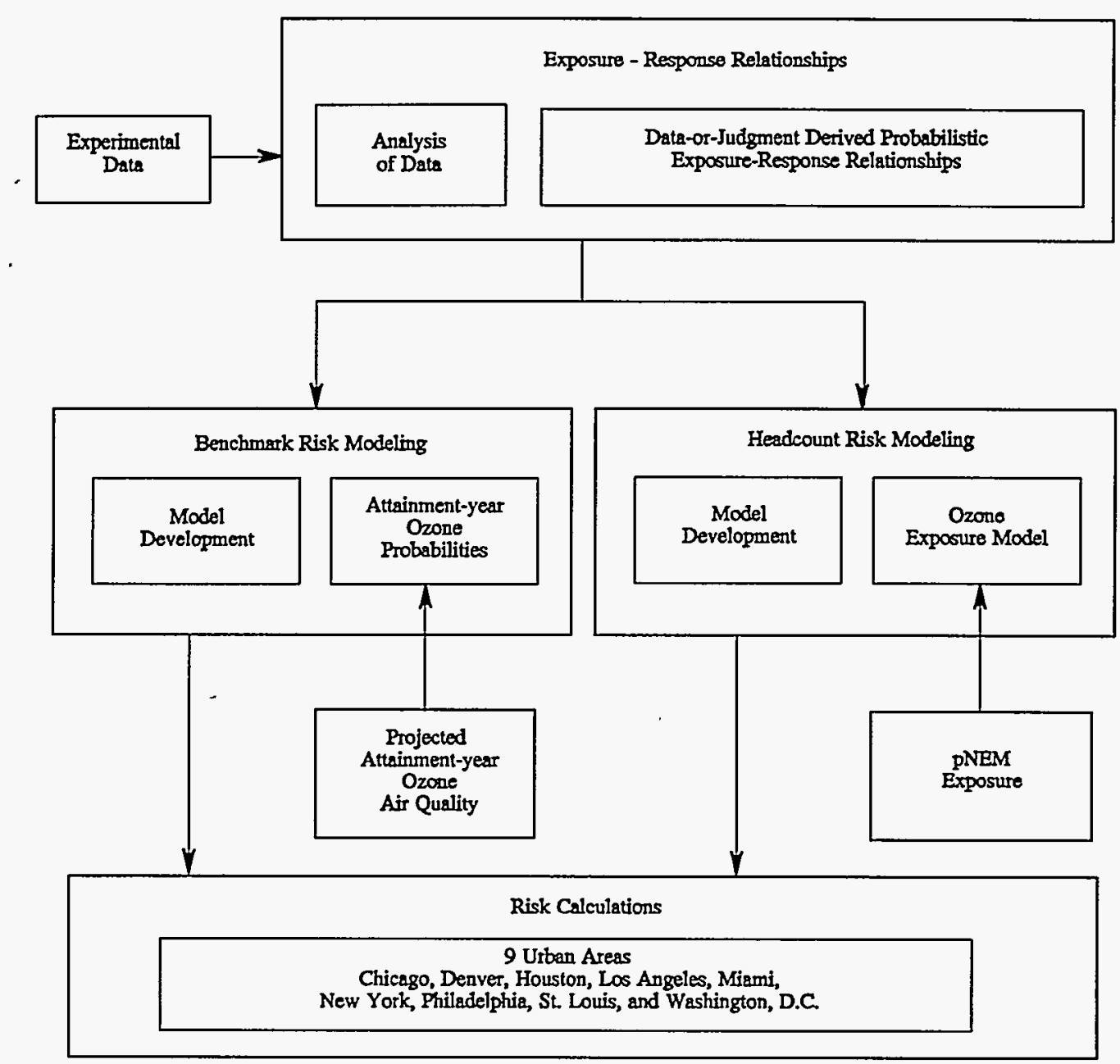

FIGURE 2 Relationship between Benchmark and Headcount Risk Models

is counted the same as 10 occurrences for 1 person. The maximum number of hourly exposure occurrences is equal to the population multiplied by the number of hours in the ozone season. This report includes both types of measures.

A health model accounts for human reaction to contact with a criteria pollutant. Reactions include symptoms or physiological changes (e.g., reduced pulmonary function or lower respiratory symptoms). A health model can be based on either data, judgment, or a combination of both. One important aspect of a health model is the "most at-risk population" - people believed to be most at risk because they are either highly reactive (e.g., children whose physiological development may be impaired by exposure to ozone) or more frequently exposed (e.g., outdoor children and outdoor workers). The Clean Air Act requires NAAQS to be set at a level that protects the most at-risk populations with an adequate margin of safety.

In this study, the exposure-response relationships that characterize the effects of ozone exposure on pulmonary function and the respiratory system are based on controlled human 
exposure data obtained in clinical studies. Controlled human exposure studies, in contrast to epidemiological or field studies, were thought to be most appropriate for specifying the data needed for estimating exposure response.

\subsection{REPORT ORGANIZATION}

This report is organized into eight sections. Section 2 discusses the $\mathrm{pNEM} / \mathrm{O}_{3}$ model that generates the exposure probabilities used in the headcount risk models and the air quality data used in the benchmark risk and hospital admissions models. It also describes the $\mathrm{pNEM}_{3}$ methodology and simulated air quality data. Section 3 discusses controlled human exposureresponse data sets used in the headcount and benchmark risk models. Section 4 discusses headcount risk. It describes the general method used to calculate risk estimates and presents results for acute endpoints. Section 5 discusses the benchmark risk model and presents results for acute endpoints. Section 6 describes the hospital admissions model, which is applied to two cases: asthmatics and patients from the general population hospitalized for respiratory conditions. Section 7 discusses limitations of the analysis. Section 8 contains a list of references cited in this report.

Details of modeling techniques are presented in Appendix A. Most of the headcount and benchmark risk results are presented in Appendixes B-D. Sections 4 and 5 present methodology and use selected risk results to introduce the form of presentation of results used in the appendixes. Headcount risk results are limited to those for outdoor children, and then only for the more serious endpoints. Likewise, benchmark risk results are shown only for the more serious endpoints. (Complete results for all endpoints, including results for outdoor workers and total populations, are available elsewhere. ${ }^{4}$ )

\footnotetext{
${ }^{4}$ Software and data (for IBM personal computers or compatibles only) needed to generate and view all risk results can be obtained from the Energy Science and Technology Software Center, P.O. Box 1020, Oak Ridge, TN 37831, phone 423-576-2606. The complete system requires about 100 Mbytes of disk storage.
} 


\section{EXPOSURE MODEL}

Evaluating alternative NAAQS proposed for a particular pollutant involves assessing the risks to human health associated with ozone exposures that result while just attaining each of the standards under consideration. Important factors that need to be considered in an ozone exposure assessment are magnitude of ozone concentrations; duration of ozone concentrations; spatial distribution of concentrations; frequency of repeated peak concentrations; ventilation state of the individual at time of exposure; and movement of people through zones of varying air quality, which affects the actual exposure patterns of people living within a defined area.

Until 1986, the NAAQS exposure model (NEM) series did not model random processes within the exposure simulation. The latest version of the model, $\mathrm{pNEM} / \mathrm{O}_{3}$, incorporates probabilistic elements into the original NEM methodology and uses a mass balance model to estimate indoor and in-vehicle ozone concentrations (Johnson et al. 1996a-c). Figure 3 shows how the $\mathrm{pNEM} / \mathrm{O}_{3}$ methodology fits into the risk assessment. This section briefly describes the pNEM/O $\mathrm{O}_{3}$ and its methodology. A more detailed description of the model can be found in Johnson et al. (1996a-c).

\subsection{ALTERNATIVE AIR QUALITY SCENARIOS INCLUDED IN THE ANALYSIS}

The results from the $\mathrm{pNEM} / \mathrm{O}_{3}$ are available for nine alternative air quality scenarios selected by OAQPS for analysis. Eight of these scenarios represent ozone air quality when alternative NAAQS are just attained. They differ with respect to averaging time $(1$ or $8 \mathrm{~h})$, the number of expected exceedances allowed ( 1 or 5$)$, and the allowed ozone limit $(0.07,0.08,0.09$, 0.10 , or $0.12 \mathrm{ppm}$ ). A ninth scenario, representative of existing air quality, is referred to as the "As-Is" scenario. For easy reference, initialisms for the scenarios are presented in Table 1.

\subsection{EXPOSURE MODELING METHODOLOGY}

The $\mathrm{pNEM} / \mathrm{O}_{3}$ consists of two principal parts: the cohort exposure program and the exposure extrapolation program. The cohort exposure program estimates the sequence of ozone exposures experienced by defined population groups. The exposure extrapolation program estimates the number of persons within a particular study who are represented by each cohort. The program combines this information with the cohort exposure sequences to determine the distribution of exposures over a defined population of interest.

The general NEM methodology consists of five steps. Step 1 defines the study area, appropriate subdivisions of the study area, the exposure period, and the population of interest. Step 2 divides the population of interest into the exhaustive set of cohorts, while step 3 develops 


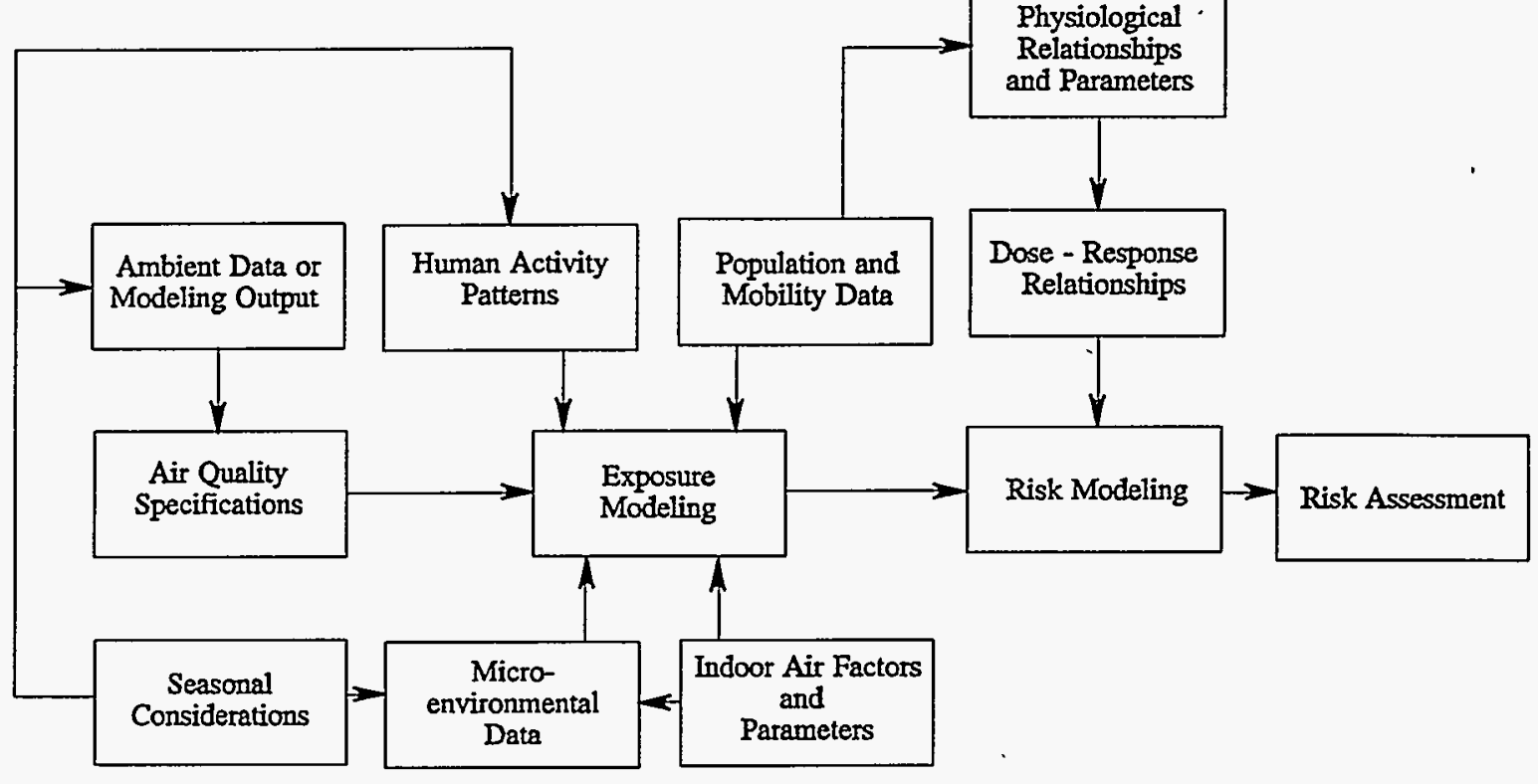

FIGURE 3 Major Components of the NEM Series of Exposure Models and Associated Health Risk Assessment Procedures

TABLE 1 Characteristics of Alternative Air Quality Scenarios

\begin{tabular}{cccc}
\hline Scenario & $\begin{array}{c}\text { Averaging } \\
\text { Time (h) }\end{array}$ & $\begin{array}{c}\text { Expected } \\
\text { Exceedance }\end{array}$ & $\begin{array}{c}\text { Ozone } \\
\text { Concentration } \\
(\mathrm{ppm})^{\mathrm{a}}\end{array}$ \\
\hline As-Is & $\mathrm{NA}^{\mathrm{b}}$ & $\mathrm{NA}$ & $\mathrm{NA}$ \\
1112 & 1 & 1 & 0.12 \\
1110 & 1 & 1 & 0.10 \\
8110 & 8 & 1 & 0.10 \\
8109 & 8 & 1 & 0.09 \\
8108 & 8 & 1 & 0.08 \\
8107 & 8 & 1 & 0.07 \\
8509 & 8 & 5 & 0.09 \\
8508 & 8 & 5 & 0.08 \\
\hline
\end{tabular}

a Daily maximum 1- or 8-h average value.

b Not applicable. 
an exposure event sequence (EES) for each cohort for the exposure period. Step 4 estimates the pollutant concentration, ventilation rate, and physiological indicator, if applicable, associated with each exposure event. Step 5 extrapolates the cohort exposures to the population of interest and to particular at-risk population groups. Each of these steps is explained in greater detail in the following sections.

\subsubsection{Define the Study Area, Subdivisions of the Study Area, the Exposure Period, and the Population of Interest}

The study area is an aggregation of exposure districts. Each exposure district is a contiguous set of geographic census units (GCUs). Each GCU consists of one or more census tracts, as defined by the 1990 census. No census tract is split between two or more GCUs. All GCUs assigned to a particular exposure district are located within a specified radius $(15 \mathrm{~km})$ of a fixed ozone monitor.

The exposure period is a series of months within a particular calendar year. The months conform to the ozone season specified by the EPA for the urban area. The exposure season is the annual period when high ambient ozone levels are likely to occur. Three ozone seasons are evaluated in this report: January-December, March-September, and April-October. The ozone periods for the nine urban areas are listed in Table 2.

The population of interest is typically defined as either all residents of a specific study area or residents of the study area who belong to the populations at risk.

TABLE 2 Ozone Seasons for the Nine Urban Areas

\begin{tabular}{|c|c|c|}
\hline \multirow[b]{2}{*}{ Study Area } & \multicolumn{2}{|c|}{ Ozone Season } \\
\hline & Calendar Months & $\begin{array}{l}\text { Number } \\
\text { of Days }\end{array}$ \\
\hline Chicago & April - October & 214 \\
\hline Denver & March - September & 214 \\
\hline Houston & January - December & 365 \\
\hline Los Angeles & January - December & 365 \\
\hline Miami & January - December & 365 \\
\hline New York City & April - October & 214 \\
\hline Philadelphia & April - October & 214 \\
\hline St. Louis & April - October & 214 \\
\hline Washington, D.C. & April - October & 214 \\
\hline
\end{tabular}




\subsubsection{Divide the Population of Interest into an Exhaustive Set of Cohorts}

The population of interest is divided into a set of cohorts. Each person is assigned to only one cohort. Each cohort is assumed to contain persons who received identical exposures during the specified exposure period. Cohort exposure is typically assumed to be a function of (1) demographic group, (2) location of residence, and (3) location of workplace. Specifying the demographic group links cohort exposure to activity patterns that vary with age, work status, and other demographic variables. Specifying the home and work district of each cohort links cohort exposure to ambient pollutant concentrations.

The exposure of each cohort is determined by an EES specific to the cohort. Each EES consists of a series of events with durations from 1 to $60 \mathrm{~min}$. To determine average exposures for specific clock hours, exposure events are defined such that no event falls within more than one clock hour. Each exposure event assigns the cohort to a particular combination of geographic area and microenvironment. Each event also indicates respiration rate. The respiration rates are classified as sleeping, slow, medium, and fast.

\subsubsection{Develop an Exposure Event Sequence for Each Cohort for the Exposure Period}

Each EES is determined by assembling activity diary records that relate individual 24-h periods into a series of records that span the ozone season of the associated study area. Because each subject of a typical activity diary study provides data for one to three days, the construction of a multimonth EES requires either repeating data from one subject or using data from multiple subjects. The latter approach is used in $\mathrm{pNEM} / \mathrm{O}_{3}$ analyses because it better represents the variability of exposure expected to occur among persons included in each cohort. The activity diary data used in the general population version of $\mathrm{pNEM} / \mathrm{O}_{3}$ were obtained from CADS - the Cincinnati Activity Diary Study (Johnson 1987). During the study, more than 900 subjects completed three-day activity diaries and detailed background questionnaires. A new diary page was completed whenever the location or activity was changed.

A distinct EES is developed for each cohort. The exposure event within an EES is defined by the district, the microenvironment, and the breathing rate associated with the individual's activity.

The district is defined as the home or work location associated with the cohort. The microenvironments are as follows:

- Indoor residence with a central air conditioning system,

- Indoor residence with window air conditioning units, 
- Indoor residence without an air conditioning system,

- Indoor nonresidential location,

- Outdoor location near a road,

- Outdoors - other locations, and

- In a vehicle.

The $C D$ identifies outdoor workers and outdoor children as population groups particularly at risk for experiencing ozone-related health effects. These groups were identified on the basis of the increased time they spend outdoors engaged in moderate and heavy exertion, which increases the likelihood of experiencing ozone-induced health effects. Although outdoor workers and outdoor children were included in the general population version of $\mathrm{pNEM} / \mathrm{O}_{3}$, EPA analysts felt that the procedures used did not adequately represent exposures for workers or children who regularly spend considerable time outdoors. Therefore, special versions of $\mathrm{pNEM} / \mathrm{O}_{3}$ were developed to estimate population exposures for outdoor workers and outdoor children.

For the outdoor worker version of $\mathrm{pNEM} / \mathrm{O}_{3}$, additional data from six time/activity studies were combined with the CADS database and processed to form a unified time/activity database representative of outdoor workers. The activity data selected to represent outdoor workers were based on data from subjects who spend at least $4 \mathrm{~h}$ at work and at least $50 \%$ of their work time outdoors. The final pool contained 89 outdoor workers with 136 person-days of diary data. City-specific outdoor worker estimates were derived on the basis of city-specific 1990 census data and judgments by a panel of researchers about the percentage of outdoor workers in each of 37 census occupation groups. Section 6 of Johnson et al. (1996b) describes in detail the procedures used to develop the outdoor worker time/activity database and population extrapolation.

For the outdoor children version of $\mathrm{pNEM} / \mathrm{O}_{3}$, additional data from six other time/ activity studies were combined with the CADS database and processed to form a unified time/activity database representative of outdoor children. The pool of activity patterns used to represent outdoor children was based on data for children who met the following conditions:

- During a non-summer weekday, the child had at least one diary day on which he or she spent $\geq 2 \mathrm{~h}$ outdoors, or

- During a non-summer weekend, the child had at least one diary day on which he or she spent $\geq 3 \mathrm{~h}$ outdoors, or 
- During a summer weekday or weekend, the child had at least one diary day on which he or she spent $\geq 4.5 \mathrm{~h}$ outdoors.

For this analysis, "summer" was defined as June through August and "non-summer" as all other months. This procedure produced a pool of 479 outdoor children with 792 person-days of activity diary data. Outdoor children included in the analysis fell into two demographic groups: 6- to 13-year-old children ("preteens") and 14- to 18-year-old children ("teens"). The city-specific percentages of outdoor children were derived on the basis of city-specific 1990 census data for the two demographic groups and the percentages of outdoor preteens and teens in three of the time/activity studies conducted in Cincinnati and California that used a random selection procedure to enroll subjects (Johnson 1987; Wiley et al. 1991a,b). About 47\% of preteens and $31 \%$ of teens met the selection criteria for outdoor children.

\subsubsection{Estimate the Pollutant Concentration and Ventilation Rate Associated with Each Exposure Event}

The year-long sequence of hourly average ozone values for indoor and in-vehicle microenvironments is generated by a mass balance algorithm. This algorithm estimates the hourly average indoor ozone concentrations during the hour as a function of (1) indoor ozone concentration at the end of the preceding hour, (2) outdoor ozone concentration during the hour, (3) air exchange rate (AER) during the hour, and (4) ozone decay factor. Values for the AER and the ozone decay factor are sampled from an appropriate distribution on a daily basis. The AER is permitted to change hourly in the three residential microenvironments; it changes based on window status (open or closed). A probabilistic model assigns a window status each clock hour. The assignment is assumed to be a function of air conditioning system, temperature range, and window status during the previous clock hour. In the $\mathrm{pNEM} / \mathrm{O}_{3}$ simulation, the ozone concentration in a microenvironment during a particular clock hour is assumed to be constant. For indoor and in-vehicle microenvironments, this value is determined by using a mass balance model to calculate average ozone concentration for the clock hour under the following conditions:

- Indoor sources of ozone are not present.

- The indoor ozone concentration at the end of the preceding hour is known.

- The outdoor ozone concentration during the clock hour is constant and known.

- The AER during the clock hour is constant at a specified value.

- Ozone decays at a rate proportional to the indoor ozone concentration. 
The $\mathrm{pNEM} / \mathrm{O}_{3}$ mass balance model requires representative ambient air quality data for each exposure district in the form of a time series that contains one value for each hour in the specified ozone season. From 6 to 16 monitoring sites are selected to represent the geographic variation of ozone levels in each of 9 study areas. The number of monitors chosen in an area depends on a data completeness criterion for each monitor and whether home-to-work data are available for a district.

All monitors reporting valid data (i.e., $75 \%$ complete) in an urban area are used to characterize ozone air quality. In no case is an area with fewer than three valid stations included because the ozone concentration surface cannot be computed from only two monitors. If an area has numerous monitors, the total number is capped at 16 because of limitations on computational resources. This case occurs only in the Los Angeles urban area, where 30 possible monitors were pared to 16 by removing one of the nearby pairs of monitors that had similar ozone air quality distributions. Otherwise, all monitors in an area were used..$^{5}$

- After the number of monitors was chosen, the districts were defined. Districts must be nonoverlapping areas associated with one monitor. In addition, home-to-work trip information must be available to include a district in a $\mathrm{pNEM} / \mathrm{O}_{3}$ study area. If this information is not available, the district is removed from further analysis. This restriction excludes low-density rural areas from $\mathrm{pNEM} / \mathrm{O}_{3}$ exposure assessments.

Ventilation rate depends on the type of activity in which individuals are engaged. The $\mathrm{pNEM} / \mathrm{O}_{3}$ exposure probabilities used in this analysis were computed only for individuals who reached the following ventilation rates corrected for (i.e., divided by) body surface area - called equivalent ventilation rates (EVRs):

- $\geq 30$ EVR for 1-h exposures at heavy exertion,

- 16-30 EVR for 1-h exposures at moderate exertion, and

- 13-27 EVR for 8-h exposures at moderate exertion.

\subsubsection{Extrapolate the Cohort Exposures to the Population of Interest and to At-Risk Groups}

The final step extrapolates the cohort exposures to the population of interest and to at-risk groups. The cohort-specific exposure estimates are extrapolated to the general population

5 The only exception was in the New York City study area, where one monitor (the World Trade Center) was replaced with a nearby monitor because air quality data from the World Trade Center were not considered to be representative of breathed air (i.e., the monitors were too high to be of use). 
of each study area by estimating the population size of each cohort. A three-step procedure estimates cohort populations:

- Estimate by census tract the number of persons associated with each demographic group.

- Estimate by census tract the fraction of homes falling into each of the three air conditioning categories.

- Multiply the demographic group populations by the air conditioning fractions to estimate the number of persons in each combination of demographic group and air conditioning category. This step gives the cohort population estimates.

An iterative adjustment procedure simulates just attaining the eight alternative NAAQS. This procedure involves fitting Weibull equations to the As-Is ozone ambient monitoring data, modifying parameters of the equations so that the "worst" monitor achieves the desired standard, and respecifying the entire ozone pattern for all districts in an urban area. The adjustment procedure and the exposure analysis methodology are discussed in detail in the individual exposure analysis reports (Johnson et al. 1996a-c).

The $\mathrm{pNEM} / \mathrm{O}_{3}$ contains many stochastic variables; therefore, exposure estimates vary from run to run. For the general population, outdoor worker, and outdoor children exposure analyses, the $\mathrm{pNEM} / \mathrm{O}_{3}$ was simulated 10 times for each scenario in each of the 9 urban areas to better characterize the uncertainty in the exposure estimates. On the basis of a previous analysis of sets of 10-run results compared with a 108-run result, McCurdy (1994a) showed that results from only 10 runs adequately predict the mean and variance observed in 100 or more runs of $\mathrm{pNEM} / \mathrm{O}_{3}$. Additional runs of the model would increase the range of possible outcomes, but limited resources preclude undertaking more runs.

In any $\mathrm{pNEM} / \mathrm{O}_{3}$ analysis, several different indicators estimate exposure of people to various levels of air pollution. One indicator of population exposure is "people-exposed" (i.e., the number of people who experience a given level of air pollution, or higher, at least once during the analysis). Another indicator is "occurrences of exposure" (i.e., the number of times individuals from the population of interest experience a given level of pollution; individuals can be counted more than once in an ozone season).

The model estimates exposure in terms of highest concentrations (exposures) or highest dose. The exposure estimates summarized here pertain to "daily maximum dose" (DMAX), where dose is the product of ozone concentration and ventilation rate over a defined period. The DMAX does not necessarily occur at the same time as the maximum ozone concentration in a given urban area. The DMAX indicator was selected because it is a better surrogate for the number of 
ozone molecules that enter the oral-nasal cavities per unit time period. Therefore, it is likely to be more relevant from a health risk viewpoint than is maximum concentration exposure.

A number of caveats must be acknowledged concerning the $\mathrm{pNEM} / \mathrm{O}_{3}$ results. Probably the most significant caveat is that considerable uncertainty exists about a number of important inputs to the model. Listed below are the most important caveats and limitations in the current versions of the exposure model:

- The algorithm used to construct a season-long EES for each cohort is constrained because none of the available time/activity studies provides more than three days of diary data for any one subject. To maximize use of the available diary data, the $\mathrm{pNEM} / \mathrm{O}_{3}$ sequencing algorithm constructs each EES by sampling data from more than one subject.

- The subjects who contributed to the human activity database may not provide a balanced representation of U.S. outdoor children or outdoor workers. Most of the subjects resided in either California or Cincinnati. Although the algorithm that constructs EESs attempts to account for the effects of local climate on activity, it is unlikely that this adjustment procedure corrects for all intercity differences in outdoor children's or outdoor workers' activities. Time/activity patterns are likely to be affected by many local factors, including topography, land use, traffic patterns, mass transit systems, and recreational opportunities.

- The average subject in the time/activity studies provided less than two days of diary data. For this reason, the construction of each season-long EES required either repeating data from one subject or using data from multiple subjects. The latter approach was used in the outdoor children and outdoor worker $\mathrm{pNEM} / \mathrm{O}_{3}$ analyses to better represent the variability of exposure expected to occur among the children in each cohort. The main deficiency of this approach is that it may not adequately account for the day-to-day repetition of activities common to individual children or workers. Consequently, $\mathrm{pNEM} / \mathrm{O}_{3}$ may underestimate the number of people who experience multiple occurrences of high ozone exposure. For example, the outdoor children analysis does not adequately reflect exposures for children who attend residential summer camps because this activity pattern is not included in the database used in the outdoor children exposure analysis.

- Exposure estimates are presented separately for outdoor children, outdoor workers, and the general population and are not aggregated. Any aggregation would have to adjust the general population exposure estimates to avoid double counting exposures for workers and children. 
- The algorithm that assigns the EVR associated with each exposure is based on an analysis of data from several studies conducted by Dr. Hackney and associates in Los Angeles. Because of the small sample sizes (e.g., 39 children and 36 outdoor workers) in these studies and the lack of subjects below age 10 or above age 50 , uncertainty cannot be quantified about these EVR estimates. The pNEM/O ${ }_{3}$ model also uses an EVRlimiting algorithm that limits the maximum EVR that can be maintained for a given duration by an individual who exercises regularly at a high ventilation rate. This algorithm allows a small probability that the EVR chosen is higher than that seen for some individuals in the population analyzed, since not all individuals exercise regularly at a high ventilation rate.

- The air quality adjustment procedures used to simulate just attaining alternative NAAQS were based on statistical analyses of ozone data from sites that experienced moderate reductions in ozone levels during the 1980s. These procedures assume that (1) the Weibull distribution provides a good fit to most ozone data, and (2) the parameters of the Weibull distribution fitting data from a particular monitoring site will change over time in a predictable fashion. Because of the empirical basis for the adjustment procedure, less confidence is placed in the predicted air quality levels for just attaining alternative standards in Los Angeles, where significant reductions would have to take place to attain any of the alternative standards analyzed. Uncertainty also exists about the impact on the spatial pattern and shape of ozone air quality distributions if any significant changes in control strategies were to be made to attain the ozone NAAQS in the future. Some regional oxidant modeling work is under way to examine the possible impact of alternative control strategies on the spatial and temporal pattern of ozone concentrations.

- The $\mathrm{pNEM} / \mathrm{O}_{3}$ model uses a mass balance model to estimate ozone levels in residential buildings (windows open or closed), nonresidential buildings, and inside vehicles. For some of these microenvironments, the database on AERs and ozone decay rates, which are key inputs to the mass balance model, is rather sparse. For example, the AER and ozone decay rate for vehicles is a point estimate based on data for a single vehicle. In contrast, data on AER values for residential buildings with closed windows are based on a lognormal distribution fit to AER data from 312 residences across the United States. The uncertainties about ozone levels in these "indoor" microenvironments should not significantly affect exposure estimates at moderate and high exertion, where exposure levels exceed $0.08 \mathrm{ppm}$, because these levels are likely due to outdoor exposures. 


\subsection{EXAMPLE OF THE EXPOSURE ESTIMATES USED IN THE RISK ASSESSMENT}

The $\mathrm{pNEM} / \mathrm{O}_{3}$ exposure estimates were generated for the nine urban areas listed in Table 2 for the total population, outdoor children, and outdoor workers. The $\mathrm{pNEM} / \mathrm{O}_{3}$ output for the ozone concentrations given in Table 3 includes results for 10 runs. The ozone concentrations of interest for calculating exposure-response relationships are listed in Table 4 . These concentrations are approximately the midpoints of the intervals listed in Table 3.

This risk assessment focuses on risk estimates in excess of background concentrations. "Background" ozone is defined for the purposes of this analysis as the ozone concentrations that would be observed in the United States in the absence of anthropogenic precursor emissions of volatile organic compounds and nitrogen oxides in North America (EPA 1996a). The CD (EPA 1996b) estimates that during the summer ozone season in the United States, the typical background daily maximum $1-\mathrm{h}$ level is between 0.03 and $0.05 \mathrm{ppm}$.

Exposure-response probabilities for each run are computed by dividing the number of children at each ozone concentration by the number of children who reached a specific exertion level in a run. For example, the data in Table 5 (which are for just attaining standard 1112, Philadelphia, outdoor children, 8-h exposures at moderate exertion, $\mathrm{pNEM} / \mathrm{O}_{3}$ run 2) show that the number of children for this particular run is 268,569. A total of 37,953 children were exposed to ozone at concentrations of $0.041-0.060 \mathrm{ppm}$. Dividing this number by the total number of children at moderate exertion results in a probability of 0.1413 . The same computations are performed for the remaining ozone concentrations. The sum of the calculated probabilities does not equal 1 because exposures at estimated background or lower are not included. The probabilities for this example are presented in Table 5. Such probabilities are needed to estimate . risk distributions (Section 5). 
TABLE 3 Ozone Concentration Intervals for Which $\mathrm{pNEM} / \mathrm{O}_{3}$ Data Have Been Generated

\begin{tabular}{cc}
\hline 1-h Exposures & 8-h Exposures \\
\hline 0.000 & 0.000 \\
$0.001-0.020$ & $0.001-0.020$ \\
$0.021-0.040$ & $0.021-0.040$ \\
$0.041-0.060$ & $0.041-0.060$ \\
$0.061-0.080$ & $0.061-0.070$ \\
$0.081-0.100$ & $0.071-0.080$ \\
$0.101-0.120$ & $0.081-0.090$ \\
$0.121-0.140$ & $0.091-0.100$ \\
$0.141-0.160$ & $0.101-0.110$ \\
$0.161-0.180$ & $0.111-0.120$ \\
$0.181-0.200$ & $0.121-0.130$ \\
$0.201-0.220$ & $0.131-0.140$ \\
$0.221-0.240$ & $0.141-0.150$ \\
$0.241-0.260$ & $0.151-0.160$ \\
$0.261-0.280$ & $0.161-0.170$ \\
$0.281-0.300$ & $0.171-0.180$ \\
$0.301-0.320$ & $0.181-0.190$ \\
$0.321-0.340$ & $0.191-0.200$ \\
$0.341-0.360$ & $0.201+$ \\
$0.361-0.380$ & \\
$0.381-0.400$ & \\
$0.401+$ & \\
\hline
\end{tabular}

TABLE 4 Ozone Concentrations for Which Response Rates Need to Be Estimated (ppm) ${ }^{a}$

1-h Exposures 8-h Exposures

$\begin{array}{ll}0.040^{\mathrm{b}} & 0.040^{\mathrm{b}} \\ 0.051 & 0.051 \\ 0.071 & 0.066 \\ 0.091 & 0.076 \\ 0.111 & 0.086 \\ 0.131 & 0.096 \\ 0.151 & 0.106 \\ 0.171 & 0.116 \\ 0.191 & 0.126 \\ 0.211 & 0.136 \\ 0.231 & 0.146 \\ 0.251 & 0.156 \\ 0.271 & 0.166 \\ 0.291 & 0.176 \\ 0.311 & 0.186 \\ 0.331 & 0.196 \\ 0.351 & \\ 0.371 & \\ 0.391 & \end{array}$

a Except for $0.040 \mathrm{ppm}$, these concentrations are approximately the midpoints of intervals $>0.040 \mathrm{ppm}$ listed in Table 3.

b The estimated short-term background level for ozone is $0.040 \mathrm{ppm}$. 
TABLE 5 Calculating Exposure Probabilities for Outdoor Children Exposed for Eight Hours at Moderate Exertion from pNEM/O ${ }_{3}$ Estimates for Run 2, Philadelphia, Scenario 1112, Just Attained

\begin{tabular}{|c|c|c|c|}
\hline \multirow[b]{2}{*}{$\begin{array}{l}\text { Ozone Interval } \\
\qquad(\mathrm{ppm})\end{array}$} & \multicolumn{2}{|c|}{ Number of Children } & \multirow[b]{2}{*}{ Probability $^{c}$} \\
\hline & $\begin{array}{l}\text { In Interval } \\
\text { or Higher }\end{array}$ & In Interval ${ }^{b}$ & \\
\hline 0.000 & 268,569 & 0 & $N^{d}$ \\
\hline $0.001-0.020$ & 268,569 & 1,322 & $\mathrm{NR}$ \\
\hline $0.021-0.040$ & 267,247 & 17,644 & NR \\
\hline $0.041-0.060$ & 249,603 & 37,953 & 0.1413 \\
\hline $0.061-0.070$ & 211,650 & 46,879 & 0.1746 \\
\hline $0.071-0.080$ & 164,771 & 101,972 & 0.3797 \\
\hline $0.081-0.090$ & 62,799 & 50,181 & 0.1868 \\
\hline $0.091-0.100$ & 12,618 & 11,338 & 0.0422 \\
\hline $0.101-0.110$ & 1,280 & 1,280 & 0.0048 \\
\hline $0.111-0.120$ & 0 & 0 & 0.0000 \\
\hline $0.121-0.130$ & 0 & 0 & 0.0000 \\
\hline $0.131-0.140$ & 0 & 0 & 0.0000 \\
\hline $0.141-0.150$ & 0 & 0 & 0.0000 \\
\hline $0.151-0.160$ & 0 & 0 & 0.0000 \\
\hline $0.161-0.170$ & 0 & 0 & 0 \\
\hline $0.171-0.180$ & 0 & 0 & 0 \\
\hline $0.181-0.190$ & 0 & 0 & 0 \\
\hline $0.191-0.200$ & 0 & 0 & 0 \\
\hline $0.201+$ & 0 & 0 & 0 \\
\hline
\end{tabular}

a A total of 268,569 children reached a moderate exertion level in run 2.

b Number in interval $i$ equals number in interval $i$ or higher minus the number in interval $i+1$ or higher (e.g., $1,322=268,569$. 267,247).

c Probability of interval $i$ equals the number in interval $i$ divided by 268,569 . This probability is also the fraction of children who reached a moderate exertion level while exposed to the ozone concentration for interval $i$ in run 2 .

d NR means not required. Calculations were not made for these concentrations because they are less than or equal to the estimated background level (0.04 ppm). 


\section{EXPOSURE-RESPONSE DATA AND RELATIONSHIPS}

This section describes sources of information (data) about acute health effects attributable to ozone exposure and lists the mathematical functions fit to the data to represent exposure-response relationships. Acute health effects include changes in lung function (i.e., decreases in forced expiratory volume) and the onset of various symptoms (e.g., cough, chest pain on deep inspiration). The information is used to develop a series of models. The models for acute effects are based on controlled human exposure studies. ${ }^{6}$ The criteria used to select the laboratory studies are discussed, and the individual studies used for the acute health effects models are described.

Although the controlled human exposure studies discussed in this section only included 18- to 35-year-old adults, exposure-response relationships derived from these studies are applied to both outdoor children and outdoor workers. Recent findings support the use of adult-based results to describe children. These findings include results from other chamber studies (e.g., McDonnell et al. 1985a) and summer camp field studies in at least six different locations in northeastern United States, Canada, and southern California (Kinney et al. 1996). These locations reported changes in lung function in healthy children similar to those observed in healthy adults exposed to ozone under controlled chamber conditions. As stated in the $C D$, "although direct comparisons cannot be made because of incompatible differences in experimental design and analytical approach," the range of response in the summer camp studies "is comparable to the range of response seen in chamber studies at low $\mathrm{O}_{3}$ concentrations" (EPA 1996b, pp. 9-7 and 9-8).

\subsection{CRITERIA FOR SELECTING LABORATORY DATA}

Controlled human exposure studies are thought to be most appropriate for developing exposure-response relationships for acute health effects that result from exposure to ozone. Controlled human exposure studies based on 1-, 2-, and 6.6-h exposures are used to develop the health models. The following criteria were used to select the particular studies:

- Applicability to the population groups potentially at greatest risk. Studies of persons exposed while engaged in moderate or heavy exertion are of greatest interest, because such subjects are thought to be at greater risk than those at rest.

- Comparability. The total dose must be compared with the level of exertion and the exercise protocol of particular interest.

${ }^{6}$ Hospital admissions (Section 6) are also acute effects; however, the model described in Section 6 is not based on controlled human exposure studies. 
- Number of subjects. To limit the effects of small sample size, studies with at least 10 subjects per exposure level are desired.

- Exposure concentrations. Studies with multiple concentration levels in the range of ambient levels are desired.

- Individual subject data. These data are needed to develop exposureresponse relationships.

\subsection{DISCUSSION OF 1-HOUR DATA FOR POPULATIONS ENGAGED IN HEAVY EXERTION}

Exposure-response relationships for populations engaged in heavy exertion are based on controlled human exposure studies (McDonnell et al. 1983; Avol et al. 1984; Kulle et al. 1985). These studies provide sufficient information to define nine health endpoints for risk calculations: (1) decreases in forced expiratory volume in $1 \mathrm{~s}\left(\mathrm{DFEV}_{1}\right)^{7}$ that are $\geq 10 \%\left(\mathrm{DFEV}_{1} \geq 10 \%\right)$, (2) decreases in forced expiratory volume in $1 \mathrm{~s}$ that are $\geq 15 \%$ (DFEV $1 \geq 15 \%$ ), (3) decreases in forced expiratory volume in $1 \mathrm{~s}$ that are $\geq 20 \%\left(\mathrm{DFEV}_{1} \geq 20 \%\right.$ ), (4) any cough (ACF), (5) any chest pain on deep inspiration (APD), (6) any lower respiratory symptom (ALR), (7) moderate or severe cough (MCF), (8) moderate or severe chest pain on deep inspiration (MPD), and (9) moderate or severe lower respiratory symptoms (MLR). None of the studies supports all of the endpoints. Table 6 indicates the endpoints associated with each study, and Table 7 summarizes the characteristics of each study.

TABLE 6 Health Endpoints Associated with Each Study

\begin{tabular}{cccc}
\hline Endpoint & $\begin{array}{c}\text { McDonnell et al. } \\
(1983)\end{array}$ & $\begin{array}{c}\text { Avol et al. } \\
(1984)\end{array}$ & $\begin{array}{c}\text { Kulle et al. } \\
(1985)\end{array}$ \\
\hline$D_{\text {FEV }} \geq 10 \%$ & Yes & Yes & Yes \\
$\mathrm{DFEV}_{1} \geq 15 \%$ & Yes & Yes & Yes \\
$\mathrm{DFEV}_{1} \geq 20 \%$ & Yes & Yes & Yes \\
ACF & Yes & No & Yes \\
APD & Yes & No & Yes \\
ALR & No & Yes & No \\
MCF & Yes & No & Yes \\
MPD & Yes & No & Yes \\
MLR & No & Yes & No \\
\hline
\end{tabular}

7 Forced expiratory volume in $1 \mathrm{~s}$ is the maximum volume of air that an individual can expel from the lungs in $1 \mathrm{~s}$. 
TABLE 7 Summary of the Studies Used in Developing 1-h Exposure-Response Relationships for Populations Engaged in Heavy Exertion

\begin{tabular}{|c|c|c|c|}
\hline Study Protocol & Avol et al. (1984) & Kulle et al. (1985) & McDonnell et al. (1983) \\
\hline Number of subjects & $\begin{array}{l}50 \text { bicyclists: } 42 \text { male } \\
\text { and } 8 \text { female; } \\
\text { complete data were } \\
\text { available for } 48 \text { of the } \\
\text { subjects }\end{array}$ & $\begin{array}{l}20 \text { healthy males; } \\
8 \text { of } 20 \text { subjects } \\
\text { exposed to } \\
0.30 \text { ppm }^{\mathrm{a}}\end{array}$ & $\begin{array}{l}135 \text { healthy males; } \\
\text { complete data were } \\
\text { available for } 132 \text { of the } \\
\text { subjects }\end{array}$ \\
\hline $\begin{array}{l}\text { Exposure concentrations } \\
\text { (ppm) }\end{array}$ & $\begin{array}{l}0.00,0.08,0.16,0.24 \\
\text { and } 0.32\end{array}$ & $\begin{array}{l}0.00,0.10,0.15 \\
0.20,0.25, \text { and } 0.30\end{array}$ & $\begin{array}{l}0.00,0.12,0.18,0.24 \\
0.30, \text { and } 0.40\end{array}$ \\
\hline $\begin{array}{l}\text { Ventilation rate } \\
\text { (L/min) }\end{array}$ & $57.6 \pm 12.5$ & $67.8 \pm 8.2$ & $65.6 \pm 7.4$ \\
\hline $\operatorname{EVR}^{c}\left(\mathrm{~L} / \mathrm{min} / \mathrm{m}^{2}\right)$ & 30.3 & 35.7 & $34.3 \pm 3.1$ \\
\hline Exercise pattern & $\begin{array}{l}\text { Continuous (10-min } \\
\text { warm-up, } 60 \text { min of } \\
\text { continuous exercise, } \\
10 \text {-min cooldown and } \\
\text { measurement) }\end{array}$ & $\begin{array}{l}\text { Intermittent ( } 4 \text { cycles } \\
\text { of } 14 \mathrm{~min} \text { of exercise } \\
\text { and } 16 \mathrm{~min} \text { of rest) }\end{array}$ & $\begin{array}{l}\text { Intermittent ( } 4 \text { cycles of } \\
15 \text { min of exercise and } \\
15 \mathrm{~min} \text { of rest) }\end{array}$ \\
\hline $\begin{array}{l}\text { Exercise duration } \\
\text { (heavy) (min) }\end{array}$ & 60 & 56 & 60 \\
\hline Exposure duration (h) & 1.33 & 2 & $2.5^{\mathrm{d}}$ \\
\hline Subject exposures & $\begin{array}{l}\text { Exposed to all } \\
\text { concentrations }\end{array}$ & $\begin{array}{l}\text { Exposed to all } \\
\text { concentrations }\end{array}$ & $\begin{array}{l}\text { Divided about equally } \\
\text { into } 6 \text { groups, each } \\
\text { exposed to a single } \\
\text { concentration }\end{array}$ \\
\hline
\end{tabular}

a Data for exposures of $0.30 \mathrm{ppm}$ not reported in Kulle et al. (1985) were taken from Hayes et al. (1987).

b Mean \pm standard deviation; averages of group (based on ozone concentration) means.

c Estimated for Avol et al. and Kulle et al. by dividing ventilation rate by $1.9 \mathrm{~m}^{2}$, the approximate human body surface area, to obtain equivalent liters per minute; calculated for McDonnell et al. from available data.

d Includes a final 30-min period during which subjects rested, and spirometric and symptoms measurements were made.

The exercise protocols differed significantly among the three studies. The Avol et al. study required 60 min of continuous exercise with no rest; the Kulle et al. study consisted of 4 cycles of $14 \mathrm{~min}$ of treadmill exercise and $16 \mathrm{~min}$ of rest; and the McDonnell et al. study required 4 cycles of $15 \mathrm{~min}$ of exercise and $15 \mathrm{~min}$ of rest. The mean ventilation rates varied 
from 58 to $68 \mathrm{~L} / \mathrm{min}$. These rates compare favorably with $30 \mathrm{~L} / \mathrm{min} / \mathrm{m}^{2}$, the lower bound of the range on EVR used to define heavy exertion for $1-\mathrm{h}$ exposures in $\mathrm{pNEM} / \mathrm{O}_{3}$. The same individuals were exposed at all concentration levels in the Avol et al. and Kulle et al. studies, whereas different subjects were exposed at each level in the McDonnell et al. study. The number of subjects ranged from 20 to 135 . Females and males participated in the Avol et al. study; only males participated in the Kulle et al. and McDonnell et al. studies.

Kulle et al. and McDonnell et al. inquired about cough and chest pain on deep inspiration. Avol et al. inquired about lower respiratory, upper respiratory, and nonrespiratory symptoms. Subjects in the Kulle et al. and McDonnell et al. studies ranked individual symptoms separately as either none, mild, moderate, or severe. The Avol et al. subjects rated symptom severity as none; mild, but noticeable only upon questioning; mild; moderate; severe; or incapacitating.

Because of the significant differences among the studies, each study is treated separately in this risk assessment. Retaining the identity of each study for risk calculations makes it possible to investigate an additional source of uncertainty attributable to differences among the studies. In spite of the differences, these studies were matched with 1-h exposures for two related reasons. First, the duration of the exercise for all three studies is about $1 \mathrm{~h}$. Second, McKittrick and Adams (1995) reported that for producing $\mathrm{FEV}_{1}$ decrements in healthy individuals, 2-h ozone exposures under intermittent heavy exercise are nearly equivalent to 1-h ozone exposures under continuous exercise.

\subsection{DISCUSSION OF 1-HOUR DATA FOR POPULATIONS ENGAGED IN MODERATE EXERTION}

Exposure-response relationships for populations engaged in moderate exertion are based on the Seal et al. (1993) study. The following health endpoints were derived from the laboratory data: (1) $\mathrm{DFEV}_{1} \geq 10 \%$, (2) $\mathrm{DFEV}_{1} \geq 15 \%$, (3) $\mathrm{DFEV}_{1} \geq 20 \%$, (4) ACF, (5) MCF, (6) APD, and (7) MPD.

Unlike other studies in which only males were exposed, this study exposed Caucasian and African-American males and females. In total, 372 subjects, 18-35 years old, were exposed once for $2.33 \mathrm{~h}$ to ozone concentrations of $0.00,0.12,0.18,0.24,0.30$, or $0.40 \mathrm{ppm}$. Potential subjects were excluded from the study for any of the following reasons: family histories of cardiovascular disease; personal history of chronic cardiovascular or chronic respiratory disease; an acute respiratory infection within six weeks of the study; deficiency in glucose-6-phosphate dehydrogenase; sickle-cell disease; pregnancy; use of birth control pills within two weeks of the study; use of marijuana one week before exposure; consumption of alcohol $24 \mathrm{~h}$ before exposure; or smoking more than one pack of cigarettes per year within the five years preceding the study. 
Before exposure, subjects completed baseline pulmonary function tests and symptoms questionnaires. The first $2 \mathrm{~h}$ of exposure alternated 15 -min periods of rest with 15 -min periods of exercise on a treadmill. Pulmonary function tests and questionnaires were re-administered after exposure. The questions asked about the presence of a cough, shortness of breath, pain on deep inspiration, headache, eye itching, and sweating. The symptoms were rated as 1 (none), 2 (mild), 3 (moderate), or 4 (severe). The only symptoms analyzed in this report are cough and chest pain on deep inspiration. Table 8 summarizes the study.

TABLE 8 Summary of the Study Used to Develop 1-h Exposure-Response Relationships for Populations Engaged in Moderate Exertion

\begin{tabular}{|c|c|}
\hline Study Protocol & Seal et al. (1993) \\
\hline Number of subjects & $\begin{array}{l}372 \text { African-American and } \\
\text { Caucasian males and females }\end{array}$ \\
\hline $\begin{array}{l}\text { Exposure } \\
\text { concentration (ppm) }\end{array}$ & $\begin{array}{l}0.00,0.12,0.18,0.24,0.30 \text {, or } \\
0.40\end{array}$ \\
\hline $\begin{array}{l}\text { Mean ventilation rate } \\
(\mathrm{L} / \mathrm{min})\end{array}$ & 45 \\
\hline $\mathrm{EVR}^{\mathrm{b}}\left(\mathrm{L} / \mathrm{min} / \mathrm{m}^{2}\right)$ & $23.8 \pm 2.8$ \\
\hline Exercise pattern & $\begin{array}{l}\text { Intermittent ( } 4 \text { periods of } 15 \text {-min } \\
\text { exercise, } 15 \text {-min rest) }\end{array}$ \\
\hline Exercise duration $(\mathrm{h})$ & 1 \\
\hline Exposure duration (h) & $2.33^{c}$ \\
\hline Subject exposures & $\begin{array}{l}\text { About } 60 \text { subjects exposed at } \\
\text { each level; each subject exposed } \\
\text { to only } 1 \text { concentration level }\end{array}$ \\
\hline
\end{tabular}

a Calculated from mean EVR by multiplying by $1.9 \mathrm{~m}^{2}$, the approximate body surface area.

b Mean \pm standard deviation; averages of group means.

c Includes a final 20-min period during which subjects rested, and spirometric and symptom measurements were made. 


\subsection{DISCUSSION OF 8-HOUR DATA FOR POPULATIONS ENGAGED IN MODERATE EXERTION}

The Folinsbee et al. (1988), Horstman et al. (1990), and McDonnell et al. (1991) studies provided laboratory data for exposure-response relationships for 8-h exposures. These studies were conducted in EPA's controlled human exposure chambers in Chapel Hill, North Carolina. The data from the studies are combined and treated as a single study because the studies are similar with respect to exercise protocols, recruitment of subjects, ozone concentrations to which subjects were exposed, grouping of symptoms, and rating of symptom severity. The health endpoints developed from the combined studies for use in the risk calculations include (1) $\mathrm{DFEV}_{1} \geq 10 \%$, (2) $\mathrm{DFEV}_{1} \geq 15 \%$, (3) $\mathrm{DFEV}_{1} \geq 20 \%$, (4) ACF, (5) MCF, (6) APD, and (7) MPD. Table 9 summarizes the studies.

TABLE 9 Summary of the Studies Used to Develop 8-h Exposure-Response Relationships

\begin{tabular}{|c|c|c|c|}
\hline Study Protocol & Folinsbee et al. (1988) & Horstman et al. (1990) & McDonnell et al. (1991) \\
\hline Number of subjects & 10 nonsmoking males & 22 nonsmoking males & 38 nonsmoking males \\
\hline $\begin{array}{l}\text { Exposure } \\
\text { concentration (ppm) }\end{array}$ & 0.00 or 0.12 & $\begin{array}{l}0.00,0.08,0.10, \text { or } \\
0.12\end{array}$ & $0.00,0.08$, or 0.10 \\
\hline $\begin{array}{l}\text { Ventilation rate } \\
\text { (L/min) }\end{array}$ & $39-42^{\mathfrak{a}}$ & $37-41^{a}$ & $\begin{array}{l}(40.3,40.5,39.6) \pm(4.3 \\
4.3,6.3)^{b}\end{array}$ \\
\hline $\operatorname{EVR}\left(\mathrm{L} / \mathrm{min} / \mathrm{m}^{2}\right)$ & $20.5-22.1^{c}$ & $19.5-21.6^{\mathrm{C}}$ & $\begin{array}{l}(20.1,20.2,19.9) \pm(1.8 \\
1.8,2.3)^{b}\end{array}$ \\
\hline Exercise pattern & $\begin{array}{l}50 \mathrm{~min} \text { of exercise, } \\
10 \mathrm{~min} \text { of rest for each } \\
\text { hour, and } 35 \mathrm{~min} \text { of rest } \\
\text { after } 3 \mathrm{~h}\end{array}$ & $\begin{array}{l}50 \mathrm{~min} \text { of exercise, } \\
10 \mathrm{~min} \text { of rest for each } \\
\text { hour and } 35 \mathrm{~min} \text { of rest } \\
\text { after } 3 \mathrm{~h}\end{array}$ & $\begin{array}{l}50 \mathrm{~min} \text { of exercise, } 10 \mathrm{~min} \\
\text { of rest for each hour and } \\
35 \mathrm{~min} \text { of rest after } 3 \mathrm{~h}\end{array}$ \\
\hline Exercise duration (h) & 5 & 5 & 5 \\
\hline Exposure duration (h) & 6.6 & 6.6 & 6.6 \\
\hline Subject exposures & $\begin{array}{l}\text { Exposed to all } \\
\text { concentrations }\end{array}$ & $\begin{array}{l}\text { Exposed to all } \\
\text { concentrations, except } \\
\text { for } 1 \text { subject who } \\
\text { experienced respiratory } \\
\text { problems at } 0.10 \mathrm{ppm}\end{array}$ & $\begin{array}{l}28 \text { subjects exposed to } 0.00 \\
\text { and } 0.08 \mathrm{ppm} ; 10 \text { subjects } \\
\text { exposed to } 0.00,0.08 \text {, and } \\
0.10 \mathrm{ppm}\end{array}$ \\
\hline
\end{tabular}

a Range of group means.

b Means \pm standard deviation for $0.00,0.08$, and $0.10 \mathrm{ppm}$, respectively.

c Range of group means estimated by dividing the ventilation rate by $1.9 \mathrm{~m}^{2}$, the approximate human body surface area, to obtain equivalent liters per minute. 
The 10 subjects, 18-33 years old, for the Folinsbee et al. (1988) study were exposed once to clean air and once to an ozone concentration of $0.12 \mathrm{ppm}$. These sessions were separated by a minimum of 1 week, and the exposure sequence was randomized. The exercise protocol included 6 sessions of 50 min of exercise on a treadmill or cycle ergometer. Each session was followed by a 10-min rest and measurement period. Each subject was given an additional $35 \mathrm{~min}$ to eat lunch after the third period. Subjects received physiologic testing before exposure, during and after each of the 6 exercise periods, and at the end of exposure. Subjects were asked to evaluate cough, pain on deep inspiration (PDI), eye irritation, and pain/discomfort in the legs. Symptoms were rated as 0 (none), 1 (just noticeable), 2 (mild discomfort), 3 (moderate discomfort), or 4 (severe discomfort/pain). The rating scale for cough was 0 (none), 1 (cough during spirometry only), 2 (spontaneous single cough), 3 (spontaneous. multiple cough), and 4 (repeated spontaneous multiple cough).

Twenty-two males participated in the Horstman et al. (1990) study. Candidates were not allowed to participate if they had a condition that could worsen as a result of ozone exposure or exercise, or if they had an acute respiratory infection within the previous 4 weeks. Exposures were separated by a minimum of 1 week, and the exposure sequence was randomized. With few exceptions, all subjects were exposed to all 4 ozone concentrations. Spirometric measurements and symptoms ratings were obtained during 10 -min rest periods after each session. One of the 22 subjects did not complete all of the 6.6-h exposures; he was not exposed at $0.12 \mathrm{ppm}$ because he experienced severe respiratory symptoms at an ozone level of $0.10 \mathrm{ppm}$ (although his pulmonary function responses at ozone levels of 0.08 and $0.10 \mathrm{ppm}$ were not markedly greater than the means of the group). In addition to the primary spirometric measurements, the subjects also rated the same symptoms as in the Folinsbee et al. (1988) study.

Of the 38 males who participated in the McDonnell et al. (1991) study, 28 subjects were exposed twice: once to clean air and once to an ozone level of $0.08 \mathrm{ppm}$. The remaining 10 subjects were exposed to ozone a third time, at $0.10 \mathrm{ppm}$. Exposures were separated by at least 2 weeks. Candidates were excluded if they had smoked regularly; had a history of asthma, allergic rhinitis, or cardiac disease; or had an acute respiratory illness within the preceding 4 weeks. Five minutes immediately following the last exercise period, airway resistance, forced expiratory volume, and symptoms were measured. Subjects rated cough, PDI, and shortness of breath. Symptom severity was rated as 0 (none), 1 (barely noticeable), 2 (mild), 3 (moderate), or 4 (severe).

These 6.6-h studies were matched with 8-h exposure estimates to calculate risk. This procedure seems reasonable because response appears to "level off" after several hours of exposure. Thus, it is unlikely that, even if the studies had been conducted for $8 \mathrm{~h}, \mathrm{FEV}_{1}$ decrements would have been appreciably different. 


\subsection{EXPOSURE-RESPONSE RELATIONSHIPS FOR ACUTE ENDPOINTS}

Developing exposure-response relationships for acute endpoints is a three-step process. (Appendix A gives details of this process.) The process starts with data from the laboratory experiments described in the preceding sections. Before developing the probabilistic exposureresponse relationships, "the data were corrected for exercise in clean air," which means that any systematic bias in the data that might be attributable to an exercise effect was removed (Appendix A). Tables 10 and 11 list the results of any corrections made to the laboratory data for lung function and symptoms, respectively. These data become the "observations" shown in Figure 4 (step 1). In step 2, a function is fit to the data via regression techniques. This step is necessary to estimate response rates at ozone concentrations that differ from those at which laboratory data are available. Step 3 develops, for example, the $90 \%$ credible interval (CI) about the fitted (predicted) response rate at ozone concentrations needed for the risk assessment calculations (i.e., those used in $\mathrm{pNEM} / \mathrm{O}_{3}$ ). This last step is accomplished by applying the inverse beta function with parameters $X$ and $N-X$, where $X$ is the predicted response rate at a particular ozone concentration, and $N$ is the number of subjects associated with the chosen ozone concentration. The $90 \% \mathrm{CI}$ is defined by the 0.05 and 0.95 fractiles. For the risk assessment, response rates are calculated for 21 fractiles (for cumulative probabilities from 0.05 to 0.95 in steps of 0.05 , plus probabilities of 0.01 and 0.99 ) at a number of ozone concentrations that depend on the health endpoint. The function chosen fit the data best according to the following principles and rules:

- Linear functions were favored, especially when the number of observation points (i.e., ozone concentrations at which laboratory data are available) was small. As few as two usable observation points and as many as six observation points for endpoints are listed in Tables 10 and 11.

- Functions with high regression $r^{2}$ values were more desirable than functions with low $r^{2}$ values. This principle allowed choosing a nonlinear function over a linear function - even if the number of observation points was small - if the $r^{2}$ value of the nonlinear function was considerably larger than that for the linear function.

- All functions for each of the fractiles must be monotonic increasing (i.e., they must never decrease) as ozone concentration increases. This factor is a logical rule, and it came into play when the number of subjects varied considerably at different ozone concentrations. Such a condition made it necessary to use an average number of subjects at all ozone concentrations.

- Related groups of functions presented special problems. For example, the function for $\mathrm{DFEV}_{1} \geq 10 \%$ ' should never intersect the function for 
TABLE 10 Summary of Experimental Data - Forced Expiratory Volume - Corrected for Exercise in Clean Air

\begin{tabular}{|c|c|c|c|c|c|c|c|c|c|c|c|c|c|}
\hline \multirow[b]{2}{*}{$\begin{array}{c}\text { Study and } \\
\text { Length of Exposure }\end{array}$} & \multirow[b]{2}{*}{$\begin{array}{c}\text { Health } \\
\text { Endpoint }^{\mathrm{a}}\end{array}$} & \multicolumn{12}{|c|}{ Ozone Level (ppm) } \\
\hline & & 0.08 & 0.10 & 0.12 & 0.15 & 0.16 & 0.18 & 0.20 & 0.24 & 0.25 & 0.30 & 0.32 & 0.40 \\
\hline $\begin{array}{l}\text { Avol et al. (1984), } \\
1.33 \mathrm{~h}\end{array}$ & $\begin{array}{l}\operatorname{DFEV}_{1} \geq 10 \% \\
\operatorname{DFEV}_{1} \geq 15 \% \\
\operatorname{DFEV}_{1} \geq 20 \%\end{array}$ & $\begin{array}{l}1 / 48 \\
0 / 48 \\
0 / 48\end{array}$ & & & & $\begin{array}{l}14 / 48 \\
10 / 48 \\
7 / 48\end{array}$ & & & $\begin{array}{l}32 / 48 \\
25 / 48 \\
20 / 48\end{array}$ & & & $\begin{array}{l}39 / 48 \\
33 / 48 \\
30 / 48\end{array}$ & \\
\hline $\begin{array}{l}\text { Kulle et al. (1985), } \\
2 \mathrm{~h}\end{array}$ & $\begin{array}{l}\mathrm{DFEV}_{1} \geq 10 \% \\
\operatorname{DFEV}_{1} \geq 15 \% \\
\operatorname{DFEV}_{1} \geq 20 \%\end{array}$ & & $\begin{array}{l}0 / 20 \\
0 / 20 \\
0 \%\end{array}$ & & $\begin{array}{l}0 / 20 \\
0 / 20 \\
0 / 20\end{array}$ & & & $\begin{array}{l}4 / 20 \\
1 / 20 \\
0 / 20\end{array}$ & & $\begin{array}{l}5 / 20 \\
2 / 20 \\
2 / 20\end{array}$ & $\begin{array}{l}3 / 8 \\
2 / 8 \\
1 / 8\end{array}$ & & \\
\hline $\begin{array}{l}\text { McDonnell et al. (1983), } \\
2.5 h^{b}\end{array}$ & $\begin{array}{l}\mathrm{DFEV}_{1} \geq 10 \% \\
\mathrm{DFEV}_{1} \geq 15 \% \\
\operatorname{DFEV}_{1} \geq 20 \%\end{array}$ & & & $\begin{array}{c}2.3 / 22 \\
0.5 / 22 \\
0 / 22\end{array}$ & & & $\begin{array}{l}3.55 / 20 \\
2 / 20 \\
1.65 / 20\end{array}$ & & $\begin{array}{l}10.1 / 21 \\
8.45 / 21 \\
6.5 / 21\end{array}$ & & $\begin{array}{l}11.85 / 20 \\
9.6 / 20 \\
6.35 / 20\end{array}$ & & $\begin{array}{l}18.3 / 29 \\
14.6 / 29 \\
10.15 / 29\end{array}$ \\
\hline $\begin{array}{l}\text { Seal et al. (1993), } \\
2.33 \mathrm{~h}^{\mathrm{b}}\end{array}$ & $\begin{array}{l}\mathrm{DFEV}_{1} \geq 10 \% \\
\mathrm{DFEV}_{1} \geq 15 \% \\
\mathrm{DFEV}_{1} \geq 20 \%\end{array}$ & & & $\begin{array}{l}5.90 / 61 \\
3.63 / 61 \\
2.39 / 61\end{array}$ & & & $\begin{array}{c}18.47 / 64 \\
10.19 / 64 \\
6.32 / 64\end{array}$ & & $\begin{array}{l}30.71 / 64 \\
17.05 / 64 \\
11.11 / 64\end{array}$ & & $\begin{array}{l}34.27 / 60 \\
23.34 / 60 \\
14.47 / 60\end{array}$ & & $\begin{array}{l}46.29 / 60 \\
38.71 / 60 \\
30.71 / 60\end{array}$ \\
\hline $\begin{array}{l}\text { Folinsbee et al. (1988), } \\
\text { Horstman et al. (1990), } \\
\text { and McDonnell et al. } \\
\text { (1991), } 8 \text { h }\end{array}$ & $\begin{array}{l}\operatorname{DFEV}_{1} \geq 10 \% \\
\operatorname{DFEV}_{1} \geq 15 \% \\
\operatorname{DFEV}_{1} \geq 20 \%\end{array}$ & $\begin{array}{l}18 / 60 \\
11 / 60 \\
5 / 60\end{array}$ & $\begin{array}{l}13 / 32 \\
9 / 32 \\
5 / 32\end{array}$ & $\begin{array}{l}15 / 30 \\
15 / 30 \\
6 / 30\end{array}$ & & & & & & & & & \\
\hline
\end{tabular}

a DFEV $_{1}$ means change in forced expiratory volume in $1 \mathrm{~s}$.

b The characteristics of 20 additional subjects for McDonnell et al. and 63 additional subjects for Seal et al. exposed at 0 ppm were used to correct the responses of other subjects exposed at $>0 \mathrm{ppm}$ for exercise in clean air.

c Because of logical inconsistencies caused by this data point, it was not included in the regression to determine an exposure-response relationship. See the main text for more discussion. 
TABLE 11 Summary of Experimental Data - Symptoms - Corrected for Exercise in Clean Air

\begin{tabular}{|c|c|c|c|c|c|c|c|c|c|c|c|c|c|}
\hline \multirow{2}{*}{$\begin{array}{c}\text { Study and } \\
\text { Length of Exposure }\end{array}$} & \multirow{2}{*}{$\begin{array}{c}\text { Health } \\
\text { Endpoint }^{a}\end{array}$} & \multicolumn{12}{|c|}{ Ozone Level (ppm) } \\
\hline & & 0.08 & 0.10 & 0.12 & 0.15 & 0.16 & 0.18 & 0.20 & 0.24 & 0.25 & 0.30 & 0.32 & 0.40 \\
\hline $\begin{array}{l}\text { Avol ct al. (1984), } \\
1.33 \mathrm{~h}\end{array}$ & $\begin{array}{l}\text { Any LR } \\
\text { M/S LR }\end{array}$ & $\begin{array}{l}\% / 48 \\
\% / 48\end{array}$ & & & & $\begin{array}{l}10 / 48 \\
0 \% 48\end{array}$ & & & $\begin{array}{l}23 / 48 \\
1 / 48\end{array}$ & & & $\begin{array}{l}30 / 48 \\
4 / 48\end{array}$ & \\
\hline $\begin{array}{l}\text { Kulle et al. (1985), } \\
2 \mathrm{~h}\end{array}$ & $\begin{array}{l}\text { Any cough } \\
\text { Any PDI } \\
\text { M/S cough } \\
M / S \text { PDI }\end{array}$ & & $\begin{array}{l}0 / 20 \\
0 / 20 \\
0 / 20 \\
0 / 20\end{array}$ & & $\begin{array}{l}4 / 20 \\
2 / 20 \\
0 / 20 \\
0 / 20\end{array}$ & & & $\begin{array}{l}8 / 20 \\
6 / 20 \\
0 / 20 \\
1 / 20\end{array}$ & & $\begin{array}{l}9 / 20 \\
5 / 20 \\
3 / 20 \\
1 / 20\end{array}$ & $\begin{array}{l}5 / 8 \\
7 / 8 \\
0 / 8 \\
4 / 8\end{array}$ & & \\
\hline $\begin{array}{l}\text { McDonnell et al. } \\
(1983), 2.5 \mathrm{~h}^{\mathrm{b}}\end{array}$ & $\begin{array}{l}\text { Any cough } \\
\text { Any PDI } \\
\text { M/S cough } \\
\text { M/S PDI }\end{array}$ & & & $\begin{array}{l}12.24 / 22 \\
7.53 / 21 \\
3.77 / 22 \\
1.94 / 21\end{array}$ & & & $\begin{array}{l}12.24 / 19 \\
9.65 / 19 \\
2.82 / 19 \\
2.59 / 19\end{array}$ & & $\begin{array}{c}16 / 21 \\
15.53 / 21 \\
6.59 / 21 \\
5.53 / 21\end{array}$ & & $\begin{array}{c}16 / 18 \\
12.53 / 19 \\
9.41 / 18 \\
6.24 / 19\end{array}$ & & $\begin{array}{l}25.41 / 28 \\
21.18 / 28 \\
12.24 / 28 \\
11.06 / 28\end{array}$ \\
\hline $\begin{array}{l}\text { Scal et al. (1993), } \\
2 \mathrm{~h}\end{array}$ & $\begin{array}{l}\text { Any cough } \\
\text { Any PDI } \\
M / S \text { cough } \\
M / S \text { PDI }\end{array}$ & & & $\begin{array}{c}7.35 / 59 \\
11.21 / 60 \\
0 / 59 \\
2 / 60\end{array}$ & & & $\begin{array}{c}18.39 / 61 \\
21.49 / 59 \\
6 / 61 \\
8 / 59\end{array}$ & & $\begin{array}{c}35.85 / 61 \\
29.90 / 60 \\
14 / 61 \\
10 / 60\end{array}$ & & $\begin{array}{c}32.18 / 58 \\
36.44 / 57 \\
15 / 58 \\
13 / 57\end{array}$ & & $\begin{array}{c}45.05 / 59 \\
38.31 / 57 \\
23 / 59 \\
29 / 57\end{array}$ \\
\hline $\begin{array}{l}\text { Folinsbec et al. (1988), } \\
\text { Horstman et al. (1990), } \\
\text { and McDonnell et al. } \\
\text { (1991), } 8 \text { h }\end{array}$ & $\begin{array}{l}\text { Any cough } \\
\text { Any PDI } \\
\text { M/S cough } \\
\text { M/S PDI }\end{array}$ & $\begin{array}{l}9 / 54 \\
4 / 54 \\
1 / 54 \\
0 / 54\end{array}$ & $\begin{array}{l}3 / 28 \\
8 / 28 \\
1 / 28 \\
1 / 28\end{array}$ & $\begin{array}{l}10 / 27 \\
13 / 27 \\
3 / 27 \\
4 / 27\end{array}$ & & & & & & & & & \\
\hline
\end{tabular}

a LR means lower respiratory, M/S means moderate or severe, and PDI means pain on deep inspiration.

b The characteristics of 20 additional subjects for McDonnell et al. and 63 additional subjects for Seal et al. exposed at 0 ppm were used to correct the responses of other subjects exposed at $>0 \mathrm{ppm}$ for exercise in clean air. 


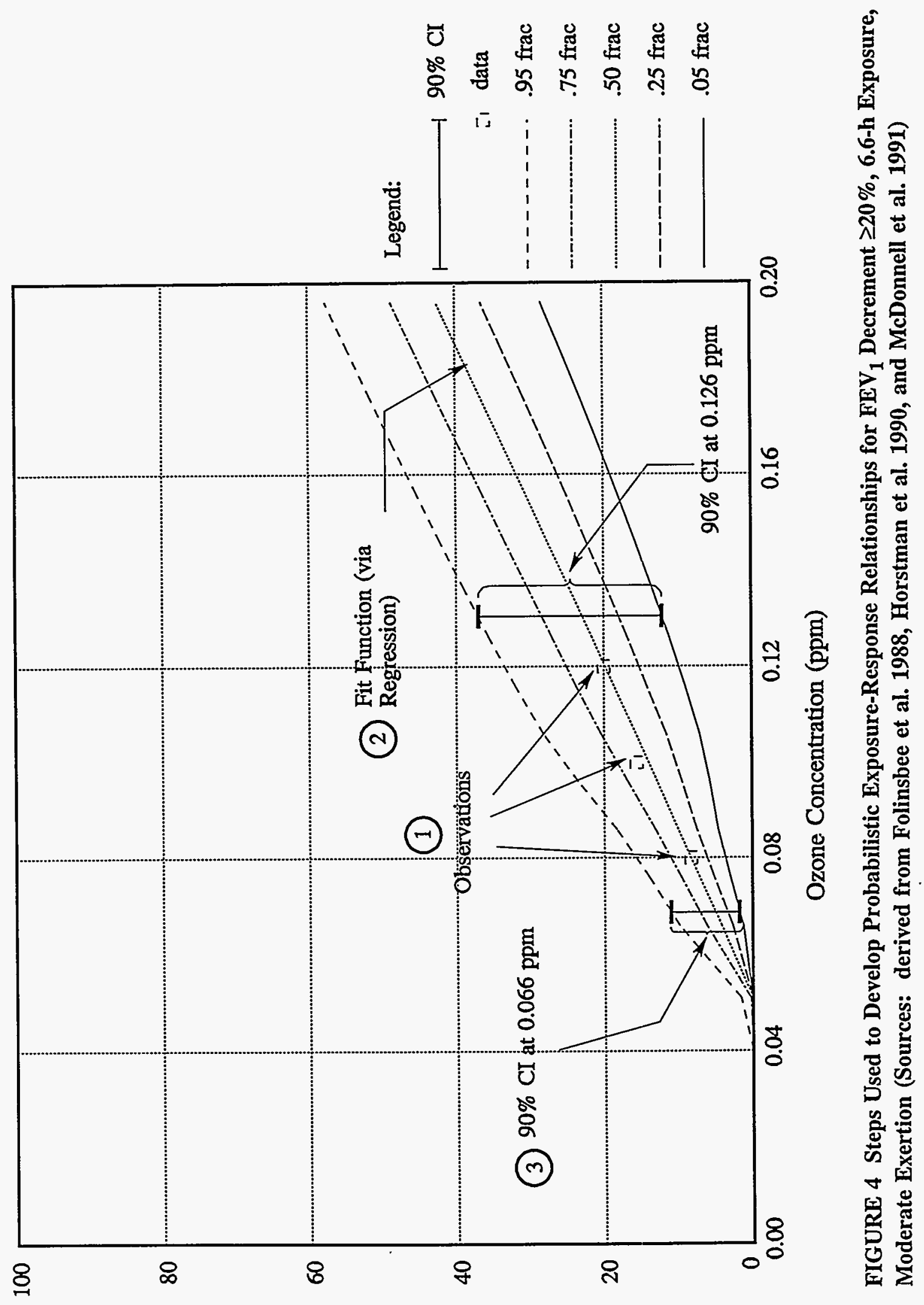

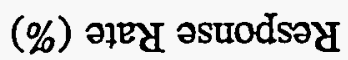


$\mathrm{DFEV}_{1} \geq 15 \%$ in the range of ozone concentrations used in the risk assessment. When functions intersected, those functions that did not intersect were chosen. These conditions arose most frequently when attempts were made to fit linear functions to related sets of data.

One particularly bothersome case involved the lung function endpoints for 6.6-h exposures of subjects engaged in moderate exertion (the combined data of the studies by Folinsbee et al. 1988, Horstman et al. 1990, and McDonnell et al. 1991). The observed response rate at $0.12 \mathrm{ppm}$ for the $\mathrm{FEV}_{1}$ decrement $\geq 15 \%$ endpoint was judged to be unreasonable and was not used in the regression.

The results of fitting functions to the laboratory data are listed in Tables 12 and 13 for lung function and symptoms, respectively. The tables indicate the type of function fit to the data (linear, logistic, probit, or lognormal) for each endpoint, parameters of the functions, and the regression $r^{2}$ value. Parameter $a$ is the intercept of the linear function and the geometric mean of the lognormal function. Parameter $b$ is the slope of the linear function and the geometric standard deviation (s.d.) of the lognormal function. The mathematical definitions of the functional forms are given in Appendix A.

Data for a total of 33 health endpoints are described in Tables 12 and 13. The headcount and benchmark risk results presented in Sections 4 and 5, respectively, focus on three endpoints for outdoor children that are, in a sense, more serious than the others and therefore of greater interest to EPA staff. These are $\mathrm{FEV}_{1}$ decrements $\geq 15$ and $\geq 20 \%$ for 8 -h exposures, and moderate-to-severe PDI for 1-h exposures, all at moderate exercise. Appendixes $\mathrm{C}$ and $\mathrm{D}$ provide results for 19 of the endpoints $\left(\mathrm{FEV}_{1}\right.$ decrements $\geq 10 \%$ and "any" symptoms are excluded). 
TABLE 12 Summary of Functions Fit to Experimental Data - FEV 1 Decrement - Corrected for Exercise in Clean Air

\begin{tabular}{|c|c|c|c|c|c|c|c|}
\hline \multirow[b]{2}{*}{$\begin{array}{l}\text { Study and } \\
\text { Length of Exposure }\end{array}$} & \multirow[b]{2}{*}{ Endpoint $^{b}$} & \multirow[b]{2}{*}{ Function } & \multicolumn{4}{|c|}{ Parameters $^{\mathbf{a}}$} & \multirow[b]{2}{*}{$r^{2}$} \\
\hline & & & $a$ & $b$ & $d$ & $e$ & \\
\hline \multirow{3}{*}{$\begin{array}{l}\text { Avol et al. (1984), } \\
1.33 \mathrm{~h}\end{array}$} & $\mathrm{DFEV}_{1} \geq 10 \%$ & Lincar & -0.2395 & 3.4388 & \multirow{3}{*}{$\begin{array}{l}-c \\
- \\
-\end{array}$} & \multirow{3}{*}{ - } & 0.98 \\
\hline & $\mathrm{DFEV}_{1} \geq 15 \%$ & Linear & -0.2400 & 2.9713 & & & 0.99 \\
\hline & $\mathrm{DFEV}_{1} \geq 20 \%$ & Linear & -0.2395 & 2.6825 & & & 0.99 \\
\hline \multirow{3}{*}{$\begin{array}{l}\text { Kulle et al. (1985), } \\
2 \mathrm{~h}\end{array}$} & $\mathrm{DFEV}_{1} \geq 10 \%$ & Linear & -0.3225 & 2.3500 & \multirow{3}{*}{$\begin{array}{l}- \\
-\end{array}$} & \multirow{3}{*}{$\begin{array}{l}- \\
- \\
-\end{array}$} & 0.95 \\
\hline & $\mathrm{DFEV}_{1} \geq 15 \%$ & Linear & -0.2600 & 1.6000 & & & 0.93 \\
\hline & $\mathrm{DFEV}_{1} \geq 20 \%$ & Linear & -0.2375 & 1.2500 & & & 0.89 \\
\hline \multirow{3}{*}{$\begin{array}{l}\text { McDonnell et al. (1983), } \\
2.5 \mathrm{~h}\end{array}$} & $\mathrm{DFEV}_{1} \geq 10 \%$ & Logistic & - & 0.6420 & 5.5996 & -27.2927 & 0.99 \\
\hline & $\mathrm{DFEV}_{1} \geq 15 \%$ & Logistic & - & 0.4968 & 9.4948 & -45.3838 & 1.00 \\
\hline & $\mathrm{DFEV}_{1} \geq 20 \%$ & Logistic & - & 0.3347 & 12.0073 & -60.4547 & 1.00 \\
\hline \multirow{3}{*}{$\begin{array}{l}\text { Seal et al. (1993), } \\
2.33 \mathrm{~h}\end{array}$} & $\mathrm{DFEV}_{1} \geq 10 \%$ & Probit & -1.0276 & 0.7917 & \multirow{3}{*}{ - } & \multirow{3}{*}{$\begin{array}{l}- \\
-\end{array}$} & 0.99 \\
\hline & DFEV $_{1} \geq 15 \%$ & Probit & -0.6639 & 0.8401 & & & 0.99 \\
\hline & $\mathrm{DFEV}_{1} \geq 20 \%$ & Probit & -0.3259 & 0.9192 & & & 0.97 \\
\hline \multirow{3}{*}{$\begin{array}{l}\text { Folinsbee et al. (1988), } \\
\text { Horstman et al. (1990), } \\
\text { and McDonnell et al. } \\
\text { (1991), } 8 \text { h }\end{array}$} & $\operatorname{DFEV}_{1} \geq 10 \%$ & Linear & -0.0980 & 5.0000 & \multirow{3}{*}{$\begin{array}{l}- \\
- \\
-\end{array}$} & \multirow{3}{*}{ - } & 1.00 \\
\hline & $\mathrm{DFEV}_{1} \geq 15 \%$ & Linear & -0.2087 & 4.9000 & & & 1.00 \\
\hline & $\mathrm{DFEV}_{1} \geq 20 \%$ & Lincar & -0.1462 & 2.9250 & & & 0.98 \\
\hline
\end{tabular}

a No functional form uses more than three parameters. The letters used to designate parameters are consistent with earlier work by Hayes et al. (1987, 1989).

b DFEV $_{1}$ means change in forced expiratory volume in $1 \mathrm{~s}$.

c These parameters are not used in this function. 
TABLE 13 Summary of Functions Fit to Experimental Data - Symptoms - Corrected for Exercise in Clean Air

\begin{tabular}{|c|c|c|c|c|c|c|c|}
\hline \multirow{2}{*}{$\begin{array}{l}\text { Study and } \\
\text { Length of Exposure }\end{array}$} & \multirow[b]{2}{*}{ Endpoint $^{b}$} & \multirow[b]{2}{*}{ Function } & \multicolumn{4}{|c|}{ Parameters $^{a}$} & \multirow[b]{2}{*}{$r^{2}$} \\
\hline & & & $a$ & $b$ & $d$ & $e$ & \\
\hline \multirow{3}{*}{$\begin{array}{l}\text { Avol et al. (1984), } \\
1.33 \mathrm{~h}\end{array}$} & \multirow{3}{*}{$\begin{array}{l}\text { Any lower } \\
\text { respiratory } \\
\text { M/S lower resp }\end{array}$} & Linear & -0.2084 & 2.6824 & $-c$ & - & 0.99 \\
\hline & & Linear & -0.0902 & 0.5206 & - & - & 0.94 \\
\hline & & & & & & & \\
\hline \multirow{4}{*}{$\begin{array}{l}\text { Kulle et al. (1985), } \\
2 \mathrm{~h}\end{array}$} & Any cough & Linear & -0.2650 & 3.0000 & - & - & 0.97 \\
\hline & Any PDI & Linear & -0.4550 & 3.8000 & - & - & 0.79 \\
\hline & $\mathrm{M} / \mathrm{S}$ cough & Linear & -0.1626 & 0.8675 & - & $\begin{array}{l}- \\
-\end{array}$ & \multirow{2}{*}{$\begin{array}{c}-0.33^{d} \\
0.72\end{array}$} \\
\hline & $\mathrm{M} / \mathrm{S}$ PDI & Linear & -0.5250 & 3.0000 & - & - & \\
\hline \multirow{4}{*}{$\begin{array}{l}\text { McDonnell et al. (1983), } \\
2.5 \mathrm{~h}\end{array}$} & Any cough & Probit & -2.0954 & 1.2098 & - & - & 0.99 \\
\hline & Any PDI & Probit & -1.6071 & 1.5124 & - & - & 0.96 \\
\hline & $\mathrm{M} / \mathrm{S}$ cough & Linear & 0.0062 & 1.2604 & $\begin{array}{l}- \\
-\end{array}$ & $\begin{array}{l}- \\
-\end{array}$ & 0.70 \\
\hline & M/S PDI & Linear & -0.0427 & 1.1512 & - & - & 0.96 \\
\hline \multirow{4}{*}{$\begin{array}{l}\text { Seal et al. (1993), } \\
2.33 \mathrm{~h}\end{array}$} & Any cough & Lognormal & 0.2469 & 1.9248 & - & - & 0.97 \\
\hline & Any PDI & Lognormal & 0.2464 & 2.3641 & - & - & 0.99 \\
\hline & $\mathrm{M} / \mathrm{S}$ cough & Linear & -0.1445 & 1.3704 & - & $\begin{array}{l}- \\
-\end{array}$ & 0.97 \\
\hline & M/S PDI & Probit & -0.3209 & 0.9317 & - & - & 0.96 \\
\hline \multirow{4}{*}{$\begin{array}{l}\text { Folinsbee et al. (1988), } \\
\text { Horstman et al. (1990), } \\
\text { and McDonnell et al. } \\
\text { (1991), } 8 \text { h }\end{array}$} & Any cough & Linear & -0.2928 & 5.0750 & - & - & 0.54 \\
\hline & Any PDI & Linear & 0.7372 & 10.1750 & - & - & 1.00 \\
\hline & $\mathrm{M} / \mathrm{S}$ cough & Linear & -0.1747 & 2.3000 & - & $\begin{array}{l}- \\
-\end{array}$ & 0.88 \\
\hline & M/S PDI & Linear & -0.3087 & 3.7000 & - & - & 0.93 \\
\hline
\end{tabular}

a No functional form uses more than three parameters. The letters used to designate parameters are consistent with earlier work by Hayes et al. $(1987,1989)$.

b M/S means moderate to severe, and PDI means pain on deep inspiration.

c These parameters are not used in this function.

d The data do not support a meaningful exposure-response relationship for this health endpoint. The negative $r^{2}$ value flags this situation. 


\section{HEADCOUNT RISK MODELS AND ILLUSTRATIVE RESULTS}

This section uses one health endpoint to (1) illustrate the calculations needed to develop headcount risk distributions and (2) present the main output format for the risk results. This format makes it easy to investigate the effectiveness of several air quality standards in each of the nine urban areas included in this report. An acute endpoint (8-h exposure, moderate exertion) is used, but the general computational approach and output format apply to all acute endpoints.

\subsection{CALCULATION OF HEADCOUNT RISK}

The risk computations (which comprise the risk model) are conceptually simple and based on exposure and health models. In general, the risk (which is an expected fractional response rate) for the $k^{\prime}$ th fractile $R_{k}$ is

$$
R_{k}=\sum_{j=1}^{N} P_{j} \times\left[\left(R R_{k} \mid e_{j}\right)-\left(R R_{k} \mid \text { background }\right)\right],
$$

or

$$
R_{k}=\sum_{j=1}^{N} P_{j} \times R R_{k} \mid e_{j}-\sum_{j=1}^{N} P_{j} \times\left(R R_{k} \mid \text { background }\right),
$$

where:

$$
\begin{aligned}
P_{j}= & \begin{array}{l}
\text { fraction of the population having personal exposures at an } \\
\text { ozone concentration of } e_{j} \text { ppm in a single pNEM/O }
\end{array} \text { run; } \\
R R_{k} \mid e_{j}= & k \text {-fractile response at ozone concentration } e_{j} ; \\
R R_{k} \mid \text { background }= & k \text {-fractile response at background; and } \\
N= & \text { number of ozone concentrations. }
\end{aligned}
$$

As discussed in Section 3, it was possible to correct exposure-response relationships for exercise in clean air. The $R R_{k} \mid e_{j}$ values reflect this correction for the $\mathrm{FEV}_{1}$ decrements $\geq 20 \%$ for 8-h exposures at moderate exertion (based on study results from Folinsbee et al. 1988, Horstman et al. 1990, and McDonnell et al. 1991). 
Table 14 shows the risk computations for the 0.5 fractile (the median) $\mathrm{pNEM} / \mathrm{O}_{3}$ run 2 exposures to outdoor children based on air quality in Philadelphia that just attains the current standard (1112) for the people measure (i.e., the fraction of people exposed one or more times during the ozone season). The table indicates eight ozone concentrations ${ }^{8}$ above background involved in the computations. Background ozone is $\leq 0.04 \mathrm{ppm}$. The entries in column $\mathrm{C}$ are the fractions of the population engaged in moderate exertion who are exposed to the corresponding ozone concentrations in column $\mathrm{B}$. The entries in column $\mathrm{D}$ are the expected fractional response rates at the corresponding ozone concentrations for the 0.5 fractile. The result is about 0.06 , or $6 \%$. It happens that $R R_{0.5} \mid$ background is exactly zero. Therefore, this result is unchanged after correcting for background. Column $\mathrm{E}$ shows the results of multiplying the fractional response rate by the number of outdoor children who achieved moderate exertion for $8 \mathrm{~h}$ to obtain, in this case, the median estimate of the number of individuals who experience $\mathrm{FEV}_{1}$ decrements $\geq 20 \%$. For $\mathrm{pNEM} / \mathrm{O}_{3}$ run 2, 268,569 outdoor children achieved the heavy exertion level, so the median estimate is about 15,900 outdoor children.

To develop a probability distribution over outdoor children that accounts for all of the conditions mentioned earlier, the above computations are repeated for any number of fractiles. Results usually appear "smoother" if a large number of fractiles are used. In this analysis, 21 fractiles are used to obtain the results presented in Section 4.2 and Appendix C (for acute endpoints).

TABLE 14 Calculating the Median of a Risk Distribution

\begin{tabular}{ccccc}
\hline $\mathrm{A}$ & $\mathrm{B}$ & $\mathrm{C}$ & $\mathrm{D}$ & $\mathrm{E}$ \\
\hline Index $j$ & $e_{j}(\mathrm{ppm})$ & $P_{j}$ & $R R_{0.5} \mid e_{j}$ & $R R_{0.5}=\mathrm{C} \times \mathrm{D}$ \\
\hline & & 0.1413 & 0.000233 & 0.0000 \\
1 & 0.051 & 0.1746 & 0.041839 & 0.0073 \\
2 & 0.066 & 0.071412 & 0.0271 \\
3 & 0.076 & 0.3797 & 0.100205 & 0.0187 \\
4 & 0.086 & 0.1868 & 0.128108 & 0.0054 \\
5 & 0.096 & 0.0422 & 0.156623 & 0.0008 \\
6 & 0.106 & 0.0048 & 0.186317 & 0.0000 \\
7 & 0.116 & 0.0000 & 0.216072 & $\underline{0.0000}$ \\
8 & 0.126 & 0.0000 & & \\
& & & Column E Sum: & 0.0593 \\
\hline
\end{tabular}

8 The concentrations indicated in Table 14 are the midpoints of intervals above $0.06 \mathrm{ppm}$ that are $0.01 \mathrm{ppm}$ wide; the first interval is $0.02 \mathrm{ppm}$ wide. 


\subsection{HEADCOUNT RISK DISTRIBUTIONS FOR 10 pNEIM/O ${ }_{3}$ RUNS AND 2 AIR QUALITY SCENARIOS}

Headcount risk results are presented in a set of figures. These results have been corrected for background, that is, $0.04 \mathrm{ppm}$ for acute exposures. As explained in Section 3, correcting for background involves subtracting the probability distribution over response at the background concentration from the probability distribution over response for ozone concentrations above background. As with many computations, performing this function assumes perfect correlation and allows corrections to be made by simply subtracting corresponding responses on a fractile-by-fractile basis. If no response occurs at a particular background concentration, the "uncorrected" and "corrected" results are identical.

One acute endpoint $\left(\mathrm{FEV}_{1}\right.$ decrement $\geq 20 \%$, 8-h exposure, moderate exertion, outdoor children, based on the Folinsbee et al. 1988, Horstman et al. 1990, and McDonnell et al. 1991 data) illustrates the computations and different forms of risk output. The final result is an output format that captures central (median, or 0.5 fractile) and extreme ( 0.05 and 0.95 fractiles) properties of risk distributions. This format allows investigation of results across nine urban areas for several air quality scenarios (this report shows nine).

Table 15 lists risk results for this endpoint while just attaining standard 1112 in

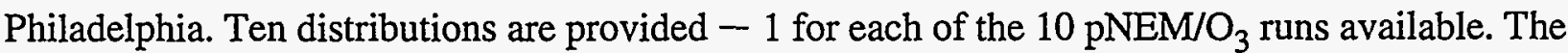
first column lists cumulative probabilities for the distributions. Each row in the table, except for those in the first column, lists the number of children having $\mathrm{FEV}_{1}$ decrements $\geq 20 \%$ for each

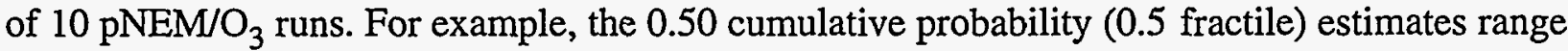
from about 15,000 (run 5) to about 16,000 (run 2) outdoor children. The mean, standard deviation, and total number of people (TotPop) are listed at the bottom of the table. For run 1 , the mean is about 17,000, the standard deviation is about 8,000, and TotPop is about 270,000. TotPop is based on the total number of outdoor children who reached a moderate level of exertion in each $\mathrm{pNEM} / \mathrm{O}_{3}$ run. This number varies from run to run. The significance (if any) of this variation is one of the issues specifically addressed in Section 4.4 .

Figure 5 is a plot of the data for just attaining standard 1112 given in Table 15. It also includes results for the As-Is scenario. Note that the risks are much lower if the current standard is met (indicated by the fact that all distributions for the current standard are closer to the $\mathrm{Y}$ axis than all distributions for the As-Is scenario, 8110. Note also that variation in results is about the same for each standard (evidenced by the fact that the "spread" within each group of 10 distributions is about the same).

On this scale, it is not helpful to plot the distributions for the other scenarios, because they are even closer to the $\mathrm{Y}$ axis than are those for 1112, and, if they were included, the figure would be quite cluttered. Figure 6 gives an idea of the range of results among each set of 10 distributions. Nine plots are shown, one for each air quality scenario. Each plot is 
TABLE 15 Probability Distributions over the Number of Outdoor Children Having FEV 1

Decrements $\geq 20 \%$ during One Ozone Season, Philadelphia, 8-Hour Exposure, Moderate Exertion, Just Attaining Standard 1112, Corrected for Background Ozone ${ }^{\mathrm{a}}$

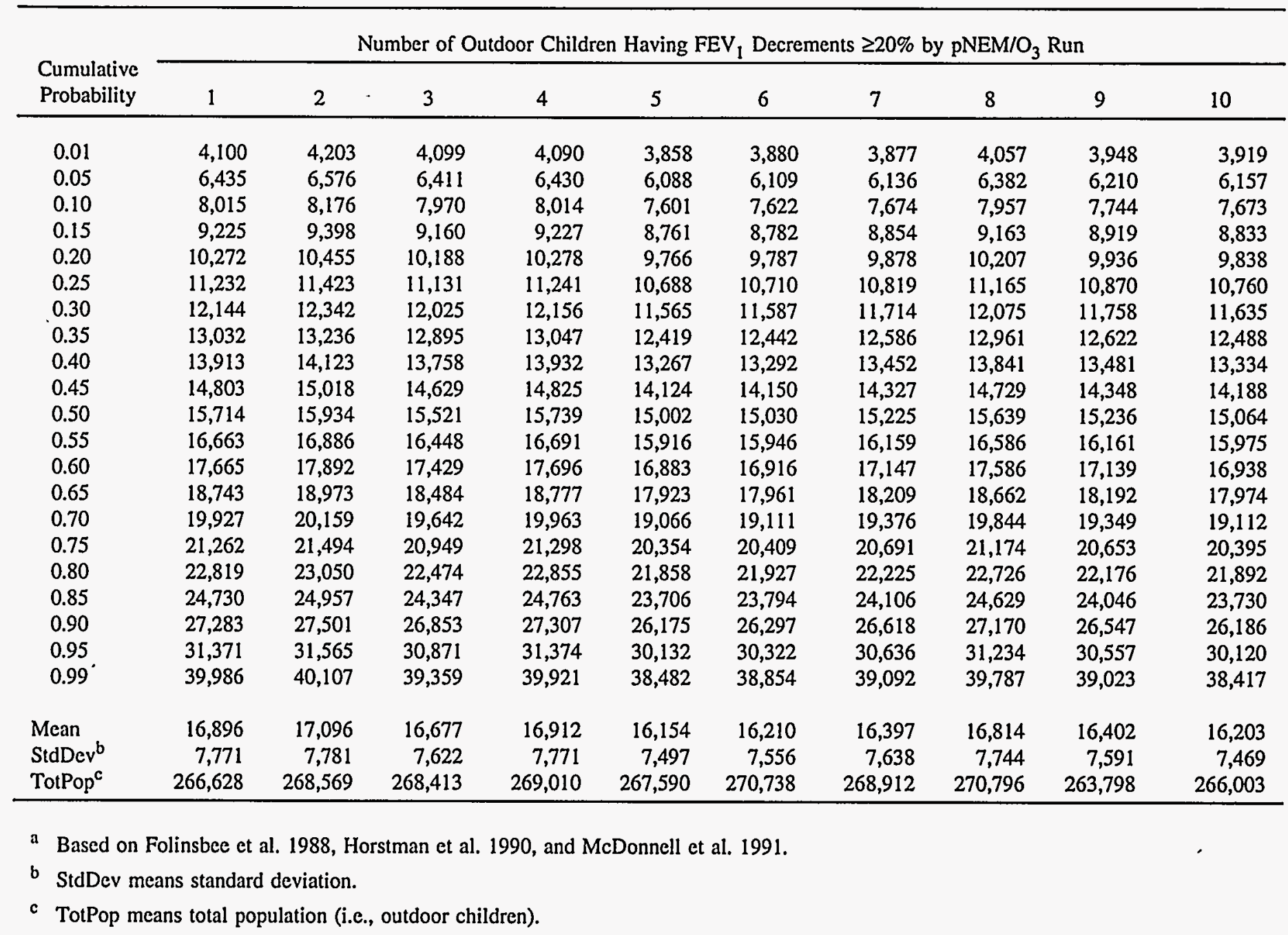




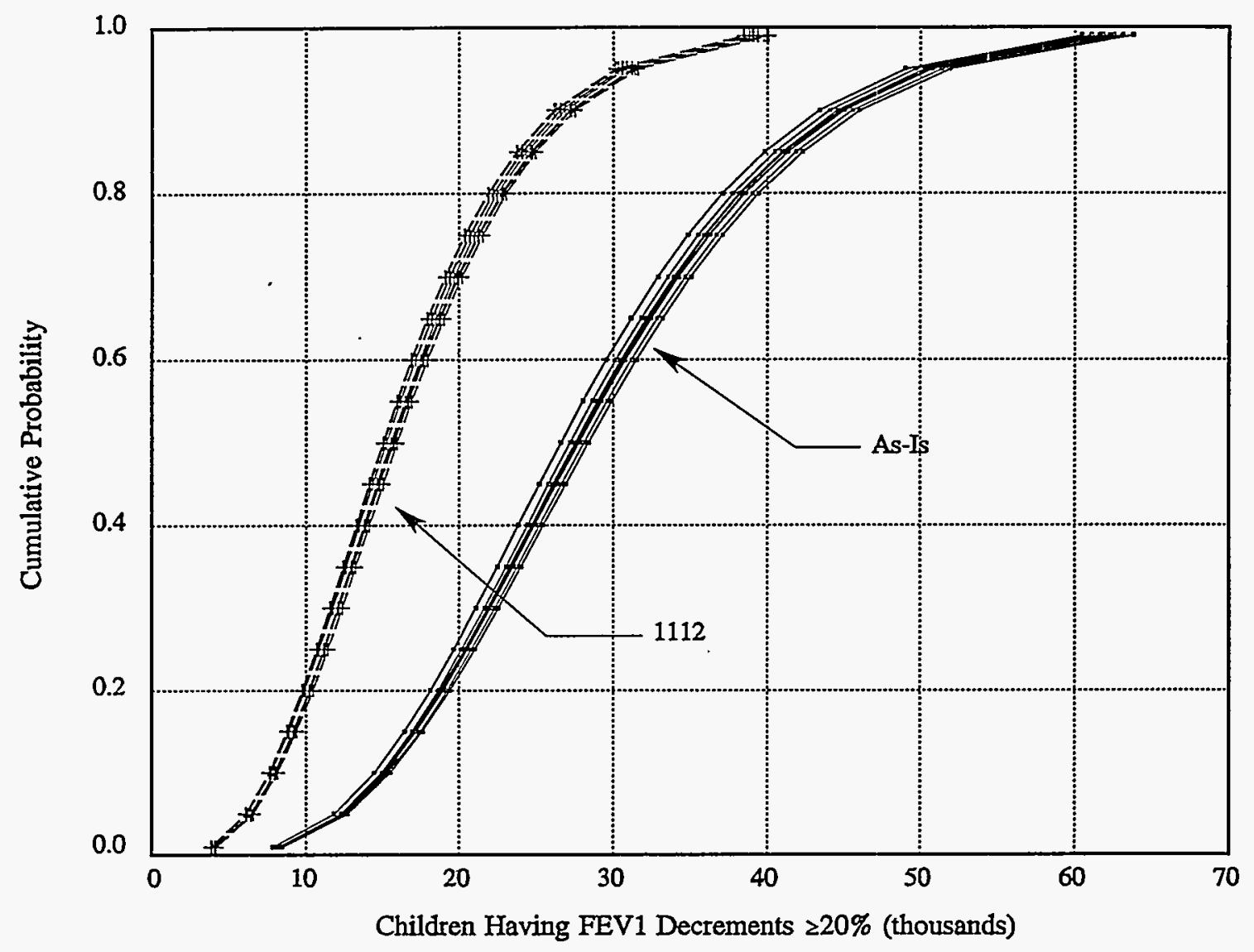

FIGURE 5 Comparison of 2 Sets of 10 Risk Distributions (As-Is air quality and just attaining scenario $1112, \mathrm{FEV}_{1}$ decrements $\geq 20 \%$, Philadelphia, outdoor children, 8-h exposure, moderate exertion level corrected for background ozone) (Sources: based on Folinsbee et al. 1988; Horstman et al. 1990; McDonnell et al. 1991)

"representative" of the 10 distributions for a particular scenario. Because only 9 plots are shown rather than 90 , it is easier to see patterns. Each of these plots is a valid cumulative probability distribution. ${ }^{9}$ The following insights can be gained about the effects of attaining each standard by examining Figure 6:

- The distributions for scenarios 1110 and 8108 are indistinguishable.

- Attaining the current standard results in lower risk estimates than attaining scenario 8110 (because the representative risk distribution for 1112 lies to the left of the distribution for 8110 ).

9 The representative distribution is obtained by computing the average cumulative probability at selected points along the $\mathrm{X}$ axis. This calculation, like the risk calculations described earlier, implicitly assumes that the distributions are perfectly correlated. It may be argued that perfect correlation, while not correct, is more reasonable than perfect independence, and no basis exists for choosing any other degree of correlation between these two extremes. 
- Standard 8107 results in the lowest risk.

- The risk estimates for scenarios 8508 and 8108 are between those for 8509 and 8107.

\subsection{SUMMARY OF RISK DISTRIBUTIONS FOR NINE URBAN AREAS AND NINE AIR QUALITY SCENARIOS}

The median estimates across $10 \mathrm{pNEM} / \mathrm{O}_{3}$ runs range from 15,000 to 16,000 Philadelphia outdoor children having $\mathrm{FEV}_{1}$ decrements $\geq 20 \%$, when standard 1112 is just attained (Section 4.2). Table 15 further shows that the 0.05 fractiles of the risk distributions range from 6,100 to 6,600 and that the 0.95 fractiles range from 30,000 to 32,000 . These estimates are

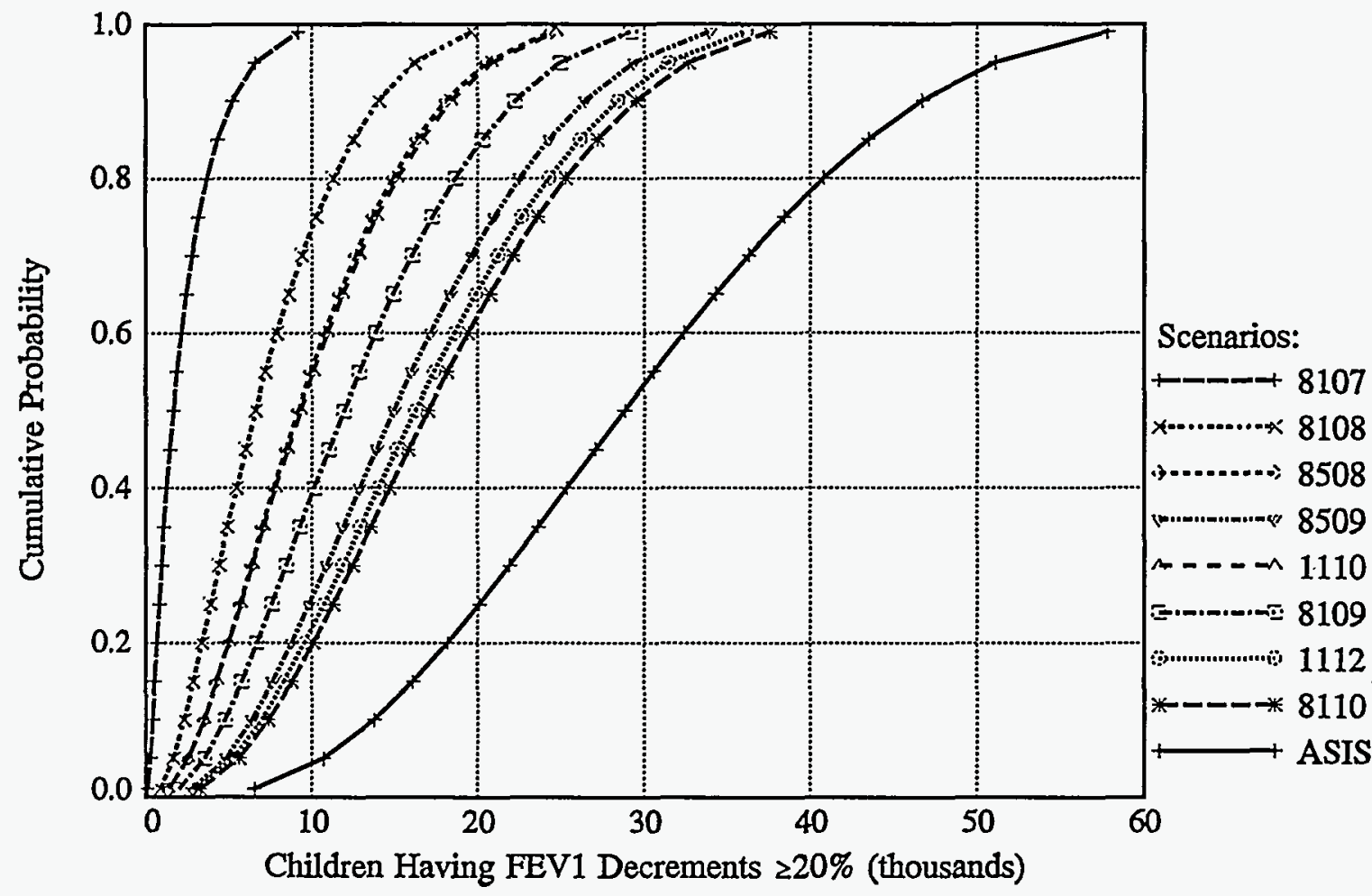

FIGURE 6 Representative Distributions for 9 Sets of 10 Risk Distributions (As-Is and just attaining 9 standards, $\mathrm{FEV}_{1}$ decrements $\geq 20 \%$, Philadelphia, outdoor children, 8-h exposure, moderate exertion) (Sources: based on Folinsbee et al. 1988; Horstman et al. 1990; McDonnell et al. 1983) 
indicated in Figure $7,{ }^{10}$ which uses a variation of the Tukey box plot described in Morgan and Henrion (1990) to indicate uncertainty in the risk distribution. Three rectangles above letter A (the code letter for standard 1112) are associated with Philadelphia. The top rectangle represents the range of the 0.95 fractiles; the middle rectangle represents the range of the medians; and the bottom rectangle represents the range of the 0.05 fractiles. A line connects the bottom of the 0.95 -fractile rectangle and the top of the 0.05 -fractile rectangle and passes through the 0.5 -fractile rectangle.

If the risk distributions for a particular air quality scenario are quite "similar," the rectangles are small. If the variance of a risk distribution is small, the rectangles are close together. If the distributions are spaced far enough apart (indicative of widely varying risk estimates for different $\mathrm{pNEM} / \mathrm{O}_{3}$ runs), the rectangles overlap.

Figure 8 shows 81 sets of rectangles and lines -9 sets for each of 9 cities, 1 set for each air quality standard. This figure is an example of the format used in Appendix $C$ to indicate features of the risk distributions for a single health endpoint associated with As-Is air quality and just attaining each of 8 air quality standards. The meanings of the letter codes are indicated in the legends of Figures 7 and 8 and defined in Table 16.

Figure 9 illustrates and explains the four patterns of rectangles in Figure 8. Pattern 1 is the most common; the rectangles do not overlap because the risk distributions are relatively close. Pattern 2 occurs when the lower tails of the risk distributions are relatively steep in slope and close enough that the 0.05 - and 0.5 -fractile ranges overlap. Pattern 3 occurs when all fractile ranges have the same lower bound (usually 0 ), which happens when 1 or more of $10 \mathrm{pNEM} / \mathrm{O}_{3}$ runs result in no exposures to the population of interest. Pattern 4, which is similar to pattern 2, occurs when the ranges of the 0.5 and 0.95 fractiles overlap. None of the scenarios in Figure 8 exhibits patterns $2-4$. When a particular air quality scenario presents no risk, which happens for some of the "more severe" health endpoints (e.g., $\mathrm{FEV}_{1}$ decrements $\geq 20 \%$, moderate or severe chest pain on deep inspiration, moderate or severe cough), no rectangles are present. In some cases, the entire figure can be blank.

\subsection{SIGNIFICANCE TESTS}

An important question concerning risk results for each air quality scenario is how to assess the significance of relatively small differences. For example, the representative risk distributions shown in Figure 6 are distinguishable, but is this statistically significant, especially those distributions that are close to one another? Recall that the distributions do not necessarily

10 The legend in Figure 7 shows a letter code for each air quality scenario. These codes, which are fully explained in Table 16, are needed to meet file specification length restrictions of the personal computer disk operating system. 


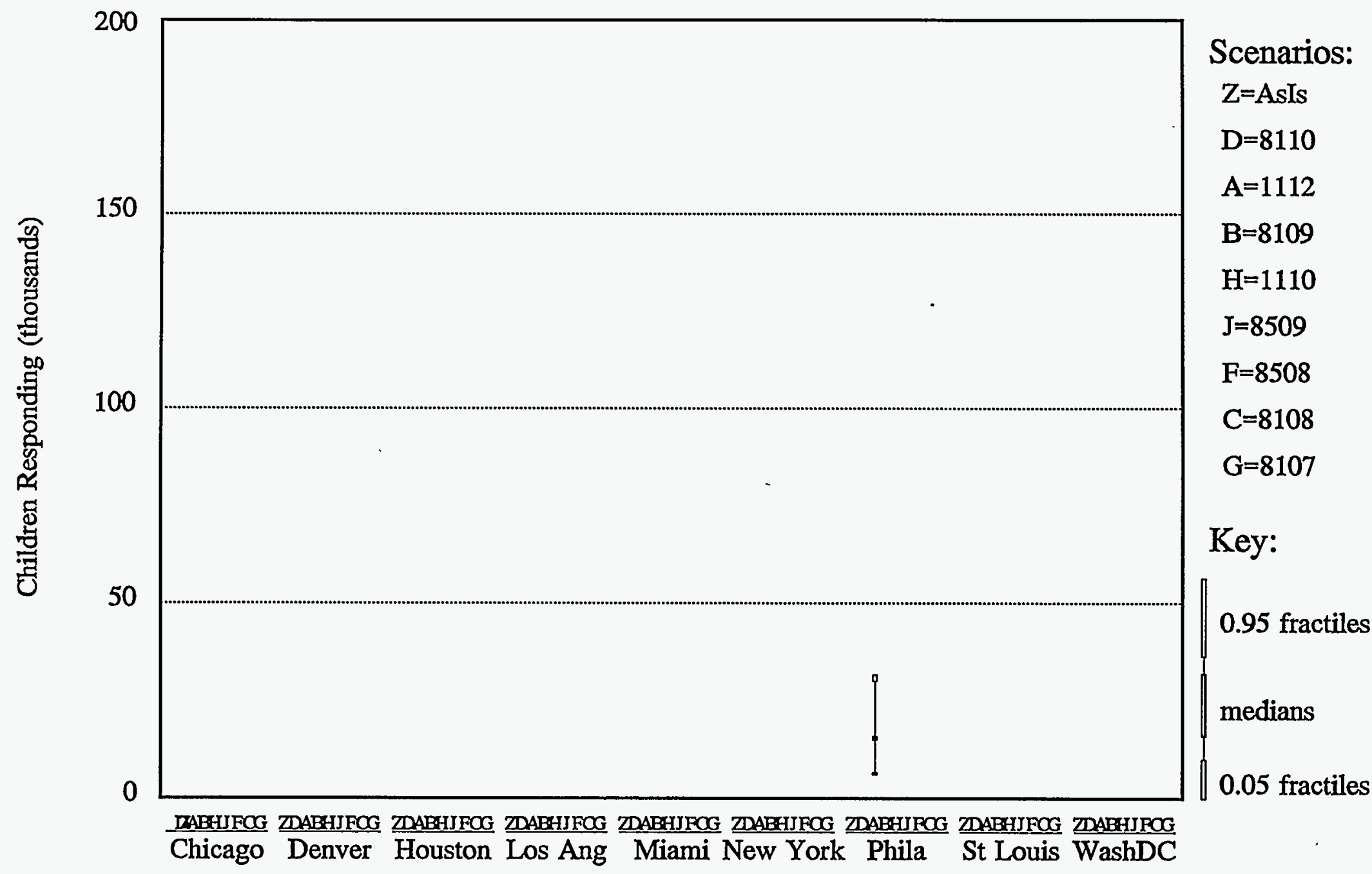

FIGURE 7 Using Rectangles to Represent Variability in 1 Set of 10 Risk Distributions Attributable to Differences in pNEM/O Exposure Estimates 


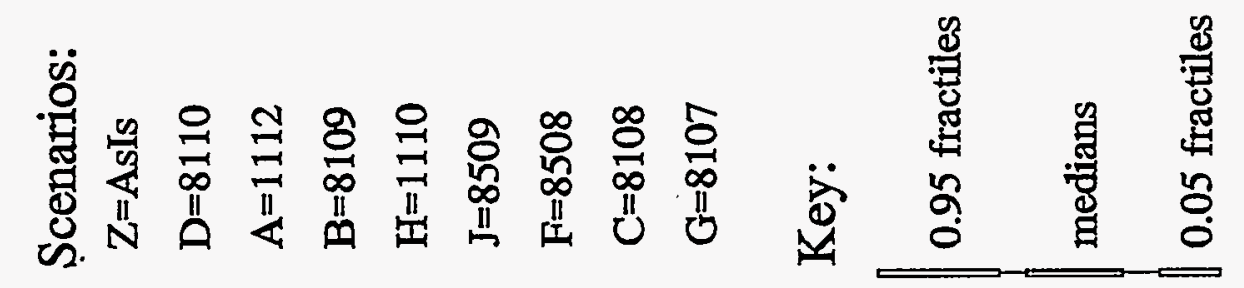

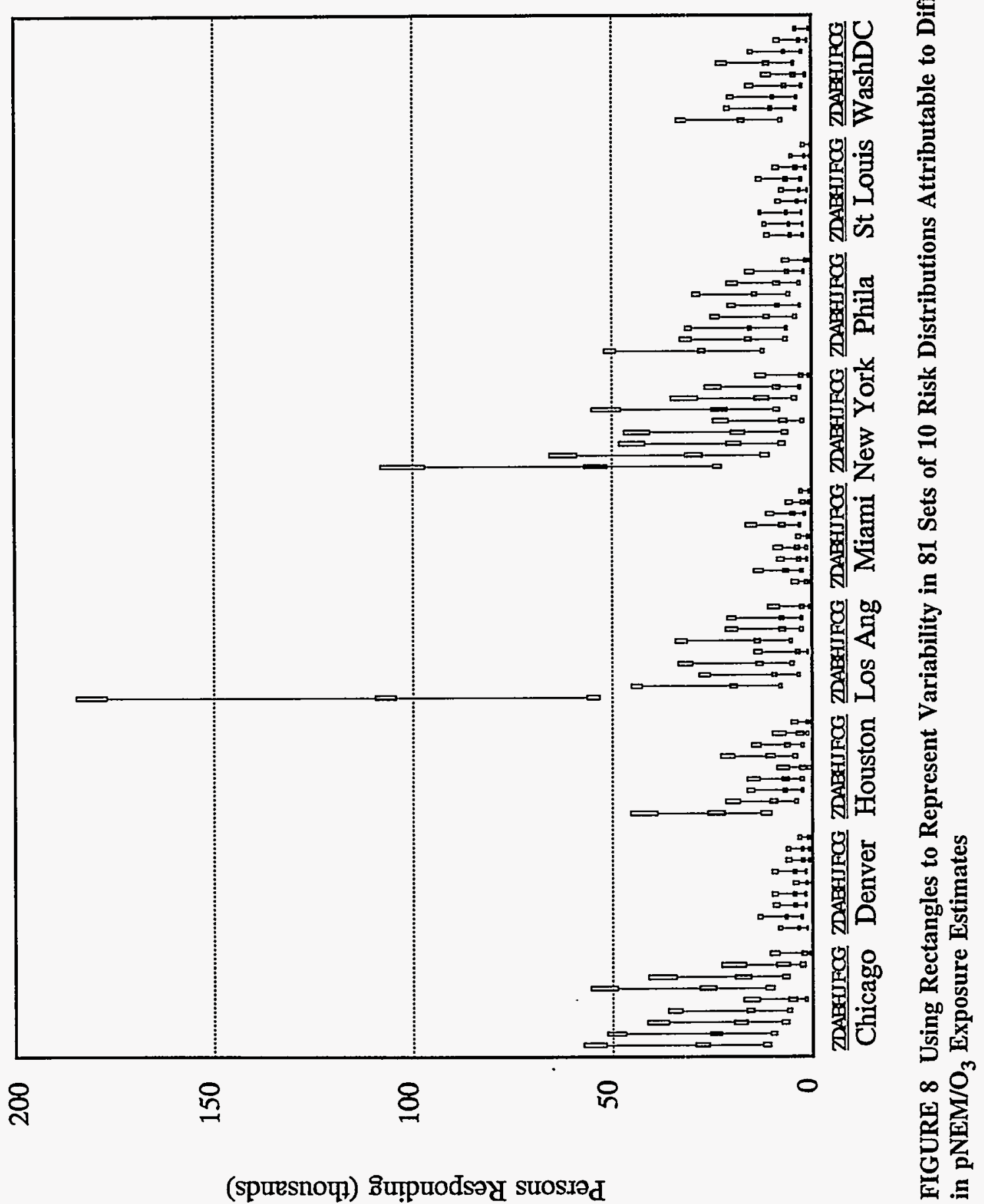


TABLE 16 Definitions of Letter Codes for Air Quality Scenarios

\begin{tabular}{|c|c|c|}
\hline $\begin{array}{l}\text { Letter } \\
\text { Code }\end{array}$ & Abbreviation & Definition of Scenario \\
\hline $\mathrm{Z}$ & As-Is & Existing air quality \\
\hline $\mathrm{D}$ & 8110 & $8 \mathrm{~h}, 1$ expected exceedance, $0.10 \mathrm{ppm}$ standard \\
\hline $\mathrm{A}$ & 1112 & $1 \mathrm{~h}, 1$ expected exceedance, $0.12 \mathrm{ppm}$ standard \\
\hline $\mathrm{B}$ & 8109 & $8 \mathrm{~h}, 1$ expected exceedance, $0.09 \mathrm{ppm}$ standard \\
\hline $\mathrm{H}$ & 1110 & $1 \mathrm{~h}, 1$ expected exceedance, $0.10 \mathrm{ppm}$ standard \\
\hline $\mathrm{J}$ & 8509 & $8 \mathrm{~h}, 5$ expected exceedances, $0.09 \mathrm{ppm}$ standard \\
\hline $\mathrm{F}$ & 8508 & $8 \mathrm{~h}, 5$ expected exceedances, $0.08 \mathrm{ppm}$ standard \\
\hline $\mathrm{C}$ & 8108 & $8 \mathrm{~h}, 1$ expected exceedance, $0.08 \mathrm{ppm}$ standard \\
\hline G & 8107 & $8 \mathrm{~h}, 1$ expected exceedance, $0.07 \mathrm{ppm}$ standard \\
\hline
\end{tabular}

(1) No overlap:

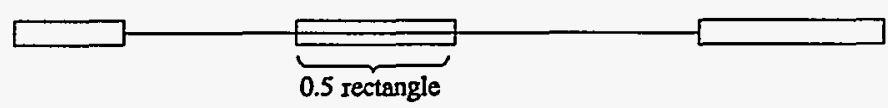

(2) 0.05 and 0.5 rectangles overlap:

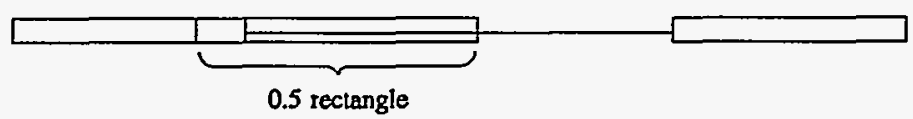

(3) All rectangles have the same (e.g., 0 ) lower bound:

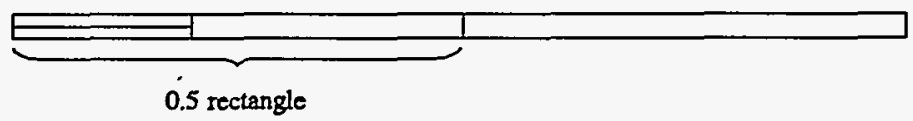

(4) 0.5 and 0.95 rectangles overlap:

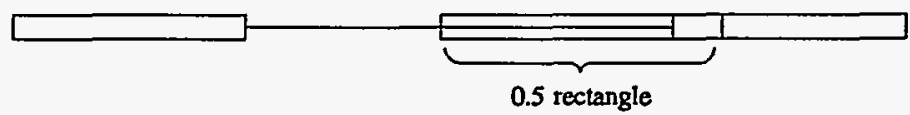

FIGURE 9 Patterns of Rectangles That Appear in Summary Figures for Headcount Risk Distributions 
represent the variability among the 10 risk distributions available for each air quality scenario, and this variability should be considered when addressing the issue of statistical significance.

To address significance, the Kolmogorov-Smirnoff (KS) test was applied. This twosample nonparametric test assumes that:

- There are two independent random samples, and

- The level of measurement is at least an ordinal scale.

The principle behind the KS test is as follows: if the null hypothesis is correct (that independent random samples have been drawn from identical populations), the cumulative frequency distributions for the two samples are expected to be similar. The test statistic is the maximum difference between two cumulative distributions. If the maximum difference is larger than would be expected by chance under the null hypothesis, the difference between the distributions is large enough to justify rejecting the null hypothesis.

The KS test was applied to the means of the 10 risk distributions available for each air quality scenario for the following:

- A 0.05 level of significance;

- Three health endpoints (MPD, 1-h exposures, moderate exertion; and FEV decrements $\geq 15$ and $\geq 20 \%$, 8-h exposures, moderate exertion);

- Children;

- Nine urban areas; and

- Five standards of particular interest $(8509,8508,8109,8108$, and 8107$)$.

These standards have 10 pairs of comparisons that can be tested. Of the 270 pairs of tests made in this study,

- 252 were significantly different (i.e., $P<0.05$ ), and

- 18 were not significantly different (i.e., cannot reject the null hypothesis).

Of the 18 pairs that were not significantly different, 3 had $P$ values very close to 0.05 . In a sense, these values may be "too close to call" because the KS test is weaker than other tests. Therefore, a stronger t-test was also applied (which was appropriate because the data for these cases were approximately normally distributed), and all 3 pairs were significantly different. Tables 17 and 18 summarize these results in detail. 
Of the 15 cases that were not significantly different (for which we accept the null hypothesis through either a KS test or a clarifying t-test), 7 are for Denver, and 3 are for Los Angeles; no other urban area has more than 2 cases that are not significantly different, and all cases are significantly different for 4 urban areas (Miami, New York, Philadelphia, and St. Louis). Furthermore, 5 of these cases are for the 8508 and 8109 scenarios and 7 compare 1 and 5 exceedances at the same ozone level, all of which were expected to produce similar risk estimates. None of the cases that are not significantly different involves a comparison with standard 8107 (the only standard that could reasonably be similar is 8108). Of these comparisons, 3 involve the M/S PDI endpoint, 6 involve $\mathrm{FEV}_{1}$ decrements $\geq 15 \%$, and 6 involve $\mathrm{FEV}_{1}$ decrements $\geq 20 \%$.

This analysis concludes that, for the majority of comparisons of interest, the standards produce significantly different risk results. This conclusion results from $\mathrm{KS}$ or t-tests of mean values that result from the $10 \mathrm{pNEM} / \mathrm{O}_{3}$ exposure distributions available for each standard. About 5\% of the comparisons of interest are not significantly different, and of these, the results are not unexpected for 12 of the cases. This conclusion is important because it is based on risk results that account for two principal sources of uncertainty: exposure levels and response given exposure level (i.e., exposure-response uncertainty).

\subsection{RATIOS OF MEDIAN NUMBERS OF BOTH OCCURRENCES AND RESPONDERS}

Results discussed so far have focused on the people measure for headcount risks, which addresses the fraction of individuals who may experience a specific health effect one or more times during an ozone season. For this measure, an individual can be counted at most one time. The "person-occurrences" measure counts the number of times in an ozone season that individuals experience a specific health effect. For this measure, an individual can be counted more than one time. Individuals who respond one or more times are called responders. The total number of person-occurrences divided by the number of responders yields an estimate of the number of times on average in an ozone season that a responder experiences a specific health effect. 
TABLE 18 Details about Scenarios That Are Not Significantly Different

\begin{tabular}{|c|c|c|c|c|c|c|}
\hline \multirow[b]{2}{*}{ Case } & \multirow[b]{2}{*}{ Endpoint $^{\mathrm{a}}$} & \multirow[b]{2}{*}{ Urban Area } & \multirow{2}{*}{\multicolumn{2}{|c|}{ Scenarios Compared }} & \multicolumn{2}{|c|}{$P$ Value } \\
\hline & & & & & $\mathrm{KS}$ test & t-test \\
\hline 1 & M/S PDI, 1-h & Denver & 8509 & 8109 & 0.951 & $-b$ \\
\hline 2 & M/S PDI, 1-h & Denver & 8108 & 8109 & 0.797 & - \\
\hline 3 & M/S PDI, 1-h & Denver & 8108 & 8509 & 0.991 & - \\
\hline 4 & $\mathrm{DFEV}_{1} \geq 15 \%, 8-\mathrm{h}$ & Chicago & 8508 & 8109 & 0.084 & - \\
\hline 5 & $\mathrm{DFEV}_{1} \geq 15 \%, 8-\mathrm{h}$ & Denver & 8509 & 8109 & 0.919 & - \\
\hline 6 & $\mathrm{DFEV}_{1} \geq 15 \%, 8-\mathrm{h}$ & Denver & 8108 & 8508 & 0.247 & - \\
\hline 7 & $\mathrm{DFEV}_{1} \geq 15 \%, 8-\mathrm{h}$ & Houston & 8508 & 8109 & 0.920 & - \\
\hline 8 & $\mathrm{DFEV}_{1} \geq 15 \%, 8-\mathrm{h}$ & Los Angeles & 8509 & 8109 & 0.055 & 0.012 \\
\hline 9 & $\mathrm{DFEV}_{1} \geq 15 \%, 8-\mathrm{h}$ & Los Angeles & 8108 & 8508 & 0.361 & - \\
\hline 10 & $\mathrm{DFEV}_{1} \geq 15 \%, 8-\mathrm{h}$ & St. Louis & 8508 & 8109 & 0.054 & 0.017 \\
\hline 11 & $\mathrm{DFEV}_{1} \geq 15 \%, 8-\mathrm{h}$ & Washington, D.C. & 8508 & 8109 & 0.901 & - \\
\hline 12 & $\mathrm{DFEV}_{1} \geq 20 \%, 8-\mathrm{h}$ & Denver & 8509 & 8109 & 0.837 & - \\
\hline 13 & $\mathrm{DFEV}_{1} \geq 20 \%, 8-\mathrm{h}$ & Denver & 8108 & 8508 & 0.240 & - \\
\hline 14 & $\mathrm{DFEV}_{1} \geq 20 \%, 8-\mathrm{h}$ & Houston & 8508 & 8109 & 1 & - \\
\hline 15 & $\mathrm{DFEV}_{1} \geq 20 \%, 8-\mathrm{h}$ & Los Angeles & 8509 & 8109 & 0.153 & - \\
\hline 16 & $\mathrm{DFEV}_{1} \geq 20 \%, 8-\mathrm{h}$ & Los Angeles & 8108 & 8508 & 1 & - \\
\hline 17 & $\operatorname{DFEV}_{1} \geq 20 \%, 8-h$ & St. Louis & 8508 & 8109 & 0.054 & 0.012 \\
\hline 18 & DFEV $_{1} \geq 20 \%, 8-\mathrm{h}$ & Washington, D.C. & 8508 & 8109 & 0.814 & - \\
\hline
\end{tabular}

a All endpoints are for individuals engaged in activities at a moderate level of exertion. M/S PDI denotes moderate-to-severe PDI, and $\mathrm{DFEV}_{1}$ denotes $\mathrm{FEV}_{1}$ decrement.

b Denotes that a t-test was not needed because the KS test $P$ value was sufficiently $>0.05$. 
Figures 10-12 display ratios of median numbers of occurrences and median numbers of responders for three of the "more severe" health endpoints ${ }^{11}$ (i.e., $\mathrm{FEV}_{1}$ decrements $\geq 15$ and $\geq 20 \%$ for 8-h exposure, moderate-to-severe chest pain on deep inspiration for 1-h exposures, all for outdoor children at moderate exertion). The ratio for a particular urban area is indicated by the first letter of the area name. For example, the ratio for Philadelphia and the M/S PDI endpoint is about 8 for scenario 8107 .

In general, the number of responders is lowest for the M/S PDI (1-h exposures, moderate exertion, outdoor children) endpoint and highest for $\mathrm{FEV}_{1}$ decrements $\geq 15 \%$ (8-h exposures, moderate exertion), as implied by Figures C.44, C.46, and C.47 in Appendix C (which show percent responses). However, M/S PDI have the highest frequency of repeated occurrences (the ratios range from 3 to 12 across the selected air quality scenarios), and $\mathrm{FEV}_{1}$ decrements $\geq 20 \%$ responders have the lowest frequency of repeated occurrences (the ratios range from 1 to 4). Thus, no consistent pattern is found among endpoints with respect to the number of responders and frequency of repeated occurrences.

With respect to cities, Chicago, Houston, and Miami tend to have the lowest ratios, and Philadelphia, St. Louis, and Washington, D.C., tend to have the highest ratios. Denver, Los Angeles, and New York City tend to be in between. Scenario 1112, which usually results in the second largest number of responders among the air quality scenarios considered in this section (scenario 8509 usually results in the largest number of responders), also tends to result in the highest ratios for each of the urban areas for all of the endpoints. Scenario 8107, which produces the lowest number of responders, tends to result in the lowest ratio for each city with two exceptions: the ratios for Philadelphia and Washington, D.C., for $\mathrm{FEV}_{1}$ decrements $\geq 15 \%$ are higher for scenario 8107 than they are for scenario 1112.

\subsection{AGGREGATE RESULTS FOR NINE URBAN AREAS}

This section describes an analysis that explicitly includes more than one urban area. Results for children in nine urban areas were combined for selected air quality scenarios and health endpoints to gain insights about the implications of different standards on a scale larger than one urban area. Results show that, among the air quality scenarios considered in this section:

- The 8-h, 1 expected exceedance, $0.07 \mathrm{ppm}$ of ozone standard results in the lowest number of responders;

- The 8-h, 5 expected exceedances, $0.09 \mathrm{ppm}$ of ozone standard results in the highest number of responders; and

11 These are not the endpoints associated with the highest levels of risk. 


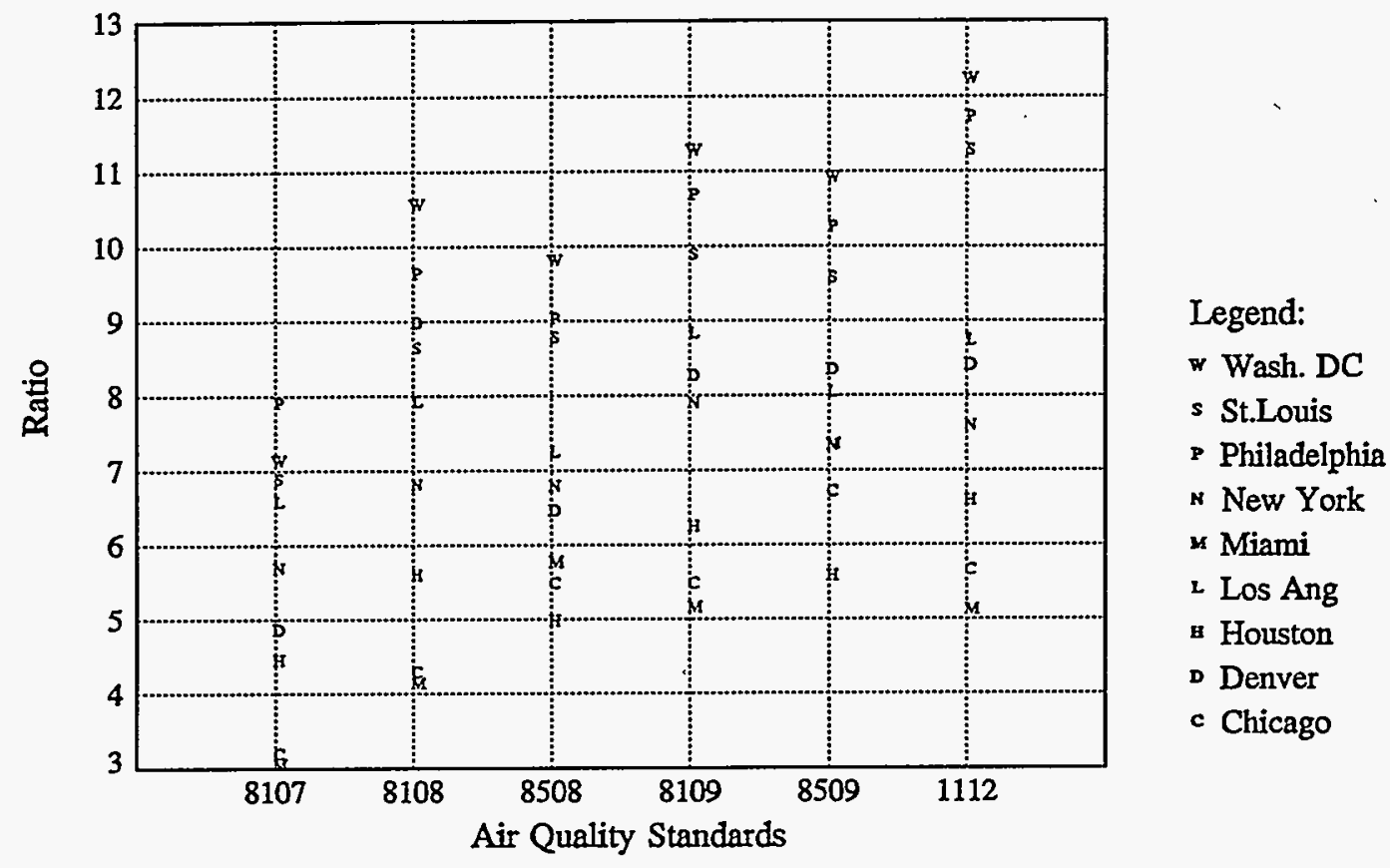

FIGURE 10 Ratios of Median Numbers of Occurrences and Responders Experiencing Moderate-to-Severe Pain on Deep Inspiration, for Nine Urban Areas, Outdoor Children, 1-h Exposures, and Moderate Exertion

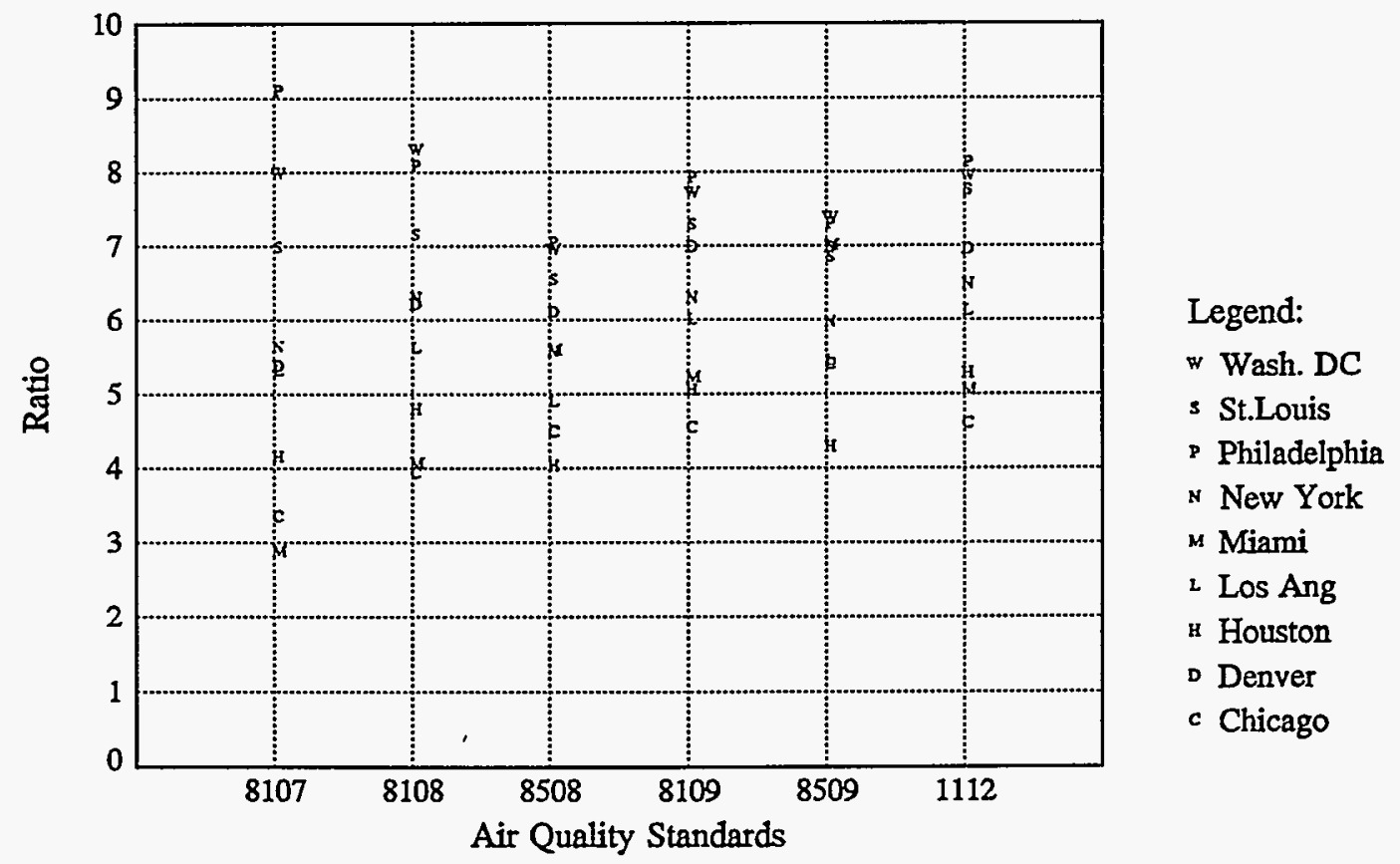

FIGURE 11 Ratios of Median Numbers of Occurrences and Responders for $\mathrm{FEV}_{1}$ Decrements $\geq 15 \%$, Nine Urban Areas, Outdoor Children, 8-h Exposures, and Moderate Exertion 


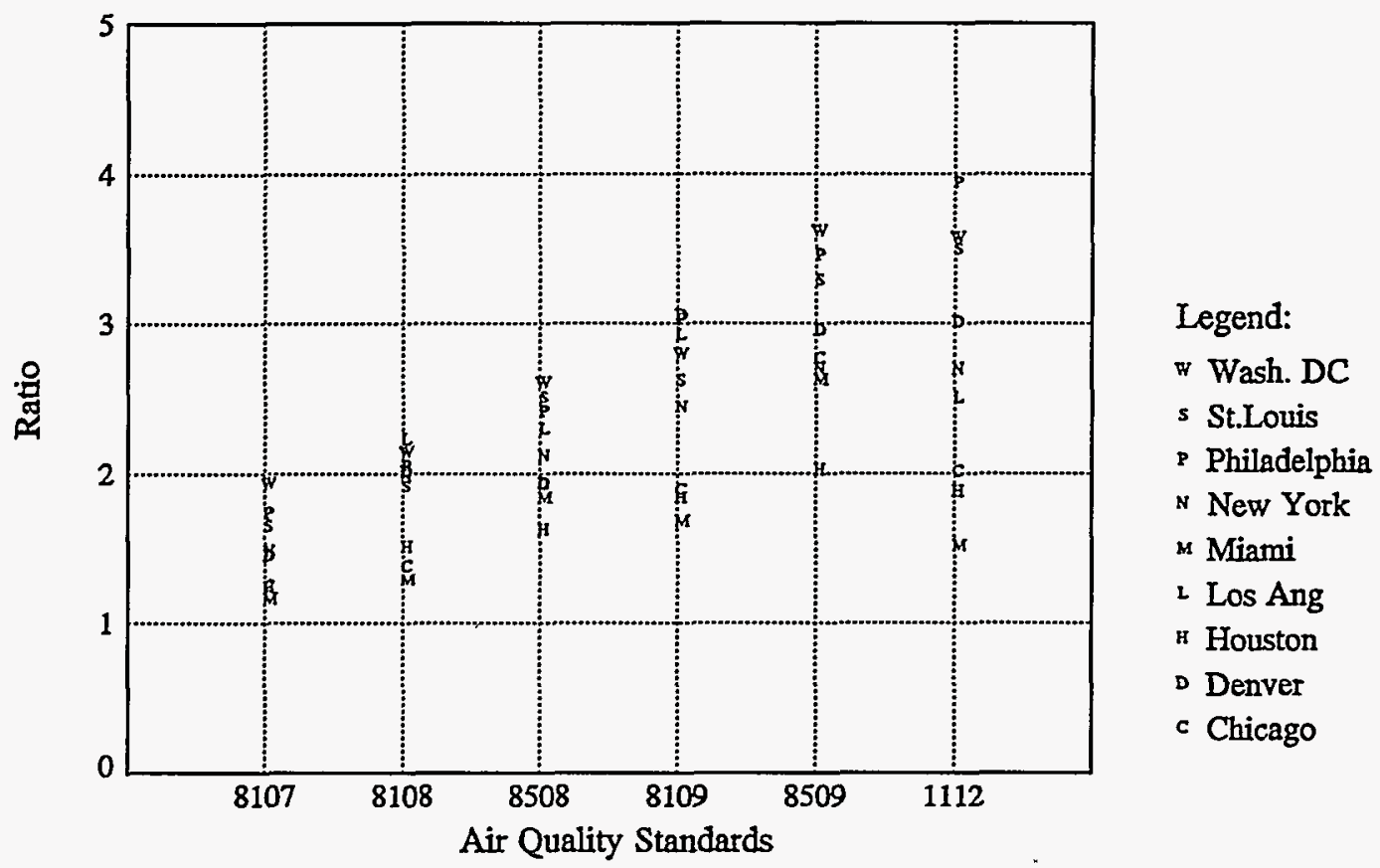

FIGURE 12 Ratios of Median Numbers of Occurrences and Responders for $\mathrm{FEV}_{1}$ Decrements $\geq 20 \%$, Nine Urban Areas, Outdoor Children, 8-h Exposures, and Moderate Exertion

- The 8-h, 1 expected exceedance, $0.09 \mathrm{ppm}$ and the current 1-h, 1 expected exceedance, $0.12 \mathrm{ppm}$ ozone standards result in similar numbers of responders.

For a specific health endpoint and air quality scenario, the analysis approach was to (1) determine the smallest and largest 0.05-, 0.5- (median), and 0.95-fractile results among the $10 \mathrm{pNEM} / \mathrm{O}_{3}$ runs available for each urban area, and (2) add each of these sets of values to obtain low and high 0.05-, 0.5-, and 0.95-fractile estimates for an aggregate risk distribution.

Results for three of the more severe health endpoints ${ }^{12}$ (i.e., $\mathrm{FEV}_{1}$ decrements $\geq 15$ and $\geq 20 \%$ for 8 -h exposure, moderate-to-severe chest pain on deep inspiration for 1-h exposures, all for outdoor children at moderate exertion) are shown for selected air quality scenarios in Figure 13. For all endpoints, the lowest responses are associated with the 8-h, 1 expected exceedance, $0.07 \mathrm{ppm}$ scenario, and the highest responses are associated with the 8-h, 5 expected exceedances, $0.09 \mathrm{ppm}$ scenario. Median responses across the range of standards examined are about $10,000-40,000,100,000-300,000$, and 10,000-120,000 children for the M/S PDI, FEV

12 These are not the endpoints associated with the highest levels of risk. 

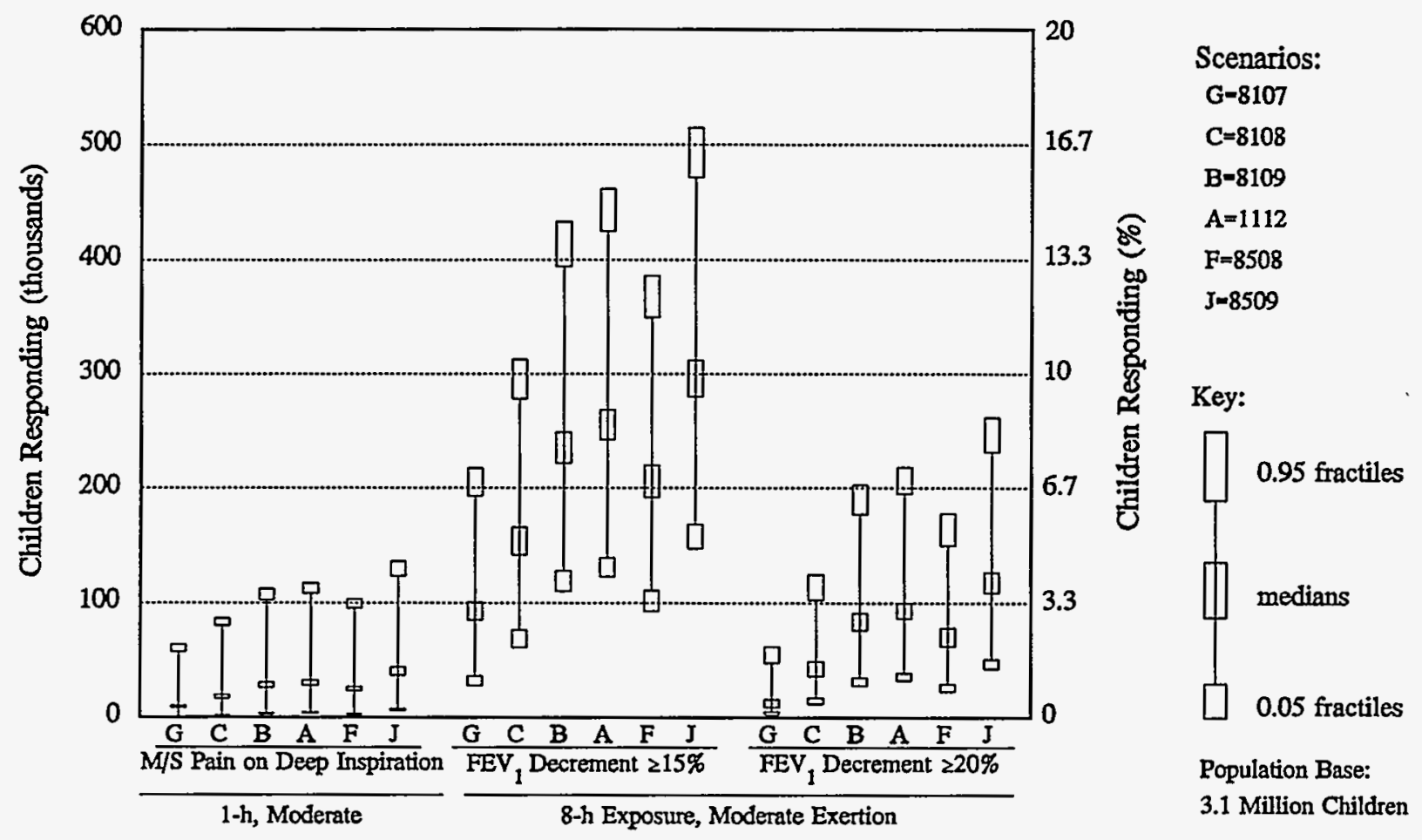

FIGURE 13 Aggregate Risk Results across Nine Urban Areas for Selected Health Endpoints and Air Quality Scenarios

decrements $\geq 15$ and $\geq 20 \%$ endpoints, respectively. Because the population base for these urban areas is about 3.1 million children, these response ranges correspond to percentage rates of about $0.3-1.3,3.3-10$, and $0.3-4 \%$, respectively. The risks associated with the current standard ( $1 \mathrm{~h}$, 1 exceedance, $0.12 \mathrm{ppm}$ ) are slightly higher than those for the 8-h, 1 exceedances, $0.09 \mathrm{ppm}$ standard and slightly lower than those for the 8-h, 5 exceedances, $0.09 \mathrm{ppm}$ standard. These results are consistent with those for individual urban areas shown in Figures C.45-C.47 in Appendix C.

\subsection{ANALYSIS ADDRESSING MULTIYEAR FORMS OF OZONE 8-HOUR NAAQS}

As noted earlier, all risk estimates in this report are based on a single year of air quality data or exposure estimates. Furthermore, the current ozone NAAQS are actually specified in terms of a three-year period. Therefore, the risk estimates discussed to this point represent the risks expected in a typical or average year in an area that just attains a given standard. To gain some insight into the possible impact of assessing risks based on a single year, EPA analyses of air quality data show that very few areas $(<4 \%)$ would have reported more than 10 exceedances during any single ozone season in which an $0.08 \mathrm{ppm}, 8-\mathrm{h}, 5$ expected exceedances standard 
would have been met (EPA 1996a). Recent discussions about a different form for the ozone NAAQS include limiting the average value of the fifth-highest 8-h daily maximum ozone concentration during a three-year period to $0.08 \mathrm{ppm}$ (designated as 8-h AvgMax5-80). Such a standard would not have an explicit limit to the number of exceedances that could occur in a given year. Again, recent air quality analyses suggest that few areas $(<8 \%)$ would have had more than 10 exceedances in the worst year of a three-year period that just attained this standard. Therefore, a tenth-highest, 8-h daily maximum, $0.08 \mathrm{ppm}$ scenario (designated as $10 \mathrm{xx} 08$, letter code $\mathrm{X}$ ) should lead to an upper bound of the risks in the highest year of a three-year period that just attains either an 8-h AvgMax5-80 or an 8-h, 5 expected exceedances, 0.08 ppm standard (EPA 1996a).

As a sensitivity analysis, the modified adjustment procedure was applied to ozone monitoring data used in previous $\mathrm{pNEM} / \mathrm{O}_{3}$ analyses of seven study areas: Chicago, Houston, Los Angeles, New York City, Philadelphia, St. Louis, and Washington, D.C. (Two other pNEM/O ${ }_{3}$ study areas, Denver and Miami, were omitted from the analysis because the ozone levels in these areas are low relative to the levels permitted by an 8-h AvgMax5-80 standard.) As for the nine air quality scenarios discussed earlier, the $\mathrm{pNEM} / \mathrm{O}_{3}$ model was run 10 times to produce exposure estimates for outdoor children in the selected study areas (Johnson 1996).

Figures 14-16 show risk results (box plots for percent of children responding) for three health endpoints for outdoor children at moderate exertion: MPD after 1-h exposure and FEV 1 decrements $\geq 15$ and $\geq 20 \%$ after 8 -h exposure, respectively. Several air quality scenarios are included to frame results for this special scenario. Of particular interest are comparisons with scenarios F (8508), B (8109), and $\mathrm{J}$ (8509). For these three endpoints, risk results for scenario $\mathrm{X}$ are, as expected, (1) higher than those for scenario $F$ and (2) lower than those for scenario $J$ in all seven urban areas. ${ }^{13}$ In addition, the risk estimates for scenario $B$ are lower than those for scenario $\mathrm{X}$ in five study areas and higher in two study areas (Los Angeles and Philadelphia).

13 The only possible exceptions to these general statements involve the New York City study area: the risk distributions for scenarios $\mathrm{J}$ and $\mathrm{X}$ are similar. 


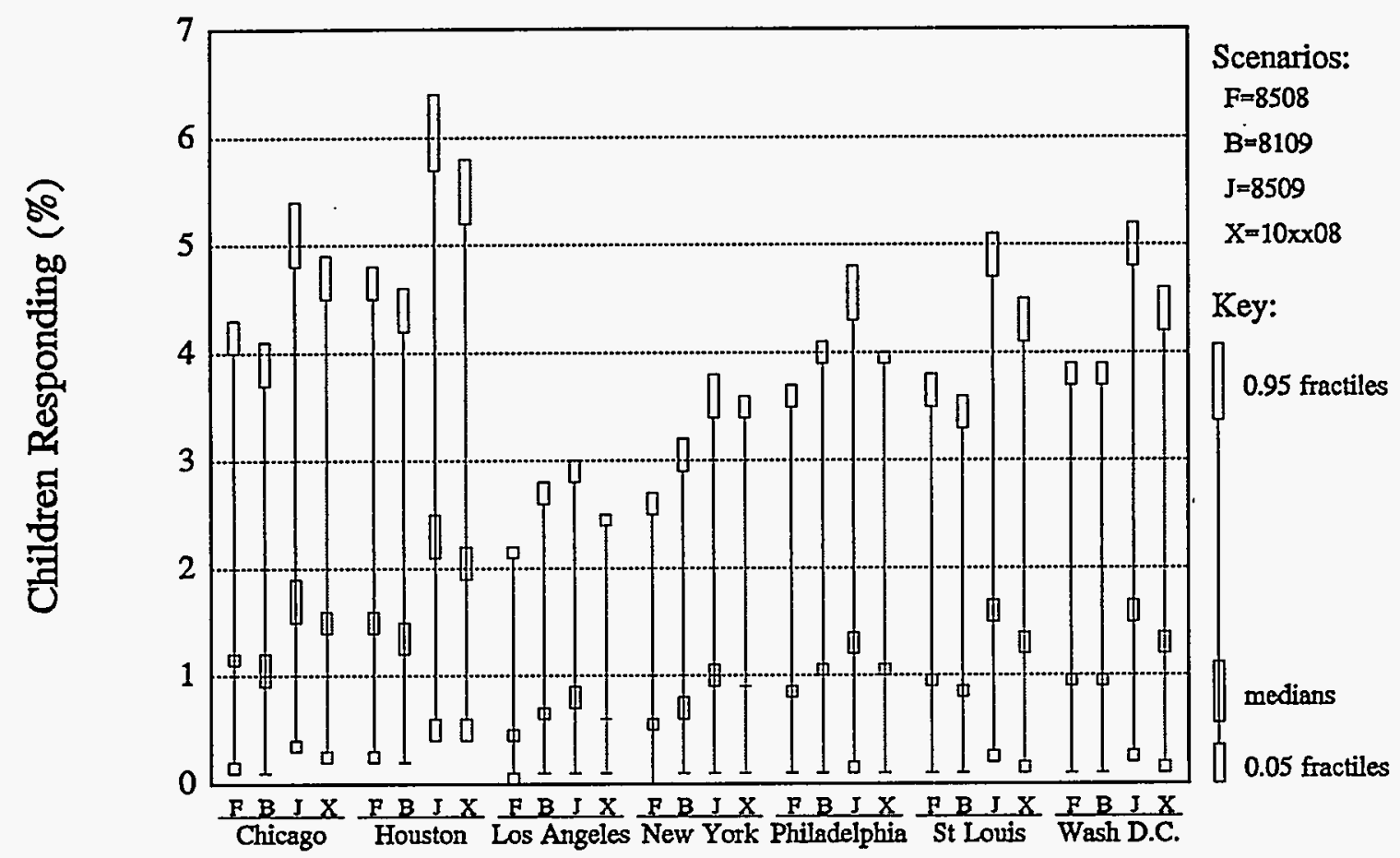

FIGURE 14 Risk Results for the MPD Endpoint and a Tenth-Highest, 1-h Daily Maximum Air Quality Scenario (outdoor children, 1-h exposure, moderate exertion)

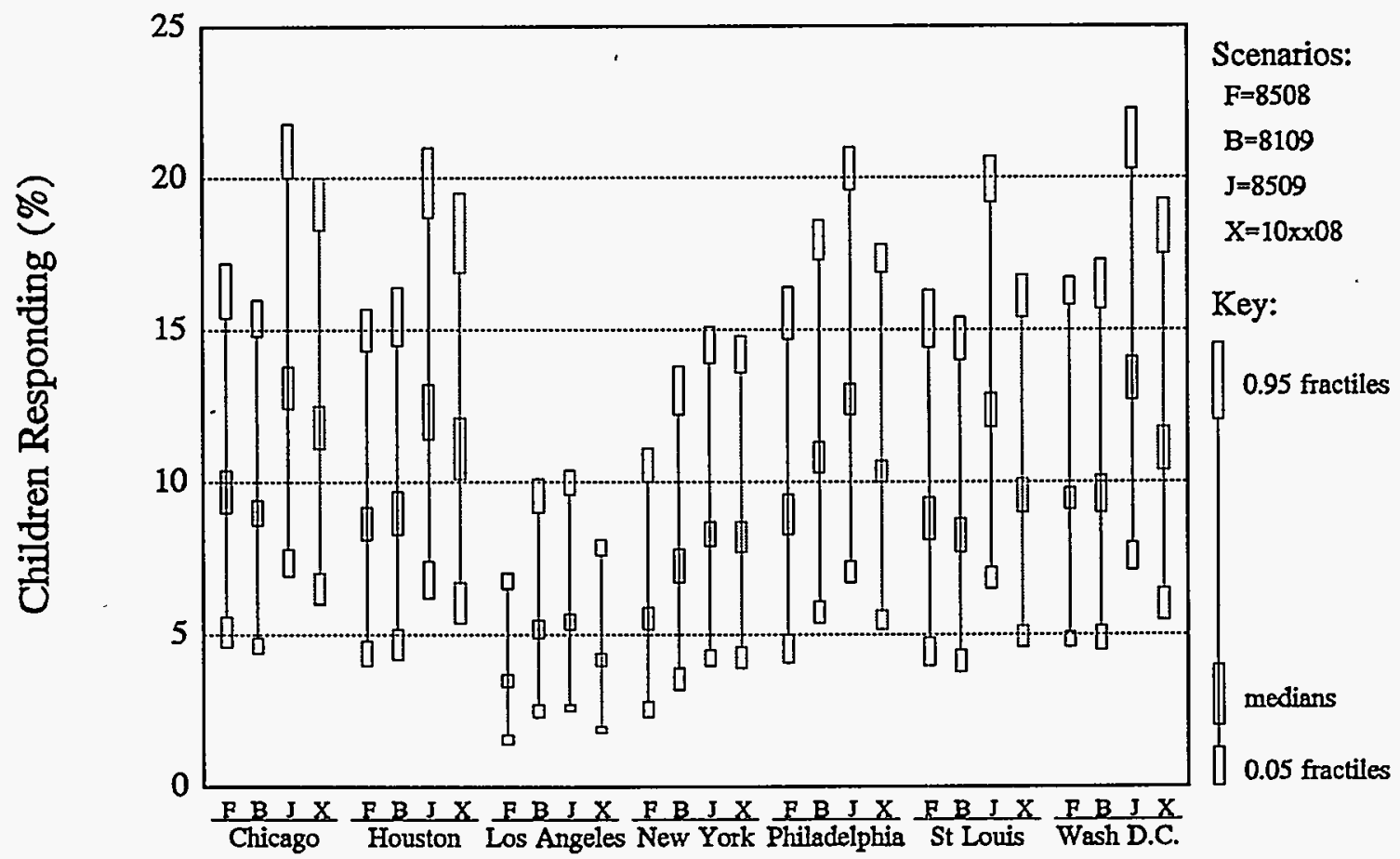

FIGURE 15 Risk Results for the FEV $_{1}$ Decrement $\geq 15 \%$ Endpoint and a Tenth-Highest, 8-h Daily Maximum Air Quality Scenario (outdoor children, 8-h exposure, moderate exertion) 


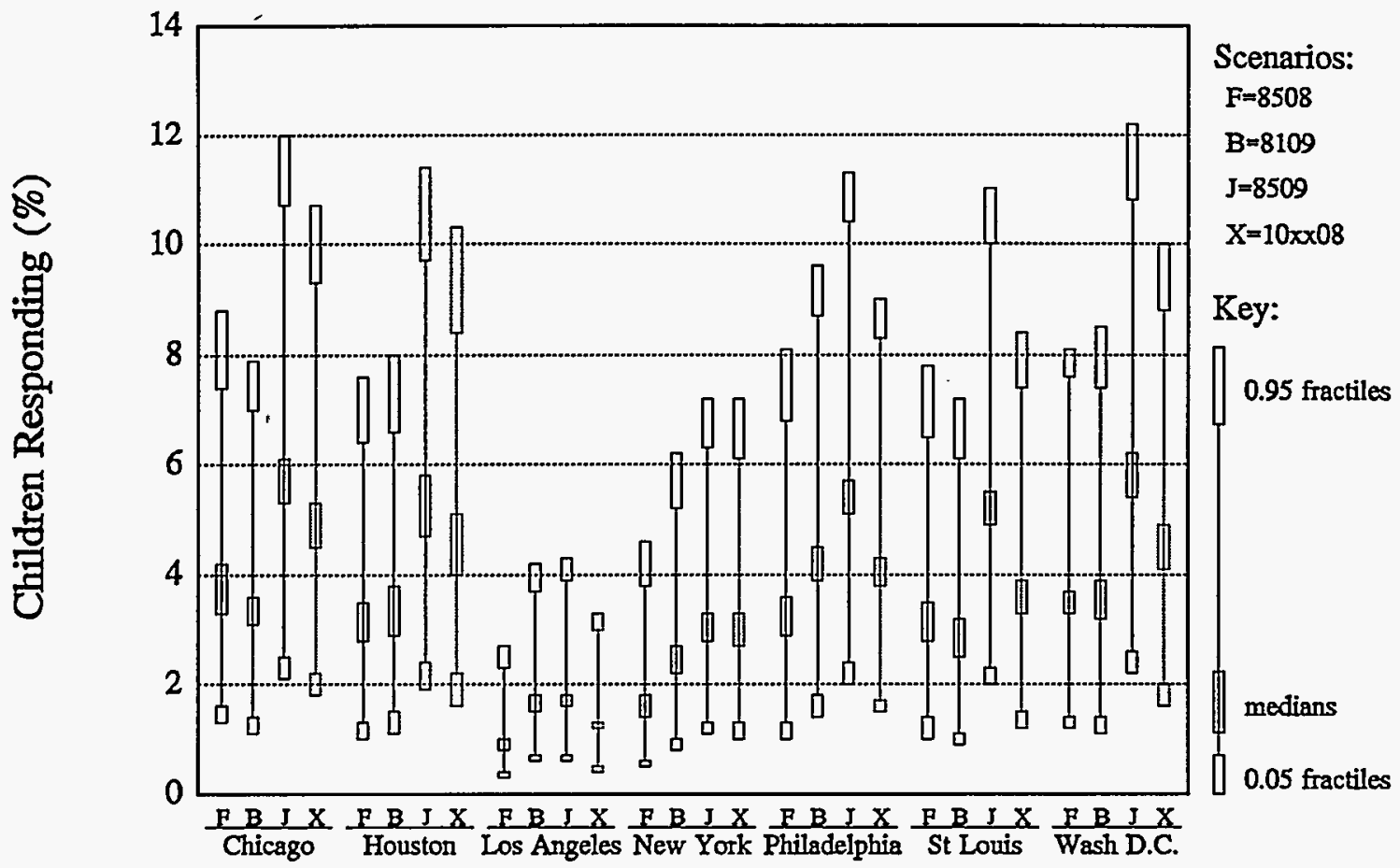

FIGURE 16 Risk Results for the $\mathrm{FEV}_{1}$ Decrement $\geq 20 \%$ Endpoint and a Tenth-Highest, 8-h Daily Maximum Air Quality Scenario (outdoor children, 8-h exposure, moderate exertion) 


\section{BENCHMARK RISK MODEL AND SELECTED RESULTS}

The second type of risk measure provided in this ozone health risk assessment is benchmark risk (Feagans and Biller 1981; Hayes et al. 1987; Whitfield et al. 1994). The first measure, headcount risk, focuses on the number of people affected and the number of incidences of a given health effect considering individuals' personal exposures as they go about their daily activities (e.g., going from indoors to outdoors, moving from place to place, and engaging in activities at different exertion levels). In contrast, benchmark risk focuses on the probability or risk of unhealthful air. Headcount risk measures the risk posed to a population by exposure to ozone. Benchmark risk treats the risk of unhealthful air as a hazard or the presence of a source of danger without regard to the number of people who may or do come in contact with it.

Air quality data and exposure-response probabilities (derived from the same exposureresponse relationships used to compute headcount risks for acute endpoints) are combined to obtain benchmark risk estimates. Unlike the headcount risk estimates, for which results of $10 \mathrm{pNEM} / \mathrm{O}_{3}$ runs are available, the benchmark risk calculations have only one estimate for air quality data.

\subsection{EQUATION FOR BENCHMARK RISK}

Benchmark response $r$ is the fraction of the population that experiences the specified health effect when exposed to ozone. Benchmark risk is the probability that the benchmark response is $\geq r, n$ or more times in a given period (e.g., one ozone season) at some location within a geographic region, given a specific condition of air quality (e.g., that standard 1112 is just attained). This report uses $r$ values of 0.05 and 0.1 (sometimes referred to as 0.05 and 0.1 benchmarks, or 5 and $10 \%$ benchmarks). Results for a 0.01 benchmark are also available.

Benchmark risk $B$ is calculated by applying the following equation:

$$
B(r, n, m, S)=\int_{c=0}^{\infty}[1-\Psi(r \mid c)] \cdot f_{n}(c \mid m, S) \mathrm{d} c
$$

where:

$$
\begin{aligned}
& m=\text { number of contiguous exposure periods in the ozone season; } \\
& S=\text { air quality scenario under consideration; } \\
& c=\text { ozone concentration; }
\end{aligned}
$$




$$
\begin{aligned}
\Psi(r \mid c)= & \begin{array}{l}
\text { exposure-response probability function (i.e., the probability } \\
\text { that up to an } r \text { fraction of the population will experience the } \\
\text { specified health effect, given that they are exposed to an } \\
\text { ozone concentration of } c \text { ppm; and }
\end{array} \\
f_{n}(c \mid m, S)= & \begin{array}{l}
\text { probability density function for the } n^{\prime} \text { th highest }(1-\text { or } 8-\mathrm{h} \\
\text { average) ozone concentration in the ozone season. }
\end{array}
\end{aligned}
$$

For 1-h averages or 8-h running averages, $m$ is the number of hours in the ozone season; for daily maximum 1-h averages or 8 - $\mathrm{h}$ running averages, $m$ is the number of days in the ozone season.

The result of the calculation is a probability estimate. The benchmark risk was calculated for all combinations of the following factors:

- 9 urban areas;

- 33 acute health endpoints;

- 6 air quality scenarios (i.e., alternative air quality standards);

- 2 ozone concentration sets (24 concentrations per day, or 1 daily maximum concentration) for each day in the ozone season;

- 3 values of $r(0.01,0.05$, and 0.1$)$; and

- 2 levels for the $n^{\prime}$ th highest ozone concentration (namely, first- and fifthhighest values).

This report does not show results for the 24 running averages per day, the $r=0.01$ cases, or all endpoints. Results for all other combinations of factors for 19 endpoints are shown in the figures in Appendix D.

\subsection{GRAPHIC FORMAT FOR DISPLAYING BENCHMARK RISK RESULTS}

Figure 17 shows the graphic format used to display benchmark risk results. The figure is for the probability that the benchmark response for the 8-h exposure, moderate exertion, FEV decrement $\geq 20 \%$ endpoint will be exceeded 5 or more times in an ozone season for 0.05 and 0.10 benchmarks. 

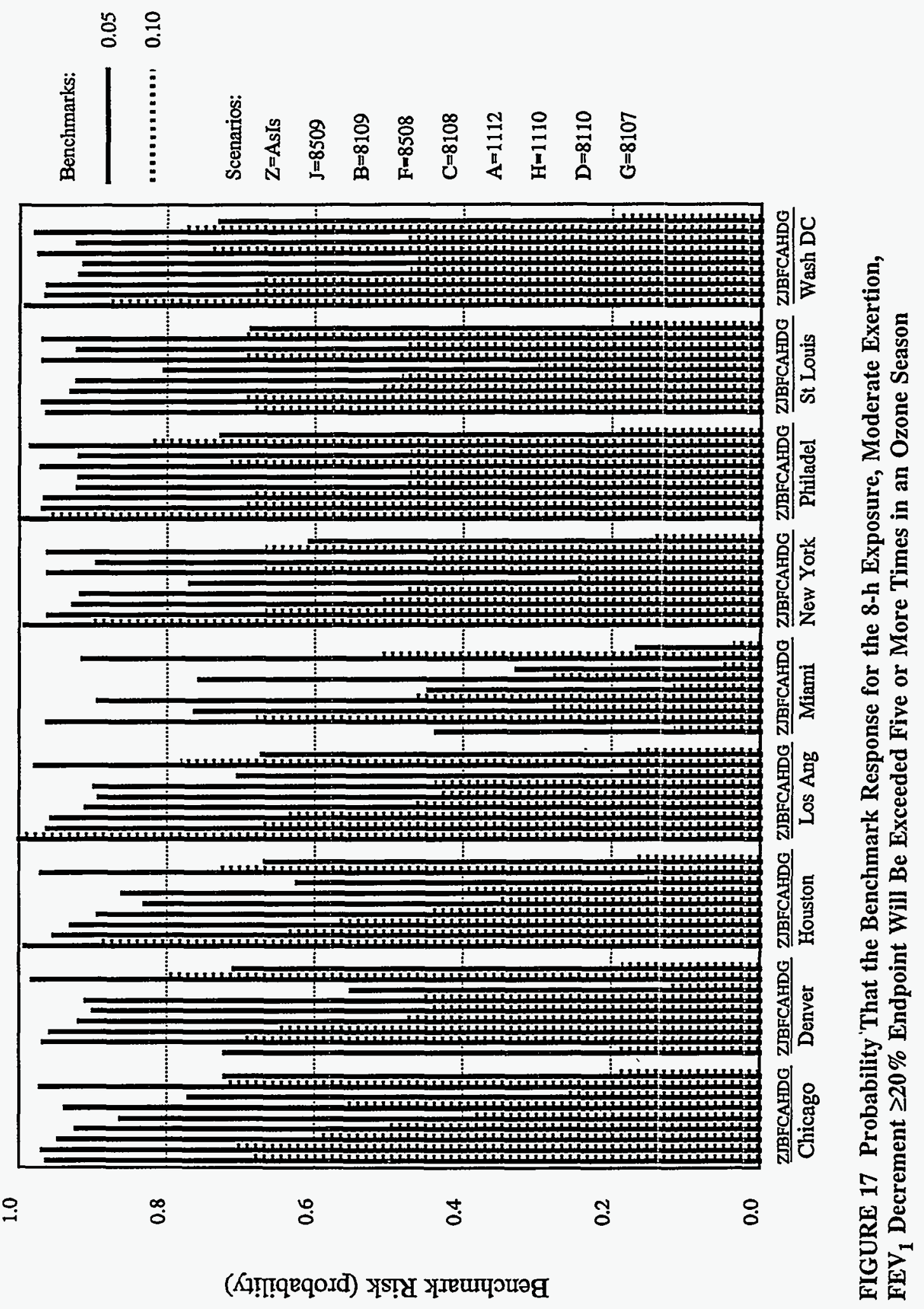
Figure 17 includes results for nine urban areas and nine air quality scenarios for each urban area. A letter code above the name of each area indicates the air quality scenario. The letter code is explained in the right side of the figure. Two vertical lines are shown for each air quality scenario: the solid line denotes the 0.05 benchmark, and the dotted line denotes the 0.10 benchmark. The height of the line indicates the benchmark risk. The benchmark risk for the 0.05 benchmark is, logically, greater than or equal to the benchmark risk for the 0.10 benchmark. For example, for Chicago, scenario G, daily maximum 8-h running average ozone concentrations (using the distribution for the highest ozone concentration), the benchmark risk for the 0.05 benchmark is about 0.5 , and the benchmark risk for the 0.10 benchmark is about 0.1 . In other words, if standard 8107 were just attained in Chicago, the benchmark risk (i.e., probability) would be 0.5 that the air quality would be sufficient to cause $\geq 5 \%$ of the population to experience $\mathrm{FEV}_{1}$ decrements $\geq 20 \%$ one or more times in an ozone season.

Table 19 summarizes one aspect of the contents in Figure 17: it lists the number of urban areas that have benchmark risk estimates $\leq 0.1,0.2$, and 0.3 at each alternative NAAQS for 5 and $10 \%$ benchmarks for the following endpoint: $\mathrm{FEV}_{1}$ decrement $\geq 20 \%$, 8-h exposure of outdoor children engaged in activities at moderate exertion for one or more times in an ozone season. For the 5\% benchmark, which is addressed in the left side of the table, only one urban area (Miami) has benchmark risk estimates $\leq 0.3$, and then only for scenario $G(8107$ ).

The second set of entries in the right side of the table is for the same endpoint, but for a $10 \%$ benchmark; as expected, more urban areas have benchmark risks less than or equal to the levels of interest under these conditions because ozone levels are less likely to exceed the benchmarks five or more times compared to one or more times. For scenario $G$ (8107), all nine urban areas have benchmark risks $\leq 0.2$ (but not $\leq 0.1$ ); only two areas (Chicago and Miami) have benchmark risks $\leq 0.1$.

Similar results for the 19 benchmark risk figures shown in Appendix D (and 47 other cases that are not discussed further in this report) are given in Table D.1. The results in Table 19 correspond to case 58 in Table D.1. 
TABLE 19 Summary of Benchmark Risk Results: Number of Urban Areas Having Benchmark Risks $\leq \mathbf{0 . 1}, 0.2$, and 0.3 for As-Is Air Quality and Just Attaining Eight Alternative NAAQS for 5 and $10 \%$ Benchmarks (outdoor children, $\mathrm{FEV}_{1}$ decrements $\geq 20 \%$, 8-h exposures, moderate exertion, fifth highest of 8-h daily maximum concentrations) (Sources: based on Folinsbee et al. 1988; Horstman et al. 1990; McDonnell et al. 1991) ${ }^{\mathrm{a}}$

\begin{tabular}{|c|c|c|c|c|c|c|}
\hline \multirow[b]{2}{*}{ NAAQS } & \multicolumn{3}{|c|}{$5 \%$ Benchmark } & \multicolumn{3}{|c|}{$10 \%$ Benchmark } \\
\hline & $\leq 0.1$ & 0.2 & 0.3 & $\leq 0.1$ & 0.2 & 0.3 \\
\hline $\mathrm{Z}=\mathrm{As}-\mathrm{Is}$ & $-b$ & - & - & - & 2 & 2 \\
\hline $\mathrm{J}=8509$ & - & - & - & - & - & - \\
\hline$B=8109$ & - & - & - & - & - & 1 \\
\hline$F=8508$ & - & - & - & - & - & - \\
\hline$C=8108$ & - & - & - & - & 1 & 4 \\
\hline$A=1112$ & - & - & - & - & - & 1 \\
\hline $\mathrm{H}=1110$ & - & - & - & 1 & 4 & 5 \\
\hline $\mathrm{D}=8110$ & - & - & - & - & - & - \\
\hline$G=8107$ & - & 1 & 1 & 2 & 9 & 9 \\
\hline
\end{tabular}

a Three benchmark risk values $(\leq 0.1,0.2$, and 0.3 ) have represented three different "degrees of risk" of interest to OAQPS staff in the past.

b The dash is used rather than 0 to make the table easier to read. 


\section{MODEL FOR EXCESS HOSPITAL ADMISSIONS}

The hospital admissions model is based on (1) regression coefficients and corresponding standard errors developed by Thurston et al. (1992) and (2) 1-h daily maximum ozone concentrations developed by Johnson et al. (1996a-c). The model applies only to New York City and includes two types of respiratory admissions: asthmatics and members of the general population (including asthmatics) for any of a number of respiratory ailments (i.e., acute bronchitis or bronchiolitis, pneumonia, chronic obstructive pulmonary disease not related to asthma).

\subsection{MODEL DEVELOPMENT}

Regression coefficients and corresponding standard errors (Table 20) define "concentration-response" relationships that include related uncertainties. Figure 18 is a graph of the relationship (which is a set of 21 "curves," one for each of 21 fractiles) for asthmatics. Only the $0.05,0.50$ (median), and 0.95 fractiles are shown to avoid clutter. Although the concentrationresponse relationship is defined over the range of $0-0.04 \mathrm{ppm}$ ozone, the risk calculations in this section pertain, unless otherwise stated, to ozone levels greater than the estimated background $(0.04 \mathrm{ppm})$. The fractiles at each ozone concentration are obtained from the normal probability distribution.

One-hour daily maximum ozone concentrations for one ozone season under eight air quality scenarios from four monitor sites in New York City were used to estimate the number of excess (i.e., attributable to ozone concentrations higher than background) admissions. Table 21 lists the monitor numbers, names, and reasons for inclusion (in this analysis) of air quality data from these monitor sites in the admissions model.

TABLE 20 Regression Coefficients and Standard Errors for the Hospital Admissions Model $^{\mathrm{a}}$

\begin{tabular}{lcc}
\hline $\begin{array}{c}\text { Type of } \\
\text { Admission }\end{array}$ & $\begin{array}{c}\text { Regression } \\
\text { Coefficient }^{\mathrm{b}}\end{array}$ & $\begin{array}{c}\text { Standard } \\
\text { Error }^{\mathrm{b}}\end{array}$ \\
\hline Asthmatics & 11.7 & 4.7 \\
Total respiratory & 13.7 & 5.3 \\
\hline
\end{tabular}

a From Thurston et al. (1992), for 1988 air quality and admissions data.

b Units are admission/ppm-ozone/million people. 


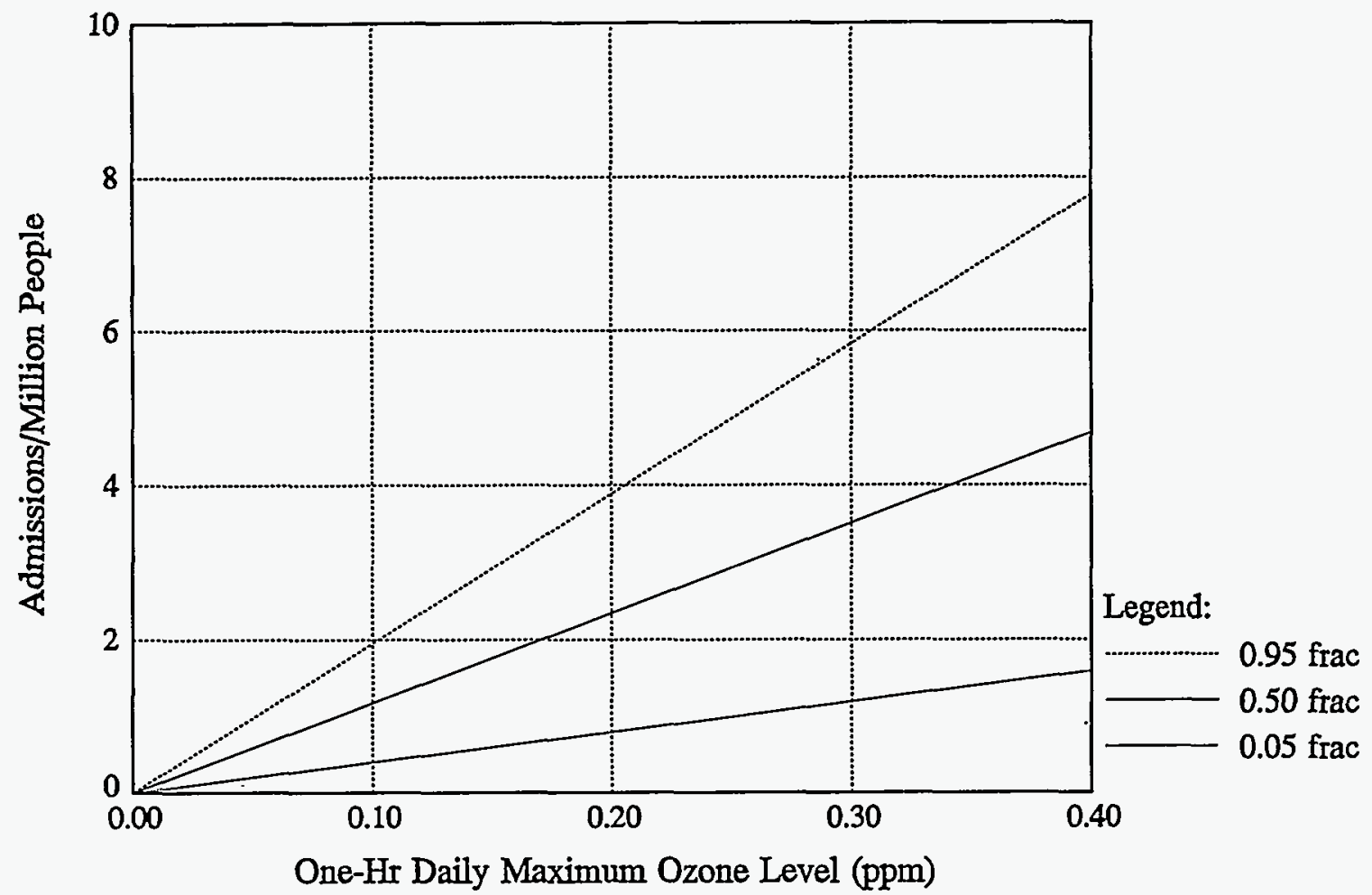

FIGURE 18 Uncertainty about Daily Hospital Admissions of Asthmatics in Relation to Ozone Concentration

TABLE 21 Monitor Site Numbers, Names, and Reasons for Inclusion in the Hospital Admissions Model

\begin{tabular}{|c|c|c|}
\hline No. & Name & Reason for Inclusion \\
\hline 1 & Greenwich & Design monitor site for 1 - and 8-h standards. \\
\hline 9 & Queens County & $\begin{array}{l}\text { Thurston et al. (1992) used Queens County ozone data in } \\
\text { their regressions. }\end{array}$ \\
\hline 11 & Babylon & Design monitor site for As-Is air quality. \\
\hline 12 & White Plains & $\begin{array}{l}\text { Thurston et al. (1992) used White Plains aerosol strong } \\
\text { acidity (particulate phase) ion }\left(\mathrm{H}^{+}\right) \text {and sulfate ion }\left(\mathrm{SO}_{4}=\right) \\
\text { data in their regressions. }\end{array}$ \\
\hline
\end{tabular}


Equation 4 gives the number of excess admissions (at a specific fractile) in an ozone season $X$ :

$$
X=P \times \sum_{i=1}^{N} \max \left[r\left(c_{i}\right)-r\left(c_{b g}\right), 0\right]
$$

where:

$$
\begin{aligned}
P & =\text { population (in millions); } \\
N & =\text { number of days in the ozone season (214 for New York City); } \\
c_{i} & =1 \text {-h daily maximum ozone concentration on day } i \\
r\left(c_{i}\right) & =\text { admission rate at concentration } c_{i} \text { on day } i \\
r\left(c_{b g}\right) & =\text { admission rate at the background concentration; and } \\
c_{b g} & =\text { background ozone concentration. }
\end{aligned}
$$

The incremental result is zero for days on which the 1-h daily maximum ozone concentration is less than or equal to the background concentration.

\subsection{RESULTS}

Calculations of Equation 4 were performed for a number of fractiles to define a probability distribution over admissions for an entire ozone season. Figure 19 and Table 22 give the results for asthmatics, a New York City population of 7.3 million, a background ozone concentration of $0.04 \mathrm{ppm}$, and air quality data at monitor 9 (the most relevant site). Figure 19 shows a cumulative probability function over excess annual admissions, corrected for background ozone of $0.04 \mathrm{ppm}$, for each of the nine air quality scenarios, eight of which are alternative NAAQS. The median estimate of the reduced number of excess admissions for the most stringent standard $G(60)$ compared to the median estimate for As-Is air quality (390) is about 330 .

Figures 20-22 give the results for asthmatics and additional monitors, and Figures 23-26 give the results for total respiratory admissions and all monitors. Figures 27 and 28 show another form of the results. This form is a variation of the Tukey box plot described in Morgan and Henrion (1990) and allows investigation of characteristics of the uncertainty in admissions results for all four monitors on one graph. A vertical line intersected by three short horizontal lines 


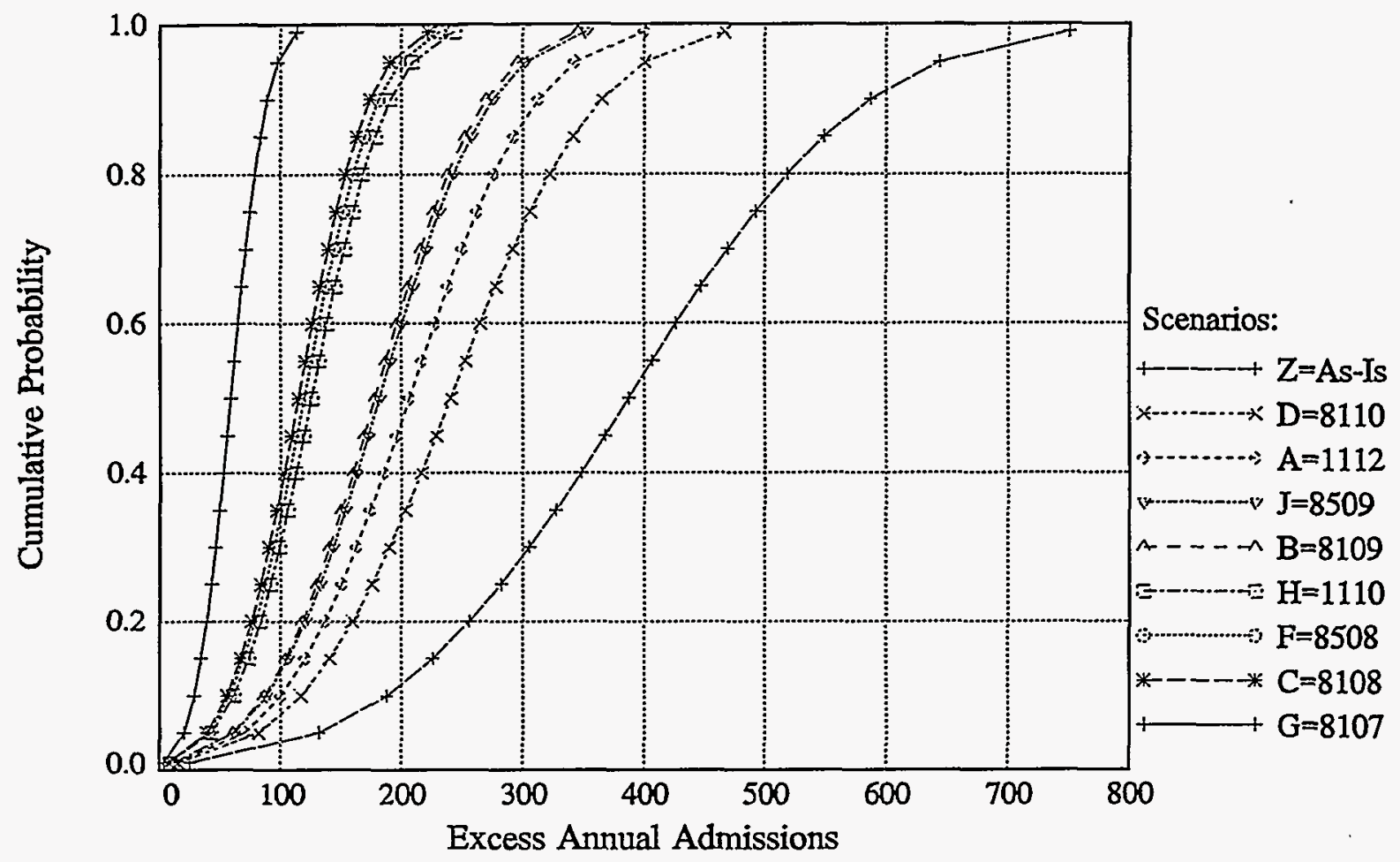

FIGURE 19 Excess Annual Hospitalizations of New York City Asthmatics for Nine Air Quality Scenarios and Monitor 9 Air Quality Data with Background of 0.04 ppm

indicates three points on a distribution over admissions for a specific air quality scenario: the top horizontal line indicates the 0.95 fractile of the distribution; the bottom horizontal line indicates the 0.05 fractile; and the short horizontal line between these two lines indicates the 0.5 fractile (i.e., the median). A letter code below each box plot indicates the air quality scenario. Table 16 defines the letter codes.

\subsection{DISCUSSION}

Thurston et al.'s (1992) results imply a mean number of 5.9 (standard error [s.e.] 2.4) excess daily admissions ${ }^{14}$ in New York City during June-August 1988, one of the highest ozone seasons on record. This estimate includes admissions attributable to ozone concentrations less than background levels. In comparision, the median estimate of the admissions model for monitor 9, As-Is air quality, and the 1991 ozone season (214 days) is nearly 390 excess annual

14 Thurston et al. (1992) report an average of 49.4 (s.e. 1.2) unscheduled admissions per day during the summer of 1988. Of these admissions, $11.9 \%$ (s.e. $4.8 \%$ ) are attributable to ozone exposure. (Thurston et al. call the $11.9 \%$ figure the "pollutant mean effect.") These estimates imply a daily average of 5.9 (s.e. 2.4) unscheduled admissions attributable to ozone exposure. 
TABLE 22 Annual Excess Hospital Admissions of Asthmatics for Monitor 9 Data (background = $0.04 \mathrm{ppm}$; basis 1-h daily maximum ozone level)

\begin{tabular}{|c|c|c|c|c|c|c|c|c|c|}
\hline \multirow[b]{2}{*}{ Fractile } & \multicolumn{9}{|c|}{ Air Quality Scenario } \\
\hline & As-Is & $8110^{a}$ & 1112 & 8509 & 8109 & 1110 & 8508 & 8108 & 8107 \\
\hline 0.01 & 25 & 16 & 14 & 12 & 12 & 8 & 8 & 8 & 4 \\
\hline 0.05 & 132 & 82 & 70 & 62 & 61 & 43 & 41 & 39 & 20 \\
\hline 0.10 & 188 & 117 & 100 & 88 & 87 & 61 & 58 & 56 & 28 \\
\hline 0.15 & 226 & 141 & 121 & 106 & 104 & 74 & 70 & 67 & 34 \\
\hline 0.20 & 257 & 160 & 137 & 121 & 118 & 83 & 79 & 76 & 39 \\
\hline 0.25 & 283 & 176 & 151 & 133 & 130 & 92 & 87 & 84 & 43 \\
\hline 0.30 & 306 & 190 & 163 & 144 & 141 & 99 & 94 & 91 & 46 \\
\hline 0.35 & 328 & 204 & 175 & 154 & 151 & 106 & 101 & 97 & 50 \\
\hline 0.40 & 348 & 217 & 186 & 164 & 160 & 113 & 107 & 103 & 53 \\
\hline 0.45 & 368 & 229 & 196 & 173 & 169 & 120 & 114 & 109 & 56 \\
\hline 0.50 & 388 & 241 & 207 & 182 & 179 & 126 & 120 & 115 & 59 \\
\hline 0.55 & 407 & 253 & 217 & 191 & 188 & 132 & 126 & 121 & 62 \\
\hline 0.60 & 427 & 266 & 228 & 201 & 197 & 139 & 132 & 127 & 65 \\
\hline 0.65 & 448 & 279 & 239 & 210 & 206 & 145 & 138 & 133 & 68 \\
\hline 0.70 & 469 & 292 & 250 & 221 & 216 & 153 & 145 & 139 & 71 \\
\hline 0.75 & 493 & 307 & 263 & 232 & 227 & 160 & 152 & 146 & 75 \\
\hline 0.80 & 519 & 323 & 277 & 244 & 239 & 169 & 160 & 154 & 79 \\
\hline 0.85 & 549 & 342 & 293 & 258 & 253 & 178 & 169 & 163 & 83 \\
\hline 0.90 & 587 & 365 & 313 & 276 & 270 & 191 & 181 & 174 & 89 \\
\hline 0.95 & 644 & 401 & 344 & 303 & 296 & 209 & 199 & 191 & 97 \\
\hline 0.99 & 750 & 467 & 400 & 352 & 345 & 244 & 231 & 222 & 114 \\
\hline Mean & 388 & 241 & 207 & 182 & 179 & 126 & 120 & 115 & $59^{\circ}$ \\
\hline StdDev & 156 & 97 & 83 & 73 & 72 & 51 & 48 & 46 & 24 \\
\hline
\end{tabular}

a Key to scenario code: character 1 defines averaging time; character 2 defines the number of exceedances; characters 3 and 4 define the ozone level (e.g., 8110 means 8-h averaging time, 1 exceedance, and $0.10 \mathrm{ppm})$.

admissions (corrected for background), which corresponds to a daily average of 1.8 (s.d. 0.7) admissions. When the model is restricted to the 92 days of June-August 1991, the median estimate is 2.9 (s.d. 1.2) admissions per day. Furthermore, when the background level of ozone in the model is set at $0 \mathrm{ppm}$ (which more closely matches the conditions modeled by Thurston et al.), the median results are 5.0 (s.d. 2.0) and 5.9 (s.d. 2.5) admissions per day for 214 days (April-October 1991) and 92 days (June-August 1991), respectively. Thus, model results for 92 days and background set at $0 \mathrm{ppm}$, which most closely match the conditions modeled by Thurston et al., are nearly identical to the results of Thurston et al. 


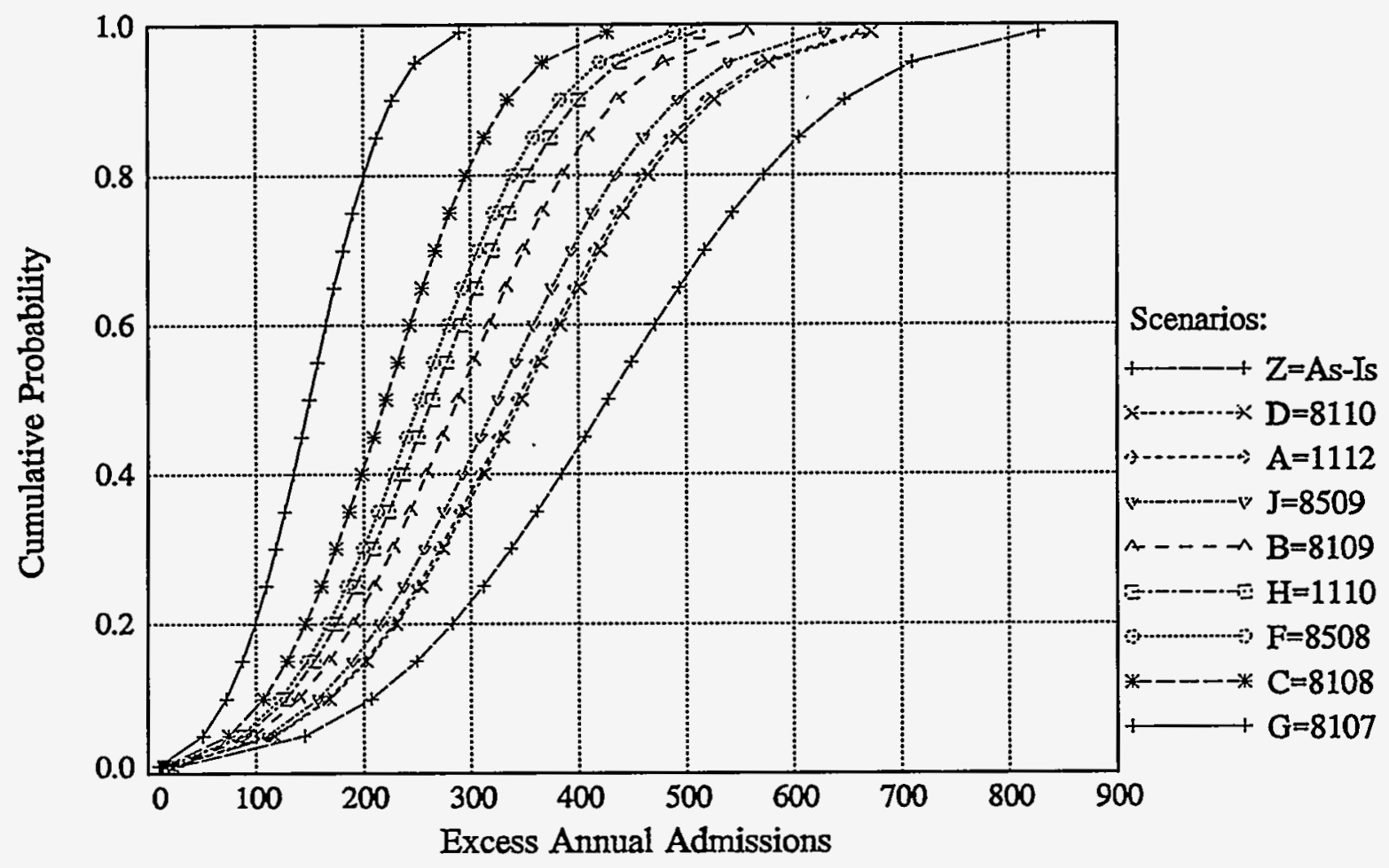

FIGURE 20 Excess Annual Hospitalizations of New York City Asthmatics for Nine Air Quality Scenarios and Monitor 1 Air Quality Data with Background of 0.04 ppm

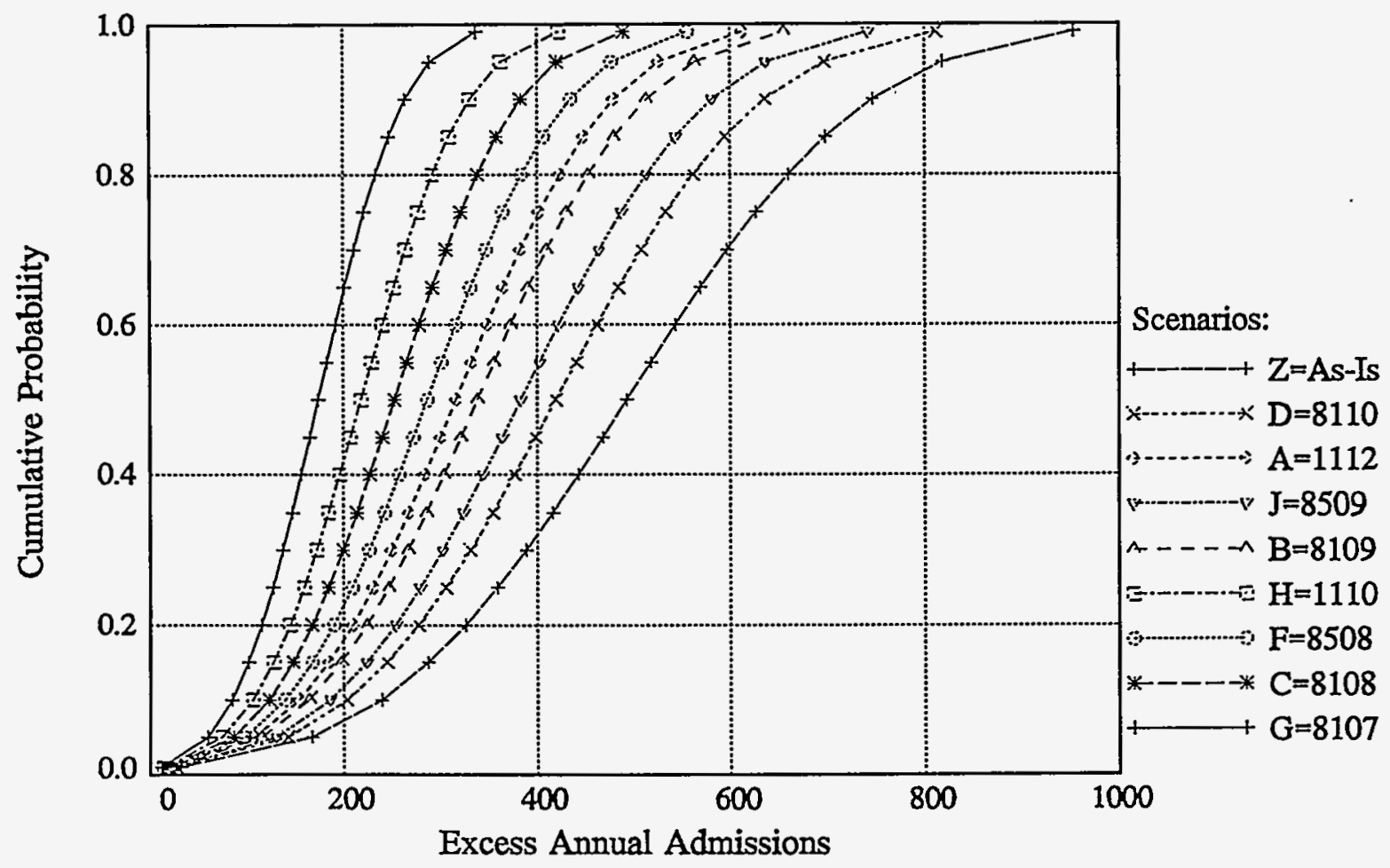

FIGURE 21 Excess Annual Hospitalizations of New York City Asthmatics for Nine Air Quality Scenarios and Monitor 11 Air Quality Data with Background of 0.04 ppm 


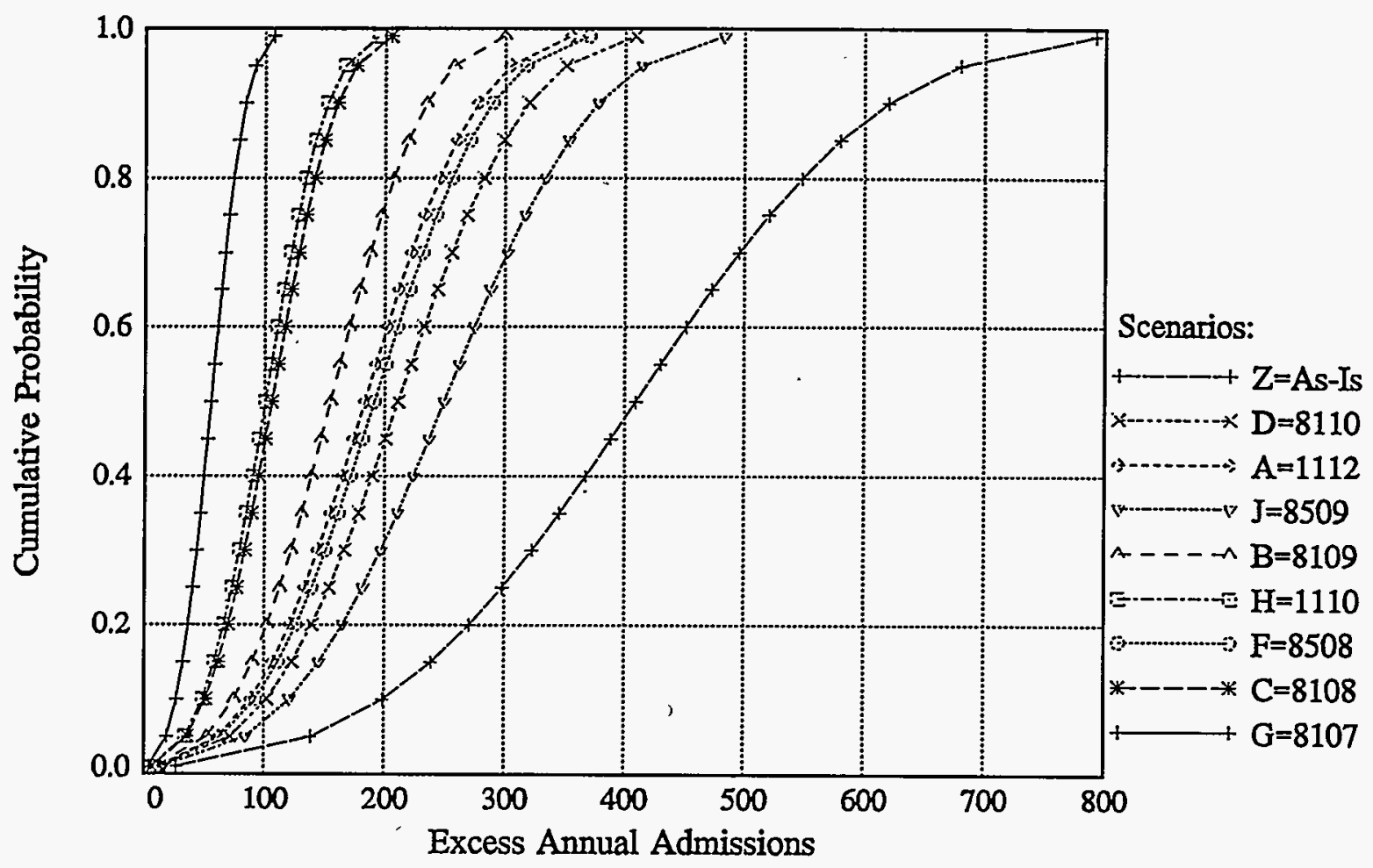

FIGURE 22 Excess Annual Hospitalizations of New York City Asthmatics for Nine Air Quality Scenarios and Monitor 12 Air Quality Data with Background of 0.04 ppm

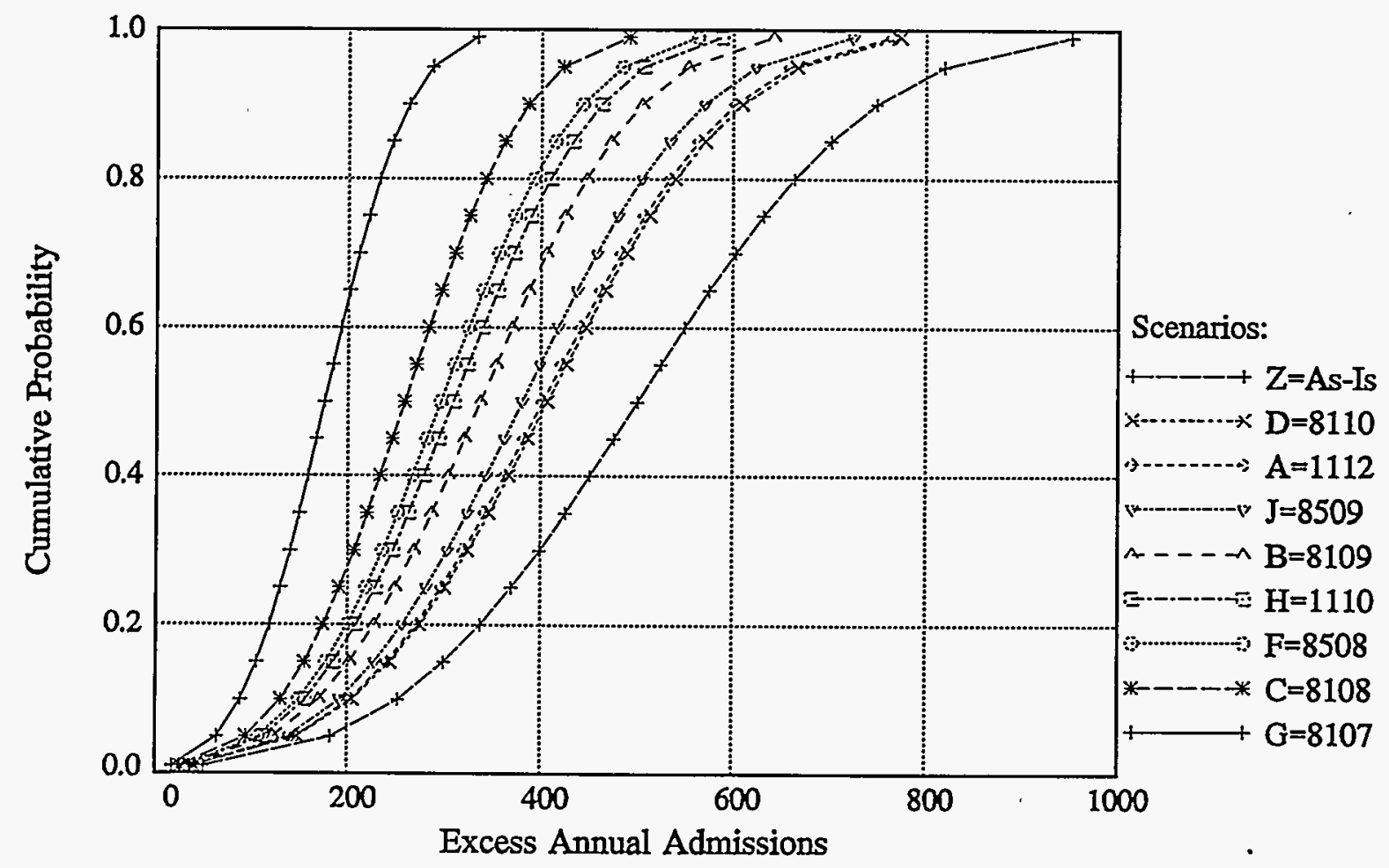

FIGURE 23 Excess Annual Hospitalizations of New York City Respiratory Patients for Nine Air Quality Scenarios and Monitor 1 Air Quality Data with Background of 0.04 ppm 


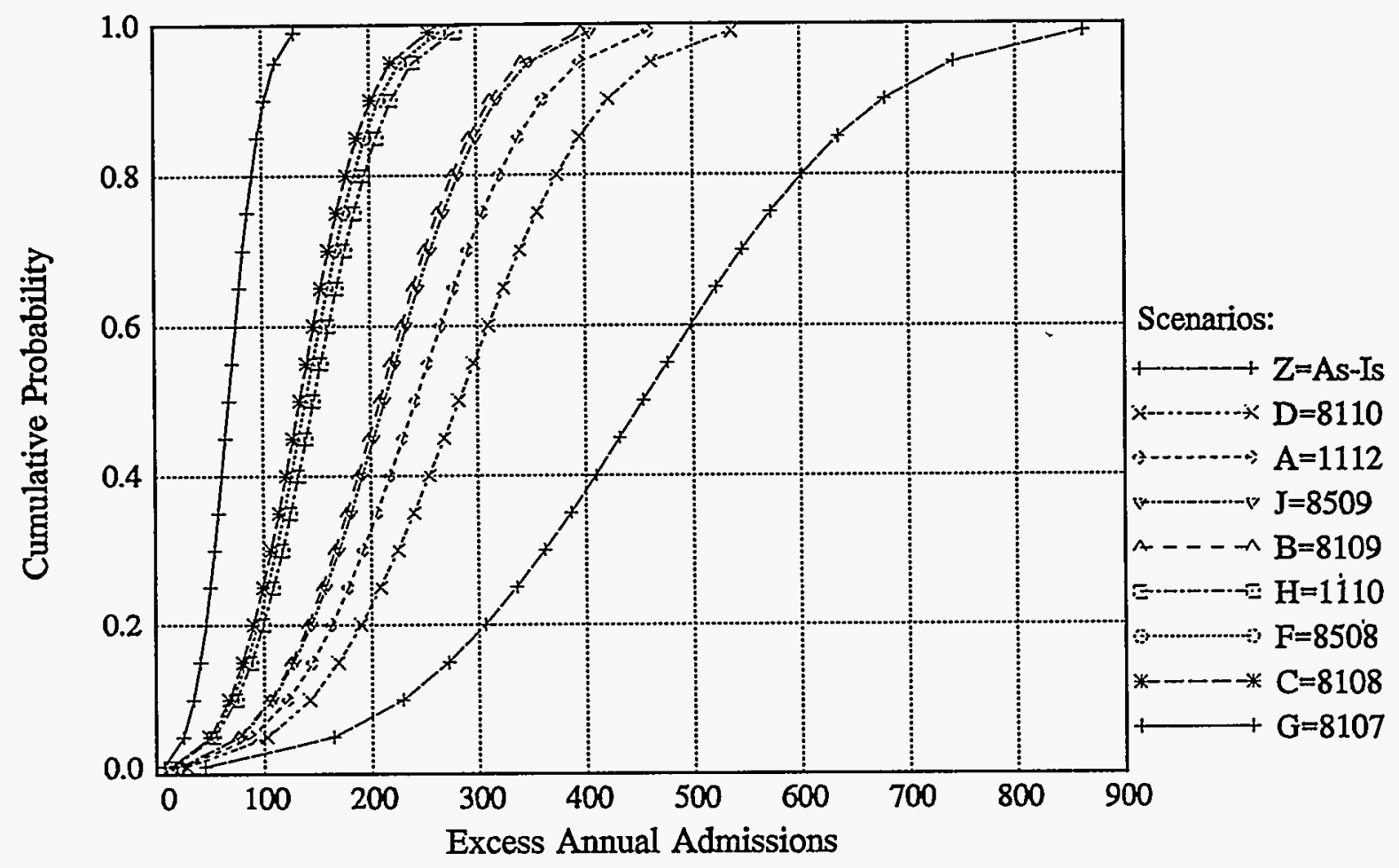

FIGURE 24 Excess Annual Hospitalizations of New York City Respiratory Patients for Nine Air Quality Scenarios and Monitor 9 Air Quality Data with Background of 0.04 ppm

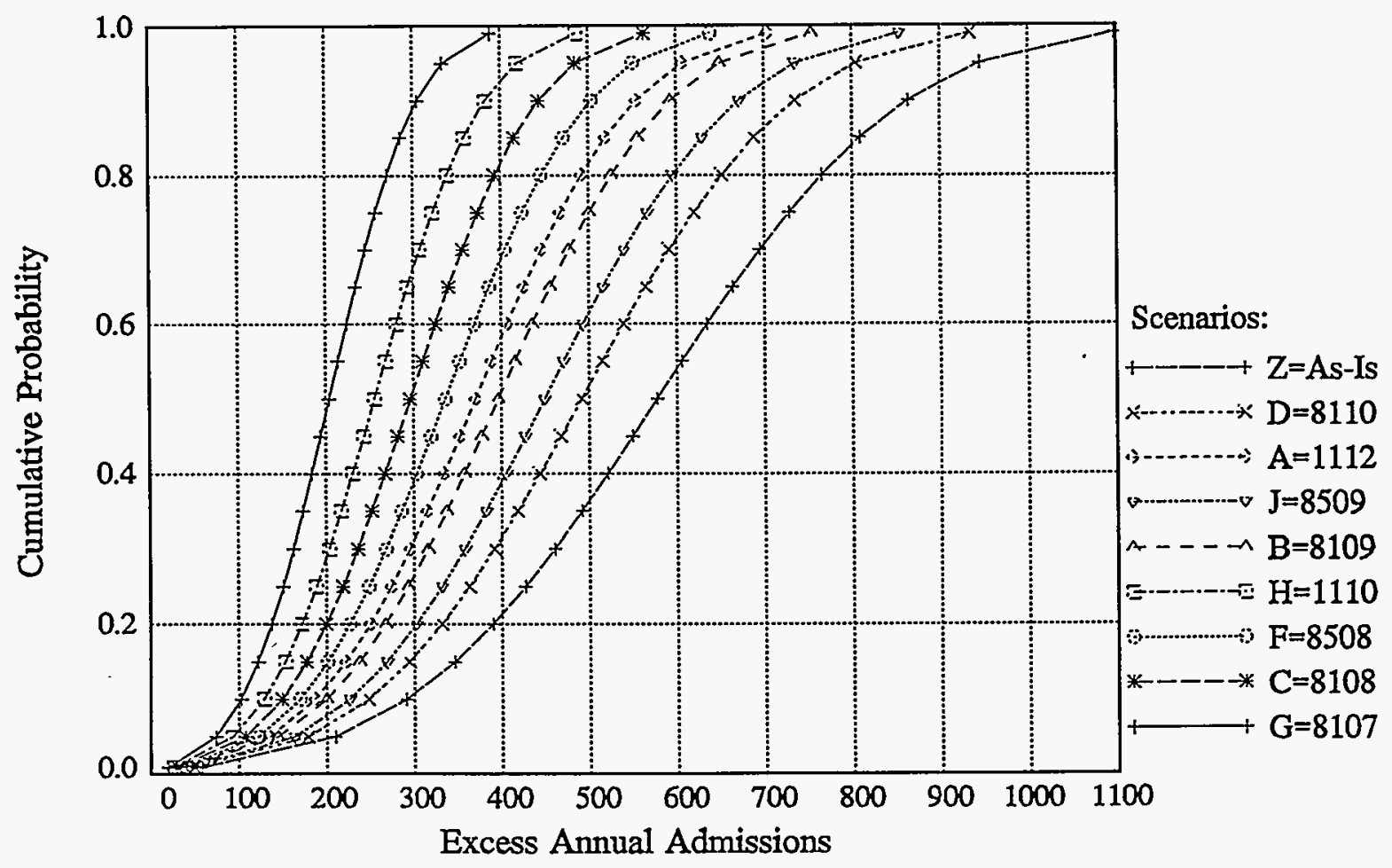

FIGURE 25 Excess Annual Hospitalizations of New York City Respiratory Patients for Nine Air Quality Scenarios and Monitor 11 Air Quality Data with Background of 0.04 ppm 


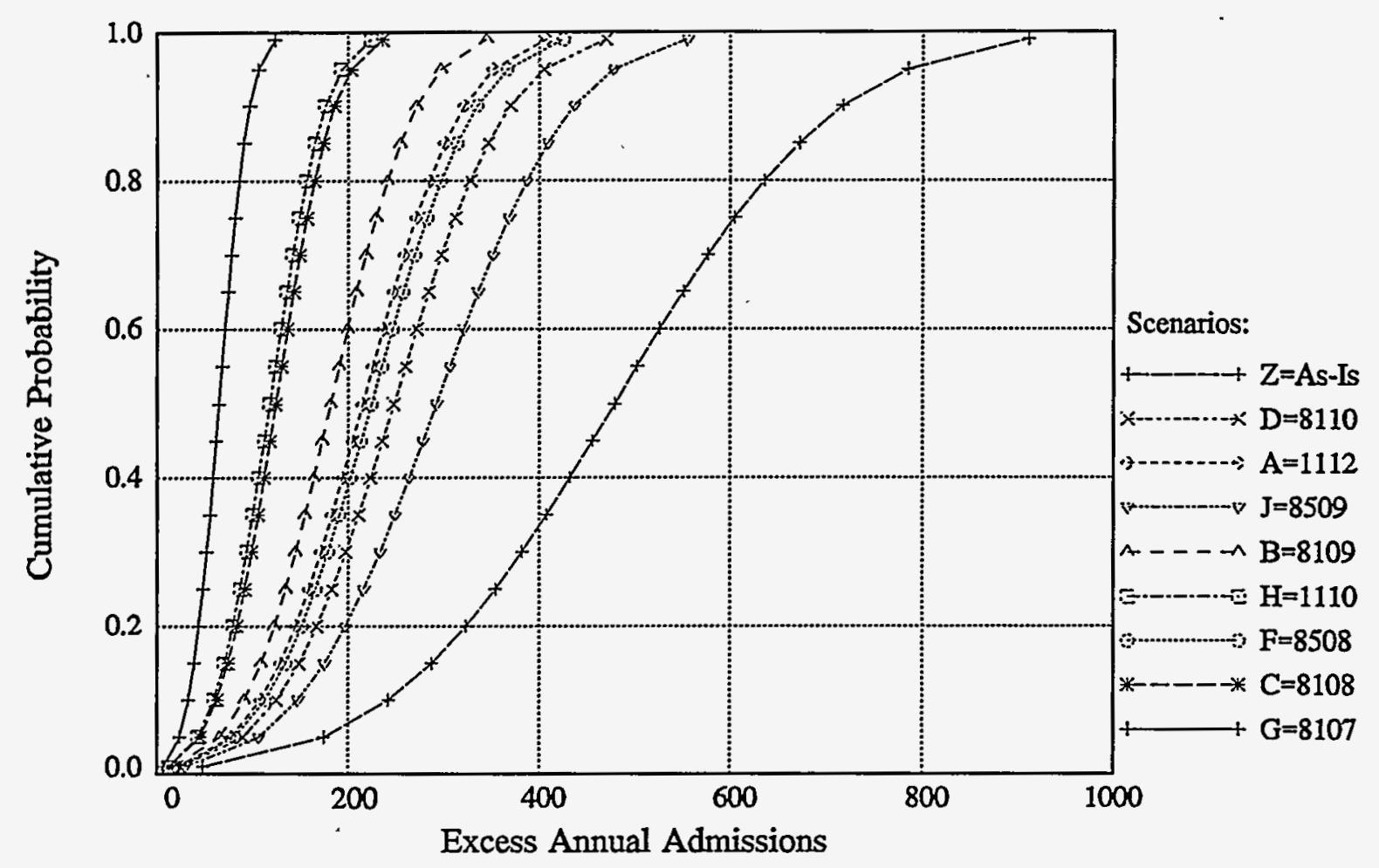

FIGURE 26 Excess Annual Hospitalizations of New York City Respiratory Patients for Nine Air Quality Scenarios and Monitor 12 Air Quality Data with Background of 0.04 ppm

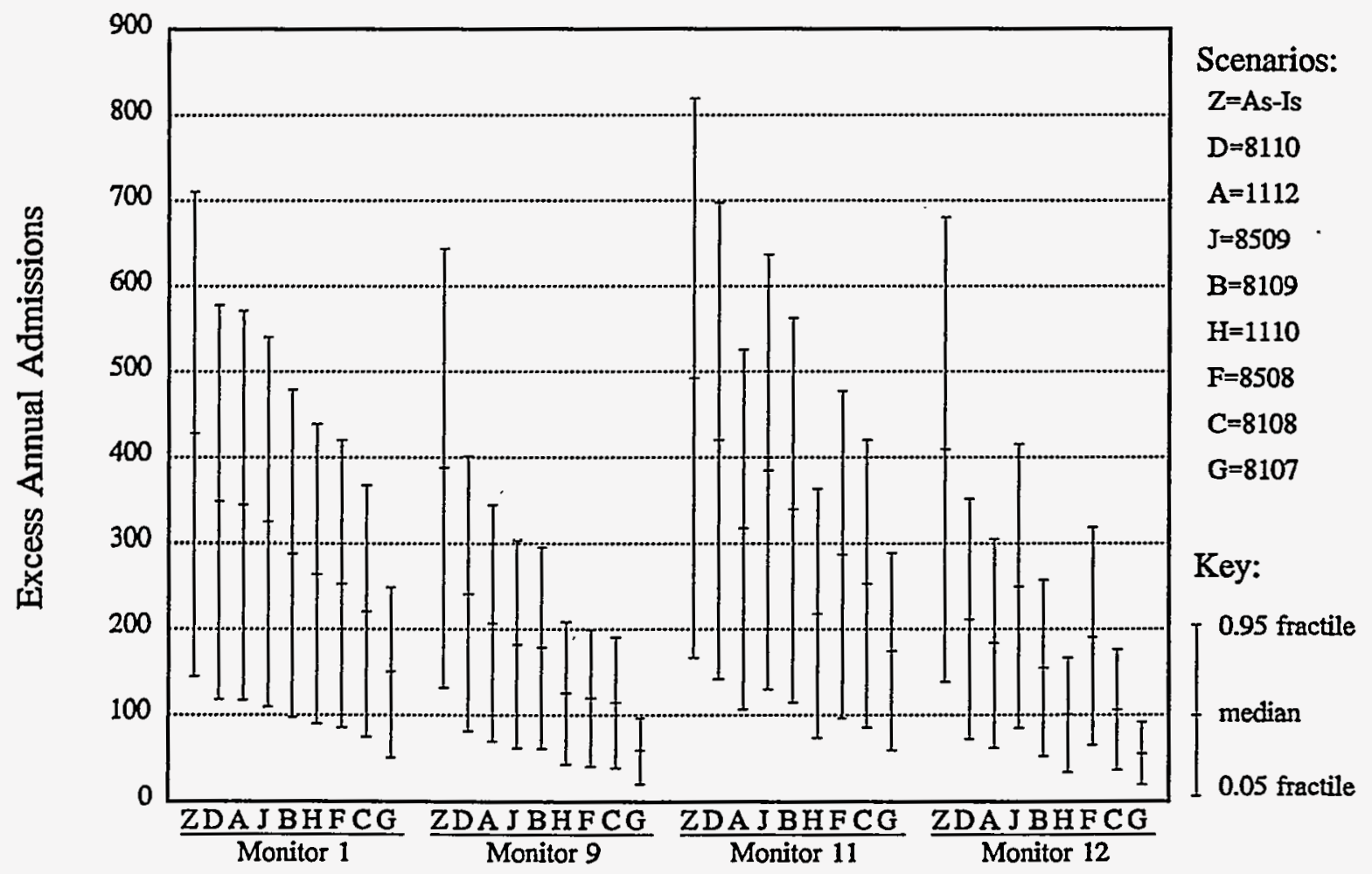

FIGURE 27 Median and 90\% Credible Interval Estimates of Excess Annual Hospitalizations of New York City Asthmatics for Nine Air Quality Scenarios and Air Quality Data for All Monitors 


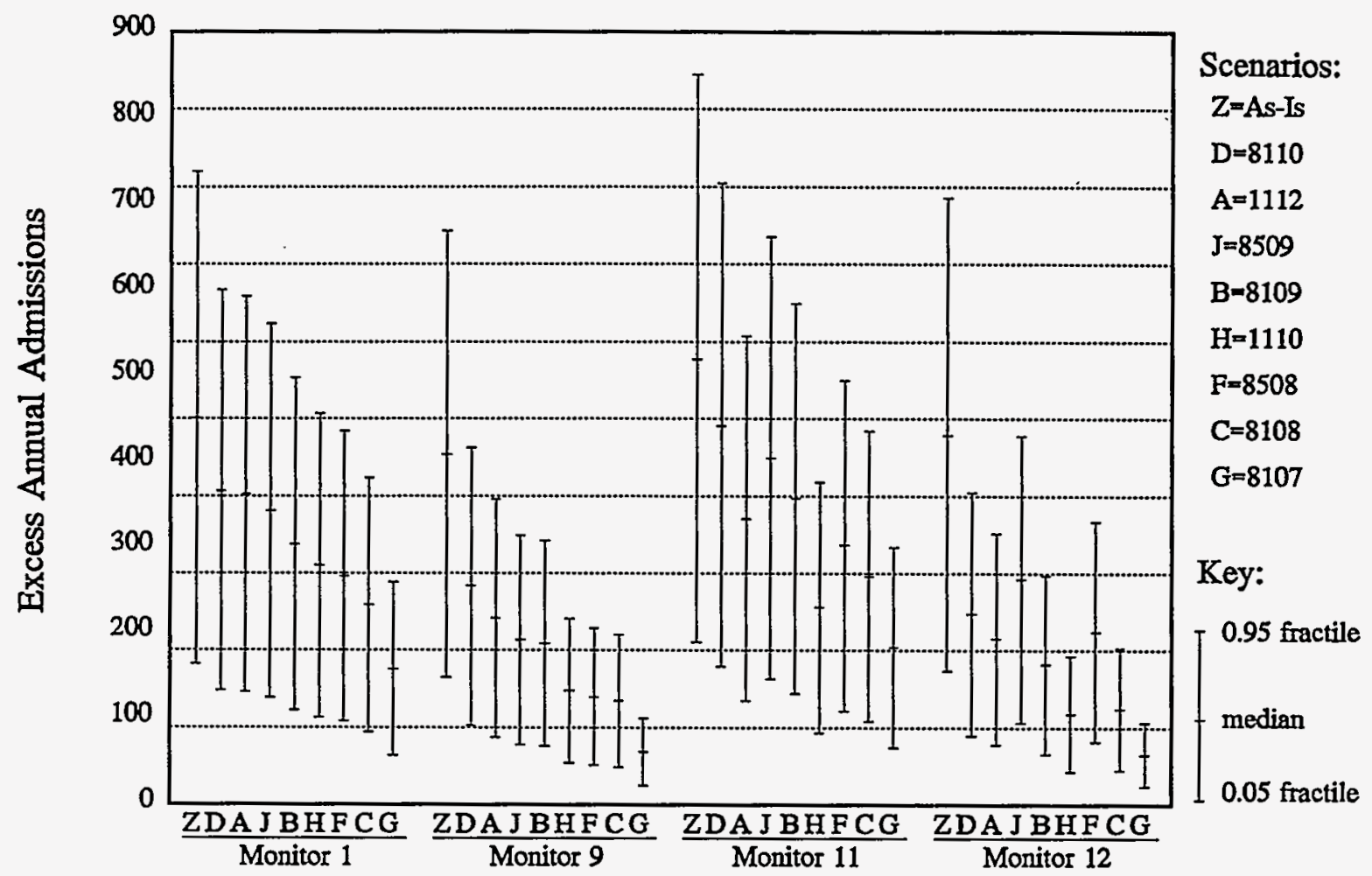

FIGURE 28 Median and 90\% Credible Interval Estimates of Excess Annual Hospitalizations of Respiratory Patients for Nine Air Quality Scenarios and Air Quality Data for All Monitors

The number of respiratory-related admissions of asthmatics in New York City during 1988-1990 was 14,000-16,000 per ozone season (Thurston 1995). By using the middle of this range, a reduction of about 90 excess admissions (the difference between just attaining the current 1-h standard versus just attaining a $0.08 \mathrm{ppm}, 8-\mathrm{h}, 1$ expected exceedance standard) represents a decrease of about $0.6 \%$ in overall admissions for asthmatics. In terms of reductions in ozoneinduced excess admissions, the differences between the As-Is scenario and just attaining the current standard or the $0.08 \mathrm{ppm}, 8-\mathrm{h}, 5$ expected exceedances standard are decreases of about 50 or $70 \%$, respectively, in ozone-induced admissions attributable to exposure at concentrations in excess of the estimated 0.04 ppm background level for ozone.

Another way to examine the results, which use the current standard (1112) as a reference, is shown in Table 23. The excess admissions estimates come from the hospital admissions model. The estimates for all admissions are based on (1) the 14,000-16,000 admissions per ozone season estimates provided by Thurston (1995) and (2) excess admissions attributable to exposures at ozone levels $>0.04 \mathrm{ppm}$. For example, for the current standard $(1 \mathrm{~h}$, 1 expected exceedance, $0.12 \mathrm{ppm}$ ), the median number of admissions of asthmatics for any respiratory-related reason is 14,800 (which is approximately equal to 15,000 [the median of total 
TABLE 23 Admissions of New York City Asthmatics - Comparison Relative to Meeting the Current Standard ( $1 \mathrm{~h}, 1$ expected exceedance, $0.12 \mathrm{ppm}$ )

\begin{tabular}{|c|c|c|c|c|c|}
\hline \multirow{2}{*}{\multicolumn{2}{|c|}{ Case No./Issue }} & \multicolumn{4}{|c|}{ Air Quality Scenario } \\
\hline & & $\begin{array}{c}A=1112 \\
\left(1 \mathrm{~h}, 1 \mathrm{ex},{ }^{\mathrm{a}}\right. \\
0.12 \mathrm{ppm})\end{array}$ & $\begin{array}{c}\mathrm{C}=8108 \\
(8 \mathrm{~h}, 1 \mathrm{ex} \\
0.08 \mathrm{ppm})\end{array}$ & $\begin{array}{l}F=8508 \\
(8 \mathrm{~h}, 5 \mathrm{ex} \\
0.08 \mathrm{ppm})\end{array}$ & $\mathrm{Z}=\mathrm{As}$-Is \\
\hline \multirow[t]{2}{*}{1.} & $\begin{array}{l}\text { Excess admissions }^{\mathrm{b}} \\
\text { (background }=0.04 \mathrm{ppm} \text { ) }\end{array}$ & $\begin{array}{c}207^{\mathrm{c}} \\
(70,344)^{d}\end{array}$ & $\begin{array}{c}115 \\
(39,191)\end{array}$ & $\begin{array}{c}120 \\
(41,199)\end{array}$ & $\begin{array}{c}388 \\
(132,644)\end{array}$ \\
\hline & $\begin{array}{l}\text { Percent change from } \\
\text { current standard }\end{array}$ & 0 & -44 & -42 & 87 \\
\hline & $\begin{array}{l}\text { Excess admissions }^{\mathrm{b}} \\
\text { (background }=0 \mathrm{ppm} \text { ) }\end{array}$ & $\begin{array}{c}909 \\
(308,1,509)\end{array}$ & $\begin{array}{c}804 \\
(273,1,336)\end{array}$ & $\begin{array}{c}797 \\
(270,1,324)\end{array}$ & $\begin{array}{c}1,065 \\
(361,1,768)\end{array}$ \\
\hline & $\begin{array}{l}\text { Percent change from } \\
\text { current standard }^{e}\end{array}$ & 0 & -12 & -12 & 17 \\
\hline & $\begin{array}{l}\text { All admissions }{ }^{f} \\
\text { (thousands) }\end{array}$ & $\begin{array}{c}14.8 \\
(13.8-15.8)\end{array}$ & $\begin{array}{c}14.7 \\
(13.7-15.7)\end{array}$ & $\begin{array}{c}14.7 \\
(13.7-15.7)\end{array}$ & $\begin{array}{c}15^{g} \\
(14-16)^{g}\end{array}$ \\
\hline & $\begin{array}{l}\text { Percent change from } \\
\text { current standard }\end{array}$ & 0 & $\begin{array}{c}-0.6 \\
(-0.2,1.1)\end{array}$ & $\begin{array}{c}-0.6 \\
(-0.2,-1.1)\end{array}$ & $\begin{array}{c}1.2 \\
(-0.4,2.2)\end{array}$ \\
\hline
\end{tabular}

a ex means expected exceedance.

b Admissions of asthmatics because of exposure to ozone.

c Median estimate.

d $90 \%$ credible interval (about the median).

e Because of the necessary assumption that results across scenarios are highly correlated (i.e., if admissions are high for one scenario, they are high for all scenarios), very little variation occurs in the percentage change from the current standard.

f Admissions of asthmatics for any respiratory-related reason; for scenario $i$, based on estimates of all admissions and excess admissions attributable to ozone levels $>0.04 \mathrm{ppm}$ for the As-Is scenario, and estimates of excess admissions attributable to ozone levels $>0.04 \mathrm{ppm}$ for scenario $i$ (e.g., for scenario $1112: 14,800 \approx 15,000-$ $388+207)$.

g Admissions of New York City asthmatics for any respiratory-related reason during the 1988-1990 ozone seasons (Thurston 1995).

h Variation in these results is attributable to the different numbers of admissions of New York City asthmatics for any respiratory-related reason during the 1988-1990 ozone seasons (Thurston 1995). 
admissions in the As-Is scenario] minus 388 [the median number of excess admissions in the As-Is scenario attributable to ozone exposure at concentrations greater than the estimated $0.04 \mathrm{ppm}$ background level] plus 207 [the median number of excess admissions in the As-Is scenario attributable to ozone exposures at concentrations $>0.04 \mathrm{ppm}]$ ).

As expected, as the excess admissions base increases (i.e., the number of excess admissions under scenario 1112 for the three cases listed in Table 23), the percentage change relative to admissions under scenario 1112 (current standard) decreases substantially. For example, excess admissions of asthmatics attributable to exposures to ozone levels $>0.04 \mathrm{ppm}$ decrease from 207 for scenario 1112 to 115 for scenario 8108 - a decrease of 92 admissions, or $44 \%$. For exposures to any level of ozone, the decrease for the same scenarios is 105 admissions, or $12 \%$; for admissions for any respiratory-related reason, the decrease is 100 admissions, or $0.6 \%$. 


\section{LIMITATIONS OF THIS RISK ASSESSMENT}

To complete this risk assessment, it was necessary to make a number of assumptions that could have systematically biased the results. This section discusses some of the more important assumptions in terms of issues. The issues are grouped into three areas, and each is discussed in a separate section: exposure modeling, exposure-response modeling, and risk modeling.

\subsection{ISSUES PERTAINING TO EXPOSURE MODELING}

The risk estimates depend on estimates of air quality and subsequent exposures that populations may experience. Many factors could significantly affect these estimates. Because the nature of deficiencies for many of these factors cannot be identified, it is impossible to say how the results may have been biased (i.e., are they too high or too low). Some of the more important factors involve the following issues:

- Representativeness of diary data subjects. Most subjects who provided diary data used to develop human activity patterns resided in either Cincinnati or California. These subjects may not be representative of residents of the nine urban areas for which risk estimates were developed. The proportions of time that individuals from different areas spend in different microenvironments (i.e., indoors, outdoors, vehicles) and their levels of exertion may differ significantly.

- Representativeness of diary data. The average subject provided less than two days of diary data; some subjects provided data for as many as three days. To develop exposure estimates for an entire ozone season, it was necessary to repeatedly sample data from the small number of days available. The legitimacy of applying data obtained in the short term to extrapolate to an entire ozone season is unknown.

- Adjustment procedures. Adjustment procedures may not adequately account for the day-to-day activities of many outdoor children. and may cause the model to underestimate the number of outdoor children exposed multiple times to high ozone concentrations.

- Estimation of ozone concentrations upon attainment of alternative standards. Complex algorithms were developed to estimate ozone concentrations upon attainment of alternative standards. These algorithms made it possible to develop estimates not only for the monitor with the highest measured ozone concentrations, but for other monitors in the area as well. The algorithms used to model air quality upon attainment were 
developed with special attention to the behavior of the tails of $1-$ and 8-h ozone levels. As a result, it is uncertain how well the adjustment procedure characterizes longer averaging times (especially seasonal 8-h averages) and 1- and 8-h daily maximum values in the middle of the distribution. In addition, the current (or any new) standard actually addresses a three-year period in determining compliance. Air quality data were adjusted to simulate just attaining a given standard. Therefore, exposures and risk estimates are what is expected, on average, when air quality in an area just attains a given standard. Actual exposures and risks can be lower or higher in different years during a three-year period used to judge compliance with a standard.

- Special circumstances in Los Angeles. Ozone levels are considerably higher in the Los Angeles area compared with those in other urban areas. Therefore, larger ozone reductions would be needed in the Los Angeles area to attain the alternative standards considered in this analysis. The adequacy of the adjustment algorithms is more uncertain for Los Angeles than for the other urban areas analyzed because the procedures were developed and tested by using empirical ozone air quality data from urban areas where ozone levels had already been reduced.

- Air exchange rate. Air exchange rate data are limited for some microenvironments (e.g., the sample size for in-vehicle data). This factor could be a significant source of uncertainty, affecting exposure estimates for total populations. It probably is less important for outdoor children and outdoor workers because the exposures of greatest interest occur at moderate or heavy exertion; it is unlikely that those exposures will be inside vehicles.

\subsection{ISSUES PERTAINING TO EXPOSURE-RESPONSE MODELING}

In addition to uncertainties about exposure estimates, uncertainties also occur about responses given exposures. Some of these uncertainties have been accounted for (e.g., by explicitly including sample size at each ozone concentration to develop CIs about mean estimates), but several other issues contribute to uncertainty:

- Length of exposure. Data from 1- to 2.5-h controlled human exposure studies were matched with $1-\mathrm{h}$ exposures at moderate or heavy exertion levels. This procedure is reasonable because the $>1-\mathrm{h}$ exposures were intermittent. McKittrick and Adams (1995) reported that lung function responses were very similar for subjects exposed either continuously for $1 \mathrm{~h}$ or exposed for $2 \mathrm{~h}$ with intermittent exercise. One-hour exposure EVR 
levels were matched with the hourly average EVRs of the health effects studies used to develop exposure-response relationships.

The 8-h at moderate exertion risk estimates are based on three controlled human exposure studies conducted with $6.6-\mathrm{h}$ exposures. This procedure is also reasonable because lung function response appears to level off after exposure for 4-6 h. Therefore, it is unlikely that the exposure-response relationships would have been appreciably different had the studies been conducted for $8 \mathrm{~h}$.

- Extrapolation of exposure-response relationships. To develop probabilistic exposure-response relationships useful for risk assessment calculations, it was necessary to estimate response at ozone concentrations below the lowest exposure levels used in the laboratory studies (i.e., $0.08 \mathrm{ppm}$ for the 8-h exposures at moderate exertion and 0.08 or $0.12 \mathrm{ppm}$ for the $1-\mathrm{h}$ exposures at heavy and moderate exertion). This extrapolation is necessary, because for all urban areas, $\mathrm{pNEM} / \mathrm{O}_{3}$ estimates that most exposures (even for existing air quality) will be less than these levels. Estimating response rates below the lowest levels available from the laboratory studies involves extrapolating "downward" to estimated background ozone levels.

- Reproducibility of ozone-induced responses. This study assumed that ozoneinduced responses for individuals are reproducible. This assumption is supported by the CD, which cites studies by Gliner et al. (1983) and McDonnell et al. (1985b), and by analysis of the Avol et al. (1984) and Kulle et al. (1985) data sets by Hayes et al. (1987).

- Age and lung function. A number of summer camp studies of outdoor children in the Northeast show ozone-induced lung function changes similar to those observed in controlled human exposure studies. For example, McDonnell et al. (1985a) reported that children 8-11 years old experienced $\mathrm{FEV}_{1}$ changes similar to adults 18-35 years old and exposed to concentrations of $0.12 \mathrm{ppm}$ of ozone at an EVR of $35 \mathrm{~L} / \mathrm{min} / \mathrm{m}^{2}$.

- Age and symptoms. Exposure-response relationships were developed for symptoms for the general population, outdoor children, and outdoor workers. None of the controlled human exposure studies includes outdoor children, and none of the summer camp studies reports symptoms. Therefore, the same exposure-response relationships involving symptoms for children are used for adults.

- Interaction between ozone and other pollutants. Because the controlled human exposure studies used in this risk assessment involved only ozone 
exposures, it was assumed that estimates of ozone-induced health effects would not be affected by the presence of other pollutants (e.g, sulfur dioxide, nitrogen dioxide, carbon monoxide, sulfuric acid, or other aerosols). Some evidence supports that other pollutants may enhance the respiratory effects of ozone, but the evidence is not consistent across studies.

It is also possible that other pollutants may cause the same respiratory effects as ozone. If that were the case, studies such as the summer camp studies (in which subjects breathe ambient air) might be expected to show higher response rates than controlled chamber studies. However, they do not.

- Smoking status. Some evidence shows that smokers may be less responsive to ozone than nonsmokers. The exposure-response relationships in this report do not account for smoking status. The extent to which smokers are less responsive than nonsmokers and the relative proportion of smokers among general populations and outdoor worker populations could affect the accuracy of exposure-response relationships. It is possible that risk estimates in this report are overstated.

- Exposure history. It was assumed that the ozone-induced response in any particular hour is not affected by previous ozone exposures. It is possible that ozone-induced responses can be enhanced or attenuated by previous exposures. The degree to which either of these possibilities is true cannot be quantified and thus is an additional source of uncertainty.

\subsection{ISSUES PERTAINING TO RISK MODELING}

Naturally, the uncertainties inherent in modeling exposure and exposure-response relationships propagate to the subsequent risk estimates. In addition, several issues related to the risk calculations add uncertainty:

- Naturally occurring ozone. The risk results (for benchmark and headcount risk models, including hospital admissions) account for background ozone levels. "Background ozone" is defined as the ozone concentrations that would be observed in the United States in the absence of anthropogenic precursor emissions of VOCs and $\mathrm{NO}_{\mathrm{x}}$ in North America. Correcting for background was done by subtracting responses attributable to uncontrollable ozone. The choice of background level is critical. The CD estimates that the summer, 1-h average range for background ozone is $0.03-0.05 \mathrm{ppm}$. The 
midpoint of this range is used as the background ozone level for acute endpoints.

- Correction for background ozone. The process of correcting risk results to account for responses attributable to uncontrollable ozone involves subtracting response at background from "total response." (This process is mathematically equivalent to correcting the exposure-response relationships before calculating the risk; however, it was desirable to know about total response. Therefore, the correction was performed on the intermediate, total response results.) This correction step required an assumption about the correlation between distributions over total response and response at background. It is logical that the correlation should be $\geq 0$. The zero (i.e., uncorrelated) was dismissed because it does not seem likely that responses at background would be unrelated to total responses (i.e., if response at background is high, total response should'also be high). Therefore, a correlation of 1 (i.e., perfect correlation) was used because it seems logical and likely that the correlation, if it were known how to determine it, would be quite high and that results for a correlation of, for example, 0.75 , would not differ significantly from results for a correlation of 1 .

- Output graphs. The output graphs, particularly the plots that show the

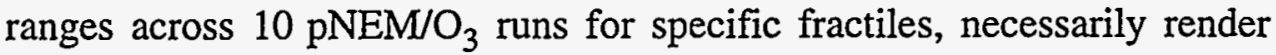
indistinguishable characteristics of results for individual $\mathrm{pNEM} / \mathrm{O}_{3}$ runs. Therefore, the graphs should primarily be used as a guide. The plots should be useful for identifying possible trends and developing insights that can be verified only by investigating detailed results. 


\section{REFERENCES}

Avol, E.L., et al., 1984, "Comparative Respiratory Effects of Ozone and Ambient Oxidant Pollution Exposure during Heavy Exercise," Journal of the Air Pollution Control Association 34:804-809.

EPA: see U.S. Environmental Protection Agency.

Feagans, T.B., and W.F. Biller, 1981, "Describing the Protection Provided by Ambient Air Quality Standards," Environmental Professional 3:235-247.

Folinsbee, L.J., et al., 1988, "Pulmonary Function and Symptom Responses after 6.6-Hour Exposure to 0.12 ppm Ozone with Moderate Exercise," Journal of the Air Pollution Control Association 38:28-35.

Gliner, J.A., et al., 1983, "Preexposure to Low Ozone Concentrations Does Not Diminish the Pulmonary Function Response on Exposure to Higher Ozone Concentrations," American Review of Respiratory Disease 127:51-55.

Hayes, S.R., et al., 1987, Assessment of Lung Function and Symptom Health Risks Associated with Attainment of Alternative Ozone NAAQS, Draft Final Report, SYSAPP-87-171, Systems Applications, Inc., San Rafael, Calif. (For copies, contact H.M. Richmond, U.S. Environmental Protection Agency, Office of Air Quality Planning and Standards, MD-15, Research Triangle Park, N.C. 27711; phone 919-541-5271.)

Hayes, S.R., et al., 1989, "A Health Risk Assessment for Use in Setting the U.S. Primary Ozone Standard," in Atmospheric Ozone Research and Its Policy Implications: Proceedings of the 3rd U.S.-Dutch International Symposium, T. Schneider et al. (eds.), May 1988, Nijmegen, the Netherlands, Elsevier Science Publishers, Amsterdam, the Netherlands, pp. 851-867; see also Studies in Environmental Science 35.

Horstman, D.H., et al., 1990, "Ozone Concentration and Pulmonary Response Relationships for 6.6-Hour Exposures with Five Hours of Moderate Exercise to 0.08, 0.10, and $0.12 \mathrm{ppm}$," American Review of Respiratory Disease 142:1158-1163.

Johnson, T.R., 1987, A Study of Human Activity Patterns in Cincinnati, Ohio, prepared by PEI Associates, Inc., for Electric Power Research Institute, Palo Alto, Calif. (For copies, contact T. Johnson, IT Corporation, 3710 University Drive, Durham, N.C. 27707; phone 919-493-3661.)

Johnson, T., 1994, Enhancements to the pNEM Summer Camp Methodology, prepared by IT/Air Quality Services for U.S. Environmental Protection Agency, Office of Air Quality Planning and Standards, Research Triangle Park, N.C., March 21. (For copies, contact H.M. Richmond, 
U.S. Environmental Protection Agency, Office of Air Quality Planning and Standards, MD-15, Research Triangle Park, N.C. 27711; phone 919-541-5271.)

Johnson, T., 1996, Estimation of Ozone Exposures in Outdoor Children for Special 8H5AVG-80 Scenario, IT Air Quality Services letter report to H.M. Richmond, U.S. Environmental Protection Agency, Feb. 23. (For copies, contact H.M. Richmond, U.S. Environmental Protection Agency, Office of Air Quality Planning and Standards, MD-15, Research Triangle Park, N.C. 27711; phone 919-541-5271.)

Johnson, T., et al., 1996a, Estimation of Ozone Exposures Experienced by Urban Residents Using a Probabilistic Version of NEM and 1990 Population Data, prepared by IT/Air Quality Services for U.S. Environmental Protection Agency, Office of Air Quality Planning and Standards, Research Triangle Park, N.C., April.

Johnson, T., et al., 1996b, Estimation of Ozone Exposures Experienced by Outdoor Workers in Nine Urban Areas Using a Probabilistic Version of NEM, prepared by IT/Air Quality Services for U.S. Environmental Protection Agency, Office of Air Quality Planning and Standards, Research Triangle Park, N.C., April.

Johnson, T., et al., 1996c, Estimation of Ozone Exposures Experienced by Outdoor Children in Nine Urban Areas Using a Probabilistic Version of NEM, prepared by IT/Air Quality Services for U.S. Environmental Protection Agency, Office of Air Quality Planning and Standards, Research Triangle Park, N.C., April.

Kinney, P.L., et al., 1996, "The Effects of Ambient Ozone on Lung Function in Children: A Reanalysis of Six Summer Camp Studies," Environmental Health Perspectives 104:170-174.

Kulle, T.J., et al., 1985, “Ozone Response Relationships in Healthy Nonsmokers," American Review of Respiratory Disease 132:36-41.

McCurdy, T., 1994a, Testing pNEM/O 3 Runs to Determine If a Set of 10 Runs Is "Representative," U.S. Environmental Protection Agency Memorandum, April 19.

McCurdy, T.R., 1994b, "Human Exposure to Ambient Ozone," in Tropospheric Ozone: Human Health and Agricultural Impacts, D.J. McKee (ed.), Lewis Publishers, Boca Raton, Fla., pp. 85-127.

McDonnell, W.F., et al., 1983, "Pulmonary Effects of Ozone Exposure during Exercise: DoseResponse Characteristics," Journal of Applied Physiology: Respiratory Environmental Exercise Physiology 54:1345-1352.

McDonnell, W.F., et al., 1985a, "Respiratory Responses of Vigorously Exercising Children to 0.12 ppm Ozone Exposure," American Review of Respiratory Disease 132:875-879. 
McDonnell, W.F., III, et al., 1985b, "Reproducibility of Individual Responses to Ozone Exposure," American Review of Respiratory Disease 131:36-40.

McDonnell, W.F., et al., 1991, "Respiratory Response of Humans Exposed to Low Levels of Ozone for 6.6 Hours," Archives of Environmental Health 46:145-150.

McKittrick, T., and W.C. Adams, 1995, "Pulmonary Function Response to Equivalent Doses of Ozone Consequent to Intermittent and Continuous Exercise," Archives of Environmental Health 50:153-158.

Morgan, M.G., and M. Henrion, 1990, Uncertainty: A Guide to Dealing with Uncertainty in Quantitative Risk and Policy Analysis, Cambridge University Press, New York, N.Y.

Nagda, N.L., et al., 1987, Guidelines for Monitoring Air Quality, Hemisphere Publishing Company, Washington, D.C.

National Institutes of Health, 1991, Guidelines for the Diagnosis and Management of Asthma, publication no. 91-3042, U.S. Department of Health and Human Services, National Heart, Lung, and Blood Institute, National Asthma Education Program, Bethesda, Md.

Seal, E., Jr., et al., 1993, "The Pulmonary Response of White and Black Adults to Six Concentrations of Ozone," American Review of Respiratory Disease 147:804-810.

Thurston, G.D., et al., 1992, “A Multi-Year Study of Air Pollution and Respiratory Hospital Admissions in Three New York State Metropolitan Areas: Results for 1988 and 1989 Summers," Journal of Exposure Analysis and Environmental Epidemiology 2:429-450.

Thurston, G.D., 1995, New -York University Medical Center, personal communication to R.G. Whitfield, Argonne National Laboratory, Argonne, Ill., Dec. 5.

U.S. Environmental Protection Agency, 1986, Air Quality Criteria for Ozone and Other Photochemical Oxidants, EPA-600/8-84-020aF-eF.5v, Office of Health and Environmental Assessment, Environmental Criteria and Assessment Office, Research Triangle Park, N.C.; available as report No. PB87-142949 from the National Technical Information Service, Springfield, Va.

U.S. Environmental Protection Agency, 1994, National Air Quality and Emissions Trends Report, 1993, EPA-454/R-94-026, Office of Air Quality Planning and Standards, Research Triangle Park, N.C.; available from the National Technical Information Service, Springfield, Va. 
U.S. Environmental Protection Agency, 1996a, Review of National Ambient Air Quality Standards for Ozone: Assessment of Scientific and Technical Information, draft Office of Air Quality Planning and Standards (OAQPS) Staff Paper, EPA-450/R-96-007, June. (For copies, contact David McKee, U.S. Environmental Protection Agency, OAQPS, MD-15, Research Triangle Park, N.C. 27711; phone 919-541-5288.)

U.S. Environmental Protection Agency, 1996b, Air Quality Criteria for Ozone and Related Photochemical Oxidants (Review Draft), EPA/600/P-93/004 af, bf, cf, National Center for Environmental Assessment, Office of Research and Development, Research Triangle Park, N.C.

Wallsten, T.S., and R.G. Whitfield, 1986, Assessing the Risks to Young Children of Three Effects Associated with Elevated Blood-Lead Levels, final report prepared for U.S. Environmental Protection Agency, Office of Air Quality Planning and Standards, Research Triangle Park, N.C., by Argonne National Laboratory, Argonne, Ill., Dec.

Whitfield, R.G., and T.S. Wallsten, 1989, "A Risk Assessment for Selected Lead-Induced Health Effects: An Example of a General Methodology," Risk Analysis 9(2):197-208.

Whitfield, R.G., et al., 1994, "Health Risk Assessment of Ozone," in Tropospheric Ozone: Human Health and Agricultural Impacts, D.J. McKee (ed.), Lewis Publishers, Boca Raton, Fla., pp. 129-173.

Wiley, J.A., et al., 1991a, Activity Patterns of California Residents, Research Division, California Air Resources Board, Sacramento, Calif., May.

Wiley, J.A., et al., 1991b, Study of Children's Activity Patterns, Research Division, California Air Resources Board, Sacramento, Calif., Sept. 
APPENDIX A:

MODELING TECHNIQUES 


\section{APPENDIX A:}

\section{MODELING TECHNIQUES}

This appendix discusses four topics: (1) correction of forced expiratory volume in $1 \mathrm{~s}$ $\left(\mathrm{FEV}_{1}\right)$ decrement calculations for the effects of exercising in clean air, (2) mathematical functions used to represent mean exposure-response relationships, (3) development of probabilistic exposure-response relationships for acute endpoints, and (4) the beta distribution.

\section{A.1 CORRECTION OF FEV $_{1}$ DECREMENT CALCULATIONS FOR THE EFFECTS OF EXERCISING IN CLEAN AIR}

Exercising in clean air can result in small increases or decreases in $\mathrm{FEV}_{1}$. In principle, only the effect of ozone is of interest, and the impact that exercising in clean air has on FEV 1 should not be considered. The exposure-response relationships for changes in lung function are based on $\mathrm{FEV}_{1}$ measurements from the laboratory studies discussed in Section 3 of the main text. The data required to compute the correction for exercising in clean air are the pre- and postexposure $\mathrm{FEV}_{1}$ measurements in clean air and ozone. Two cases were measured: (1) all subjects were subjected to all ozone concentrations (including 0 parts per million [ppm] — clean air), and (2) all subjects were not subjected to all ozone concentrations. In the latter case, data are used for subjects who exercised at $0 \mathrm{ppm}$ to correct the results for subjects exposed at $>0 \mathrm{ppm}$. In both cases, the results were corrected on an individual subject basis.

\section{A.1.1 Case 1: All Subjects Were Subjected to All Ozone Concentrations}

The process for correcting the $\mathrm{FEV}_{1}$ decrement (for a specific subject) for the effects of exercising in clean air is explained by using the most complicated data - that for 6.6-h exposures and moderate exertion (Folinsbee et al. 1988; Horstman et al. 1990; McDonnell et al. 1991). The first step is to compute the change in $\mathrm{FEV}_{1}$, which is the difference between the $\mathrm{FEV}_{1}$ measurement before (pre) exposure and after (post) exposure. This change is computed in clean air (i.e., $0 \mathrm{ppm}$ ozone) and after exposure to ozone (i.e., $\Delta \mathrm{FEV}_{1 \text {, air }}$ and $\triangle \mathrm{FEV}_{1 \text {, ozone }}$ ). The second step is to compute the average of the pre-exposure $\mathrm{FEV}_{1}$ measurements $\left(\mathrm{AFEV}_{1 \text {, pre }}\right)$ for a specific subject, including the $\mathrm{FEV}_{1}$ measurement at $0 \mathrm{ppm}$ ozone. The corrected decrement $\left(\mathrm{DFEV}_{1} \mathrm{C}\right)$ is computed by subtracting the change in $\mathrm{FEV}_{1}$ in clean air from the change after exposure to ozone and dividing the result by the average of all pre-exposure $\mathrm{FEV}_{1}$ measurements. The following set of equations demonstrates this process:

$$
\Delta \mathrm{FEV}_{1, \text { ozone }}=\mathrm{FEV}_{1, \text { pre, ozone }}-\mathrm{FEV}_{1, \text { post, ozone }} \text {, }
$$




$$
\begin{gathered}
\Delta \mathrm{FEV}_{1, \text { air }}=\mathrm{FEV}_{1, \text { pre, air }}-\mathrm{FEV}_{1, \text { post, air }} \\
\mathrm{AFEV}_{1, \text { pre }}=\frac{1}{\mathrm{~N}} \sum_{j=1}^{N} \mathrm{FEV}_{1, \text { pre, ppm, }},
\end{gathered}
$$

and

$$
\mathrm{DFEV}_{1} \mathrm{C}=\left(\frac{\Delta \mathrm{FEV}_{1, \text { ozone }}-\mathrm{FEV}_{1, \text { air }}}{\mathrm{AFEV}_{1, \text { pre }}}\right) \cdot 100 \%
$$

where $\mathrm{ppm}_{j}$ denotes a particular ozone level, and $N$ denotes the number of ozone concentrations for which data are available for a specific subject. These calculations are performed separately for each subject and each nonzero ozone concentration. After all the computations are completed, it is simple to determine the number of subjects that have corrected $F E V_{1}$ decrements $\geq 10, \geq 15$, and $\geq 20 \%$ at each ozone concentration.

The laboratory data from the McDonnell et al. (1991) study illustrate the calculations. The $\mathrm{FEV}_{1}$ measurements for Subject 12 are given in Table A.1. The decrement experienced by Subject 12 at an ozone concentration of $0.10 \mathrm{ppm}$ is:

$$
\begin{gathered}
\Delta \mathrm{FEV}_{1, \text { ozone }}=3,004-2,535=469, \\
\Delta \mathrm{FEV}_{1, \text { air }}=2,883-2,939=-56, \\
\mathrm{AFEV}_{1, \text { pre }}=\frac{(2,883+2,925+3,004+2,846)}{4}=2,914.5,
\end{gathered}
$$

and

$$
\Delta \mathrm{FEV}_{1} \mathrm{C}=\left[\frac{469-(-56)}{2,914.5}\right] \cdot 100 \%=18 \%
$$

The negative value for $\Delta \mathrm{FEV}_{1 \text {,air }}$ indicates that the subject experienced an increase in lung function after exercise in clean air. If this information had not been used to correct the calculation at $0.10 \mathrm{ppm}$, the result would have been a decrement of $16 \%$. So, in this case, the correction does not affect ${ }^{1}$ the additional calculations for $\mathrm{FEV}_{1}$ decrements $\geq 10, \geq 15$, and $\geq 20 \%$.

1 The correction would have affected the result for $\mathrm{FEV}_{1}$ decrements $\geq 15 \%$ if the uncorrected result had been $<15 \%$. 
TABLE A.1 $\mathrm{FEV}_{1}$ Data from McDonnell et al. (1991) - Subject 12

\begin{tabular}{ccccc}
\hline & & \multicolumn{2}{c}{ Exposure } & \\
\cline { 3 - 4 } Obs & Subject & Pre & Post & $\begin{array}{c}\text { Concentration } \\
\text { (ppm) }\end{array}$ \\
\hline 67 & 12 & 2,883 & 2,939 & 0.00 \\
68 & 12 & 2,925 & 2,712 & 0.08 \\
69 & 12 & 3,004 & 2,535 & 0.10 \\
70 & 12 & 2,846 & 2,349 & 0.12 \\
\hline
\end{tabular}

The same approach was also used to correct the symptoms data for exercise in clean air. In all cases, this correction slightly reduced or increased response rates for both lung function and symptoms. The number of increases and the number of decreases were about equal.

\section{A.1.2 Case 2: All Subjects Were Not Subjected to All Ozone Concentrations}

Some of the protocols did not include $\mathrm{FEV}_{1}$ measurements at both $0 \mathrm{ppm}$ and nonzero ozone concentrations for individual subjects. For such studies, a probabilistic correction was made. Rather than using a subject's actual performance (pre- and post-FEV $\mathrm{F}_{1}$ measurements) in clean air (because it did not exist), the study used data from all subjects who exercised in clean air. The percentage change in $\mathrm{FEV}_{1}$ was corrected for a subject exposed to an ozone level $>0$ ppm by systematically subtracting, one by one, the percentage change in $F V_{1}$ for a subject who did exercise in clean air.

If 20 subjects exercised in clean air, which was the case for the 1-h, heavy-exertion subjects of McDonnell et al. (1983), 20 results were obtained for each subject exposed at a nonzero ozone level. If all of these results for a particular subject were $\geq 10 \%$, the subject was counted as a responder with a "likelihood" (or probability) of 1 . If half of these results for a particular subject were $\geq 10 \%$, the subject was counted as a responder with a likelihood of 0.5 , and so on. In this manner, the number of subjects who had corrected FEV $\mathrm{Fecrements}_{1} \geq 10 \%$ was "counted" by adding the likelihoods at each nonzero ozone concentration at which subjects were exposed. That is why the corrected $\mathrm{FEV}_{1}$ decrements results for the McDonnell et al. (1983) and Seal et al. (1993) studies listed in Table 10 in the main text have nonintegers for the numbers responding. For example, the sum of the likelihoods related to $\mathrm{FEV}_{1}$ decrements $\geq 10 \%$ for the 22 subjects exposed at $0.12 \mathrm{ppm}$ was 2.3. (Without correction, the result would have been 3 .)

The same approach was also used to correct the symptoms data for exercising in clean air. In all cases, this correction slightly reduced or increased response rates for both lung function and symptoms. The number of increases and the number of decreases were about equal. 


\section{A.2 MATHEMATICAL FUNCTIONS USED TO REPRESENT MEAN EXPOSURE-RESPONSE RELATIONSHIPS}

Denote the $n$ data points (one for each nonzero ozone concentration at which subjects were exposed) as $\left(C_{i}, R_{i}\right)$, for $i=1, \ldots, n$, where $R_{i}$ is the percentage response rate at concentration $C_{i}$. A function is fit to these data points to obtain an estimate $\hat{R}_{i}$ for each of the $R_{i}$ values and compute the average $\bar{R}$ of the $R_{i}$ values. These values are then used to calculate goodness of fit for each function from the standard regression $r^{2}$ statistic:

$$
r^{2}=1-\left[\frac{\sum_{i=1}^{n}\left(R_{i}-\hat{R}_{i}\right)^{2}}{\sum_{i=1}^{n}\left(R_{i}-\bar{R}_{i}\right)^{2}}\right] \text {. }
$$

The following subsections define the four functions used to represent mean exposure-response relationships: linear, probit, logistic, and lognormal.

\section{A.2.1 Linear Function}

Linear functions were fit to 22 of the 33 acute endpoints. Tables 12 and 13 in the main text list the parameters ( $a$ is the intercept, and $b$ is the slope) of the functions and the regression $r^{2}$ values of the fits to the laboratory data. The equation for the linear function is:

$$
R_{C}(C)=(a+b C) \cdot 100 \% \text {, }
$$

where $R(C)$ denotes the percentage response rate as a function of $C$, the ozone concentration in parts per million, and

$$
R_{C}(C)=0 \% \text {, if }(a+b C) \text { is }<0,
$$

and

$$
R_{C}(C)=100 \% \text {, if }(a+b C) \text { is }>100 \%,
$$

which means that $R_{C}(C)$ must be $\geq 0$ and $\leq 100 \%$. 


\section{A.2.2 Probit Function}

For 11 of the 33 endpoints, the exposure-response relationship was sufficiently S-shaped to justify fitting a nonlinear function to the data, and enough data were available to perform a meaningful nonlinear regression. Three nonlinear functions were used: probit, logistic, and lognormal. The cumulative probability function was fit to exposure-response data, which ideally should also be monotonically increasing. The probit function was obtained by fitting a cumulative normal probability function to the log-odds of ozone concentration. Thus,

$$
X=\left(\frac{C}{1-C}\right), \text { for } 0.04 \mathrm{ppm} \leq C \leq 0.40 \mathrm{ppm},
$$

and

$$
Y=\ln (X), \quad-3.18 \leq Y \leq-0.42,
$$

where $X$ is the odds variable, and $Y$ is the log-odds variable. The range on $C$ is from the assumed background ozone level $(0.04 \mathrm{ppm})$ to $0.40 \mathrm{ppm}$, the highest concentration at which human exposure studies included in this report were conducted. The variable $Y$ is assumed to be normally distributed with mean $a$ and variance $b^{2}$. The degree to which this assumption is appropriate can be investigated with the exposure-response data for each endpoint. If $Y$ is normally distributed, $X$ is lognormally distributed. Although no closed-form expression is readily available for $R(C)$, all probabilistic and statistical results of interest for $R(C)$ can be obtained through $F_{Y}(Y)$, the distribution on $Y$, because:

$$
\begin{aligned}
& R_{C}\left(C_{o}\right)=\operatorname{pr}\left(C \leq C_{o}\right) \cdot 100 \% \\
= & p r\left[X \leq\left(\frac{C_{o}}{1-C_{o}}\right)\right] \cdot 100 \% \\
= & \operatorname{pr}\left[Y \leq \ln \left(\frac{C_{o}}{1-C_{o}}\right)\right] \cdot 100 \%,
\end{aligned}
$$

and

$$
F_{Y}\left(Y_{o}\right)=\operatorname{pr}\left(Y \leq Y_{o}\right)
$$




$$
\begin{aligned}
& =p r\left[Z \leq\left(\frac{Y_{o}-a}{b}\right)\right] \\
& =\Phi\left[z=\left(\frac{Y_{o}-a}{b}\right)\right] \\
& =\Phi\left[\left(\frac{Y_{o}-a}{b}\right)\right],
\end{aligned}
$$

where:

$$
\begin{aligned}
\operatorname{pr}[\cdot] & =\text { probability } \\
Z & =\text { unit normal random deviate, and } \\
\Phi(Z) & =\text { cumulative probability function over } Z .
\end{aligned}
$$

Thus,

$$
\begin{aligned}
& R_{C}\left(C_{o}\right)=\operatorname{pr}\left[X\left(\frac{C_{o}}{1-C_{o}}\right)\right] \cdot 100 \% \\
& =\operatorname{pr}\left[Y \ln \left(\frac{C_{o}}{1-C_{o}}\right)\right] \cdot 100 \% \\
& =\Phi\left[\frac{\ln \left(\frac{C_{o}}{1-C_{o}}\right)-a}{b}\right] \cdot 100 \% .
\end{aligned}
$$

Denote the $n$ data points (one for each nonzero ozone concentration at which subjects were exposed) as $\left(C_{i}, R_{i}\right)$, for $i=1, \ldots, n$, where $R_{i}$ is the percentage response rate at concentration $C_{i}$, and let:

$$
z_{i}=\left(\frac{R_{i}}{100-R_{i}}\right)
$$


Least-squares estimates $\hat{a}$ and $\hat{b}_{i}$ for $a$ and $b$, respectively, can be obtained by linearly regressing the $z_{i}$ on the $y_{i}$. The reciprocal of the slope of the regression equation is $\hat{b}_{i}$, and the intercept is $\hat{a}$ (i.e., the value of $y$ corresponding to $z=0$ in the regression equation).

\section{A.2.3 Lognormal Function}

The lognormal function is related to the probit function in the sense that its determination is similar: rather than two transformations, we define $Y=\ln (C)$ and proceed from that point in a fashion identical to that for the probit function. After regression, the intercept is $\hat{a}$, and the reciprocal of the slope is $\hat{b}_{i}$. The geometric mean (parameter $a$ in Table 13 in the main text) is $e^{\hat{a}}$ and the geometric standard deviation (parameter $b$ in Table 13) is $e^{\hat{b}}$. The exposure-response relationship also involves $\Phi(Z)$ :

$$
R_{C}\left(C_{o}\right)=\Phi\left[\frac{\ln \left(C_{o}\right)-\hat{a}}{\hat{b}}\right] .
$$

\section{A.2.4 Logistic Function}

The logistic function used is a special case of the generalized four-parameter logistic. The special case is that the function is forced through the origin $(0 \mathrm{ppm}, 0 \%$ response) to reflect the fact that the exposure-response relationships have been corrected to account for exercise in clean air (Hayes et al. 1987). The function is:

$$
R_{C}\left(C_{o}\right)=b \cdot \exp (d)\left\{\frac{1-\exp \left(e C_{o}\right)}{[1+\exp (d)] \cdot\left[1+\exp \left(e C_{o}+d\right)\right]}\right\} \cdot 100 \%
$$

This function was only used to fit 1 -h exposure, heavy-exertion, $\mathrm{FEV}_{1}$ decrement data (McDonnell et al. 1983). The logistic was fit with a nonlinear solver tool in a spreadsheet.

\section{A.3 DEVELOPMENT OF PROBABILISTIC EXPOSURE-RESPONSE RELATIONSHIPS FOR ACUTE HEALTH ENDPOINTS FOR HEADCOUNT RISK ESTIMATES}

Section 3.6 of the main text listed principles and rules that guided development of probabilistic exposure-response relationships for acute health endpoints for headcount risk estimates. These affected the choice of function (linear, probit, lognormal, or logistic) for the mean exposure-response relationship (step 2 in Figure 4), and the choice of sample size used to 
develop the response fractiles at each ozone concentration (i.e., the probabilistic exposureresponse relationship; step 3 in Figure 4). Table A.2 lists and explains "special" steps used to develop the probabilistic exposure-response relationship for an endpoint.

TABLE A.2 Details about Probabilistic Exposure-Response Relationships for Acute Health Endpoints for Headcount Risk Estimates ${ }^{\mathrm{a}}$

Moderate or severe lower respiratory symptoms; 1-h exposures at heavy exertion; based on Avol et al. (1984)

- 3 of 4 ozone concentrations used in regression $(0.08 \mathrm{ppm}$ was excluded because response was $0 \%$ at $0.16 \mathrm{ppm}$ )

$\mathrm{FEV}_{1}$ decrement $\geq 10 \%$; 1-h exposures at heavy exertion; based on Kulle et al. (1985)

- 4 of 5 ozone concentrations used in regression $(0.10$ ppm was excluded because response was $0 \%$ at $0.15 \mathrm{ppm}$ )

$\mathrm{FEV}_{1}$ decrement $\geq 15 \%$; 1-h exposures at heavy exertion; based on Kulle et al. (1985)

- 4 of 5 ozone concentrations used in regression $(0.10 \mathrm{ppm}$ was excluded because response was $0 \%$ at $0.15 \mathrm{ppm}$ )

$\mathrm{FEV}_{1}$ decrement $\geq 20 \%$; 1 -h exposures at heavy exertion; based on Kulle et al. (1985)

- 3 of 5 ozone concentrations used in regression $(0.10$ and $0.15 \mathrm{ppm}$ were excluded because response was $0 \%$ at $0.20 \mathrm{ppm}$ )

Any cough; 1-h exposures at heavy exertion; based on Kulle et al. (1985)

- Average sample size used so that the probabilistic exposure-response relationships would be monotonic

Moderate-to-severe cough; 1-h exposures at heavy exertion; based on Kulle et al. (1985)

- 3 of 5 ozone concentrations used in regression ( 0.10 and $0.15 \mathrm{ppm}$ were excluded because response was $0 \%$ at $0.20 \mathrm{ppm})$

- Regression forced through $(0.20 \mathrm{ppm}, 0 \%)$; resultant regression line equidistant from the data points at 0.25 and $0.30 \mathrm{ppm}$

- Regression $r^{2}$ (which was negative) only included the 3 points used in the constrained regression

- Meaningful exposure-response relationship not supported by data

Moderate-to-severe pain on deep inspiration; 1-h exposures at heavy exertion; based on Kulle et al. (1985)

- 4 of 5 ozone concentrations used in regression $(0.10 \mathrm{ppm}$ was excluded because response was $0 \%$ at $0.15 \mathrm{ppm}$ ) 
$\mathrm{FEV}_{1}$ decrement $\geq 10 \%$; 1-h exposures at heavy exertion; based on McDonnell et al. (1983)

- Logistic function used to capture the threshold above $0.30 \mathrm{ppm}$

- Average sample size used so that the probabilistic exposure-response relationships would be monotonic

- Ample number of data points (5 concentrations $>0 \mathrm{ppm}$ ) support use of this function

$\mathrm{FEV}_{1}$ decrement $\geq 15 \%$; 1-h exposures at heavy exertion; based on McDonnell et al. (1983)

- Logistic function used to capture the threshold above $0.30 \mathrm{ppm}$

- Average sample size used so that the probabilistic exposure-response relationships would be monotonic

- Ample number of data points (5 concentrations $>0 \mathrm{ppm}$ ) support use of this function

$\mathrm{FEV}_{1}$ decrement $\geq 20 \%$; 1 -h exposures at heavy exertion; based on McDonnell et al. (1983)

- Logistic function used to capture the threshold above $0.24 \mathrm{ppm}$

- Average sample size used so that the probabilistic exposure-response relationships would be monotonic

- Ample number of data points (5 concentrations $>0 \mathrm{ppm}$ ) support use of this function

Any cough; 1-h exposures at heavy exertion; based on McDonnell et al. (1983)

- Probit function fit to the data to capture highly nonlinear characteristics

- Ample number of data points (5 concentrations $>0 \mathrm{ppm}$ ) support use of this function

Any pain on deep inspiration; 1-h exposures at heavy exertion; based on McDonnell et al. (1983)

- Probit function fit to the data to capture highly nonlinear characteristics

- Ample number of data points (5 concentrations $>0 \mathrm{ppm}$ ) support use of this function

Any cough; 1-h exposures at moderate exertion; based on Seal et al. (1993)

- Lognormal function fit to the data to capture highly nonlinear characteristics

- Ample number of data points (5 concentrations $>0 \mathrm{ppm}$ ) support use of this function

Any pain on deep inspiration; 1-h exposures at moderate exertion; based on Seal et al. (1993)

- Lognormal function fit to the data to capture highly nonlinear characteristics

- Ample number of data points (5 concentrations $>0 \mathrm{ppm}$ ) support use of this function

Moderate-to-severe pain on deep inspiration; 1-h exposures at moderate exertion; based on

Seal et al. (1993)

- Probit function fit to the data to capture the S-shaped characteristics

- Ample number of data points (5 concentrations $>0 \mathrm{ppm})$ support use of this function 
TABLE A.2 (Cont.)

$\mathrm{FEV}_{1}$ decrement $\geq 15 \%$; 8-h exposures at moderate exertion; based on Folinsbee et al. (1988), Horstman et al. (1990), and McDonnell et al. (1991)

- 2 of 3 ozone concentrations used in regression $(0.12 \mathrm{ppm}$ was excluded because of logical inconsistencies caused by this data point)

- Regression $r^{2}$ value (1.0) does not include $0.12 \mathrm{ppm}$; if $0.12 \mathrm{ppm}$ is included, the $r^{2}$ value is 0.73 .

${ }^{a}$ Unless otherwise stated, linear fits were used for the mean exposure-response relationships. Endpoints not listed used linear fits for the mean exposure-response relationships and linear interpolation between sample sizes at available ozone concentrations to develop the probabilistic exposure-response relationships. Sample sizes for ozone concentrations less than the lowest concentration available were set equal to the sample size at the lowest concentration; sample sizes for ozone concentrations greater than the highest concentration available were set equal to the sample size at the highest concentration.

\section{A.4 BETA DISTRIBUTION}

Step 3 in Figure 4 in the main text determines the response rate associated with specific fractiles of a probability distribution specified by the number $X$ of $N$ subjects who "respond" (and the remainder, $N-X$, do not) at a specific ozone concentration. These data provide an estimate $\hat{P}$ of the true proportion $P$ of the population experiencing the health effect at the ozone level in question. The estimate is uncertain because of sampling error. In classic probability theory, this uncertainty is handled by using the binomial distribution with parameters $\hat{P}, X$, and $N$ to calculate confidence intervals around $\hat{P}$ as an estimate of $P$, or more generally to calculate a probability distribution over $P$. Thus, the output is a probability distribution over the percentage of the population affected at each observed ozone concentration.

The Bayesian approach calculates a posterior probability distribution over $P$, given the data and a prior distribution over $P$. When the prior distribution is represented as a beta distribution with parameters $a$ and $b$, the posterior distribution is also beta, but with parameters $a+X$ and $b+(N-X)$. A conservative strategy is to assume a diffuse prior state of information characterized by $a=b=0$ (Winkler 1972). In this case, the resulting probability distribution over the fraction of the population affected at a given ozone concentration is very close to that calculated in the classic manner. The Bayesian approach appears to be best suited for purposes here. The beta distribution is defined as:

$$
\beta(R)=\frac{(N-1)}{(X-1) !(N-X-1) !}\left(R^{\prime}\right)^{X-1}\left(1-R^{\prime}\right)^{N-X-1},
$$


where $R^{\prime}$ is $0.01-R$ (i.e., $R^{\prime}$ is a fraction). The cumulative probability function for the beta distribution is:

$$
\begin{aligned}
& \beta\left(R^{\prime}\right)=\int_{R_{o}=0}^{R^{\prime}} \beta\left(R_{o}^{\prime}\right) d R_{o}^{\prime} \\
& =1-\sum_{i=0}^{X-1}\left(\begin{array}{c}
N-1 \\
i
\end{array}\right) R^{i}(1-R)^{N-1-i},
\end{aligned}
$$

where $\left(\begin{array}{c}N-1 \\ i\end{array}\right)$ is the binomial coefficient that denotes the number of possible combinations of $N-1$ distinguishable items taken $i$ at a time. From this equation, the values of $R^{\prime}$ that are determined correspond to the $0.01,0.05, \ldots, 0.50, \ldots, 0.95$, and 0.99 fractiles needed for the risk calculations.

\section{A.5 APPENDIX A REFERENCES}

Avol, E.L., et al., 1984, "Comparative Respiratory Effects of Ozone and Ambient Oxidant Pollution Exposure during Heavy Exercise," Journal of the Air Pollution Control Association 34:804-809.

Folinsbee, L.J., et al., 1988, "Pulmonary Function and Symptom Responses after 6.6-Hour Exposure to $0.12 \mathrm{ppm}$ Ozone with Moderate Exercise," Journal of the Air Pollution Control Association 38:28-35.

Hayes, S.R., et al., 1987, Assessment of Lung Function and Symptom Health Risks Associated with Attainment of Alternative Ozone NAAQS, Draft Final Report, SYSAPP-87-171, Systems Applications, Inc., San Rafael, Calif. (For copies, contact H.M. Richmond, U.S. Environmental Protection Agency, Office of Air Quality Planning and Standards, MD-15, Research Triangle Park, N.C. 27711; phone 919-541-5271.)

Horstman, D.H., et al., 1990, "Ozone Concentration and Pulmonary Response Relationships for 6.6-Hour Exposures with Five Hours of Moderate Exercise to 0.08, 0.10, and $0.12 \mathrm{ppm}$," American Review of Respiratory Disease 142:1158-1163.

Kulle, T.J., et al., 1985, "Ozone Response Relationships in Healthy Nonsmokers," American . Review of Respiratory Disease 132:36-41. 
McDonnell, W.F., et al., 1983, "Pulmonary Effects of Ozone Exposure during Exercise: DoseResponse Characteristics," Journal of Applied Physiology: Respiratory Environmental Exercise Physiology 54:1345-1352.

McDonnell, W.F., et al., 1991, "Respiratory Response of Humans Exposed to Low Levels of Ozone for 6.6 Hours,." Archives of Environmental Health 46:145-150.

Seal, E., Jr., et al., 1993, "The Pulmonary Response of White and Black Adults to Six Concentrations of Ozone," American Review of Respiratory Disease 147:804-810.

Winkler, R.L., 1972, An Introduction to Bayesian Inference and Decision, Holt, Rinehart and Winston, New York, N.Y. 
APPENDIX B:

PROBABILISTIC EXPOSURE-RESPONSE RELATIONSHIPS FOR ACUTE ENDPOINTS 


\section{APPENDIX B: \\ PROBABILISTIC EXPOSURE-RESPONSE RELATIONSHIPS FOR ACUTE ENDPOINTS}

\section{B.1 INTRODUCTION}

This appendix presents probabilistic exposure-response relationships used to develop headcount risk estimates for 33 acute endpoints (Figures B.1-B.33). Each figure shows five exposure-response relationships, data from human exposure experiments used to derive the exposure-response relationships, and the $90 \%$ credible interval (CI) around each experimental data point. The five exposure-response relationships shown are the 0.05-, 0.25-, 0.5- (median), 0.75-, and 0.95-fractile exposure-response relationships; exposure-response relationships for 16 other fractiles are also used in the risk calculations. The 0.25 and 0.75 fractiles define a $50 \%$ $\mathrm{CI}$ on response rate at each ozone concentration; the 0.05 and 0.95 fractiles define a $90 \% \mathrm{CI}$.

Figure B.1 shows the probabilistic exposure-response relationship for forced expiratory volume in $1 \mathrm{~s}\left(\mathrm{FEV}_{1}\right)$ decrement $\geq 10 \%$, derived from Avol et al. (1984), for 1-h exposures and individuals engaged in heavy exertion, corrected for exercise in clean air. The small squares indicate the response rates at the ozone concentrations at which subjects were exposed by Avol et al. $(0.08,0.16,0.24$, and 0.32 parts per million [ppm]). The short horizontal line segments above and below the data point, which are connected by a line segment, indicate the $90 \%$ CI. For example, the response rate at $0.16 \mathrm{ppm}$ is approximately $20 \%$, and the $90 \% \mathrm{CI}$ about this value is approximately $20-40 \%$; the derived $90 \%$ CI compares favorably to this range. The "derived" $90 \% \mathrm{CIs}$ and the "experimental" CIs compare less favorably at 0.24 and $0.32 \mathrm{ppm}$ because the linear regression used to fit the data does not capture the nonlinear characteristics of the data. The derived and experimental CIs for other endpoints compare more favorably (e.g., the FEV 1 endpoints for 1-h exposures at heavy exertion [Figures B.13-B.15] and the $\mathrm{FEV}_{1}$ decrements $\geq 10 \%$ endpoint for 8-h exposures at moderate exertion [Figure B.27]).

The experimental data are presented in Section 3 of the main text. Characteristics (i.e., parameters of functions fit to the data, and regression $r^{2}$ values) of functions are also tabulated in Section 3. Mathematical definitions of the functional forms are presented in Appendix A. 


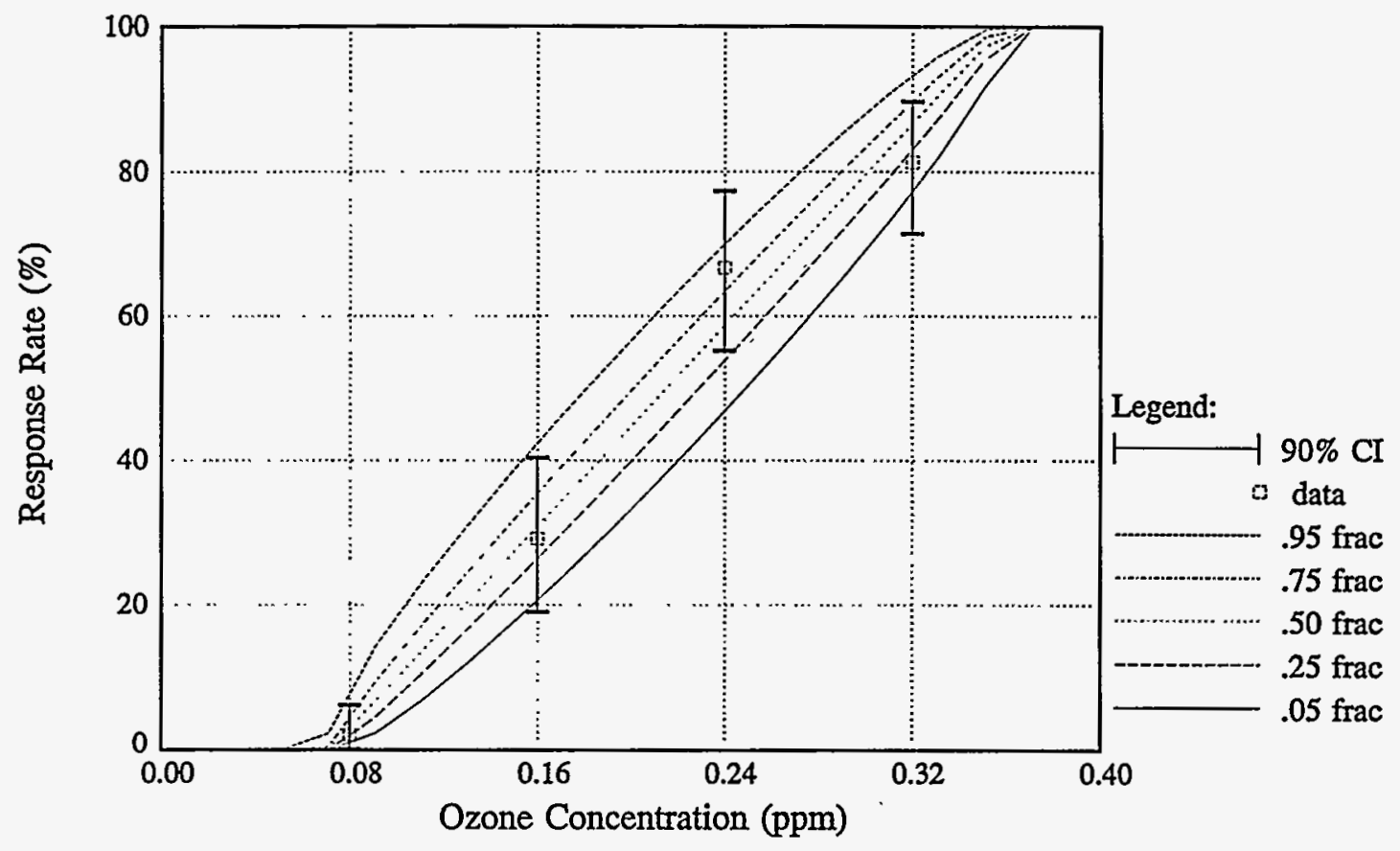

FIGURE B.1 Probabilistic Exposure-Response Relationship for FEV 1

Decrement $\geq \mathbf{1 0} \%$ for 1 -h Exposures, Heavy Exertion; Includes Data, Medians, and 90\% CIs for Data and Relationship (Source: derived from Avol et al. 1984)

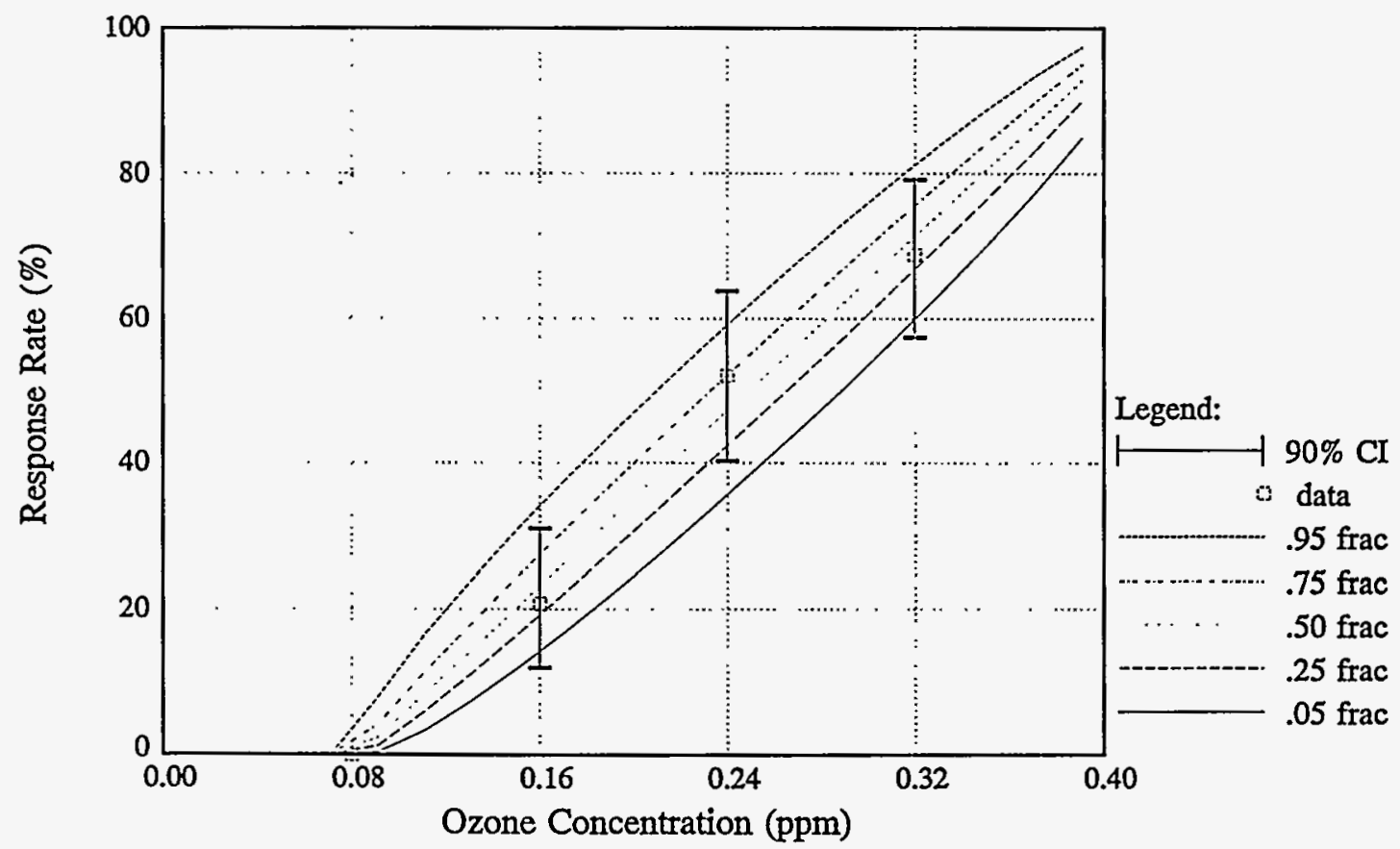

FIGURE B.2 Probabilistic Exposure-Response Relationship for FEV

Decrement $\geq 15 \%$ for 1-h Exposures, Heavy Exertion; Includes Data, Medians, and 90\% CIs for Data and Relationship (Source: derived from Avol et al. 1984) 


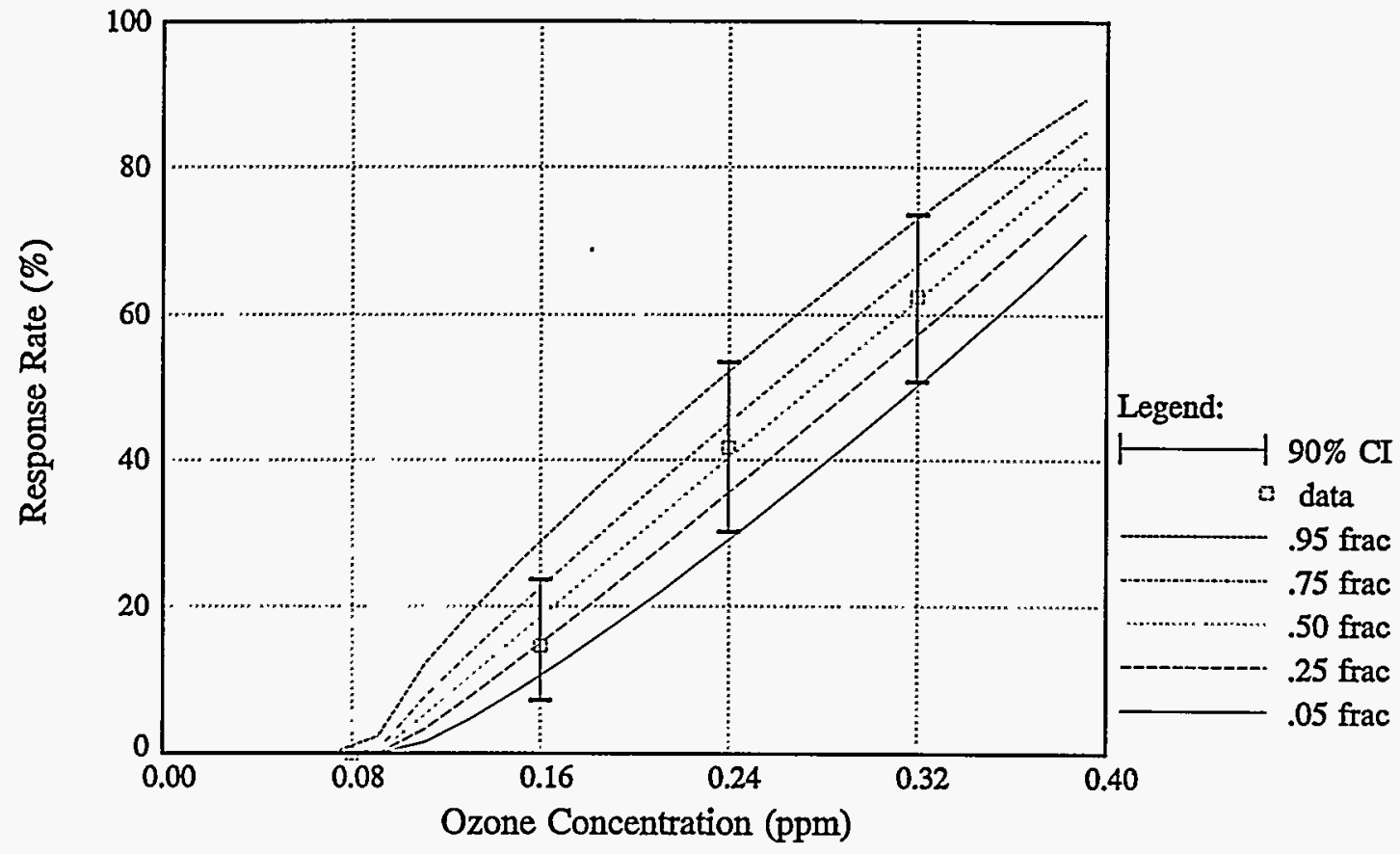

FIGURE B.3 Probabilistic Exposure-Response Relationship for FEV 1 Decrement $\geq 20 \%$ for 1 -h Exposures, Heavy Exertion; Includes Data, Medians, and 90\% CIs for Data and Relationship (Source: derived from Avol et al. 1984)

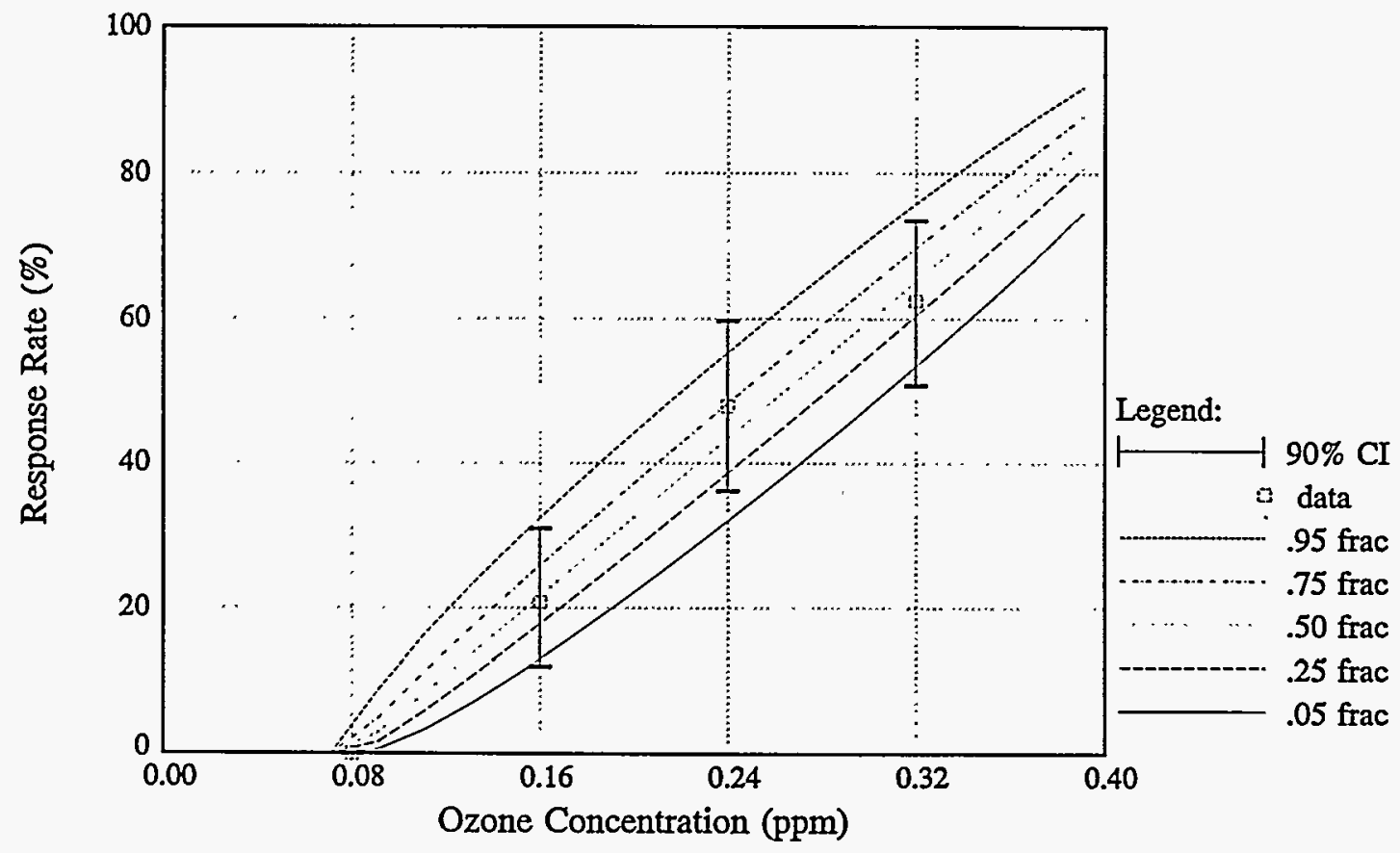

FIGURE B.4 Probabilistic Exposure-Response Relationship for Any Lower Respiratory Symptoms for 1-h Exposures, Heavy Exertion; Includes Data, Medians, and $90 \%$ CIs for Data and Relationship (Source: derived from Avol et al. 1984) 


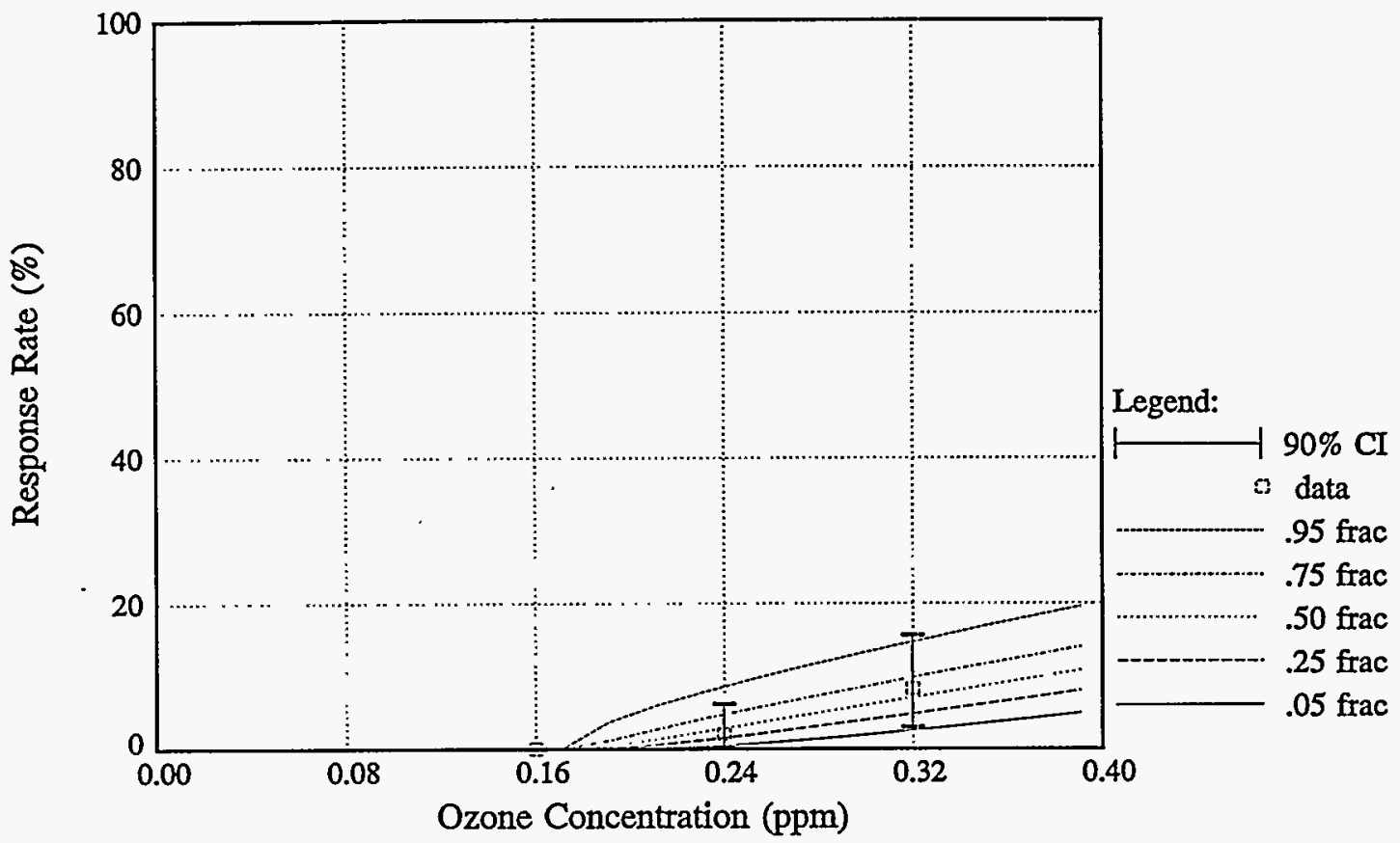

FIGURE B.5 Probabilistic Exposure-Response Relationship for Moderate-to-Severe Lower Respiratory Symptoms for 1-h Exposures, Heavy Exertion; Includes Data, Medians, and $\mathbf{9 0 \%}$ CIs for Data and Relationship (Source: derived from Avol et al. 1984)

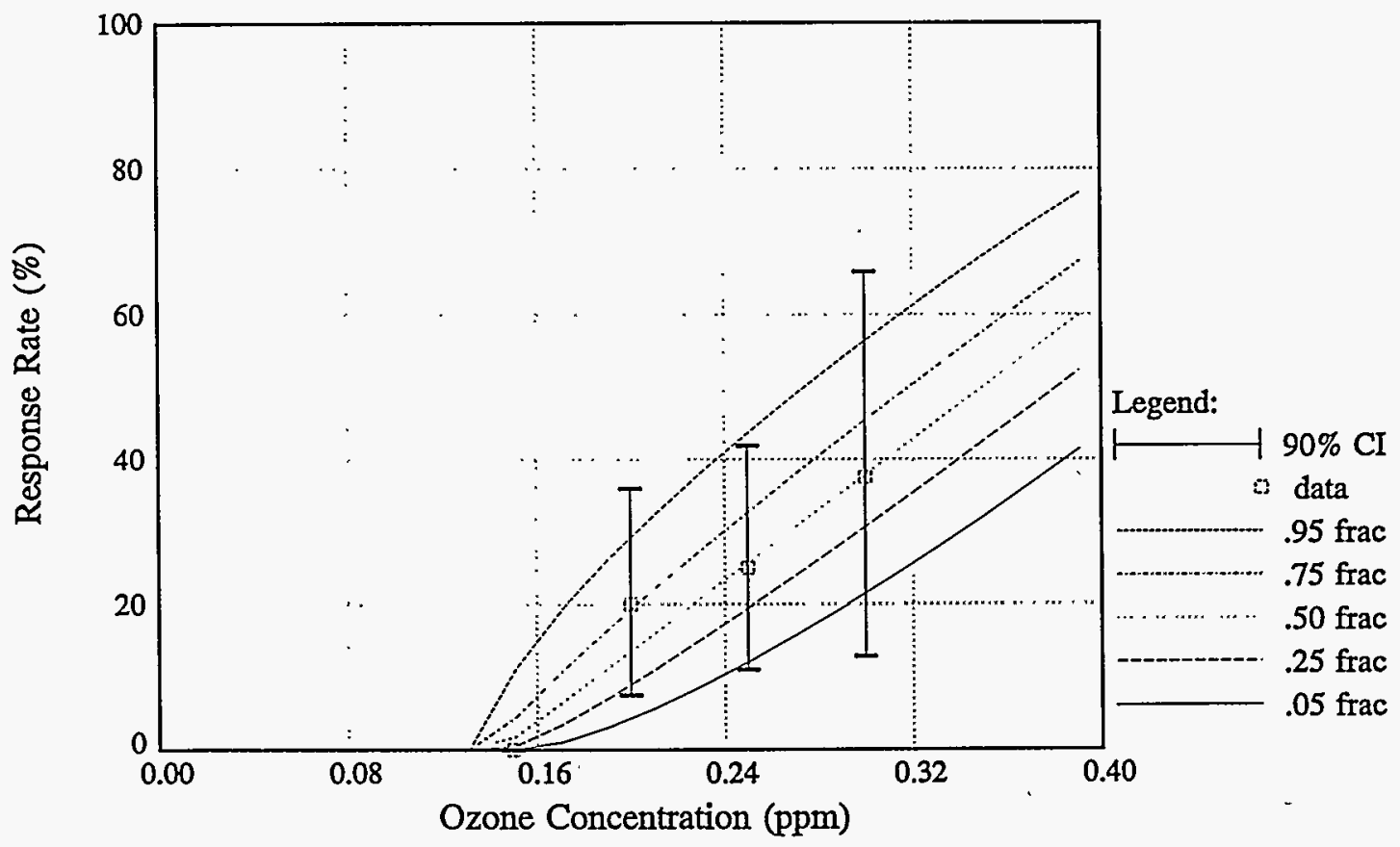

FIGURE B.6 Probabilistic Exposure-Response Relationship for FEV 1 Decrement $\geq 10 \%$ for 1-h Exposures, Heavy Exertion; Includes Data, Medians, and 90\% CIs for Data and Relationship (Source: derived from Kulle et al. 1984) 


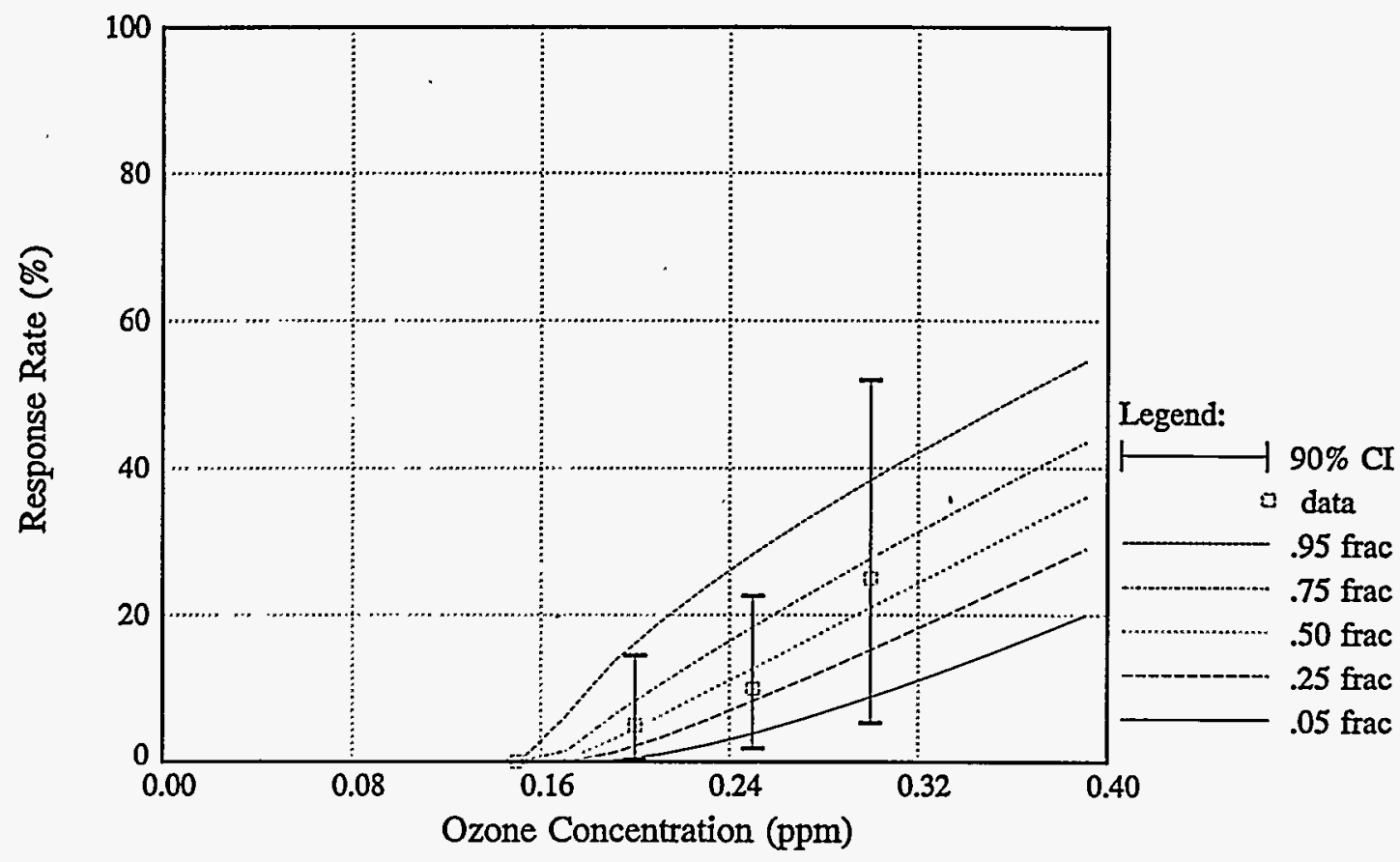

FIGURE B.7 Probabilistic Exposure-Response Relationship for FEV

Decrement $\geq 15 \%$ for 1-h Exposures, Heavy Exertion; Includes Data, Medians, and $\mathbf{9 0 \%}$ CIs for Data and Relationship (Source: derived from Kulle et al. 1984)

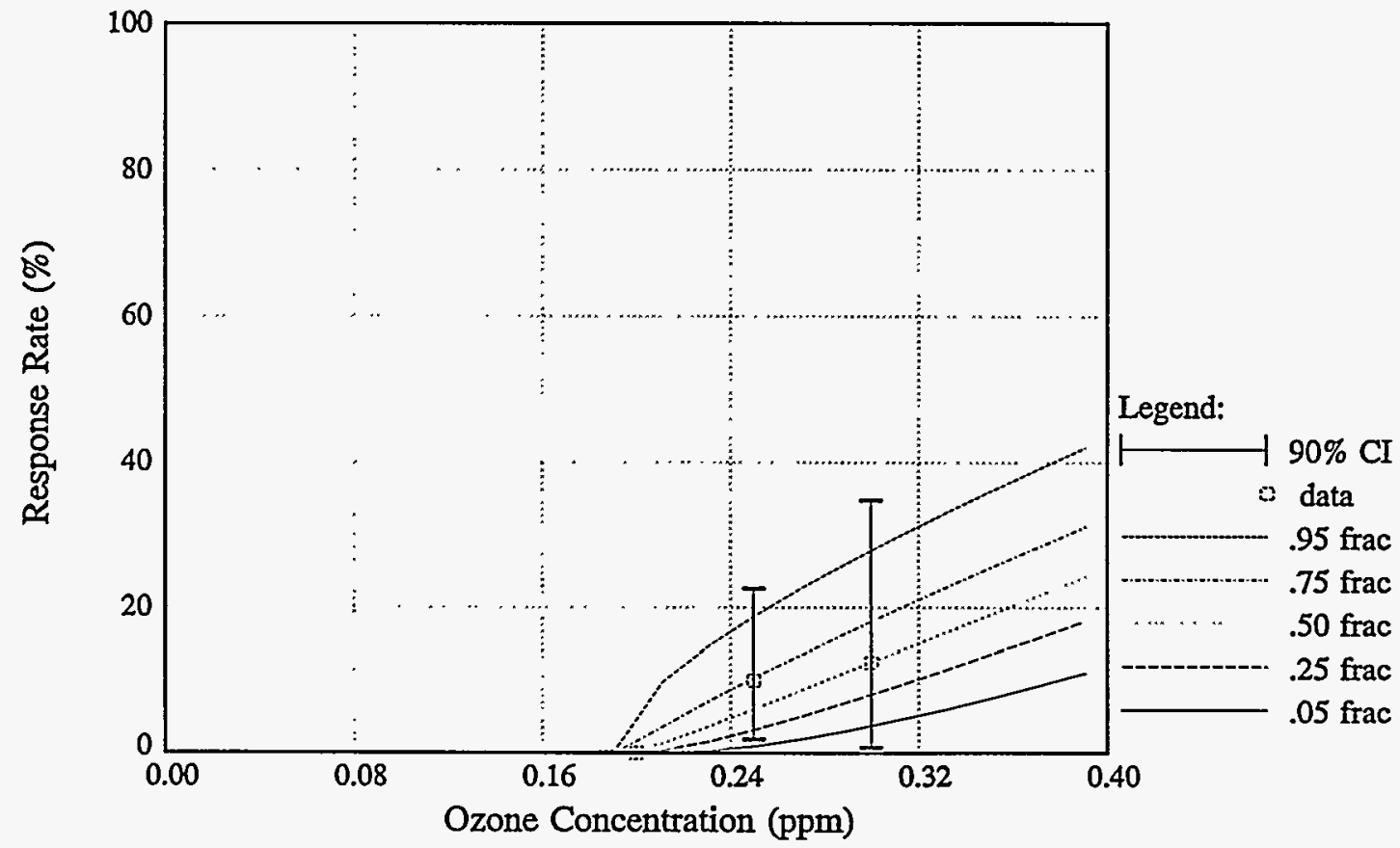

FIGURE B.8 Probabilistic Exposure-Response Relationship for FEV 1 Decrement $\geq 20 \%$ for 1-h Exposures, Heavy Exertion; Includes Data, Medians, and 90\% CIs for Data and Relationship (Source: derived from Kulle et al. 1984) 


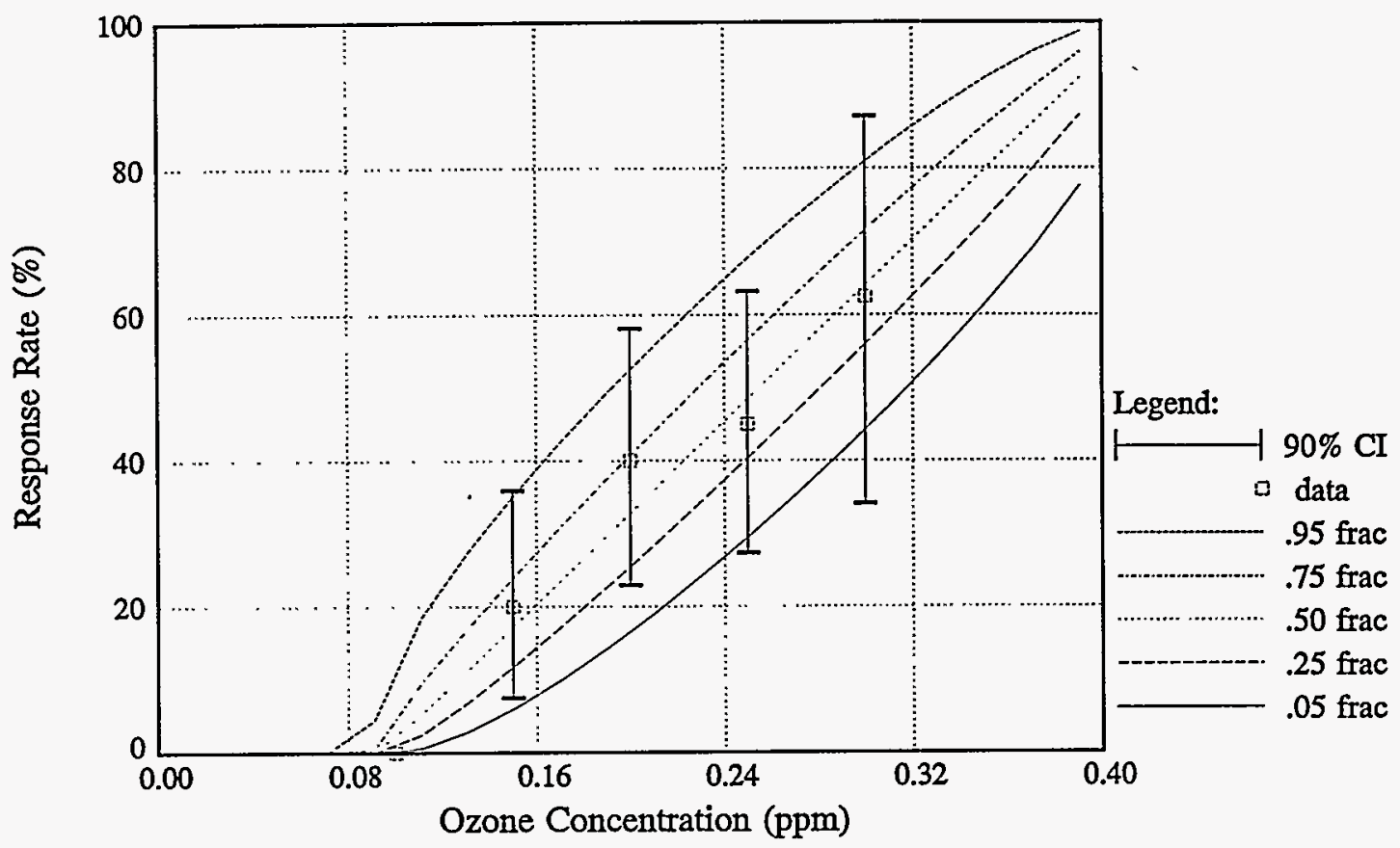

FIGURE B.9 Probabilistic Exposure-Response Relationship for Any Cough for 1-h Exposures, Heavy Exertion; Includes Data, Medians, and $90 \%$ CIs for Data and Relationship (Source: derived from Kulle et al. 1984)

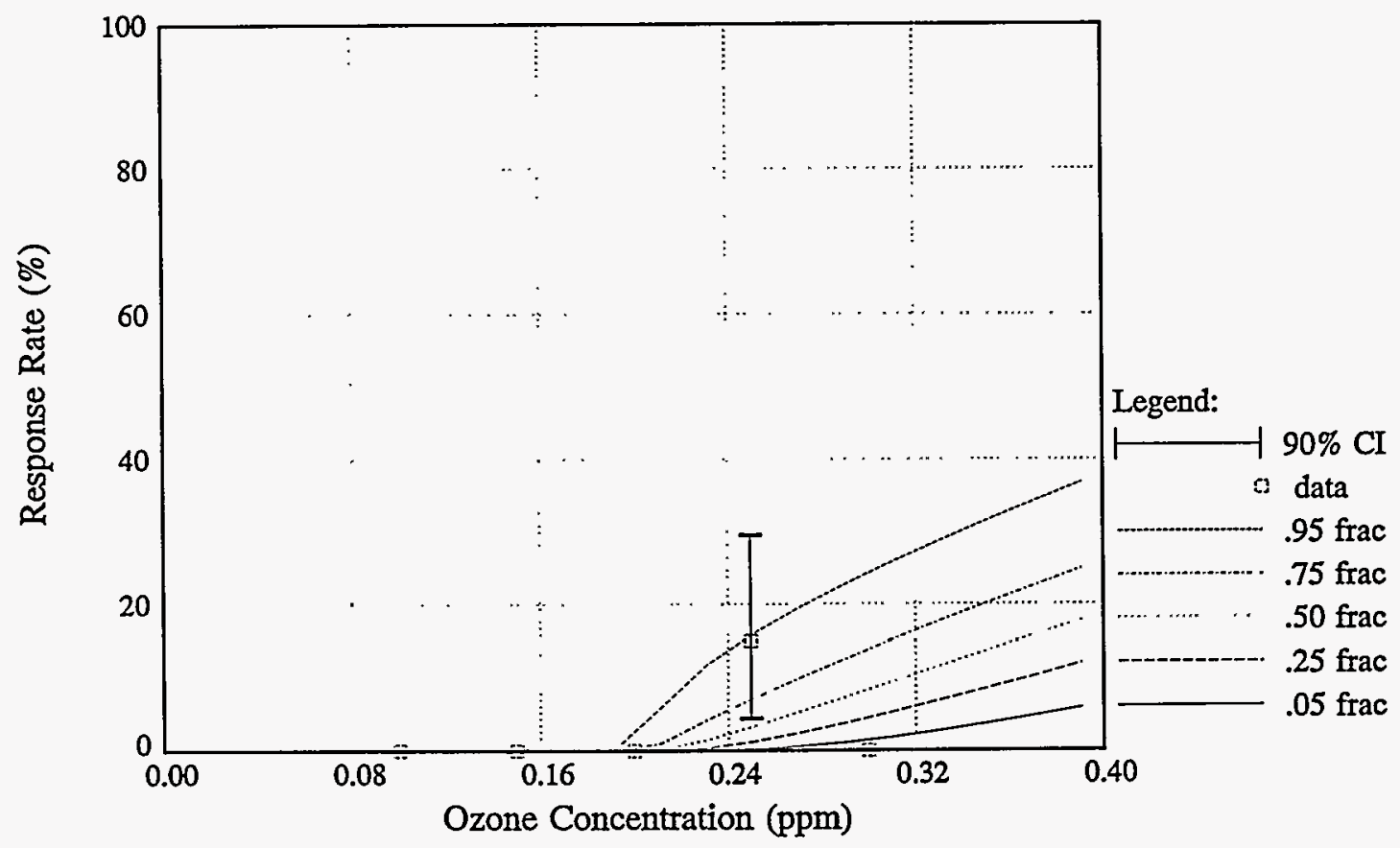

FIGURE B.10 Probabilistic Exposure-Response Relationship for Moderate-to-

Severe Cough for 1-h Exposures, Heavy Exertion; Includes Data, Medians, and $\mathbf{9 0 \%}$ CIs for Data and Relationship (Source: derived from Kulle et al. 1984) 


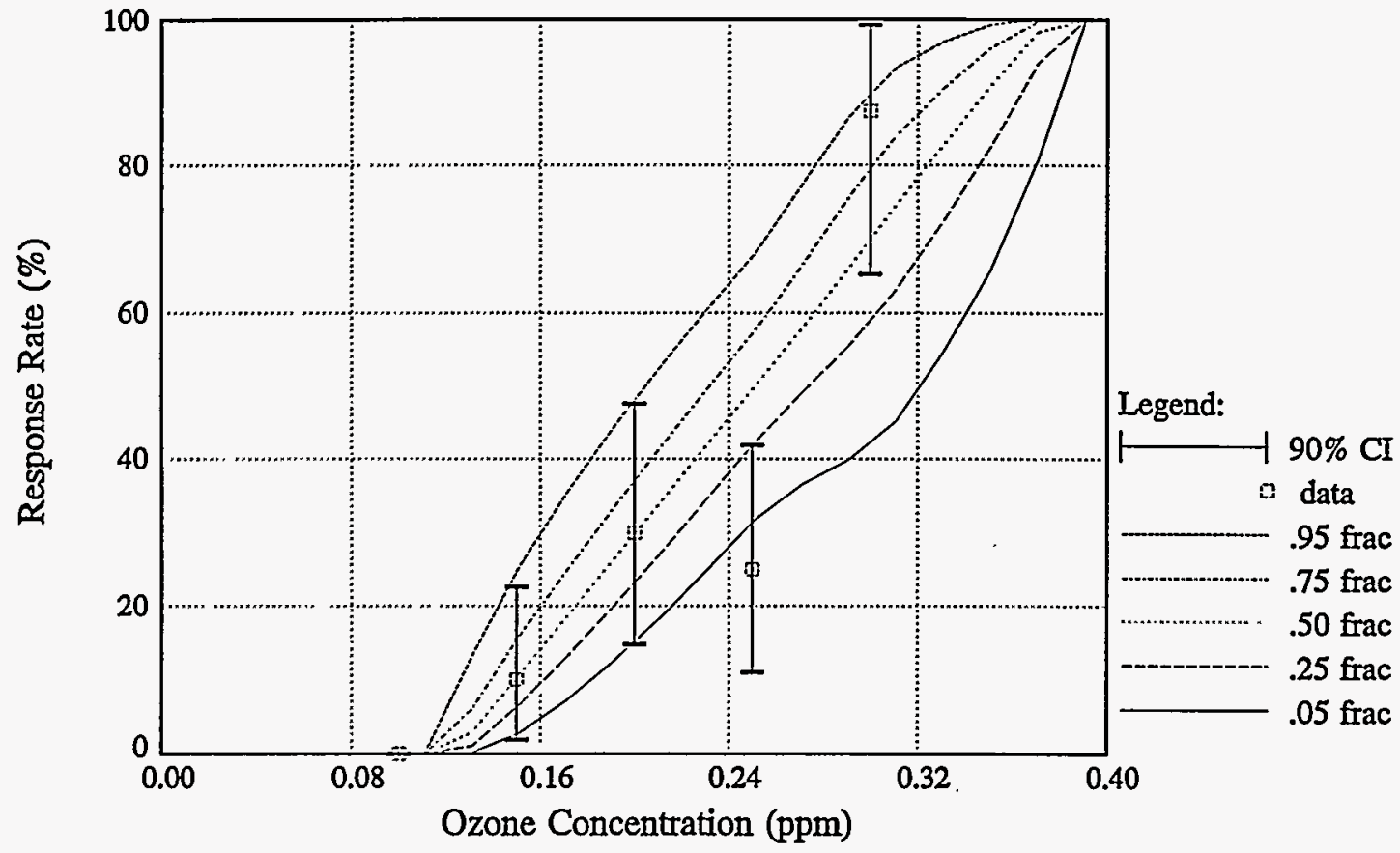

FIGURE B.11 Probabilistic Exposure-Response Relationship for Any Pain on Deep Inspiration for 1-h Exposures, Heavy Exertion; Includes Data, Medians, and $\mathbf{9 0 \%}$ CIs for Data and Relationship (Source: derived from Kulle et al. 1984)

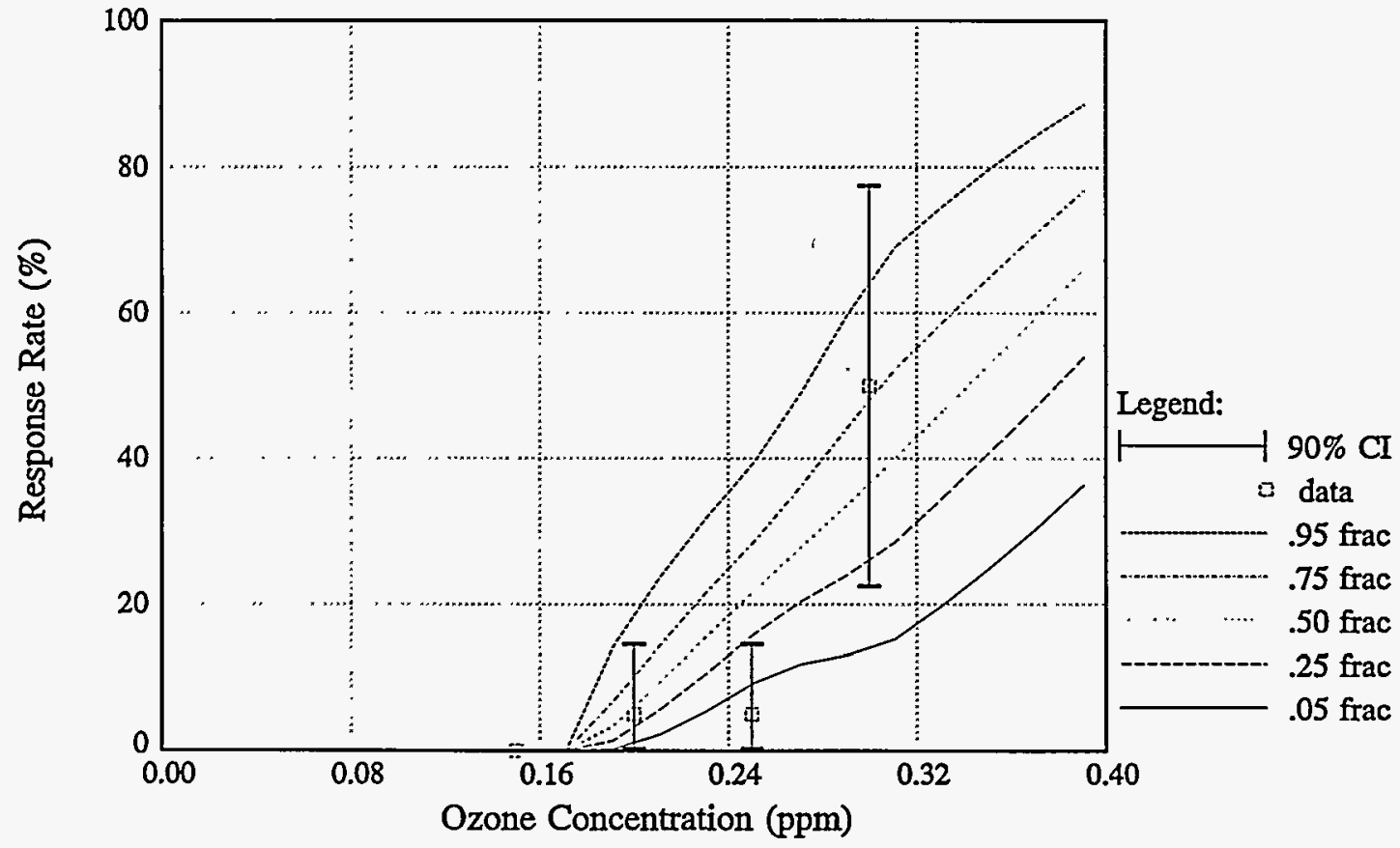

FIGURE B.12 Probabilistic Exposure-Response Relationship for Moderate-toSevere Pain on Deep Inspiration for 1-h Exposures, Heavy Exertion; Includes Data, Medians, and $\mathbf{9 0 \%}$ CIs for Data and Relationship (Source: derived from Kulle et al. 1984) 


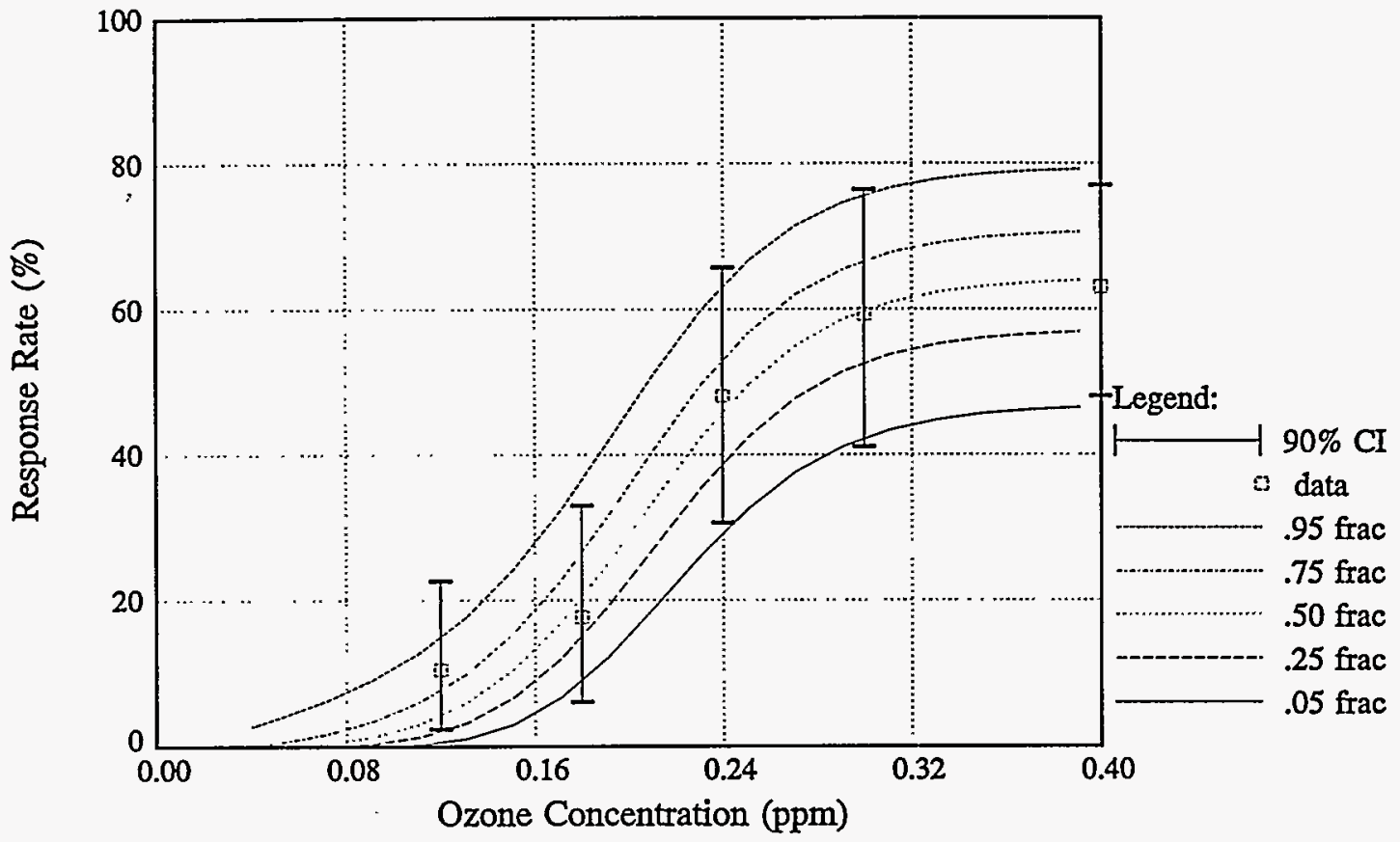

FIGURE B.13 Probabilistic Exposure-Response Relationship for FEV

Decrement $\geq 10 \%$ for 1-h Exposures, Heavy Exertion; Includes Data, Medians, and 90\% CIs for Data and Relationship (Source: derived from McDonnell et al. 1983)

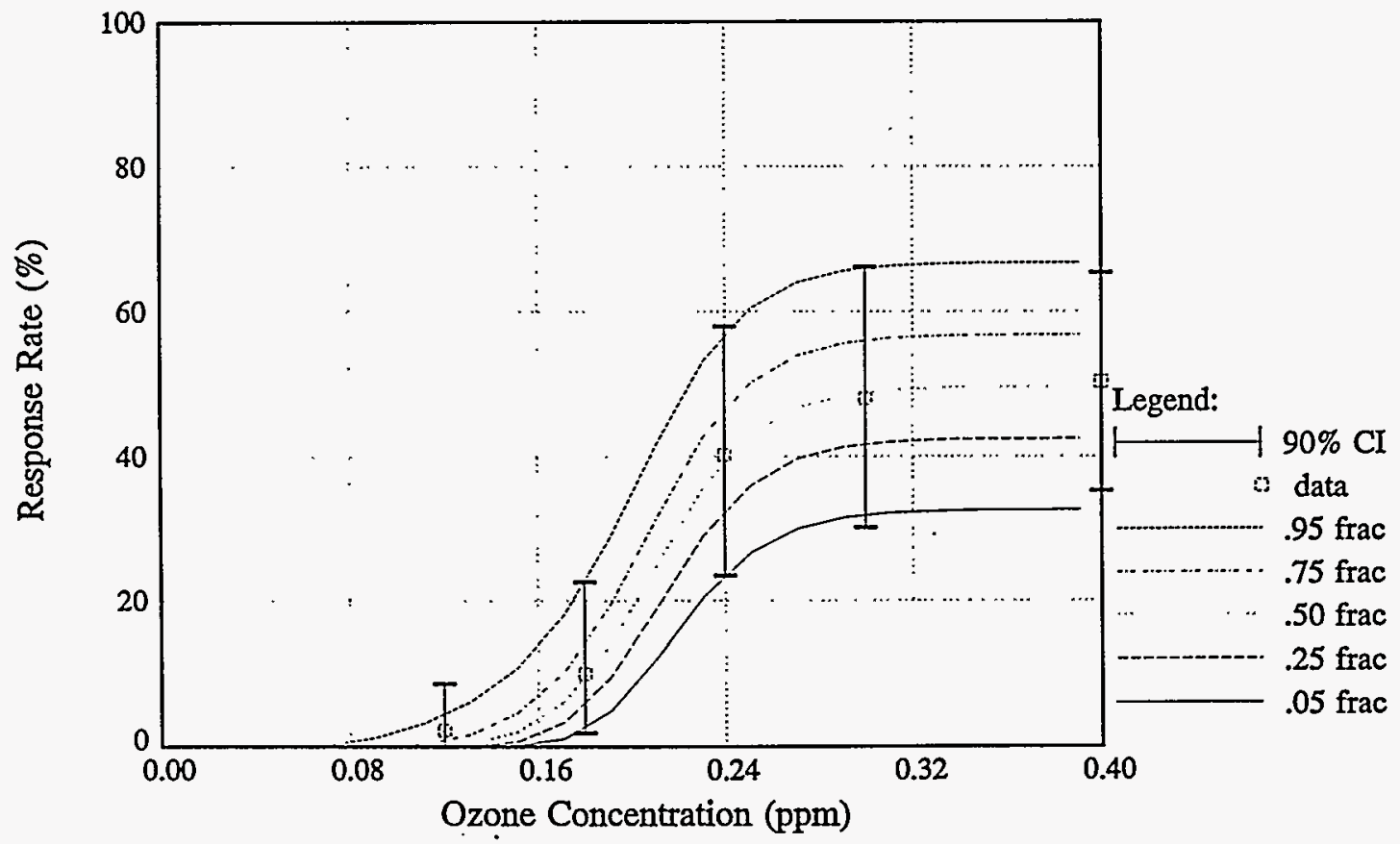

FIGURE B.14 Probabilistic Exposure-Response Relationship for FEV 1

Decrement $\geq 15 \%$ for 1-h Exposures, Heavy Exertion; Includes Data, Medians, and 90\% CIs for Data and Relationship (Source: derived from McDonnell et al. 1983) 


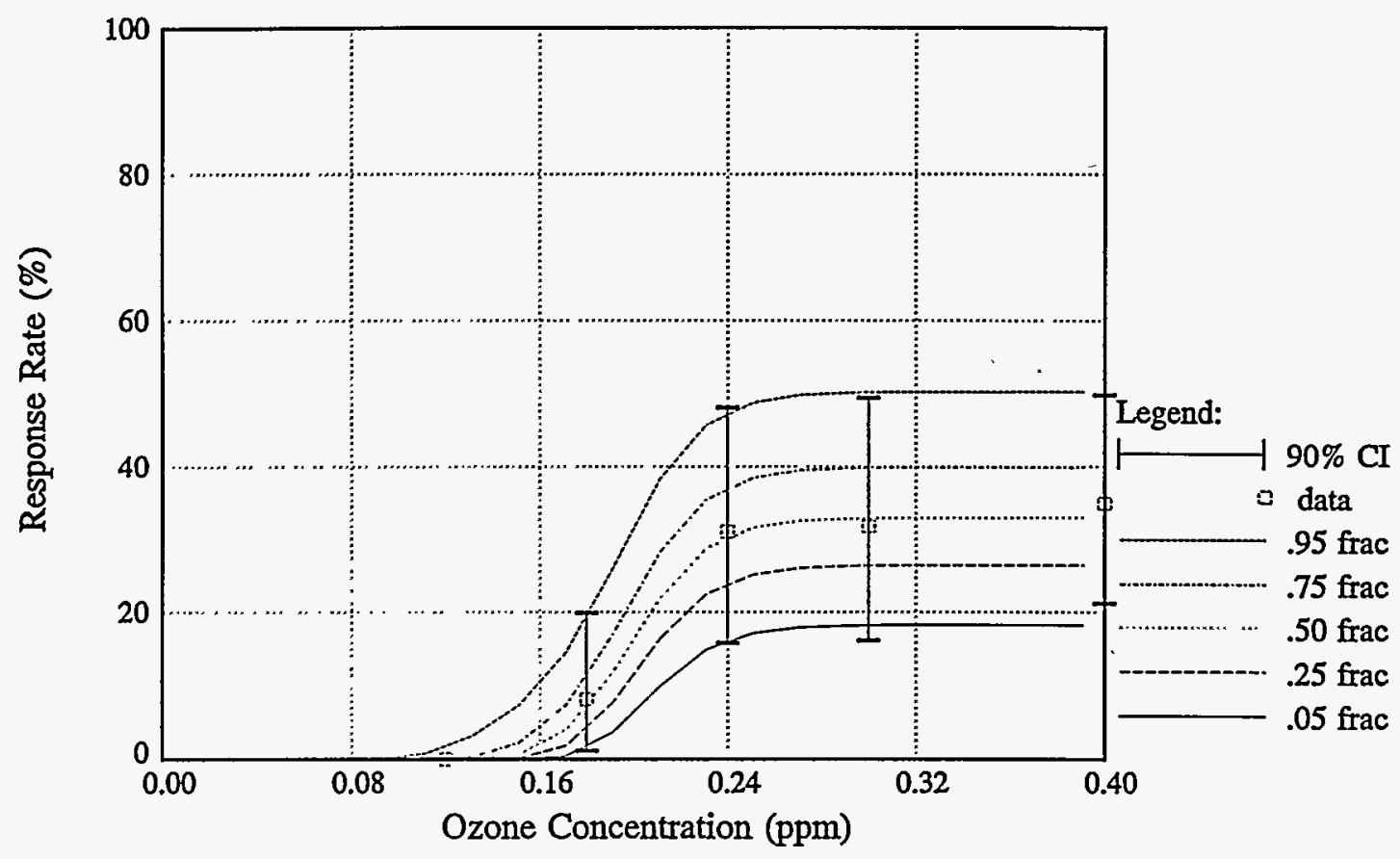

FIGURE B.15 Probabilistic Exposure-Response Relationship for FEV

Decrement $\geq 20 \%$ for 1-h Exposures, Heavy Exertion; Includes Data, Medians, and 90\% CIs for Data and Relationship (Source: derived from McDonnell et al. 1983)

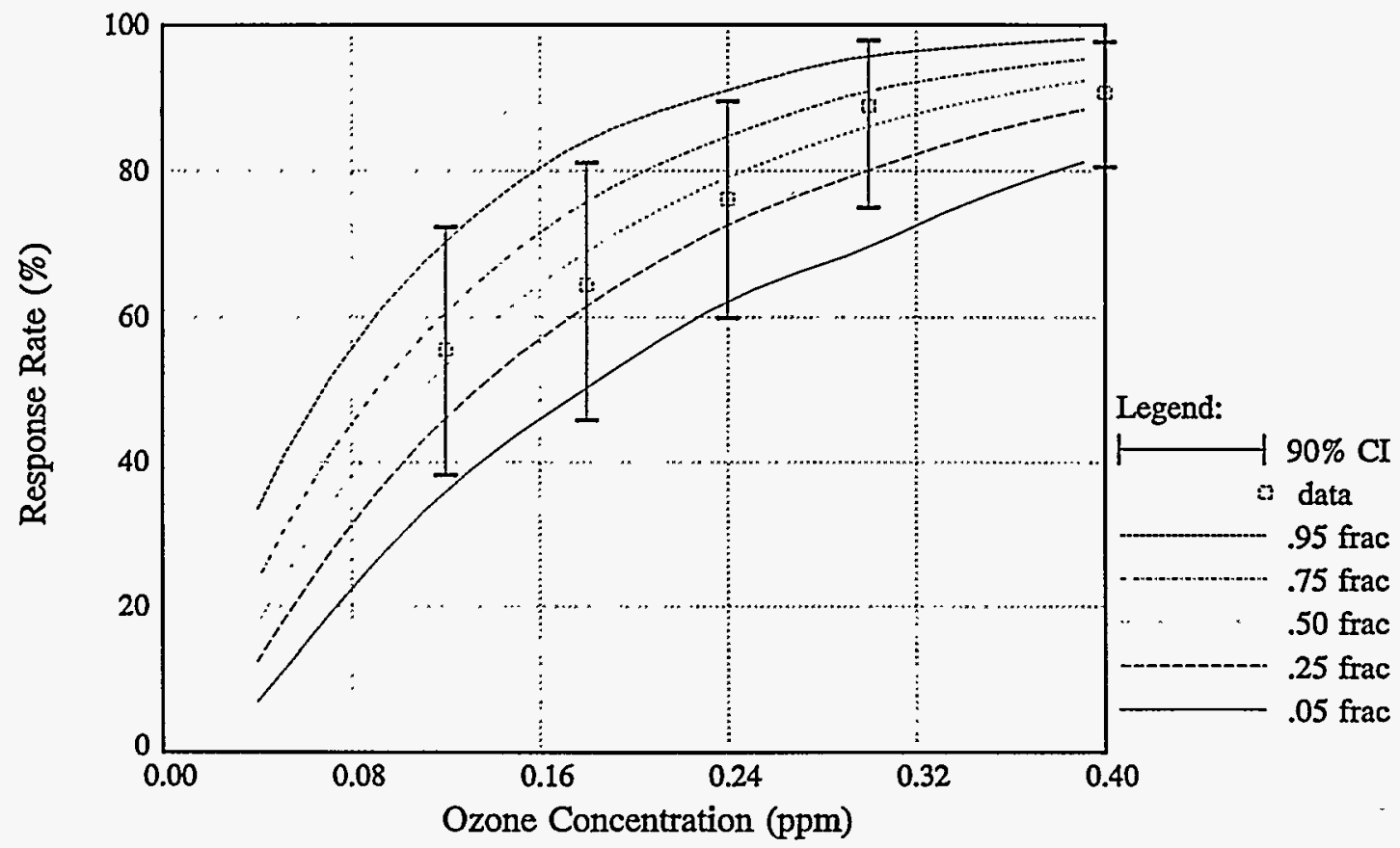

FIGURE B.16 Probabilistic Exposure-Response Relationship for Any Cough for 1-h Exposures, Heavy Exertion; Includes Data, Medians, and $\mathbf{9 0 \%}$ CIs for Data and Relationship (Source: derived from McDonnell et al. 1983) 


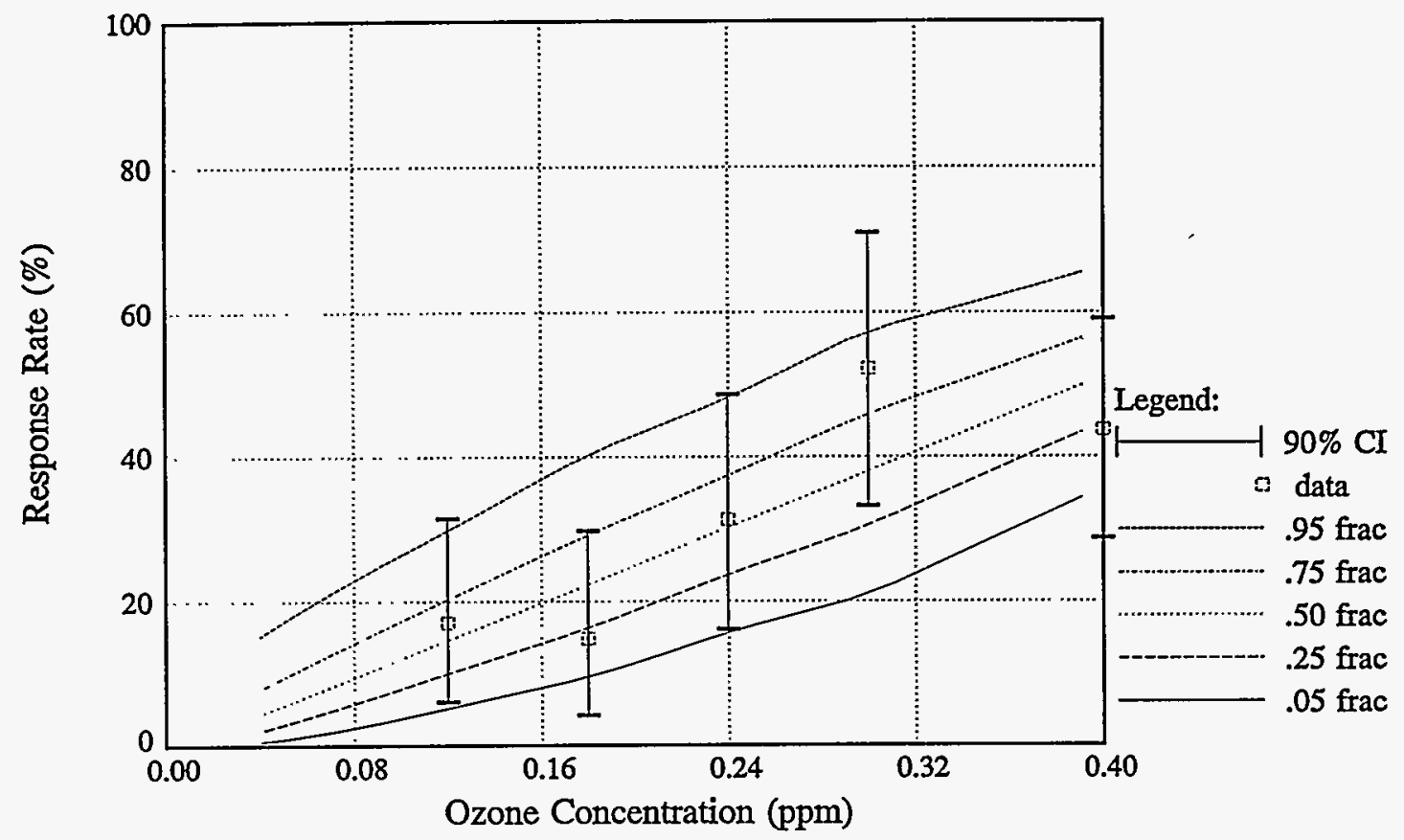

FIGURE B.17 Probabilistic Exposure-Response Relationship for Moderate-to-

Severe Cough for 1-h Exposures, Heavy Exertion; Includes Data, Medians, and $\mathbf{9 0 \%}$ CIs for Data and Relationship (Source: derived from McDonnell et al. 1983)

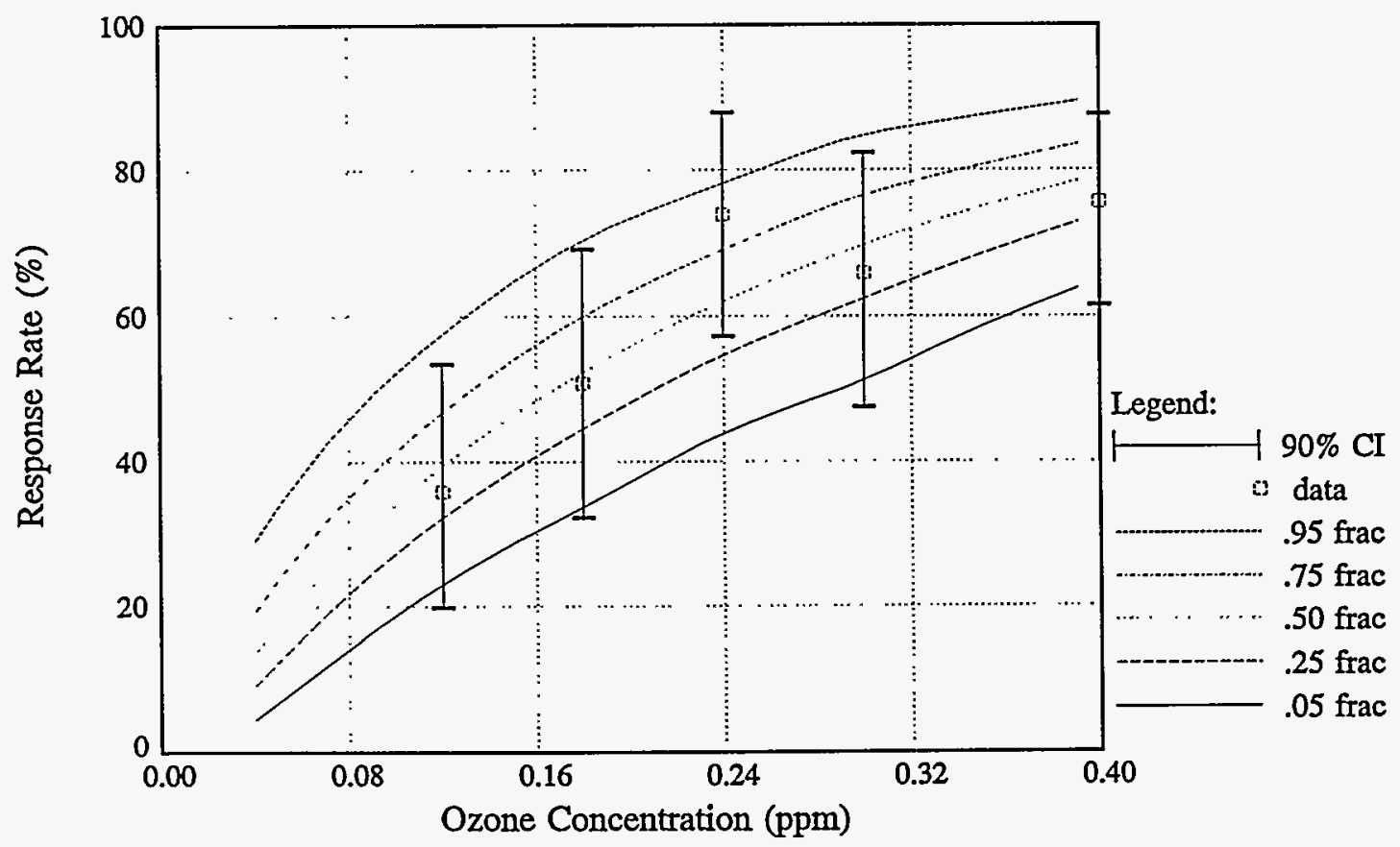

FIGURE B.18 Probabilistic Exposure-Response Relationship for Any Pain on Deep Inspiration for 1-h Exposures, Heavy Exertion; Includes Data, Medians, and $90 \%$ CIs for Data and Relationship (Source: derived from McDonnell et al. 1983) 


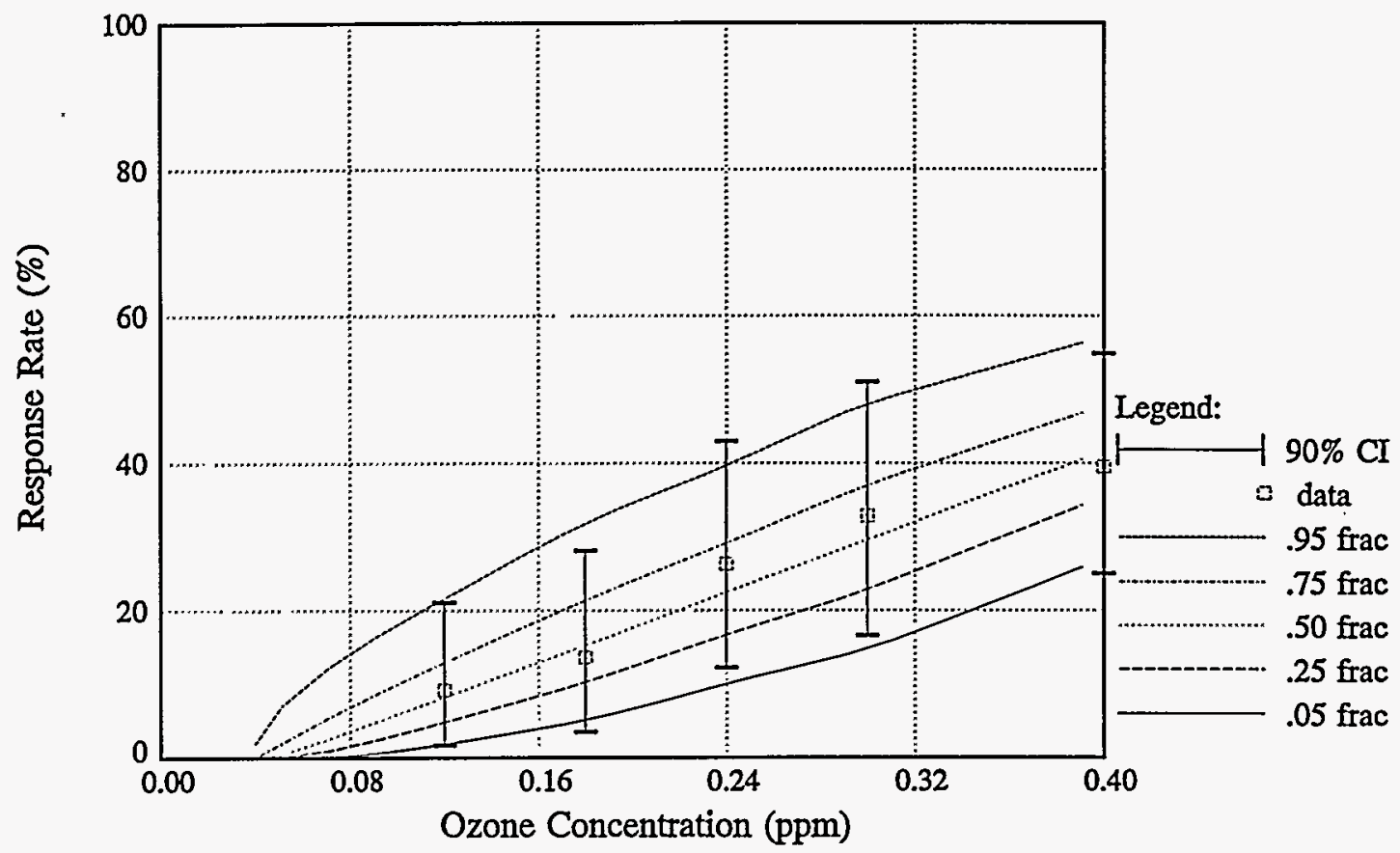

FIGURE B.19 Probabilistic Exposure-Response Relationship for Moderate-toSevere Pain on Deep Inspiration for 1-h Exposures, Heavy Exertion; Includes Data, Medians, and $\mathbf{9 0 \%}$ CIs for Data and Relationship (Source: derived from McDonnell et al. 1983)

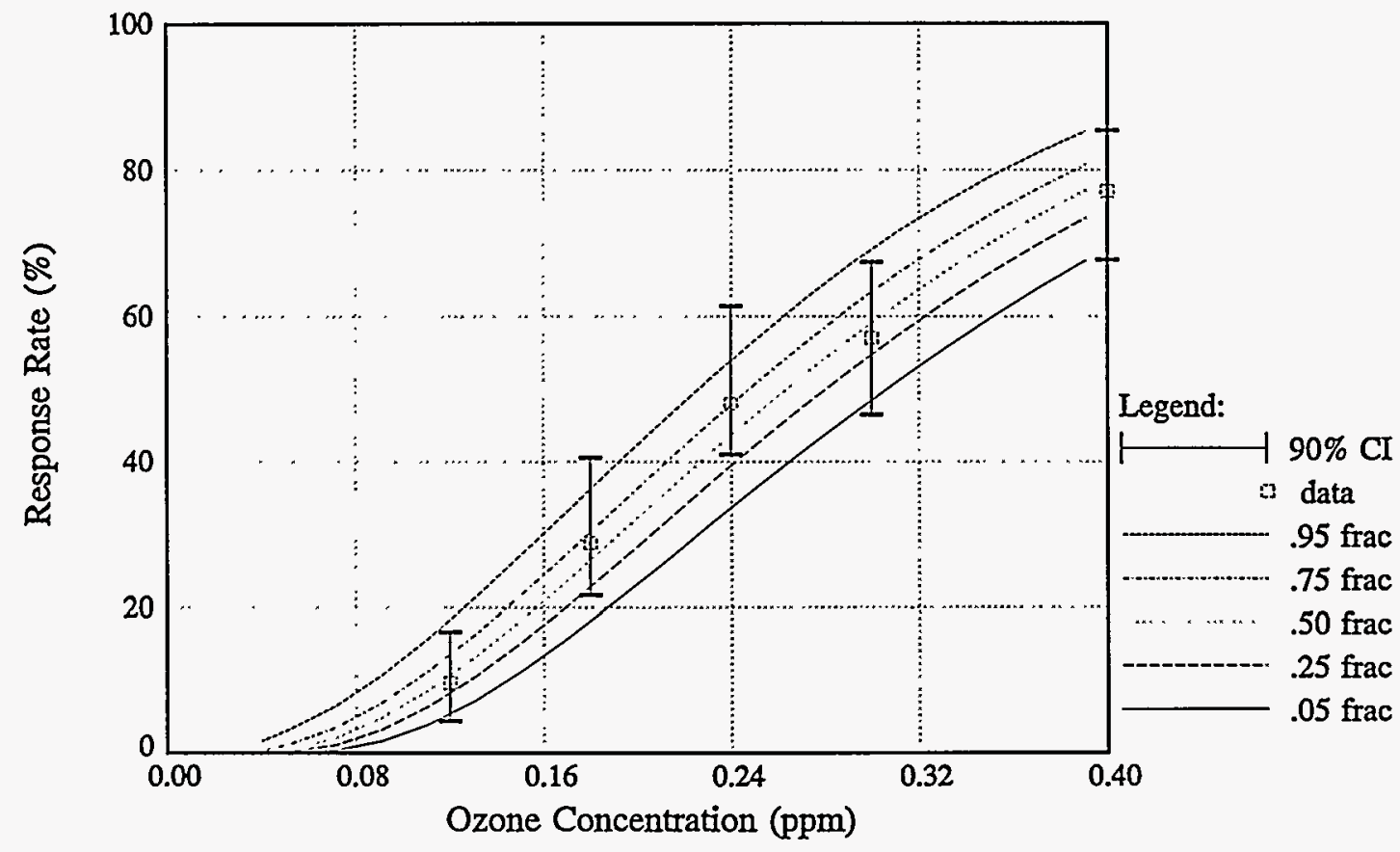

FIGURE B.20 Probabilistic Exposure-Response Relationship for FEV 1 Decrement $\geq 10 \%$ for 1-h Exposures and Moderate Exertion; Includes Data, Medians, and $90 \%$ CIs for Data and Relationship (Source: derived from Seal et al. 1993) 


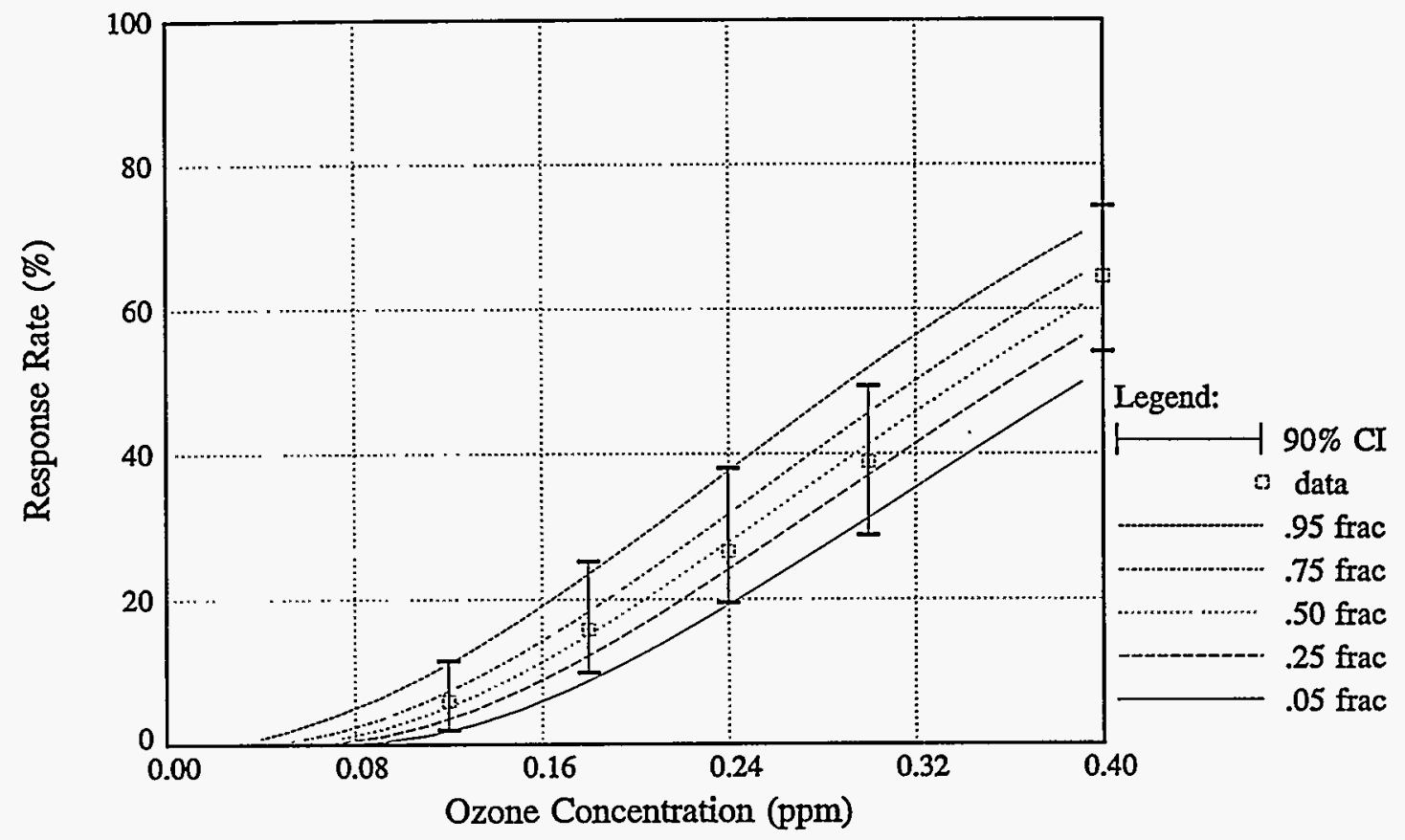

FIGURE B.21 Probabilistic Exposure-Response Relationship for FEV 1 Decrement $\geq 15 \%$ for 1-h Exposures and Moderate Exertion; Includes Data, Medians, and $90 \%$ CIs for Data and Relationship (Source: derived from Seal et al. 1993)

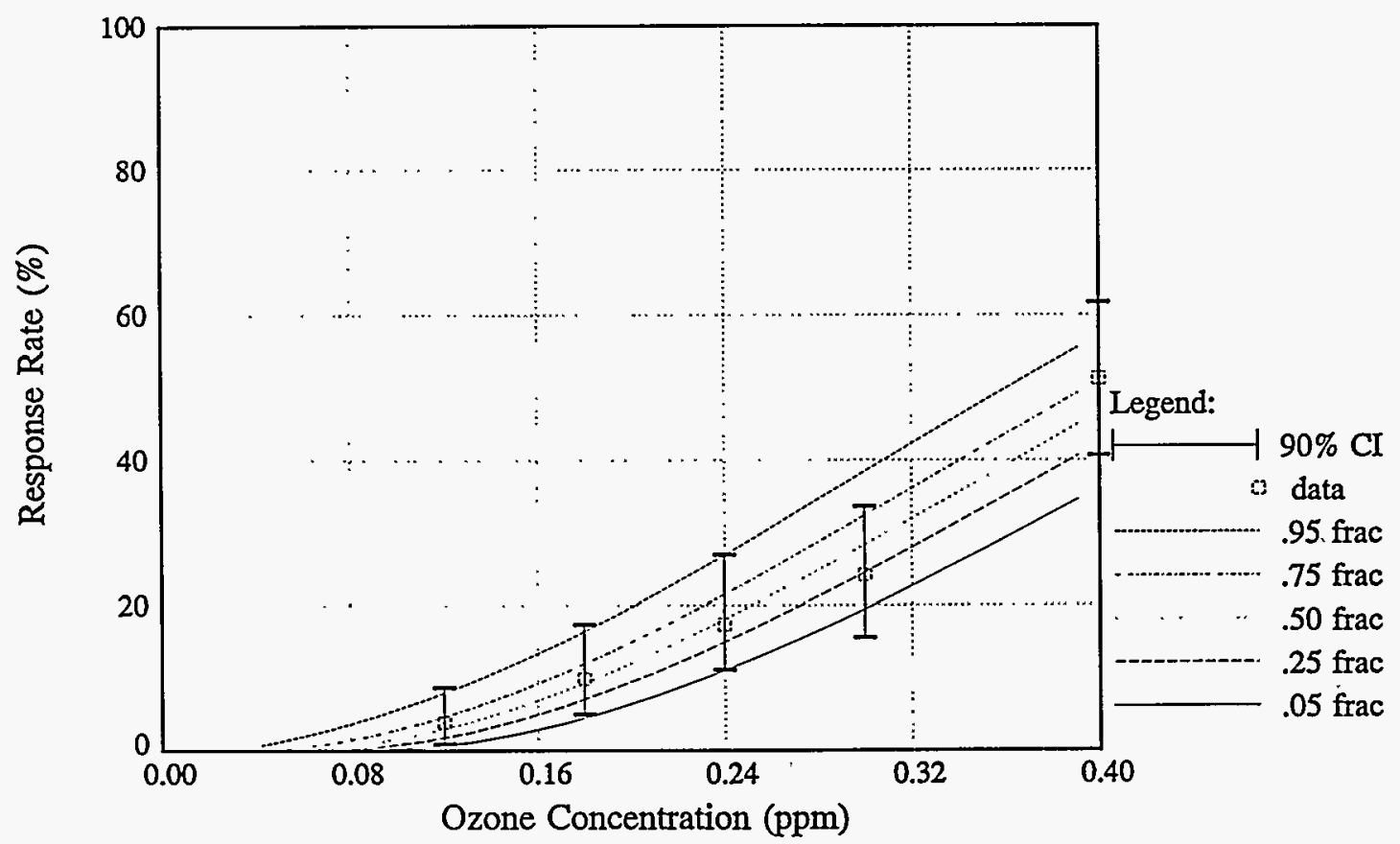

FIGURE B.22 Probabilistic Exposure-Response Relationship for FEV Decrement $\geq 20 \%$ for 1 -h Exposures and Moderate Exertion; Includes Data, Medians, and 90\% CIs for Data and Relationship (Source: derived from Seal et al. 1993) 


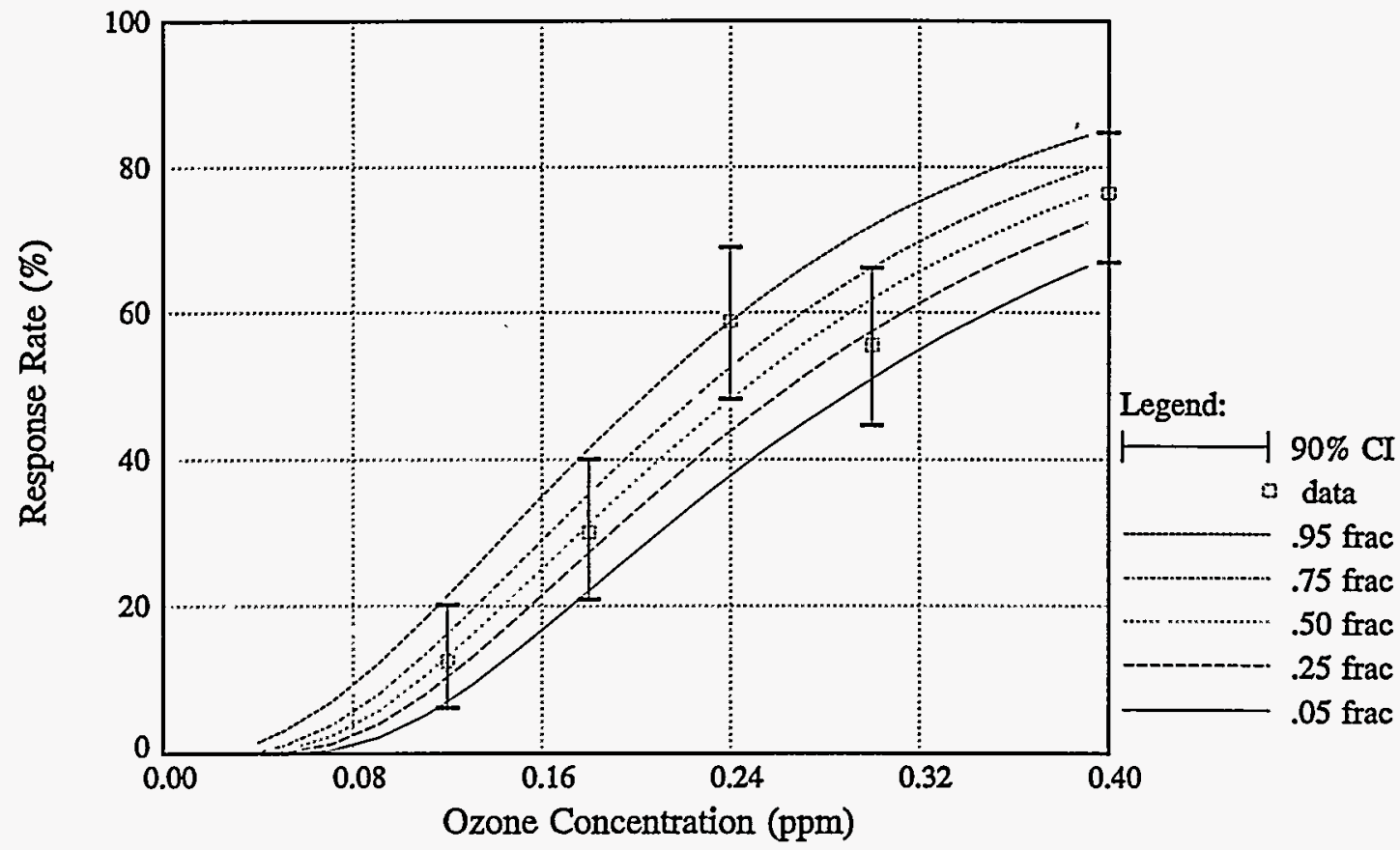

FIGURE B.23 Probabilistic Exposure-Response Relationship for Any Cough for 1-h Exposures and Moderate Exertion; Includes Data, Medians, and 90\% CIs for Data and Relationship (Source: derived from Seal et al. 1993)

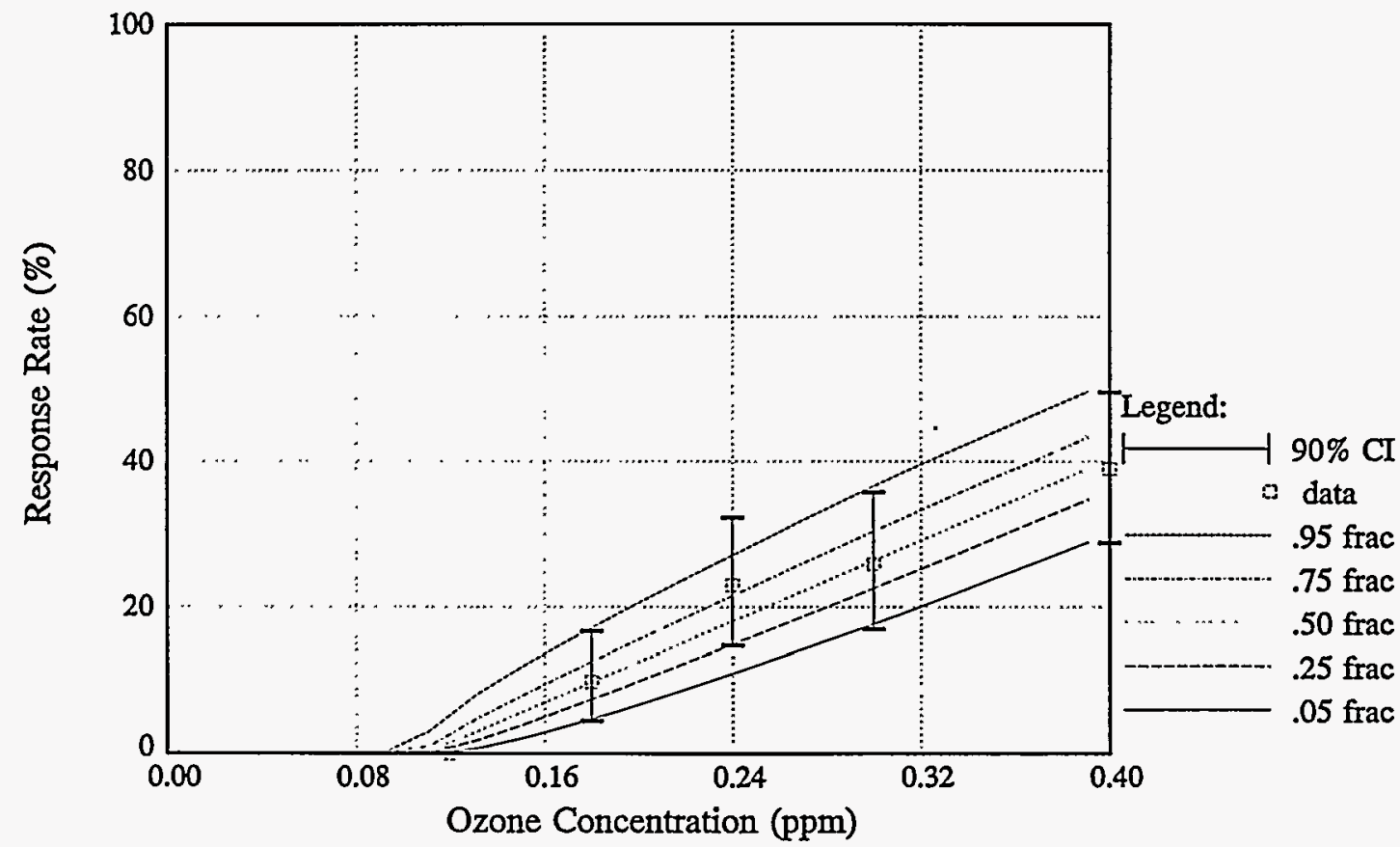

FIGURE B.24 Probabilistic Exposure-Response Relationship for Moderate-toSevere Cough for 1-h Exposures and Moderate Exertion; Includes Data, Medians, and $90 \%$ CIs for Data and Relationship (Source: derived from Seal et al. 1993) 


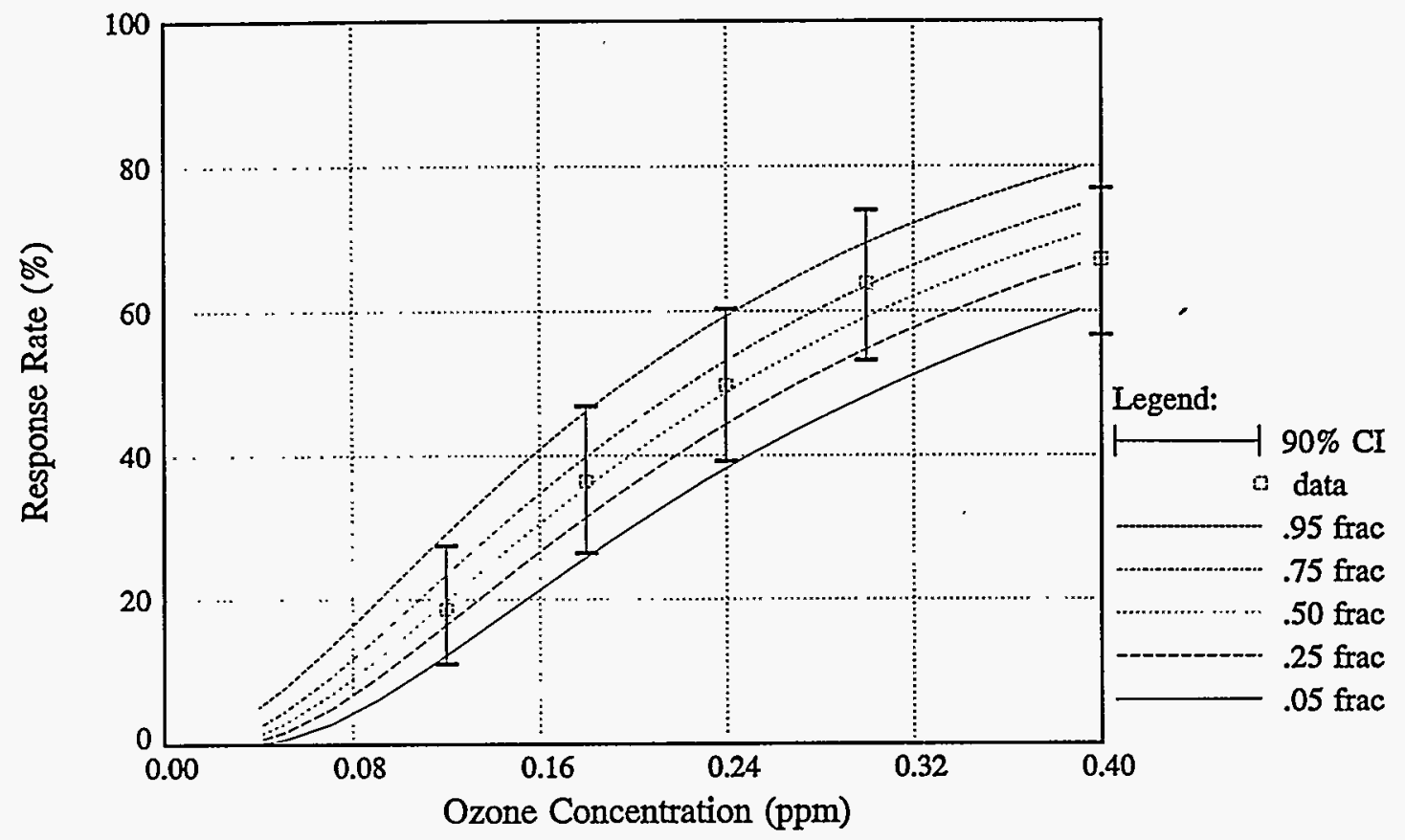

FIGURE B.25 Probabilistic Exposure-Response Relationship for Any Pain on Deep Inspiration for 1-h Exposures and Moderate Exertion; Includes Data, Medians, and 90\% CIs for Data and Relationship (Source: derived from Seal et al. 1993)

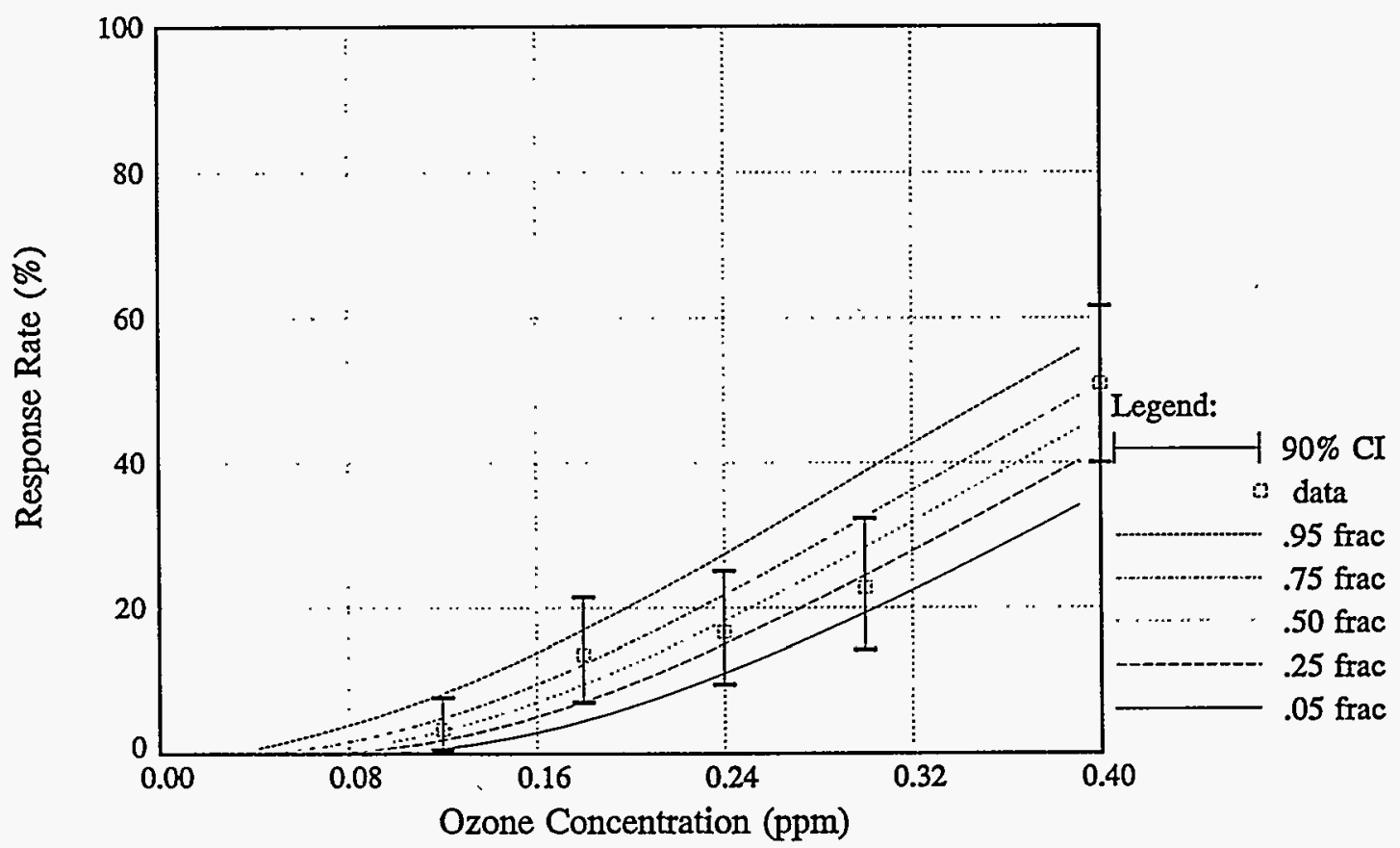

FIGURE B.26 Probabilistic Exposure-Response Relationship for Moderate-toSevere Pain on Deep Inspiration for 1-h Exposures and Moderate Exertion; Includes Data, Medians, and $90 \%$ CIs for Data and Relationship (Source: derived from Seal et al. 1993) 


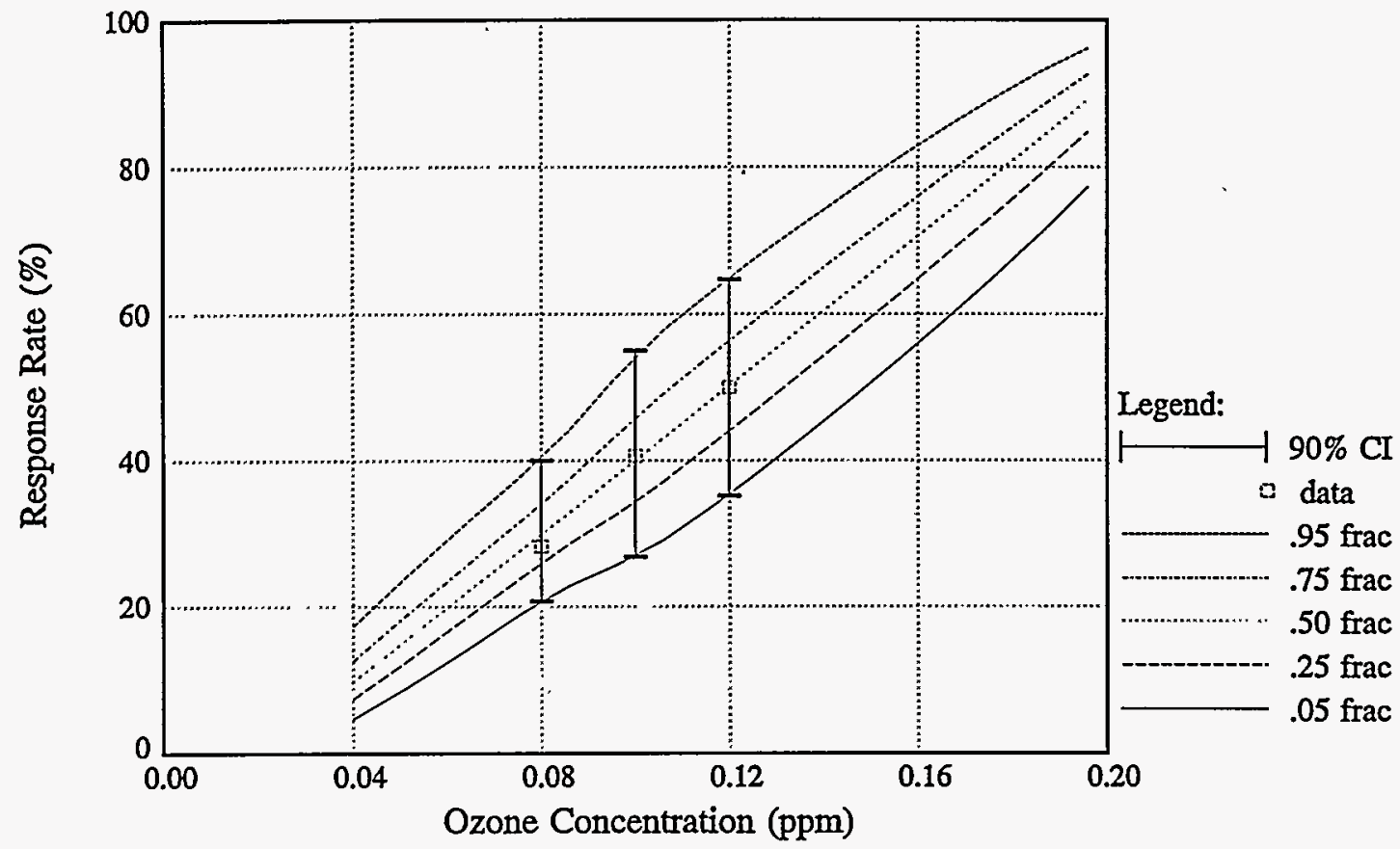

FIGURE B.27 Probabilistic Exposure-Response Relationship for FEV 1

Decrement $\geq 10 \%$ for 8 -h Exposures and Moderate Exertion; Includes Data, Medians, and $\mathbf{9 0 \%}$ CIs for Data and Relationship (Sources: derived from Folinsbee et al. 1988, Horstman et al. 1990, and McDonnell et al. 1991)

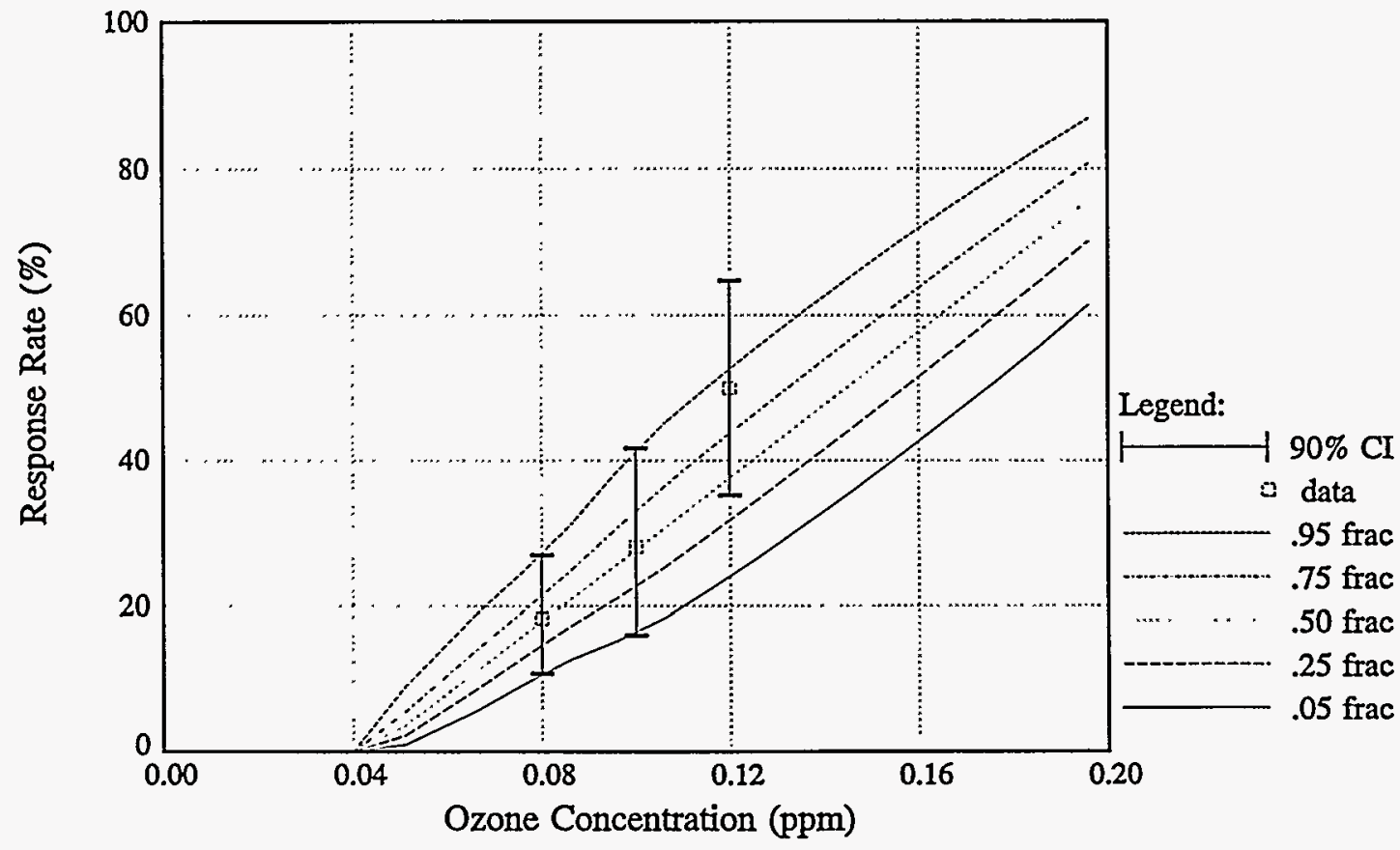

FIGURE B.28 Probabilistic Exposure-Response Relationship for $\mathrm{FEV}_{1}$ Decrement $\geq 15 \%$ for 8 -h Exposures and Moderate Exertion; Includes Data, Medians, and $90 \%$ CIs for Data and Relationship (Sources: derived from Folinsbee et al. 1988, Horstman et al. 1990, and McDonnell et al. 1991) 


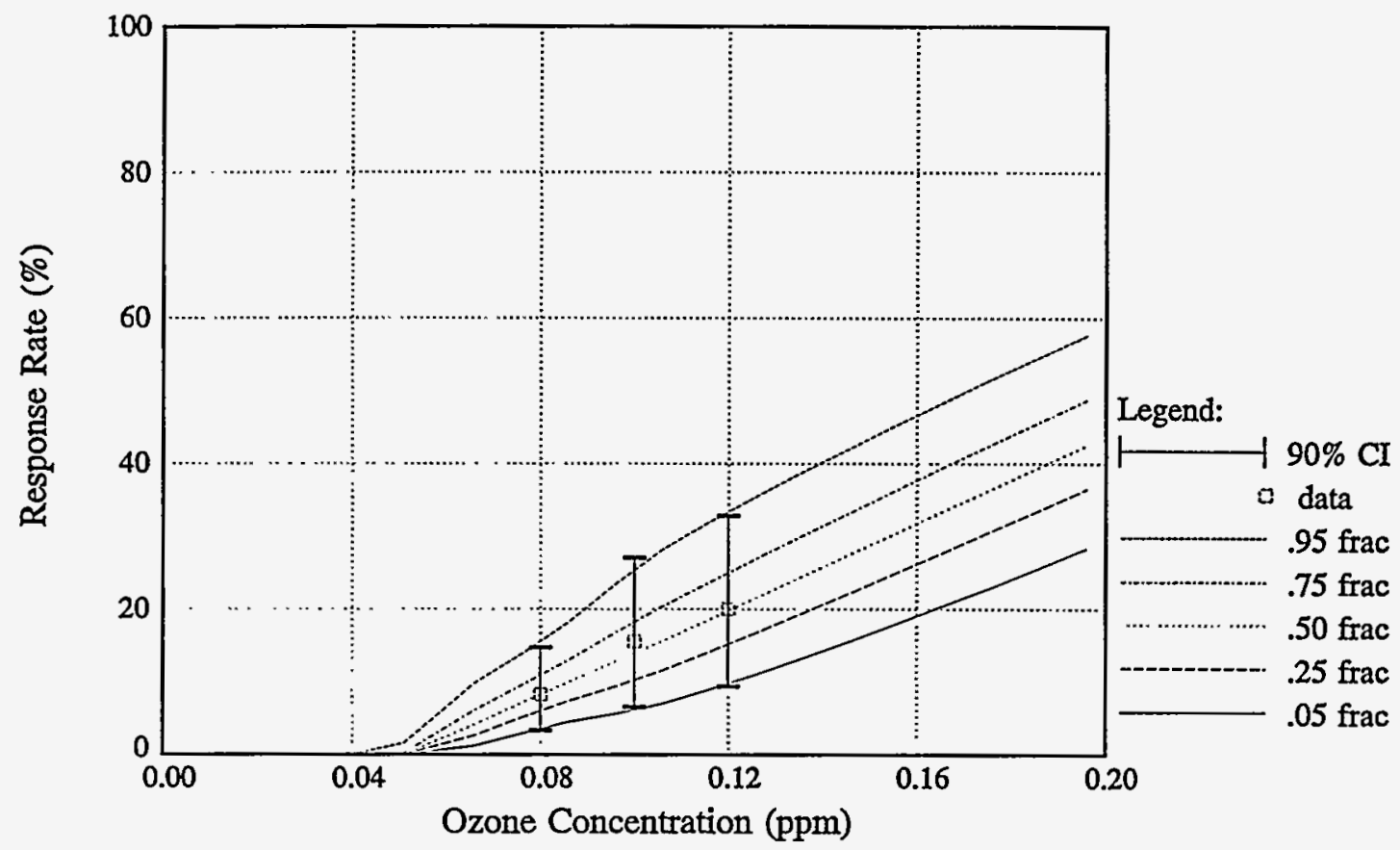

FIGURE B.29 Probabilistic Exposure-Response Relationship for FEV 1 Decrement $\geq 20 \%$ for 8 -h Exposures and Moderate Exertion; Includes Data, Medians, and $\mathbf{9 0 \%}$ CIs for Data and Relationship (Sources: derived from Folinsbee et al. 1988, Horstman et al. 1990, and McDonnell et al. 1991)

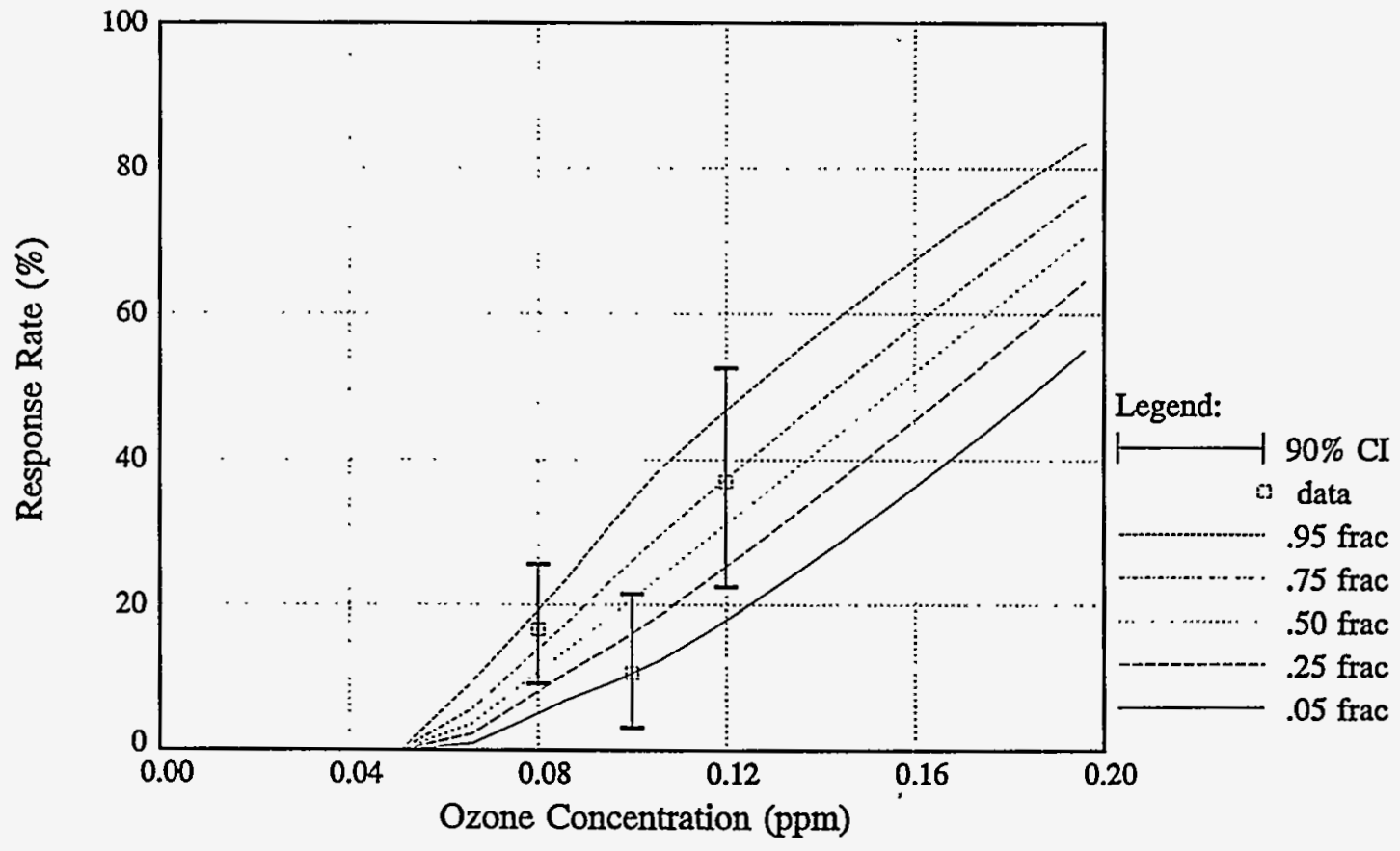

FIGURE B.30 Probabilistic Exposure-Response Relationship for Any Cough for 8-h Exposures and Moderate Exertion; Includes Data, Medians, and 90\% CIs for Data and Relationship (Sources: derived from Folinsbee et al. 1988, Horstman et al. 1990, and McDonnell et al. 1991) 


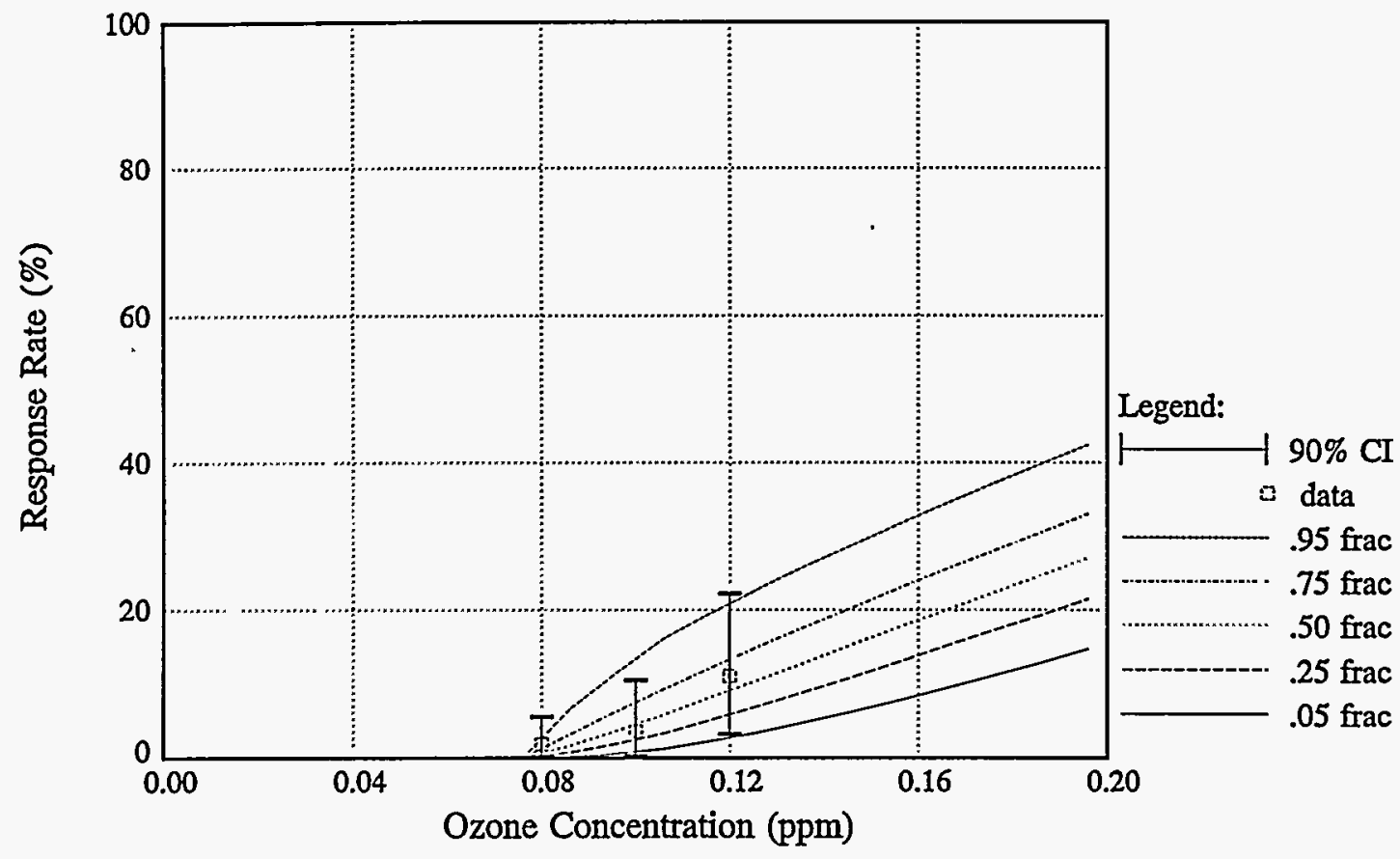

FIGURE B.31 Probabilistic Exposure-Response Relationship for Moderate-toSevere Cough for 8-h Exposures and Moderate Exertion; Includes Data, Medians, and $90 \%$ CIs for Data and Relationship (Sources: derived from Folinsbee et al. 1988, Horstman et al. 1990, and McDonnell et al. 1991)

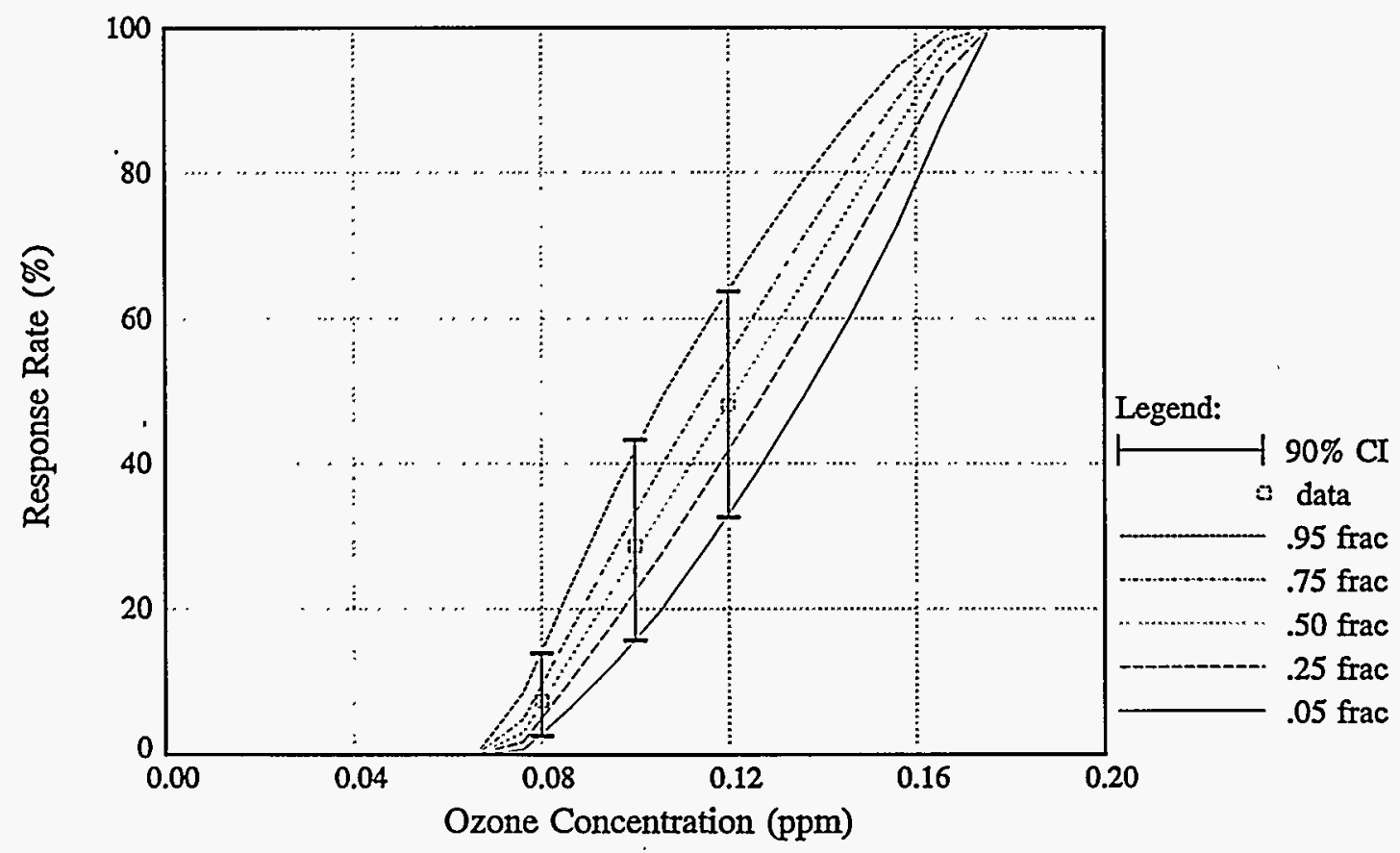

FIGURE B.32 Probabilistic Exposure-Response Relationship for Any Pain on Deep Inspiration for 8-h Exposures and Moderate Exertion; Includes Data, Medians, and 90\% CIs for Data and Relationship (Sources: derived from Folinsbee et al. 1988, Horstman et al. 1990, and McDonnell et al. 1991) 


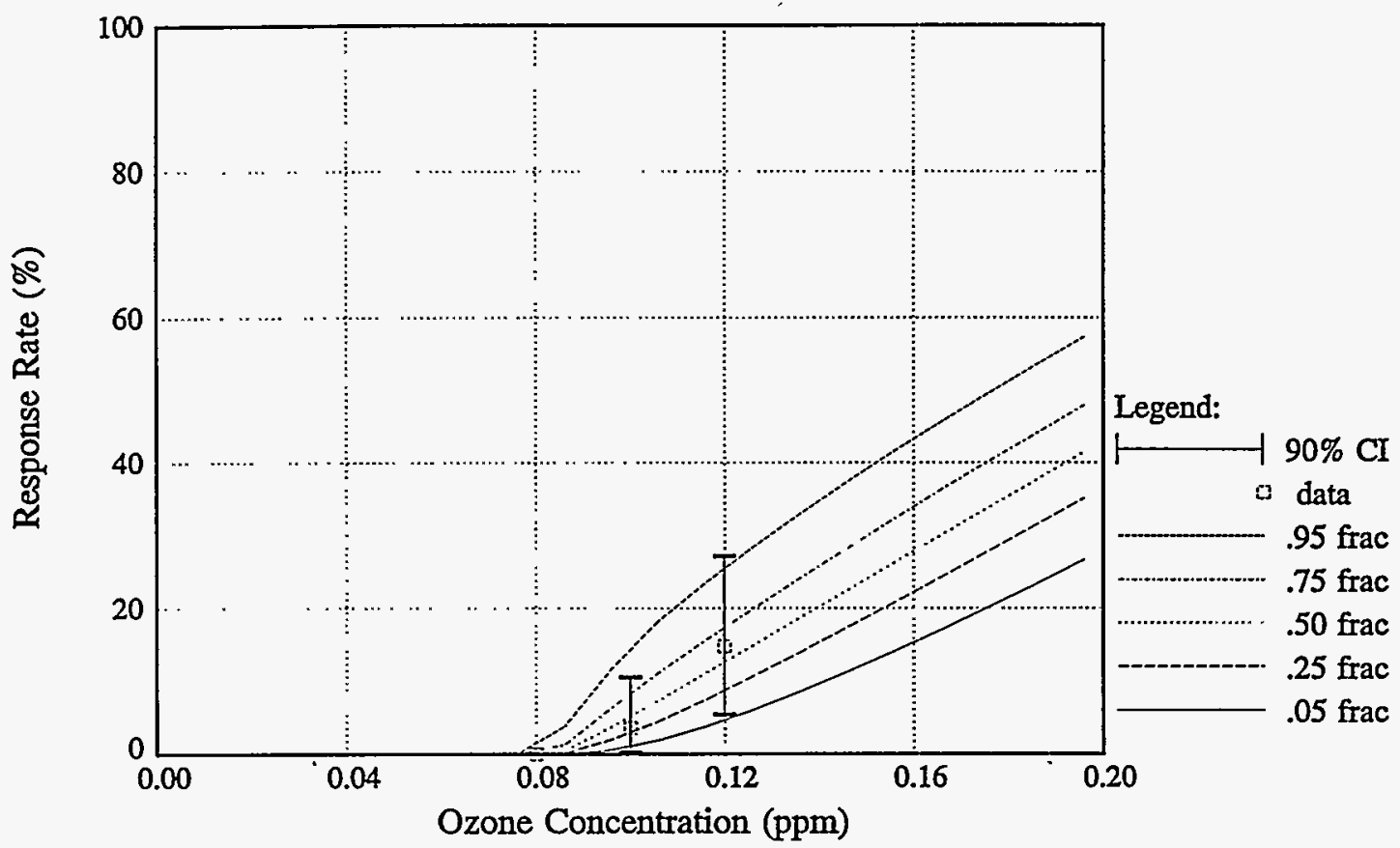

FIGURE B.33 Probabilistic Exposure-Response Relationship for Moderate-to-

Severe Pain on Deep Inspiration for 8-h Exposures and Moderate Exertion; Includes Data, Medians, and $\mathbf{9 0 \%}$ CIs for Data and Relationship (Sources: derived from Folinsbee et al. 1988, Horstman et al. 1990, and McDonnell et al. 1991) 


\section{B.2 APPENDIX B REFERENCES}

Avol, E.L., et al., 1984, "Comparative Respiratory Effects of Ozone and Ambient Oxidant Pollution Exposure during Heavy Exercise," Journal of the Air Pollution Control Association 34:804-809.

Folinsbee, L.J., et al., 1988, "Pulmonary Function and Symptom Responses after 6.6-Hour Exposure to $0.12 \mathrm{ppm}$ Ozone with Moderate Exercise," Journal of the Air Pollution Control Association 38:28-35.

Horstman, D.H., et al., 1990, "Ozone Concentration and Pulmonary Response Relationships for 6.6-Hour Exposures with Five Hours of Moderate Exercise to 0.08, 0.10, and $0.12 \mathrm{ppm}$," American Review of Respiratory Disease 142:1158-1163.

Kulle, T.J., et al., 1985, "Ozone Response Relationships in Healthy Nonsmokers," American Review of Respiratory Disease 132:36-41.

McDonnell, W.F., et al., 1983, "Pulmonary Effects of Ozone Exposure during Exercise: DoseResponse Characteristics," Journal of Applied Physiology: Respiratory Environmental Exercise Physiology 54:1345-1352.

McDonnell, W.F., et al., 1991, "Respiratory Response of Humans Exposed to Low Levels of Ozone for 6.6 Hours," Archives of Environmental Health 46:145-150.

Seal, E., Jr., et al., 1993, "The Pulmonary Response of White and Black Adults to Six Concentrations of Ozone," American Review of Respiratory Disease 147:804-810. 

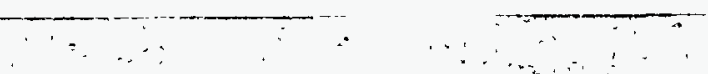
APPENDIX C:

HEADCOUNT RISK RESULTS FOR ACUTE ENDPOINTS 


\section{APPENDIX C:}

\section{HEADCOUNT RISK RESULTS FOR ACUTE ENDPOINTS}

\section{C.1 INTRODUCTION}

This appendix contains Figures C.1-C.49 organized in three groups. The first group is composed of 27 figures for representative risk distributions: one figure for each of nine urban areas and three of the more severe health endpoints $\left(\mathrm{FEV}_{1}\right.$ decrement $\geq 15 \%, \mathrm{FEV}_{1}$ decrement $\geq 20 \%$, and moderate-to-severe pain on deep inspiration [M/S PDI] among outdoor children; the $\mathrm{FEV}_{1}$ endpoints are for 8-h exposures at moderate exertion, and the M/S PDI endpoint is for 1-h exposures at moderate exertion).

The second group is composed of 3 figures that summarize one aspect of the first group of figures: the ratios of the mean number of occurrences and the mean number of responders, which is an estimate of the times that responders respond over an ozone season. The last group is composed of 19 figures; each summarizes risk results for all 9 urban areas, all 9 air quality scenarios, and 1 health endpoint.

\section{C.2 REPRESENTATIVE RISK DISTRIBUTIONS}

Risk results for each endpoint are available in the form of 10 probability distributions for each air quality scenario. Because the study has nine scenarios, it is not practical to plot all of the distributions in one figure because the figure would be quite "messy." To gain insight about the risk implications of the air quality scenarios, "representative distributions" and "box plots" were developed. Both are shown in Figures C.1-C.27 and C.31-C.49, respectively. Figures C.28-C.30 estimate the number of times that responders (i.e., outdoor children who experience a specific condition, such as having an $\mathrm{FEV}_{1}$ decrement $\geq 15 \%$ ) respond.

Figure C.1, which contains representative distributions, shows two sets of nine plots for $\mathrm{FEV}_{1}$ decrement $\geq 15 \%$, 8-h exposures, moderate exertion, Chicago, and children. The top half of the figure shows representative distributions over the number of children experiencing the effect one or more times (i.e., persons basis), and the bottom half shows representative distributions over the number of times any child experiences the effect (i.e., person-occurrences basis). Each representative distribution gives some idea of the range of results among each set of 10 distributions. Each of nine air quality scenarios has one representative distribution. Each plot is "representative" of the 10 distributions for a particular scenario. Because we have only 9 plots rather than 90 , it is easier to see patterns. Each of these plots is a valid cumulative 
probability distribution. ${ }^{1}$ The plot indicates, for example, that the median number of children in Chicago who may experience $\mathrm{FEV}_{1}$ decrements $\geq 15 \%$ under As-Is air quality is around 65,000 . When the most stringent standard (8107) is just attained, the median estimate is about 15,000 children.

\section{C.3 RATIOS OF MEAN NUMBERS OF OCCURRENCES AND MEAN NUMBERS OF RESPONDERS}

Figures C.28-C.30 show the ratios for the mean numbers of occurrences and mean numbers of outdoor children responding for three endpoints, nine urban areas, and five air quality scenarios. The endpoints are $\mathrm{FEV}_{1}$ decrements $\geq 15$ and $\geq 20 \%$ for 8 -h exposures at moderate exertion, and moderate-to-severe pain on deep inspiration for 1-h exposures at moderate exertion. The following letter codes identify the urban areas: $\mathrm{CH}=$ Chicago, $\mathrm{DE}=$ Denver, $\mathrm{HO}=$ Houston, $\mathrm{LA}=$ Los Angeles, $\mathrm{MI}=$ Miami, $\mathrm{NY}=$ New York City, $\mathrm{PH}=$ Philadelphia, $\mathrm{SL}=\mathrm{St}$. Louis, and DC $=$ Washington, D.C.

The ratios were computed in the following way. For a specific endpoint, available risk results include 10 probability distributions (one for each of $10 \mathrm{pNEM} / \mathrm{O}_{3}$ runs) over the number of persons who respond one or more times, and 10 probability distributions over the number of person-occurrences (which allows for the possibility that an individual may respond more than one time). The ratio of interest is the sum of the expected values of the person-occurrences distributions divided by the sum of the expected values of the persons distributions. The ratio is, in a sense, an estimate of the average number of times that a responder responds during an ozone season.

\section{C.4 BOX PLOTS FOR RISK RESULTS}

Box plots provide another perspective about risk results. Each box plot displays the ranges of the medians (or 0.5 fractile), 0.05 fractiles, and 0.95 fractiles of 10 risk distributions that result from the $10 \mathrm{pNEM} / \mathrm{O}_{3}$ exposure distributions available. These ranges are represented by rectangles in the figures (unless there is no range, in which case the rectangle "collapses" into a horizontal line). Three rectangles are above the code letter for each standard. The top rectangle represents the range of the 0.95 fractiles, the middle rectangle represents the range of the medians, and the bottom rectangle represents the range of the 0.05 fractiles. A line connects the bottom of the 0.95 -fractile rectangle and the top of the 0.05 -fractile rectangle and passes through

1 The representative distribution is obtained by computing the average cumulative probability at selected points along the $\mathrm{X}$ axis. This calculation, like the risk calculations described earlier, implicitly assumes that the distributions are perfectly correlated. It may be argued that perfect correlation, while not correct, is more reasonable than perfect independence, and there is no basis for choosing any other degree of correlation between these two extremes. 
the 0.5 -fractile rectangle. With this format, results for 81 scenarios (9 scenarios for each of 9 urban areas) can be displayed in one figure. For these plots, however, we switched from numbers of persons or person-occurrences to percentage of persons responding. As shown in Figure C.1, under As-Is conditions in Chicago, the median risk estimates for the percentage of children having $\mathrm{FEV}_{1}$ decrements $\geq 15 \%$ vary from 13 to $14 \%$, the 0.95 fractiles vary from 21 to $22 \%$, and the 0.05 fractiles vary from 7 to $8 \%$. Box plots have the following characteristics. If the risk distributions for a particular air quality scenario are quite "similar," the rectangles are small. If the variance of a risk distribution is small, the rectangles are close together. If the distributions are spaced far enough apart (indicative of widely varying risk estimates for different $\mathrm{pNEM} / \mathrm{O}_{3}$ runs), rectangles overlap. 

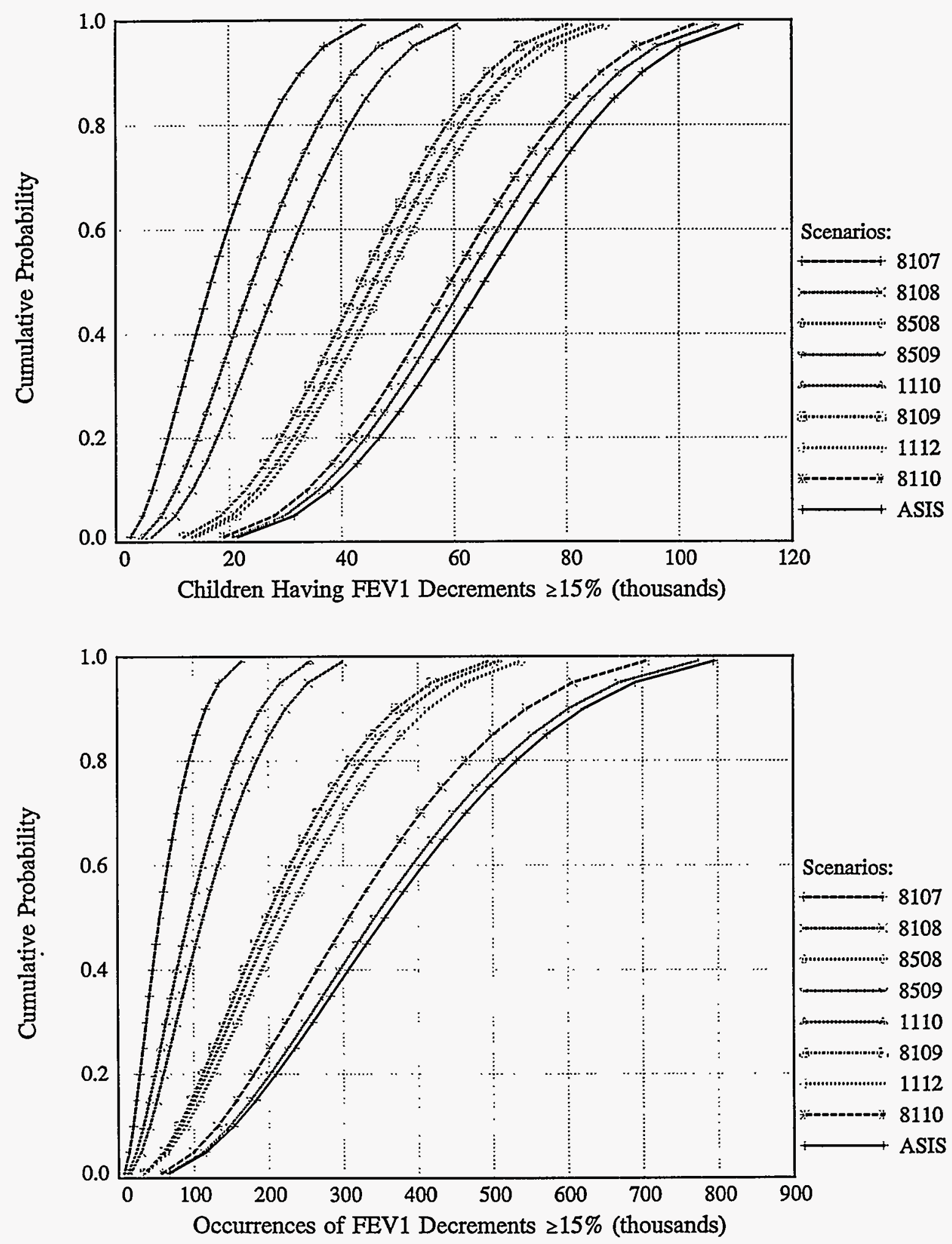

FIGURE C.1 Representative Risk Distributions for Alternative Air Quality Scenarios for FEV $_{1}$ Decrements $\geq 15 \%$, Chicago, Outdoor Children, 8-h Exposures, and Moderate Exertion (underlying exposure-response relationship based on Folinsbee et al. 1988, Horstman et al. 1990, and McDonnell et al. 1991) 

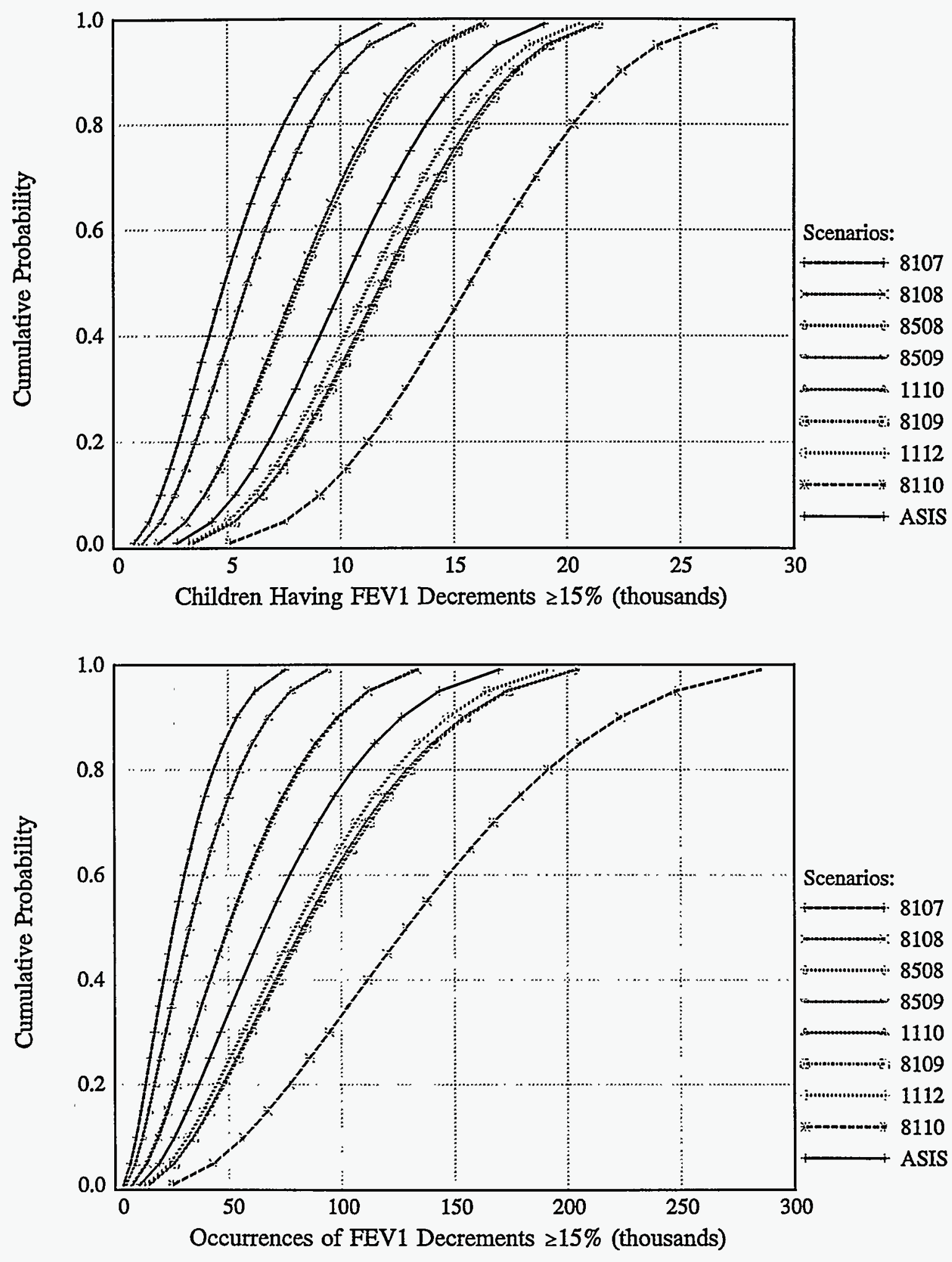

FIGURE C.2 Representative Risk Distributions for Alternative Air Quality Scenarios for FEV $_{1}$ Decrements $\geq 15 \%$, Denver, Outdoor Children, 8-h Exposures, and Moderate Exertion (underlying exposure-response relationship based on Folinsbee et al. 1988, Horstman et al. 1990, and McDonnell et al. 1991) 

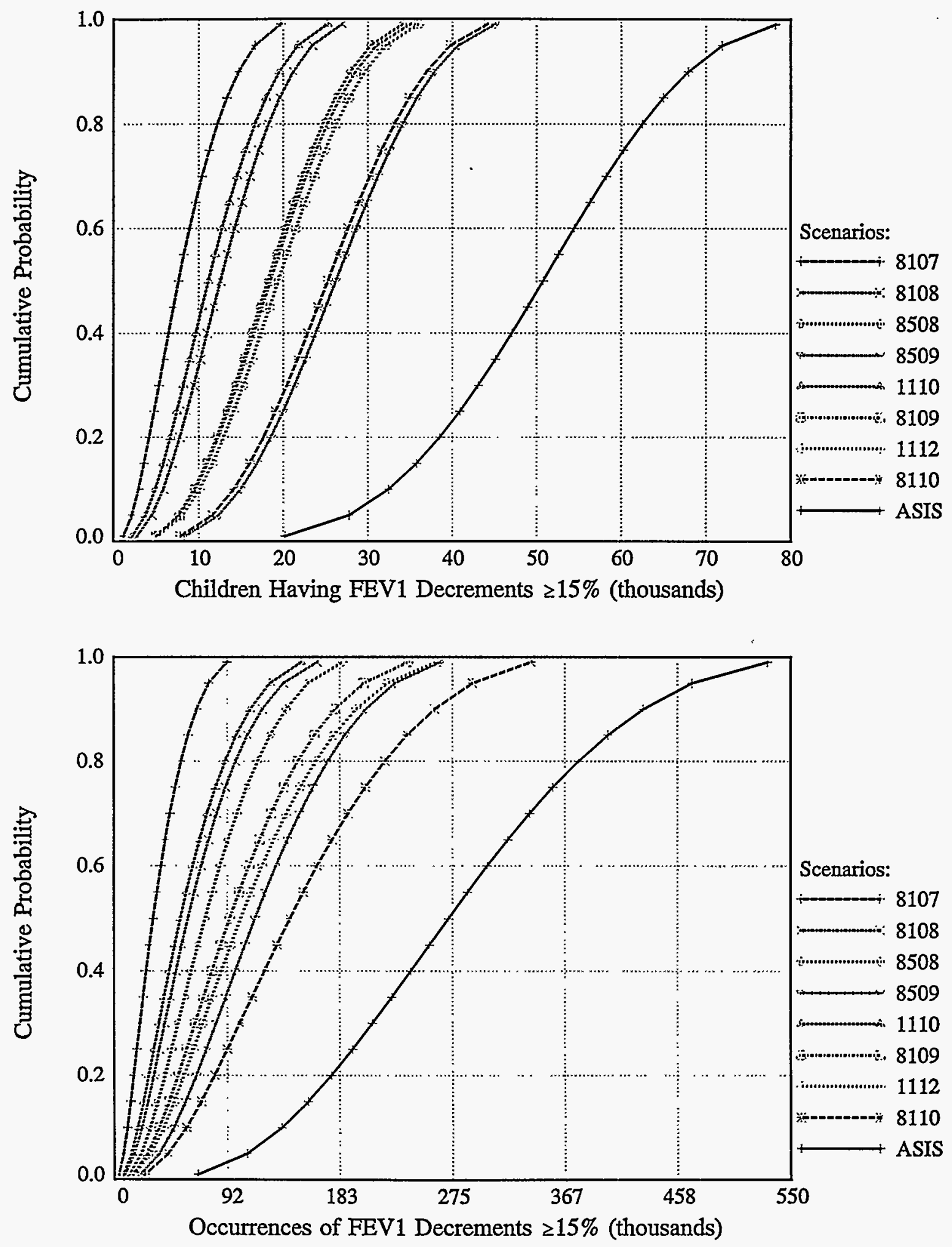

FIGURE C.3 Representative Risk Distributions for Alternative Air Quality Scenarios for FEV $_{1}$ Decrements $\geq 15 \%$, Houston, Outdoor Children, 8-h Exposures, and Moderate Exertion (underlying exposure-response relationship based on Folinsbee et al. 1988, Horstman et al. 1990, and McDonnell et al. 1991) 

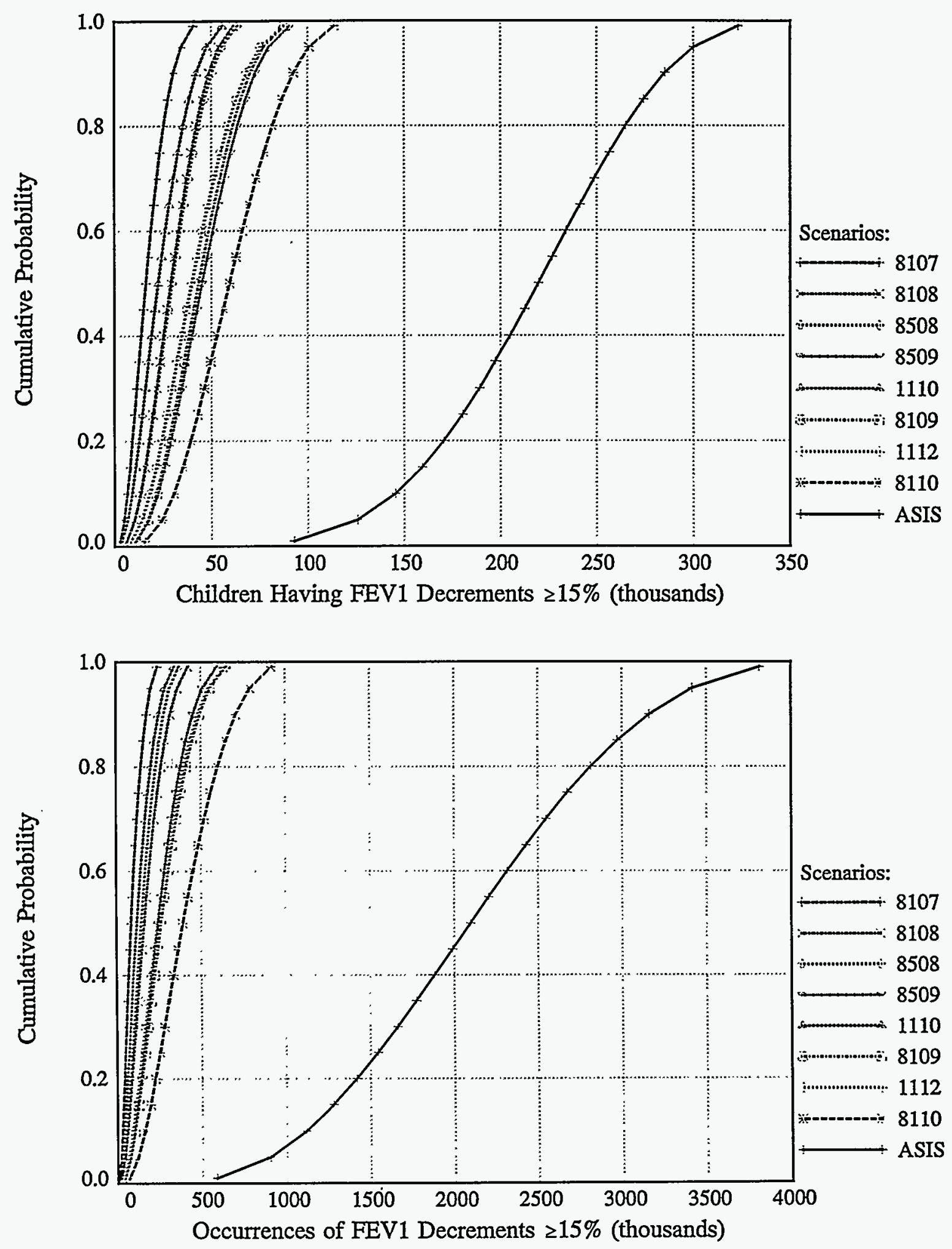

FIGURE C.4 Representative Risk Distributions for Alternative Air Quality Scenarios for FEV $_{1}$ Decrements $\geq 15 \%$, Los Angeles, Outdoor Children, 8-h Exposures, and Moderate Exertion (underlying exposure-response relationship based on Folinsbee et al. 1988, Horstman et al. 1990, and McDonnell et al. 1991) 

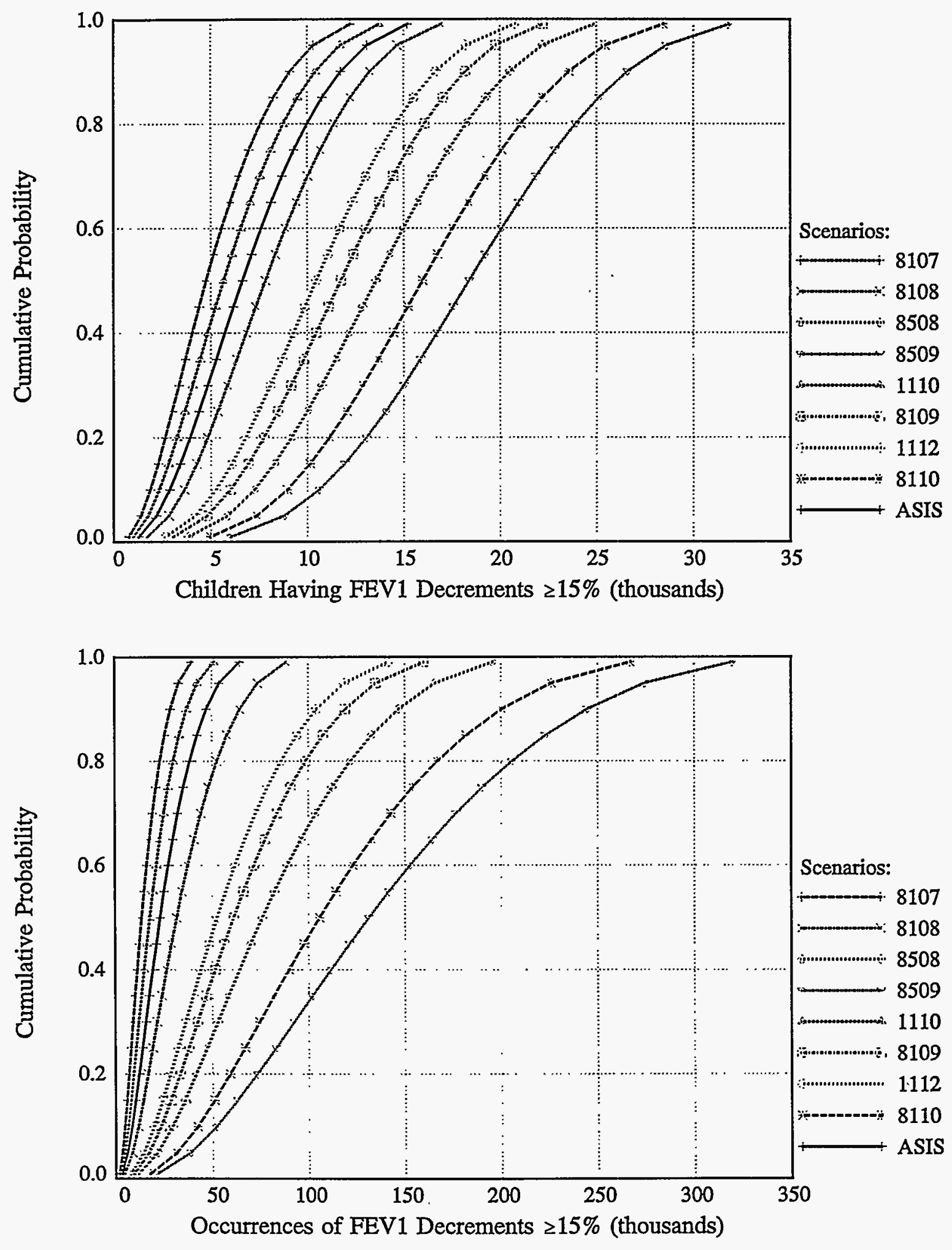

FIGURE C.5 Representative Risk Distributions for Alternative Air Quality Scenarios for FEV $_{1}$ Decrements $\geq 15 \%$, Miami, Outdoor Children, 8-h Exposures, and Moderate Exertion (underlying exposure-response relationship based on Folinsbee et al. 1988, Horstman et al. 1990, and McDonnell et al. 1991) 

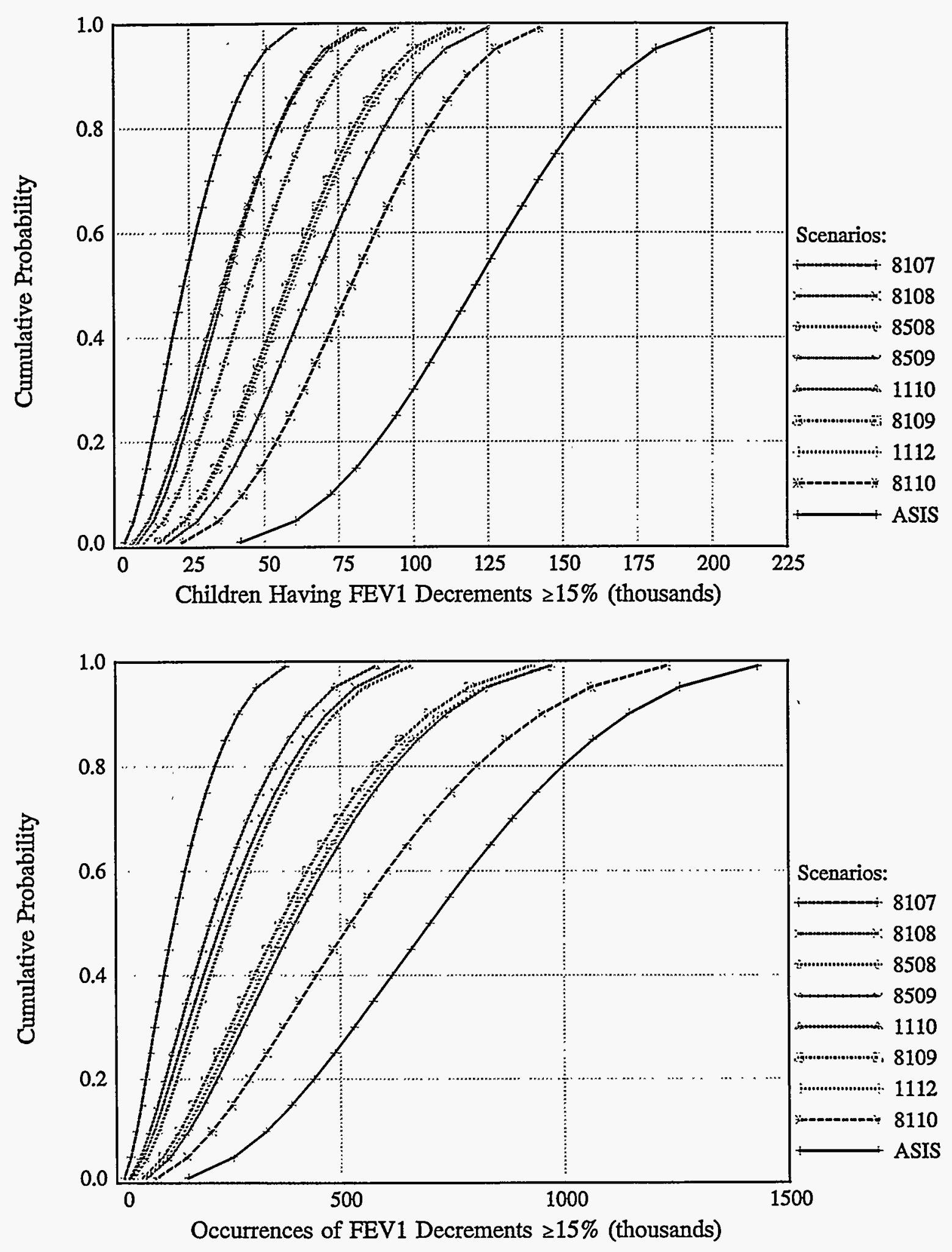

FIGURE C.6 Representative Risk Distributions for Alternative Air Quality Scenarios for FEV $_{1}$ Decrements $\geq 15 \%$, New York, Outdoor Children, 8-h Exposures, and Moderate Exertion (underlying exposure-response relationship based on Folinsbee et al. 1988, Horstman et al. 1990, and McDonnell et al. 1991) 

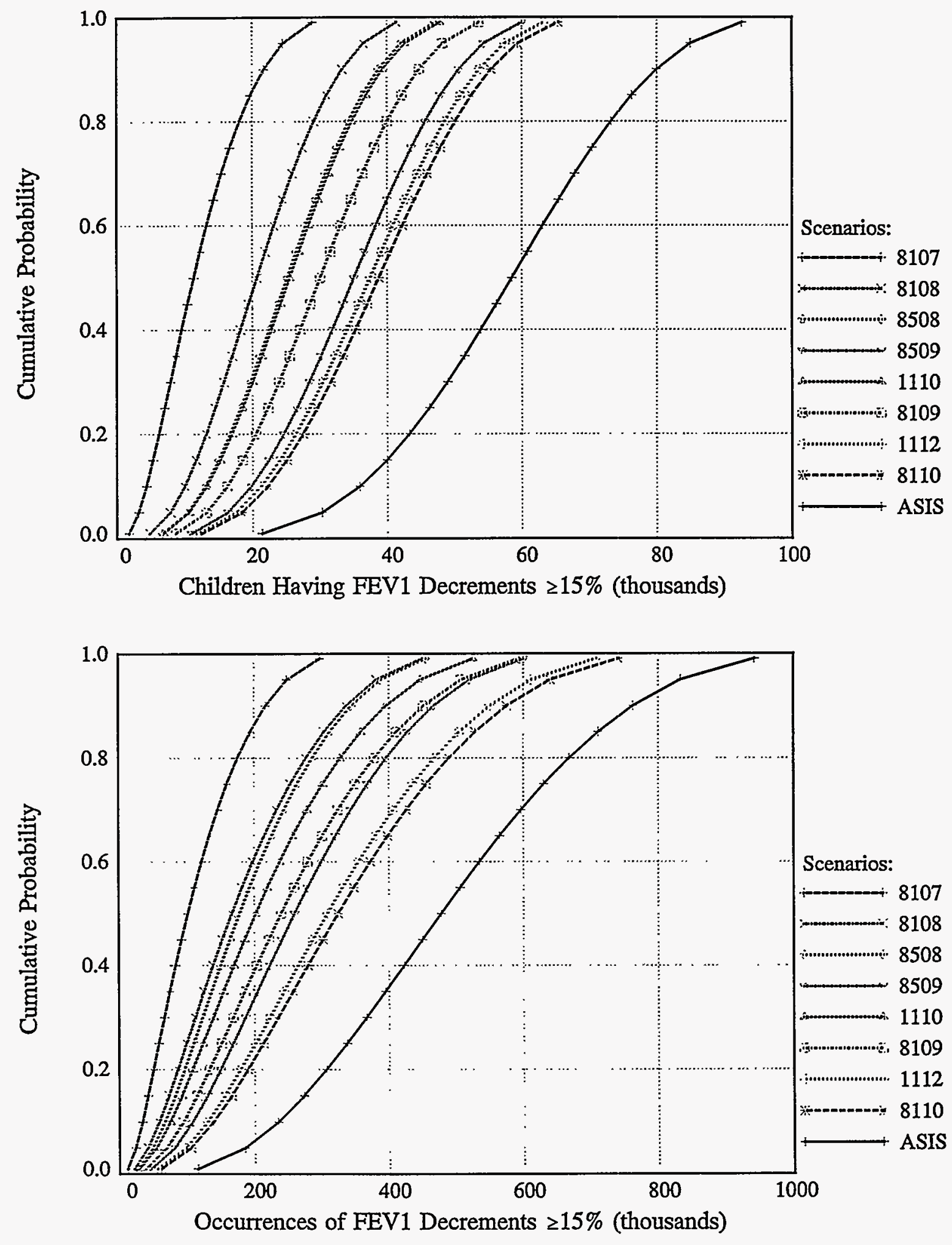

FIGURE C.7 Representative Risk Distributions for Alternative Air Quality Scenarios for FEV $_{1}$ Decrements $\geq 15 \%$, Philadelphia, Outdoor Children, 8-h Exposures, and Moderate Exertion (underlying exposure-response relationship based on Folinsbee et al. 1988, Horstman et al. 1990, and McDonnell et al. 1991) 

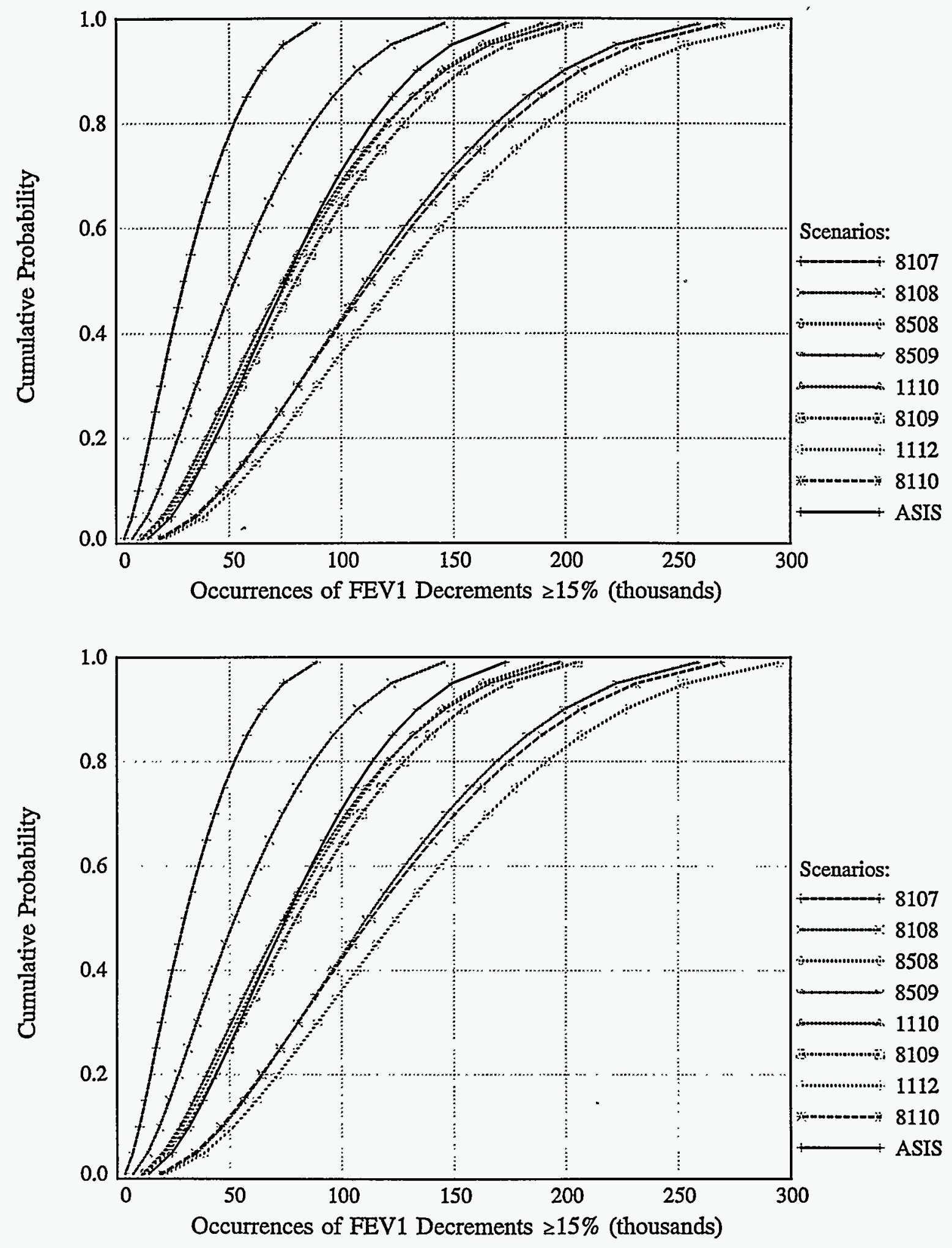

FIGURE C.8 Representative Risk Distributions for Alternative Air Quality Scenarios for $\mathrm{FEV}_{1}$ Decrements $\geq 15 \%$, St. Louis, Outdoor Children, 8-h Exposures, and Moderate Exertion (underlying exposure-response relationship based on Folinsbee et al. 1988, Horstman et al. 1990, and McDonnell et al. 1991) 

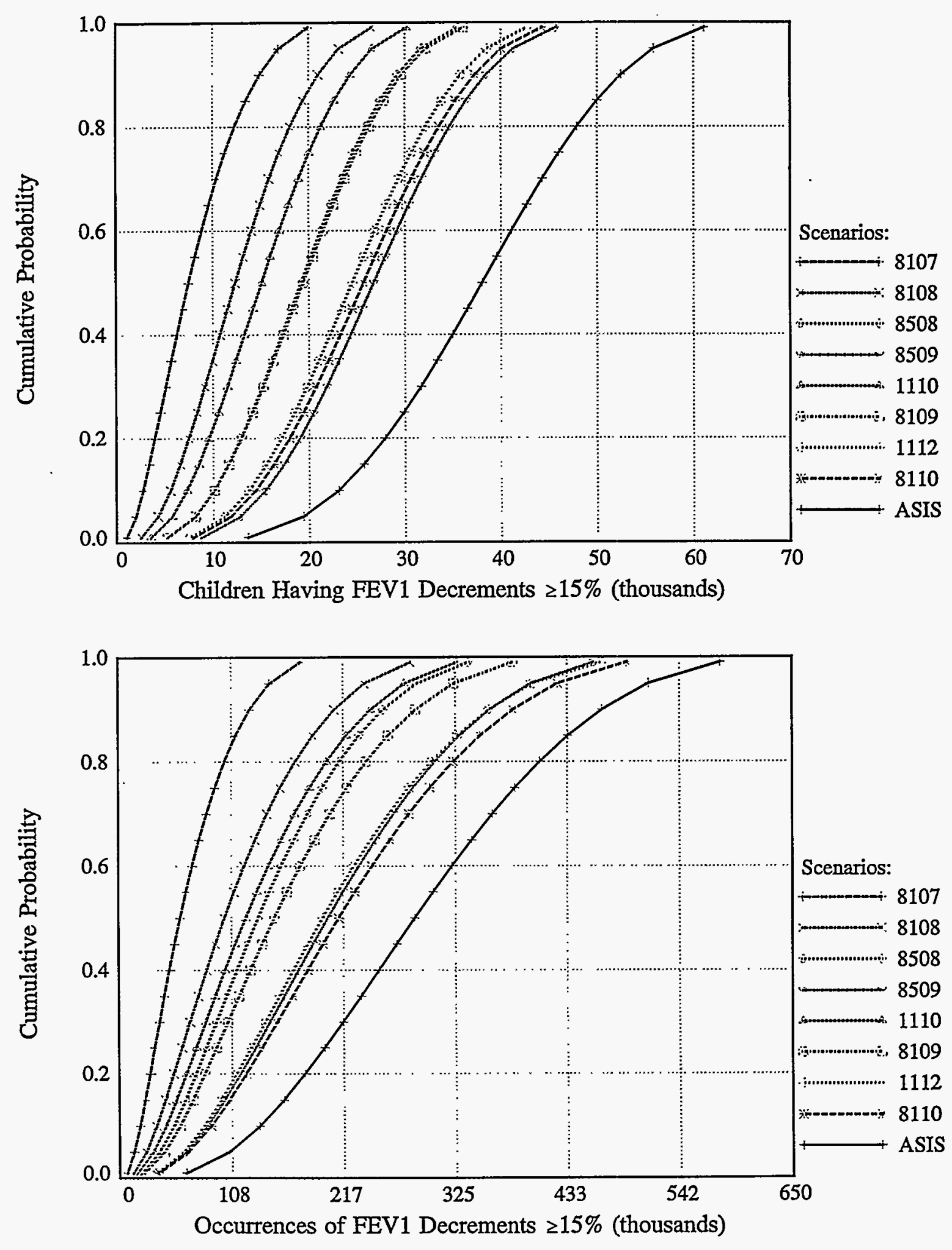

FIGURE C.9 Representative Risk Distributions for Alternative Air Quality Scenarios for FEV 1 Decrements $\geq 15 \%$, Washington DC, Outdoor Children, 8-h Exposures, and Moderate Exertion (underlying exposure-response relationship based on Folinsbee et al. 1988, Horstman et al. 1990, and McDonnell et al. 1991) 


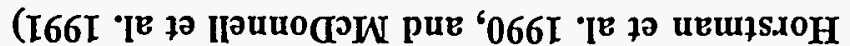

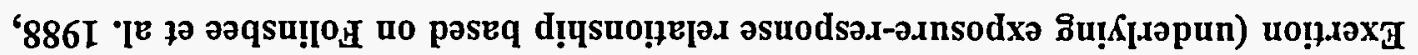

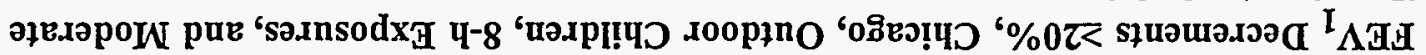

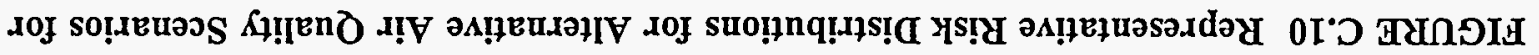

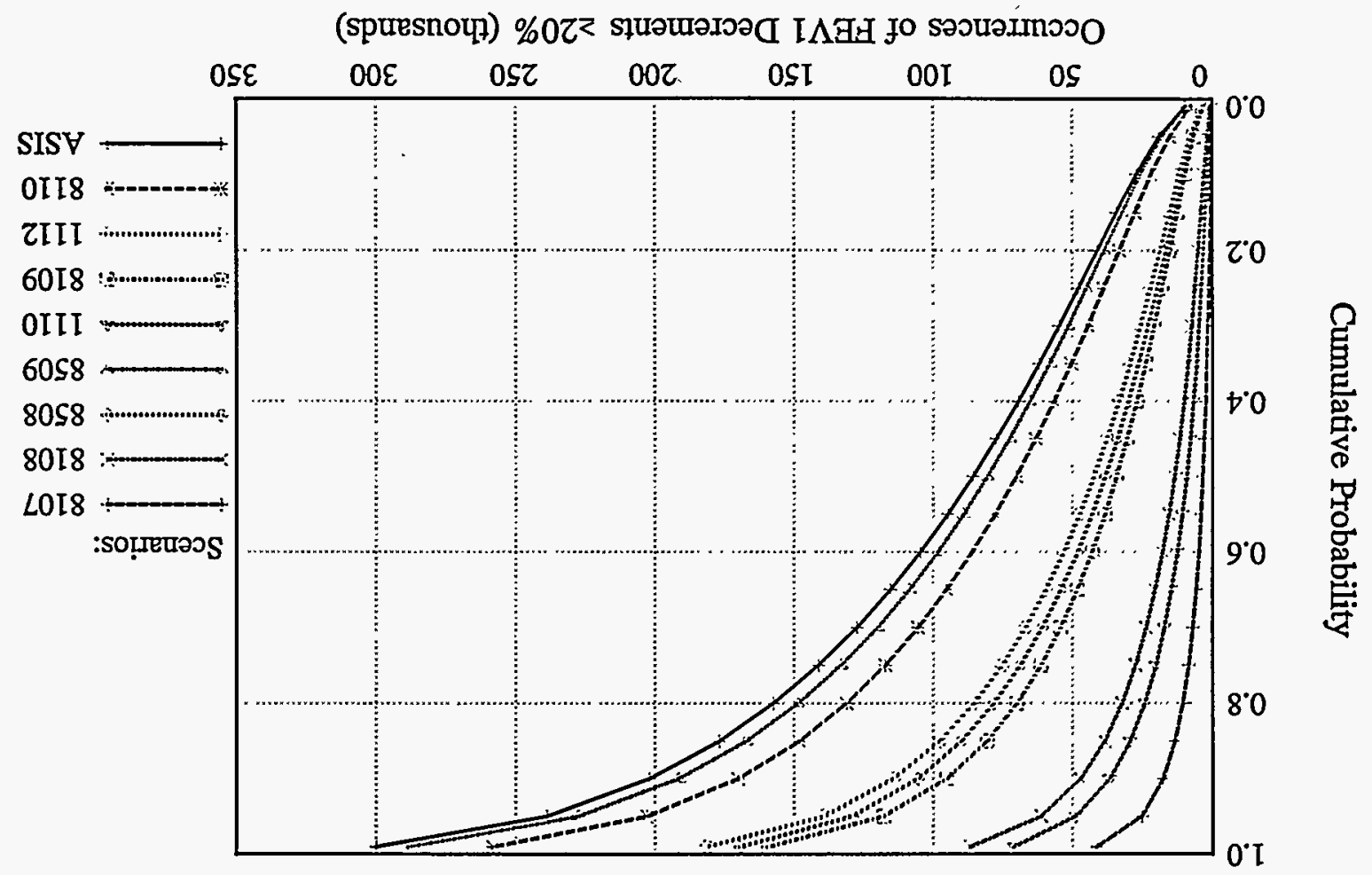

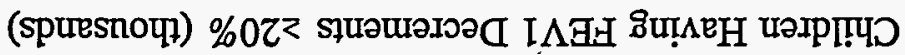

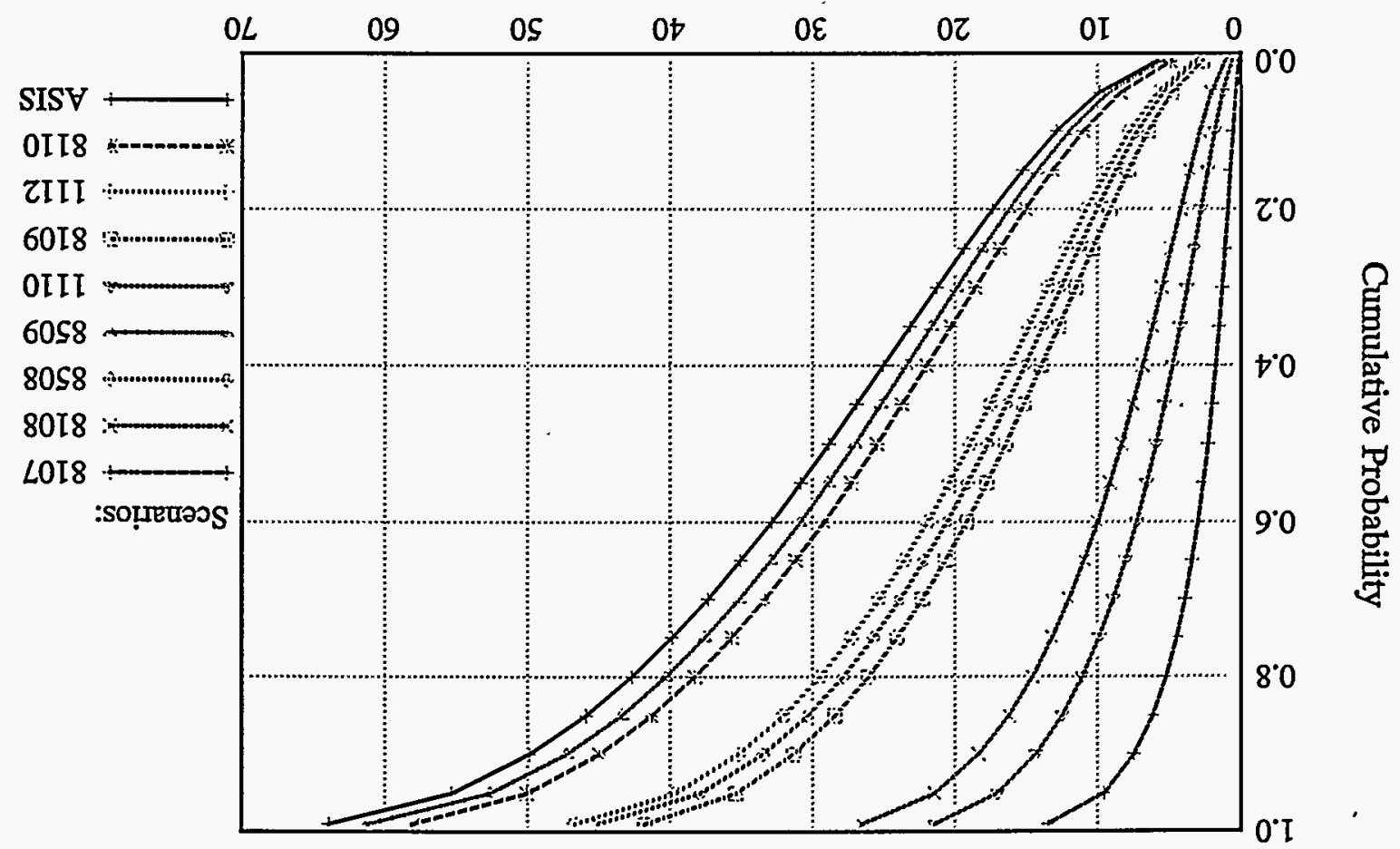



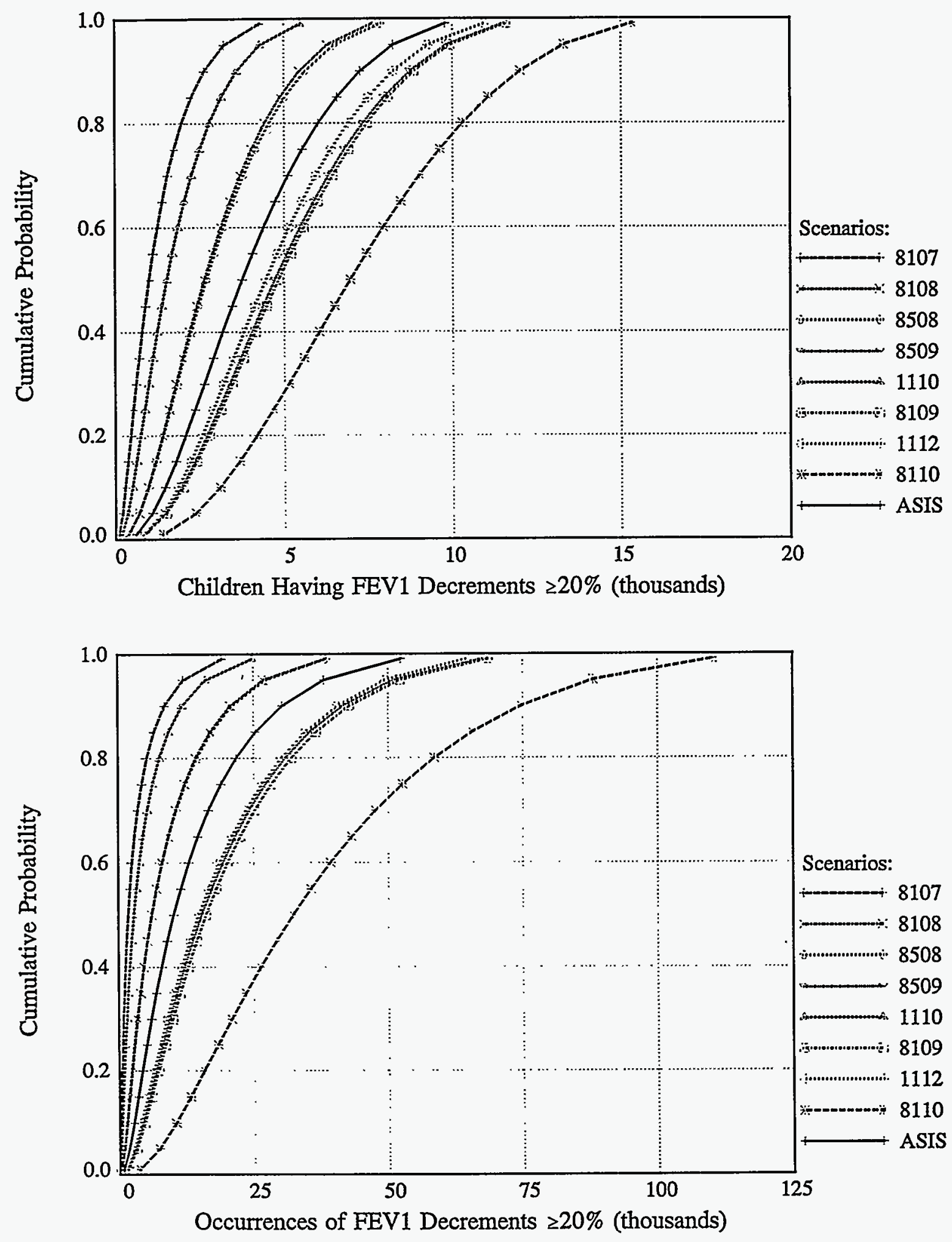

FIGURE C.11 Representative Risk Distributions for Alternative Air Quality Scenarios for FEV 1 Decrements $\geq 20 \%$, Denver, Outdoor Children, 8-h Exposures, and Moderate Exertion (underlying exposure-response relationship based on Folinsbee et al. 1988, Horstman et al. 1990, and McDonnell et al. 1991) 

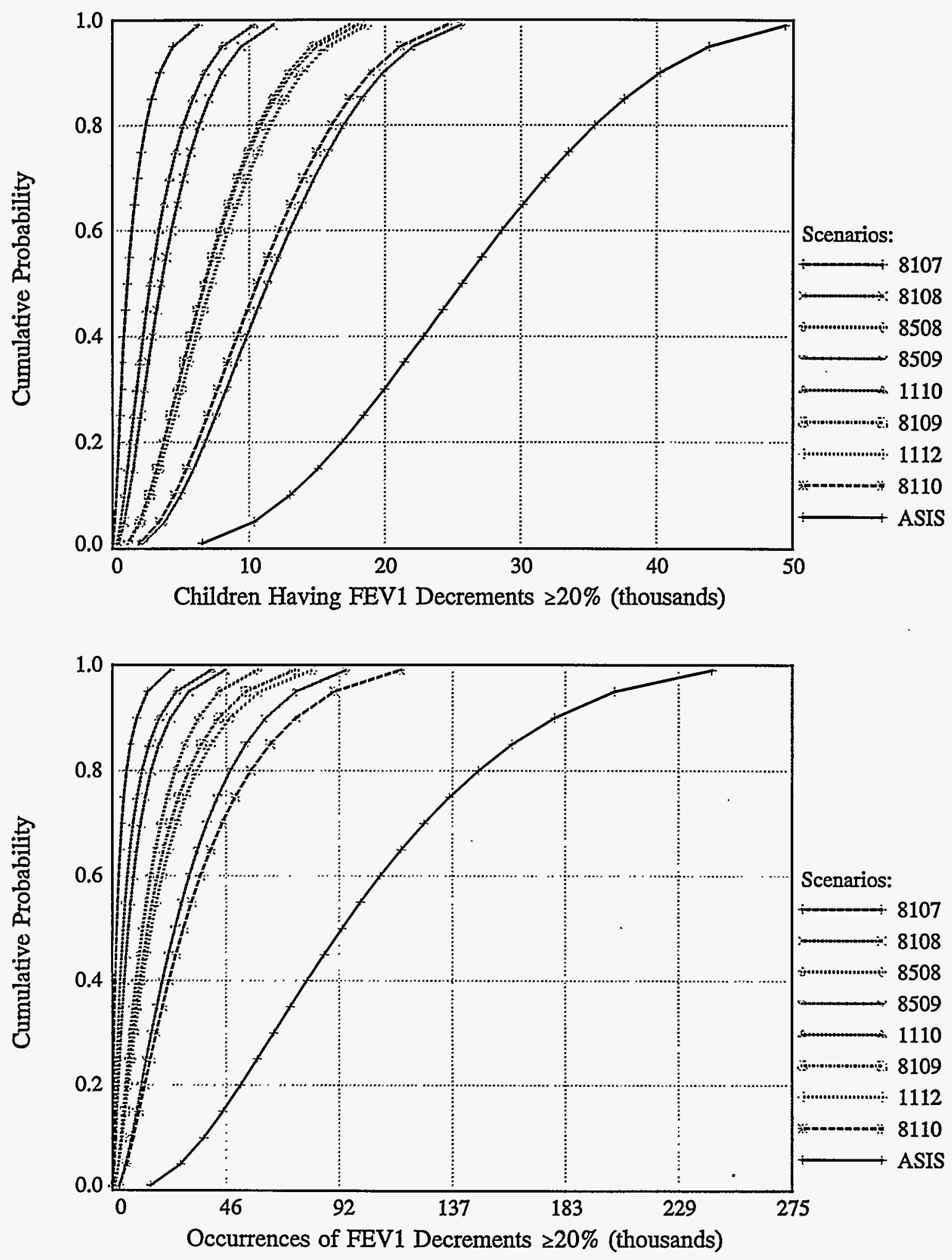

FIGURE C.12 Representative Risk Distributions for Alternative Air,Quality Scenarios for FEV $_{1}$ Decrements $\geq 20 \%$, Houston, Outdoor Children, 8-h Exposures, and Moderate Exertion (underlying exposure-response relationship based on Folinsbee et al. 1988, Horstman et al. 1990, and McDonnell et al. 1991) 

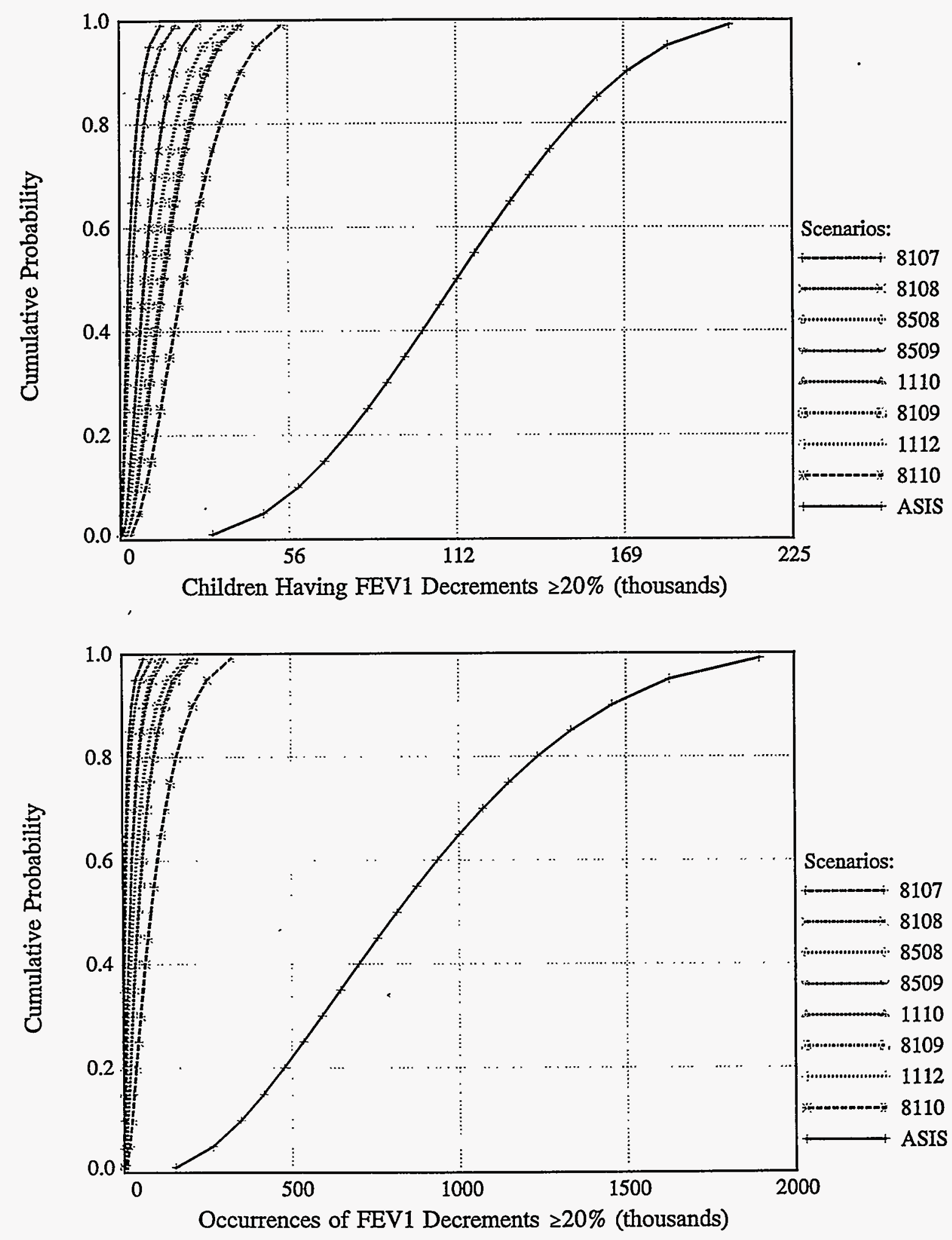

FIGURE C.13 Representative Risk Distributions for Alternative Air Quality Scenarios for FEV $_{1}$ Decrements $\geq \mathbf{2 0} \%$, Los Angeles, Outdoor Children, 8-h Exposures, and Moderate . Exertion (underlying exposure-response relationship based on Folinsbee et al. 1988, Horstman et al. 1990, and McDonnell et al. 1991) 

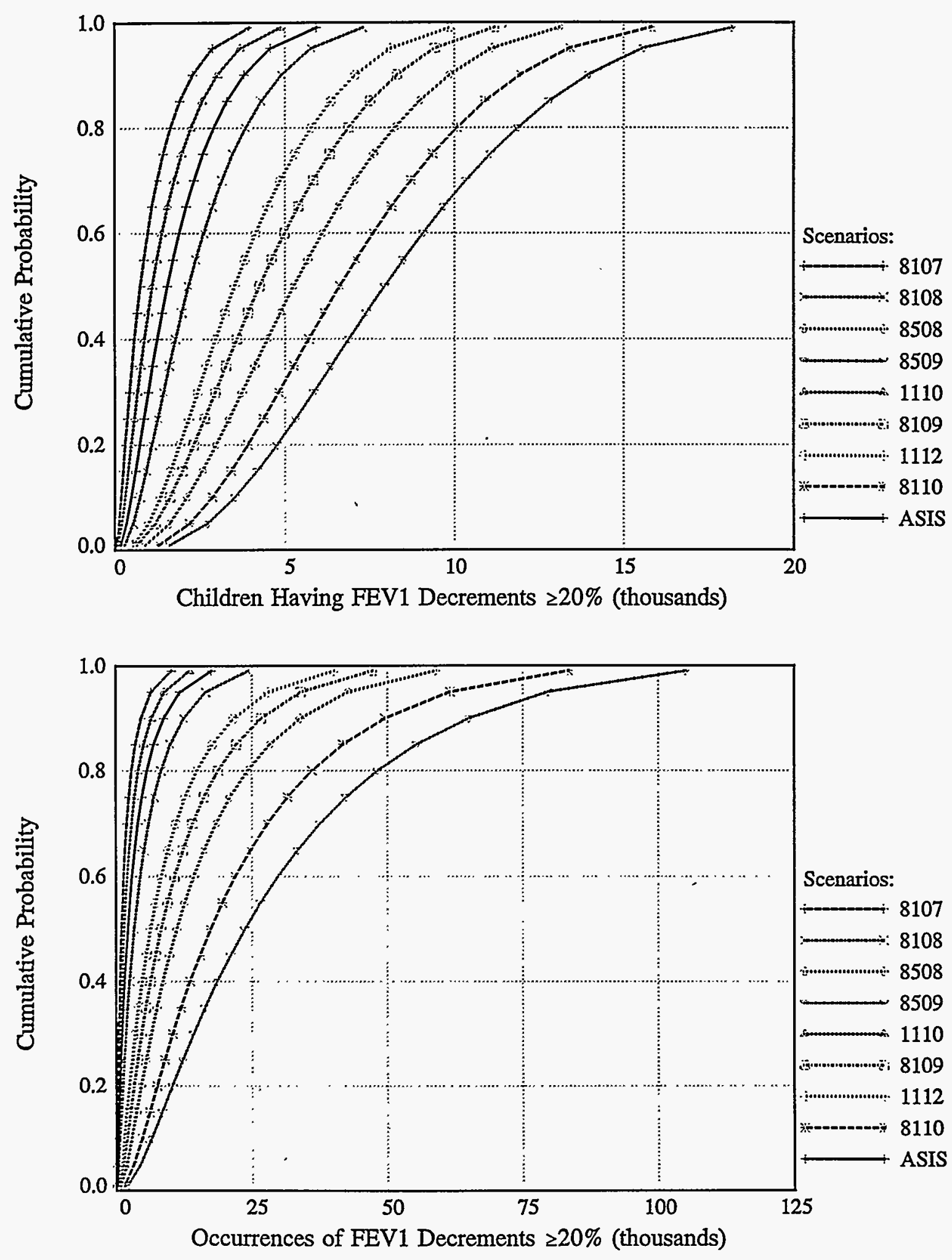

FIGURE C.14 Representative Risk Distributions for Alternative Air Quality Scenarios for FEV $_{1}$ Decrements $\geq 20 \%$, Miami, Outdoor Children, 8-h Exposures, and Moderate Exertion (underlying exposure-response relationship based on Folinsbee et al. 1988, Horstman et al. 1990, and McDonnell et al. 1991) 

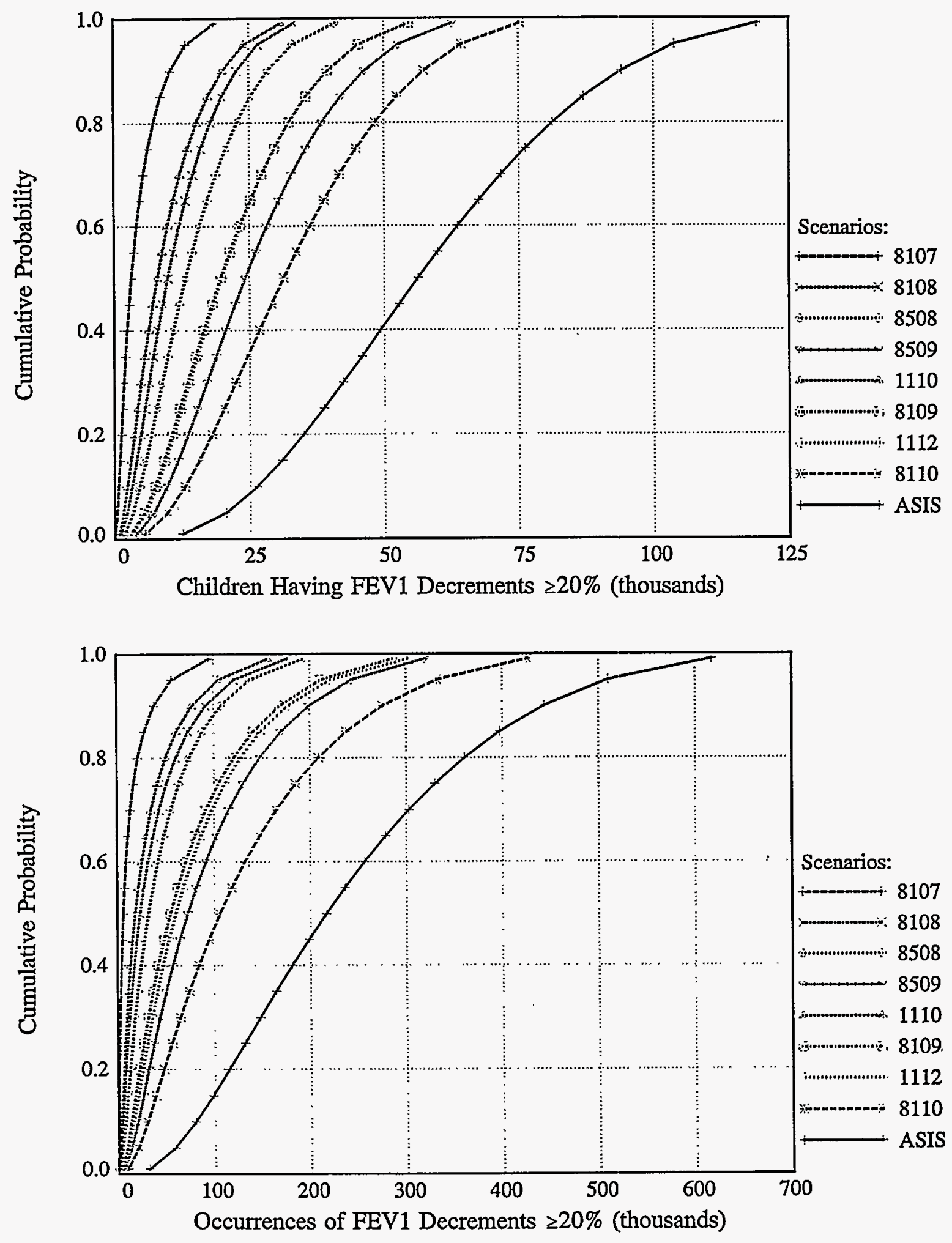

FIGURE C.15 Representative Risk Distributions for Alternative Air Quality Scenarios for FEV $_{1}$ Decrements $\geq 20 \%$, New York, Outdoor Children, 8-h Exposures, and Moderate Exertion (underlying exposure-response relationship based on Folinsbee et al. 1988, Horstman et al. 1990, and McDonnell et al. 1991) 

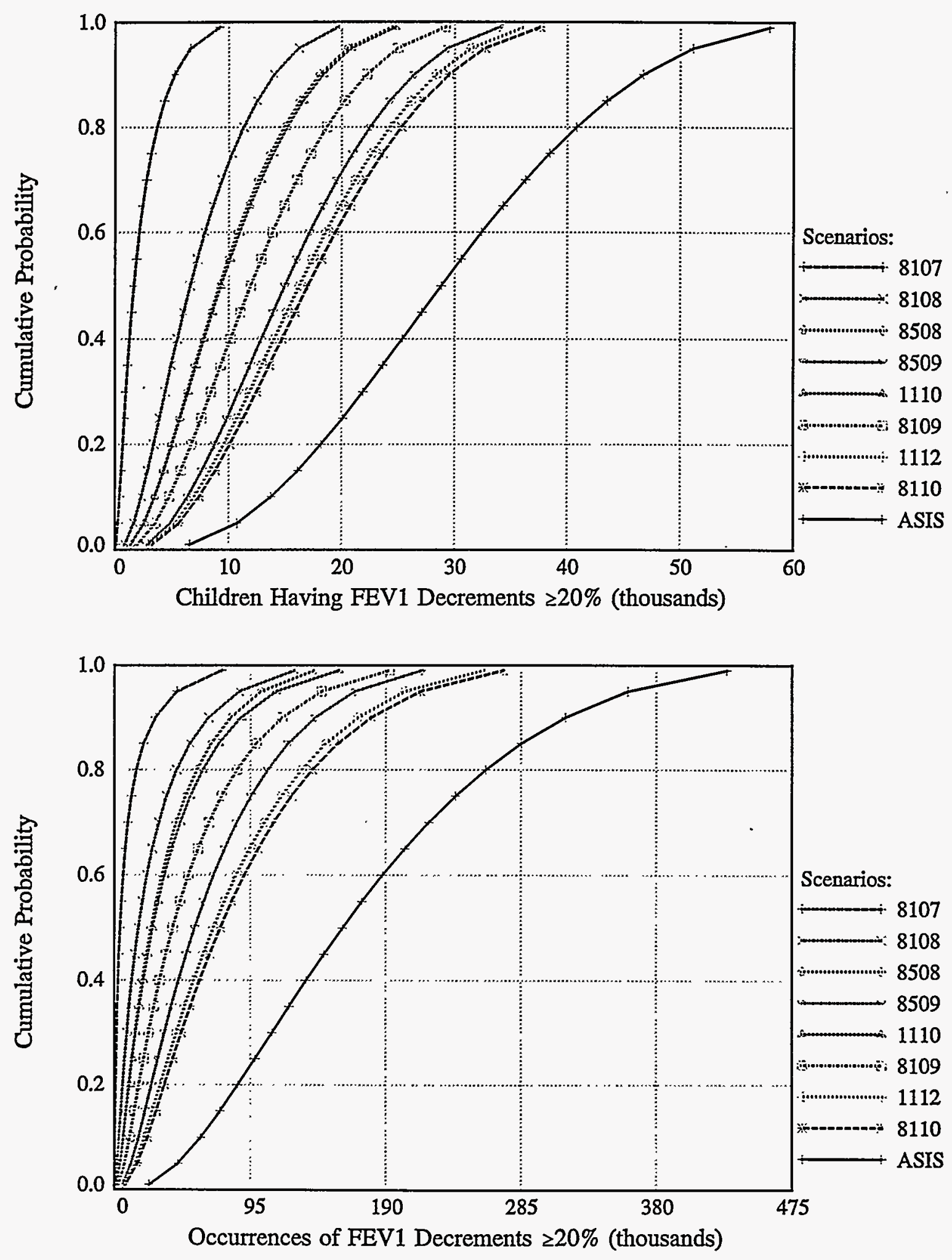

FIGURE C.16 Representative Risk Distributions for Alternative Air Quality Scenarios for FEV $_{1}$ Decrements $\geq 20 \%$, Philadelphia, Outdoor Children, 8-h Exposures, and Moderate Exertion (underlying exposure-response relationship based on Folinsbee et al. 1988, Horstman et al. 1990, and McDonnell et al. 1991) 

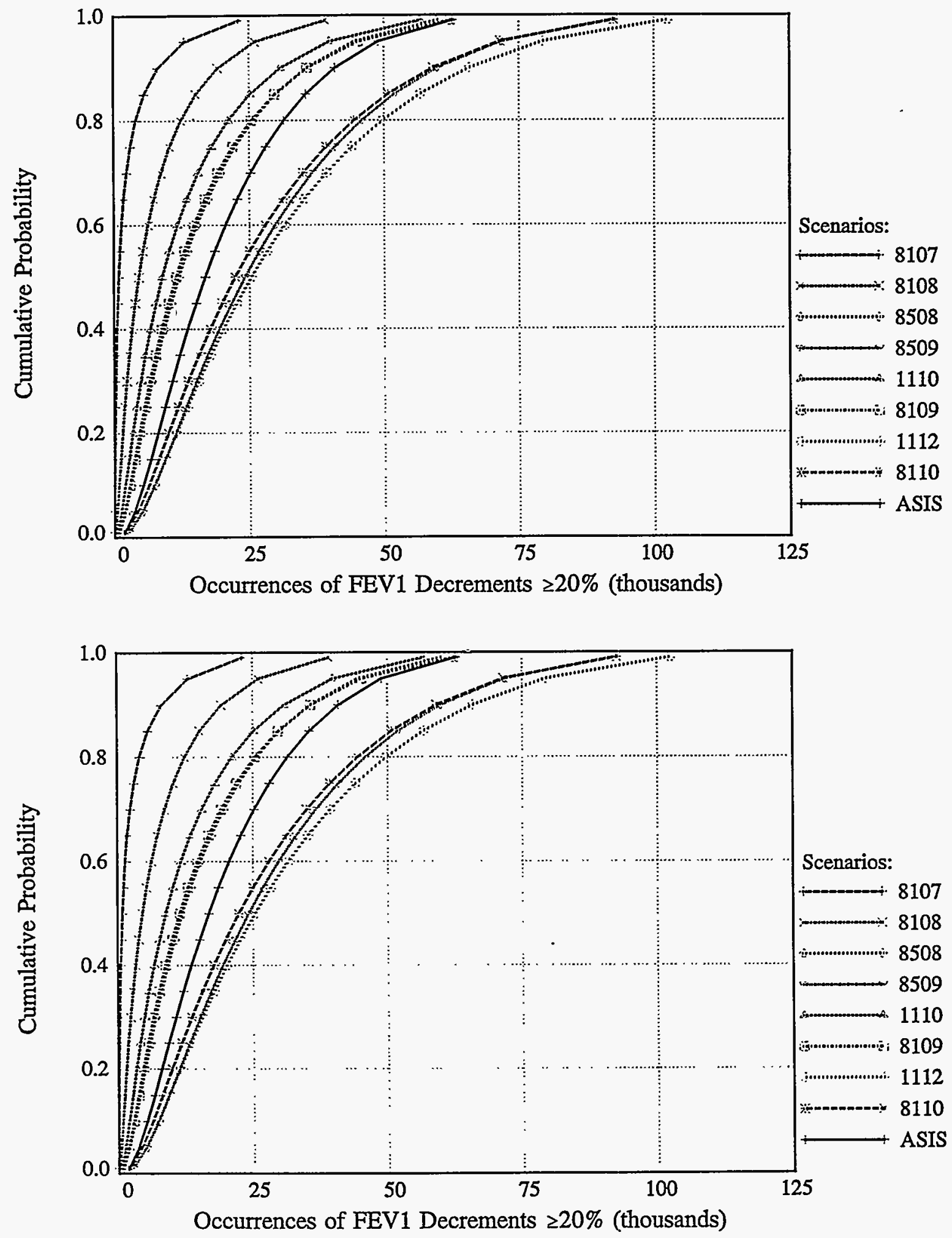

FIGURE C.17 Representative Risk Distributions for Alternative Air Quality Scenarios for FEV $_{1}$ Decrements $\geq 20 \%$, St. Louis, Outdoor Children, 8-h Exposures, and Moderate Exertion (underlying exposure-response relationship based on Folinsbee et al. 1988, Horstman et al. 1990, and McDonnell et al. 1991) 

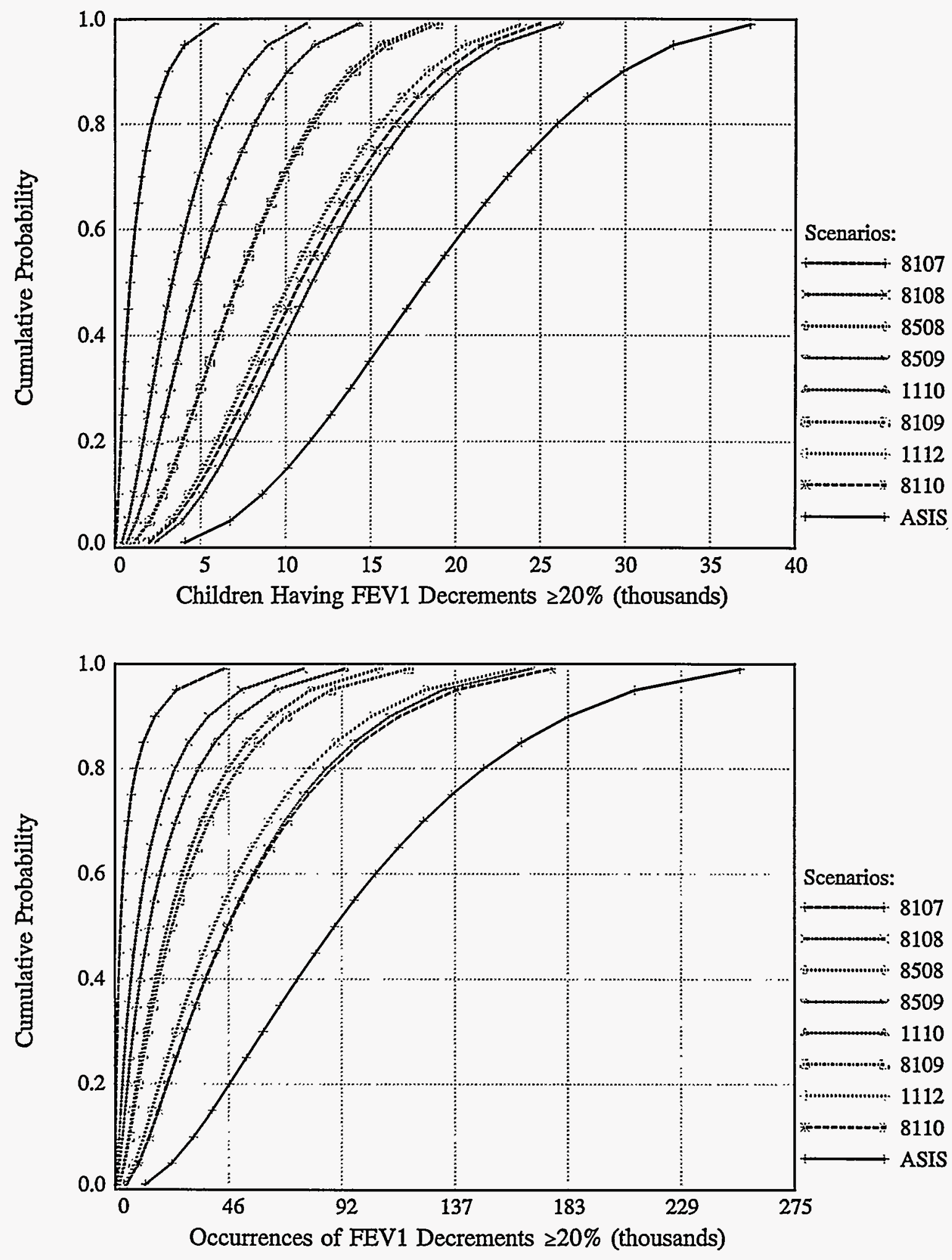

FIGURE C.18 Representative Risk Distributions for Alternative Air Quality Scenarios for FEV $_{1}$ Decrements $\geq 20 \%$, Washington DC, Outdoor Children, 8-h Exposures, and Moderate Exertion (underlying exposure-response relationship based on Folinsbee et al. 1988, Horstman et al. 1990, and McDonnell et al. 1991) 

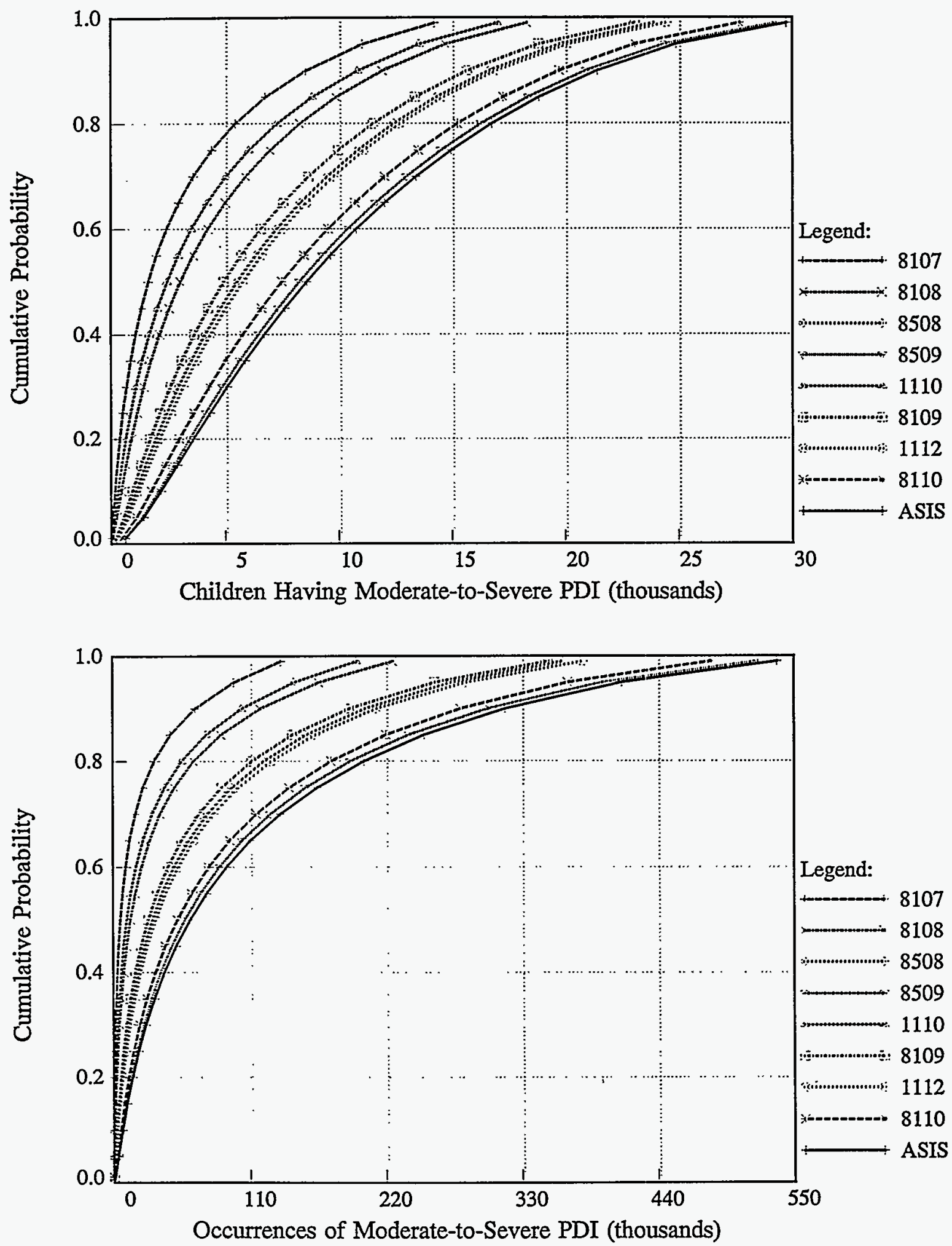

FIGURE C.19 Representative Risk Distributions for Alternative Air Quality Scenarios for Moderate-to-Severe Pain on Deep Inspiration, Chicago, Outdoor Children, 1-h Exposures, and Moderate Exertion (underlying exposure-response relationship based on Seal et al. 1993) 

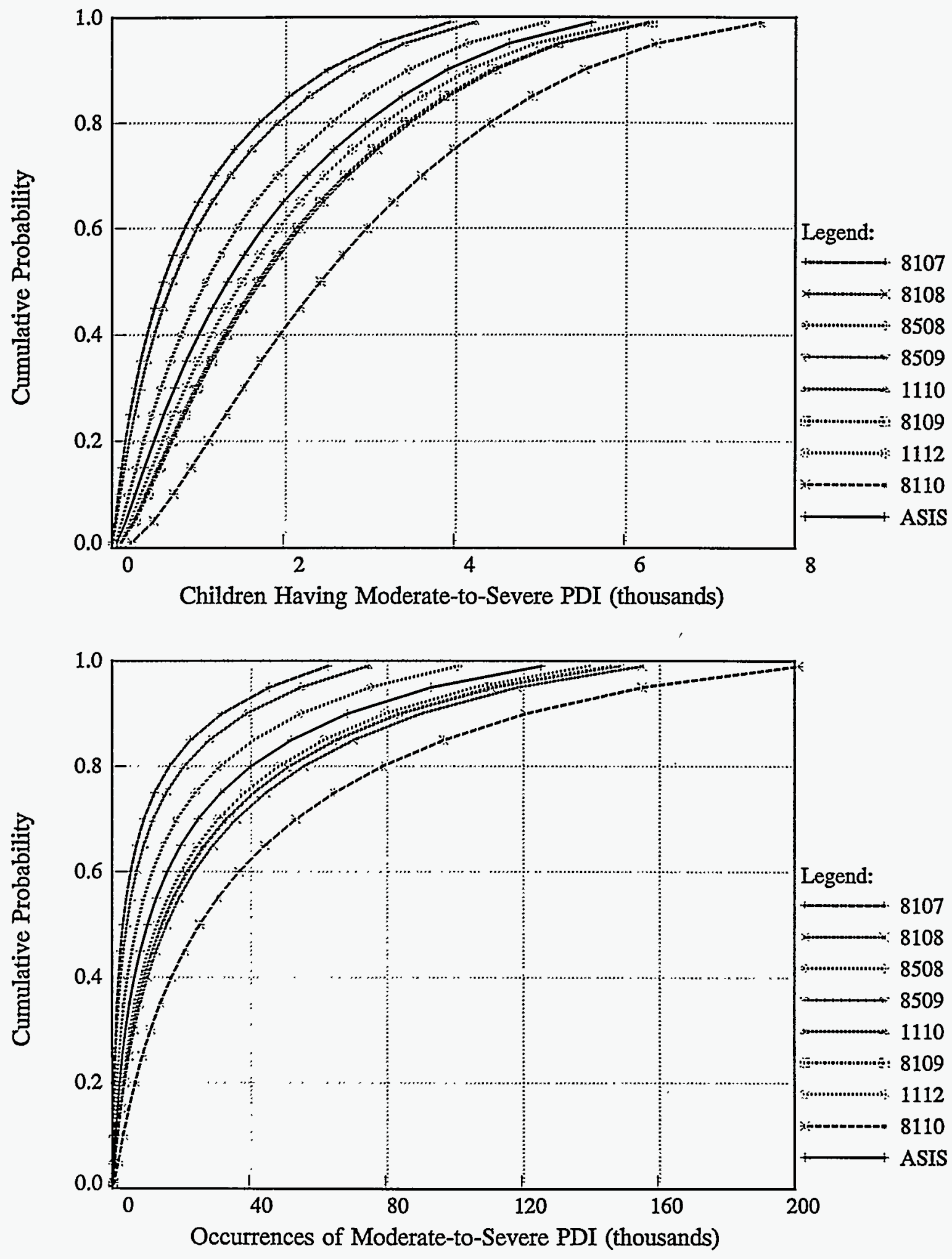

FIGURE C.20 Representative Risk Distributions for Alternative Air Quality Scenarios for Moderate-to-Severe Pain on Deep Inspiration, Denver, Outdoor Children, 1-h Exposures, and Moderate Exertion (underlying exposure-response relationship based on Seal et al. 1993) 

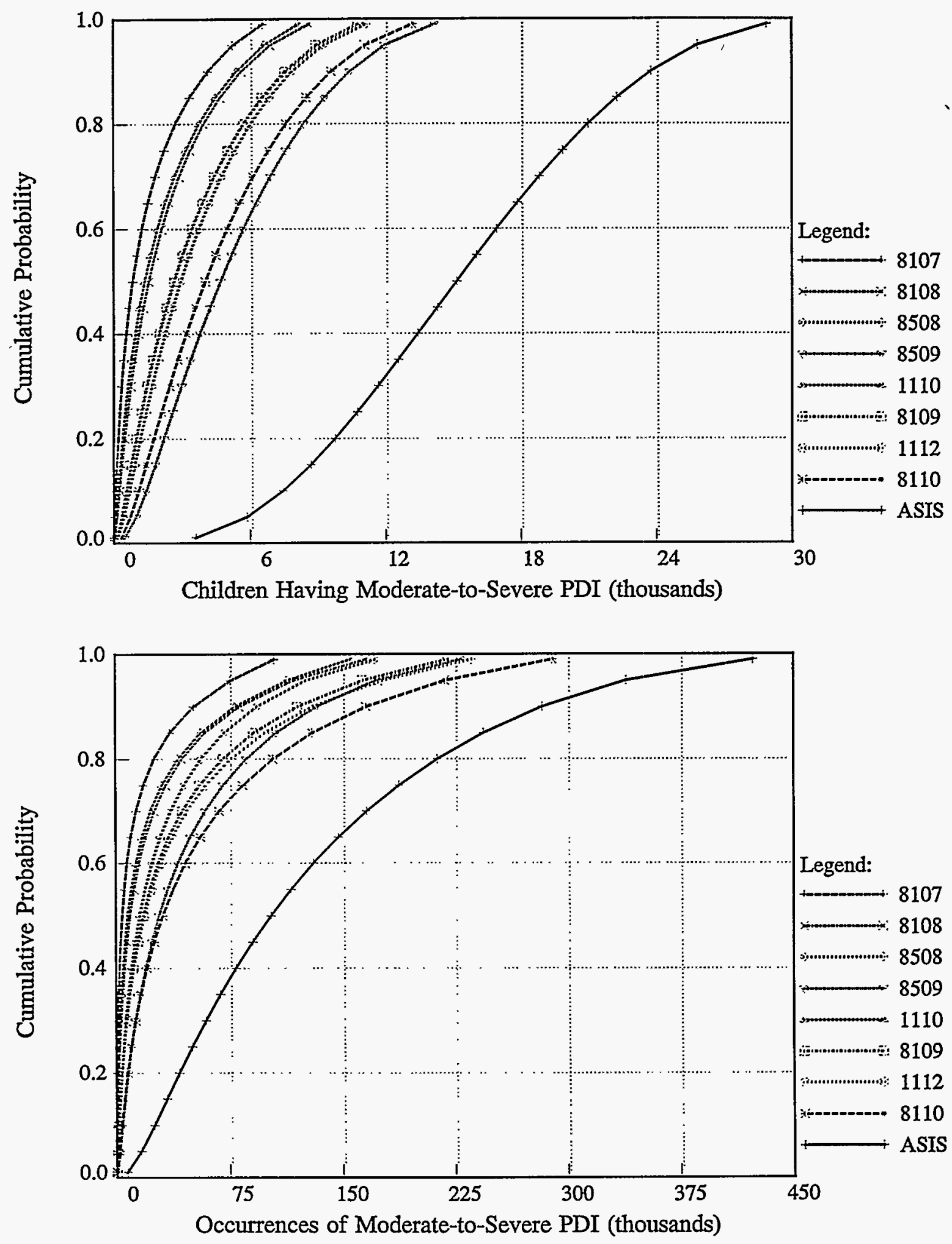

FIGURE C.21 Representative Risk Distributions for Alternative Air Quality Scenarios for Moderate-to-Severe Pain on Deep Inspiration, Houston, Outdoor Children, 1-h Exposures, and Moderate Exertion (underlying exposure-response relationship based on Seal et al. 1993) 

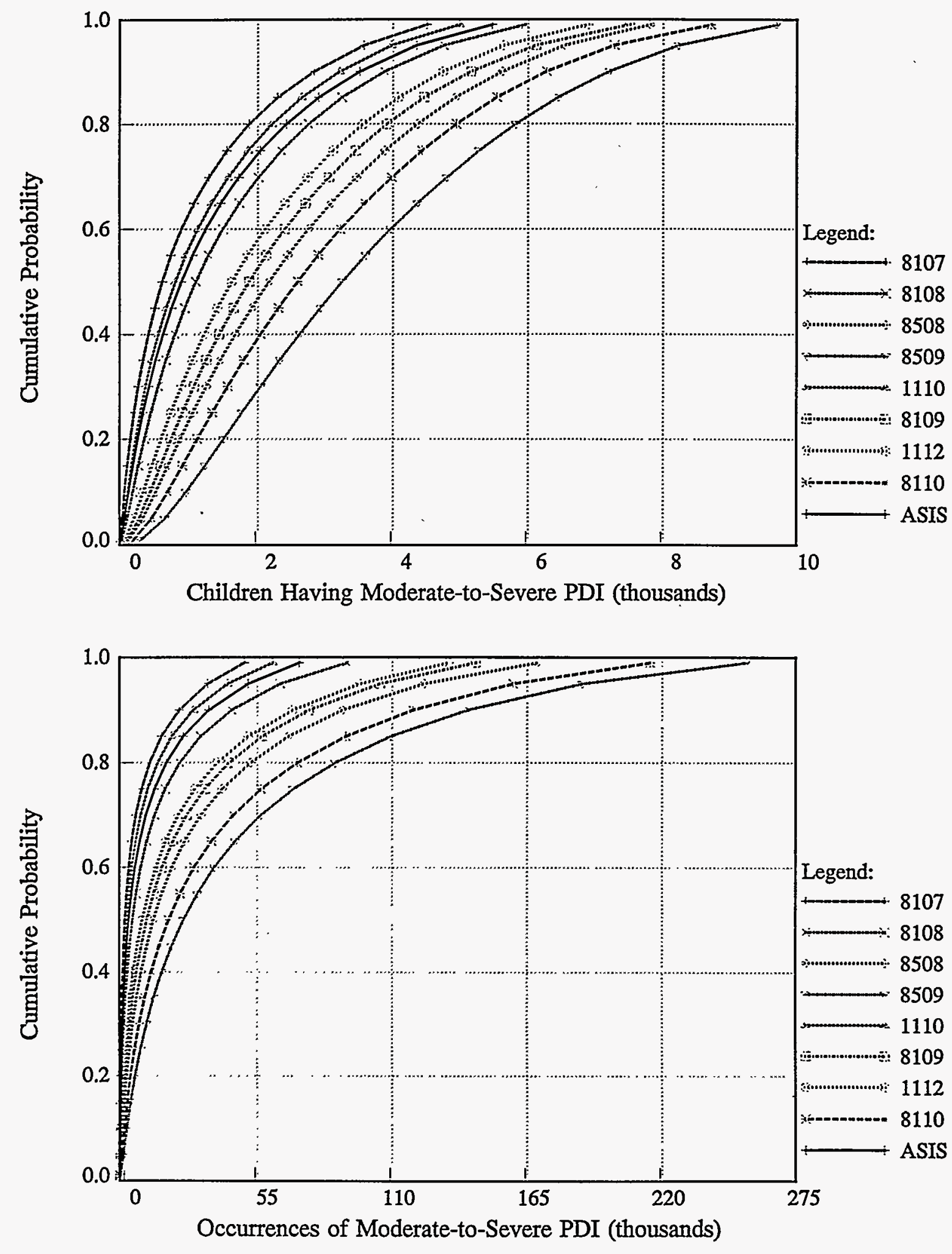

FIGURE C.22 Representative Risk Distributions for Alternative Air Quality Scenarios for Moderate-to-Severe Pain on Deep Inspiration, Los Angeles, Outdoor Children, 1-h Exposures, and Moderate Exertion (underlying exposure-response relationship based on Seal et al. 1993) 

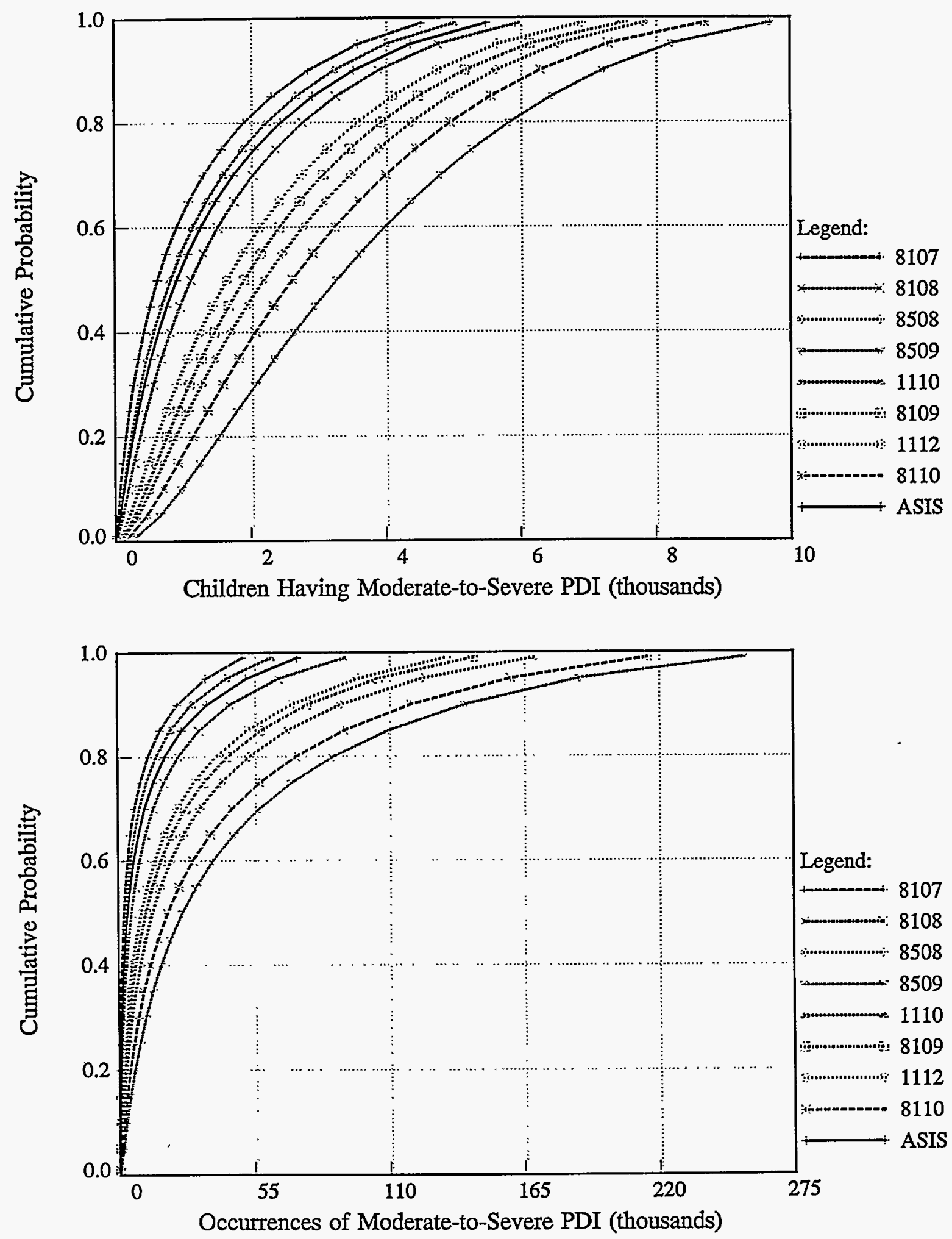

FIGURE C.23 Representative Risk Distributions for Alternative Air Quality Scenarios for Moderate-to-Severe Pain on Deep Inspiration, Miami, Outdoor Children, 1-h Exposures, and Moderate Exertion (underlying exposure-response relationship based on Seal et al. 1993) 

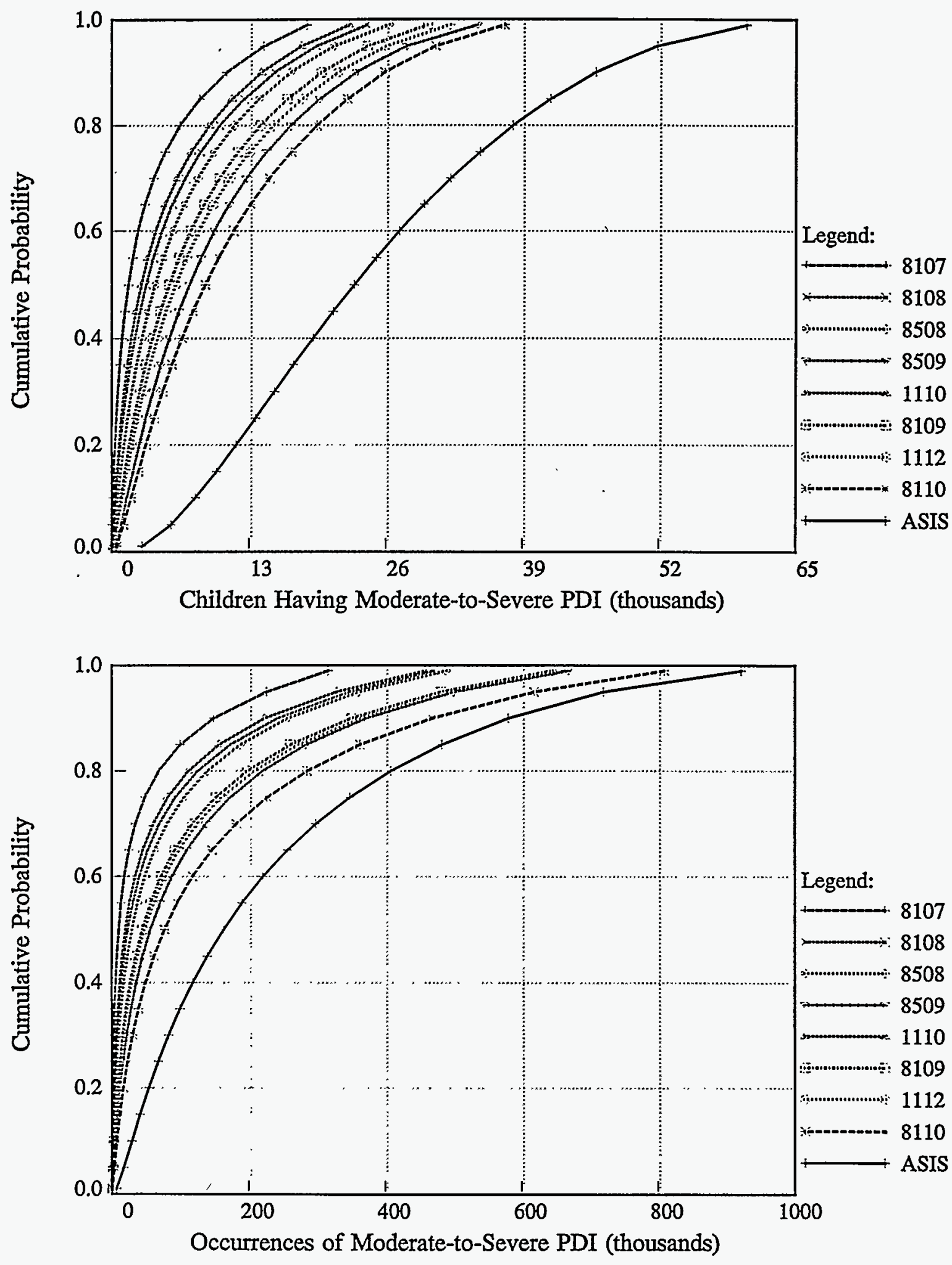

FIGURE C.24 Representative Risk Distributions for Alternative Air Quality Scenarios for Moderate-to-Severe Pain on Deep Inspiration, New York, Outdoor Children, 1-h Exposures, and Moderate Exertion (underlying exposure-response relationship based on Seal et al. 1993) 
(E66I 'je pa jeəs uo paseq

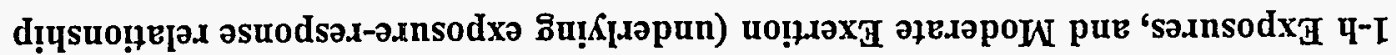

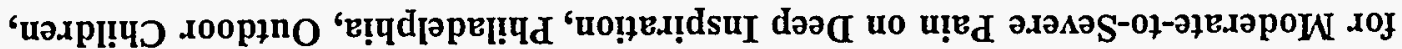

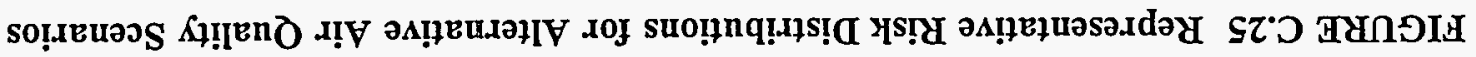

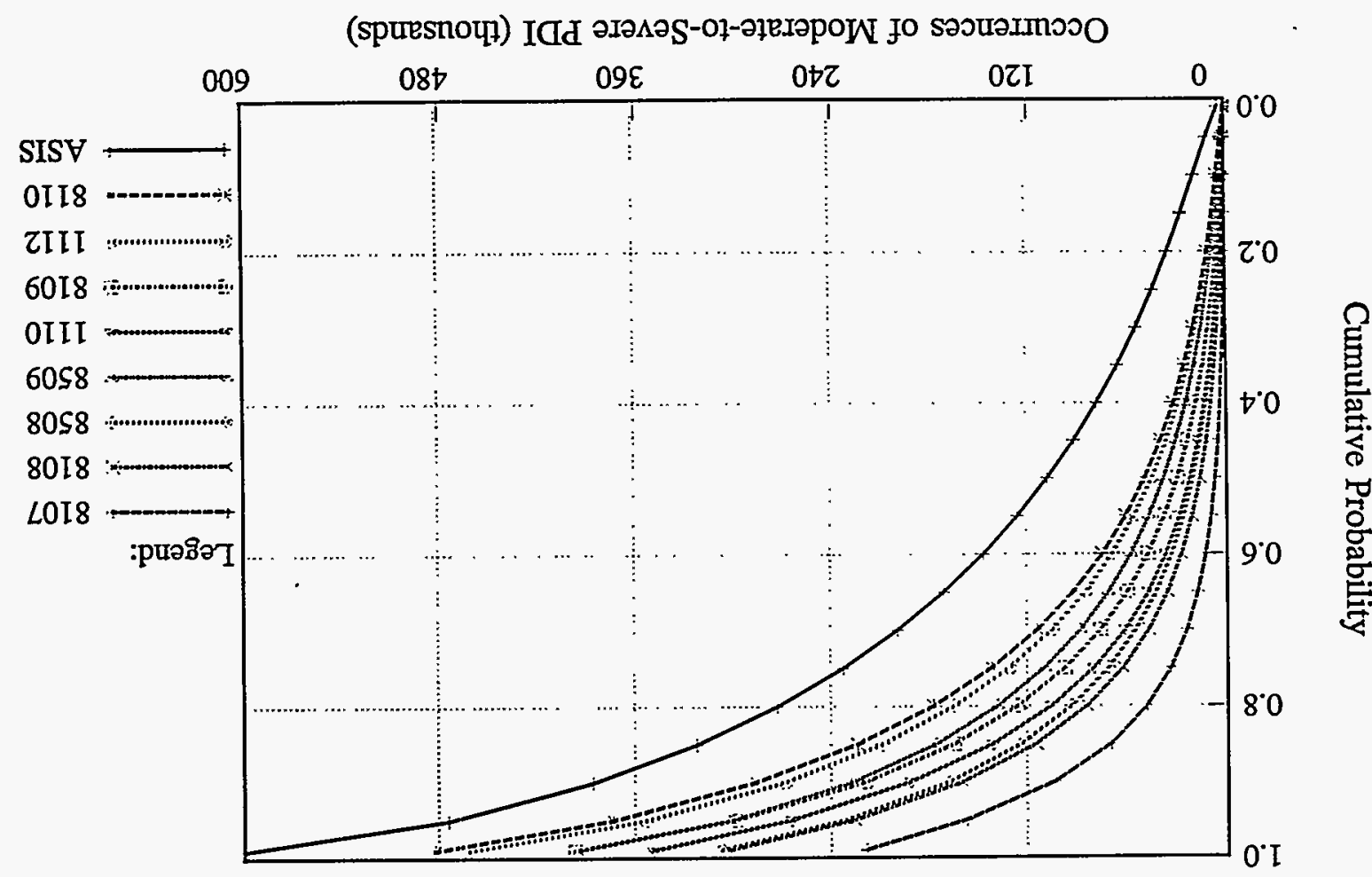

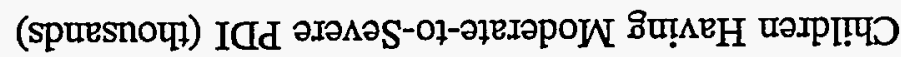

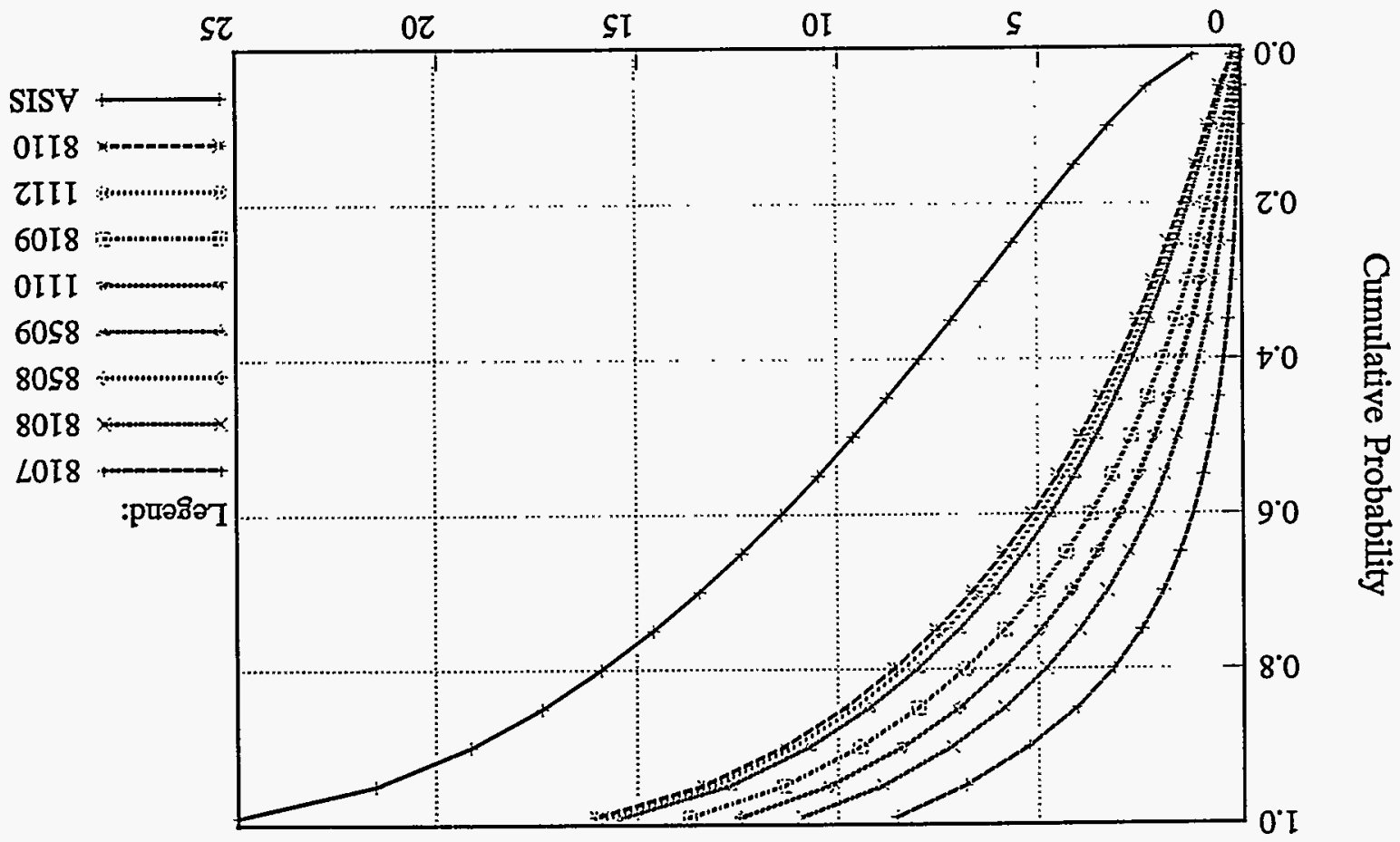



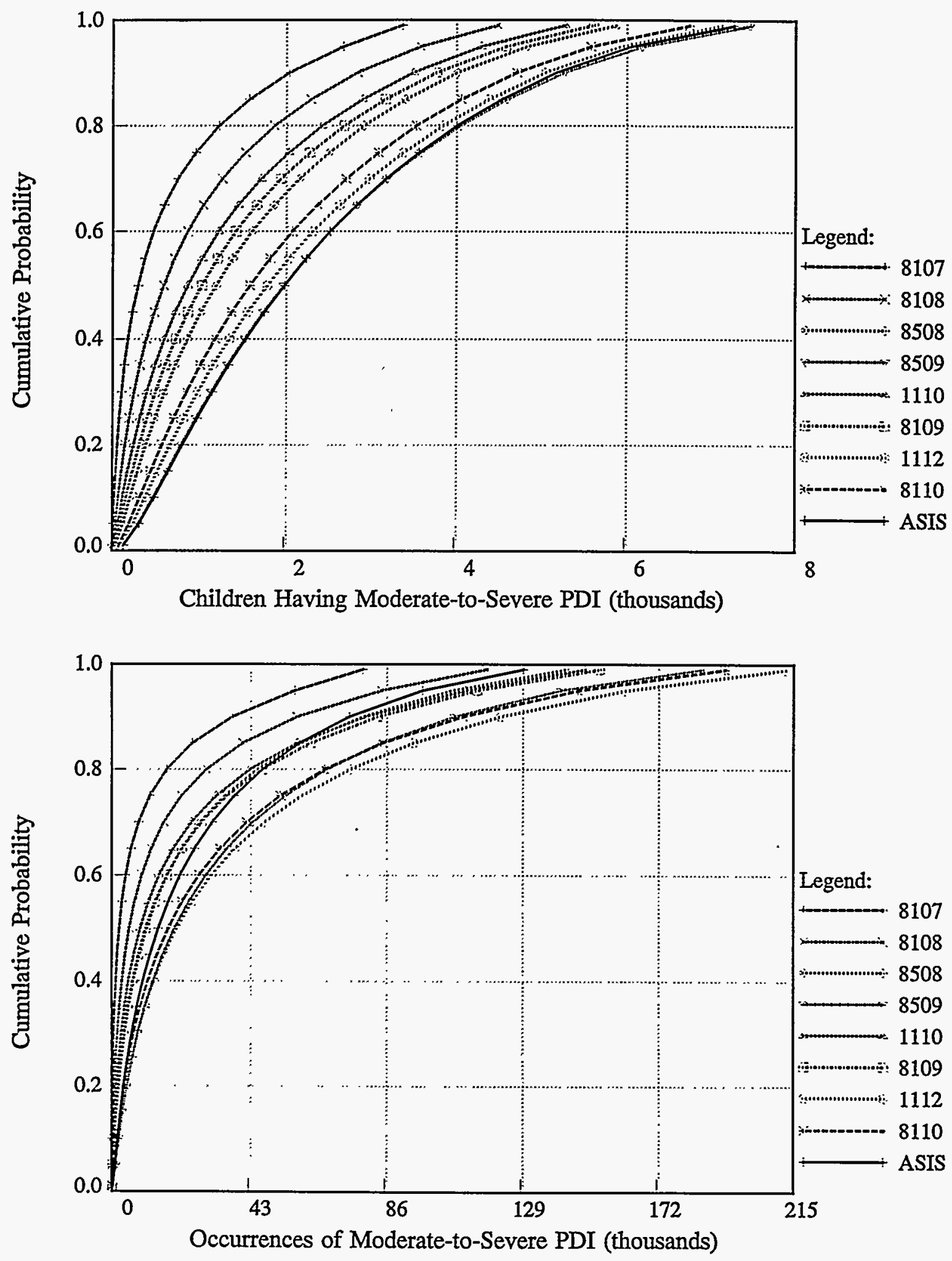

FIGURE C.26 Representative Risk Distributions for Alternative Air Quality Scenarios for Moderate-to-Severe Pain on Deep Inspiration, St. Louis, Outdoor Children, 1-h Exposures, and Moderate Exertion (underlying exposure-response relationship based on Seal et al. 1993) 

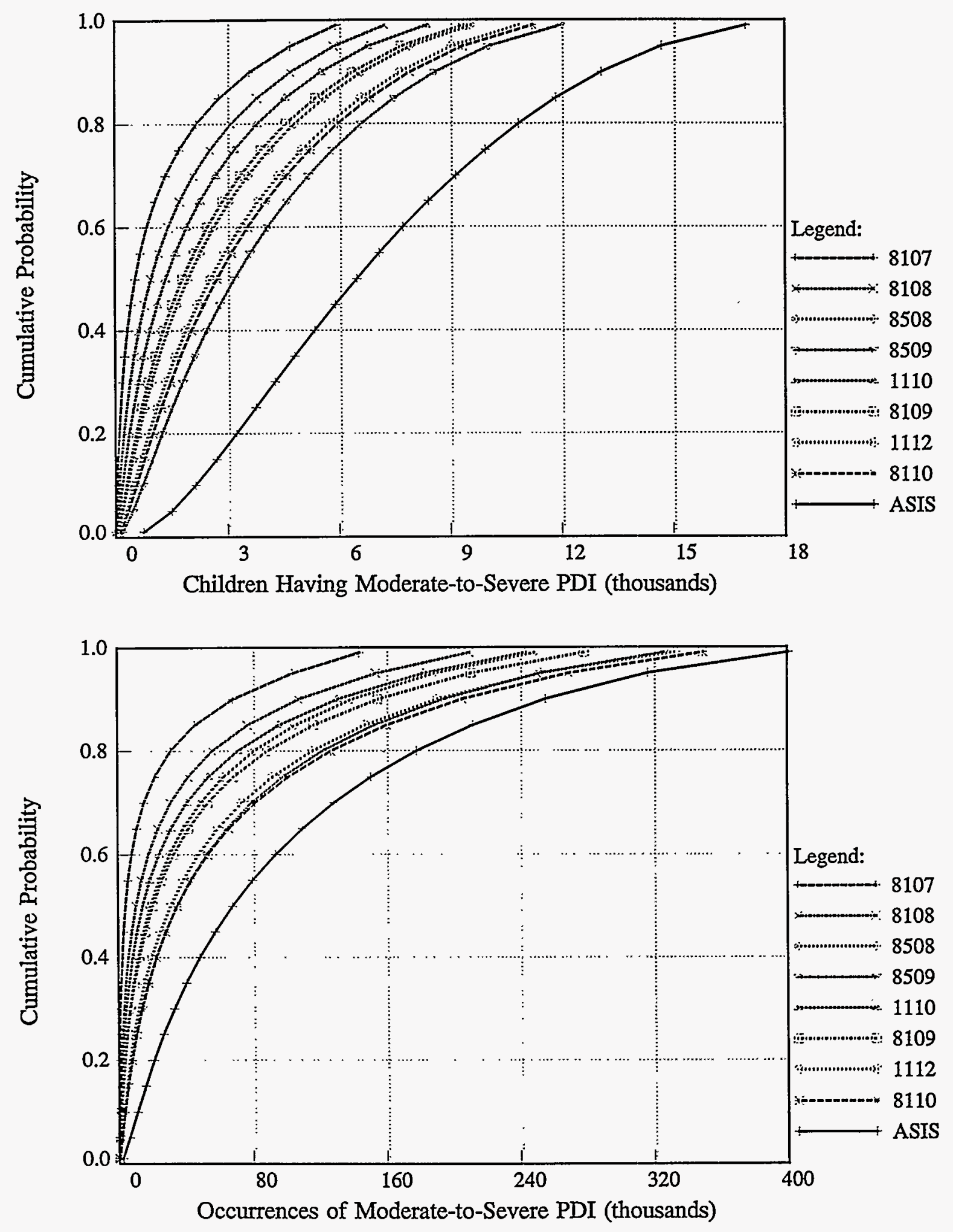

FIGURE C.27 Representative Risk Distributions for Alternative Air Quality Scenarios for Moderate-to-Severe Pain on Deep Inspiration, Washington DC, Outdoor Children, 1-h Exposures, and Moderate Exertion (underlying exposure-response relationship based on Seal et al. 1993) 


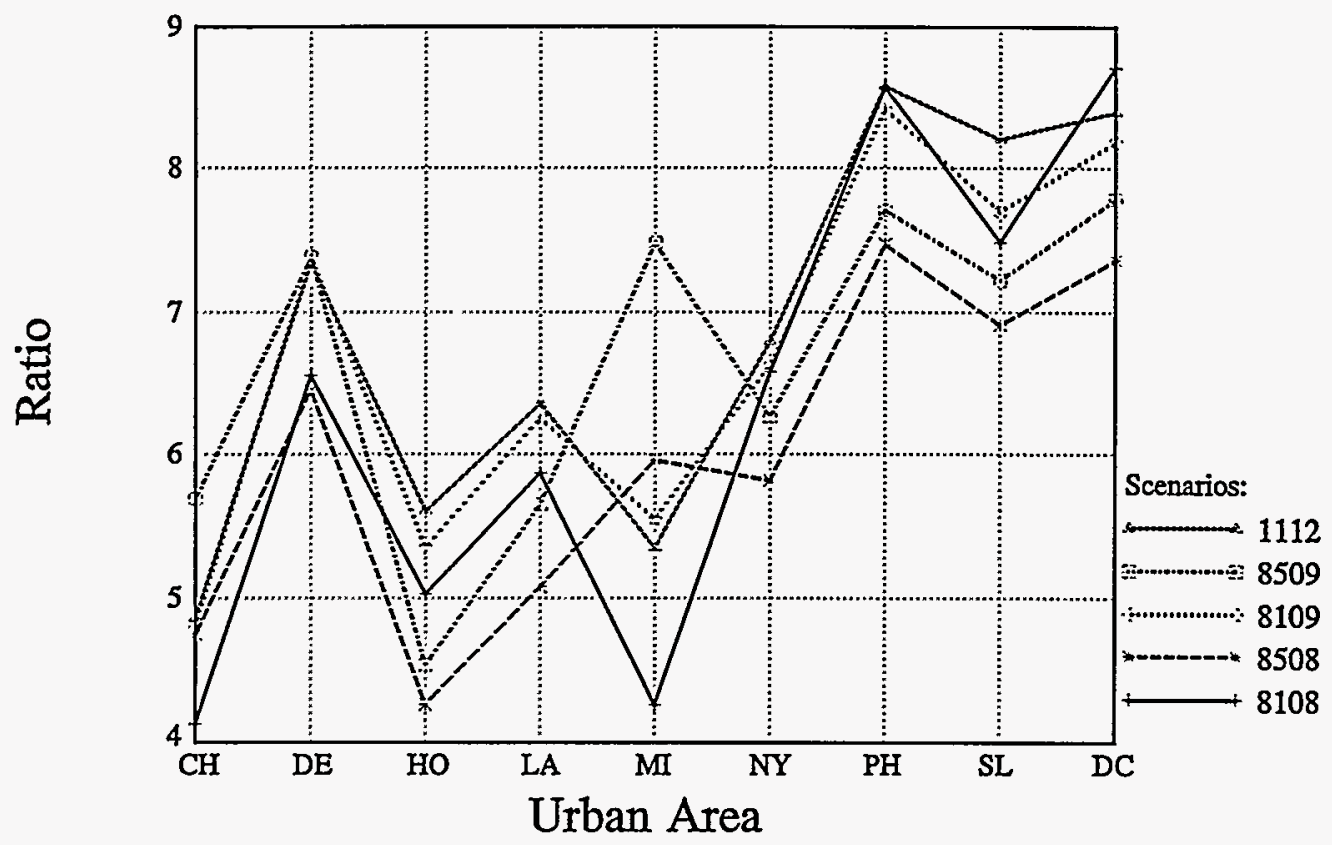

FIGURE C.28 Ratios of Mean Number of Occurrences and Mean Number of Responders for $\mathrm{FEV}_{1}$ Decrements $\geq 15 \%, 9$ Urban Areas, Outdoor Children, 8-h Exposures, and Moderate Exertion (underlying exposureresponse relationship based on Folinsbee et al. 1988, Horstman et al. 1990, and McDonnell et al. 1991)

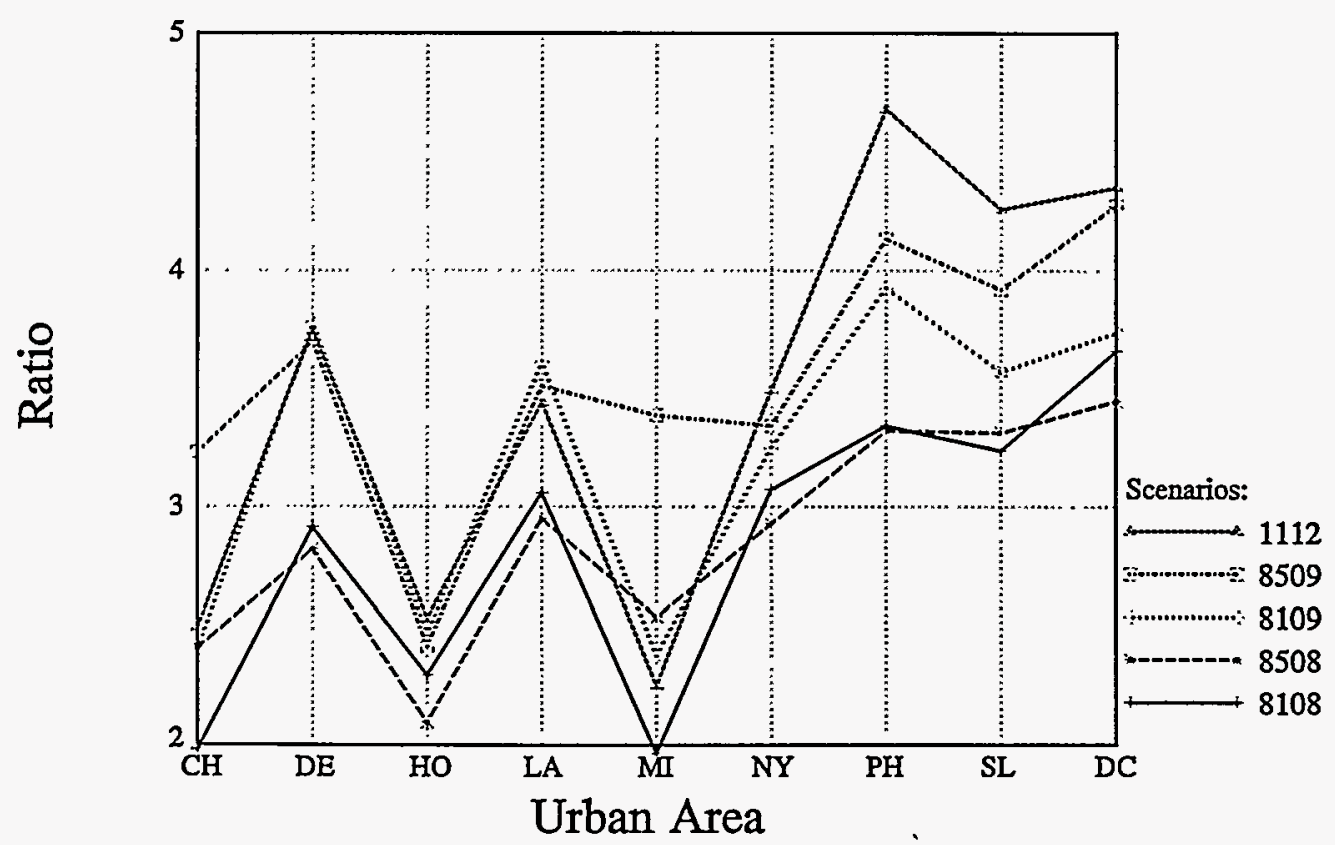

FIGURE C.29 Ratios of Mean Number of Occurrences and Mean Number of Responders for $\mathrm{FEV}_{1}$ Decrements $\geq 20 \%, 9$ Urban Areas, Outdoor Children, 8-h Exposures, and Moderate Exertion (underlying exposureresponse relationship based on Folinsbee et al. 1988, Horstman et al. 1990, and McDonnell et al. 1991) 


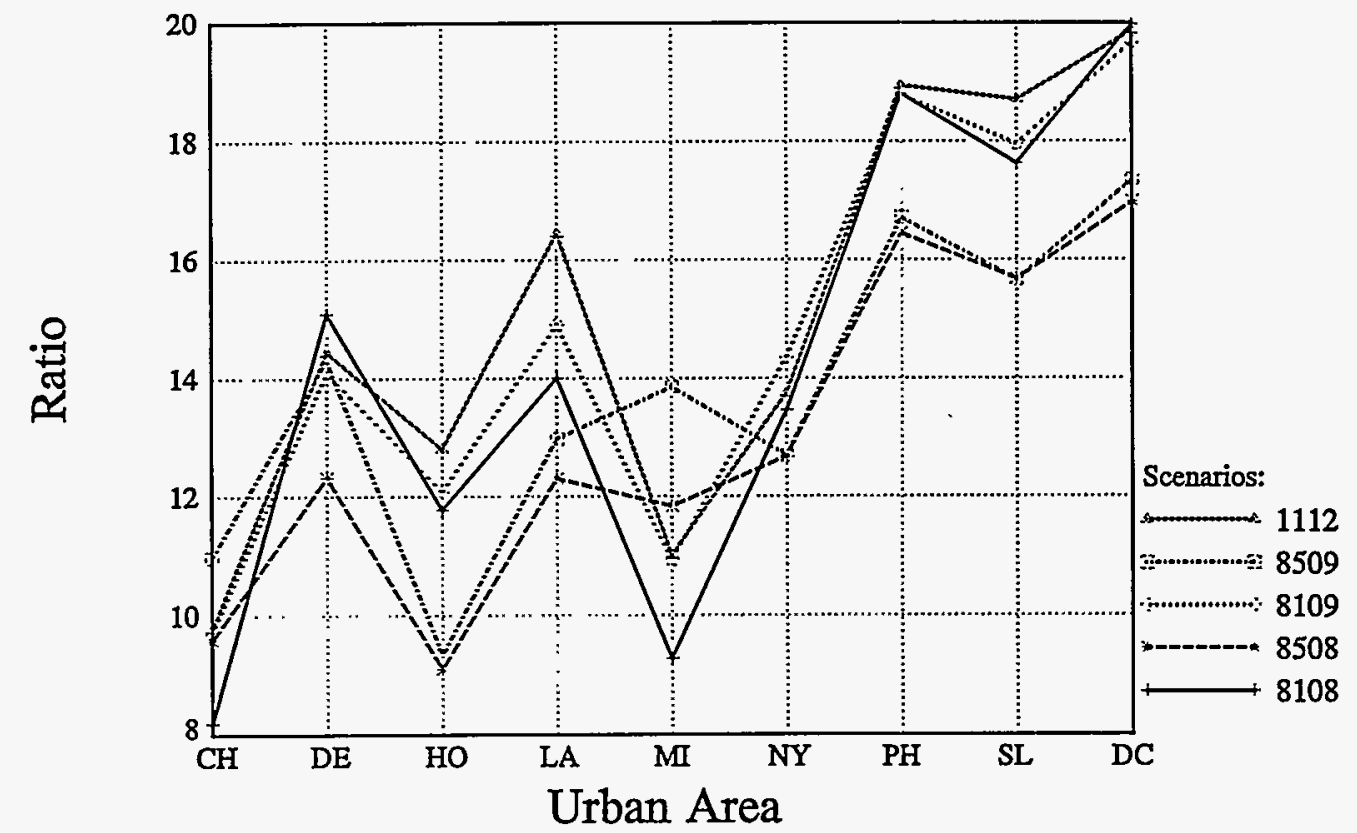

FIGURE C.30 Ratios of Mean Number of Occurrences and Mean Number of Responders for Moderate-to-Severe Pain on Deep Inspiration, 9 Urban Areas, Outdoor Children, 1-h Exposures, and Moderate Exertion (underlying exposure-response relationship based on Seal et al. 1993) 


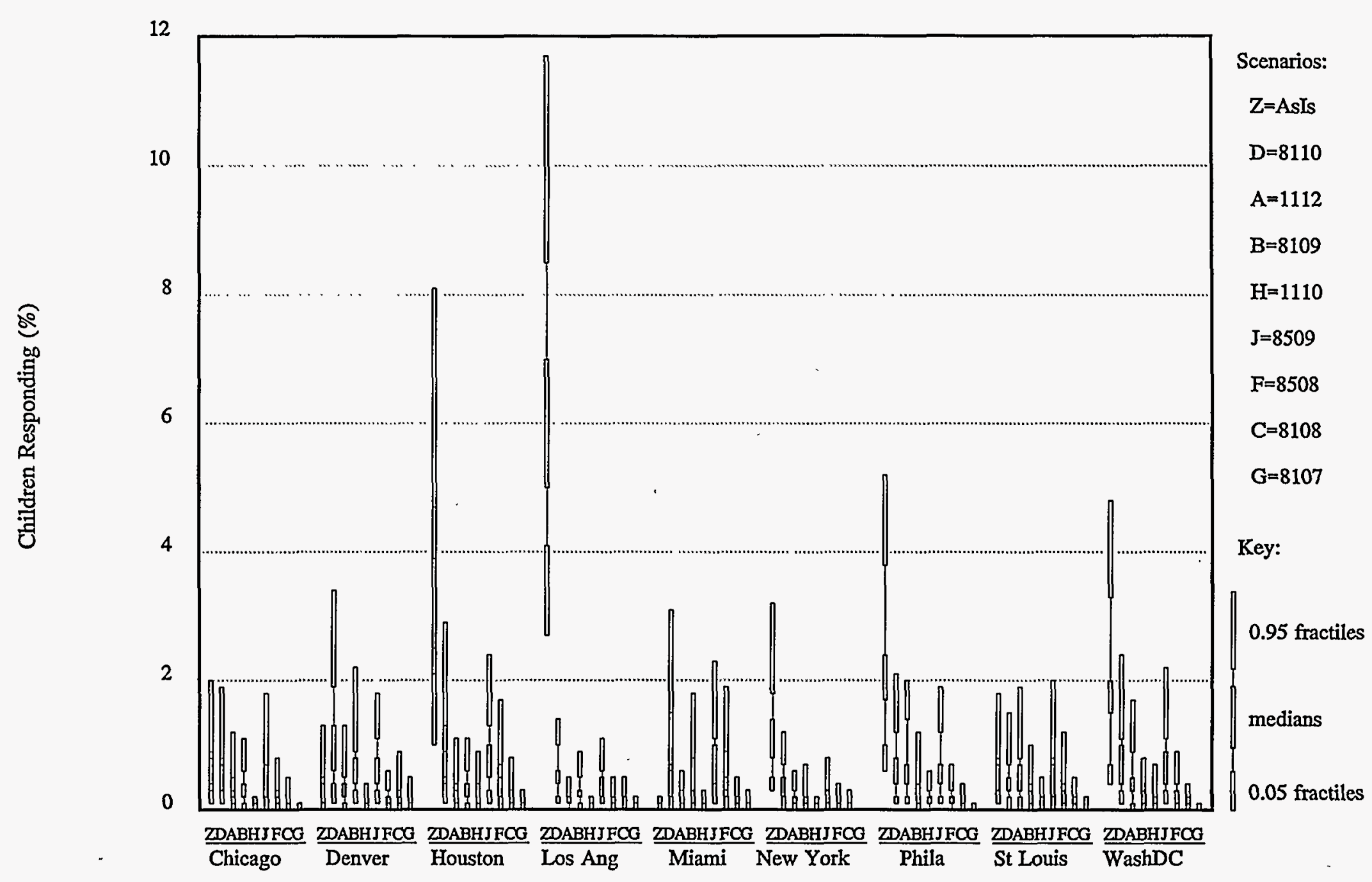

FIGURE C.31 Headcount Risk Results for Percentage of Children Responding for FEV 1 Decrement $\geq 15 \%$, 1-h Exposures, and Heavy Exertion (persons basis; underlying exposure-response relationship based on Avol et al. 1984) 


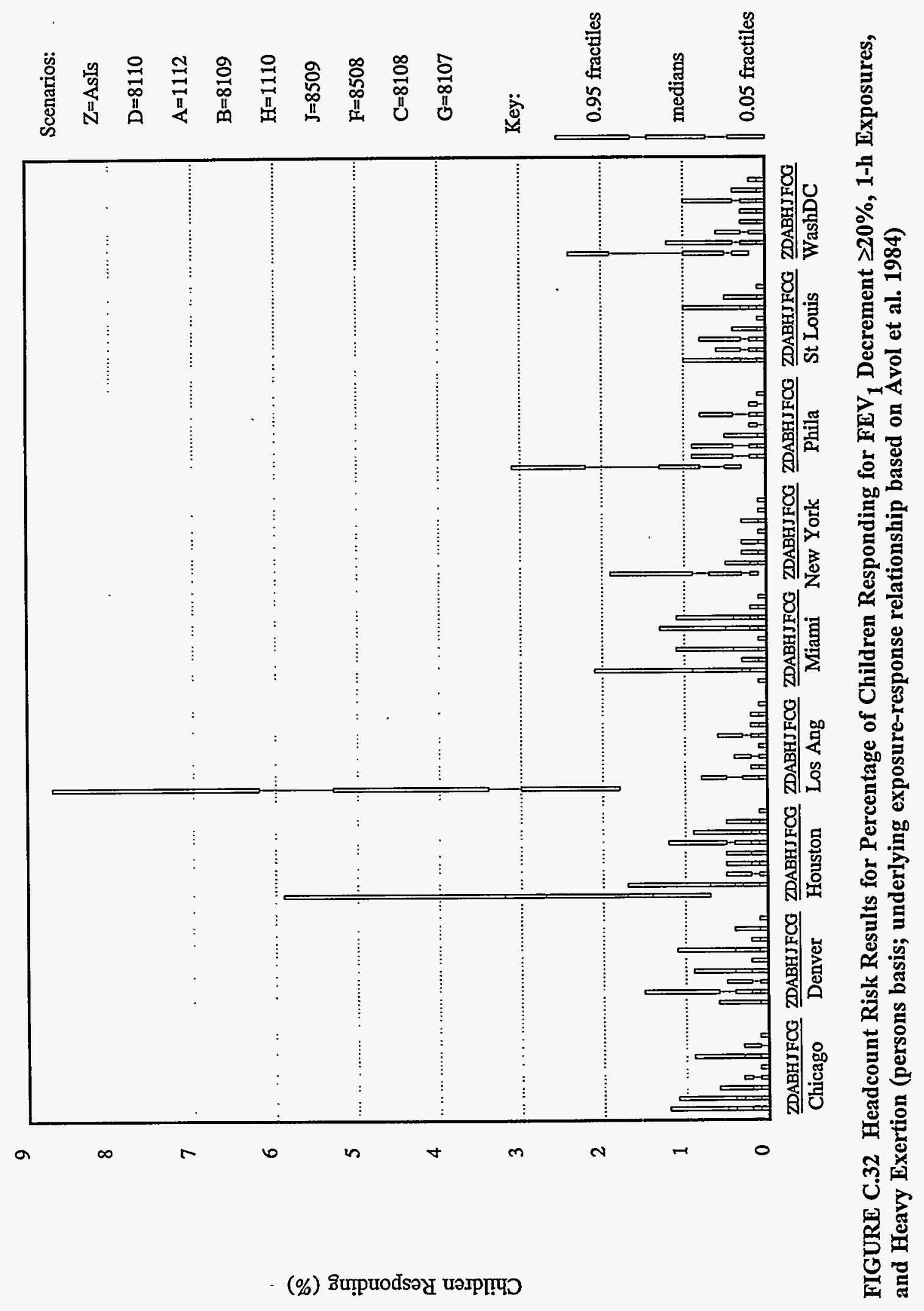




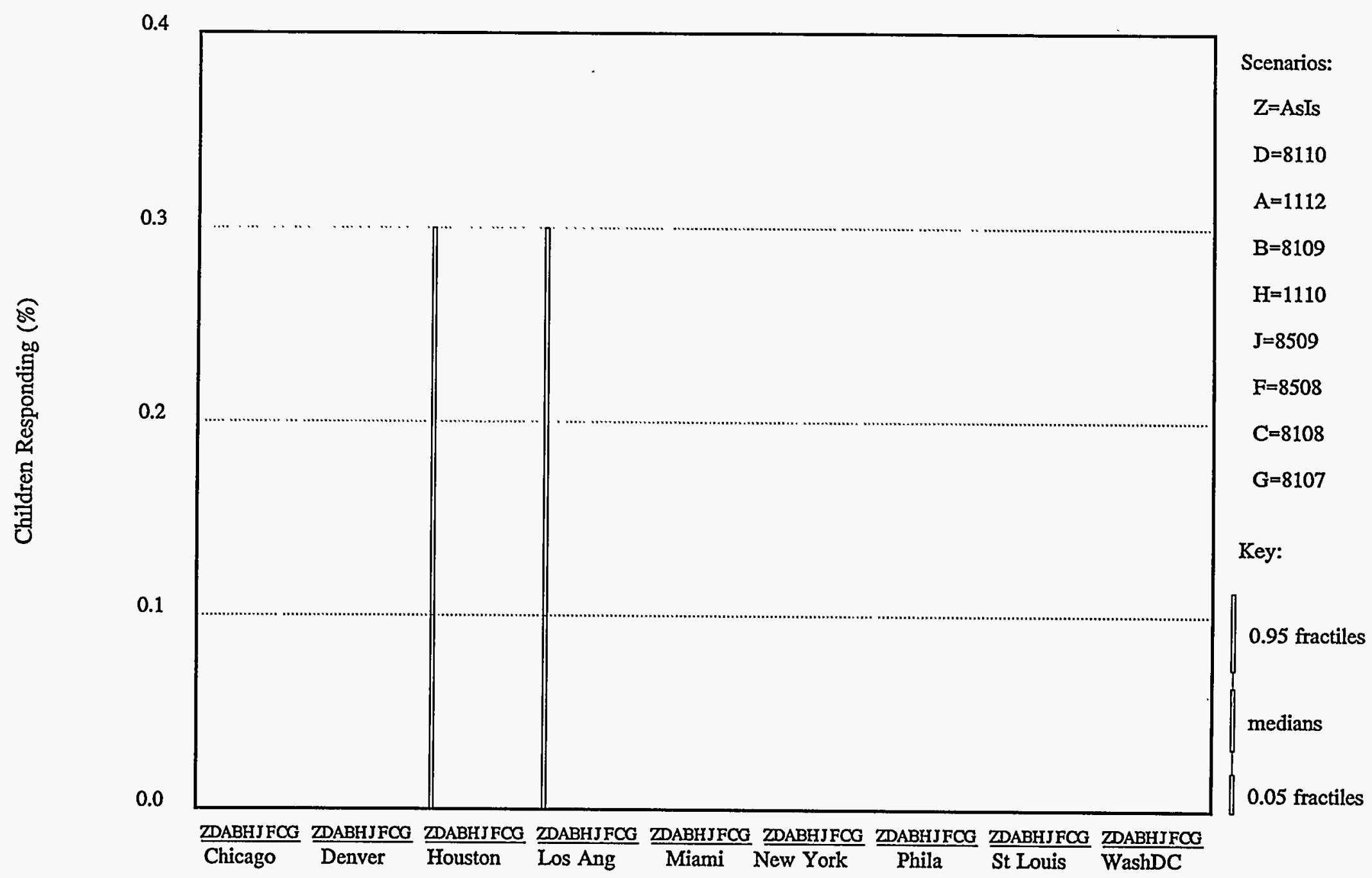

FIGURE C.33 Headcount Risk Results for Percentage of Children Responding for Moderate-to-Severe Lower Respiratory Symptoms, 1-h Exposures, and Heavy Exertion (persons basis; underlying exposure-response relationship based on Avol et al. 1984) 


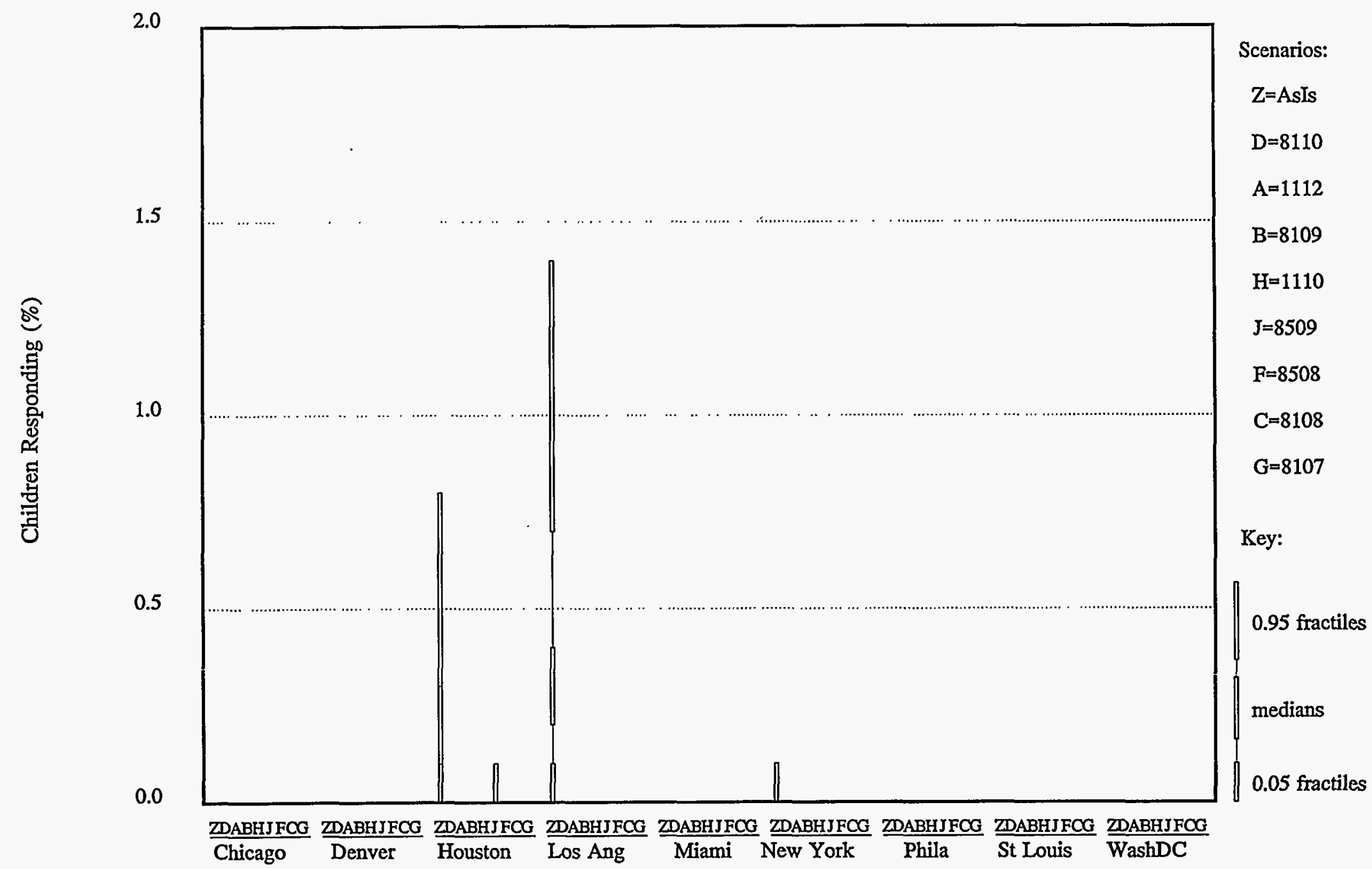

FIGURE C.34 Headcount Risk Results for Percentage of Children Responding for FEV 1 Decrement $\geq 15 \%$, 1-h Exposures, and Heavy Exertion (persons basis; underlying exposure-response relationship based on Kulle et al. 1985) 


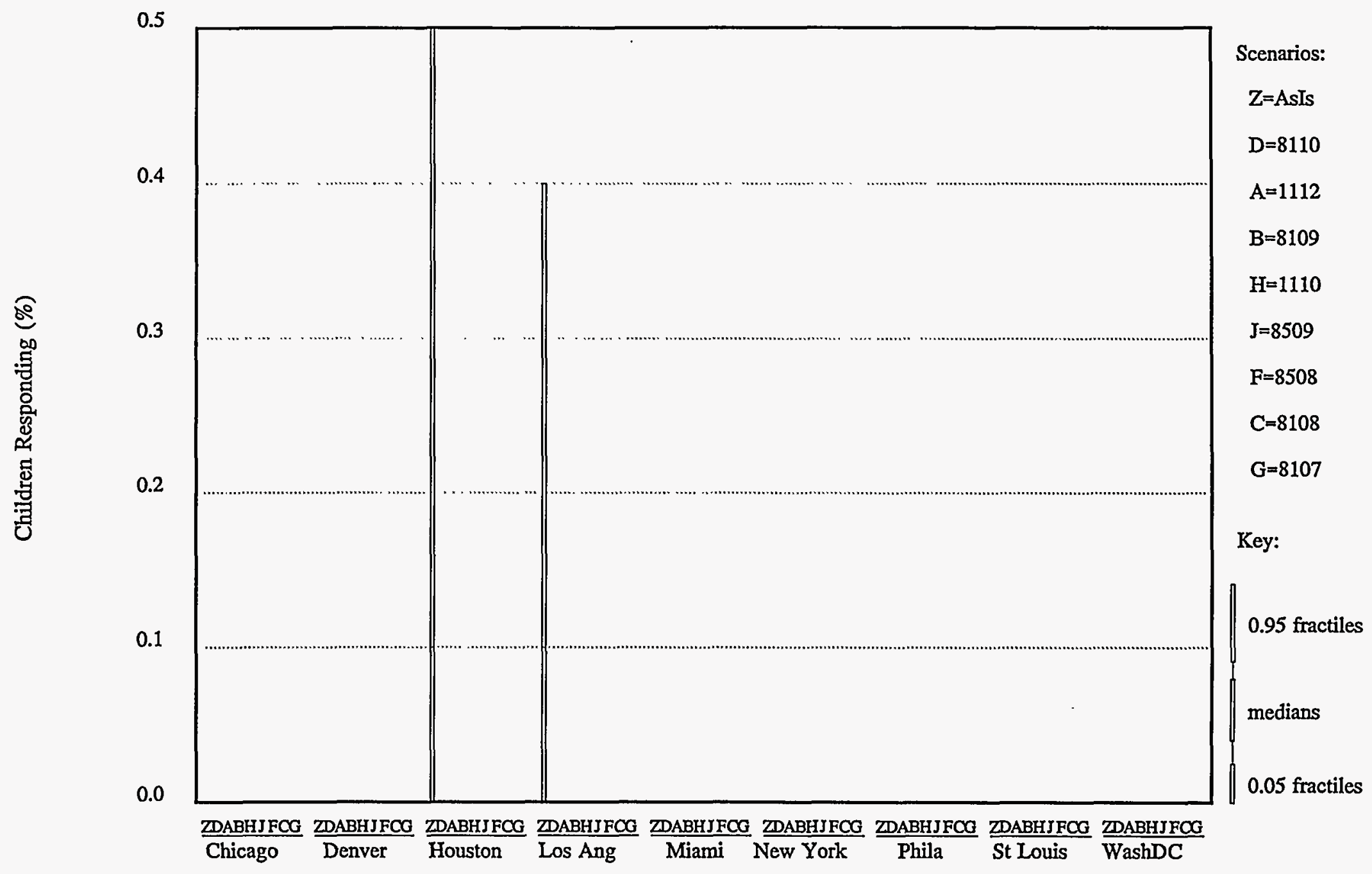

FIGURE C.35 Headcount Risk Results for Percentage of Children Responding for FEV 1 Decrement $\geq 20 \%$, 1-h Exposures, and Heavy Exertion (persons basis; underlying exposure-response relationship based on Kulle et al. 1985) 


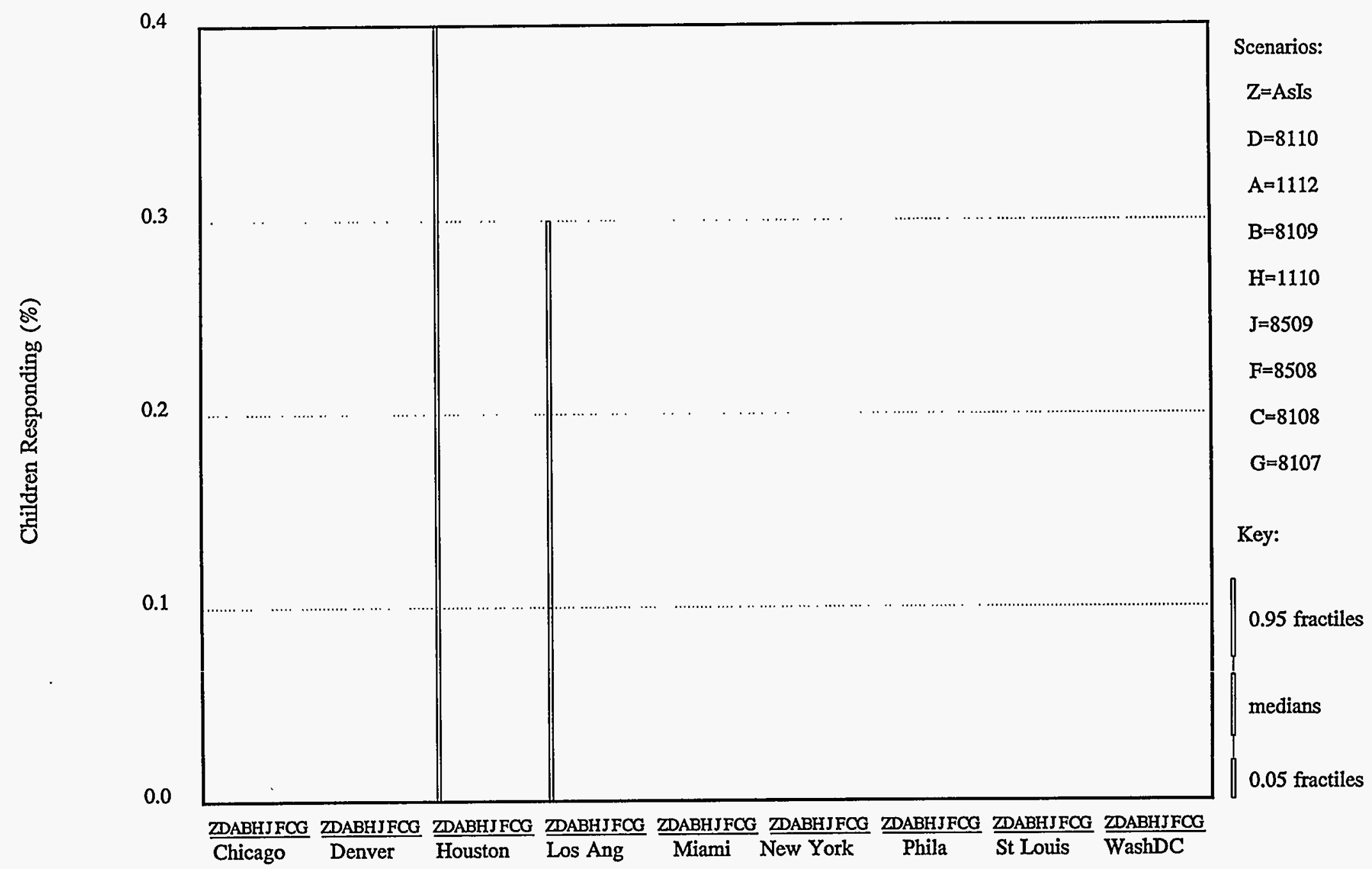

FIGURE C.36 Headcount Risk Results for Percentage of Children Responding for Moderate-to-Severe Cough,

1-h Exposures, and Heavy Exertion (persons basis; underlying exposure-response relationship based on Kulle et al. 1985) 


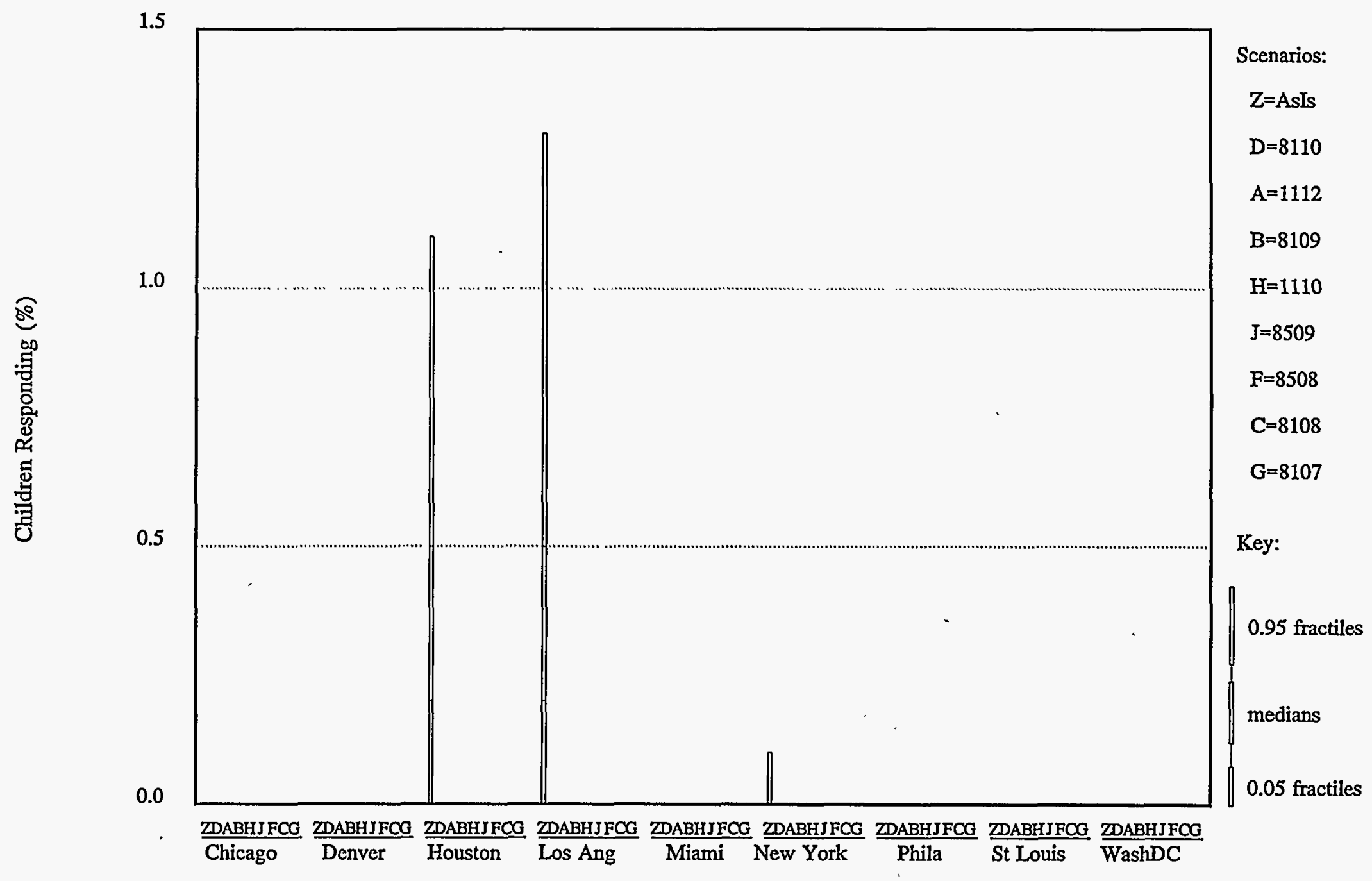

FIGURE C.37 Headcount Risk Results for Percentage of Children Responding for Moderate-to-Severe PDI, 1-h Exposures, and Heavy Exertion (persons basis; underlying exposure-response relationship based on Kulle et al. 1985) 


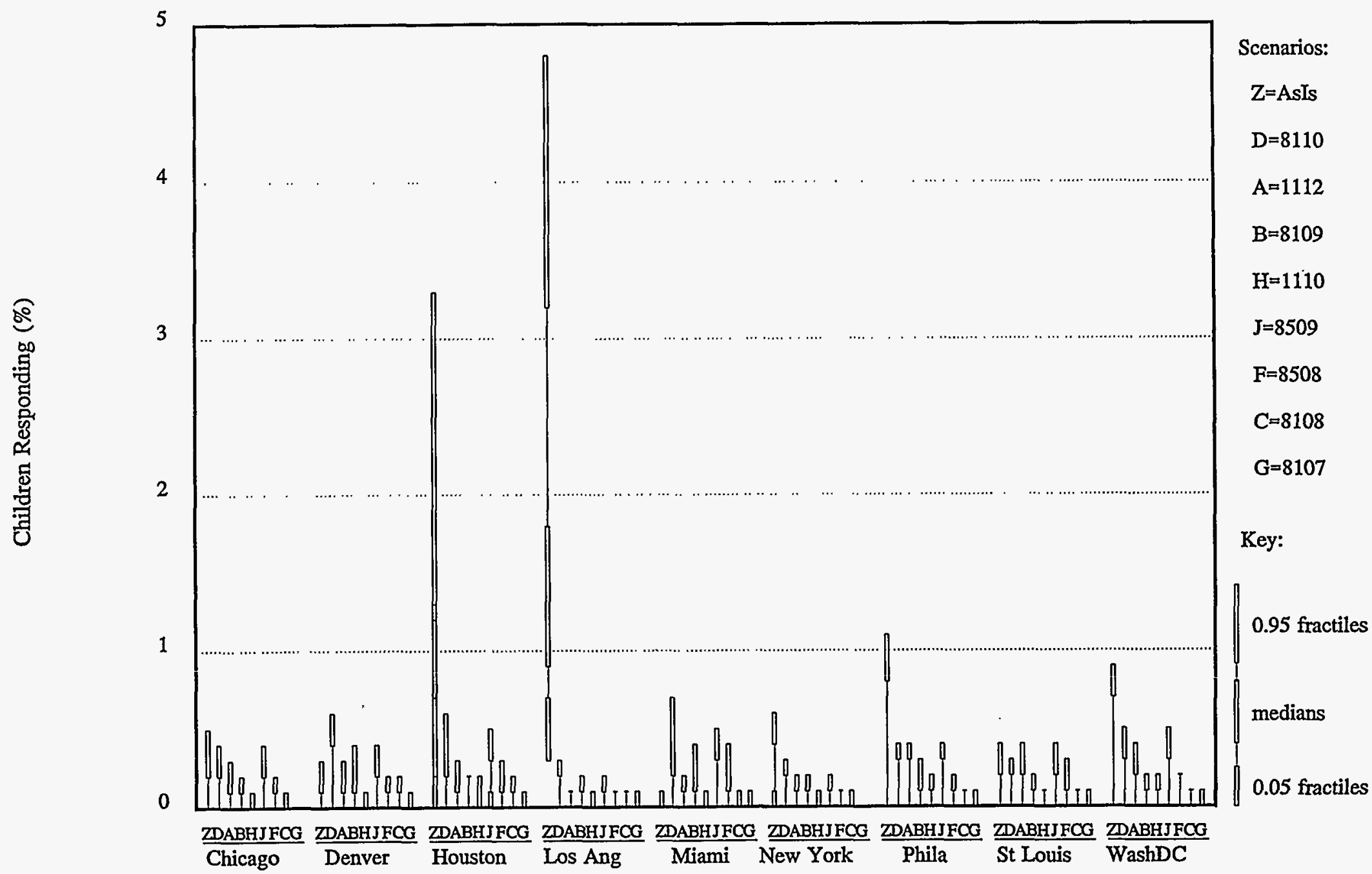

FIGURE C.38 Headcount Risk Results for Percentage of Children Responding for FEV 1 Decrement $\geq 15 \%$, 1-h Exposures, and Heavy Exertion (persons basis; underlying exposure-response relationship based on McDonnell et al. 1983) 


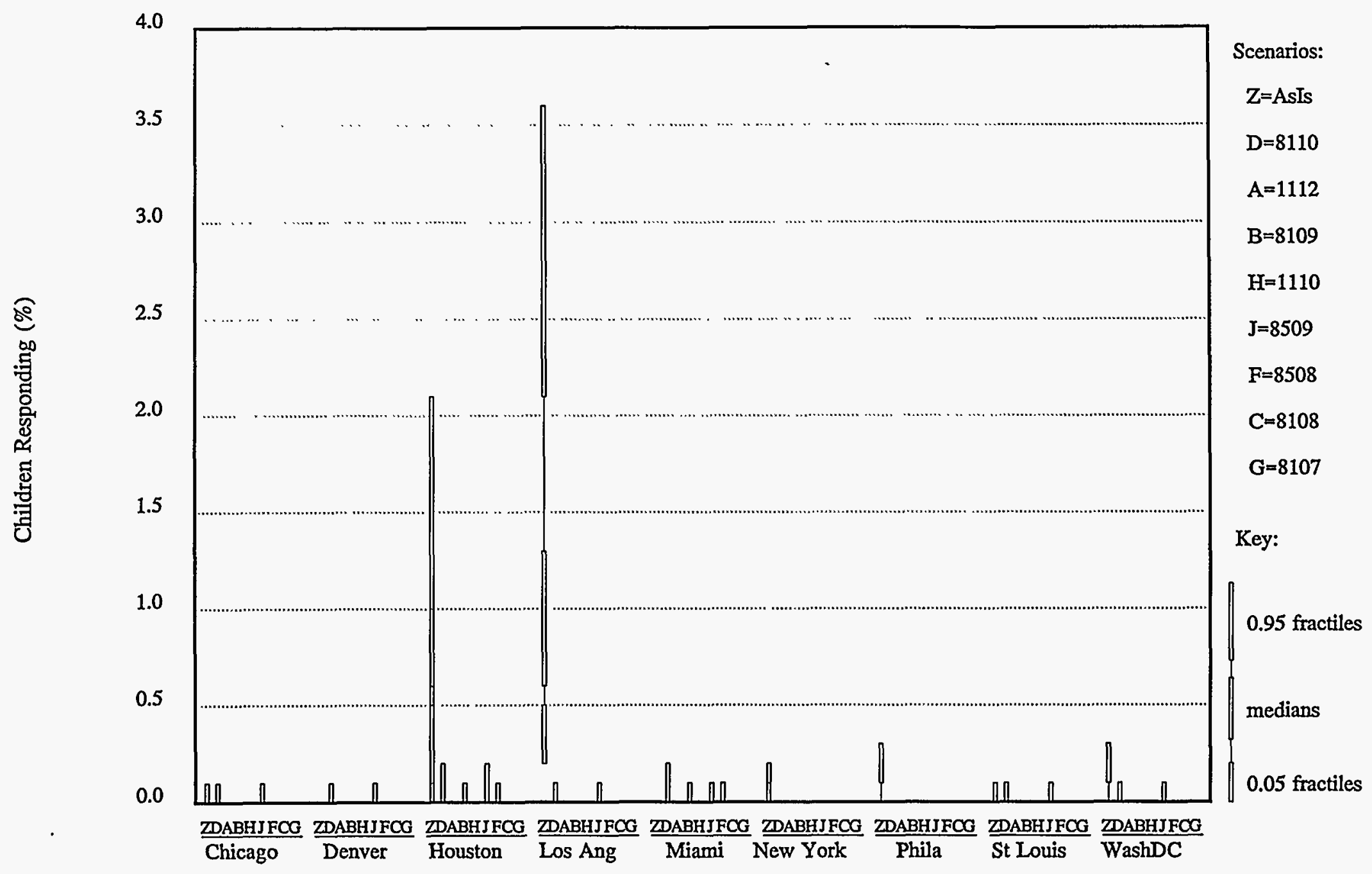

FIGURE C.39 Headcount Risk Results for Percentage of Children Responding for FEV 1 Decrement $\geq 20 \%$, 1-h Exposures, and Heavy Exertion (persons basis; underlying exposure-response relationship based on McDonnell et al. 1983) 


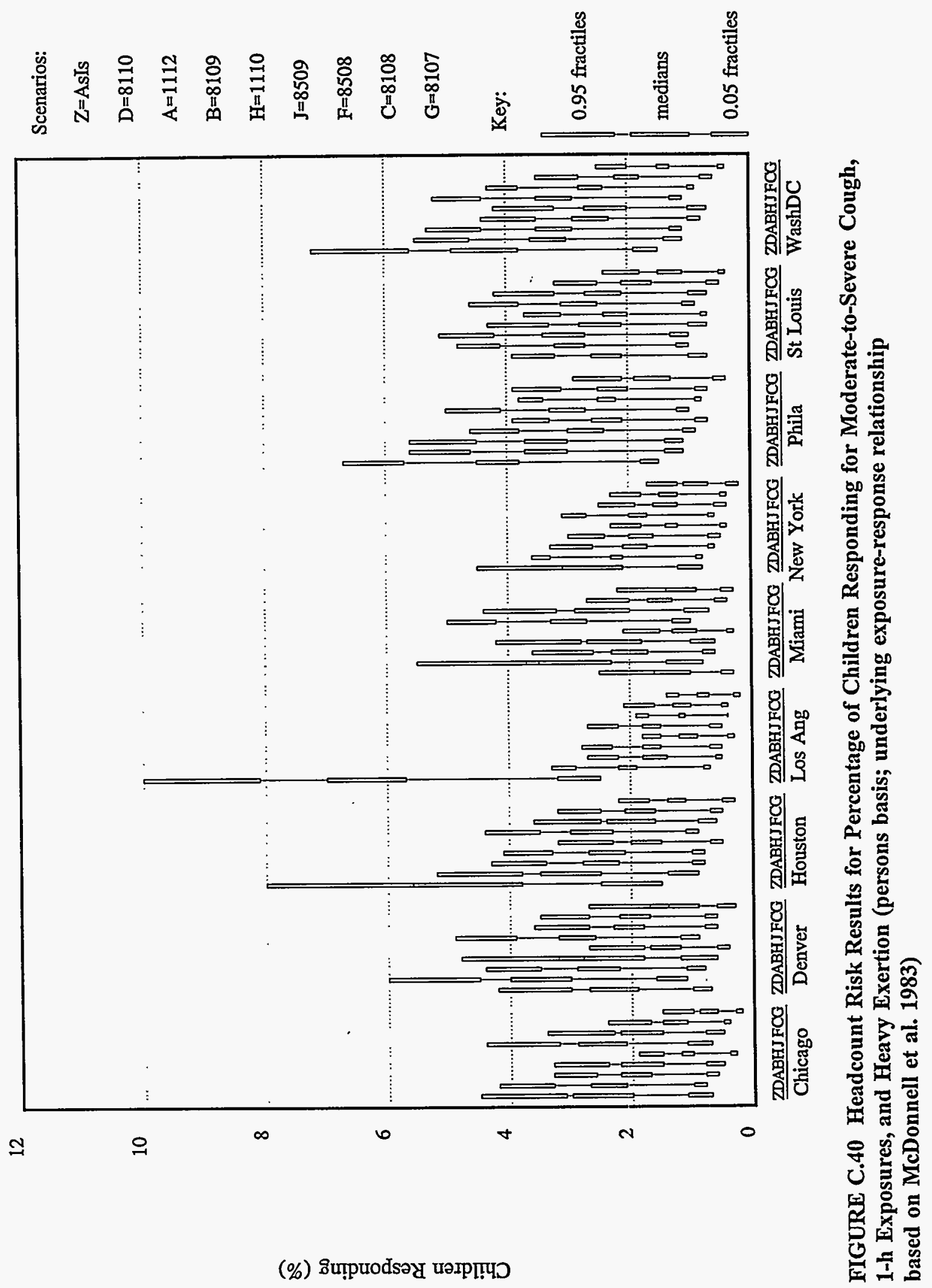




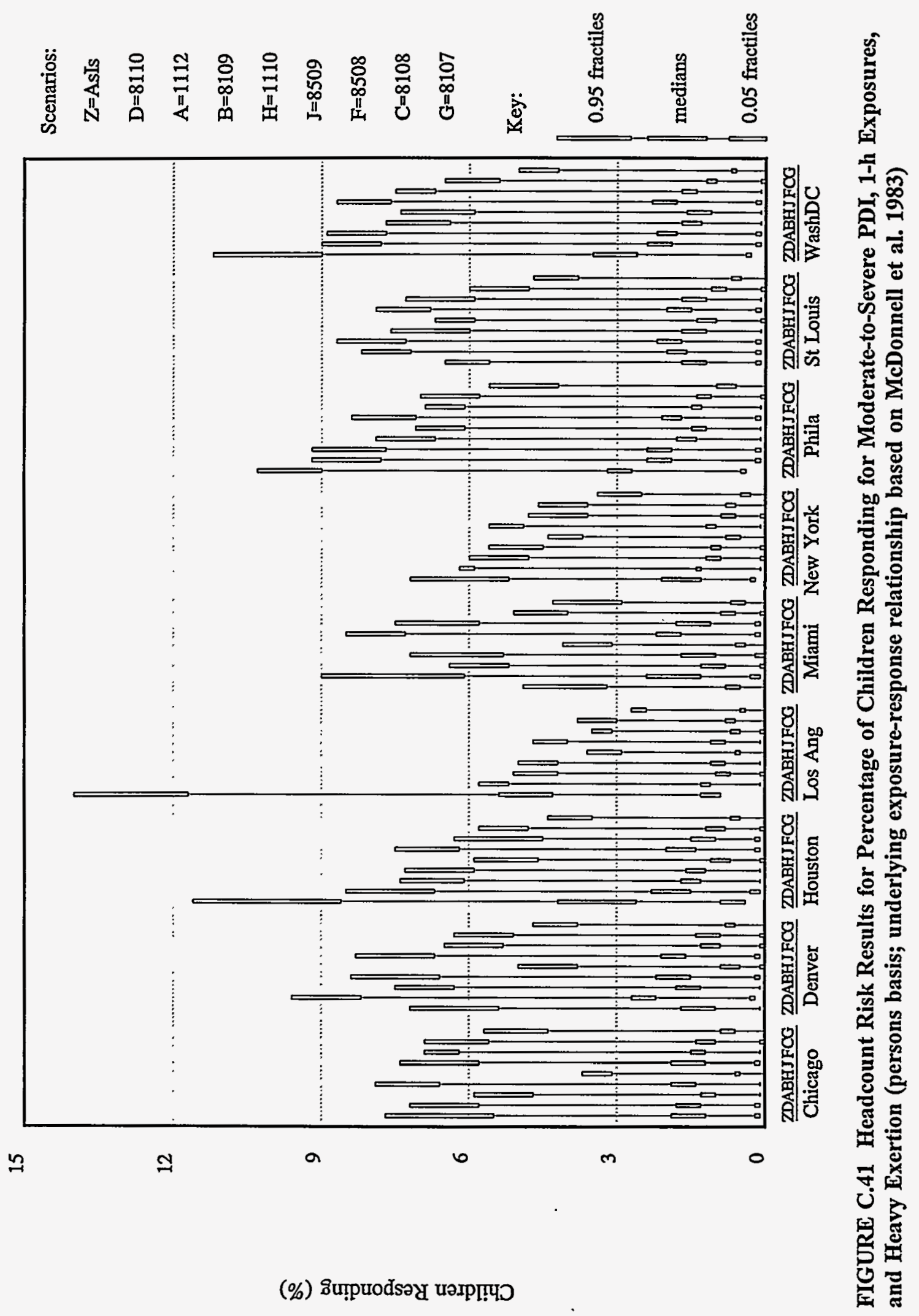




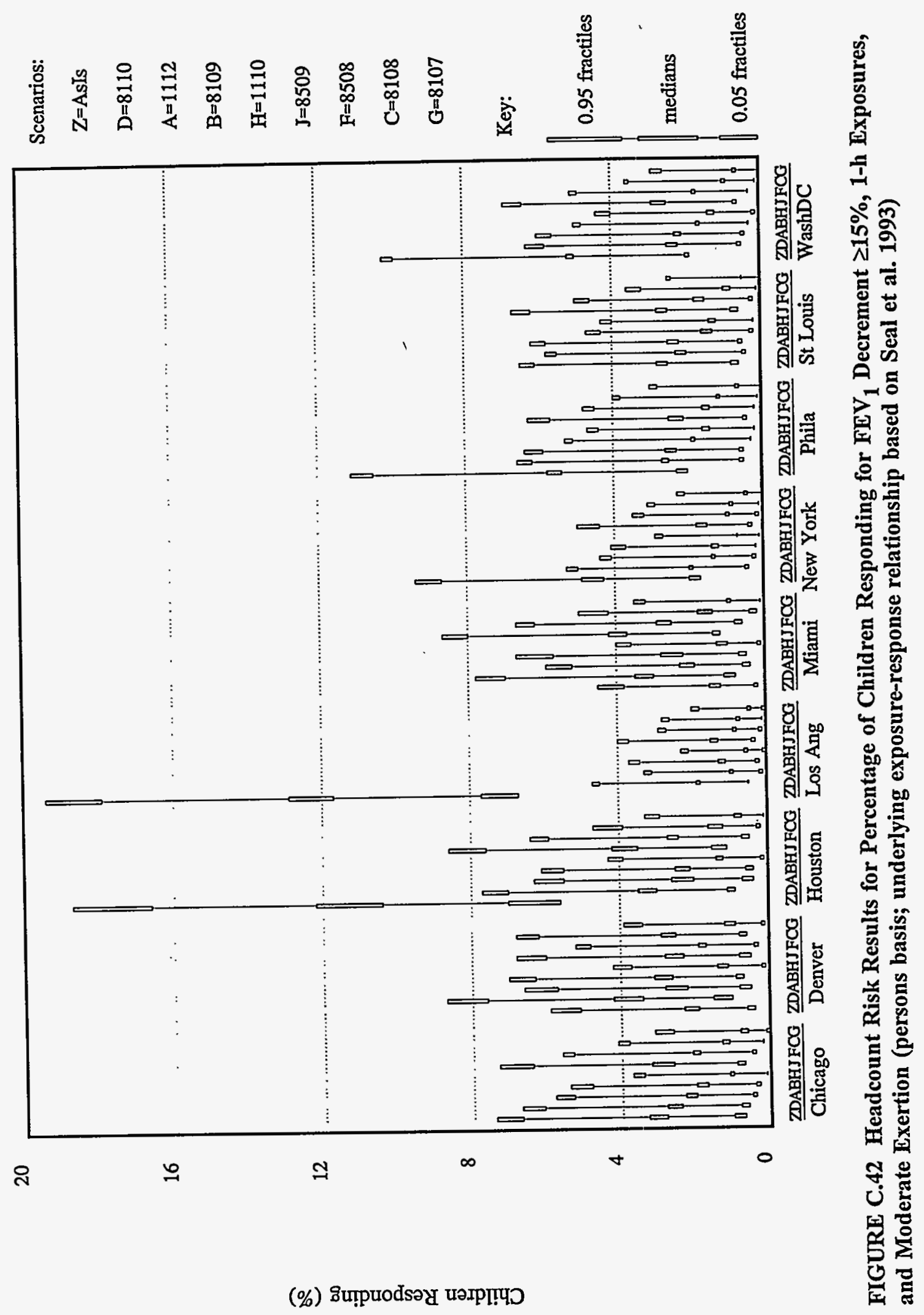




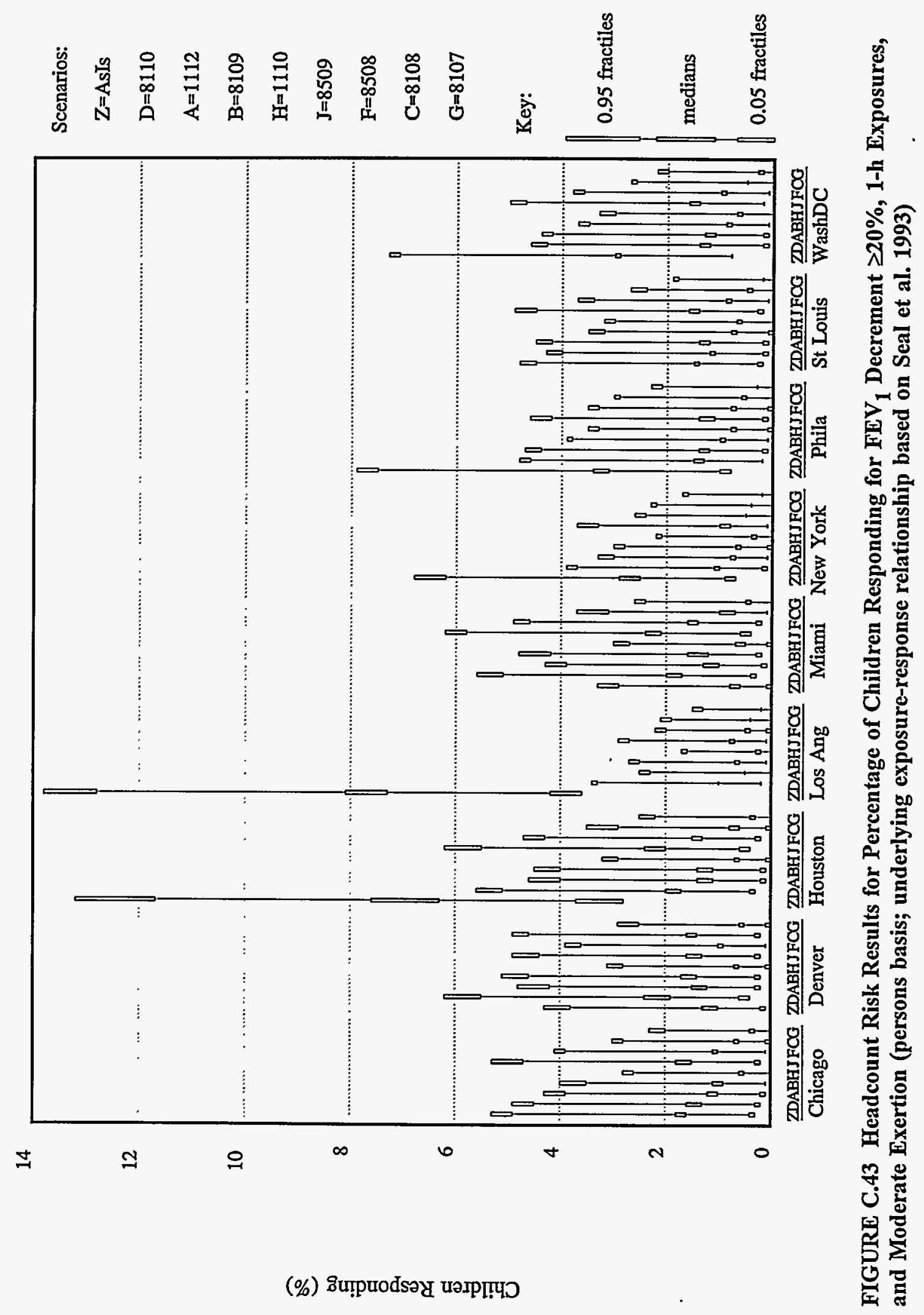




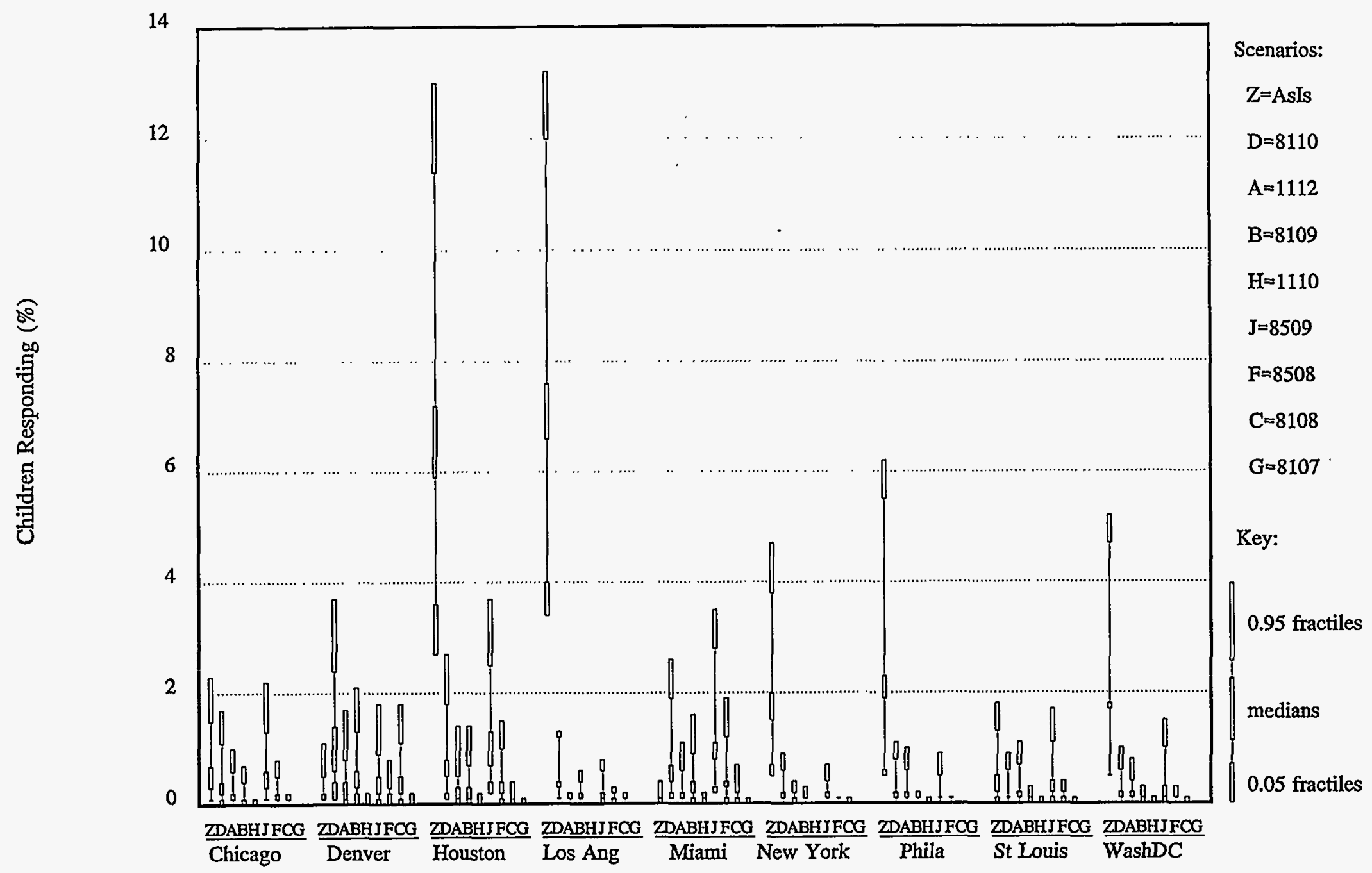

FIGURE C.44 Headcount Risk Results for Percentage of Children Responding for Moderate-to-Severe Cough,

1-h Exposures, and Moderate Exertion (persons basis; underlying exposure-response relationship based on Seal et al. 1993) 


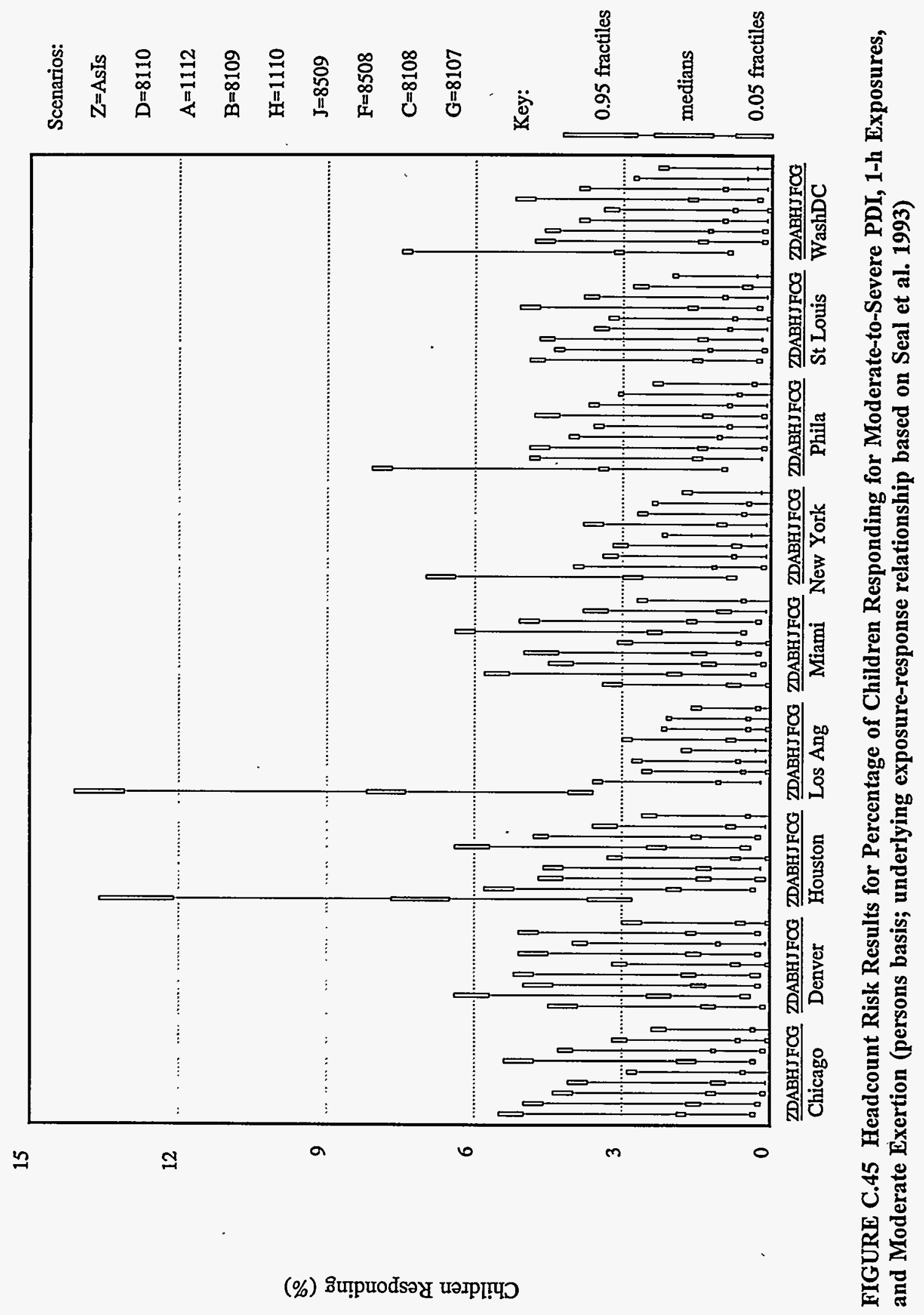




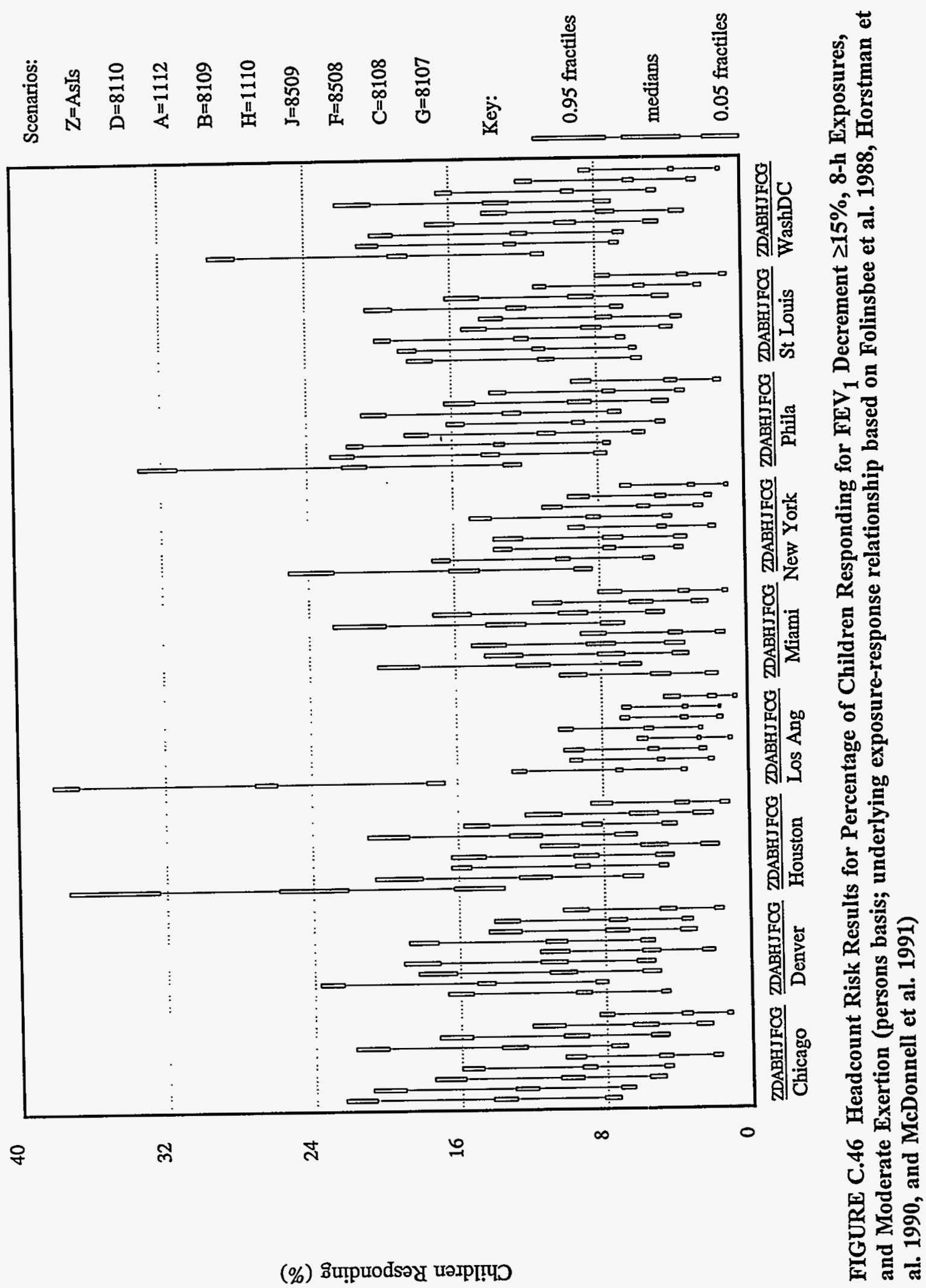




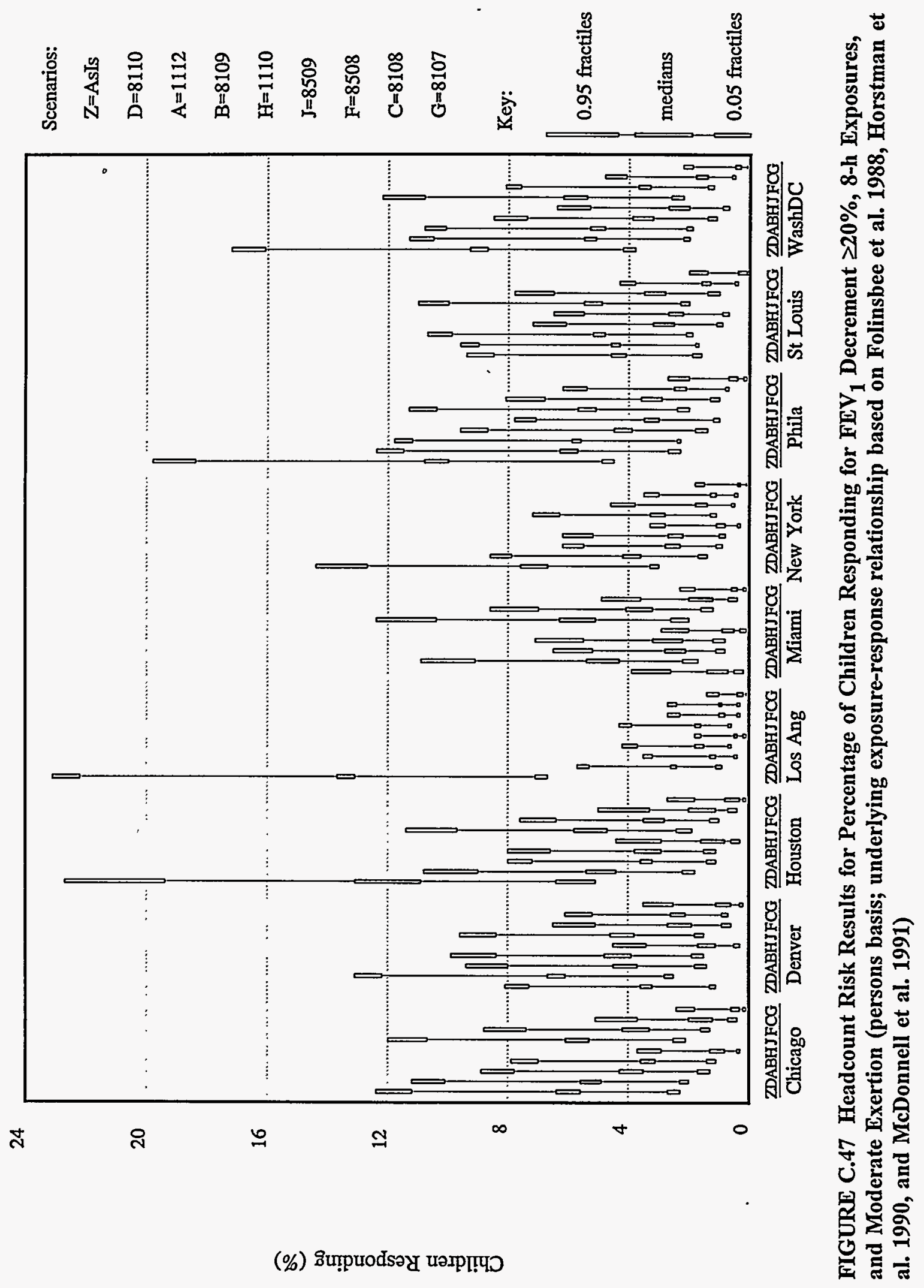




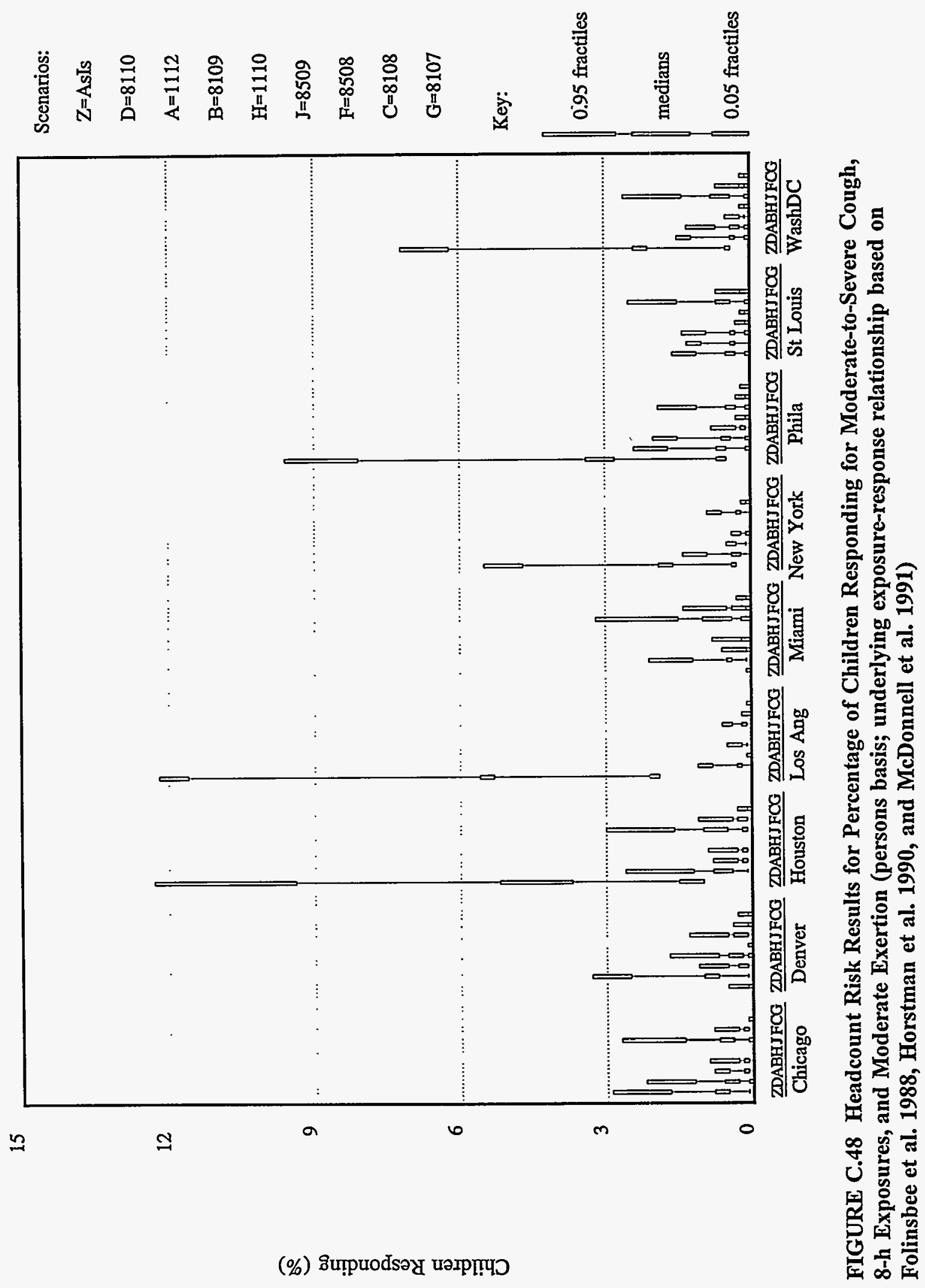




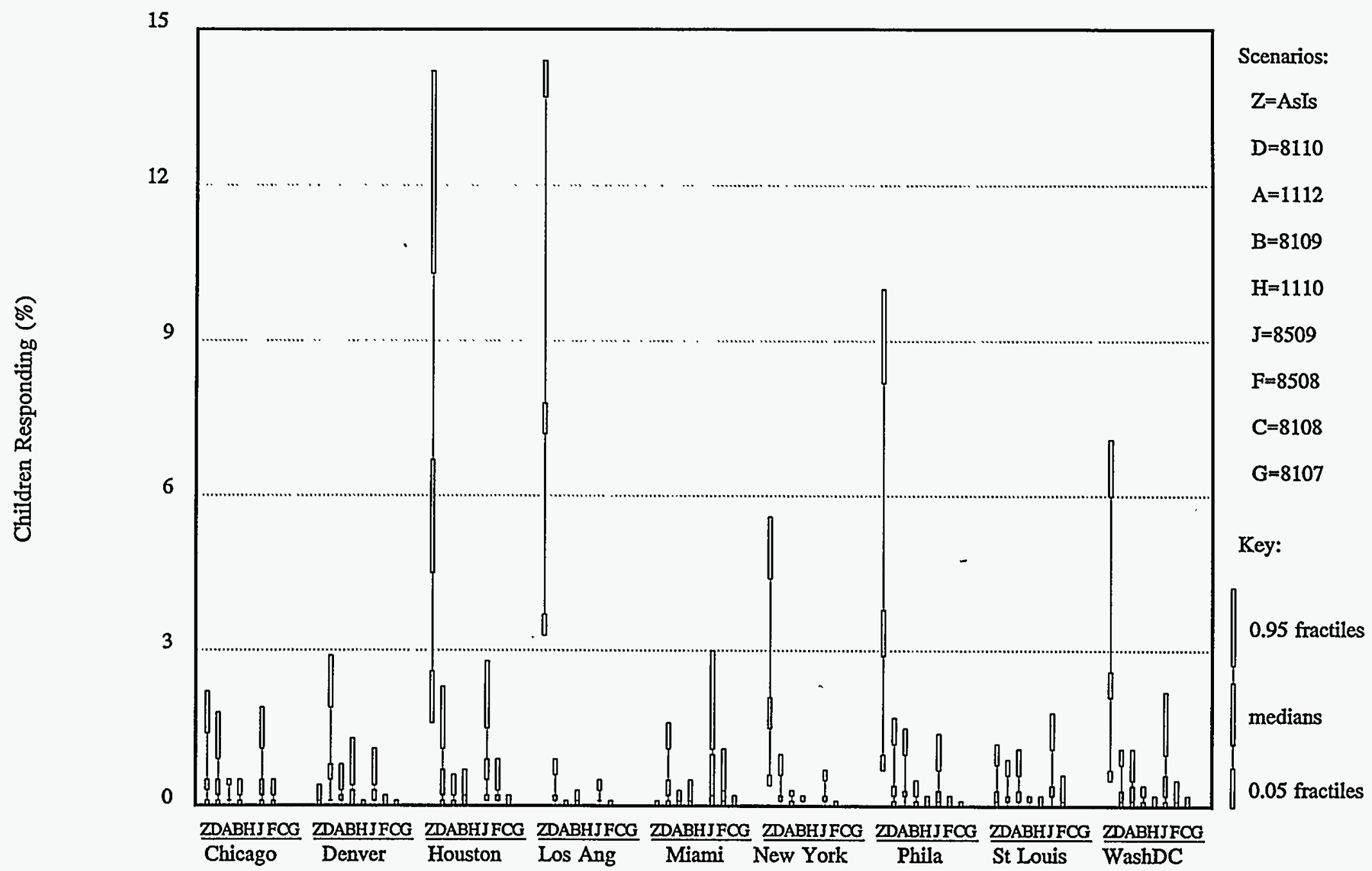

FIGURE C.49 Headcount Risk Results for Percentage of Children Responding for Moderate-to-Severe PDI, 8-h Exposures, and Moderate Exertion (persons basis; underlying exposure-response relationship based on Folinsbee et al. 1988, Horstman et al. 1990, and McDonnell et al. 1991) 


\section{C.3 APPENDIX C REFERENCES}

Avol, E.L., et al., 1984, "Comparative Respiratory Effects of Ozone and Ambient Oxidant Pollution Exposure during Heavy Exercise," Journal of the Air Pollution Control Association 34:804-809.

Folinsbee, L.J., et al., 1988, "Pulmonary Function and Symptom Responses after 6.6-Hour Exposure to 0.12 ppm Ozone with Moderate Exercise," JAPCA 38:28-35.

Horstman, D.H., et al., 1990, "Ozone Concentration and Pulmonary Response Relationships for 6.6-Hour Exposures with Five Hours of Moderate Exercise to 0.08, 0.10, and 0.12 ppm," American Review of Respiratory Disease 142:1158-1163.

Kulle, T.J., et al., 1985, "Ozone Response Relationships in Healthy Nonsmokers," American Review of Respiratory Disease 132:36-41.

McDonnell, W.F., et al., 1983, "Pulmonary Effects of Ozone Exposure during Exercise: DoseResponse Characteristics," Journal of Applied Physiology: Respiratory Environmental Exercise Physiology 54:1345-1352.

McDonnell, W.F., et al., 1991, "Respiratory Response of Humans Exposed to Low Levels of Ozone for 6.6 Hours," Archives of Environmental Health 46:145-150.

Seal, E., Jr., et al., 1993, "The Pulmonary Response of White and Black Adults to Six Concentrations of Ozone," American Review of Respiratory Disease 147:804-810. 
APPENDIX D:

BENCHMARK RISK RESULTS 

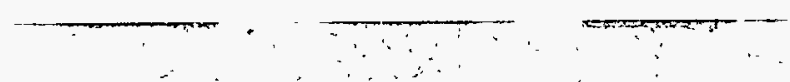


\section{APPENDIX D:}

\section{BENCHMARK RISK RESULTS}

\section{D.1 INTRODUCTION}

This appendix contains Figures D.1-D.19 and Table D.1, which summarize the benchmark risk results for acute health endpoints. Each figure addresses one of the 33 more severe acute endpoints and indicates the probability that the benchmark response will be exceeded five or more times in an ozone season.

All of the results shown are based on 1-h (for 1-h endpoints) or running 8-h (for 8-h endpoints) daily maximum average ozone concentrations. Results for other health endpoints and for exceeding the benchmark response one or more times in an ozone season are available elsewhere. ${ }^{1}$

\section{D.2 BENCHMARK RISK FIGURES FOR SELECTED HEALTH ENDPOINTS}

Benchmark response is $r$, the fraction of the population that experiences the specified health effect when exposed to ozone. Benchmark risk is defined as the probability that the benchmark response is $\geq r, n$ or more times in a given period (one ozone season) at some location within a geographic region, given a specific condition of air quality (e.g., that standard 1112 is just attained). This report uses $r$ values of 0.05 and 0.1 (sometimes referred to as 0.05 and 0.1 benchmarks, or 5 and $10 \%$ benchmarks).

Figure D.1 is an example of the graphic format used to display benchmark risk results. The figure shows the probability that the benchmark response for the Avol et al. (1984) $\mathrm{FEV}_{1}$ decrement $\geq 15 \%$ endpoint will be exceeded five or more times in an ozone season for 0.05 and 0.1 benchmarks. It includes results for nine urban areas and nine air quality scenarios for each urban area. The air quality scenarios are indicated by a letter code above the name of each area. The letter code is explained in the right side of the figure. Two vertical lines are used for each air quality scenario: the solid line is for the 0.05 benchmark, and the dotted line is for the 0.1 benchmark. The height of the line indicates the benchmark risk. The benchmark risk for the 0.05 benchmark is, logically, less than or equal to the benchmark risk for the 0.1 benchmark.

For example, for Chicago, scenario D, daily maximum 8-h running average ozone concentrations, and using the distribution for the fifth-highest ozone concentration, the benchmark

${ }^{1}$ Software and data (for IBM personal computers or compatibles only) needed to generate and view all risk results can be obtained from the Energy Science and Technology Software Center, P.O. Box 1020, Oak Ridge, TN 37831, phone 423-576-2606. The complete system requires about 100 Mbytes of disk storage. 
For example, for Chicago, scenario D, daily maximum 8-h running average ozone concentrations, and using the distribution for the fifth-highest ozone concentration, the benchmark risk for the 0.05 benchmark is about 0.92 ; the benchmark risk for the 0.1 benchmark is about 0.55. In other words, if standard 8110 is just attained in Chicago, the benchmark risk (i.e., probability) is 0.92 that $\geq 5 \%$ of the population will experience $\mathrm{FEV}_{1}$ decrements $\geq 15 \%$ five or more times in an ozone season.

The 17 figures included in this appendix address the fifth-highest of 1- or 8-h daily maximum concentrations. Results for the highest and the fifth-highest concentrations are listed in Table D.1.

\section{D.3 BENCHMARK RISK TABLES FOR SELECTED HEALTH ENDPOINTS}

Table D. 1 summarizes one aspect of the contents of the 19 benchmark risk figures shown here (and 47 other cases not discussed further in this report). It lists the number of urban areas that have benchmark risk estimates $\leq 0.1,0.2$, and 0.3 at alternative national ambient air quality standards (NAAQS) for 5 and 10\% benchmarks. Figure D.17 relates to the entries for case 58 in Table D.1. The entries for a $5 \%$ benchmark, which are in the left side of the table, indicate that only Miami has benchmark risk estimates $\leq 0.3$ and then solely for scenario $G$ (8107), for the $\mathrm{FEV}_{1}$ decrements $\geq 20 \%$ endpoint for 8 -h exposures of individuals engaged in activities at moderate exertion for 1 or more times in a year.

The second set of entries, in the right side of the table, is for the same endpoint, but for a $10 \%$ benchmark; as expected, more urban areas have benchmark risks less than or equal to the levels of interest under these conditions because ozone levels are less likely to exceed the benchmarks five or more times compared with one or more times. For scenario $G$ (8107), all nine urban areas have benchmark risks $\leq 0.2$ (but not $\leq 0.1$ ); only two areas (Chicago and Miami) have benchmark risks $\leq 0.1$. 


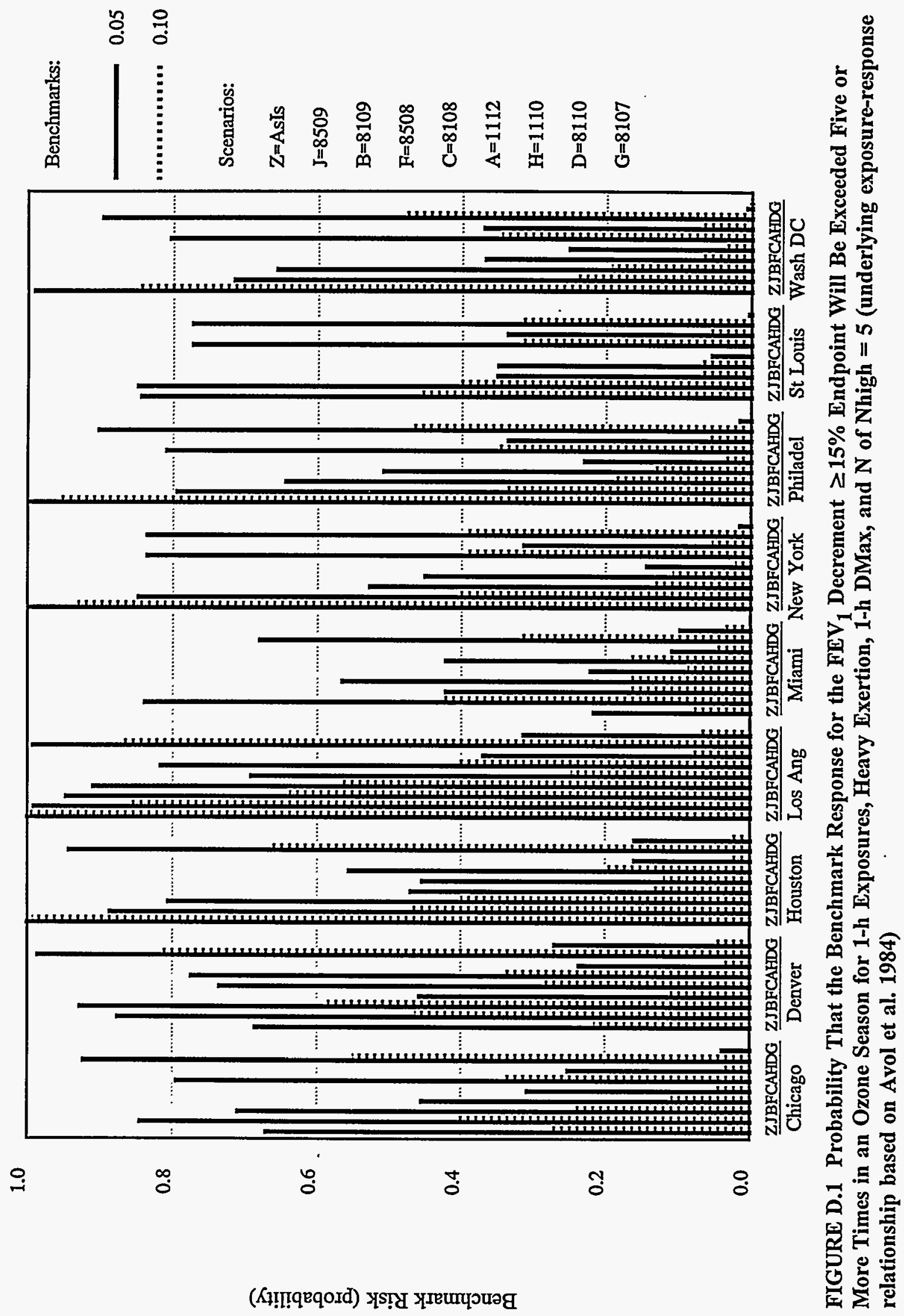




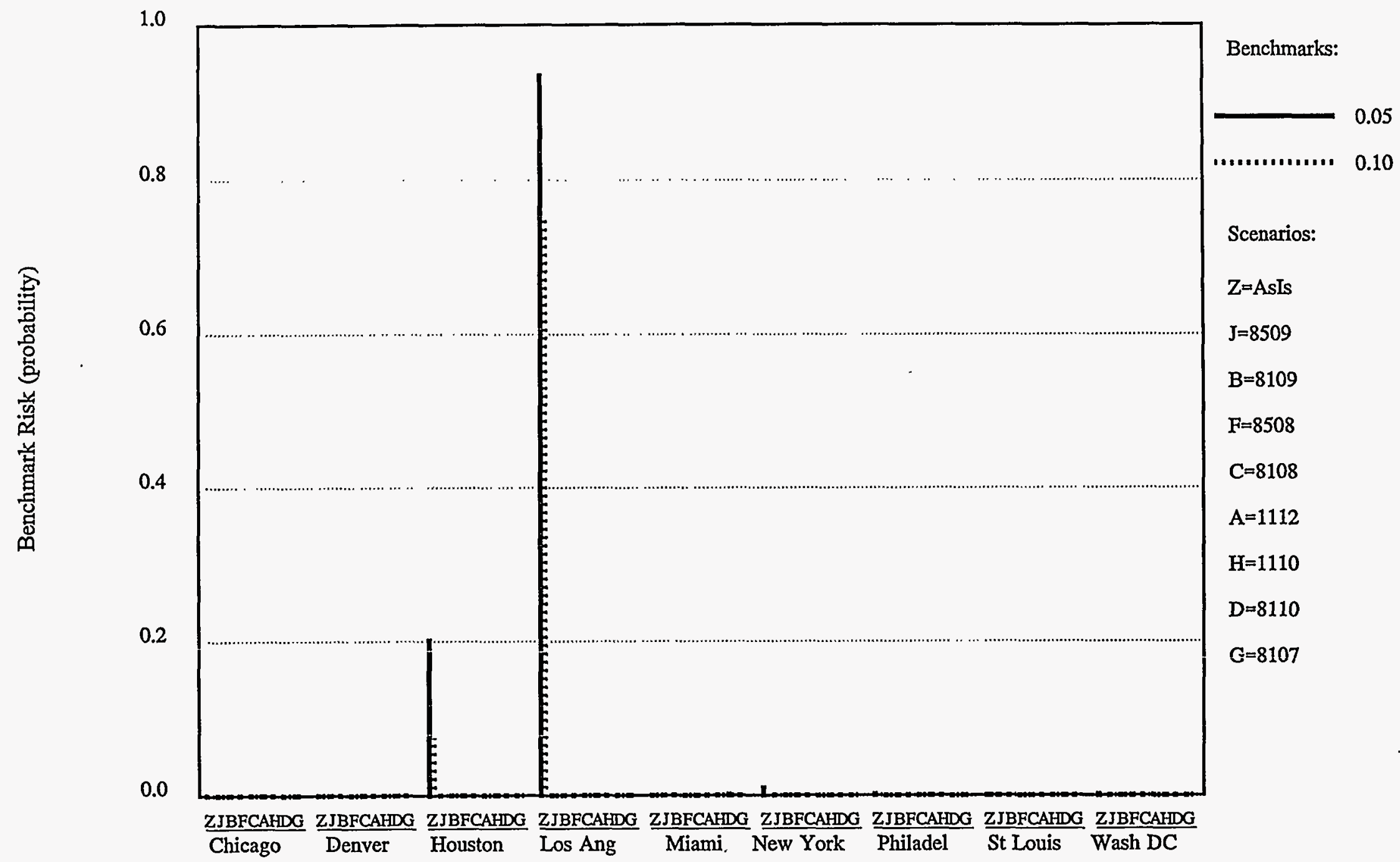

FIGURE D.2 Probability That the Benchmark Response for the $\mathrm{FEV}_{1}$ Decrement $\geq 15 \%$ Endpoint Will Be Exceeded Five or More Times in an Ozone Season for 1-h Exposures, Heavy Exertion, 1-h DMax, and $\mathrm{N}$ of Nhigh $=5$ (underlying exposure-response relationship based on Kulle et al. 1985) 


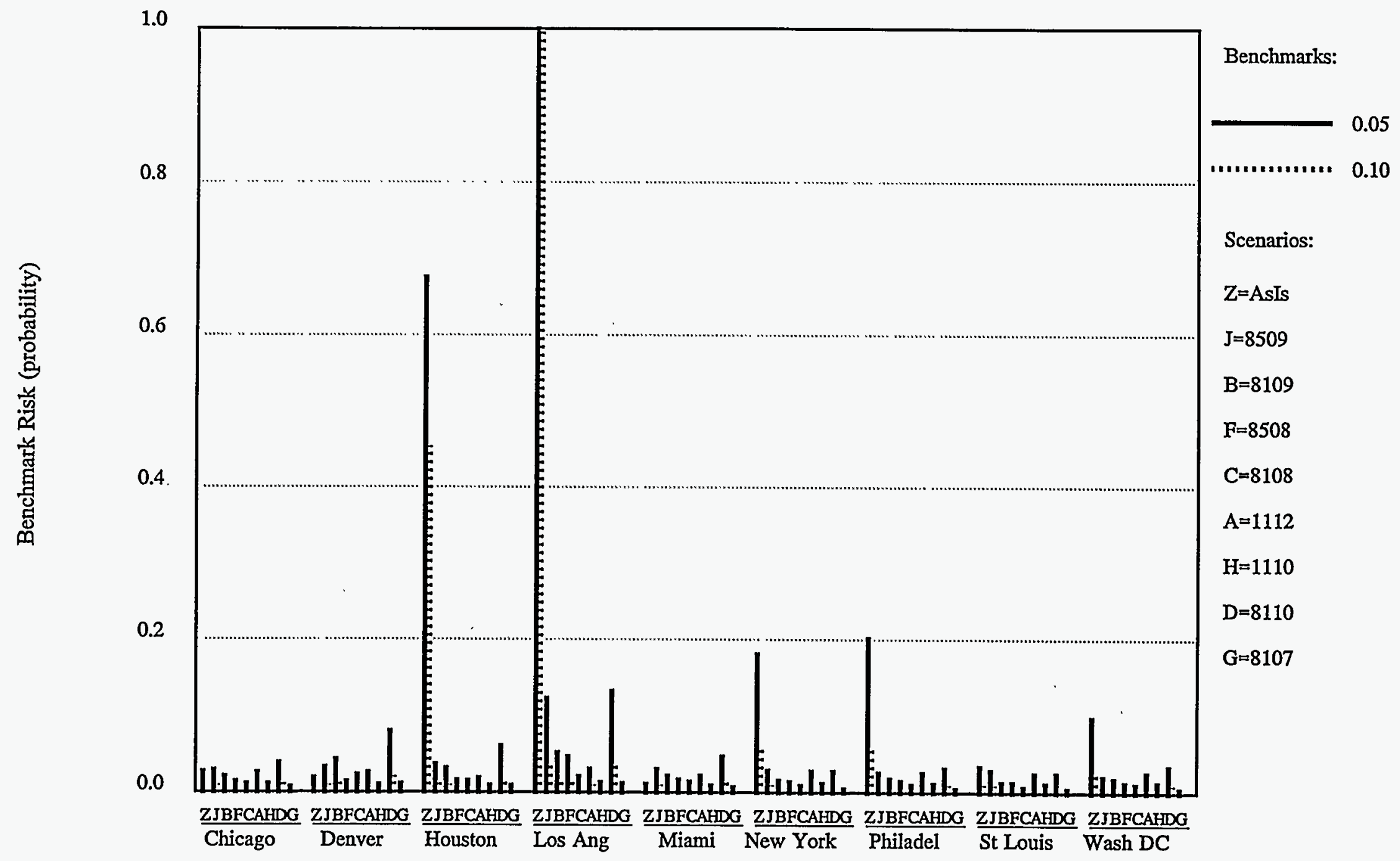

FIGURE D.3 Probability That the Benchmark Response for the FEV $_{1}$ Decrement $\geq 15 \%$ Endpoint Will Be Exceded Five or More Times in an Ozone Season for 1-h Exposures, Heavy Exertion, 1-h DMax, and $\mathrm{N}$ of Nhigh $=5$ (underlying exposure-response relationship based on McDonnell et al. 1983) 

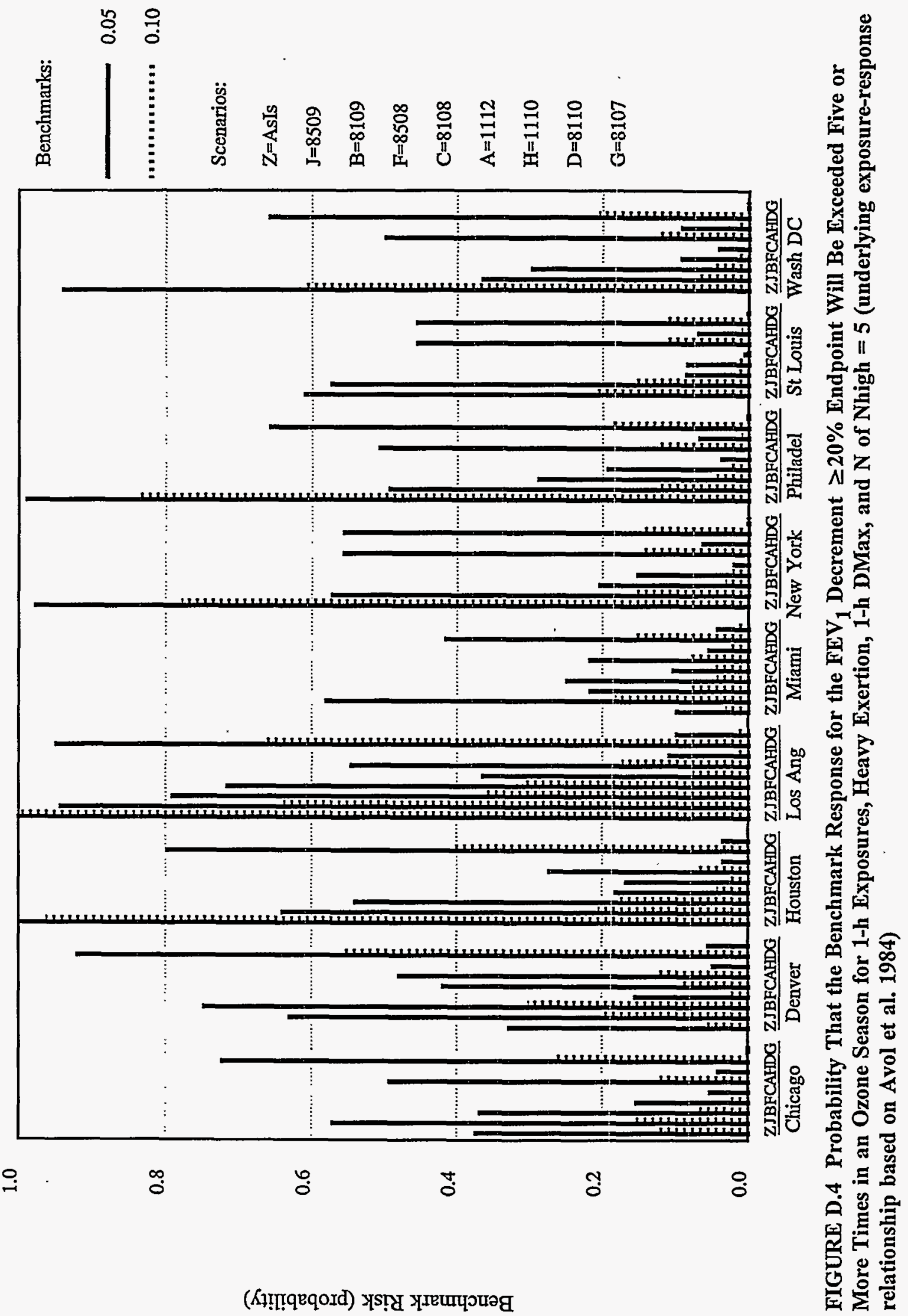

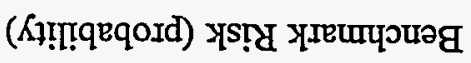




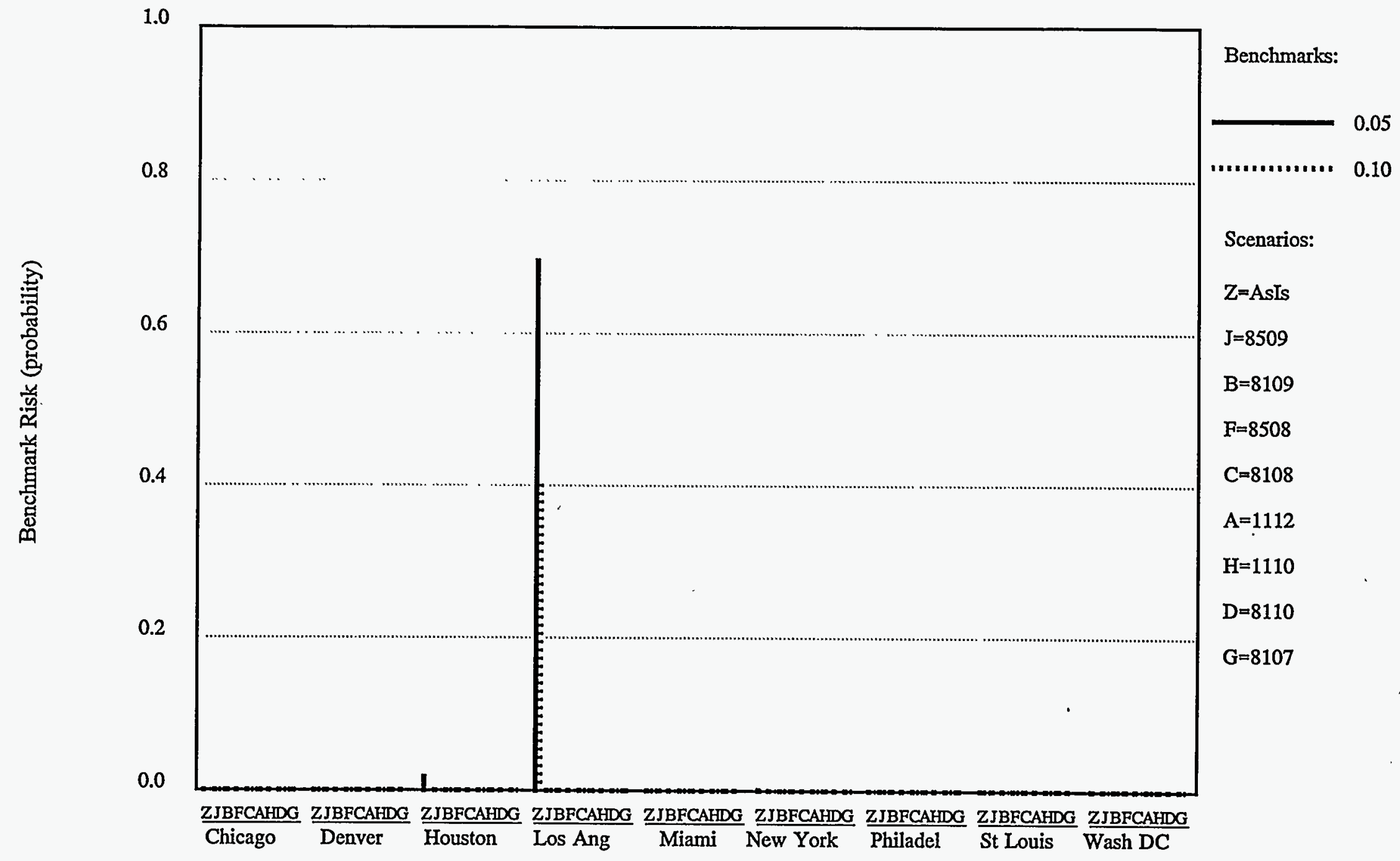

FIGURE D.5 Probability That the Benchmark Response for the FEV 1 Decrement $\geq 20 \%$ Endpoint Will Be Exceeded Five or More Times in an Ozone Season for 1-h Exposures, Heavy Exertion, 1-h DMax, and $\mathrm{N}$ of Nhigh $=5$ (underlying exposure-response relationship based on Kulle et al. 1985) 


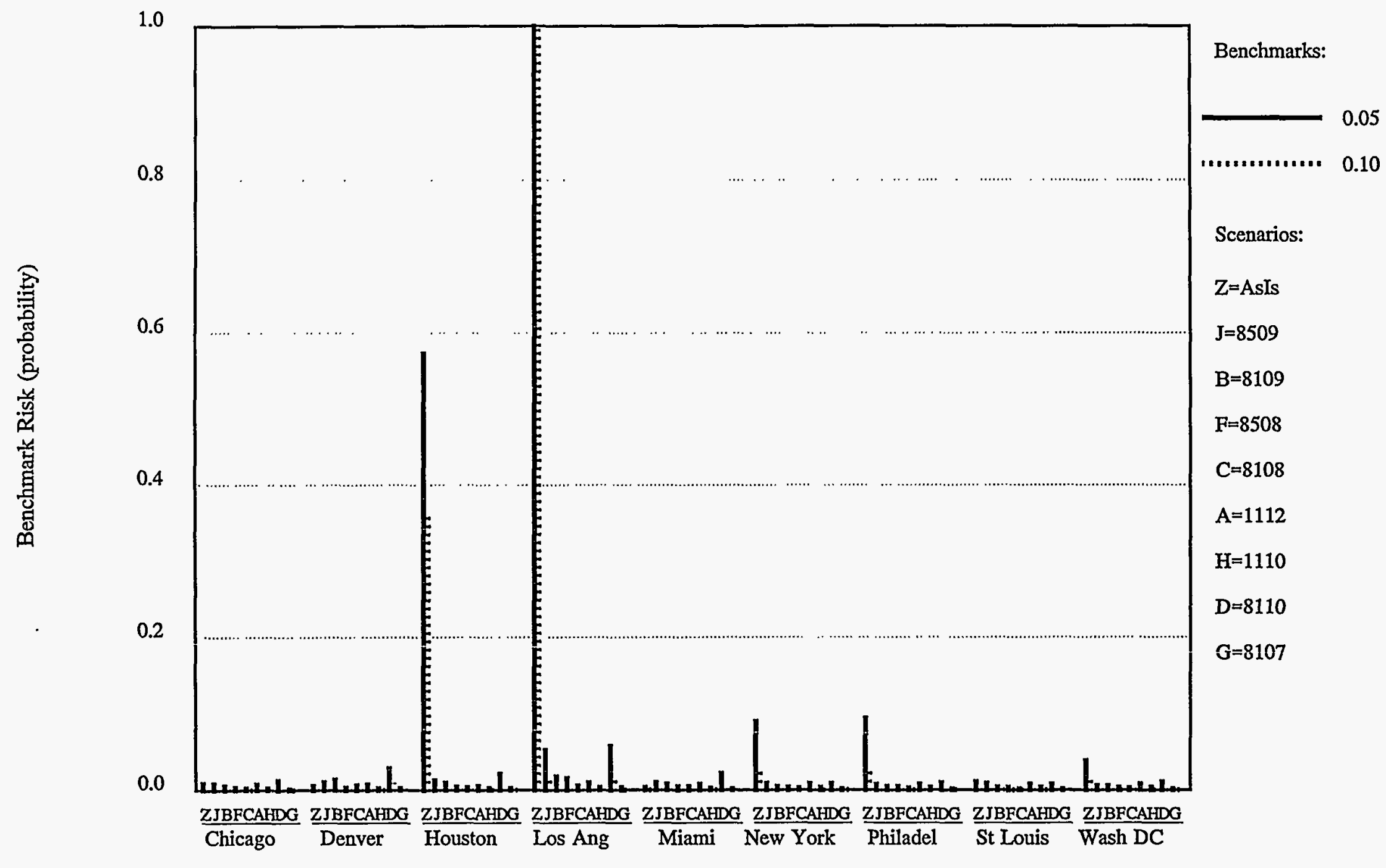

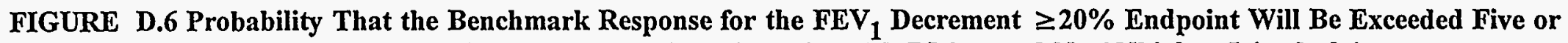
More Times in an Ozone Season for 1-h Exposures, Heavy Exertion, 1-h DMax, and $N$ of Nhigh $=5$ (underlying exposure-response relationship based on McDonnell et al. 1983) 


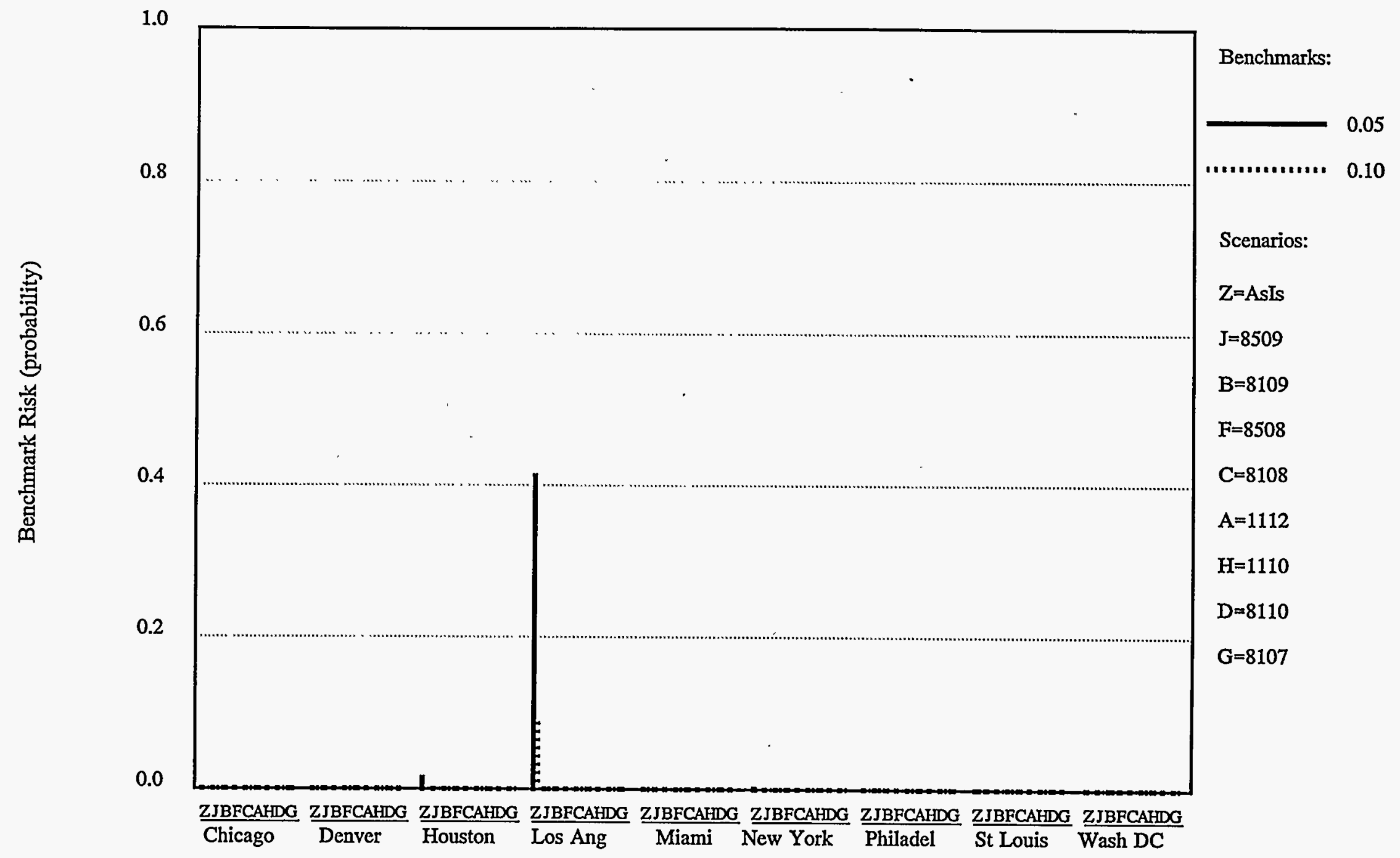

FIGURE D.7 Probability That the Benchmark Response for the Moderate-to-Severe Lower Respiratory Endpoint Will Be Exceded Five or More Times in an Ozone Season for 1-h Exposures, Heavy Exertion, 1-h DMax, and N of Nhigh $=5$ (underlying exposure-response relationship based on Avol et al. 1984) 


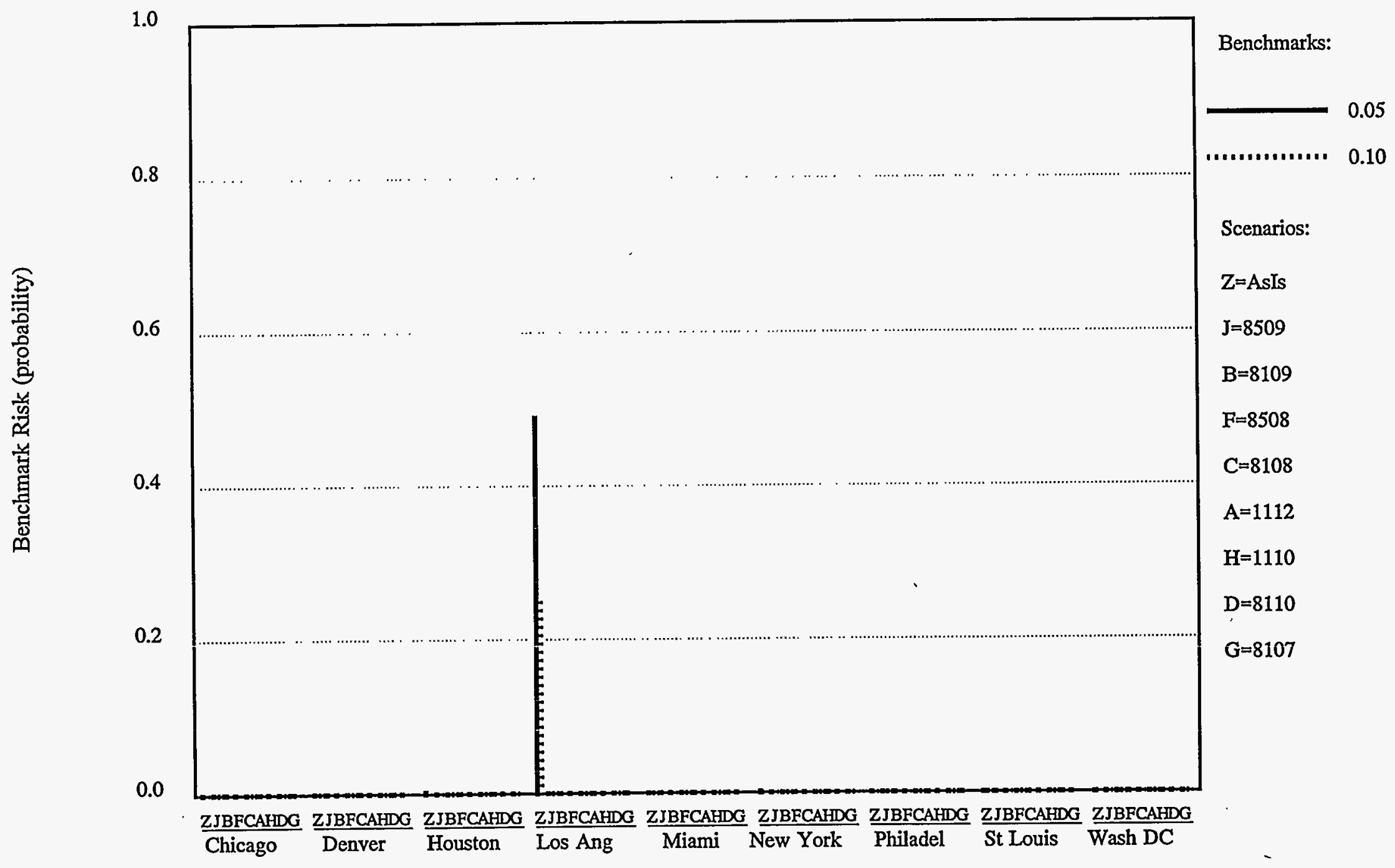

FIGURE D.8 Probability That the Benchmark Response for the Moderate-to-Severe Cough Endpoint Will Be Exceeded Five or More Times in an Ozone Season for 1-h Exposures, Heavy Exertion, 1-h DMax, and $\mathrm{N}$ of Nhigh $=5$ (underlying exposure-response relationship based on Kulle et al. 1985) 


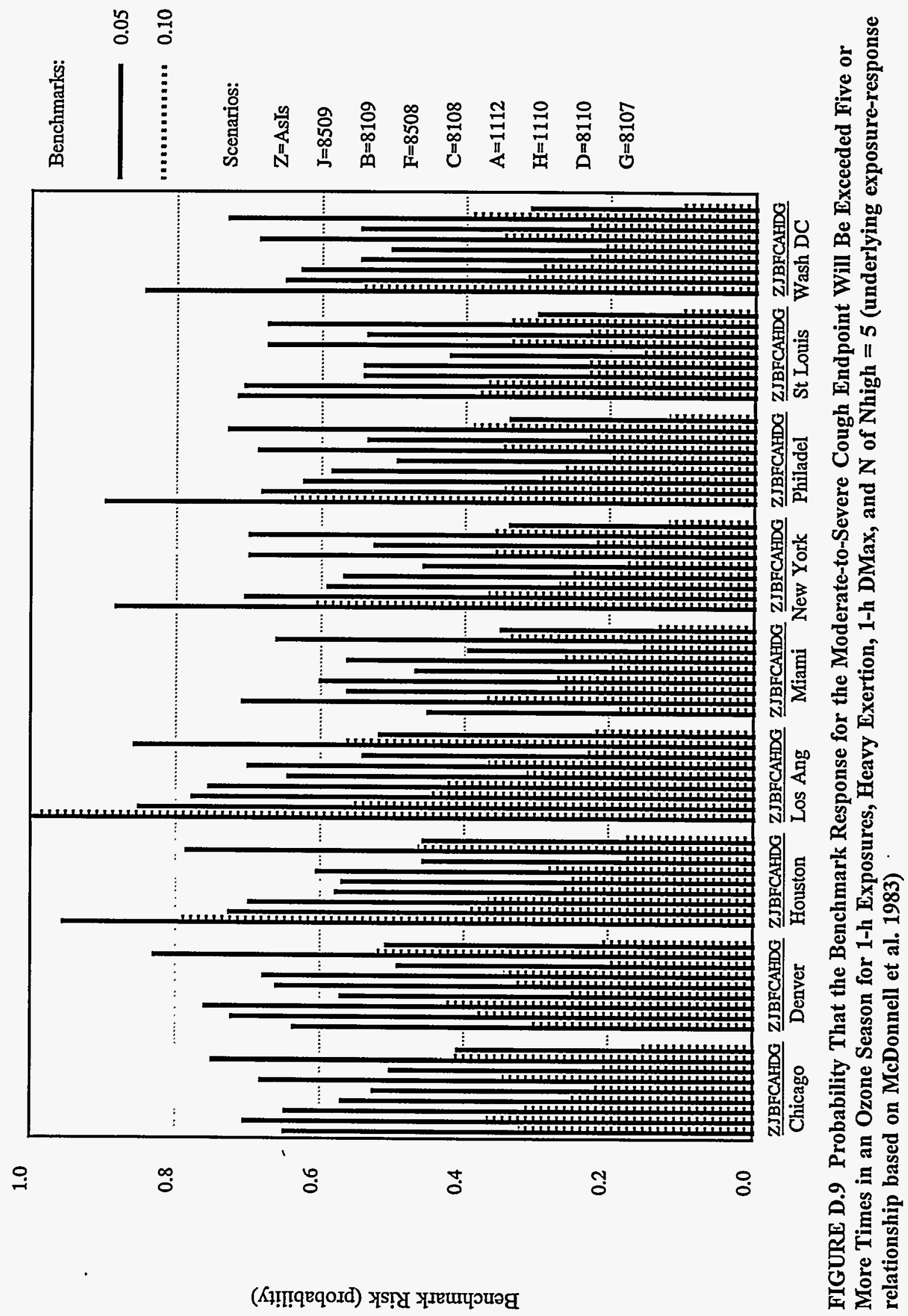




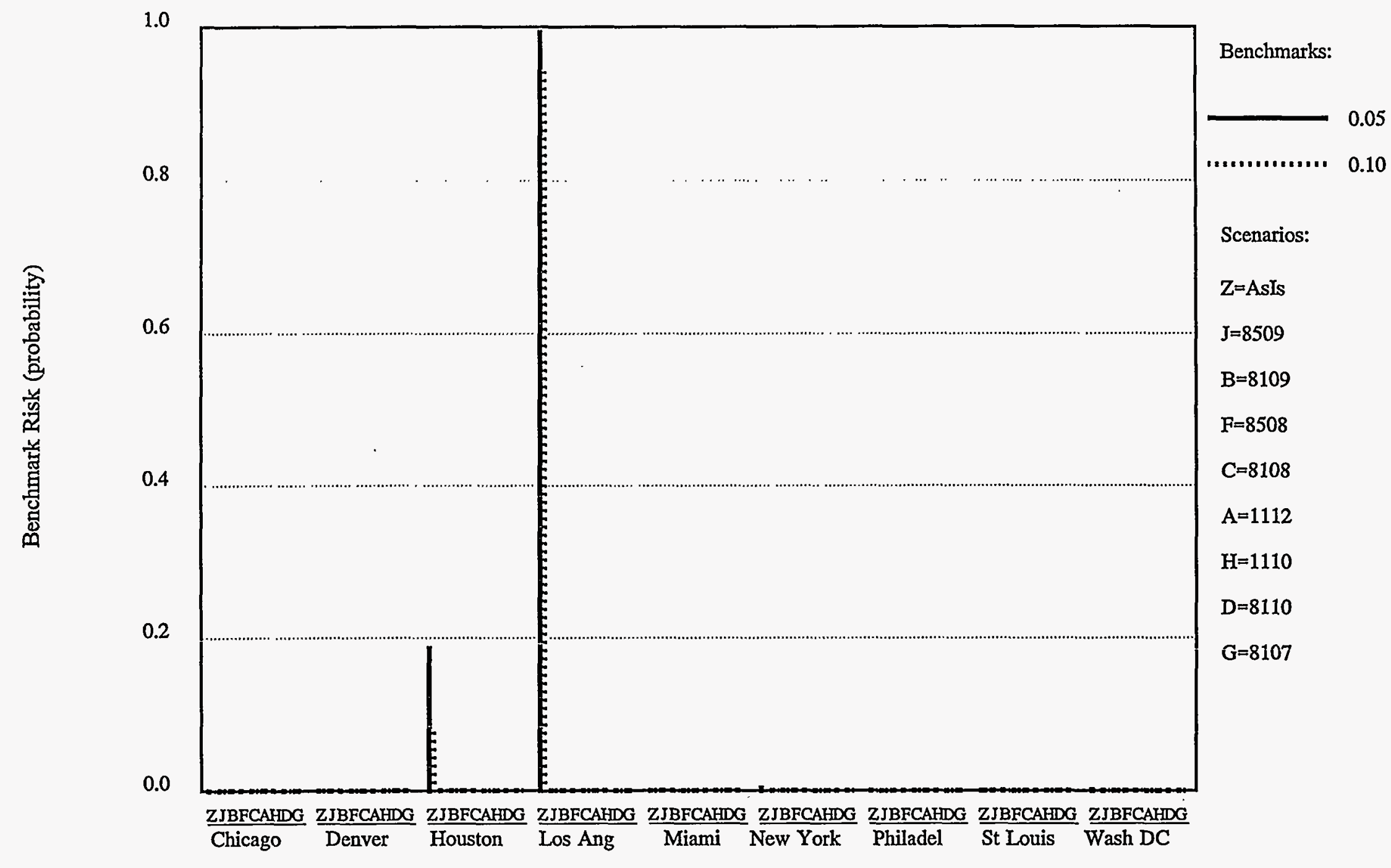

FIGURE D.10 Probability That the Benchmark Response for the Moderate-to-Severe Pain on Deep Inspiration Endpoint Will Be Exceeded Five or More Times in an Ozone Season for 1-h Exposures, Heavy Exertion, 1-h DMax, and N of Nhigh = 5 (underlying exposure-response relationship based on Kulle et al. 1985) 


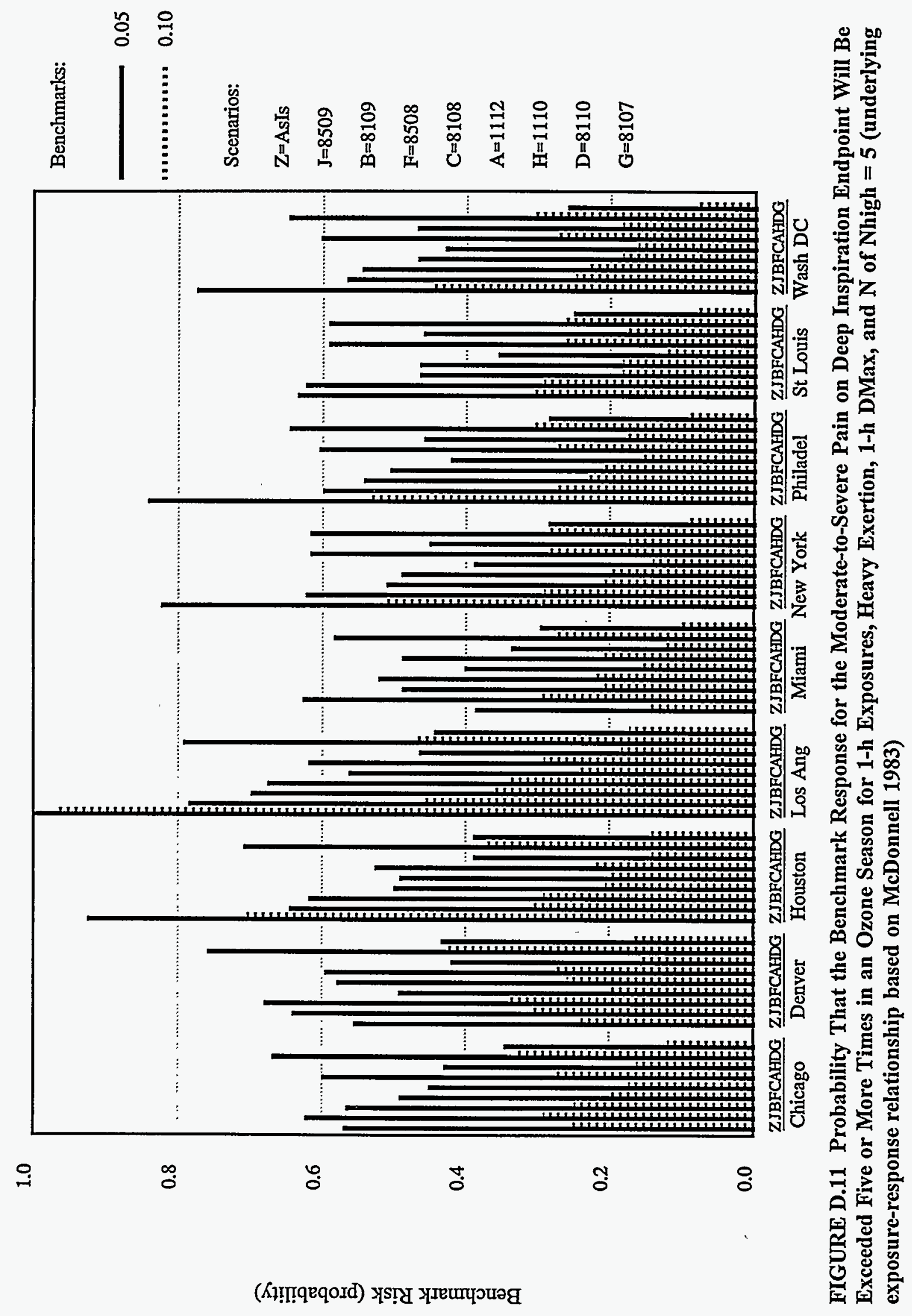




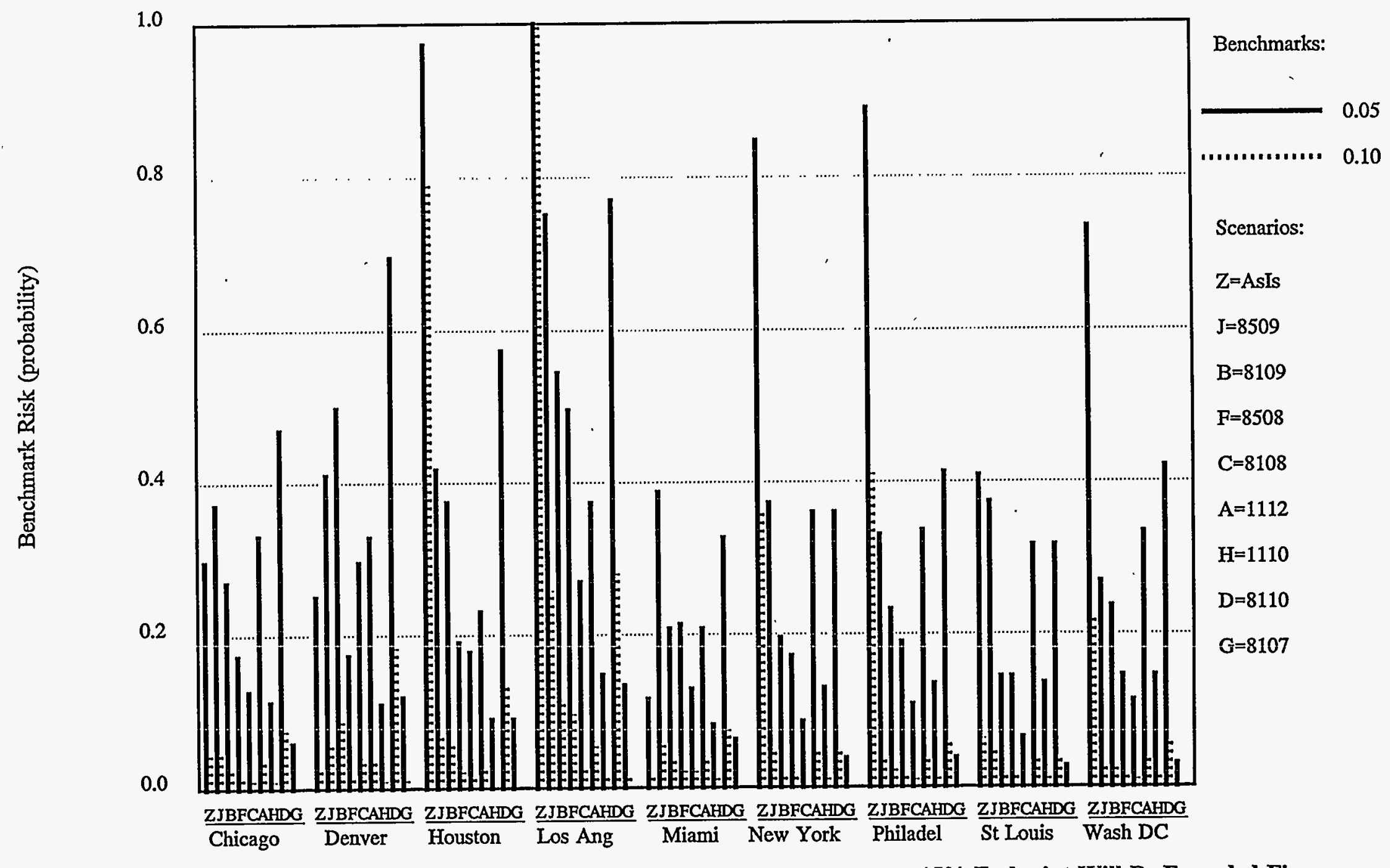

FIGURE D.12 Probability That the Benchmark Response for the FEV 1 Decrement $\geq 15 \%$ Endpoint Will Be Exceeded Five or More Times in an Ozone Season for 1-h Exposures, Moderate Exertion, 1-h DMax, and $\mathrm{N}$ of Nhigh $=5$ (underlying exposureresponse relationship based on Seal et al. 1993) 


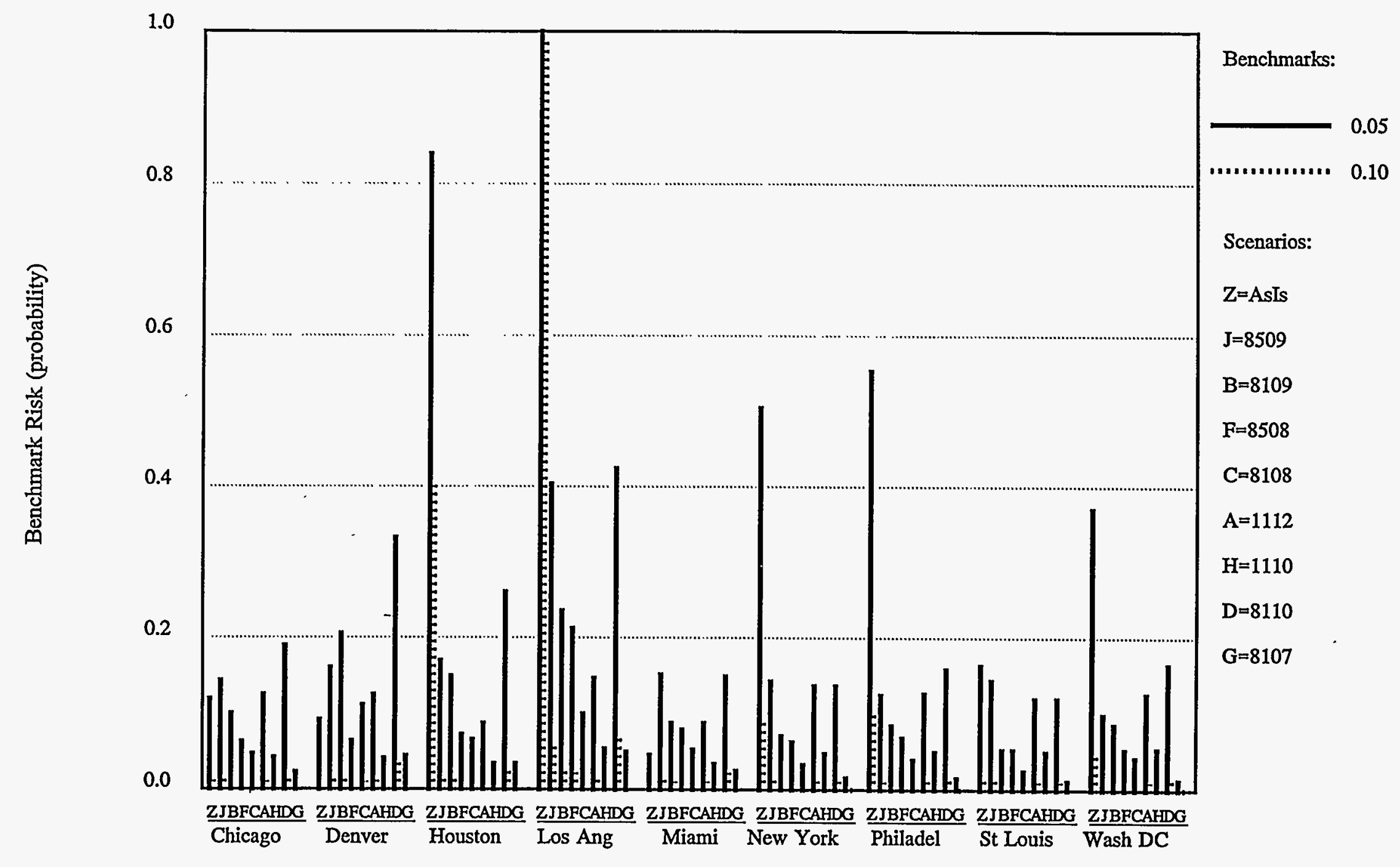

FIGURE D.13 Probability That the Benchmark Response for the $\mathrm{FEV}_{1}$ Decrement $\geq 20 \%$ Endpoint Will Be Exceeded Five or More Times in an Ozone Season for 1-h Exposures, Moderate Exertion, 1-h DMax, and $\mathrm{N}$ of Nhigh $=5$ (underlying exposureresponse relationship based on Seal et al. 1993) 


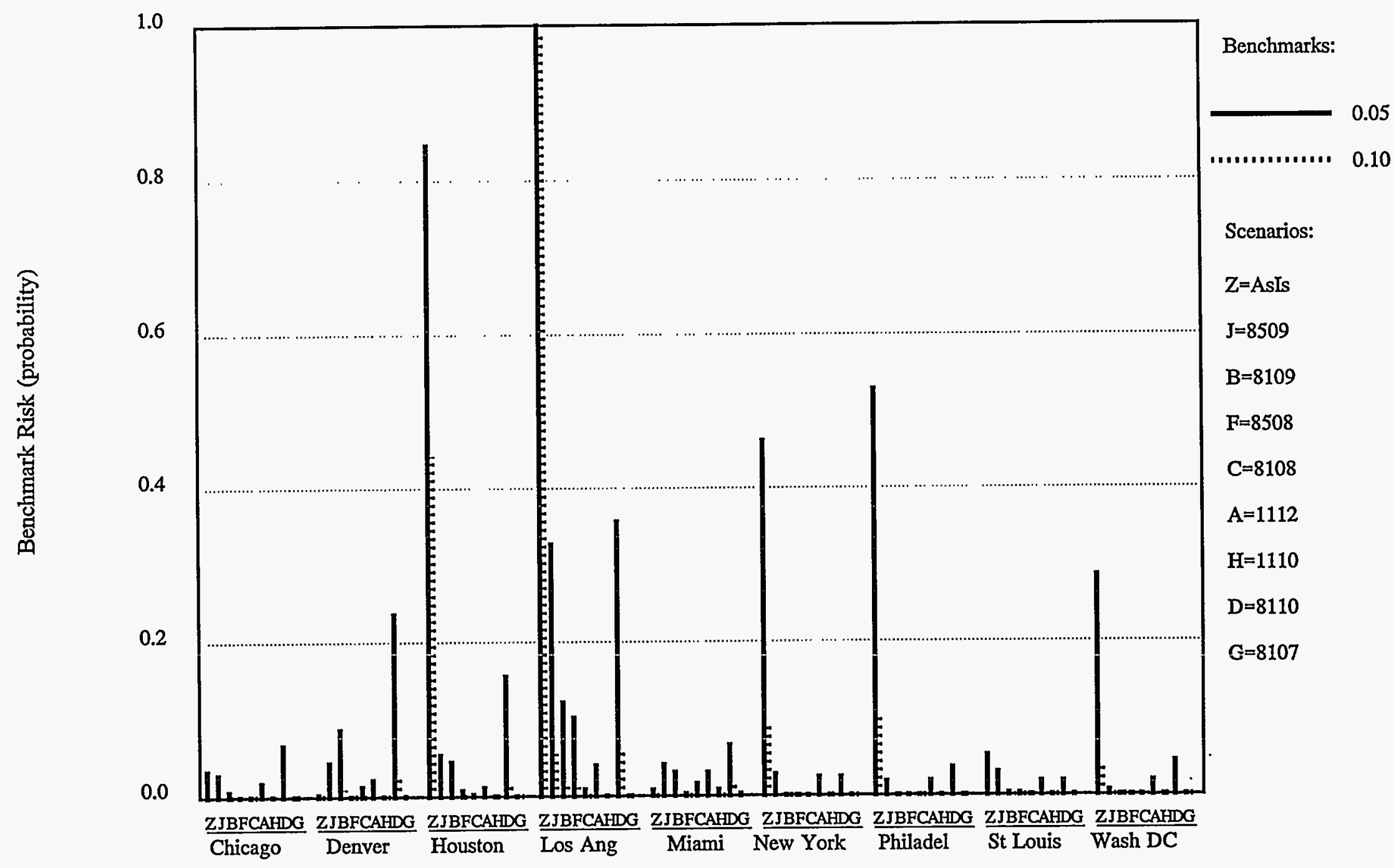

FIGURE D.14 Probability That the Benchmark Response for the Moderate-to-Severe Cough Endpoint Will Be Exceeded Five or More Times in an Ozone Season for 1-h Exposures, Moderate Exertion, 1-h DMax, and $\mathrm{N}$ of Nhigh $=5$ (underlying exposureresponse relationship based on Seal et al. 1993) 


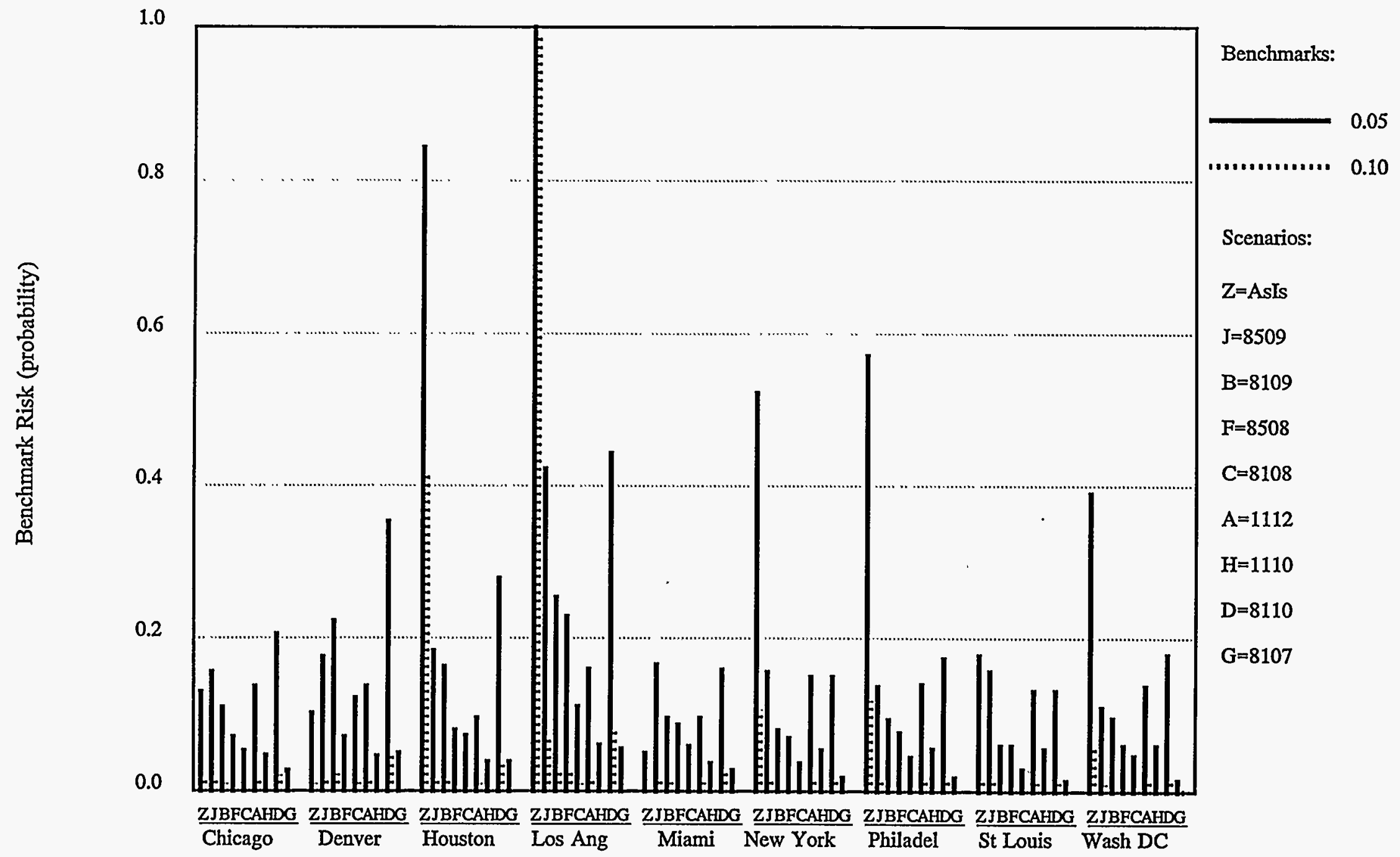

FIGURE D.15 Probability That the Benchmark Response for the Moderate-to-Severe Pain on Deep Inspiration Endpoint Will Be Exceeded Five or More Times in an Ozone Season for 1-h Exposures, Moderate Exertion, 1-h DMax, and N of Nhigh = 5 (underlying exposure-response relationship based on Seal et al. 1993) 


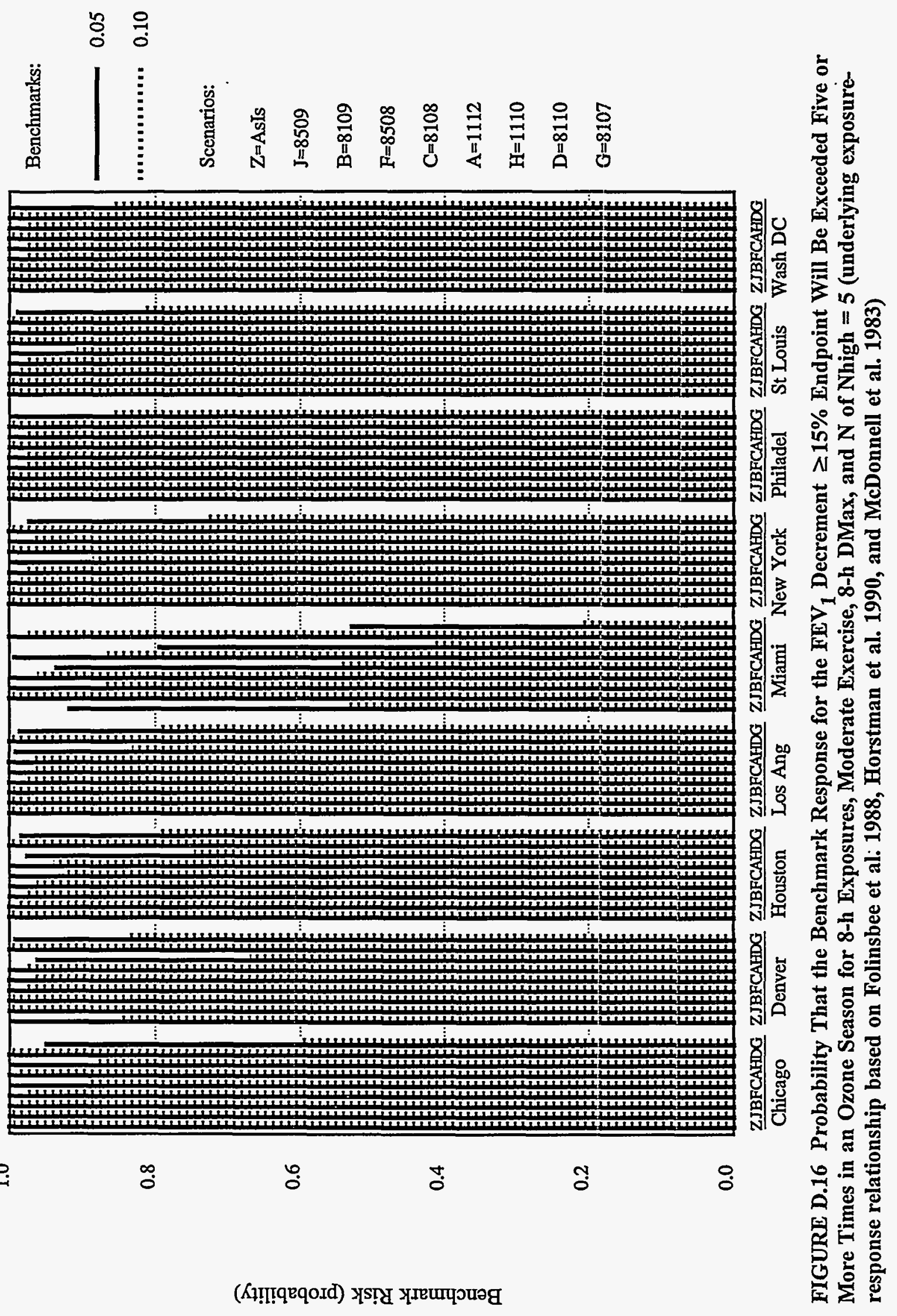




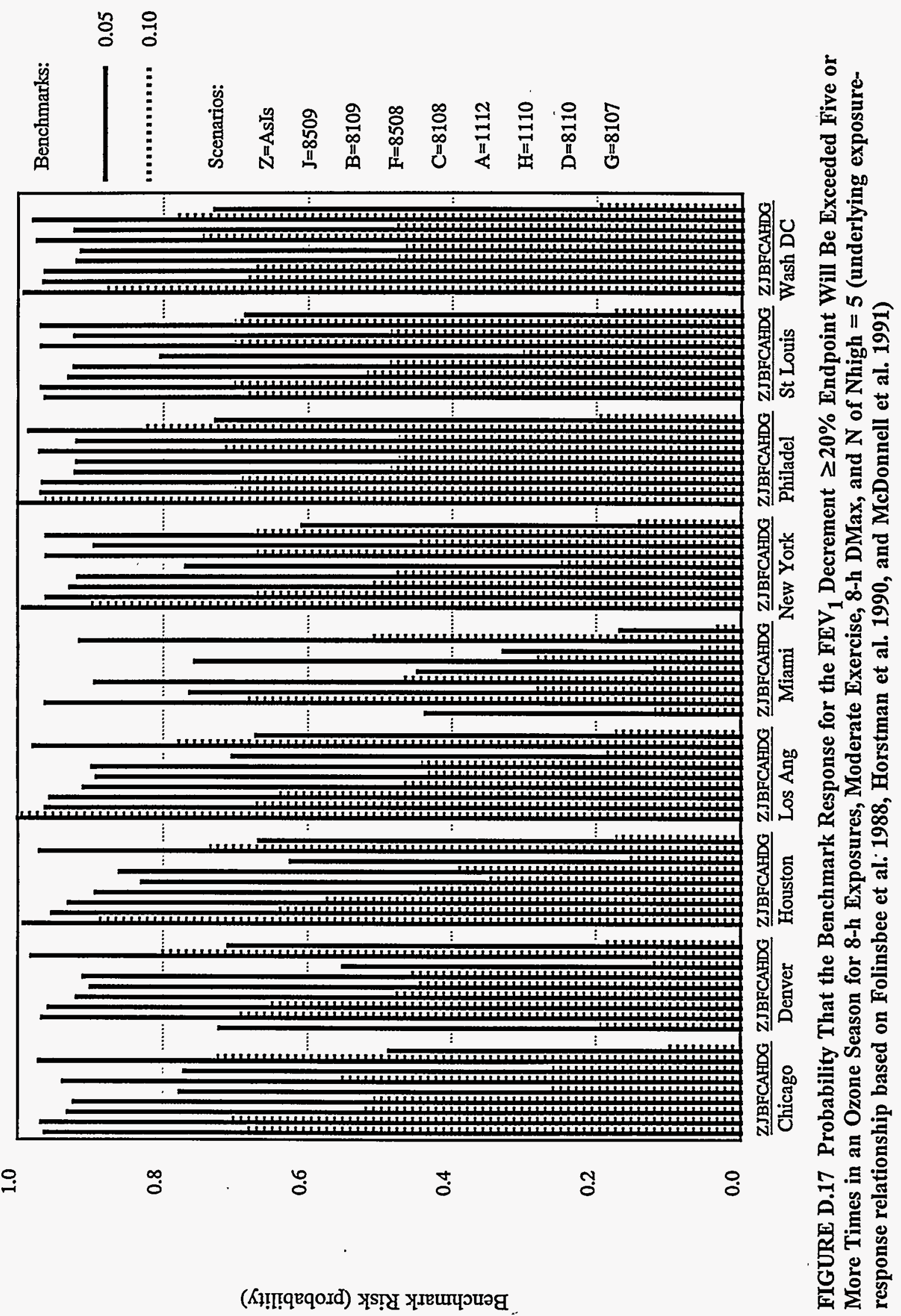




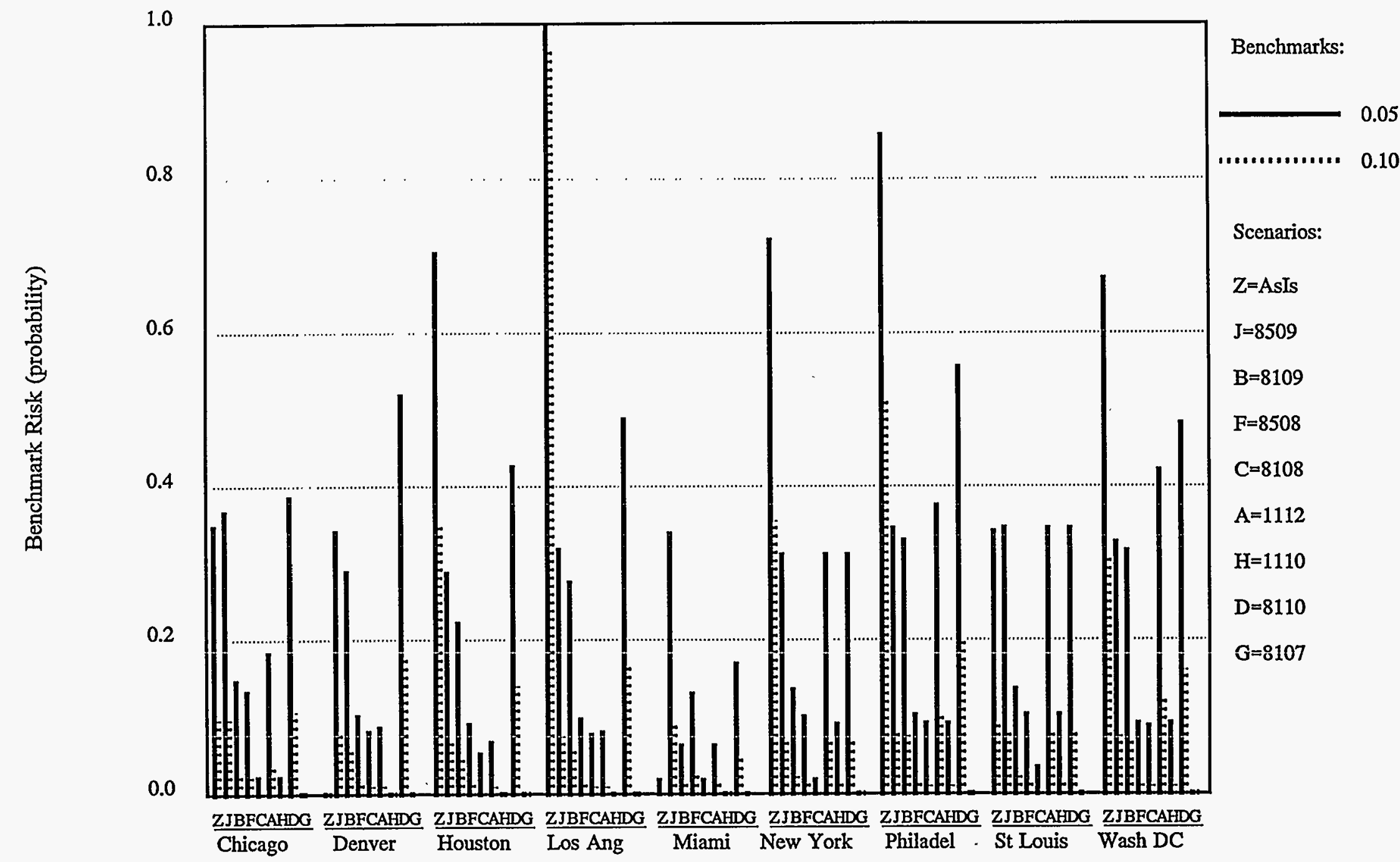

FIGURE D.18 Probability That the Benchmark Response for the Moderate-to-Severe Cough Endpoint Will Be Exceeded Five or More Times in an Ozone Season for 8-h Exposures, Moderate Exercise, 8-h DMax, and N of Nhigh $=5$ (underlying exposureresponse relationship based on Folinsbee et al: 1988, Horstman et al. 1990, and McDonnell et al. 1991) 


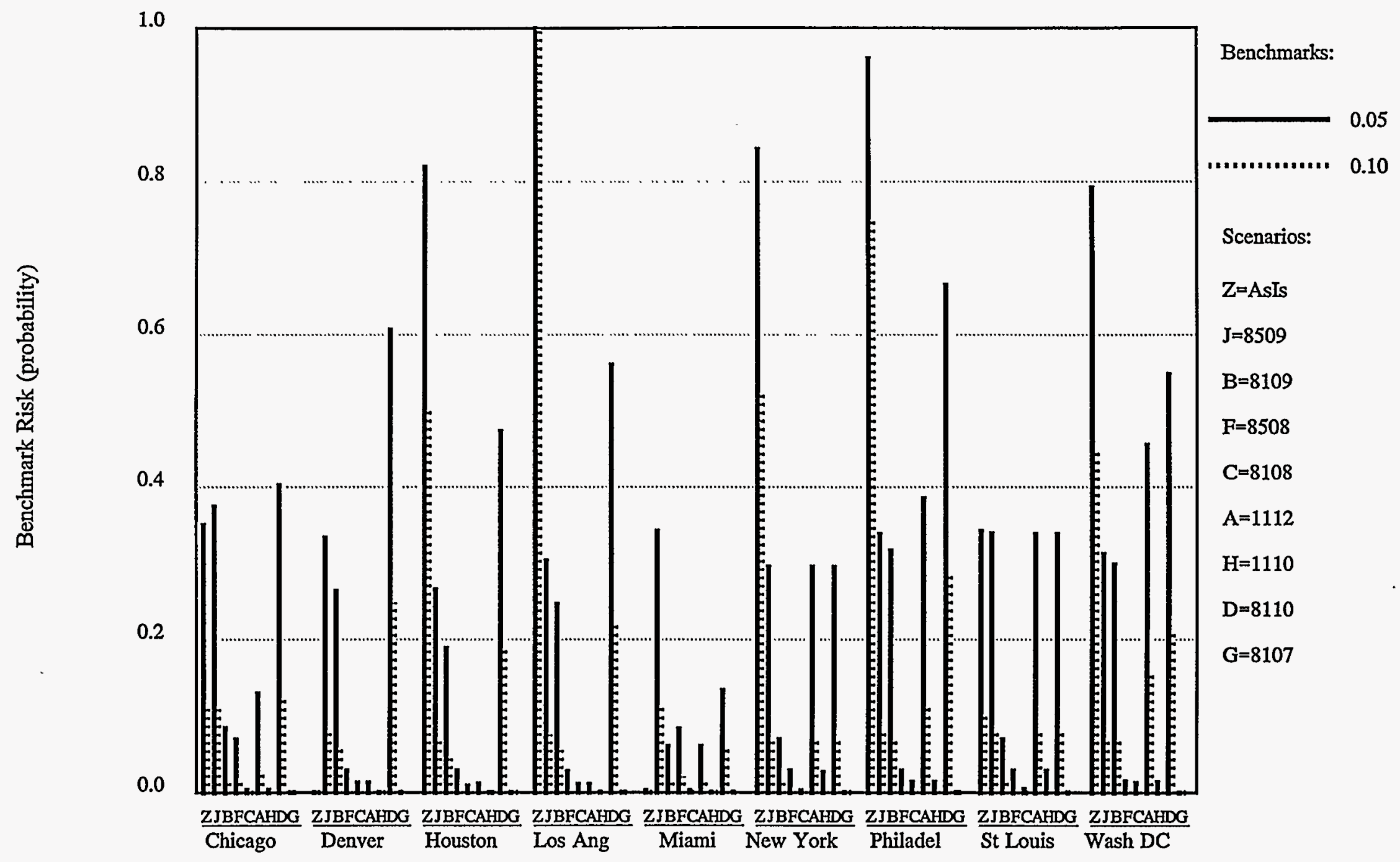

FIGURE D.19 Probability That the Benchmark Response for the Moderate-to-Severe Pain on Deep Inspiration Endpoint Will Be Exceeded Five or More Times in an Ozone Season for 8-h Exposures, Moderate Exercise, 8-h DMax, and $\mathrm{N}$ of Nhigh $=5$ (underlying exposure-response relationship based on Folinsbee et al. 1988, Horstman et al. 1990, and McDonnell et al. 1991) 
TABLE D.1 Summary of Benchmark Risk Results: Number of Urban Areas Having Benchmark Risks $\leq 0.1,0.2$, and 0.3 for AsIs Air Quality and Just Attaining Eight Alternative NAAQS for 5 and $10 \%$ Benchmarks $^{\mathrm{a}}$

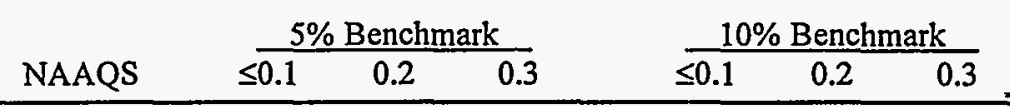

(1) $\mathrm{FEV}_{1}$ Decrements $\geq 10 \%$, 1-h Exposures, Heavy Exertion, Highest of 1-h Daily Maximum Concentrations (underlying exposure-response relationship based on Avol et al. 1984)

$$
\begin{aligned}
& Z=\text { As-Is } \\
& J=8509 \\
& B=8109 \\
& F=8508 \\
& C=8108 \\
& A=1112 \\
& H=1110 \\
& D=8110 \\
& G=8107
\end{aligned}
$$

$\begin{array}{lll}-b & - & - \\ - & - & - \\ - & - & - \\ - & - & - \\ - & - & - \\ - & - & - \\ - & - & - \\ - & - & - \\ - & - & -\end{array}$

$\begin{array}{lll}- & - & - \\ - & - & - \\ - & - & - \\ - & - & - \\ - & - & - \\ - & - & - \\ - & - & - \\ - & - & - \\ 4 & 4 & 5\end{array}$

(2) $\mathrm{FEV}_{1}$ Decrements $\geq 10 \%$, 1-h Exposures, Heavy Exertion, Fifth Highest of 1-h Daily Maximum Concentrations (underlying exposure-response relationship based on Avol et al. 1984)

$\begin{array}{lllllll}Z=A s-I s & - & - & - & - & - & 1 \\ J=8509 & - & - & - & - & - & - \\ B=8109 & - & - & - & - & - & - \\ F=8508 & - & - & - & - & - & - \\ C=8108 & - & - & - & 1 & 2 & 5 \\ A=1112 & - & - & - & - & - & - \\ H=1110 & - & - & - & - & 2 & 4 \\ D=8110 & - & - & - & - & - & - \\ G=8107 & 1 & 2 & 5 & 5 & 7 & 8\end{array}$

(3) FEV 1 Decrements $\geq 10 \%$, 1-h Exposures, Heavy Exertion, Highest of 1-h Daily Maximum Concentrations (underlying exposure-response relationship based on Kulle et al. 1985)

$\begin{array}{lllllll}\mathrm{Z}=\mathrm{As}-\mathrm{Is} & 3 & 4 & 4 & 4 & 5 & 5 \\ \mathrm{~J}=8509 & 7 & 7 & 8 & 8 & 9 & 9 \\ \mathrm{~B}=8109 & 9 & 9 & 9 & 9 & 9 & 9 \\ \mathrm{~F}=8508 & 9 & 9 & 9 & 9 & 9 & 9 \\ \mathrm{C}=8108 & 9 & 9 & 9 & 9 & 9 & 9 \\ \mathrm{~A}=1112 & 9 & 9 & 9 & 9 & 9 & 9 \\ \mathrm{H}=1110 & 9 & 9 & 9 & 9 & 9 & 9 \\ \mathrm{D}=8110 & 7 & 7 & 7 & 7 & 9 & 9 \\ \mathrm{G}=8107 & 9 & 9 & 9 & 9 & 9 & 9\end{array}$


TABLE D.1 (Cont.)

\begin{tabular}{|c|c|c|c|c|c|c|}
\hline \multirow[b]{2}{*}{ NAAQS } & \multicolumn{3}{|c|}{$5 \%$ Benchmark } & \multicolumn{3}{|c|}{$10 \%$ Benchmark } \\
\hline & $\leq 0.1$ & 0.2 & 0.3 & $\leq 0.1$ & 0.2 & 0.3 \\
\hline
\end{tabular}

(4) FEV 1 Decrements $\geq 10 \%$, 1-h Exposures, Heavy Exertion, Fifth Highest of 1-h Daily Maximum Concentrations (underlying exposure-response relationship based on Kulle et al. 1985)

$\begin{array}{lllllll}Z=\text { As-Is } & 5 & 7 & 7 & 7 & 7 & 7 \\ \mathrm{~J}=8509 & 9 & 9 & 9 & 9 & 9 & 9 \\ \mathrm{~B}=8109 & 9 & 9 & 9 & 9 & 9 & 9 \\ \mathrm{~F}=8508 & 9 & 9 & 9 & 9 & 9 & 9 \\ \mathrm{C}=8108 & 9 & 9 & 9 & 9 & 9 & 9 \\ \mathrm{~A}=1112 & 9 & 9 & 9 & 9 & 9 & 9 \\ \mathrm{H}=1110 & 9 & 9 & 9 & 9 & 9 & 9 \\ \mathrm{D}=8110 & 9 & 9 & 9 & 9 & 9 & 9 \\ \mathrm{G}=8107 & 9 & 9 & 9 & 9 & 9 & 9\end{array}$

(5) $\mathrm{FEV}_{1}$ Decrements $\geq 10 \%$, 1-h Exposures, Heavy Exertion, Highest of 1-h Daily Maximum Concentrations (underlying exposure-response relationship based on McDonnell et al. 1983)

$\begin{array}{lllllll}Z=A s-I s & - & - & - & - & 2 & 3 \\ J=8509 & - & - & - & - & 3 & 6 \\ B=8109 & - & - & 4 & 5 & 5 & 7 \\ F=8508 & - & - & 5 & 5 & 7 & 9 \\ C=8108 & - & 5 & 6 & 6 & 8 & 9 \\ A=1112 & - & - & - & 1 & 6 & 8 \\ H=1110 & - & 1 & 8 & 8 & 9 & 9 \\ D=8110 & - & - & - & - & 2 & 5 \\ G=8107 & 4 & 5 & 9 & 9 & 9 & 9\end{array}$

(6) $\mathrm{FEV}_{1}$ Decrements $\geq 10 \%, 1$-h Exposures, Heavy Exertion, Fifth Highest of 1-h Daily Maximum Concentrations (underlying exposure-response relationship based on McDonnell et al. 1983)

$\begin{array}{lllllll}\mathrm{Z}=\mathrm{As}-\mathrm{Is} & - & 1 & 3 & 3 & 4 & 5 \\ \mathrm{~J}=8509 & - & - & 5 & 6 & 8 & 8 \\ \mathrm{~B}=8109 & - & 3 & 7 & 7 & 9 & 9 \\ \mathrm{~F}=8508 & - & 8 & 8 & 8 & 9 & 9 \\ \mathrm{C}=8108 & 1 & 7 & 9 & 9 & 9 & 9 \\ \mathrm{~A}=1112 & - & 1 & 9 & 9 & 9 & 9 \\ \mathrm{H}=1110 & 1 & 9 & 9 & 9 & 9 & 9 \\ \mathrm{D}=8110 & - & - & 3 & 3 & 7 & 8 \\ \mathrm{G}=8107 & 6 & 9 & 9 & 9 & 9 & 9\end{array}$


TABLE D.1 (Cont.)

\begin{tabular}{|c|c|c|c|c|c|c|}
\hline \multirow[b]{2}{*}{ NAAQS } & \multicolumn{3}{|c|}{$5 \%$ Benchmark } & \multicolumn{3}{|c|}{$10 \%$ Benchmark } \\
\hline & $\leq \overline{0.1}$ & 0.2 & $\overline{0.3}$ & $\leq 0.1$ & 0.2 & 0.3 \\
\hline
\end{tabular}

(7) $\mathrm{FEV}_{1}$ Decrements $\geq 15 \%$, 1-h Exposures, Heavy Exertion, Highest of 1-h Daily Maximum Concentrations (underlying exposure-response relationship based on Avol et al. 1984)

$$
\begin{aligned}
& Z=A s-I s \\
& J=8509 \\
& B=8109 \\
& F=8508 \\
& C=8108 \\
& A=1112 \\
& H=1110 \\
& D=8110 \\
& G=8107
\end{aligned}
$$

$\begin{array}{llllll}- & - & - & - & - & - \\ - & - & - & - & - & - \\ - & - & - & - & - & 1 \\ - & - & - & - & - & 1 \\ - & - & - & 2 & 5 & 5 \\ - & - & - & - & - & - \\ - & - & - & - & 6 & 6 \\ - & - & - & - & - & - \\ 4 & 4 & 5 & 5 & 8 & 8\end{array}$

(8) $\mathrm{FEV}_{1}$ Decrements $\geq 15 \%$, 1-h Exposures, Heavy Exertion, Fifth Highest of 1-h Daily Maximum Concentrations (underlying exposure-response relationship based on Avol et al. 1984)

$\begin{array}{lllllll}Z=A s-I s & - & - & 1 & 1 & 1 & 3 \\ J=8509 & - & - & - & - & - & 1 \\ \text { B }=8109 & - & - & - & 1 & 5 & 6 \\ \text { F }=8508 & - & - & - & 2 & 8 & 8 \\ \text { C }=8108 & 1 & 2 & 5 & 6 & 7 & 9 \\ \text { A }=1112 & - & - & - & - & 2 & 2 \\ H=1110 & - & 2 & 4 & 9 & 9 & 9 \\ \text { D }=8110 & - & - & - & - & - & - \\ \text { G }=8107 & 6 & 7 & 8 & 9 & 9 & 9\end{array}$

(9) FEV 1 Decrements $\geq 15 \%$, 1-h Exposures, Heavy Exertion, Highest of 1-h Daily Maximum Concentrations (underlying exposure-response relationship based on Kulle et al. 1985)

$\begin{array}{lllllll}\mathrm{Z}=\mathrm{As}-\mathrm{Is} & 5 & 6 & 6 & 6 & 6 & 7 \\ \mathrm{~J}=8509 & 9 & 9 & 9 & 9 & 9 & 9 \\ \mathrm{~B}=8109 & 9 & 9 & 9 & 9 & 9 & 9 \\ \mathrm{~F}=8508 & 9 & 9 & 9 & 9 & 9 & 9 \\ \mathrm{C}=8108 & 9 & 9 & 9 & 9 & 9 & 9 \\ \mathrm{~A}=1112 & 9 & 9 & 9 & 9 & 9 & 9 \\ \mathrm{H}=1110 & 9 & 9 & 9 & 9 & 9 & 9 \\ \mathrm{D}=8110 & 9 & 9 & 9 & 9 & 9 & 9 \\ \mathrm{G}=8107 & 9 & 9 & 9 & 9 & 9 & 9\end{array}$


TABLE D.1 (Cont.)

\begin{tabular}{|c|c|c|c|c|c|c|}
\hline \multirow[b]{2}{*}{ NAAQS } & \multicolumn{3}{|c|}{$5 \%$ Benchmark } & \multicolumn{3}{|c|}{$10 \%$ Benchmark } \\
\hline & $\leq 0.1$ & 0.2 & 0.3 & $\leq 0.1$ & 0.2 & 0.3 \\
\hline
\end{tabular}

(10) FEV 1 Decrements $\geq 15 \%$, 1-h Exposures, Heavy Exertion, Fifth Highest of 1-h Daily Maximum Concentrations (underlying exposure-response relationship based on Kulle et al. 1985)

$\begin{array}{lllllll}\mathrm{Z}=\text { As-Is } & 7 & 7 & 8 & 8 & 8 & 8 \\ \mathrm{~J}=8509 & 9 & 9 & 9 & 9 & 9 & 9 \\ \mathrm{~B}=8109 & 9 & 9 & 9 & 9 & 9 & 9 \\ \mathrm{~F}=8508 & 9 & 9 & 9 & 9 & 9 & 9 \\ \mathrm{C}=8108 & 9 & 9 & 9 & 9 & 9 & 9 \\ \mathrm{~A}=1112 & 9 & 9 & 9 & 9 & 9 & 9 \\ \mathrm{H}=1110 & 9 & 9 & 9 & 9 & 9 & 9 \\ \mathrm{D}=8110 & 9 & 9 & 9 & 9 & 9 & 9 \\ \mathrm{G}=8107 & 9 & 9 & 9 & 9 & 9 & 9\end{array}$

(11) $\mathrm{FEV}_{1}$ Decrements $\geq 15 \%$, 1-h Exposures, Heavy Exertion, Highest of 1-h Daily Maximum Concentrations (underlying exposure-response relationship based on McDonnell et al. 1983)

$\begin{array}{lllllll}\mathrm{Z}=\mathrm{As} \text {-Is } & 3 & 3 & 4 & 4 & 5 & 5 \\ \mathrm{~J}=8509 & 6 & 7 & 8 & 8 & 9 & 9 \\ \mathrm{~B}=8109 & 7 & 9 & 9 & 9 & 9 & 9 \\ \mathrm{~F}=8508 & 9 & 9 & 9 & 9 & 9 & 9 \\ \mathrm{C}=8108 & 9 & 9 & 9 & 9 & 9 & 9 \\ \mathrm{~A}=1112 & 8 & 9 & 9 & 9 & 9 & 9 \\ \mathrm{H}=1110 & 9 & 9 & 9 & 9 & 9 & 9 \\ \mathrm{D}=8110 & 5 & 7 & 7 & 7 & 9 & 9 \\ \mathrm{G}=8107 & 9 & 9 & 9 & 9 & 9 & 9\end{array}$

(12) $\mathrm{FEV}_{1}$ Decrements $\geq 15 \%$, 1-h Exposures, Heavy Exertion, Fifth Highest of 1 -h Daily Maximum Concentrations (underlying exposure-response relationship based on McDonnell et al. 1983)

$\begin{array}{lllllll}\mathrm{Z}=\mathrm{As}-\mathrm{Is} & 5 & 6 & 7 & 7 & 7 & 7 \\ \mathrm{~J}=8509 & 8 & 9 & 9 & 9 & 9 & 9 \\ \mathrm{~B}=8109 & 9 & 9 & 9 & 9 & 9 & 9 \\ \mathrm{~F}=8508 & 9 & 9 & 9 & 9 & 9 & 9 \\ \mathrm{C}=8108 & 9 & 9 & 9 & 9 & 9 & 9 \\ \mathrm{~A}=1112 & 9 & 9 & 9 & 9 & 9 & 9 \\ \mathrm{H}=1110 & 9 & 9 & 9 & 9 & 9 & 9 \\ \mathrm{D}=8110 & 8 & 9 & 9 & 9 & 9 & 9 \\ \mathrm{G}=8107 & 9 & 9 & 9 & 9 & 9 & 9\end{array}$


TABLE D.1 (Cont.)

\begin{tabular}{|c|c|c|c|c|c|c|}
\hline \multirow[b]{2}{*}{ NAAQS } & \multicolumn{3}{|c|}{$5 \%$ Benchmark } & \multicolumn{3}{|c|}{$10 \%$ Benchmark } \\
\hline & $\leq \overline{0.1}$ & 0.2 & $\overline{0.3}$ & $\leq 0.1$ & 0.2 & $\overline{0.3}$ \\
\hline
\end{tabular}

(13) $\mathrm{FEV}_{1}$ Decrements $\geq 20 \%, 1$-h Exposures, Heavy Exertion, Highest of 1-h Daily Maximum Concentrations (underlying exposure-response relationship based on Avol et al. 1984)

$\begin{array}{lllllll}Z=A s-I s & - & - & - & - & - & - \\ J=8509 & - & - & - & - & - & - \\ B=8109 & - & - & - & 1 & 5 & 5 \\ F=8508 & - & - & - & 1 & 5 & 6 \\ C=8108 & 2 & 2 & 5 & 5 & 6 & 6 \\ A=1112 & - & - & - & - & 1 & 1 \\ H=1110 & - & - & 6 & 6 & 8 & 8 \\ D=8110 & - & - & - & - & - & - \\ G=8107 & 5 & 5 & 8 & 8 & 9 & 9\end{array}$

(14) $\mathrm{FEV}_{1}$ Decrements $\geq 20 \%$, 1-h Exposures, Heavy Exertion, Fifth Highest of 1-h Daily Maximum Concentrations (underlying exposure-response relationship based on Avol et al. 1984)

$\begin{array}{lllllll}Z=\text { As-Is } & 1 & 1 & 1 & 2 & 3 & 4 \\ \mathrm{~J}=8509 & - & - & - & 1 & 7 & 8 \\ \mathrm{~B}=8109 & 1 & 1 & 5 & 6 & 7 & 8 \\ \mathrm{~F}=8508 & 2 & 7 & 8 & 8 & 8 & 8 \\ \mathrm{C}=8108 & 5 & 7 & 7 & 9 & 9 & 9 \\ \mathrm{~A}=1112 & - & - & 2 & 2 & 9 & 9 \\ \mathrm{H}=1110 & 8 & 9 & 9 & 9 & 9 & 9 \\ \mathrm{D}=8110 & - & - & - & - & 4 & 6 \\ \mathrm{G}=8107 & 9 & 9 & 9 & 9 & 9 & 9\end{array}$

(15) $\mathrm{FEV}_{1}$ Decrements $\geq 20 \%$, 1-h Exposures, Heavy Exertion, Highest of 1-h Daily Maximum Concentrations (underlying exposure-response relationship based on Kulle et al. 1985)

$\begin{array}{lllllll}\mathrm{Z}=\text { As-Is } & 6 & 6 & 7 & 7 & 8 & 8 \\ \mathrm{~J}=8509 & 9 & 9 & 9 & 9 & 9 & 9 \\ \mathrm{~B}=8109 & 9 & 9 & 9 & 9 & 9 & 9 \\ \mathrm{~F}=8508 & 9 & 9 & 9 & 9 & 9 & 9 \\ \mathrm{C}=8108 & 9 & 9 & 9 & 9 & 9 & 9 \\ \mathrm{~A}=1112 & 9 & 9 & 9 & 9 & 9 & 9 \\ \mathrm{H}=1110 & 9 & 9 & 9 & 9 & 9 & 9 \\ \mathrm{D}=8110 & 9 & 9 & 9 & 9 & 9 & 9 \\ \mathrm{G}=8107 & 9 & 9 & 9 & 9 & 9 & 9\end{array}$


TABLE D.1 (Cont.)

\begin{tabular}{|c|c|c|c|c|c|c|}
\hline \multirow[b]{2}{*}{ NAAQS } & \multicolumn{3}{|c|}{$5 \%$ Benchmark } & \multicolumn{3}{|c|}{$10 \%$ Benchmark } \\
\hline & $\leq 0.1$ & 0.2 & 0.3 & $\leq 0.1$ & 0.2 & 0.3 \\
\hline
\end{tabular}

(16) $\mathrm{FEV}_{1}$ Decrements $\geq 20 \%$, 1-h Exposures, Heavy Exertion, Fifth Highest of 1-h Daily Maximum Concentrations (underlying exposure-response relationship based on Kulle et al. 1985)

$\begin{array}{lllllll}\mathrm{Z}=\text { As-Is } & 8 & 8 & 8 & 8 & 8 & 8 \\ \mathrm{~J}=8509 & 9 & 9 & 9 & 9 & 9 & 9 \\ \mathrm{~B}=8109 & 9 & 9 & 9 & 9 & 9 & 9 \\ \mathrm{~F}=8508 & 9 & 9 & 9 & 9 & 9 & 9 \\ \mathrm{C}=8108 & 9 & 9 & 9 & 9 & 9 & 9 \\ \mathrm{~A}=1112 & 9 & 9 & 9 & 9 & 9 & 9 \\ \mathrm{H}=1110 & 9 & 9 & 9 & 9 & 9 & 9 \\ \mathrm{D}=8110 & 9 & 9 & 9 & 9 & 9 & 9 \\ \mathrm{G}=8107 & 9 & 9 & 9 & 9 & 9 & 9\end{array}$

(17) FEV 1 Decrements $\geq 20 \%$, 1-h Exposures, Heavy Exertion, Highest of 1-h Daily Maximum Concentrations (underlying exposure-response relationship based on McDonnell et al. 1983)

$\begin{array}{lllllll}\mathrm{Z}=\mathrm{As}-\mathrm{Is} & 4 & 4 & 5 & 5 & 5 & 6 \\ \mathrm{~J}=8509 & 7 & 8 & 9 & 9 & 9 & 9 \\ \mathrm{~B}=8109 & 9 & 9 & 9 & 9 & 9 & 9 \\ \mathrm{~F}=8508 & 9 & 9 & 9 & 9 & 9 & 9 \\ \mathrm{C}=8108 & 9 & 9 & 9 & 9 & 9 & 9 \\ \mathrm{~A}=1112 & 9 & 9 & 9 & 9 & 9 & 9 \\ \mathrm{H}=1110 & 9 & 9 & 9 & 9 & 9 & 9 \\ \mathrm{D}=8110 & 7 & 7 & 9 & 9 & 9 & 9 \\ \mathrm{G}=8107 & 9 & 9 & 9 & 9 & 9 & 9\end{array}$

(18) FEV $_{1}$ Decrements $\geq 20 \%$, 1-h Exposures, Heavy Exertion, Fifth Highest of 1-h Daily Maximum Concentrations (underlying exposure-response relationship based on McDonnell et al. 1983)

$\begin{array}{lllllll}\mathrm{Z}=\text { As-Is } & 7 & 7 & 7 & 7 & 7 & 7 \\ \mathrm{~J}=8509 & 9 & 9 & 9 & 9 & 9 & 9 \\ \mathrm{~B}=8109 & 9 & 9 & 9 & 9 & 9 & 9 \\ \mathrm{~F}=8508 & 9 & 9 & 9 & 9 & 9 & 9 \\ \mathrm{C}=8108 & 9 & 9 & 9 & 9 & 9 & 9 \\ \mathrm{~A}=1112 & 9 & 9 & 9 & 9 & 9 & 9 \\ \mathrm{H}=1110 & 9 & 9 & 9 & 9 & 9 & 9 \\ \mathrm{D}=8110 & 9 & 9 & 9 & 9 & 9 & 9 \\ \mathrm{G}=8107 & 9 & 9 & 9 & 9 & 9 & 9\end{array}$


TABLE D.1 (Cont.)

\begin{tabular}{|c|c|c|c|c|c|c|}
\hline \multirow[b]{2}{*}{ NAAQS } & \multicolumn{3}{|c|}{$5 \%$ Benchmark } & \multicolumn{3}{|c|}{$10 \%$ Benchmark } \\
\hline & $\leq \overline{0.1}$ & 0.2 & $\overline{0.3}$ & $\leq 0.1$ & 0.2 & $\overline{0.3}$ \\
\hline
\end{tabular}

(19) Any Lower Respiratory Symptoms, 1-h Exposures, Heavy Exertion, Highest of 1-h Daily Maximum Concentrations (underlying exposure-response relationship based on Avol et al. 1984)

$$
\begin{aligned}
& Z=\text { As-Is } \\
& J=8509 \\
& B=8109 \\
& F=8508 \\
& C=8108 \\
& A=1112 \\
& H=1110 \\
& D=8110 \\
& G=8107
\end{aligned}
$$

$\begin{array}{lll}- & - & - \\ - & - & - \\ - & - & - \\ - & - & - \\ - & - & - \\ - & - & - \\ - & - & - \\ 4 & 4 & -\end{array}$

$\begin{array}{lll}- & - & - \\ - & - & - \\ - & - & 1 \\ - & - & 1 \\ 2 & 5 & 5 \\ - & - & - \\ - & 6 & 6 \\ - & - & - \\ 5 & 8 & 8\end{array}$

(20) Any Lower Respiratory Symptoms, 1-h Exposures, Heavy Exertion, Fifth Highest of 1-h Daily Maximum Concentrations (underlying exposure-response relationship based on Avol et al. 1984)

$\begin{array}{lllllll}\mathrm{Z}=\text { As-Is } & - & - & 1 & 1 & 1 & 3 \\ \mathrm{~J}=8509 & - & - & - & - & - & 1 \\ \mathrm{~B}=8109 & - & - & - & 1 & 5 & 6 \\ \mathrm{~F}=8508 & - & - & - & 2 & 8 & 8 \\ \mathrm{C}=8108 & 1 & 2 & 4 & 6 & 7 & 9 \\ \mathrm{~A}=1112 & - & - & - & - & 2 & 2 \\ \mathrm{H}=1110 & - & 1 & 3 & 9 & 9 & 9 \\ \mathrm{D}=8110 & - & - & - & - & - & - \\ \mathrm{G}=8107 & 5 & 6 & 7 & 9 & 9 & 9\end{array}$

(21) Any Cough, 1-h Exposures, Heavy Exertion, Highest of 1-h Daily Maximum Concentrations (underlying exposure-response relationship based on Kulle et al. 1985)

$$
\begin{aligned}
& Z=A s-I s \\
& J=8509 \\
& B=8109 \\
& F=8508 \\
& C=8108 \\
& A=1112 \\
& H=1110 \\
& D=8110 \\
& G=8107
\end{aligned}
$$

$\begin{array}{lll}- & - & - \\ - & - & - \\ - & - & - \\ - & - & - \\ - & 2 & 5 \\ - & - & - \\ - & - & 1 \\ - & - & - \\ 5 & 5 & 5\end{array}$

$\begin{array}{lll}- & - & - \\ - & - & - \\ - & 1 & 5 \\ - & 1 & 5 \\ 3 & 5 & 6 \\ - & - & 1 \\ - & 6 & 8 \\ - & - & - \\ 5 & 8 & 9\end{array}$


TABLE D.1 (Cont.)

\begin{tabular}{|c|c|c|c|c|c|c|}
\hline \multirow[b]{2}{*}{ NAAQS } & \multicolumn{3}{|c|}{$5 \%$ Benchmark } & \multicolumn{3}{|c|}{$10 \%$ Benchmark } \\
\hline & $\leq 0.1$ & 0.2 & 0.3 & $\leq 0.1$ & 0.2 & 0.3 \\
\hline
\end{tabular}

(22) Any Cough, 1-h Exposures, Heavy Exertion, Fifth Highest of 1-h Daily Maximum Concentrations (underlying exposure-response relationship based on Kulle et al. 1985)

$\begin{array}{lllllll}\mathrm{Z}=\text { As-Is } & - & 1 & 1 & 1 & 3 & 4 \\ \mathrm{~J}=8509 & - & - & - & - & 1 & 7 \\ \mathrm{~B}=8109 & - & 1 & 3 & 2 & 6 & 7 \\ \mathrm{~F}=8508 & - & 2 & 8 & 7 & 8 & 8 \\ \mathrm{C}=8108 & 4 & 6 & 7 & 7 & 9 & 9 \\ \mathrm{~A}=1112 & - & - & 1 & - & 2 & 9 \\ \mathrm{H}=1110 & 4 & 9 & 9 & 9 & 9 & 9 \\ \mathrm{D}=8110 & - & - & - & - & - & 3 \\ \mathrm{G}=8107 & 7 & 9 & 9 & 9 & 9 & 9\end{array}$

(23) Any Cough, 1-h Exposures, Heavy Exertion, Highest of 1-h Daily Maximum Concentrations (underlying exposure-response relationship based on McDonnell et al. 1983)

$$
\begin{aligned}
& Z=\text { As-Is } \\
& J=8509 \\
& B=8109 \\
& F=8508 \\
& C=8108 \\
& A=1112 \\
& H=1110 \\
& D=8110 \\
& G=8107
\end{aligned}
$$

(24) Any Cough, 1-h Exposures, Heavy Exertion, Fifth Highest of 1-h Daily Maximum Concentrations (underlying exposure-response relationship based on McDonnell et al. 1983)

$$
\begin{aligned}
& Z=\text { As-Is } \\
& J=8509 \\
& B=8109 \\
& F=8508 \\
& C=8108 \\
& A=1112 \\
& H=1110 \\
& D=8110 \\
& G=8107
\end{aligned}
$$


TABLE D.1 (Cont.)

\begin{tabular}{|c|c|c|c|c|c|c|}
\hline \multirow[b]{2}{*}{ NAAQS } & \multicolumn{3}{|c|}{$5 \%$ Benchmark } & \multicolumn{3}{|c|}{$10 \%$ Benchmark } \\
\hline & $\leq 0.1$ & 0.2 & 0.3 & $\leq 0.1$ & 0.2 & 0.3 \\
\hline
\end{tabular}

(25) Moderate-to-Severe Lower Respiratory Symptoms, 1-h Exposures, Heavy Exertion, Highest of 1-h Daily Maximum Concentrations (underlying exposureresponse relationship based on Avol et al. 1984)

$\begin{array}{lllllll}\mathrm{Z}=\text { As-Is } & 7 & 8 & 8 & 8 & 8 & 9 \\ \mathrm{~J}=8509 & 9 & 9 & 9 & 9 & 9 & 9 \\ \mathrm{~B}=8109 & 9 & 9 & 9 & 9 & 9 & 9 \\ \mathrm{~F}=8508 & 9 & 9 & 9 & 9 & 9 & 9 \\ \mathrm{C}=8108 & 9 & 9 & 9 & 9 & 9 & 9 \\ \mathrm{~A}=1112 & 9 & 9 & 9 & 9 & 9 & 9 \\ \mathrm{H}=1110 & 9 & 9 & 9 & 9 & 9 & 9 \\ \mathrm{D}=8110 & 9 & 9 & 9 & 9 & 9 & 9 \\ \mathrm{G}=8107 & 9 & 9 & 9 & 9 & 9 & 9\end{array}$

(26) Moderate-to-Severe Lower Respiratory Symptoms, 1-h Exposures, Heavy Exertion, Fifth Highest of 1-h Daily Maximum Concentrations (underlying exposure-response relationship based on Avol et al. 1984)

$\begin{array}{lllllll}\mathrm{Z}=\mathrm{As}-\mathrm{Is} & 8 & 8 & 8 & 9 & 9 & 9 \\ \mathrm{~J}=8509 & 9 & 9 & 9 & 9 & 9 & 9 \\ \mathrm{~B}=8109 & 9 & 9 & 9 & 9 & 9 & 9 \\ \mathrm{~F}=8508 & 9 & 9 & 9 & 9 & 9 & 9 \\ \mathrm{C}=8108 & 9 & 9 & 9 & 9 & 9 & 9 \\ \mathrm{~A}=1112 & 9 & 9 & 9 & 9 & 9 & 9 \\ \mathrm{H}=1110 & 9 & 9 & 9 & 9 & 9 & 9 \\ \mathrm{D}=8110 & 9 & 9 & 9 & 9 & 9 & 9 \\ \mathrm{G}=8107 & 9 & 9 & 9 & 9 & 9 & 9\end{array}$

(27) Moderate-to-Severe Cough, 1-h Exposures, Heavy Exertion, Highest of 1-h Daily Maximum Concentrations (underlying exposure-response relationship based on Kulle et al. 1985)

$\begin{array}{llll}\mathrm{Z}=\text { As-Is } & 6 & 8 & 8 \\ \mathrm{~J}=8509 & 9 & 9 & 9 \\ \mathrm{~B}=8109 & 9 & 9 & 9 \\ \mathrm{~F}=8508 & 9 & 9 & 9 \\ \mathrm{C}=8108 & 9 & 9 & 9 \\ \mathrm{~A}=1112 & 9 & 9 & 9 \\ \mathrm{H}=1110 & 9 & 9 & 9 \\ \mathrm{D}=8110 & 9 & 9 & 9 \\ \mathrm{G}=8107 & 9 & 9 & 9\end{array}$

$\begin{array}{lll}8 & 8 & 8 \\ 9 & 9 & 9 \\ 9 & 9 & 9 \\ 9 & 9 & 9 \\ 9 & 9 & 9 \\ 9 & 9 & 9 \\ 9 & 9 & 9 \\ 9 & 9 & 9 \\ 9 & 9 & 9\end{array}$


TABLE D.1 (Cont.)

\begin{tabular}{cccccccc}
\hline & \multicolumn{3}{c}{$5 \%$ Benchmark } & & \multicolumn{3}{c}{ 10\% Benchmark } \\
\cline { 3 - 5 } NAAQS & $\leq 0.1$ & 0.2 & 0.3 & & $\leq 0.1$ & 0.2 & 0.3 \\
\hline
\end{tabular}

(28) Moderate-to-Severe Cough, 1-h Exposures, Heavy Exertion, Fifth Highest of 1-h Daily Maximum Concentrations (underlying exposure-response relationship based on Kulle et al. 1985)

$\begin{array}{lllllll}\mathrm{Z}=\text { As-Is } & 8 & 8 & 8 & 8 & 8 & .9 \\ \mathrm{~J}=8509 & 9 & 9 & 9 & 9 & 9 & 9 \\ \mathrm{~B}=8109 & 9 & 9 & 9 & 9 & 9 & 9 \\ \mathrm{~F}=8508 & 9 & 9 & 9 & 9 & 9 & 9 \\ \mathrm{C}=8108 & 9 & 9 & 9 & 9 & 9 & 9 \\ \mathrm{~A}=1112 & 9 & 9 & 9 & 9 & 9 & 9 \\ \mathrm{H}=1110 & 9 & 9 & 9 & 9 & 9 & 9 \\ \mathrm{D}=8110 & 9 & 9 & 9 & 9 & 9 & 9 \\ \mathrm{G}=8107 & 9 & 9 & 9 & 9 & 9 & 9\end{array}$

(29) Moderate-to-Severe Cough, 1-h Exposures, Heavy Exertion, Highest of 1-h Daily Maximum Concentrations (underlying exposure-response relationship based on McDonnell et al. 1983)

$$
\begin{aligned}
& Z=\text { As-Is } \\
& J=8509 \\
& B=8109 \\
& F=8508 \\
& C=8108 \\
& A=1112 \\
& H=1110 \\
& D=8110 \\
& G=8107
\end{aligned}
$$

$\begin{array}{lll}- & - & - \\ - & - & - \\ - & - & - \\ - & - & - \\ - & - & - \\ - & - & - \\ - & - & - \\ - & - & - \\ - & - & -\end{array}$

$\begin{array}{lll}- & - & - \\ - & - & - \\ - & - & 1 \\ - & - & 1 \\ - & - & 5 \\ - & - & - \\ - & - & 6 \\ - & - & - \\ - & 5 & 8\end{array}$

(30) Moderate-to-Severe Cough, 1-h Exposures, Heavy Exertion, Fifth Highest of 1-h Daily Maximum Concentrations (underlying exposure-response relationship based on McDonnell et al. 1983)

$$
\begin{aligned}
& Z=\text { As-Is } \\
& J=8509 \\
& B=8109 \\
& F=8508 \\
& C=8108 \\
& A=1112 \\
& H=1110 \\
& D=8110 \\
& G=8107
\end{aligned}
$$

$\begin{array}{lll}- & - & - \\ - & - & - \\ - & - & - \\ - & - & - \\ - & - & - \\ - & - & - \\ - & - & - \\ - & - & - \\ - & - & 1\end{array}$

$\begin{array}{lll}- & 1 & 1 \\ - & - & - \\ - & - & 5 \\ - & - & 8 \\ - & 4 & 7 \\ - & - & 2 \\ - & 3 & 9 \\ -. & - & - \\ 1 & 7 & 9\end{array}$


TABLE D.1 (Cont.)

\begin{tabular}{|c|c|c|c|c|c|c|}
\hline \multirow[b]{2}{*}{ NAAQS } & \multicolumn{3}{|c|}{$5 \%$ Benchmark } & \multicolumn{3}{|c|}{$10 \%$ Benchmark } \\
\hline & $\leq 0.1$ & 0.2 & 0.3 & $\leq 0.1$ & 0.2 & 0.3 \\
\hline
\end{tabular}

(31) Any PDI, 1-h Exposures, Heavy Exertion, Highest of 1-h Daily Maximum Concentrations (underlying exposure-response relationship based on Kulle et al. 1985)

$\begin{array}{lllllll}\mathrm{Z}=\text { As-Is } & 1 & 2 & 2 & 2 & 3 & 3 \\ \mathrm{~J}=8509 & 1 & 2 & 3 & 2 & 6 & 7 \\ \mathrm{~B}=8109 & 5 & 5 & 5 & 5 & 7 & 8 \\ \mathrm{~F}=8508 & 7 & 7 & 7 & 7 & 9 & 9 \\ \mathrm{C}=8108 & 8 & 8 & 8 & 8 & 9 & 9 \\ \mathrm{~A}=1112 & 3 & 6 & 7 & 6 & 8 & 8 \\ \mathrm{H}=1110 & 8 & 9 & 9 & 9 & 9 & 9 \\ \mathrm{D}=8110 & 1 & 2 & 3 & 2 & 5 & 5 \\ \mathrm{G}=8107 & 9 & 9 & 9 & 9 & 9 & 9\end{array}$

(32) Any PDI, 1-h Exposures, Heavy Exertion, Fifth Highest of 1-h Daily Maximum Concentrations (underlying exposure-response relationship based on Kulle et al. 1985)

$\begin{array}{lllllll}Z=A s-I s & 4 & 4 & 4 & 4 & 5 & 5 \\ \mathrm{~J}=8509 & 8 & 8 & 8 & 8 & 8 & 9 \\ \mathrm{~B}=8109 & 8 & 9 & 9 & 9 & 9 & 9 \\ \mathrm{~F}=8508 & 8 & 9 & 9 & 9 & 9 & 9 \\ \mathrm{C}=8108 & 9 & 9 & 9 & 9 & 9 & 9 \\ \mathrm{~A}=1112 & 9 & 9 & 9 & 9 & 9 & 9 \\ \mathrm{H}=1110 & 9 & 9 & 9 & 9 & 9 & 9 \\ \mathrm{D}=8110 & 6 & 6 & 7 & 7 & 8 & 9 \\ \mathrm{G}=8107 & 9 & 9 & 9 & 9 & 9 & 9\end{array}$

(33) Any PDI, 1-h Exposures, Heavy Exertion, Highest of 1-h Daily Maximum Concentrations (underlying exposure-response relationship based on McDonnell et al. 1983)

$$
\begin{aligned}
& Z=A s-I s \\
& J=8509 \\
& B=8109 \\
& F=8508 \\
& C=8108 \\
& A=1112 \\
& H=1110 \\
& D=8110 \\
& G=8107
\end{aligned}
$$


TABLE D.1 (Cont.)

\begin{tabular}{|c|c|c|c|c|c|c|}
\hline \multirow[b]{2}{*}{ NAAQS } & \multicolumn{3}{|c|}{$5 \%$ Benchmark } & \multicolumn{3}{|c|}{$10 \%$ Benchmark } \\
\hline & $\leq 0.1$ & 0.2 & 0.3 & $\leq 0.1$ & 0.2 & 0.3 \\
\hline
\end{tabular}

(34) Any PDI, 1-h Exposures, Heavy Exertion, Fifth Highest of 1-h Daily Maximum Concentrations (underlying exposure-response relationship based on McDonnell et al. 1983)

$$
\begin{aligned}
& Z=\text { As-Is } \\
& J=8509 \\
& B=8109 \\
& F=8508 \\
& C=8108 \\
& A=1112 \\
& H=1110 \\
& D=8110 \\
& G=8107
\end{aligned}
$$

(35) Moderate-to-Severe PDI, 1-h Exposures, Heavy Exertion, Highest of 1-h Daily Maximum Concentrations (underlying exposure-response relationship based on Kulle et al. 1985)

$\begin{array}{lllllll}\mathrm{Z}=\text { As-Is } & 5 & 6 & 6 & 6 & 6 & 6 \\ \mathrm{~J}=8509 & 9 & 9 & 9 & 9 & 9 & 9 \\ \mathrm{~B}=8109 & 9 & 9 & 9 & 9 & 9 & 9 \\ \mathrm{~F}=8508 & 9 & 9 & 9 & 9 & 9 & 9 \\ \mathrm{C}=8108 & 9 & 9 & 9 & 9 & 9 & 9 \\ \mathrm{~A}=1112 & 9 & 9 & 9 & 9 & 9 & 9 \\ \mathrm{H}=1110 & 9 & 9 & 9 & 9 & 9 & 9 \\ \mathrm{D}=8110 & 9 & 9 & 9 & 9 & 9 & 9 \\ \mathrm{G}=8107 & 9 & 9 & 9 & 9 & 9 & 9\end{array}$

(36) Moderate-to-Severe PDI, 1-h Exposures, Heavy Exertion, Fifth Highest of 1-h Daily Maximum Concentrations (underlying exposure-response relationship based on Kulle et al. 1985)

$\begin{array}{lllllll}\mathrm{Z}=\mathrm{As}-\mathrm{Is} & 7 & 8 & 8 & 8 & 8 & 8 \\ \mathrm{~J}=8509 & 9 & 9 & 9 & 9 & 9 & 9 \\ \mathrm{~B}=8109 & 9 & 9 & 9 & 9 & 9 & 9 \\ \mathrm{~F}=8508 & 9 & 9 & 9 & 9 & 9 & 9 \\ \mathrm{C}=8108 & 9 & 9 & 9 & 9 & 9 & 9 \\ \mathrm{~A}=1112 & 9 & 9 & 9 & 9 & 9 & 9 \\ \mathrm{H}=1110 & 9 & 9 & 9 & 9 & 9 & 9 \\ \mathrm{D}=8110 & 9 & 9 & 9 & 9 & 9 & 9 \\ \mathrm{G}=8107 & 9 & 9 & 9 & 9 & 9 & 9\end{array}$


TABLE D.1 (Cont.)

\begin{tabular}{|c|c|c|c|c|c|c|}
\hline \multirow[b]{2}{*}{ NAAQS } & \multicolumn{3}{|c|}{$5 \%$ Benchmark } & \multicolumn{3}{|c|}{$10 \%$ Benchmark } \\
\hline & $\leq 0.1$ & 0.2 & 0.3 & $\leq 0.1$ & 0.2 & 0.3 \\
\hline
\end{tabular}

(37) Moderate-to-Severe PDI, 1-h Exposures, Heavy Exertion, Highest of 1-h Daily Maximum Concentrations (underlying exposure-response relationship based on McDonnell et al. 1983)

$$
\begin{aligned}
& Z=\text { As-Is } \\
& J=8509 \\
& B=8109 \\
& F=8508 \\
& C=8108 \\
& A=1112 \\
& H=1110 \\
& D=8110 \\
& G=8107
\end{aligned}
$$

$\begin{array}{llllll}- & - & - & - & - & - \\ - & - & - & - & - & - \\ - & - & - & - & - & 4 \\ - & - & - & - & - & 5 \\ - & - & - & - & 2 & 6 \\ - & - & - & - & - & - \\ - & - & - & - & - & 8 \\ - & - & - & - & - & - \\ - & - & - & - & 5 & 9\end{array}$

(38) Moderate-to-Severe PDI, 1-h Exposures, Heavy Exertion, Fifth Highest of 1-h Daily Maximum Concentrations (underlying exposure-response relationship based on McDonnell et al. 1983)

$\begin{array}{lllllll}Z=A s-I s & - & - & - & - & 1 & 3 \\ J=8509 & - & - & - & - & - & 6 \\ B=8109 & - & - & - & - & 1 & 7 \\ F=8508 & - & - & - & - & 5 & 8 \\ C=8108 & - & - & - & - & 7 & 9 \\ A=1112 & - & - & - & - & - & 9 \\ H=1110 & - & - & - & - & 9 & 9 \\ D=8110 & - & - & - & - & - & 3 \\ G=8107 & - & - & 5 & 5 & 9 & 9\end{array}$

(39) FEV 1 Decrements $\geq 10 \%$, 1-h Exposures, Moderate Exertion, Highest of 1-h Daily Maximum Concentrations (underlying exposure-response relationship based on Seal et al. 1993)

$$
\begin{aligned}
& Z=A s-I s \\
& J=8509 \\
& B=8109 \\
& F=8508 \\
& C=8108 \\
& A=1112 \\
& H=1110 \\
& D=8110 \\
& G=8107
\end{aligned}
$$

$\begin{array}{lll}- & - & - \\ - & - & - \\ - & - & 1 \\ - & - & 2 \\ 2 & 5 & 5 \\ - & - & - \\ - & 6 & 7 \\ - & - & - \\ 5 & 8 & 8\end{array}$


TABLE D.1 (Cont.)

\begin{tabular}{cccccccc} 
& \multicolumn{3}{c}{$5 \%$ Benchmark } & & \multicolumn{3}{c}{ 10\% Benchmark } \\
\cline { 3 - 6 } NAAQS & $\leq 0.1$ & 0.2 & 0.3 & & $\leq 0.1$ & 0.2 & 0.3 \\
\hline
\end{tabular}

(40) FEV 1 Decrements $\geq 10 \%$, 1-h Exposures, Moderate Exertion, Fifth Highest of 1-h Daily Maximum Concentrations (underlying exposure-response relationship based on Seal et al. 1993)

$\begin{array}{lllllll}Z=\text { As-Is } & - & - & - & 1 & 1 & 3 \\ J=8509 & - & - & - & - & - & 1 \\ B=8109 & - & - & - & 1 & 5 & 6 \\ F=8508 & - & - & - & 2 & 8 & 8 \\ C=8108 & - & - & 1 & 6 & 7 & 9 \\ A=1112 & - & - & - & - & 2 & 3 \\ H=1110 & - & - & 1 & 8 & 9 & 9 \\ D=8110 & - & - & - & - & - & 1 \\ G=8107 & - & 4 & 6 & 9 & 9 & 9\end{array}$

(41) FEV 1 Decrements $\geq 15 \%$, 1-h Exposures, Moderate Exertion, Highest of 1-h Daily Maximum Concentrations (underlying exposure-response relationship based on Seal et al. 1993)

$\begin{array}{lllllll}Z=A s-I s & - & - & - & 2 & 3 & 3 \\ J=8509 & - & - & - & 1 & 6 & 7 \\ B=8109 & - & - & 1 & 5 & 7 & 8 \\ F=8508 & - & - & 1 & 7 & 9 & 9 \\ C=8108 & - & 3 & 5 & 8 & 9 & 9 \\ A=1112 & - & - & - & 3 & 8 & 8 \\ H=1110 & - & - & 6 & 9 & 9 & 9 \\ D=8110 & - & - & - & 1 & 5 & 5 \\ G=8107 & 4 & 5 & 8 & 9 & 9 & 9\end{array}$

(42) $\mathrm{FEV}_{1}$ Decrements $\geq 15 \%$, 1-h Exposures, Moderate Exertion, Fifth Highest of 1-h Daily Maximum Concentrations (underlying exposure-response relationship based on Seal et al. 1993)

$\begin{array}{lllllll}\mathrm{Z}=\mathrm{As} \text {-Is } & - & 1 & 3 & 4 & 4 & 5 \\ \mathrm{~J}=8509 & - & - & 1 & 8 & 8 & 9 \\ \mathrm{~B}=8109 & - & 2 & 6 & 8 & 9 & 9 \\ \mathrm{~F}=8508 & - & 7 & 8 & 9 & 9 & 9 \\ \mathrm{C}=8108 & 2 & 7 & 9 & 9 & 9 & 9 \\ \mathrm{~A}=1112 & - & - & 2 & 9 & 9 & 9 \\ \mathrm{H}=1110 & 2 & 9 & 9 & 9 & 9 & 9 \\ \mathrm{D}=8110 & - & - & - & 6 & 8 & 9 \\ \mathrm{G}=8107 & 7 & 9 & 9 & 9 & 9 & 9\end{array}$


TABLE D.1 (Cont.)

\begin{tabular}{cccccccc}
\hline & \multicolumn{4}{c}{ 5\% Benchmark } & & \multicolumn{3}{c}{ 10\% Benchmark } \\
\cline { 3 - 7 } NAAQS & $\leq 0.1$ & 0.2 & 0.3 & & $\leq 0.1$ & 0.2 & 0.3 \\
\hline
\end{tabular}

(43) $\mathrm{FEV}_{1}$ Decrements $\geq 20 \%, 1-\mathrm{h}$ Exposures, Moderate Exertion, Highest of 1-h Daily Maximum Concentrations (underlying exposure-response relationship based on Seal et al. 1993)

$\begin{array}{lllllll}\mathrm{Z}=\text { As-Is } & - & - & 2 & 3 & 4 & 5 \\ \mathrm{~J}=8509 & - & - & 3 & 7 & 9 & 9 \\ \mathrm{~B}=8109 & 1 & 5 & 5 & 9 & 9 & 9 \\ \mathrm{~F}=8508 & 1 & 6 & 7 & 9 & 9 & 9 \\ \mathrm{C}=8108 & 5 & 6 & 8 & 9 & 9 & 9 \\ \mathrm{~A}=1112 & - & 1 & 7 & 9 & 9 & 9 \\ \mathrm{H}=1110 & 6 & 8 & 9 & 9 & 9 & 9 \\ \mathrm{D}=8110 & - & - & 3 & 7 & 8 & 9 \\ \mathrm{G}=8107 & 8 & 9 & 9 & 9 & 9 & 9\end{array}$

(44) FEV 1 Decrements $\geq 20 \%$, 1-h Exposures, Moderate Exertion, Fifth Highest of 1-h Daily Maximum Concentrations (underlying exposure-response relationship based on Seal et al. 1993)

$\begin{array}{lllllll}\mathrm{Z}=\text { As-Is } & 2 & 4 & 4 & 6 & 7 & 7 \\ \mathrm{~J}=8509 & 1 & 8 & 8 & 9 & 9 & 9 \\ \mathrm{~B}=8109 & 6 & 7 & 9 & 9 & 9 & 9 \\ \mathrm{~F}=8508 & 8 & 8 & 9 & 9 & 9 & 9 \\ \mathrm{C}=8108 & 8 & 9 & 9 & 9 & 9 & 9 \\ \mathrm{~A}=1112 & 2 & 9 & 9 & 9 & 9 & 9 \\ \mathrm{H}=1110 & 9 & 9 & 9 & 9 & 9 & 9 \\ \mathrm{D}=8110 & - & 6 & 7 & 9 & 9 & 9 \\ \mathrm{G}=8107 & 9 & 9 & 9 & 9 & 9 & 9\end{array}$

(45) Any Cough, 1-h Exposures, Moderate Exertion, Highest of 1-h Daily Maximum Concentrations (underlying exposure-response relationship based on Seal et al. 1993)

$$
\begin{aligned}
& Z=A s-I s \\
& J=8509 \\
& B=8109 \\
& F=8508 \\
& C=8108 \\
& A=1112 \\
& H=1110 \\
& D=8110 \\
& G=8107
\end{aligned}
$$




\section{TABLE D.1 (Cont.)}

\begin{tabular}{cccccccc}
\hline & \multicolumn{4}{c}{ 5\% Benchmark } & & \multicolumn{4}{c}{ 10\% Benchmark } \\
\cline { 3 - 7 } NAAQS & $\leq 0.1$ & 0.2 & 0.3 & & $\leq 0.1$ & 0.2 & 0.3 \\
\hline
\end{tabular}

(46) Any Cough, 1-h Exposures, Moderate Exertion, Fifth Highest of 1-h Daily Maximum Concentrations (underlying exposure-response relationship based on Seal et al. 1993)

$\begin{array}{lllllll}Z=A s-I s & - & - & - & - & 1 & 1 \\ J=8509 & - & - & - & - & - & - \\ B=8109 & - & - & - & - & 1 & 3 \\ F=8508 & - & - & - & - & 2 & 7 \\ C=8108 & - & - & - & 2 & 6 & 7 \\ A=1112 & - & - & - & - & - & 1 \\ H=1110 & - & - & - & 2 & 9 & 9 \\ D=8110 & - & - & - & - & - & - \\ G=8107 & - & 2 & 5 & 7 & 9 & 9\end{array}$

(47) Any PDI, 1-h Exposures, Moderate Exertion, Highest of 1-h Daily Maximum Concentrations (underlying exposure-response relationship based on Seal et al. 1993)

$$
\begin{aligned}
& Z=A s-I s \\
& J=8509 \\
& B=8109 \\
& F=8508 \\
& C=8108 \\
& A=1112 \\
& H=1110 \\
& D=8110 \\
& G=8107
\end{aligned}
$$

$\begin{array}{llllll}- & - & - & - & - & - \\ - & - & - & - & - & - \\ - & - & - & - & - & - \\ - & - & - & - & - & - \\ - & - & - & - & - & - \\ - & - & - & - & - & - \\ - & - & - & - & - & - \\ - & - & - & - & - & - \\ - & - & - & - & - & 2\end{array}$

(48) Any PDI, 1-h Exposures, Moderate Exertion, Fifth Highest of 1-h Daily Maximum Concentrations (underlying exposure-response relationship based on Seal et al. 1993)

$$
\begin{aligned}
& Z=\text { As-Is } \\
& J=8509 \\
& B=8109 \\
& F=8508 \\
& C=8108 \\
& A=1112 \\
& H=1110 \\
& D=8110 \\
& G=8107
\end{aligned}
$$

$\begin{array}{lll}- & - & - \\ - & - & - \\ - & - & - \\ - & - & - \\ - & - & - \\ - & - & - \\ - & - & - \\ - & - & - \\ - & - & -\end{array}$

$\begin{array}{lll}- & - & - \\ - & - & - \\ - & - & - \\ - & - & - \\ - & - & - \\ - & - & - \\ - & - & 1 \\ - & - & - \\ - & 4 & 5\end{array}$


TABLE D.1 (Cont.)

\begin{tabular}{|c|c|c|c|c|c|c|}
\hline \multirow[b]{2}{*}{ NAAQS } & \multicolumn{3}{|c|}{$5 \%$ Benchmark } & \multicolumn{3}{|c|}{$10 \%$ Benchmark } \\
\hline & $\leq \overline{0.1}$ & 0.2 & 0.3 & $\leq 0.1$ & 0.2 & 0.3 \\
\hline
\end{tabular}

(49) Moderate-to-Severe Cough, 1-h Exposures, Moderate Exertion, Highest of 1-h Daily Maximum Concentrations (underlying exposure-response relationship based on Seal et al. 1993)

$\begin{array}{lllllll}\mathrm{Z}=\text { As-Is } & 1 & 2 & 3 & 3 & 4 & 5 \\ \mathrm{~J}=8509 & 1 & 3 & 6 & 7 & 8 & 9 \\ \mathrm{~B}=8109 & 5 & 5 & 7 & 9 & 9 & 9 \\ \mathrm{~F}=8508 & 7 & 7 & 9 & 9 & 9 & 9 \\ \mathrm{C}=8108 & 8 & 8 & 9 & 9 & 9 & 9 \\ \mathrm{~A}=1112 & 3 & 7 & 8 & 9 & 9 & 9 \\ \mathrm{H}=1110 & 8 & 9 & 9 & 9 & 9 & 9 \\ \mathrm{D}=8110 & 1 & 3 & 5 & 7 & 7 & 9 \\ \mathrm{G}=8107 & 9 & 9 & 9 & 9 & 9 & 9\end{array}$

(50) Moderate-to-Severe Cough, 1-h Exposures, Moderate Exertion, Fifth Highest of 1-h Daily Maximum Concentrations (underlying exposure-response relationship based on Seal et al. 1993)

$\begin{array}{lllllll}\mathrm{Z}=\text { As-Is } & 4 & 4 & 5 & 6 & 7 & 7 \\ \mathrm{~J}=8509 & 8 & 8 & 8 & 9 & 9 & 9 \\ \mathrm{~B}=8109 & 8 & 9 & 9 & 9 & 9 & 9 \\ \mathrm{~F}=8508 & 8 & 9 & 9 & 9 & 9 & 9 \\ \mathrm{C}=8108 & 9 & 9 & 9 & 9 & 9 & 9 \\ \mathrm{~A}=1112 & 9 & 9 & 9 & 9 & 9 & 9 \\ \mathrm{H}=1110 & 9 & 9 & 9 & 9 & 9 & 9 \\ \mathrm{D}=8110 & 6 & 7 & 8 & 9 & 9 & 9 \\ \mathrm{G}=8107 & 9 & 9 & 9 & 9 & 9 & 9\end{array}$

(51) Moderate-to-Severe PDI, 1-h Exposures, Moderate Exertion, Highest of 1-h Daily Maximum Concentrations (underlying exposure-response relationship based on Seal et al. 1993)

$\begin{array}{lllllll}\mathrm{Z}=\text { As-Is } & - & - & 2 & 3 & 4 & 5 \\ \mathrm{~J}=8509 & - & - & 3 & 7 & 8 & 9 \\ \mathrm{~B}=8109 & 1 & 5 & 5 & 9 & 9 & 9 \\ \mathrm{~F}=8508 & 1 & 5 & 7 & 9 & 9 & 9 \\ \mathrm{C}=8108 & 5 & 6 & 8 & 9 & 9 & 9 \\ \mathrm{~A}=1112 & - & 1 & 6 & 9 & 9 & 9 \\ \mathrm{H}=1110 & 6 & 8 & 9 & 9 & 9 & 9 \\ \mathrm{D}=8110 & - & - & 2 & 7 & 7 & 9 \\ \mathrm{G}=8107 & 8 & 9 & 9 & 9 & 9 & 9\end{array}$




\section{TABLE D.1 (Cont.)}

\begin{tabular}{|c|c|c|c|c|c|c|}
\hline \multirow[b]{2}{*}{ NAAQS } & \multicolumn{3}{|c|}{$5 \%$ Benchmark } & \multicolumn{3}{|c|}{$10 \%$ Benchmark } \\
\hline & $\leq 0.1$ & 0.2 & 0.3 & $\leq 0.1$ & 0.2 & 0.3 \\
\hline
\end{tabular}

(52) Moderate-to-Severe PDI, 1-h Exposures, Moderate Exertion, Fifth Highest of 1-h Daily Maximum Concentrations (underlying exposure-response relationship based on Seal et al. 1993)

$\begin{array}{lllllll}\mathrm{Z}=\mathrm{As}-\mathrm{Is} & 1 & 4 & 4 & 5 & 7 & 7 \\ \mathrm{~J}=8509 & - & 8 & 8 & 9 & 9 & 9 \\ \mathrm{~B}=8109 & 5 & 7 & 9 & 9 & 9 & 9 \\ \mathrm{~F}=8508 & 8 & 8 & 9 & 9 & 9 & 9 \\ \mathrm{C}=8108 & 7 & 9 & 9 & 9 & 9 & 9 \\ \mathrm{~A}=1112 & 2 & 9 & 9 & 9 & 9 & 9 \\ \mathrm{H}=1110 & 9 & 9 & 9 & 9 & 9 & 9 \\ \mathrm{D}=8110 & - & 5 & 7 & 9 & 9 & 9 \\ \mathrm{G}=8107 & 9 & 9 & 9 & 9 & 9 & 9\end{array}$

(53) $\mathrm{FEV}_{1}$ Decrements $\geq 10 \%$, 8-h Exposures, Moderate Exertion, Highest of 8-h Daily Maximum Concentrations (underlying exposure-response relationship based on Folinsbee et al. 1988, Horstman et al. 1990, and McDonnell et al. 1991)

$$
\begin{aligned}
& Z=\text { As-Is } \\
& J=8509 \\
& B=8109 \\
& F=8508 \\
& C=8108 \\
& A=1112 \\
& H=1110 \\
& D=8110 \\
& G=8107
\end{aligned}
$$

(54) FEV $_{1}$ Decrements $\geq 10 \%$, 8-h Exposures, Moderate Exertion, Fifth Highest of 8-h Daily Maximum Concentrations (underlying exposure-response relationship based on Folinsbee et al. 1988, Horstman et al. 1990, and McDonnell et al. 1991)

$$
\begin{aligned}
& Z=A s-I s \\
& J=8509 \\
& B=8109 \\
& F=8508 \\
& C=8108 \\
& A=1112 \\
& H=1110 \\
& D=8110 \\
& G=8107
\end{aligned}
$$


TABLE D.1 (Cont.)

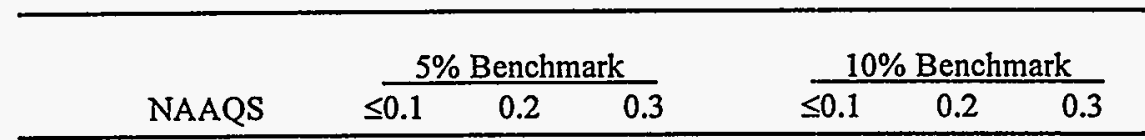

(55) FEV 1 Decrements $\geq 15 \%$, 8-h Exposures, Moderate Exertion, Highest of 8-h Daily Maximum Concentrations (underlying exposure-response relationship based on Folinsbee et al. 1988, Horstman et al. 1990, and McDonnell et al. 1991)

$$
\begin{aligned}
& Z=\text { As-Is } \\
& J=8509 \\
& B=8109 \\
& F=8508 \\
& C=8108 \\
& A=1112 \\
& H=1110 \\
& D=8110 \\
& G=8107
\end{aligned}
$$

$\begin{array}{lll}- & - & - \\ - & - & - \\ - & - & - \\ - & - & - \\ - & - & - \\ - & - & - \\ - & - & - \\ - & - & - \\ - & - & -\end{array}$

(56) $\mathrm{FEV}_{1}$ Decrements $\geq 15 \%$, 8-h Exposures, Moderate Exertion, Fifth Highest of 8-h Daily Maximum Concentrations (underlying exposure-response relationship based on Folinsbee et al. 1988, Horstman et al. 1990, and McDonnell et al. 1991)

$$
\begin{aligned}
& Z=A s-I s \\
& J=8509 \\
& B=8109 \\
& F=8508 \\
& C=8108 \\
& A=1112 \\
& H=1110 \\
& D=8110 \\
& G=8107
\end{aligned}
$$

(57) $\mathrm{FEV}_{1}$ Decrements $\geq 20 \%, 8$-h Exposures, Moderate Exertion, Highest of 8 -h Daily Maximum Concentrations (underlying exposure-response relationship based on Folinsbee et al. 1988, Horstman et al. 1990, and McDonnell et al. 1991)

$$
\begin{aligned}
& Z=A s-I s \\
& J=8509 \\
& B=8109 \\
& F=8508 \\
& C=8108 \\
& A=1112 \\
& H=1110 \\
& D=8110 \\
& G=8107
\end{aligned}
$$


TABLE D.1 (Cont.)

\begin{tabular}{|c|c|c|c|c|c|c|}
\hline \multirow[b]{2}{*}{ NAAQS } & \multicolumn{3}{|c|}{$5 \%$ Benchmark } & \multicolumn{3}{|c|}{$10 \%$ Benchmark } \\
\hline & $\leq 0.1$ & 0.2 & 0.3 & $\leq 0.1$ & 0.2 & $\overline{0.3}$ \\
\hline
\end{tabular}

(58) FEV $_{1}$ Decrements $\geq 20 \%$, 8-h Exposures, Moderate Exertion, Fifth Highest of 8-h Daily Maximum Concentrations (underlying exposure-response relationship based on Folinsbee et al. 1988, Horstman et al. 1990, and McDonnell et al. 1991)

$$
\begin{aligned}
& Z=A s-I s \\
& J=8509 \\
& B=8109 \\
& F=8508 \\
& C=8108 \\
& A=1112 \\
& H=1110 \\
& D=8110 \\
& G=8107
\end{aligned}
$$

$\begin{array}{lll}- & - & - \\ - & - & - \\ - & - & - \\ - & - & - \\ - & - & - \\ - & - & - \\ - & - & - \\ - & - & - \\ - & 1 & 1\end{array}$

$\begin{array}{lll}- & 2 & 2 \\ - & - & - \\ - & - & 1 \\ - & - & - \\ - & 1 & 4 \\ - & - & 1 \\ 1 & 4 & 5 \\ - & - & - \\ 2 & 9 & 9\end{array}$

(59) Any Cough, 8-h Exposures, Moderate Exertion, Highest of 8-h Daily Maximum Concentrations (underlying exposure-response relationship based on Folinsbee et al. 1988, Horstman et al. 1990, and McDonnell et al. 1991)

$$
\begin{aligned}
& Z=A s-I s \\
& J=8509 \\
& B=8109 \\
& F=8508 \\
& C=8108 \\
& A=1112 \\
& H=1110 \\
& D=8110 \\
& G=8107
\end{aligned}
$$

(60) Any Cough, 8-h Exposures, Moderate Exertion, Fifth Highest of 8-h Daily Maximum Concentrations (underlying exposure-response relationship based on Folinsbee et al. 1988, Horstman et al. 1990, and McDonnell et al. 1991)

$$
\begin{aligned}
& Z=\text { As-Is } \\
& J=8509 \\
& B=8109 \\
& F=8508 \\
& C=8108 \\
& A=1112 \\
& H=1110 \\
& D=8110 \\
& G=8107
\end{aligned}
$$

$\begin{array}{lll}- & - & - \\ - & - & - \\ - & - & - \\ - & - & - \\ - & - & - \\ - & - & - \\ - & - & - \\ - & - & - \\ - & - & -\end{array}$

$\begin{array}{lll}- & - & - \\ - & - & - \\ - & - & - \\ - & - & - \\ - & - & - \\ - & - & - \\ - & - & - \\ - & - & - \\ - & - & -\end{array}$


TABLE D.1 (Cont.)

\begin{tabular}{|c|c|c|c|c|c|c|}
\hline \multirow[b]{2}{*}{ NAAQS } & \multicolumn{3}{|c|}{$5 \%$ Benchmark } & \multicolumn{3}{|c|}{$10 \%$ Benchmark } \\
\hline & $\leq 0.1$ & 0.2 & 0.3 & $\leq 0.1$ & 0.2 & 0.3 \\
\hline
\end{tabular}

(61) Any PDI, 8-h Exposures, Moderate Exertion, Highest of 8-h Daily Maximum Concentrations (underlying exposure-response relationship based on Folinsbee et al. 1988, Horstman et al. 1990, and McDonnell et al. 1991)

$\begin{array}{lllllll}\mathrm{Z}=\mathrm{As}-\mathrm{Is} & - & - & - & - & - & - \\ \mathrm{J}=8509 & - & - & - & - & - & - \\ \mathrm{B}=8109 & - & - & - & - & - & - \\ \mathrm{F}=8508 & - & - & - & - & - & - \\ \mathrm{C}=8108 & - & - & - & - & - & - \\ \mathrm{A}=1112 & - & - & - & - & - & - \\ \mathrm{H}=1110 & - & 2 & 2 & 2 & 2 & 2 \\ \mathrm{D}=8110 & - & - & - & - & - & - \\ \mathrm{G}=8107 & - & 6 & 6 & 6 & 6 & 6\end{array}$

(62) Any PDI, 8-h Exposures, Moderate Exertion, Fifth Highest of 8-h Daily Maximum Concentrations (underlying exposure-response relationship based on Folinsbee et al. 1988, Horstman et al. 1990, and McDonnell et al. 1991)

$\begin{array}{lllllll}Z=\text { As-Is } & - & 2 & 2 & 1 & 2 & 2 \\ J=8509 & - & - & - & - & - & - \\ \text { B }=8109 & - & - & 1 & - & 1 & 1 \\ F=8508 & - & - & - & - & - & - \\ C=8108 & - & 1 & 2 & - & 3 & 4 \\ A=1112 & - & - & 1 & - & 1 & 1 \\ H=1110 & 3 & 4 & 4 & 4 & 5 & 5 \\ D=8110 & - & - & - & - & - & - \\ G=8107 & 3 & 9 & 9 & 9 & 9 & 9\end{array}$

(63) Moderate-to-Severe Cough, 8-h Exposures, Moderate Exertion, Highest of 8-h Daily Maximum Concentrations (underlying exposure-response relationship based on Folinsbee et al. 1988, Horstman et al. 1990, and McDonnell et al. 1991)

$\begin{array}{lllllll}\mathrm{Z}=\text { As-Is } & 2 & 2 & 2 & 2 & 2 & 3 \\ \mathrm{~J}=8509 & - & - & - & - & 5 & 8 \\ \mathrm{~B}=8109 & - & - & - & 5 & 8 & 9 \\ \mathrm{~F}=8508 & - & - & - & 6 & 9 & 9 \\ \mathrm{C}=8108 & 5 & 5 & 9 & 9 & 9 & 9 \\ \mathrm{~A}=1112 & 1 & 1 & 2 & 3 & 7 & 9 \\ \mathrm{H}=1110 & 5 & 5 & 7 & 9 & 9 & 9 \\ \mathrm{D}=8110 & - & - & - & - & 3 & 5 \\ \mathrm{G}=8107 & 9 & 9 & 9 & 9 & 9 & 9\end{array}$


TABLE D.1 (Cont.)

\begin{tabular}{|c|c|c|c|c|c|c|}
\hline \multirow[b]{2}{*}{ NAAQS } & \multicolumn{3}{|c|}{$5 \%$ Benchmark } & \multicolumn{3}{|c|}{$10 \%$ Benchmark } \\
\hline & $\leq \overline{0.1}$ & 0.2 & $\overline{0.3}$ & $\leq 0.1$ & 0.2 & 0.3 \\
\hline
\end{tabular}

(64) Moderate-to-Severe Cough, 8-h Exposures, Moderate Exertion, Fifth Highest of 8-h Daily Maximum Concentrations (underlying exposure-response relationship based on Folinsbee et al. 1988, Horstman et al. 1990, and McDonnell et al. 1991)

$\begin{array}{lllllll}\mathrm{Z}=\text { As-Is } & 2 & 2 & 2 & 4 & 4 & 4 \\ \mathrm{~J}=8509 & - & - & 1 & 9 & 9 & 9 \\ \mathrm{~B}=8109 & 1 & 4 & 7 & 9 & 9 & 9 \\ \mathrm{~F}=8508 & 5 & 9 & 9 & 9 & 9 & 9 \\ \mathrm{C}=8108 & 9 & 9 & 9 & 9 & 9 & 9 \\ \mathrm{~A}=1112 & 4 & 5 & 5 & 8 & 9 & 9 \\ \mathrm{H}=1110 & 8 & 9 & 9 & 9 & 9 & 9 \\ \mathrm{D}=8110 & - & 1 & 1 & 3 & 9 & 9 \\ \mathrm{G}=8107 & 9 & 9 & 9 & 9 & 9 & 9\end{array}$

(65) Moderate-to-Severe PDI, 8-h Exposures, Moderate Exertion, Highest of 8-h Daily Maximum Concentrations (underlying exposure-response relationship based on Folinsbee et al. 1988, Horstman et al. 1990, and McDonnell et al. 1991)

$\begin{array}{lllllll}\mathrm{Z}=\text { As-Is } & 2 & 2 & 2 & 2 & 2 & 2 \\ \mathrm{~J}=8509 & - & - & - & - & - & 5 \\ \mathrm{~B}=8109 & - & - & 1 & 5 & 5 & 8 \\ \mathrm{~F}=8508 & - & - & 5 & 6 & 9 & 9 \\ \mathrm{C}=8108 & 5 & 5 & 9 & 9 & 9 & 9 \\ \mathrm{~A}=1112 & 1 & 1 & 2 & 3 & 4 & 7 \\ \mathrm{H}=1110 & 5 & 5 & 9 & 9 & 9 & 9 \\ \mathrm{D}=8110 & - & - & - & - & - & 4 \\ \mathrm{G}=8107 & 9 & 9 & 9 & 9 & 9 & 9\end{array}$

(66) Moderate-to-Severe PDI, 8-h Exposures, Moderate Exertion, Fifth Highest of 8-h Daily Maximum Concentrations (underlying exposure-response relationship based on Folinsbee et al. 1988, Horstman et al. 1990, and McDonnell et al. 1991)

$\begin{array}{lllllll}\mathrm{Z}=\text { As-Is } & 2 & 2 & 2 & 3 & 4 & 4 \\ \mathrm{~J}=8509 & - & - & 2 & 7 & 9 & 9 \\ \mathrm{~B}=8109 & 4 & 5 & 8 & 9 & 9 & 9 \\ \mathrm{~F}=8508 & 9 & 9 & 9 & 9 & 9 & 9 \\ \mathrm{C}=8108 & 9 & 9 & 9 & 9 & 9 & 9 \\ \mathrm{~A}=1112 & 4 & 5 & 6 & 7 & 9 & 9 \\ \mathrm{H}=1110 & 9 & 9 & 9 & 9 & 9 & 9 \\ \mathrm{D}=8110 & - & 1 & 2 & 3 & 5 & 9 \\ \mathrm{G}=8107 & 9 & 9 & 9 & 9 & 9 & 9\end{array}$

a These three benchmark risk values $(0.1,0.2$, and 0.3$)$ represent three different "degrees of risk" of interest to OAQPS staff in the past.

b The hyphen is used rather than a 0 to make the table easier to read. 


\section{D.4 APPENDIX D REFERENCES}

Avol, E.L., et al., 1984, "Comparative Respiratory Effects of Ozone and Ambient Oxidant Pollution Exposure during Heavy Exercise," Journal of the Air Pollution Control Association 34:804-809.

Folinsbee, L.J., et al., 1988, "Pulmonary Function and Symptom Responses after 6.6-Hour Exposure to $0.12 \mathrm{ppm}$ Ozone with Moderate Exercise," Journal of the Air Pollution Control Association 38:28-35.

Horstman, D.H., et al., 1990, "Ozone Concentration and Pulmonary Response Relationships for 6.6-Hour Exposures with Five Hours of Moderate Exercise to 0.08, 0.10, and 0.12 ppm," American Review of Respiratory Disease 142:1158-1163.

Kulle, T.J., et al., 1985, “Ozone Response Relationships in Healthy Nonsmokers," American Review of Respiratory Disease 132:36-41.

McDonnell, W.F., et al., 1983, "Pulmonary Effects of Ozone Exposure during Exercise: DoseResponse Characteristics," Journal of Applied Physiology: Respiratory Environmental Exercise Physiology 54:1345-1352.

McDonnell, W.F., et al., 1991, "Respiratory Response of Humans Exposed to Low Levels of Ozone for 6.6 Hours," Archives of Environmental Health 46:145-150.

Seal, E., Jr., et al., 1993, "The Pulmonary Response of White and Black Adults to Six Concentrations of Ozone," American Review of Respiratory Disease 147:804-810. 


\section{DISTRIBUTION FOR ANL/DIS-3}

Internal

ANL Technical Publications Services

L. Welko (5)

R. Whitfield (188)

M. Clemmons

\section{External}

U.S. Department of Energy Office of Scientific and Technical Information (2)

Manager, U.S. Department of Energy Chicago Field Office

ANL-E Libraries

ANL-W Library 